UC-NRLF

+5 C $76 \quad 849$ 


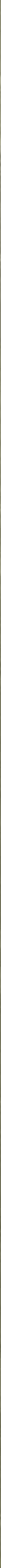




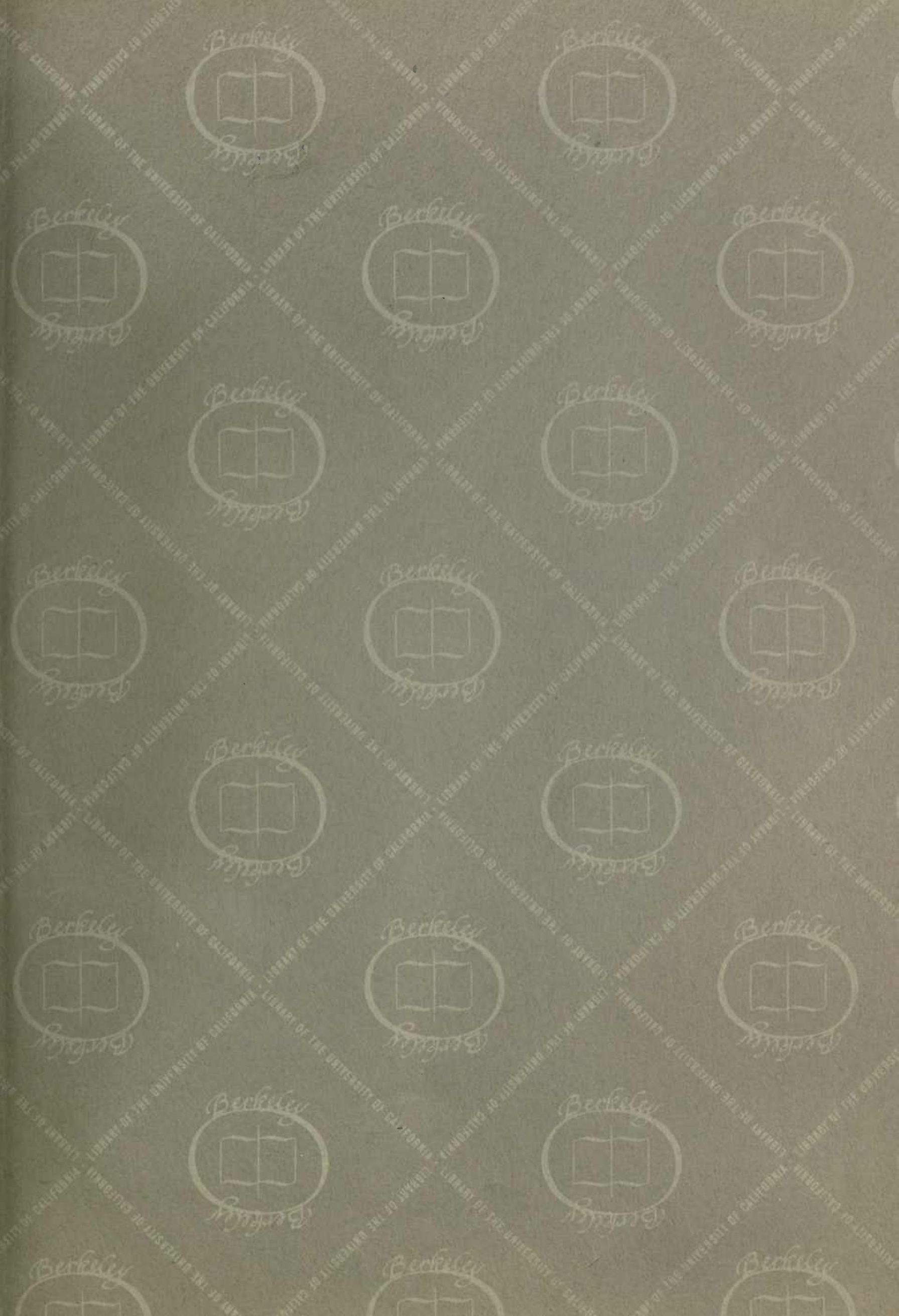





\title{
REVIEW OF THE PROGRESS
}

\section{MATHEMATICAL AND PHYSICAL SCIENCE}

\author{
IN MIORE RECENT TIMES,
}

AND PARTICULARLY BETWEEN THE YEARS $17 \% 5$ AND 1850;

BEING ONE OF THE DISSERTATIONS PREFIXED TO THE EIGHTH EDITION OF THE ENCYCLOP EDIA BRITANNICA.

BY

\section{JAMES D. FORBES, D.C.L., F.R.S.,} SEC. R.S. ED., F.G.S., \&c.

CORRESPONDING MEMBER OF THE IMPERIAL INSTITUTE OF FRANCE, ASSOCLATE OR HONORARY MEMBER OF THE BAVARIAN ACADEMY OF SCIENCES, OF THE ACADEMY OF PALERMO, OF THE DUTCH SOCIETY OF SCIENCES (HAARLEM), OF THE HELVETIC SOCIETY, OF THE PONTIFICAL ACADEMX OF " NUOVI LINCEI" AT ROME, AND OF THE NATURAL HISTORY SOCIETIES OF HEIDLEBERG, GENEVA, AND VAUD;

HONORARY MEMBER OF THE ROYAL MEDICAL SOCIETY OF EDINBURGH, OF THE CAMBRIDGE, YORKSHIRE, ST ANDREWS,

AND ISLE OF WIGHT PHIIOSOPHICAL SOCIETIES, AND OF THE PLYMOUTH AND BRISTOL INSTITUTIONS, AND PROFESSOR OF NATURAL PHILOSOPHY IN THE UNIVERSITY OF EDINBURGH.

EDINBURGH:

ADAM AND CHARLES BLACK. 



\section{A DVERTISEMENT.}

THIs Essay was written as a continuation of the Dissertations on the Progress of Mathematical and Physical Science by Professor Playratr and Sir JoHN LesLie, which appeared in the later Editions of the Encyclopedia Britannica. It is now reprinted for general circulation, in consequence of repeated applications to that effect. Only a few inconsiderable alterations have been made upon it. For information as to the plan of the Work, the reader is referred to the Introductory Chapter.

Edinburgh, January 1858. 



\section{CONTENTS.}

\section{CHAPTER I.-INTRODUCTORY.}

§ 1. On the Plan of this Dissertation.

\$2. On the Relations between Mathematics and Natural
Philosophy, and between the latter and the Mechanical

Arts.. ..page 7

\section{CHAPTER II.-PHYSICAL ASTRONOMY AND ANALYTICAL MECHANICS.}

§ 1. LAGRANGE.-Variation of Parameters-Application to Physical Astronomy. The Stability of the Planetary System ; Laplace ; Poisson. Moon's Libration...page 11

§ 2. LAPLACE.-Lunar Theory Improved.- Great Inequality of Jupiter and Saturn. Theory of the Tides -Young; Dr Whewell; Mr Airy.-Theory of Probabilities-Character of Laplace as a Physicist and Author

§ 3. LEGENDRE-IVORY.-Theory of Integration; Elliptic Transcendants (Abel, Jacobi). The Attraction of Spheroids, and Theory of the Earth's Figure. Atmospherical Refractions..............................page 24

$\S$ 4. Progress of Physical Astronomy since the publication of the Mécanique Céleste-PoIsson.-Theory of Rotation (Poinsot). - Mr AIRY-The Solar Theory.MM. PlanA and HANSEN-The Lunar Theory.Physical Astronomy in America .................page 26

§ 5. M. IEVVERRIER-Mr ADAMS.-The inverse method of Perturbations. Prediction of the place and orbit of Neptune from the motions of Uranus............... page 29

\section{CHAPTER III.-ASTRONOMY.}

§ 1. Maskeyline-Delambre.-Progress of Practical Astronomy from 1770 till 1810 - of the Lunar Theory deduced from Observation-The Density and Figure of the Globe. Cavendish; Baily.-Trigonometrical Surveys ..page 34

§ 2. Sir William Herschel.-History of Sidereal and Telescopic Astronomy to 1820. Herschel as an Optician-Planet Uranus-Solar Spots-Orbits of Double Stars-Nebulos-The Milky Way-Sun's Motion in Space ...........................................page 40

§ 3. BeSSEL-Mr AIRX.—Modern Observatories. Fixed Star Catalogues-Planetary and Lunar Observations ..............................................page 49

§ 4. BORDA-KATER-BAILY.-The Figure of the Earth from Pendulum Observations--" Reduction to a va- cunm;" Mr Stokes - Colonel Everest-M. STrOVE.-Latest Measures of the Earth. M. FoUCAULT's Pendulum Experiment ..................page 52

§ 5. M. ENCKe.-Cometary Astronomy-Periodic Comets of Halley and Encke. GAMBART's and Biela's Comet-Comets of 1811 and 1843.-Mr HIND-New Planets or Asteroids. Mr LAsselL-Newly-discovered Satellites. Mr Bond...................... page 57

§ 6. Sidereal Astronomy since 1820.-M. STRUVEDouble Stars. Observatories of Dorpat and Pulkowa. Sir John Herschel-Orbits of Double Stars. Magnitudes of Stars. Variable Stars. EARL OF ROSSE - His Telescopes. Nebula. Henderson and BesSEL-Parallax of Stars............................page 63

\section{CHAPTER IV.-MECHANICS OF SOLID AND FLUID BODIES, CIVIL ENGINEERING, AND ACOUSTICS.}

§ 1. WATT.-Condition of Practical Mechanics previous to the time of Watt. His genius for the application of Science to Practice. His successive Improvements on the Steam-Engine. Steam Navigation ......page 67

\$2. RoBison. - Application of Statical Principles to Engineering, especially to practical Masonry. CooLOMB.-Friction-Force of Torsion...............page 72

\$ 3. Thomas Young.-Strength of Materials, and Art of construction (continued).-TELFORD-Introduction of Iron into permanent Structures. Suspension Bridges-Tredgold; Mr Hodgkinson; M. Navier.Mr Robert Ste Phenson-Tubular Bridges...page 76

\$ 4. Sir M. I. BrUNEL. - Self-acting Machinery.-Thames Tunnel._Mr BABBAGE's Calculating Engines...page 80
§ 5 Trevithick.-Georae STEPhenson.-The Loco-motive Steam-Engine.-Rise and Progress of Railways.-M. de Pambour on Locomotives .........page 83

§ 6. Hydrodynamics.-Dubuat, Venturi, Professor STOKES.-Friction and Resistance of Fluids.-MM. Weber, Mr SCOT'T RUSSELL - Propagation of Waves. Their infuence on Canal Navigation.-MM. FourNeYron and PoNCELET-Improved Hydraulio Machines; Turbine. Reference to the subject of Capillary Attraction .page 88

§ 7. Progress of Acoustics. CHLADNI-SAVART.-Laplace's Correction of the Theory of Sound. Vibrating Plates and Acoustic Figures. Cagniard de la Tour's "Sirène." page 93 
CHAPTER V.-OPTICS.

§ 1. Thomas Young.-The Undulatory Theory of Light -Its History from the time of Hooke and Huygens. The Law of Interference-Its application to Diffraction-to the Rainbow-and to other subjects.-The Theory of Polarization referred to another section..page

§ 2. MALUS.-Discovery of the Polarization of Light by Reflection-Early History of Double Refraction and Polarization ... 103

§ 3. FRESNet.-The Undulatory Theory of Light continued-Diffraction-Transverse Vibrations; Young. -Polarization and Double Refraction explained.Lighthouse Illumination............................page 105

§ 4. ARAG0.-Short Account of his Scientific Career-He discovers the Colours of Polarized Light-Laws and Theory of Depolarization; M. Biot; Young; Fresnel.- Non-interference of oppositely Polarized RaysRotatory Action of Quartz.-M. Foucault's Experiment on the Velocity of Light. .page 109
§ 5. Sir David BrewSTER.-Progress of Experimental Optics - Laws of Polarization-Double Refraction pro* duced by Heat and Compression-Discovery of Biaxal Crystals-Laws of Metallic Reflection-Absorption of Light; and Lines of the Solar Spectrum; FraUnHOFER. - Seebeck; M. В10T page 113

§ 6. Mr Airy, Sir William R. Ifamilton, and Professors LLOYD and MacculLaGH.-Confirmation of Fresnel's Theory-Investigation of the Wave Surface completed; Conical Refraction.-M. CAuchY-Mechanical Theory of Elastic Media, and of Ordinary and Metallic Reflection; MI. Jamin.-Theory of Dispersion; Professor Powell ...................................page 119

§ 7. RITTER.-Chemical Rays of the Spectrum.-NIEPCE; DaUgUerre; Mr TALBot. Art of Heliography or Photography-Daguerreotype-Calotype.-Professor STokes. Chemical Rays rendered visible-Fluorescence ..............................................page 123

\section{CHAPTER VI.-HEAT, INCLUDING SOME TOPICS OF CHEMICAL PHILOSOPHY.}

§ 1. BuAck.-Latent and Specific Heat.-Irvine.-Hutton.-Doctrines of Heat applied to some Natural Phenomena .page 127

§ 2. CAVENDISH.-His Singular Character and Attainments-Eminent Chemical Discoveries-Observations on Heat and on other Branches of Physics.-LAvorSIER-The Calorimeter-Theory of Combustion and of Oxidation ..........................................pnge 130

§ 3. DALTON.-Theory of Gases and Vapours-Law of Expansion by Heat-Atomic Theory of Chemistry.GAY-LuSSAC page 135

§ 4. RuMroRD.-Economical applications of Heat.-Point of Maximum Density of Water ; Hope--Friction as a source of Heat. Theory that Heat is convertible into Mechanical Energy; Mr Joule....................page 142

§ 5. Sir JoHN LeSLIE.-Establishment of certain Laws of Radiant Heat. - Pictet. - Prevost. page 144
§ 6. FOURIER.-Mathematical Theory of the Conduction of Heat-Lambert; Poisson.-Temperature of the Earth and of Space............................page 148

§ 7. Dulong.-The Law of Cooling.-Progress of the Science of Radiant Heat between Leslie's and Melloni's Discoveries ; transmission of Radiant Heat through Glass. Herschel; De la Roche; Professor Powell. -Theory of Dew; Wells.........................page 154

§ 8. MELLONI.-Recent History of Radiant HeatTransmission and Refraction of Heat; Properties of Heat analogous to Colour-Experiments in Great Britain on the Polarization and Double Refraction of Heat ..........................................page 157

§ 9. M. REGNAULT.-Numerical Laws of Expansion by Heat ; Rudberg.-Vaporization; Dulong.-Latent Heat ; Hygrometry................................page 159

\section{CHAPTER VII.-ELECTRICITY-MAGNETISM-ELECTRO-MAGNETISM.}

§ 1. Galvani.-Discovery of Galvanism ; Proper Animal Electricity.-The subject revived by Nobili.-MM. Matteucci and Du Bois-Reymond page 160

§ 2. Volta.-Progress of Discovery in Common and Atmospheric Electricity-The Electro-motive TheoryVoltaic Pile-Chemical Analogies and Decomposition; Fabroni; Nicholson and Carlisle..................page 163

3. Sir Iunphry Davx.-Progress of Voltaic Electricity-Electro-Chemistry; Berzelius.-Davy's Invention of the Safety-Lamp.-WoLLAston; his Electrical and other Observations. Contrast of his Character with that of Davy.

§ 4. OERSTED.-AMPËre.-Discoveryof Electro-Magnetism-Electro-Dynamic Theory-Discovery of ThermoElectricity; SEЕВECK.-The Galvanometer of Schweigger and Nobili...

\$ 5. Dr FARADAY.-Progress of the Theory of Electro-Che- mical Decomposition - Volta-Electric InductionMagneto-Electricity--Diamagnetism--Optical Changes induced by Magnetism.-Professor Plücker-Magneoptic Action.......................................page 179

§ 6. OHM-DANIEL-Mr Wheatstone-M. Jacobi. -Laws of Electrical Conduction;-Constant Battery; -Applications of Electricity to Telegraphs-ClocksMotive Engines-the Electrotype...................page 184

§ 7. CAVENDISH-Coulom B.-On the Distribution of Statical Electricity, aind on the Mathematical Theory of the same.-Porsson-Mathematical Theory of Statical Electricity and of Magnetism generalized. Green; Professor William Thomson......................page 189

§ 8. Professor Hansteen-Baron A. von IUMBOLDTGAUSS-Major-General SABINE-Captain Sir J. C. Ross.-Progress of our Knowledge of Terrestrial Magnetism in the Present Century. .page 192 


\title{
CONS U L T I N G I D EX
}

TO THE

\section{PRINCIPAL NAMES AND SUBJECTS* MENTIONED IN THE FOLLOWING DISSERTATION.}

\author{
T'be References are to the Articles or Parayray,hs, not to the Pages.
}

Abel, 98.

Absorption of Light, 536 .

Acceleration of the Mloon, Secular, 62.

Acoustics, 433 , \&c.

Adams, Mr, 127, \&c.

Airy, Mr, 82,115, 228, \&c. ; 421, 549,879.

Ampère, 792, 794, \&c.

Arago, 166, 500, 792

Asteroids, 282. List of, 283, and Additions, p. 996.

Astronomy, Physical, 41, \&c.; Practical, 149, \&c.; 218, \&c. ; Sidereal, 172, \&c. ; 289, \&c.

Atomic Theory, Dalton's, 618.

Attraction of Spheroids, 99 , \&c.; of Mountains, 154, \&c.

Attractions, Theorems about, 99 , \&c.; $877,905$.

Babbage, Mr, 377.

Baily, 158, 242, \&c.

Balloon ascents, 630 , and note.

Barlow, Mr, 879.

Berzelius, 763,767 , and note.

Bessel, 221, 310 .

Biaxal Crystals, 529 ; Theory of, 493.

Biot, M., 545, 662.

Black, 318, 583.

Block Machinery, 370 .

Borda, 237, \&c.

Bowditch, 126.

Brewster, Sir David, 519, \&c.

Bridges, Suspension, 351 ; Wooden, 356 ; Girder, 358 ; Tubular, 359 , \&c.

Brinkley, 307, and note.

Brunel, Sir M. I., 366.

Cagniard de la Tour, M., 441.

Calculating Engine, 377.

Calorimeter, 88, 607 .

Calotype, The, 571.

Canal Navigation, 422.

Capillary Attraction, 88, 432

Catenary, 353.

Cauchy, M., 556.

Cavendish, 156, 593, \&c.; 871.

Cavendish Experiment, 156, \&c.

Chances, 83.

Chemical Subjects connected with Natural Philosophy, 582, 596, 618, \&c.; 763, \&c. ; 811 .

Chemical action of Light, 562 .

Chemical Equivalents, 628 .

Chemical Theory of Galvanism, 751, 763 , 811.
Chladni, 434

Civil Kingineering, 312-431.

Clairaut, 235.

Clock, Electric, 231, 862.

Coloration of Heat, 713.

Combustion, 608, 775 .

Connets, 262 ; Halley's, 26:3 ; Lncke's, 267 ; Gambart's or Biela's, 276 ; Comets of 1811 and 1843, 281.

Conduction of Heat, 661, \&c.

Conduction of Electricity, 813,842 .

Cornish Steam Engines, 323.

Conical Refraction, 551 .

Cooling, Law of, $654,694$.

Coutomb, 339, 413, 873.

\section{Daguerre, 569.}

Dalton, 610, 721, note.

Daniell, 852 .

Davy, Sir H., 758, \&c.; 792, 808.

Declination of the Needle, 881

Delambre, 164, \&c.

De la Roche, 704.

Density of the Earth, 154, \&c.

Depolarization, 505.

Detrusion, 346.

Diamagnetism, 823.

Diffraction of Light, 458,486 , \&c.

Dip, Magnetic, 882 , note ; 883 .

Discontinuous functions, 31,685 .

Dispersion of Light, ō59.

Double Refraction, 475 , \&c.

Du Bois-Reymond, M., 739.

Dubuat, 410 .

Dulong, 693, 721 .

Earth's Figure, 163, 234, 249, \&c. ; Density, 154, \&c. ; Rotation, 258.

Earth, Proper Heat of the, 675 .

Economical application of Heat, 634.

Elastic Solids, 342, \&c.

Electricity, 728, \&c.; Animal, 734, 738, 872 ; Atmospheric, 743 ; Mathematical Theory of, 869, \&c.; Distribution of, 871,874 .

Electric Telegraph and Clock, 856, \&c.

Electro-Chemistry, 751, 754, 763, 811.

Electrodynamic Machines, 866 .

Electro-Magnetisn, 786, \&c.

Electrotype, 868.

Fllipticity of the Earth, 256.

Ellis, Mr Leslie, 20, note ; 86.

Emanation of Radiant Heat, 651, 667.

Encke, M. 262, \&c. ; Encke's Comet, 267.

English Arc of Meridian, 167.
Erman, M. Ad., 885.

Evaporation, 613, 744

Everest, Colonel, 250.

Expansion of the Gases, 617, 721, and note.

Fabbroni, 751.

Fairbairn, Mrr W., 363.

Faraday, Dr, 798, 807, \&c.

Fechner, 851.

Figure of the Earth, 163, \&c. ; 234, \&c.

249 , \&c. ; Results, 256.

Flame, 770

Flexurc of Beams, 347, \&c.

Fluids, Friction and Resistance of, 410 .

Fluorescence, 579.

Foucault, M., 258, 514.

Fourier, 663, \&c.

Fourneyron's Turbine, 427.

Fraunhofer, 538.

French Arc of Meridian, 165.

French experimenters, Skill of, 720.

Fresnel, 468, 485, \&c.

Frog, Electricity of the, 734 .

Galle, M., 137.

Galloway, 212

Galvani, 728, \&c.

Galvanism, Discovery of, 732 .

Gases and Vapours, Dalton's Theory of,

$613,615,617$

Gauss, 48, 856, 896.

Gay-Lussac, 617, 625, 630 .

Green, 558 and note, 878 .

Greenwich Observatory, 150, 159, 227.

Gregory, Duncan, 31, note.

Halley, 882.

Hamilton, Sir W. R., 550, 552, note.

Hansen, M., 57, note; 122, \&c., 261.

Hansteen, Professor, 881, \&c.

Heat, 582, \&c.; Economical Applications of, 634 ; Hypothesis on the $\mathrm{Na}$ ture of, 639 ; Radiant, 641, \&c. ; 692,

\&c. ; Mathematical Theory of, 661 , \&c.; Motion in a Sphere, 673; Solar, 679 .

Heliometer, 310

Henderson, 304.

Herschel, Caroline, 269, note.

Herschel, Sir J. F. W., 294, 549.

Herschel, Sir William, 172, \&c. ; 702.

Hind, Mr, 282.

Historic periods in Science, 2, \&c.

Hodgkinson, Mr, 352, 353, 363 .

Hooke, 452 .

Hope, 636 . 


\section{INDEX.}

Humboldt, Baron A. von, 734, 892, \&c. Hutton, 591.

Huygens, 453, 475. The Principle of, 454.

Hydrodynamics, 408, \&c.

Hygrometry, 614, 727 .

Indian Arc of Meridian, 250.

Induction of Electric Currents, 816.

Inequalities, Secular and Periodic, 47.

Intensity, Magnetic, 885, 911.

Integration, 31, 97.

Interference of Light, 460 ; of Chemical Rays, 565.

Isothermal Lines, 894.

Irvine, 586, 589.

Ivory, 103.

Jacobi, C. G. I., 98.

Jacobi, Mr M. H., 848, 868.

Jamin, M., 557.

Joule, Mr, 640.

Jupiter and Saturn, Theory of, 65.

Kelland, Professor, 421, 690.

Kater, 240.

Lagrange, 20, note; 41, \&c., 90

Lalande, 162.

Lambert, 661 .

Lambton, 250

Laplace, 51, 60, \&c., 199, 235, note, 433.

Lassell, Mr, 284.

Latent Heat, 584, \&c., 599, 724.

Lavoisier, 605.

Legendre, 85, 95, \&c.

Leslie, Sir John, 641, \&c.

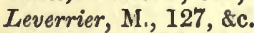

Libration of the Moon, 57.

Lines of the Spectrum, 538.

Lighthouses, 496.

Lloyd, Dr., 550, \&c., 900, 910.

Locomotive Engine, 383, \&c.

Lubbock, Sir J. W., 79, 117.

Luminous Waves, Length of, 463.

Lunar Observations, 152, 229.

Lunar Theory, 61, \&c., 118, \&c., 229.

Maccullagh, 553.

Magne-crystallic Force, 832.

Magne-optic Force, 831 .

Magnetism, Terrestrial, 880, \&c. ; Theory of, 903.

Magneto-electricity, 819

Magnifying Power, 179, 180

Malus, 474.

Maskelyne, 150.

Mathematics, Pure, 20, 24, 28, \&c.

Mathematical Theory of Heat, 661, \&c.; of Electricity, 869, \&c. ; of Magnetism, 903.

Matteucci, 739.

"Mécanique Celeste," 89.

Méchain, 166.

Mechanical Arts, 11, 32, \&c.

Mechanical Notation, 378 .

Mechanics, 312 , \&c.

Melloni, 707.

Metallic Polarization, 534.

Michell, 86, note, 156, 340 .

Milky Way, 201, \&c.

Miller, Patrick, 325.

Modulus of Elasticity, 345.

Natural Philosophy, ite Connection with
Mathematics, 24 ; with the Mechanical Arts, 32 , \&c.

Nautical Almanac, 153

Navier, M., 354.

Nebulæ, 190, 302.

Nebular Theory, 198, \&c.

Neptune, Discovery of, 127, \&c.; Elements of, 143.

Nicholson and Carlisle, 754.

Niepce, Nicephore, 569.

Nobili, 708, 738, 802.

Oersted, 786, \&c., 803, \&c.

Olbers, 161 .

Ohm, 840 .

Optics, 444, \&c.

Parallax of Stars, 188, 306, \&c.

Parameters, Variation of, 44.

Pendulum Observations, 236; M. Foucault's Pendulum, 258.

Perturbations, Planetary, 46.

Peters, M., 311 .

Photography, 567.

Pictet, 648.

Pile of Volta, 750, 752 .

Plana, M., 118, \&c.

Planets, New small, 282, \&c.

Playfair, 16, note.

Plenitude of Stars, 203.

Plïcker, Professor, 831.

Poinsot, M., 113.

Poisson, 55, 112, \&c., 689.

Polarization of Light, 474, \&c., 525, \&c. affected by Magnetic Actions, 834 ; of Heat, 717; of Chemical Rays, 565; Circular, 491 ; by Metals, 534 .

Poles, Terrestrial Maguetic, 882, 884.

Poncelet, M., 431.

Pond, 227, 307

Potential, 877, 905.

Powell, Professor, 560, 705.

Prevost, 649.

Probabilities, 83.

Proper Motions of Stars, 209, \&c.

Pulkowa, Observatory of, 293.

Quartz, Optical Properties of, 511.

Quaternions, 552, note.

Radiant Heat, 641, \&c., 692, \&c.

Railways, History of, 398.

Rainbow, Theory of the, 465 .

Reflection and Refraction of Light, 490 .

Refrangibility of Heat, 714.

Refractions, Astronomical, 107.

Regnault, M., 719, \&c.

Rheostat, 848 .

Ritter, 563 .

Rivers, Theory of, 410 .

Robison, 329, \&c., 749.

Ross, Sir J. C., 884, 910.

Rosse, Earl of, 299.

Rotation of the Earth, 258.

Royal Institution, 637.

Rumford, 632, \&c.; Rumford Prize, 637.

Russell, Mr J. S., 421.

Russo-Scandinavian Arc of Meridian,254.

Sabine, Major-General, 238, 246, 909.

Safety Lamp, 393, 770 .

Satellites, New, 284.

Savart, 441.

Schehallien Experiment, 155.

Scheutz, M., 380.
Schweigger, 802.

Seebeck, 512, 544, 801.

Ships, Magnetism of, 879 .

"Sirène," 441.

Slide-Rest, and Planing Machine, 372.

Solar Heat, 679.

Somerville, Mrs, 566 and note.

Sound, Theory of, 433 .

Specific Heat, 588, 599, 607, 727.

Spectrum, Lines of the, 538; Heat of the, 702,715 .

Stability of the Solar System, 50 .

Stars, Double, 187, 291, 296; Proper Motions of, 209, 292 ; Parallax of, 188, 306 ; Brightness of, 297 ; Variable, 298.

Steam, Elastic Force of, 699 .

Steam Carriage, 384.

Steam Engine, 317, \&c.

Steam Navigation, 325 .

Stephenson, George, 392, \&c.

Stephenson, Mr R., 355, \&c.

Stereoscope, 581.

Stokes, Professor, 235, note ; 247, 416, $579,691$.

Struve, M. W., 209, note ; 252, 290, \&c.

Sun, The, 185.

Suspension Bridges, 351.

Talbot, Mr Fox, 571.

Telegraph, Electric, 856.

Telescopes, 178, \&c., 299, \&c.

Telford, 351.

Thermo-electricity, 801.

Thermo-multiplier, 709.

Thomson, Professor W., 878.

Tides, 69 , \&c.

Torpedo, 872.

Torsion Balance, 157, 340, 874.

Transverse Vibrations, 488 .

Tredgold, 352.

Trevithick, 385, \&c.

Turbine, 425

Tunnel, The Thames, 373.

Undulatory Theory of Light, 452, \&c.

Uranus, Discovery of, 183; Perturbations of, 132 , \&c.

Variable Stars, 298.

Variation or Declination of the Needle, 881.

Variation of Parameters, 44.

Venturi, 413.

Venus and the Earth, long Inequality of, 115.

Vision, 471, 581

Volta, 740, \&c.

Volta-electric Induction, 817.

Voltaic Pile, 749.

Waves, Theory of, 419, \&c.

Water, Composition of, 597 ; Maximum Density of, 636 .

Water Wheels, 426.

Watt, James, 312, \&c., 597.

Weber, The MM., $420,856,899$.

Wells, 706.

Wheatstone, $\mathrm{Mr}, 581,853$, \&c.

Whewell, Dr, 19, 79 .

Wilson, P., 185

Wollaston, 476, 538, 564, 780, \&c.

Young, Thomas, 80, 342, \&c., 445, \&c., $488,506,780$. 


\section{DISSERTATION SIXTH.}

\section{MATHEMATICAL AND PHYSICAL SCIENCE.}

\section{CHAPTER I.}

\section{INTRODUCTORY.}

\section{$\S 1$. On the Plan of this Dissertation.}

(1.) THE year 1850 may be said to complete the Third cenModern Advances in Science

tury of modern scientific progress, or the Fourth if we include its earliest dawn. To each of these ages of discovery may be assigned a peculiarity in the character of its improvements, and even in the methods which conduced to that improvement.

(2.) Between 1450 and 1550 (a period so distinguished Period in letters and the arts), some great truths in physics 1450-1550. and mathematics had presented themselves to a few precocious minds, yet they had not received any public acknowledgment, nor perhaps an adequate demonstration. Algebra then first became a science. Leonardo da Vinci made the earliest steps since the time of Archimedes, in rational mechanics, and Copernicus almost at the close of this period promulgated the true system of the world.

(3.)

But the next centenary (1550-1650) was the first Period of true scientific activity. Its characteristic feature 1550-1650. was the vindication of observation and experiment as the prime essentials to the increase of natural knowledge, with the consequent repudiation of the dogmas of the schoolmen, and the baseless methods of a priori reasoning. The men of science formed a goodly array at this stirring time; and signal were their triumphs. Galileo was beyond all comparison the glory of his age. His sagacity, his knowledge, his versatility of talent, his ingenuity as an inventor, his success in prosecuting his discoveries, and his zeal and eloquence in making known their importance, gave him an enviable pre-eminence even amidst a mighty generation. Bacon laid down the canons of a new method in philosophy which Gilbert and Kepler, as well as Galileo, had already acted on. Napier and Descartes prepared for the general application of mathematics in the coming struggle.

The hundred years which next succeeded (1650. 1750 ) saw the triumphant application of mathema- Period tics to Mechanics and Physics, and the establishment of the greatest mechanical theory of any age, that of Gravitation. The preparatory labours of a hundred and fifty years were brought, chiefly by the unparalleled sagacity and genius of Newton, to a speedy and dazzling climax. His success brought numerous and worthy labourers into the field, but they found enough to do in gathering in the harvest which he had prepared for them.

If we look for the distinguishing characteristic of the centenary period just elapsed (1750-1850), we Period find it in this, - that it has upon Experiment as a means of arriving at truth than had previously been done. By a natural conversion of the process, the knowledge thus acquired has been applied with more freedom and boldness to the exigencies of mankind, and to the farther investigation of the secrets of nature. If we compare the now extensive subjects of Heat, Electricity, and Magnetism, with the mere rudiments of these sciences as understood in 1750 , or if we think of the astonishing revival of physical and experimental Optics (which had well nigh slumbered for more than a century) during the too short lives of Young and Fresnel, we shall be disposed to admit 
the former part of the statement; and when we recollect that the same period has given birth to the steamengine of Watt, with its application to shipping and railways, - to the gigantic telescopes of Herschel and Lord Rosse, wonderful as works of art as well as instruments of sublime discovery,- to the electric telegraph, and to the tubular bridge, - we shall be ready to grant the last part of the proposition, that science and art have been more indissolubly united than at any previous period.

(6.) The Dissertation of Professor Playfair closes with Limits of the period of Newton; that of Sir John Leslie, prothis Disser- fessedly devoted to the history of the eighteenth cen-
tation.

\section{(7.)}

Character of the close of the 18th century. tury, embraces some matters which belong more properly to those which preceded and followed it. After considering how I might best carry out the plan of these essays, I have adopted the period from about the year 1775 to 1850 as the general limit of $\mathrm{my}$ review. We may imagine this period, of three quarters of a century preceding the present time, to be divided into three lesser intervals of 25 years each, which have also some peculiar features of their owl.

From 1775 to 1800 many branches of science still continued in the comparatively inert state which characterized a great part of the eighteenth century. There were, however, two or three notable exceptions. One was the continued successful solution of the outstanding difficulties of the Theory of Gravity applied to the moon and planets, a task in which the continental mathematicians, and of these, in chief, Lagrange and Laplace, had no rivals, or even coadjutors, on this side of the channel: Another was the foundation of Sidereal Astronomy by Sir William Herschel; and the last was the commencement of a system of Chemical Philosophy based on new and important experiments, and including the laws of heat in combination with matter, which at that period very naturally ranged themselves within the province of the chemist. In this department two British and one foreign name stand conspicuous, Black, Cavendish, and Lavoisier. I do not of course mean to affirm that other branches of science were not cultivated with success within the exact period of which we speak. Electricity, for instance, first statical, afterwards that of the pile, had a share in the discoveries and speculations of the time. But these were rather the mere extension of what had previously been thought of; or the first dawn of future important results, whose development fills a large space in the succeding story. Volta and his inventions belong rather to the nineteenth than to the eighteenth century.

(8.) The first quarter of the present century attained Character a higher and more universal celebrity. Scarcely a period branch of physical science but received important 1800-1825. and even capital additions. Physical Astronomy indeed, no longer filled so large a space in the page of discovery, simply because the exhaustive labours of the geometers of the former period had brought it to a stage of perfection nearly co-ordinate with the means of observation, and because, by the publication of the Mécanique Céleste, Laplace had rendered available Mécanique and precise the masses of scattered research accu- Céleste. mulated by the labours of a century since the close of Newton's career of discovery. It was in some sense a new book of "Principia,"-not, indeerl, the work of one, but of many; nor of a few years, but of two generations at least. Still there it was, a great monument of successful toil, which, like its prototype, was for many years to be studied, even by minds of the highest order, rather than to be enlarged.

But the other branches of Natural Philosophy were now to make a stride, such as perhaps no preThe science of Optics mental was speedily expanded almost twofold, both in its facts and in its doctrines. Galvanic Electricity disclosed a series of phenomena not less brilliant and unexpected in themselves than important from the new light they threw on the still dawning science of chemistry, and from the power of the tool which they placed in the hands of philosophers. Before the first quarter of the present century closed, the important and long suspected connection between Electricity and Magnetism was revealed, and its immediate consequences had been traced out with almost unparalleled ingenuity and expedition. The basis of the science of Radiant Heat, slightly anticipated by the philosophers of the eighteenth and even the seventeenth centuries (Lambert and Mariotte), was finally laid in a distinct form, assigning to the agent, heat, an independent position dissociated from grosser matter, such as light had long enjoyed. Astronomy, though enriched on the very first night of the new century by the discovery of a small planet, the herald of so many more of the same class, made perhaps less signal progress; but Chemistry, besides the aid it received from the invention of the pile, had a triumph peculiarly its own in the addition of the comprehensive doctrine of Definite Proportions, destined to throw at some later time a steady light on the vexed question of the constitution of matter. The great number of scientific names of the first order of merit concerned in these numerous discoveries marks the extraordinary fertility of the period. They are imperfectly comprehended in the following list: Young, Malus, Sir David Brewster, Fresnel, and Arago; Volta, Dalton, Davy, and Oersted ; Prevost, Leslie, and Fourier; Gauss, Ivory, Olbers, Bessel, and Encke.

Of the twenty-five years just elapsed, it is not so easy to speak with precision. The voice of criticism Period may be fairly uttered with that reserve which every one must feel in speaking of his immediate contemporaries. Yet it may perhaps be stated without just cause either of offence or regret, that it has not on the whole been characterized by the full maturity of so many commanding minds. Of the great discoverers of the former period, several survived and continued their efficient labours during no small por- 
tion of the latter; and a few happily still remain to claim the respect and veneration of their disciples and successors. But the vast steps so recently made in Optics, in Electricity, in Magnetism, in Thermotics, and in Chemical principles, tended of necessity to call forth such an amount of laborious detail in the defining and connecting of facts and laws, and the deductions of the theories started to explain them, as seemed to render fresh and striking originality somewhat hopeless, whilst they occasioned a vast amount of useful employment to minds of every order of talent. The undulatory Theory of Light, nobly blocked out by the massive labours of Young and Fresnel, has afforded still unexhausted material to the mathematician on the one hand, and to the experimentalist on the other; and ably have they fulfilled the double task, adding at the same time discoveries whose importance and difficulty would have made them still more prominent, had they not been the legitimate consequences of a still greater discovery already in our possession. Nearly the same might have been said for the sciences of Electricity, Electromagnetism, and Electro-chemistry, had not the comFlectricity. parative newness of the whole doctrine of these sciIleat. ences, and the suddenness of their first rise, and, perhaps still more, the appearance of a philosopher of the very highest merit, Mr Faraday, who fortunately attached himself to this special department, made the last thirty years an almost unbroken period of discovery. Radiant Heat, too, has been successfully advanced by labours comparable perhaps to those which marked its first rise as a science, and some other topics connected with heat have risen into great and practical consequence. Astronomy has been prosecuted with a systematic assiduity and success, especially at the British and Russian national observatories, which yields to that of no former period, whilst physical astronomy has been cultivated by methods of still improved analysis, and has achieved one triumph which France need not grudge to England, nor England to France,- - so signal as to be placed by common consent in a position superior to any since the first publication of the theory of gravitation, more than a century and a half before. This was the prediction of the position in space of a planet whose existence was unknown except by the disturbance which it produced in the

Magnetism. movements of another. Terrestrial Magnetism has, for the first time, aspired to the rank of an exact science. In an illustrious philosopher of Germany, it has found its Kepler; and the combination of national efforts in collecting reliable data from the remotest corners of the globe is characteristic of the

Chemistry. practical energy of the age. Pure Chemistry has been cultivated with extraordinary assiduity; but though some general principles have emerged, none are comparable, from their importance, to the discovery of Dalton. To cite, then, at present, but a few names, amongst the most conspicuous benefac- tors of science of the last, or contemporary period, are MM. Airy, Cauchy, Hamilton, and MacCullagh ; MM. Faraday, Melloni, and Gauss ; Sir John Herschel, M. Struve, and Lord Rosse; MM. Plana, Poisson, Leverrier, and Adams; MM. Mitscherlich, Liebig, and Dumas.

It seems to me impossible to exclude from a review, however slight, of contemporary progress in Mechanical the exact sciences, the advantages which have accrued ${ }^{\text {arts. }}$ to them both directly, and, as it were, reflexively, by the astonishing progress of the Mechanical Arts. The causes, indeed, which called them forth are somewhat different from those which are active in more abstract, though scarcely more difficult, studies. Increasing national wealth, numbers, and enterprise, are stimulants unlike the laurels, or even the golden medals of academies, and the quiet applause of a few studious men. But the result is not less real, and the advance of knowledge scarcely more indirect. The masterpieces of civil engineering - the Steam Engine, the Locomotive Engine, and the Tubular Bridge - are only experiments on the powers of nature on a gigantic scale, and are not to be compassed without inductive skill as remarkable and as truly philosophic as any effort which the man of science exerts, save only the origination of great theories, of which one or two in a hundred years may be considered as a liberal allowance. Whilst then we claim for Watt a place amongst the eminent contributors to the progress of science in the eighteenth century, we must reserve a similar one for the Stephensons and the Brunels of the present: and whilst we are proud of the changes wrought by the increase of knowledge during the last twenty-five years on the face of society, we must recollect that these very changes, and the inventions which have occasioned them, have stamped perhaps the most characteristic feature-its intense practicalness-on the science itself of the same period.

Having thus briefly reviewed the course of discovery since the latter portion of the eighteenth century, Review of I proceed in the succeeding chapters to attempt to of Science sketch it more in detail, dividing the sciences into in this groups, and in each of these endeavouring to present Essay. a lively view of its progress by connecting it with the individnal career of the eminent men who have most contributed thereto, and introducing collaterally the chief results obtained by their contemporaries. In this manner I hope, on the one hand, to escape the formality of a history of science, and the meagre detail which our limits would prescribe to so vast a subject; and on the other to be enabled to impress upon the reader (as seems to be the design of these Essays) the leading facts and features of discovery in every age, together with the intellectual characteristics of the greatest minds which contributed to it.

It is with no overweening confidence that I lay the

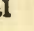

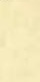


Scheme of result of this attempt before the reader. During the treatment : lengthened period of composition of this Dissertationof the sub- protracted by indisposition and untoward circumstances ject. of different kinds, I have had abundant leisure to reflect on the advantages and disadvantages of a plan which I had sketched in the previous paragraphs, at the very opening of my task. I am aware that a rigid criticism awaits every attempt like the present. I am aware also, that it is far easier to detect real faults, especially of omission, than to make sufficient allowance for the exceeding difficulty and delicacy of the undertaking. I have but one ground of confidence, and that is so strong, that I trust it will enable me calmly to meet every just critical reflection. I am conscious of having written in a spirit of absolute impartiality whether as regards persons or subjects, and that $I$ have exercised to the full amount of $\mathrm{my}$ opportunities what powers of judgment I possess. I have striven to speak judicially and historically whether of friend or stranger, the dead or the living, Englishman or Foreigner. What I have felt the most constant effort, has been the needful exclusion of meritorious names, far more numerous than those especially included and dwelt upon in these pages. But this has appeared to me the cardinal point of my whole plan. The labourers in science have been in these latter days so numerous, that had I noticed, even briefly, every one who had made a real step in science, my pages must have been crowded by names and titles of books. Even with the extension of bulk to which this essay has gradually and unavoidably grown (nearly double of its projected amount), the reader would rather have been bewildered than led by the perusal of such a catalogue. Besides, since such a brief historical synopsis forms very generally an introduction to the several articles of the Encyclopæedia, to repeat it all here would have been but a tedious redundancy. No one conversant with such matters will imagine that I have saved myself any labour by this particularity of selection. On the contrary, it would have cost no effort to enumerate under each subject the living or recently deceased authors upon it who are best known; such a detail must have left a vague and shadowy impression on the mind of the general reader, and when regard is paid to the necessary limits of the essay, and the multitude of technical details and technical words which there is no space to define and illustrate, it is plain that the perusal must have been rendered as dry and unpalatable to those who seek general and elementary yet clear ideas, as it would have been tantalizing and unsatisfactory to the accomplished student, or to the man of science in search of particular historical details.

The end at which I have aimed is to select the more striking land-marks of progress in each subject in each age, and endeavour to connect them with the character and position of all the more eminent dis- coverers, thus conveying to the general reader sufficient information on the limited number of particular subjects discussed, and interesting him not only in the science but in the individuals. Then, by a few slighter touches only, and the mention of some secondary names, to connect with one another these brighter periods of eminent progress, in which every country and every age feels a just pride.

That by many I shall be considered to have dwelt too much on some eras of invention, and to have omitted others not less important, is a difference of judgment which it is impossible not to anticipate, but equally impossible to prevent. I will only add that I have endeavoured to extend my impartiality to subjects as well as to persons; and that I have not intentionally dwelt longer on the topics of my own predilection than on those naturally considered by other persons equally or more important. Many subjects as well as persons familiarly known to me are scarcely, if at all, mentioned in these pages. To leave some definite and vivid impressions, selected solely for their importance, on the mind of my reader, has been the great object constantly before me during the composition of this work.

It will be seen from the preceding paragraphs, that I have deviated in some respects from the Compared scheme of my two distinguished predecessors in the with that of 作 (1) Dissertations on Science, Professor ing DisserPlayfair and Sir John Leslie. The essay of the for- tations. mer, which is the more finished and methodical, is admirably adapted to the period of the history of science of which he had principally to treat; the period, namely, of Galileo, Bacon, Newton, and Leibnitz. But the amount of material was smaller, and the principle of selection was also much simpler. The positive science of that age might almost be reduced to two heads, Astronomy, including its mechanical principles, and Optics. It was an age not inore distinguished for the Truths it disclosed, than for the invention and right appreciation of the Methods of Discovery. Inductive Logic, and Mathematical Logic applied for the first time to dynamics, very justly claimed a place in a dissertation on the progress of science, in a period when these preliminary doctrines and discoveries were the stepping stones by which even the basement story of the Temple of Nature could alone be reached. The Philosophy of Bacon and the discovery of Fluxions, occupied therefore, with much reason, a large portion of Professor Playfair's beautiful Dissertation; and it is impossible to regret that an intellect so admirably qualified for tracing and displaying the intimate and historical connection of branches of knowledge so varied in their principles and character, should have been thus congenially employed, to the delight and edification of readers of every degrec of acquirement from the highest to the humblest. ${ }^{1}$

1 In mentioning the name of my distinguished predecessor in the Chair of Natıral Philosophy in the University of Edinburgh, I willingly take the opportunity of noticing, in a few words, his peculiar merits, to which the Dissertation contained in 
(17.) But the extended domain of the science of the Extent and last hundred years enhances vastly the difficulty of the materials. subjects succinctly handled as one by Mr Playfair. The mechanical and experimental sciences alone constitute a body of knowledge so large that it is a responsibility sufficient for one person to attempt to grasp them all, and to set forth in order the steps of progress and improvement which have been so rapid, and even so startling. Since some of these have scarcely as yet been historically digested, and the broad features of contemporary discovery have not been gradually separated by the judgment of an impartial posterity from those slighter though praiseworthy details, which lapse of time and advance of knowledge will throw into the shadows of distance, - this difficult and most laborious task falls principally upon the reviewer. The length and breadth of the subject of Natural Philosophy, and the cumbrous and scattered depositories of knowledge in which its records must be sought, combine to render not only the undertaking an arduous one, but the result of it a good deal more bulky than might be desired, or than was easily possible, in dealing with the glorious, but compact, history of Newton's age. It might be compared to the difference between writing a history of the Jews or Romans and that of the whole of modern Europe.

The mere magnitude of the undertaking, then, might well excuse me from entering upon the cognate, but exceedingly distinct, subjects of the Logic of Inductive Discovery and the progress of the Pure
Mathematics. But an equally sound reason might be found in my consciousness of inadequacy to undertake, whatever had been the dimensions of $\mathrm{my}$ work, a threefold scheme of such magnitude and difficulty. I do not think that any one person could be found to treat the whole as it ought to be treated, and I am certain that I am not that person.

One attempt-a bold and successful one-has been made, in our own day, to unite two of the Writings of three departments, - I mean the History and the DrWhewell Philosophy of the Inductive Sciences. An English art Mill. philosopher of wonderful versatility, industry, and power has erected a permanent monument to his reputation in a voluminous work bearing the preceding title. ${ }^{1}$ A slight inspection of that work will show how impracticable and self-destructive a plan it would have been to attempt anything like a systematic abridgment of such a mass of facts and speculations within our present limits. Mr J. Stuart Mill has also published a work bearing on the origin of our scientific knowledge, diametrically opposed in principle to the preceding one, yet marked by great ability. ${ }^{2}$ Such disquisitions belong more properly to the philosophy of the human mind than of physics. After all, be it remembered that whatever has been learnt or discussed concerning the means of arriving at truth in Natural Science, it is not pretended that we have recently become possessed of any canons or rules of discovery superseding those fundamental principles of observation and experiment so well laid down by Bacon, and practised both

the present volume will bear an enduring testimony. Playfair's original contributions to science were not so marked and consi- Character derable as to justify me in including bis name in the comparatively brief catalogue of discoverers chronicled in the succeeding of Professor pages; but his efforts are, nevertheless, deserving of notice, and indirectly were perhaps hardly less beneficial. He was a most Playfair. patient and admiring student of the greatest mathematical writers of his time, and, when we consider the singularly backward state of that science in Great Britain about the end of the last and commencement of the present century, it was of no slight importance to find a man placed in the position of a public instructor able and willing to direct attention to the splendid achieve ments of the continental mathematicians. By his lectures both on Mathematics and Natural Philosophy-by his luminous articles in the Edinburgh Review-by some of his original papers in the Transactions of the Royal Society of Edinburgh-be contributed to this useful end, and would have done so still farther bad he been enabled to complete the Dissertation which he so ably commenced. He had an excellent mathematical capacity, and mathematical taste, rather than power. His explanations, even of matters of inherent difficulty, are perspicuous and popular, qualities possessed by few of his contemporaries. His style has been pronounced by the highest authorities to be a model of clearness and eloquence. He was extensively read in subjects of metaphysics and morals, as well as of pure science; and by a combination of talent rare, I am inclined to say, in a high degree, his taste, though eminently mathematical, was also directed, with signal success (at first through his intimate friendship for Dr Hutton), to the very opposite studies of Geology and Physical Geography, which may be said to have been the subjects of his predilection during the last twenty years of his life. Nor were these labours of the closet merely; be was far more intimately versed in the mineral structure of the earth, from observation, than any except a few professed geologists; and he exceeded them all in the ability with which he expounded and maintained the striking doctrines of the Hnttonian theory. Though professedly the "illustrator" of the principles specifically but obscurely laid down by Hutton, he certainly added much of his own. There is no reason to doubt that Playfair first apprehended the moving power of glaciers as geological agents in modifying the surface of Alpine countries, a matter which has of late been so earnestly discussed by the ablest geologists.

What adds to the singularity of the combination of tastes and talents to which $I$ have referred is, that he appears to bave had the slightest possible taste for that art of experiment which he eloquently advocated, with Bacon, as the grand distinction of modern science. I may be wrong in stating it broadly, but I do not now recollect a single experimental novelty, much less discovery, which we owe to Playfair, I mean in the department of Natural Philosophy; for we cannot include barometrical mea surements under this head, of which, indeed, it was the mathematical theory, and not the application to practice, which chiefly occupied bim. The same was the case in Astronomy, which, of the mechanical sciences, interested him most. In two capacities he will be remembered,-first, as the able, eloquent, and generally impartial and accurate Historian of Science; secondly, as the promoter, to so great a degree as to be considered a second founder, of modern Dynamical Geology. He was much beloved in private life, and was singularly free from the tendency to carping criticism and personal prejudices sometimes, unfortunately, found in men of letters. He was the intimate associate of Jeffrey and the other founders of the Edinburgh Review. His character has been drawn in three words by Sir James Mackintosh, and as happily contrasted with that of his illustrious friend :- . ..... Playfair and Jeffrey; the first a person very remarkable for understanding, calmness, and simplicity, the second more lively, fertile, and brilliant than any Scotchman of letters" (Life of Sir James Mackintosh, ii. 25I).

1 Whewell's History and Philosophy of the Inductive Sciences, 5 vols. 8 vo.

2 Slill's Logic, 2 vols. 8vo. 
before him and since by Galileo, Newton, and their disciples.

(20.)

Pure mathematic -their progress and tech nicality.
With regard to Pure Mathematics, and their progress during the last seventy years, to the difficulty arising froin the extent to which the review must have enlarged this Essay, and the enormous and disproportionate labour it must have cost (a labour far greater to the present writer than to one by taste and habit more addicted to the study of merely abstract Analysis), a conclusive argument against their systematic introduction into this historical sketch is to be found in the very nature of these modern improvements. All sciences-but especially the abstract sciencestend to become more intensely technical the farther they are pursued. These especially are incapable of popular treatment, although in their applications to physical science they occasionally admit of it in a very remarkable manner. The progress of analysis cannot be even enunciated or expressed but in the language of analysis, and the History becomes almost a Treatise, or, if not a Treatise, something nearly as technical. It is partly for this reason that the history of the Pure Mathematics has so seldom been even attempted to be written. ${ }^{1}$ Mathematicians have, since the time of $\mathrm{D}^{\prime} \mathrm{Al}$ lembert, been noted for being more ready themselves to publish than to become acquainted with what others have done; and one consequence of this has been the formation of a mathematical literature, able, profound, and original, but cumbrous, fragmentary, and full of repetitions. ${ }^{2}$ Besides, the seventeenth century had attained the vantage-ground of those grand and striking improvements in methods to which no subsequent improvements, however real and ingenious, can by possibility compare. We shall never have inventions comparable, in universality and importance, to the application of Algebra to Geometry, and the discovery of Fluxions. These also admit of being at least partly explained in language not obtrusively technical, and have been so explained by the facile pen of Playfair; but all subsequent discoveries have been but enlargements and improvements on these primary and distinguishing ones; and before the date at which our present discourse properly opens, even the larger generalizations of Newton's fertile calcu. lus - the method, namely, of Variations, and the integration of partial differential equations, had been established, by the genius of Euler and Lagrange, on an impregnable basis.

The intense, and praiseworthy, and successful labours of their followers have been, then, chiefly devoted to the occupation of the ficlds of conquest thus summarily opened; or, rather, to storming, one by one, fortresses still unreduced, after the main resisting army had been first routed in the open field. To quit metaphor, the efforts of mathematicians have for many years been chiefly applied to rendering possible the solution of problems involving quantities which actually occurred in the course of the rapid simultaneous advances of physical science. They are in a manner inseparable from the branches of physics in aid of which they were originally called forth, and will therefore be most properly noticed, however briefly, in the chapters of this Dissertation where their application is considered. Some farther observations on this subject will be found in the immediately succeeding section of the present Essay.

In reviewing the progress of science-physical science in particular-during the last seventy or eighty years, I have thought it advisable not to sion of Phy subdivide the subjects too minutely, and, followi sico-Matbe nearly the arrangements of Dr Whewell's excellent Sciences. treatise, already quoted, ${ }^{3}$ I shall treat, in successive chapters, of Analytical Mechanics including Physical Astronomy as their loftiest and most successful application; of Astronomy as a science of observation; of Mechanics, with reference to the intimate constitution of matter, including Hydrodynamics, Acoustics, and Civil Engineering'; of Optics, or Light; of Heat, including the Daltonian theory of the gases and chemical elements; and, finally, of the large and comprehensive science of Electro-magnetism, including ordinary and Voltaic Electricity, Terrestrial Magnetism, and Diamagnetism the discovery of Dr Faraday.

The arrangement of the chapters is thus strictly Methodical; but in the subdivision into sections, I have allowed the Biographical principle to predomi-ment partly nate, thus character to the whole, and endeavouring to intro-graphical. duce the reader to the intellectual acquaintance of the eminent men who are selected for notice on the principles which have been already detailed. In some

1 Specimens of what a history of pure mathematics would be, and must be, are to be found in the able "Reports" of Dr Peacock and Mr Leslie Ellis, in the Transactions of the British Association for 1833 and for 1846 . A glance at these profound and very technical essays will show the impossibility of a popular mode of treatment, whilst the difficulty and labour of producing such summaries may be argued from their exceeding rarity in this or any other language.

2 The celebrated Lagrange, in his later years, contrasting the mathematical works of his own generation with those which he stadied when a youth, is said to have observed :- " I pity the young mathematicians who have so many theories to wade through. If I were to begin, I would not study ; these large quartos frighten me too much."-(Thomson's Annals, vol. iv.) And it is stated that whilst his own most abstruse investigations were conducted in Paris, he kept the perusal of M. Gauss's writings for the tranquil retirement of the country, - a distinction intelligible enough between the intense effort of invention more than sustained by the vis viva of genius which prompts it, and the strain required to master the dead weight of reasoning imposed upon the mind by the discoveries of another. Dr Young, in his biography of Lagrange, observes upon the voluminous mathematical literature of his time, that " unless something be done to check the useless accumulation of weighty materials, the fabric of science will sink in a few ages under its own insupportable bulk." The fact is that a large proportion of the mathematical writings of even eminent authors are in a few years forgotten, or only casually consulted on some matter of history.

3 History and Philosophy of the Inductive Sciences. 
instances, however, it has been necessary to introduce the same individual into two or three different sections, and even into different chapters, when his pursuits have been in very various branches of science. This has been avoided, however, as much as possible, and a sacrifice has occasionally been made of the methodical order of the subjects, so as to combine in one view all that has made an eminent philosopher illustrious. Such little sacrifices of arrangement are incidental to the way of treating the whole subject; and it may be hoped that its practical advantages, in the eye of the general reader, will be found to compensate for its defects as they may appear to the more rigorous student. For the sake of the latter especially, a short but comprehensive index of Names and Terms is prefixed, by which, I believe, it will be easy to trace all that is said of any one person or subject in any of the chapters. ${ }^{1}$

\section{§2. On the relations between Matheniatics and Natural Philosophy, and between the latter and the Mechanical Arts.}

(24.) The object of this Dissertation has little in comConnection mon with an attempt formally to subdivide human of Mathe- knowledge into compartments, and to assign their matics, mechanical in their practical bearing on one another that they arts. must be considered. If one science, like Mathematics, furnish the only sure step towards the understanding or the enlargement of another, as Astronomy or Optics, a practical link is constructed between them, which renders the progress of the one not independent of the progress of the other. The intimate and reciprocal connection thus subsisting between Mathematics and Physics is to be found in almost an equal degree between Pure Physics and the Mechanical Arts, of which we take Civil Engineering to represent the department most cognate to that of Natural Philosophy, of which this Dissertation more especially treats.

(25.) The history of the last seventy or eighty years Boundaries enforces this conclusion. The boundaries of Science of Science and Art are as undefinable as those of "fact" and and Art un- "theory," or those which separate the kingdoms of

(26.) hardly be called scientific, and there are others which have contributed more to the original stock of knowledge than they ever drew from it. These last are like the shoots of those tropical plants which at first are mere buds upon the trunk, and are nourished solely by its juices, but which, when they reach the ground, plant themselves there, and become not only the props and stays of the parent stem, but supply it from an ever-increasing area with the sap which they originally borrowed.

The more closely we examine the subject, the more are we satisfied that it is impossible to teach science

rightly without teaching its applications; and that the limit to which we are to do so is a limit depending solely on the judgment of the teacher, and on the special purpose of the lesson. But the progress of science is a lesson learnt from the great book of experience; and if we are to feel the force of its teachings, we must consult, not one, but many of its pages. Looking to the history of science since 1750 , but especially during the present century, it is quite impossible not to admit how large a share the sciences of application have had in moulding the direction of men's thoughts and speculations, and in enabling, nay, compelling them to realize certain abstract notions far from easy of conception. Instances of this are to be found in the force of percussion, the coexistence of vibrations in air and other substances, and such notions of body as we derive from practical efforts of continually-increasing boldness to extend the scale of our constructions.

The analogy of the relation between Mathematics and Physics, and of the latter to civil Engineering, is Practical so close that the three subjects might almost be character presented as three terms of a continued proportion. 100 years. What the second is to the first may be affirmed of the third relatively to the second. Physics may exist, at least to a limited extent, without a mathematical basis, as the art of construction long preceded a knowledge of the principles on which it is founded. But as knowledge advances it extends in both directions towards speculation and towards practical applications, but most towards the applications. This Bacon well understood, and he has consistently maintained, that knowledge, to be profitable to its cultivators, must also be fruitful to mankind. And all the history of science since Bacon's day has read this

1 I have borrowed but sparingly, in the following pages, from the existing compilations on the bistory of science. Indeed, a writer who intends to make a subject his own by a well-considered, fundamental plan of treating it, will use such works principally as a guide to his own further inquiries, and to assist him in selecting the topics worthy of fullest discussion. In this respect Dr Whewell's excellent writings, already cited, have been of great use to me; and in the particular department of Astronomy, I have often referred to Mr Grant's valuable History of that science, as well as to the writings of Delambre, and the very elaborate Historical Essay by M. Gautier, on the problem of the Three Bodies, which is not, I think, noticed by Mr Grant, but which contains a most elaborate history of the researches of Lagrange and Laplace. In optics, I have consulted the systematic treatises of Dr Young, Sir D. Brewster, Sir J. Herschel, Dr Lloyd, M. Moigno, and M. Radicke; and so of other subjects. Gehler's Physikalisches Worterbuch, Fechner's Repertorium, and that of Dove, afford a vast amount of historical information. The Transactions and Proceedings of the various societies, British and Foreign, have of course furnished a great part of my information. Such strictly biographical details as I have made use of, have in general been very carefully taken from the best accessible authority. 
lesson more and more loudly in the ears of mankind. The era of Newton and Leibnitz was grandly distinguished by the continually increasing applications of mathematics to physics, whereof Newton was the great teacher ; the century 1750-1850, whilst profiting by the lessons of the past, has added almost a new one in the eminently practical character of its science, and in the no less scientific character of its practice. The result of these gradual modifications of human knowledge has not been in the slightest degree injurious to the real progress of the more abstract inNot inju- gredient of the mixed sciences. Did mathematics rious to ever flourish more vigorously than under Newton? abstract has pure physical science had greater triumphs than science. in the era of Volta, Watt, and Young? It was precisely because the new application of mathematics stimulated their growth, because abstract relations of quantity were vivified by concrete solutions of physical problems, that a new geometry arose. Dynamics could hardly be said to exist as a science without the invention of Fluxions as a language by which its conditions and results might be expressed; and from that time onwards, the necessities of the natural philosopher have been the prime sources of inspiration to the geometer, while the subjects have become so blended that a mere discoverer in mathematics has become a singularity. It would be hardly possible to point out any mathematician of the highest class since Newton, or but a few of the second class, who have not contributed almost as much to physical science as they have to analysis. Of purely mathematical discoveries, the great majority have been called forth by the immediate necessity arising from some problem requiring solution in astronomy, mechanics, optics, or heat. Lagrange's method of Variations of arbitrary Constants in Integration, the artifices for the computation of attractions by Laplace's coefficients ; the introduction of the method of factorials by Kramp in his solution of the problem of refraction, and numberless improvements in the Theory of Definite Integrals by Fourier and his successors, sufficiently warrant the statement, and show how richly the physical sciences have repaid to the purely mathematical ones the debt which they originally owed. One other conclusion may be drawn from these and parallel facts. It is that the combinations arising out of external phenomena are more suggestive of the possible relations of number and quantity than is the most unlimited stretch of fancy and imagination; and $I$ believe it will be conceded that, with few exceptions, theorems of the greatest value and beauty have been more frequently discovered during the attempt to solve some physical or at least geometrical problem, than in comprehensive yet indefinite attempts to generalize the relations of abstract magnitude. torical fact of the paucity of pure mathematicians, The pure and of distinct mathematical treatises of a strictly Matheoriginal character in an age distinguished by the diffusion of mathematical knowledge, and in countries (like France) most celebrated for its triumphs. There are not, perhaps, much more than half a dozen really great mathematicians of the last seventy years, who have not left treatises more numerous and more distinguished on physical science, treated mathematically, than on pure mathematics. Among the exceptions which more immediately occur, are Monge, Legendre, and Abel. And of distinct treatises, whilst we have the Mécanique Analytique, the Mécanique Céleste, the Theorie de la Chaleur, and numberless others, containing precious mathematical developments, in connection with the applications which suggested them, the purely mathematical memoirs of the same period are to be sought chiefly in the form of detached essays, in the ponderous volumes of Academical Transactions.

One point in the History of Mathematics has especial interest for the English reader, and as such may be touched upon here with reference to the progress of science for the last three quarters of a century. The national pride of England in the triumphs of Newton impelled her ablest mathematicians to attempt to carry forward the synthetic methods which he had chiefly used, at least in his published works, to the more arduous and intricate questions of $\mathrm{Me}$ chanics and Astronomy which presented themselves for solution in the course of the 18th century. Maclaurin was almost the last Englishman of that period whose mathematical writings came into direct competition with the rising schools of Germany and France. The labours of Matthew Stewart, and Simpson were mostly geometrical; those of Landen and Waring, though profound, created little general impression; and, gradually, the extent and difficulty of the foreign mathematics, increased by the use of the Leibnitzian notation of differentials which was absolutely unfamiliar in England, deterred almost every one even from perusing the writings of Clairaut and D'Alembert, Lagrange and Laplace. Of the continental mathematicians, Euler was probably the best known, owing to the lucidity of his writings and their eminently practical tendencies. Some idea may be formed of the negation of mathematical talent in Britain during the later portion of the last century, when we find D'Alembert declaring, in 1769 , that if an Englishman is to be elected one of the eight foreign associates of the Academy of Sciences, he will vote for Earl Stanhope as the best mathematician there, as he believes, not having read any of his works! If the choice was to be free, he should prefer M. de Lagrange! !1 A more cutting, though unintentional satire on the state of Mathematics in this country could not have been written. country.

.

.


(30.) The commencement of a better era originated, early At Cam- in the present century, with Woodhouse at Cambridge, Edinburgh, and Playfair in Edinburgh, by both of whom the conand Dublin. tinental methods were introduced into the studies of their respective universities; whilst Ivory, a native of Scotland, was the first to challenge, by his writings, a place in the list of great living mathematicians. The systematic form of the Mécanique Céleste rendered the subject more accessible than were the countless memoirs by men of the highest name, which then filled the Transactions of Paris, Turin, Berlin, and StPetersburg. But the notation of differentials, which could alone break down the barrier between the British and foreign mathematicians, was first introduced at Cambridge by the efforts of Sir John Herschel and Dean Peacock about 1816, soon after which the translation of Lacroix's Differential Calculus, which they superintended, came into use as an university textbook. From this time the works of foreign mathematicians began to be more generally read, particularly the writings of Laplace and Poisson; and within ten or a dozen years subsequently, a few active and undaunted men, chiefly of the Cambridge school, such as Mr Airy and Sir John Lubbock, grappled with the outstanding difficulties of physical astronomy, whilst a larger number applied themselves to the most difficult parts of pure analysis, and acquired great dexterity in its use in the solution of geometrical and mechanical problems. Such, for example, were $\mathrm{Mr}$ Babbage, $\mathrm{Mr}$ De Morgan, $\mathrm{Mr}$ Murphy, and Mr Green; and at Dublin Sir William R. Hamilton and $\mathrm{Mr}$ MacCullagh, whose names will occur in other parts of this Dissertation.

No new calculus or great general method in analysis has resulted from these persevering labours, whether of British or foreign mathematicians, but an increased facility and power in applying the existing resources of mathematics to the solution of large classes of problems previously intractable, or resolved only indirectly or by approximation. The Integral Calculus, in particular, affords an almost boundless field for research, and each branch of science in succession-not only Physical Astronomy, but Optics, Heat, Electricity, and Civil Engineering - has offered problems of great importance, which awaited only the skill of the pure mathematician to resolve in a practical and finite form. ${ }^{1}$ Every year, and every civilized community, contribute to these real improvements. The principle of discontinuity, con- spicuously introduced into the doctrine of the conduction of Heat in consequence of the abrupt variation of physical circumstances at the boundary of the conducting body, enters largely into the speculations of mathematicians of the present century; and the doctrine of definite integrals so intimately connected with it has received a proportional extension. Next, analytical geometry has acquired a very great Analytical enlargement and by attention principally to symmetry geometry. in the arrangement of the results, solutions otherwise the most intricate are obtained with facility and directness. Of this we shall find examples in our history of the Undulatory Theory of Light. Lastly, The Calcunotwithstanding the pre-eminently practical charac- lus of Opeter of the mathematics of the last age, speculative rations. geometers and analysts have found time to discuss the metaphysics of their respective sciences, both as regards the foundations of the Differential Calculus and as to the use of imaginary and other symbols in Algebra. An almost new branch of abstract science (though faintly foreshadowed by Leibnitz) has come into existence-the separation of symbols of operation from symbols of quantity, and the treatment of the former like ordinary algebraic magnitudes. In some cases remarkable simplicity is thus introduced into the solution of problems, although perhaps few mathematicians would choose to depend implicitly upon the method in untried cases. Sir John Herschel and the late Mr Gregory ${ }^{2}$ were amongst the most active introducers of this new algebra, but few of the more eminent living British or foreign mathematicians have failed to contribute their share to this more metaphysical department of analysis.

I shall now attempt to consider more particularly the reciprocal relations of pure physical science Connection and the mechanical arts.

This is evidently a very intimate oncend core dis-the arts. tich as Astronomy, Acoustics, Magnetism), are the results of either observation or experiment, and they consist in generalizations, by means of which a multitude of facts are reduced under one simple expression of a more general fact or principle. But instruments often very complicated are necessary for observation and for experiment; as telescopes in astronomy, organs in acoustics, properly magnetized and suspended steel bars in magnetism. Art is required to construct these. The highest possible degree of science, and the utmost

1 For example the Lucasian Professor at Cambridge, Mr Stokes, has effected two previously impracticable integrations, one occurring in the theory of the rainbow, the other in that of railway girder bridges.

2 Mr Duncan Gregory, a promising mathematician who died 23d February 1844, at the early age of 30 , was the youngest Mr Duncan son of Dr James Gregory, the late distinguished Professor of Medicine at Edinburgh. His name deserves a passing record, not only Gregory. from the influence he exercised on the progress of the English mathematics of his time, but as having revived the dormant character for this peculiar kind of talent, so long connected with the family of Gregory. He was, in fact, the lineal descendant of the inventor of the reflecting telescope. Mr Gregory was the first editor of the Cambridge Mathematical Journal, and author of an excellent book of Examples in the Differential and Integral Calculus, both of which have exercised a beneficial influence on the progress of science in England. He also wrote several original memoirs on the subjects referred to in the text. Mr Leslie Ellis, a man of congenial ability, has written a short but pleasing hiography of his friend. 
mechanical skill, are both indispensable to make a telescope. A telescope then, though a mechanical invention, inasmuch as it is a deduction from physical principles, not an addition to them, is yet a deduction so ingenious-so far from obvious-so impossible to be conceived accidentally or by a loose thinker, and so easily made the instrument of innumerable discoveries of the highest novelty and grandeur, that no historian of science ever thought of omitting the invention of the telescope, or of giving it an importance inferior to that of a discovery, containing as it did the germ of so many discoveries. In like manner a theory of optics might be written in which a telescope need never be mentioned; but would not the pedantry of such a work be obvious, or would any reasonable person wish to learn science after such a fashion?

(34.) The clear co-ordination of the parts of an invention Great Me- towards the attainment of a given result, with a due inventions regard to natural laws, and the properties of the subpresume a stances used, constitutes the merit of the invention; knowledge and this merit may be irrespective of the precise imlaws; portance of the end of the invention, which may be intended to promote science, or commerce, or convenience, or even to satisfy mere curiosity. A telescope would have been a telescope still, could we have imagined it invented with no other object than a deerstalker's sport; and so contrivances which in their origin and application seem remote from scientific uses, constitute nevertheless real steps in the progress of knowledge. The steam-engine is one striking example. Originally devised with an exclusively commercial object-the extrication of the Cornish mines from subterranean water, it became in the hands of Watt, first an instrument for experiments on the relation of heat to matter; next, in its improved form, a beautiful exemplification of these laws, and an enduring monument of the sagacity and skill of its author, as well as the most important inorganic agent which exists in modifying the social condition of the entire globe. Finally, to illustrate the position from which we started, it becomes the instrument of fresh discoveries. This very engine has a theory to be worked out, probably unimagined even by its sagacious author-its operation as an agent for obtaining power from matter by the application of heat, is shown to be in all probability a single case of a more general law, including all kinds of machines and all sorts of matter; and this more general theory of heat as a motive power leads, once more, to new practical deductions, to the conditions under which such machines may be most usefully constructed and employed. ${ }^{1}$

(35.) Every instrument, every construction, which is and gene- founded on a theory, and in which a certain compli-

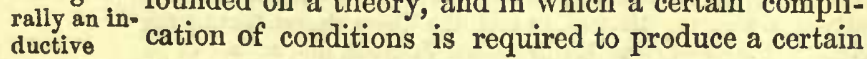

result; a telescope, for example, or a steam-engine, or a bridge, is, in the first instance at least, an experiment. Few inventions are so simple and straightforward in their plan, are so independent of the seemingly capricious behaviour of matter under untried circumstances, or depend so entirely on physical laws thoroughly understood, that the inventor can await, without the pang-at least of impatience-if not of anxiety, the moment of the realization, by actual trial, of his hopes and his calculations. We can all readily imagine the throb of anxiety with which Galileo pointed his glasses for the first time to the moon-with which Watt saw the cylinder of his As in model exhausted, and the piston descend under the Watt's action of his separate condenser-and Stephenson, gine. the stupendous iron tube at Conway resting for the first time straight as a ramrod on its two piersthese are moments of anxiety and of triumph, which place the inventor of a machine and the architect of a structure on a par with the discoverer of a planet, or with the author of a theory. "Whenever an original mind produces new combinations of thought and feeling,' says Sir James Mackintosh, with equal impartiality and truth, "whether its means be words or colours, or marble or sound, or command over the mighty agents of nature; whether the result be an epic poem, or a statue, or a steam-engine, we must equally reverence those transcendent faculties to which we give the name of genius." 2 It is almost needless to add the caution, that such praise is only applicable when the invention is such as to call forth the qualities which distinguish the Philosopher. It is not the mere command over the agents of nature which challenges our admiration, it is the foresight, the patience, the conceptive faculty, the clear-sighted and confident anticipations of what will be the results of natural laws acting in given circumstances, these circumstances being in some essential particulars new. Merely to adopt known contrivances, where experience has already anticipated the result, may exercise judgment, but hardly genius; and to make contrivances in which the result depends rather upon laws of geometry than of physics, hardly come within the scope of these remarks.

Watt's Parallel Motion, perhaps the most ingenious of his inventions, would not have made a great reputation; nor does the endless variety of machines used in the arts, as in spinning, printing, and papermaking, stand higher. It is when the inventor places Matter in new relations to Force, or derives power from new sources, or teaches Light or Electricity to act under new conditions, that he becomes really a Mechanical Philosopher.

It is not given to man to endue matter with new properties, or to prescribe the laws under which his The limit inventions are to take effect. A new motive power, of arto 
a new form of construction, are experiments on the resources of nature under new conditions. (38.) In fact, even in comparatively simple cases, we mechanical cannot set forces to act on matter, or dispose matas of ma- ter so as to resist force, without doing not only what thematical we intend to do, but also a great deal more. Man problems sometimes manifold. may put powers in motion which he is unable to control; and whilst he calculates confidently upon the effects of such and such dispositions of force or resistance, he may overlook consequences equally necessary, because resulting from laws of nature which are either unknown to him, or the magnitude of which he had overlooked, in considering those only which he required. A complicated mechanical contrivance may be compared to the mathematical solution of a problem. It represents commonly a great deal more than is meant to be derived from it. It may represent several distinct results, some possible, some impossible, and of the former only one, it may be, congruous to the real conditions of the problem proposed. In mechanics, the laws of nature are as impatient of control as the laws of quantity in geometry, and the engineer may find, too late, that nature has solved his problem differently from what he expected. But even when successful, it is to bo presumed that his own contrivance contains within it results unforeseen by himself. If he is wise he will become a student in his own workshop. The material contrivances are indeed his own, but the powers which they awaken or distribute are beyond his control. Even if his reading of the equation be strictly correct, there may remain in the background others no less important.

The considerations here imperfectly laid before the reader are intended to justify the introduction of certain practical topics into the present Dissertation, which, though many readers will see their insertion without surprise, or would have been sorry to find them omitted, others possibly may think more or less independent of, and separable from a scheme already sufficiently extensive and intricate, if confined to mere subjects of scientific doctrine, to the exclusion of its applications. My chief reason for including such subjects as the steam-engine, the strength of materials and some great examples of construction, and the electric telegraph, is that these important practical improvements are both historically and logically interwoven with the progress of pure or abstract physics. They have besides impressed upon the character of scientific discovery of the last hundred years a peculiar stamp which it would have been absurd to ignore while endeavouring, within a moderate compass, and in the plainest language, to convey a vivid though comprehensive sketch of the advancement of Natural Philosophy during this and the preceding, or rather two preceding generations.

It is not to be imagined that the difficulty of the problems which occupy the speculative philosopher, Lessons of or the comprehensiveness of mind required for their ordinary solution, diminishes in any degree as we descend from the regions of pure science to the walks of everyday life-from the vast periods and majestic motions which astronomy enables us to explain and predict, to the common details of the workshop and the railway. In fact, the former are to be regarded as the simpler investigations, whilst our terrestrial agents have their effects modified by the diversified states of aggregation and various mechanical properties of matter, and by the numerous modifications of force arising from heat, electricity, or magnetism, to which it may be exposed. We have as yet made but an insignificant advance towards that completer system of Natural Philosophy of which Newton's will form but one section, in which all the properties of matter and their consequences shall be as well understood as the particular property of gravity is at present. Many of these are to be learned by daily observation of the effects which occur in the ordinary progress of civilization amongst us. We are continually performing experiments on a great scale and on purely commercial principles, which no individual philosopher or merely scientific society could have ventured to attempt. And in the midst of these appeals to experience, unexpected results are frequently occurring which send us back once more to the study of first principles, which, indeed, while they confound the empiric, do but establish the reputation of the philosophic engineer, who seldom fails to turn them to good account, both in his theory and practice.

\section{CHAPTER II.}

\section{PHYSICAL ASTRONOMY AND ANALYTICAL MECHANICS.}

\section{\$1. Lagrange-Variation of Parameters-Application to Physical Astronomy. of the Planetary System; Laplace; Poisson. Moon's Libration.}

The Stability

(41.) The period of Lagrange's most celebrated labours Lagrange. extends so far back into the preceding century, that having been already mentioned in Sir John Leslie's
Dissertation, it might have been excusable, with so great a mass of matter before us, to have passed them over without farther notice. But they are so 
intimately connected with the most salient points of the history of physical astronomy, down even to the present time, and are so interwoven with the discoveries of Laplace, and represent altogether so much of the substantive character of the progress of the age, that I have thought it necessary to devote a small space to the recital of a few of the most prominent of them, having regard to the intellectual portraiture of the man as one of the most pre-eminent and successful reasoners of his class who have ever done honour to their race. I shall repeat as little as possible what has been said elsewhere, and confine myself to only two or three topics. (42.) Joseph Louis Lagrange was born at Turin in 1736;
birth he died at Paris 10th April 1813. His first paper His birth he died at Paris 10 th April 1813 . His first paper
and educa- was written at the age of 17 or 18 , and his end was tion; accelerated by the unremitting ardour of his labours at the age of 77. He was consequently an original author during sixty years; and for the greater part of his contem- this period he, together with Laplace, monopolized the poraries. greatest discoveries connected with analysis and physical astronomy, and exercised an almost undisputed authority in the more recondite sciences. Euler, Lagrange, and Laplace, by a singular coincidence, lived to the respective ages of 90,77 , and 79 , and all retained their activity nearly to the last. They produced, by the continuity and friendly rivalry of their labours, carried to an extent in each case which only astonishing physical vigour united to astonishing mental aptitude could have produced, during almost a century, an impression on the progress of science altogether remarkable. This coincidence was the more happy, because physical astronomy was exactly in that predicament when nothing less than such a combination of intelligence and intensity of application systematically urged, could have carried Newton's theory through the difficulties which at that time beset it-difficulties which left the Principia for so many years alone, and far in advance of the general intelligence of the age.

(43.) The pregnant suggestions of Euler were developed Euler and and applied by Lagrange, and the triumphs of LaLaplace. grange-nay, even his occasional failures-were the immediate precursors of some of Laplace's happiest efforts.
Amongst the former we reckon the method of the variation of parameters, expounded to a certain point by Euler, though, as in many other cases, his results of parame were vitiated by the haste and inaccuracy of his cal- ${ }^{\text {ters }}$; culations. That Lagrange borrowed the idea from Euler cannot admit of a doubt, any more than that he was indebted to him for the principles of the Calculus of Variations. Lagrange, with customary truthfulness, even to his latest days, always spoke of Euler as his best instructor and model, and as the chief of modern mathematicians, Newton only excepted. We know that he so regarded him in the case of the calculus of variations which he studied in Euler's "Methodus inveniendi lineas curvas, \&c.," during the first two years of his application to the higher mathematics ${ }^{1}$ whilst Euler, with equal candour, acknowledged the transcendent genius of the rising geometer, forcing its way where he himself had failed.

The method of the Variation of the arbitrary constants or Parameters, though it may be regarded in its signifione point of view as a merely analytical artifice for cation. effecting integrations, is in reality a conception purely geometrical, first introduced by Newton ${ }^{2}$ under the name of "revolving orbits," and applied by him to the explication of the conception (to use a recently introduced phrase) of the lunar inequalities. Neither the moon nor any planet really describes a mathematical ellipse (in consequence of the mutual perturbations of the heavenly bodies). They describe curves of double curvature in space, of which we could form. no intelligible idea, except by referring them to the very approximate type of the ellipse, of which the eccentricity, line of apsides, inclination, \&c., are continually varying, not only from one revolution to another, but throughout every part of a revolution. This representation is not only convenient, but strictly accurate. At each instant the moon or planet is describing a portion of an ellipse, which may be called the instantaneous ellipse, and which instantan may be defined as the particular ellipse which the ous ellips body would go on to describe if it were at that instant freed from all perturbation, and allowed to complete a revolution under the single influence of its acquired motion and the central force. To take

Lagrange's 1 The following is a list of the books be then read, taken from a paper probably little known, which appeared soon after the early death of Lagrange in the Moniteur newspaper, and which was translated in Thomson's Annals of Philosophy, vol. iv. He studies. first read Euclid's Elements, Clairaut's Algebra; then, in less than two years, and in the following order, Agnesi's Analytical Institutions, Euler's Analysis of Infinites, John. Bernouilli's Lectures, Euler's Mechanics, the two first books of Newton's Principia, D'Alembert's Dynamics, and Bougainville's Integral Calculus, Euler's Differential Calculus and Methodus Inveniendi -a precty course of mathematical reading for a youth between 17 and 19 .

From the same paper we abridge a few practical directions given by Lagrange for the study of mathematics, which, if tolerably obvious, are interesting from the extraordinary genins of the man, and from his singular reticence on subjects of a personal nature. "I never," be said, "studied more than one book at a time; if good, I read it to the end." "I did not verplex myself with difficulties, but returned to them twenty times if necessary. This failing ,I examined another author." " $I$ considered reading large treatises of purs analysus qunte useless. We ought to devote our time and labour chiefly to the applications." Thus he read Euler's Mechanics when he had acquired a very slight knowledge of the differential and integral calculus. "I always read with my pen in my hand, developing the calculations, and exercising myself on the questions."

"From the very beginning of my career, I endeavoured to make myself master of certain subjects, that I might have an opportunity of inventing improvements; and $I$ always, as far as possible, made theories to myself of the essential points, in order to fix them more completely in my mind, to render them my own, and to accustom myself to composition." "Finally, I every day assigned myself a task for the next. I learned this custom from the King of Prussia."

2. This Lagrange himself points out in his Mécanique Analytique. 
a single example; the planet Uranus has not yet completed one revolution since the time of its discovery in 1781 , yet its observed path differed so much from a true elliptic arc (even when we allow for the perturbation of Jupiter and Saturn), that the orbit which satisfied the observations from 1781 to 1800 would not satisfy those from 1800 to 1820 ; and since 1820 a new orbit had to be computed for every few years, so great were the variations of the instantaneous from any permanent ellipse. These variations led to the discovery of the planet Neptune.

(46.)

To adapt the notion of the perpetual variation of Plauetary the elliptic elements to analytical calculation, and to

(47.)

Periodic and secular inequalities. volutions now, and compare them with a similar average hereafter, provided that the orbits undergo no permanent change. This, therefore, though not strictly an inequality, because the length of the year is permanently changed by it, shows an average effect independent of the configuration of the planets. An example of a true secular inequality is the revolution of the line of apsides or major axis of any orbit, by the influence of the disturbing forces of the planets, whether interior or exterior to the one considered.

Few of the secular inequalities have been detected by Change observation throughout the entire records of Astro-apsides and nomy. It is known, however, that the apsides of the excentriplanetary orbits (at least in the case of the old ${ }^{\text {cities. }}$ planets) all progress, with the exception of those of Venus, which retrograde, and that the inclination of all is at present diminishing. The excentricity of the Earth's orbit is decreasing at the rate of 40 miles per annum. The exclusive dependence of the secular inequalities on the orbits, not on the places of the planets, may be well illustrated by a method actually employed by Gauss for computing them (though it does not appear to be attended by any special advantage). He conceives the orbit of the disturbing planet to be strewed with attractive matter, whose thickness at any point is inversely as the planet's velocity there, or directly as the time of its sojourn in any small length of the orbit. After it had been clearly recognised, principally by the solity of
The method of variations of the elements is evidently most applicable to the determination of Secular Perturbations ; for to compute by means of it the ordinary inequalities involves an apparently unnecessary labour. Theplace of a planet is completely determined by three co-ordinates-its longitude, latitude, and radius vector; whilst theelements of the orbitare six in number, and when found, a further calculation must be made to find the co-ordinates of position. The more direct method of deducing the co-ordinates at once from the conditions of perturbation, was generally followed until 1808, when Lagrange and Laplace almost simultaneously devised methods of using the variation of the elements with directness and despatch in the calculation of Planetary Perturbations. In the estimation of the second and higher orders of disturbance, it has even the advantage in these respects over the other method.

Stability and Permanence of the Solar System.- (50.) the labours of Lagrange, that the elements of the pla- system. netary orbits are in a condition of perpetual change, it came to be a most interesting question how far such variations were likely to be continuous, and ultimately so great as to modify altogether the forms of the orbits, and even endanger the separate existence of the planets. This is a question which has excited a very general, as well as scientific interest. It is evident that the variations of the different elements are not all equally important in affecting the permanence of an orbit. The five properly orbital eletricitios of the lutions) of Mars and the Earth, the attraction of the former on the latter sometimes conspires with the sun's attraction, sometimes opposes it, some urges the Earth forward in its path, and sometime pulls it back, producing numerous periodic inequalities; but it is quite evident that, in the long run, the attraction of Mars on the Earth tends to pull it away from the sun, and to diminish the effect of the solar attraction-in fact, to increase the length of our year; and that this influence will be precisely the same if we take the average of a great many re- 
ments (the sixth being the longitude of the planet on its orbit at a given time) may conveniently be considered thus: 1st, the major axis, which, for one and the same system, involves the periodic time or mean motion; $2 \mathrm{~d}$, the excentricity and position of the line of apsides; 3d, the inclination and position of the line of nodes. Of these, the stability primarily depends upon the first. If the major axis and mean period increase or diminish without limit, the planets will diverge into infinite space, or rush after myriads of ages to utter annihilation in the burning embrace of the sun. The latter alternative was the popular belief about the middle of last century, and was maintained by the grave authority of Euler; whilst Darwin, in his florid but picturesque language, described the order and beauty of the planetary system as but a little more permanent than the glowing ornaments of the gay parterre. ${ }^{1}$ The principal reason for this conclusion, and its refutation, will be mentioned in the next section.

(51.) The first person who perceived the probable staLaplace's bility of the major axes and mean motions was not share in the Lagrange but Laplace, who, in a paper published in 1773 , gave a demonstration, the sufficiency of which has not been doubted, that the major axes are invariable, so far as the influence of the principal terms of the disturbances are concerned, that is as far as terms containing the cubes of the excentricities inclusive, and the first powers of the perturbing masses. Nor does Laplace appear to have doubted that the mutual distinction of the terms, including secular changes, was not accidental, but would extend also to the farther approximations. Lagrange, however, in a celebrated though short memoir of 1776, demonstrated the truth of the conclusion for the higher powers of quantities contained in the perturbations of the first order, and that by methods peculiarly comprehensive and elegant, which he farther extended in 1781 to the other five orbital elements, showing the periodicity within certain narrow limits of the excentricity and inclination, the only elements, except the major axis, whose variations menace the stability of the system. Yet it is quite impossible to separate completely the names of Lagrange and Laplace in the effectual demonstration of this important truth, the former as frequently in- dicating the means of overcoming the more purely mathematical difficulties, as the latter was suggestive and far-sighted in anticipating their application to the peculiarities of our system.

Laplace discovered (1784) two remarkable theorems which limit the whole amount of the excentricities and inclinations of the orbits of the planetary system, showing that if once small, they must ever clinations remain so; and, in particular, that the most massive of planeremain so; and, in particular, that the most massive of plane-
planets of the system (Jupiter and Saturn) must also tary orbits undergo the most trifling variation in these respects. In the case of the small planets between Mars and Jupiter, a wider range may occur (as indeed we practically find to be the case), without endangering the permanency of the whole. It also follows that these variations, though "secular," are practically " periodic ;" that is, that the excentricities and inclinations oscillate about certain mean values and within extremely narrow limits, the periods of these oscillations being also of vast duration. Concerning such changes, theory is our only guide. The whole duration of astronomical records can barely reveal the existence of two or three of them, and tells us absolutely nothing of their remoter consequences. Lagrange calculated the superior limits of the excentricities of the larger planets, and M. Leverrier has recently, by more accurate methods, obtained results nearly coincident. According to him, the maximum excentricity of the Earth's orbit is 0.07775 , the minimum 0.003314 , so that it can never be quite a circle. It is now diminishing, and will continue (according to the same geometer) to do so for 24,000 years, when it will begin to increase. The inclinations of the Earth's orbit to its equator, and also to a fixed plane, are confined within definite limits which are not perhaps very perfectly known.

The motions of the apsides and nodes of the orbits which gradually complete the entire circumference have manifestly no tendency to affect the stability of apsides an the system. The grand cycle of the Earth's perihelion will only be completed in 110,000 years. It coincided with the vernal equinox 4089 years before Christ, a period (as Laplace remarks) nearly coincident with that assigned by chronologers to the creation.

These results may be considered as among the most astonishing with which science brings us ac-

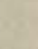

.


quainted. The range of insight which man has acquired into the past and future history of the universe throughout periods, compared to which, the whole existence of his species is but a span, enhances our admiration of the reasoning power which can attain to knowledge so high and excellent. And the sublimity of the contemplation is increased when we recollect that these recondite truths are all consequences of a law so simple as that of gravity. Observation will reveal only to a late posterity the secular modifications of the planetary orbits which geometry now predicts to us. Some of the ellipses will elongate, whilst others tend to become circles; their planes will vary in inclination, but ultimately be stayed within the limit which human sagacity had predicted myriads of years before. "These," says a French analyst, "are the pendulums of eternity, which beat ages whilst ours beat seconds." And amidst all these variations, subject to law and to impassable limits, the Major Axes of the orbits preserve a stedfast uniformity, or are subject only to transient fluctuations; and thus permanence arises in the midst of change, and the perfection of the system is demonstrated by the very nature of the disturbances which seemed at one time inevitably to limit its duration.

It remains to add, in closing this interesting Poisson's discussion, that Lagrange himself had not quitted the addition to fieory. field before his able disciple and follower, Poisson, pursued the inquiry of the stability of the system, and the permanency of the major axes particularly, to a degree of approximation not before attempted. He included the perturbations of the second order, or those which arise by correcting the elements for the disturbances first found, and including the effects of the correction in the modification of the perturbations themselves. These also are subject to the same laws as found by Laplace and Lagrange for lower degrees of approximation; and as M.M. de Pontecoulant and Leverrier have confirmed the result (at least for all the larger planets of the system), we may conclude it to be a truth as firmly established as any negative fact can be, that our system is arranged for a duration apparentlyindefinite; that if the planets cease to roll, and the sun and moon to do their office in enlightening the world, it must be in all probability by an interposition of Almighty power, as direct and immediate as the creative energy by which they were launched into space, and (our earth at least)peopled with successive races of animated beings. ${ }^{3}$

We have, in the commencement of this section, disclaimed the intention of entering at large upon the history of Lagrange's discoveries. They fell more pro- other perly under the scope of the preceding Dissertation, and an able summary and enumeration of his writings by no less competent a person than Dr Thomas Young will be found in the alphabetical part of this Encyclopædia. I will only add, that while scarcely a topic in physical astronomy, or in pure mathematics, failed to receive important additions from his pen, his memoirs on the Libration of the Moon, his solution of several problems of Sound and vibrating strings, and his methods of computing the perturbations of Comets, are amongst his contributions to science, most vividly remembered and most justly admired as models of analytical ability. He himself is stated to have preferred, amongst all his papers, one in the Turin Memoirs of 1784 , on the Integral Calculus. ${ }^{2}$

With reference to the Lunar Libration, Lagrange confirmed the singular conclusion of Newton, that the Libration moon is a spheroid, having three unequal axes, the of the longest of which is always approximately directed to the earth, and the shortest is her axis of rotation. In consequence of this, the moon, of necessity, revolves on her axis in the exact time that she circulates round the earth (supposing that at any time these periods were nearly, though not absolutely, coincident), and is subject (as Newton had divined) to a species of oscillation upon her axis, owing to the line of the earth's attraction not always coinciding (in consequence of the moon's irregular motion in longitude) with the moon's greatest diameter. This constitutes a physical libration, as inequalities in longitude, by enabling us to see more or less of the lunar hemisphere diametrically opposed to us, constitute an optical libration, or apparent to-and-fro motion on her axis. In this investigation Lagrange first used the combination of D'Alembert's Principle with that of Virtual Velocities, afterwards fully expanded in the Mécanique Analytique. ${ }^{3}$

Lagrange was happy in passing his days with
(56.) grange's writings.

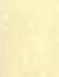


Private the tranquillity of a philosopher. He was respected character of and rewarded alike by kings and democrats-he was Lagrange. honoured and promoted in three great states, Sardinia, France, and Prussia. Though patronized by the despotic Frederick, and lodged in her palace by the gentle queen of Louis XVI., he escaped the misfortunes of almost every one of his contemporaries, including Laplace, Lavoisier, and Delambre; he retained his scientific appointments throughout all the frenzy of the French Revolution. His mildness of disposition and disinterested devotion to science, more than the European celebrity of his name, contributed to this result. He was equally fortunate in his scientific relations. Euler, D'Alembert, and Laplace, whilst they were emphatically his rivals, were also his sincere friends. If he ever felt jealousy, it was perhaps towards those who, he thought, attained too easily by circumstances to a high reputation : Monge seems to have been of this number. It is remarkable that for a series of years Lagrange diverted his mind altogether from mathematics, and studied chemistry, natural history, and even metaphysics. His reply is well known, when asked how he liked the first of these sciences; "Oh," said he, "I find it on trial as easy as algebra." It may be doubted whether in our own day he would have given as favourable an opinion!

He was unassuming in conversation, and disliked speaking of himself. His commonest answer was "I don't know." He was happy in his domestic relations, and died universally honoured and regretted, 10th April 1813.

\section{§ 2. LAPLACE.-Lunar Theory Improved.-Great Inequality of Jupiter and Saturn.-Theory of the Tides.-Young; Dr Whewell; Mr Airy.-Theory of Probabilities.-Character of Laplace as a Physicist and Author.}

(60.) Laplace.

Pierre Simon Laplace has generally, and not without reason, been considered as a sort of exemplar or type of the highest class of mathematical natural philosophers of this, or rather the immediately preceding age. The causes of this, and the degree in which it is warranted, we shall endeavour to state towards the close of this section. In the meantime, finding it quite impossible within our prescribed limits to notice, ever so briefly, all his more material investigations, we shall select three or four marked by their originality and general interest. Such are, 1. His improvements of the lunar theory. 2. His discovery of the cause of the great inequality of Jupiter and Saturn's motions. 3. His theory of the tides. 4. His work on probabilities. 5 . We shall consider his character as a general physicist, and as a writer.

I. First, then, we are to speak of the improvements of the lunar theory effected by him. The application of Newton's own principles to the perfecting of the theory of the moon's motion has been related in Sir John Leslie's Dissertation, and so far as the labours of Clairaut, D'Alembert, Euler, and Mayer, are concerned, belongs distinctly to the middle portion of the last century. The errors of Mayer's tables little exceeded one minute of space, which was twice more accurate than in Halley's time. With one important exception, the main outstanding differences between theory and observation had disappeared. The eclipses recorded in the Arabic and Chaldean annals could not (as Halley first observed) be correctly explained by the motion of the moon as given by recent tables. At length it became admitted that the mean motion of the moon has been accelerated from century to century by a minute quantity, which, in the lapse of thoussands of years, has become recognisable. It amounts to this, that the moon comes to the meridian two hours sooner than she would have done had her present period remained invariable from the earliest astronomical records of eclipses. It is at once evident how delicate a test this must be of changes otherwise imperceptible. The effect on the dimension of the moon's orbit may be thus expressed, that at each lunation she approaches nearer to the earth than during the last by one-fourteenth of an inch! thus describing a spiral of almost infinitely slow convergence. The minuteness of the effect may be illustrated by the shortening of the pendulum of a clock by an amount absolutely insensible, which yet, after days and weeks, will alter by many seconds the time shown by the hands.

After several unsuccessful speculations as to secular the cause of this anomaly, Laplace, in 1787, thus sa- tion of the tisfactorily accounts for it:- It is well known that the Moon. sun's attraction on the earth and moon lessens, on the whole, the tendency of the latter to the former, and lengthens permanently the lunar period. But, so far as this effect is uniform, it does not directly appear. The effect is greater, however, when the earth is near the sun than when it is farther off. The lunations are therefore longer in winter (when the earth is in perihelion) than in summer. This is called the annual equation, and the amount is very sensible for this reason, that (as may be easily seen) the perturbing force varies inversely as the cube of the sun's distance. Now, though the earth's mean distance from the sun has not varied in the lapse of ages, the excentricity of the earth's orbit has been diminishing from the earliest historic times, and the average inverse cube of the distance has 
been also slowly increasing. The result is that the moon's motion has been continually accelerated. Now, we have in the last section referred by anticipation to this acceleration as having led to the belief that the moon must at last fall to the earth. Laplace's discovery, however, shows that the acceleration has a limit, depending on that of the excentricity of the earth's orbit, which having reached its minimum, the lunar mean motion will begin to be retarded, and will continue so through a vast cycle of ages, and so on alternately. Theory enables us to assign, with considerable accuracy, the amount of the acceleration, which is now about $10^{\prime \prime}$ of longitude in a century. ${ }^{1}$

Besides this very satisfactory discovery, Laplace

(63.) ellipticity and solar parallax deduced from the moon's motion. investigated thrce of the lunar inequalities in a manner leading to curious and unexpected results. Two of these depend on the spheroidal figure of the earth. The nutation of the earth's axis, which is due to the attraction of the moon on the protuberant equatoreal parts of the earth, is exactly reproduced by the equivalence of action and reaction in the movements of the lunar orbit, only less perceptible in degree on account of the length of the leverage at which they are effected. The inequality of the moon's motion in latitude may be used to determine the degree of compression of our globe at the poles. Laplace deduced from the Greenwich observations of the moon the fraction $\frac{1}{304^{\cdot 6}}$, and, from a relative inequality in longitude, $\frac{\mathrm{l}}{305.05}$; a coincidence really astonishing, not only as between themselves, but also when compared with the mean result of laborious investigations by actual measurement of the earth's surface. The other result we referred to was the determination from the lunar theory of the solar parallax, - in other words, the distance of the earth from the sun, which enters into the expression of a certain inequality of the moon's motion in longitude. From the observed amount of this inequality, Laplace obtained a value of the solar parallax exactly coincident with that obtained with so much labour on occasion of the transit of Venus in 1769. Strange and admirable result (as Laplace himself remarks), that the astronomer, immured in his observatory, and watching our satellite tbrough his telescopes, and reading the re. sult by the aid of mathematical analysis and the theory of gravitation, should be able to determine the figure of our earth, and its distance from the sun, with perhaps quite as great accuracy as by any direct measurements. Truly the wonders of fact exceed those of fiction, and the divinations of true science may match the pretensions of her counterfeit, astro$\operatorname{logy}$.
In conclusion of this subject, I regret that space (64.) does not allow me to advert particularly to Laplace's Theory of remarkable success in accounting for some singular satellites. peculiarities in the system of Jupiter's satellites, arising from, and partly occasioning, an exact commensurability in the periods of some of them (which Sir John Herschel has lately observed to hold also in the Saturnian system in a somewhat different manner), a case which we have seen to be especially excluded in the instance of the planets, and which has been pronounced by a very competent judge ( $\mathrm{Mr}$ Airy) to be "the most curious and complicated system that has ever been reduced to calculation." It ought to be stated, however, that Laplace's discoveries were based upon a previous and highly original investigation of Lagrange.

II. In the second place, we shall briefly state the nature of Laplace's happy explanation of a great in-Long inequality of the solar system, to which, like the fact equalities of the lunar acceleration, especial attention had been and Sacalled by the sagacity of Halley, and which, like it, turn. resisting all the efforts of geometers to interpret, threatened the credibility of the Newtonian theory of gravity. We are therefore to look upon this step as something more than a solution of a difficult problem; it was a new, peculiar, and unsuspected combination of circumstances on which it depended, and the solution afforded a key in all time coming to difficulties depending upon a like cause.

Halley had ascertained, that by comparing modern with the most ancient observations of J upiter and History of Saturn, the mean motion of the former planet had been qualities ; accelerated, and that of the latter retarded. Lambert remarked subsequently that, if we confine ourselves to modern observations alone, an opposite change would appear to be in progress. The amount of the error of the tables was so considerable (amounting to $20^{\prime}$ or more in the middle of the eighteenth century, and capable, in fact, of becoming much larger), as to have been (along with the apsidal motion of the lunar orbit) one of the first subjects of anxiety and speculation to geometers, when the Newtonian theory came fairly into discussion. For nearly forty years this stubborn inequality was vainly attempted to be accounted for by Euler, Clairaut, D'Alembert, Lagrange, and by Laplace himself, before the latter hit upon the true cause of the anomaly. It was long, and naturally, believed to be a properly secular inequality, arising from the average mutual effects of the planets Jupiter and Saturn, though Lambert's remark rendered this less probable. It was in the course of the consequent research that Laplace proved that the mutual action of the two planets could produce

1 We here add that very recently Mr Adams has discovered that Laplace, and also his followers, in confining their attention to the radial effect of the sun's interference with the lunar motions, as affected by the excentricity of the earth's orbit, have unwarrantably assumed that the area described by the moon a unit of time is invariable. He finds, on the contrary, tangential perturbations depending on the same cause, and sensibly modifying the amount of secular mean motion deduced from theory.-(Philcsophical Transactions, 1853.) 
no permanent alteration of the mean motion of either; a conclusion which, as we have seen in the last section, he afterwards generalized for the planetary system. Several other memoirs by Lagrange and himself followed; and when the question became thus narrowed to periodic perturbations only, Laplace, with characteristic ardour and resolution, determined to search out every term which could affect the result; an irksome task, less congenial to the generalizing spirit of Lagrange. He had already noticed, in his memoir of 1773 , that Euler and Lagrange had, in their researches on this very subject, omitted terms, multiplied by sines and cosines of very small angles, which yet might, in the process of integration, become considerable by the largeness of the coefficients. their Eleven years later he detected, in the expansion of origin; the mutual perturbation of Jupiter and Saturn, terms of this kind. The coefficient (or maximum value of the term) is in this case divided by the square of the same quantity which renders the angle under the sine or cosine small. These terms were indeed likewise multiplied by the cubes of the excentricity, or like small quantities; but notwithstanding this, by reason of the small divisor just mentioned, they were capable of attaining a formidable magnitude; in the case of Jupiter, to $21^{\prime}$, and of Saturn, to $48^{\prime}$ or $49^{\prime}$. That so small a force should produce so large an effect is due to the very long period of the most considerable portion of this inequality, which, in fact, led to its being confounded with perturbations properly secular. The period of complete recurrence of the effects is about 920 years; and during half this time the motion of one planet is being constantly accelerated, and that of the other retarded; during the other half the action is reversed. An effect continually increasing or diminishing for so long a time, and between the two most massive of the planetary bodies, is evidently liable to become considerable. The maximum displacement of Jupiter and Saturn Laplace found by calculation to have occurred in 1560 , explaining the peculiarity above mentioned in the comparison of ancient with modern observations. Their

When we look to the physical cause of the largeness of these particular perturbative terms, it is found to be this ;-that the period of revolution of Jupiter compared to that of Saturn, is almost as the numbers 2 and 5 :-in other words to the near commensurability of the mean motions. Were they exactly in proportion to these numbers, formidable and permanent changes would possibly result in the orbits. As it is, the planets comeinto conjunction when Jupiter has completed 5 revolutions, and about $\frac{1}{4}$ th more; Saturn 2 revolutions, and $\frac{1}{4}$ th more. Consequently the point of conjunction travels round the circumference after about 44 conjunctions have occurred, which requires nearly 2700 years. But a little considera- tion will show that conjunctions occur successively at three nearly equidistant points of the circumference; consequently the two planets will have been presented to one another in every possible variety of configuration, when the point of conjunction has travelled one-third round the circumference, that is in about 900 years.

The effect of this great improvement in the Theory of Jupiter and Saturn was, that the most ancient observations were completely reconciled with the modern, and the modern with one another; the errors of the tables were immediately reduced to one-tenth of their former amount, and soon after to much less.

III. The third topic which I must shortly discuss in connection with the career of Laplace, is the Theory of the Tides.

The Newtonian Theory of the Tides has been explained in Mr Playfair's Dissertation, but its progress Newton's during the 18th century has not been adverted to in $\mathrm{D}$. Berthe continuation by Sir John Leslie. It will be sufficient to state here, that it was pursued into its conse- brium quences with ability and success by Daniel Bernouilli, Theory. who in 1740 shared a prize of the French Academy of Sciences on this subject, along with Euler, Maclaurin, and Cavalleri, a Jesuit, the last a supporter of the Cartesian vortices. It was, perhaps, the concluding honour paid to that once popular theory.

The Tidal Theory of Newton and Bernouilli presumes the earth to be at rest; and also the waters of the ocean to be at rest, and at every moment in a state of equilibrium between the force of gravity, tending to the earth's centre, and the lesser forces tending towards the sun and moon. That a theory, founded on suppositions so far from the truth (not to mention the irregular distribution of sea and land on the earth's surface), should in any manner or degree represent correctly what happens, may be matter of just surprise. The leading phenomena are however tolerably consistent with it; the dependence of the great tides on the moon's position with respect to the meridian of the port; the spring and neap tides when the sun's action and that of the moon conspire with or oppose one another; the priming and lagging of the tides depending on the displacement of the vertex of the compound ellipsoid due to the combined effect of the sun's and moon's attraction, depending therefore on the moon's elongation from the sun; the effects of the moon being in the nearer or remoter part of her orbit; all these facts are indicated by the Equilibrium Theory (as it has been termed), and are also results of observation. The theory, however, does not give the true depth of tide, nor (except in casual instances) does the time of high and low water coincide with theory; besides many minor imperfections.

Laplace had the singular boldness to attempt the solution of a problem, which is more one of hydro-
(69.) heory of s, or uili- 
Laplace's dynamics than of astronomy, and to estimate all the Dynamical causes of movement of the particles of a heavy fluid, Theory. surrounding a spheroidal rotating nucleus exposed to the attractions of the sun and moon. This he did in a series of memoirs, more systematically condensed in the Traité de Mécanique C'éleste, and it may safely be affirmed that no other mathematician of his day was equal to the labours and disappointments of an investigation attended with every species of difficulty, in which each result must be attained by a combination of general sagacity with mathematical rigour, and for the verification of which observations were yet in a great measure wanting. The Theory of the Tides was, upon the whole, the most arduous and complicated problem which could well be conceived, in a branch of science (hydrodynamics) hitherto remarkably little successful in predicting the results of the most simple and arbitrarily selected experiments.

That Laplace has been in a measure successful in such an undertaking must be considered the highest test of his genius, especially in reducing his mathematics to practical application; but the result has been a treatise so profound and obscure (I mean as regards the tide theory), that very few persons have attempted to master its difficulties. Mr Airy, the present astronomer royal, has done a great service to men of science, and to that far wider community whom the laws of the tides nearly interest, by giving a connected and tolerably elementary view of Laplace's investigation, which he states confidently to be " the most obscure of the Mécanique Céleste."

(74.) Its incom-

In this theory the figure of the ocean at any moment is considered as a dynamical problem; and that figure as a momentary state arising from the internal movements of the fluid itsclf, as well as from the variation of the external forces. The resulting differential equations, expressing the attractions of the sun, moon, and earth, the rotatory movement of the earth, and the pressure of the water itself in motion, are abundantly complex, and the solutions only partial and imperfect. The inferences from these solutions, too, partake not only of their imperfection, but, since they take no cognisance of the irregular distribution of land and water, present cases almost impossible to verify by observation. Some of the results are indeed so paradoxical, that without better evidence of their truth we do not further allude to them.

(75.) The tidal effects are divided by Laplace into three classes of classes; the distinction of which, however, cannot be Tides. called a discovery of his. The first class are independent of the earth's rotation, and are practically insignificant. The second class includes the diurnal tide occurring once in about 24 hours. Concerning it, Laplace draws this conclusion, that its rise and fall (not, however, its horizontal motion) are insensible if the depth of the ocean is uniform ${ }^{I}$ and being practically insensible in most latitudes, we have thence an argument of more or less weight for a general tendency to uniformity in the depth of the sea. The third class of tides are the ordinary semi-diurnal tides. They afford, as Newton acutely perceived, the most direct and attainable measure of the relative attractions of the sun and moon. We have the sum of these attractions at the conjunctions and oppositions of the luminaries, and the difference when they are $90^{\circ}$ apart; and a higher maximum when both bodies are without latitude. From the observation of the tides on the Severn near Bristol, Newton computed the relative action of the moon and sun to be as 4.48 to 1 ; but this value is much too great, and gave far too large a relative mass to the moon. The result in the harbour of Brest, from observations made under Laplace's direction, is about 2.90 to 1 ; and the moon's mass $\frac{x}{7} \frac{1}{5}$ th that of the earth, agreeing almost identically with that deduced from the nutation of the earth's axis caused by her attraction. Observations at London and Liverpool reduced by Sir John Lubbock and Dr Whewell give about 2.66 to $1 .^{2}$

From the general theory of Laplace, the following results have been deduced with confidence:-(1.) Laplace's That the stability of the ocean is secure, whilst the results. density of the ocean is inferior to that of the earth generally; which it is about five or six times. That the phenomenon of precession is not modified by the fluid covering of the globe.

In the application of his theory to special cases, Laplace is compelled to have recourse to an assumption entirely arbitrary-namely, that the periodic fluctuations, however otherwise modified by circumstances, recur in the same periods as the causes to which they are due. In this manner he conciliates the results of observation with his theory, which the latter would have been altogether incompetent to predict.

The general merits of Laplace's theory we will sum up in the words of Mr Airy, who, of all his succes- Character sors, has probably most attentively studied it:- "If of Ler putting from our thoughts the details of the investi- vestigation. gation, we consider its general plan and objects, we must allow it to be one of the most splendid works of the greatest mathematician of the past age. To appreciate this, the reader must consider-first, the boldness of the writer who, having a clear understanding of the gross imperfection of the methods of his predecessors, had also the courage deliberately to take up the problem on grounds fundamentally correct (however it might be limited by suppositions afterwards introduced); secondly, the general diffi-

1 Dr Young asserts that the conclusion will not hold unless the depth be also evanescent. Laplace has shown in his Fifth Book that the disappearance of diurnal tides will take place only when the nucleus is completely covered.

2 These ratios, however, being found to depend upon the configuration of the coast or estuary, cannot be used directly to de termine the relative action of the sun and moon. See Phil. Trans., 1845, p. 42. 
culty of treating the motions of fluids; thirdly, the peculiar difficulty of treating the motions when the fluids cover an area which is not plane but convex; and, fourthly, the sagacity of perceiving that it was necessary to consider the earth as a revolving body, and the skill of correctly introducing this consideration. The last point alone, in our opinion, gives a greater claim for reputation than the boasted explana. tion of the long inequality of Jupiter and Saturn." 1 We must, however, qualify this eulogy by adding, in the words of the same writer, that Laplace's theory, though based on sounder principles than the equilibrium theory, " has far too little regard to the actual state of the earth to serve for the explanation of even the principal phenomena of the tides." It is, in fact, like many other productions of the same age and school, a great display of ingenuity and mathematical skill, which hardly yields a single result worthy of confidence, or agreeing with nature, except by the abandonment of its deductive rigour, or a concealed induction backwards from the phenomena to be accounted for. The same amount of skill and resource which $\mathrm{Mr}$ Airy has shown in adapting it to his own views, and to recent observations, would probably have sufficed to construct a theory from the foundation. By others the attempt seems to have been abandoned as hopeless.

Since our limits will not permit us to return to Laws of the subject of tides, we shall here briefly state the Whewell-progress of the subject since the time of Laplace. Sir J. Lub-The chief steps have consisted in co-ordinating the bock. results of observation and analyzing them into their partial phenomena, by the help of Newton's and Bernouilli's theory. This labour has been greatly advanced by Dr Whewell, and also by Sir John Lub-

Cotidal bock. The former has constructed maps of " cotidal lines. lines," which, indicating the relative time of high water in different parts of the globe, give us a graphic conception of the course and propagation of the tidal wave. The tides of the Eastern Pacific are but little known; but a vast wave advances northwards between Australia and Africa, diverted or retarded by the obstacles it meets with in the Indian Archipelago. Another (and to us the most important) branch sets from south to north up the vast canal of the Atlantic, where it is gradually complicated by local tides, having their origin in the wide expanse between Africa and the Gulf of Mexico. The two sets of waves sometimes reinforce, sometimes oppose, one another; they are prolonged to the western shores of England and Norway, where the tidal impulse arrives 24 hours after it passed the Cape of Good Hope. It is propagated most rapidly at a distance from coasts, and is retarded in narrows and shallows. It sends offshoots into every bay and strait, always greatly retarded in point of time (ap- parently by friction), but often increased in elevation by concentration of the effect in a gradually narrowing channel, as we see in the exaggerated tides of the river Amazon, the Severn, and the Bay of Fundy. The same place may be the seat of several tides at once, which may increase or destroy one another; thus, a small tide is propagated through the Straits of Dover as far as the Dutch coast, where it only arrives simultaneously with the principal wave, which has made the entire tour of Great Britain.

As regards the progress of theory, Dr Thomas (80.) Young, whose character as one of the greatest $\mathrm{Dr}$ Young philosophers of the past age we shall have to consider in another chapter, next after Laplace grappled with the difficulties of this arduous subject. Employing mathematical methods of inferior power but greater directness, and taking into account causes of local action which Laplace had not ventured to include in his analysis, he gradually matured a theory adequate to represent many of the results of experience, of which Laplace gives no account.

He distinguishes the results of the forced and free oscillations of the sea; the former resulting from the Free an direct action of the sun and moon combined with the waves. rotation of the earth, and whose periods of rise and fall are determined solely by those external causes (external, I mean, to the mass of the ocean); the free waves, on the contrary, derived from the former, are transmitted with velocities depending on the mechanism of the fluid itself, on its depth, and on the resistances arising from friction to which those motions are exposed. These all-important modifications of the dynamical Theory of the Tides were deduced by Dr Young from the general theory of oscillations and resistances, and from the laws of fluids detected by Dubuat, ${ }^{2}$ and he applied them with no ordinary skill to the solution of the problems of tides in oceans, estuaries, and rivers. It is an extraordinary fact, and not without significance, in the history of science, that these researches of Young, published anonymously in the Supplement to the Sixth Edition of the Encyclopædia Britannica, and in the Seventh Edition of this work, and likewise in several journals and reviews, so generally escaped notice as to have been almost unknown till Dr Peacock, in his recently published "Life and Miscellaneous Works of Dr Thomas Young" has fortunately recalled attention to their existence and their important results.

In doing so, Dr Peacock has communicated with Mr Airy, whose very valuable article on Tides and Mr Airy on Waves has been above referred to (78), and has lides and ascertained from him that Dr Young's researches had escaped his notice when he undertook that elaborate recension of Laplace's theory, and made those important additions to it to which I have

I Encyclopadia Metropolitana, "Tides and Waves," art. 117.

2 See Chapter IV., Section 6, where we shall return to some portion of this subject. 
called attention. ${ }^{1}$ It is very satisfactory to find that, by their independent and very different modes of analysis, $\mathrm{Mr}$ Airy and $\mathrm{Dr}$ Young have arrived at results genorally coincident. It is in the essay of the former that most readers will now seek for an acquaintance with Laplace's abstruse investigations, whilst they will find in it the bearing of experiments more recent than the time of Young on the propagation of waves in canals, the theory of $\mathrm{Mr}$ Airy, beginning as it were at the opposite end from that of Laplace, and offering far more points of contact with actual observation, particularly in the Tides of Rivers and Estuaries. The theory of Young will naturally be best studied in his own article TIDES, in this Encyclopædia.

(83.) IV. In the fourth place, we connect the name of Doctrine of Laplace with the progress, during the period we are Probabili- considering, of the curious doctrine of probability, or the laws of chance and expectation. These he discussed in two works, the Theorie Analytique and the Essai Philosophique sur les Probabilités-the first the most mathematically profound, the last the most popular and elegant, account of the subject which has yet been given. Nearly all mathematicians are agreed on two points-first, in considering this the " most subtle" and "difficult to handle" of all the applications of their science, involving a perpetual recurrence to contingencies, and to elements of the argument easily left out of account, and in which, more than in any other, it is dangerous to let sleep for a moment the severely reasoning faculty, or permit it to be Iulled to security amidst the maze of symbolic transformation. In truth, from experience, I am disposed to receive with doubt the solution of even a tolerably simple problem of chances, unless two competent persons at least have concurred in verifying it. Secondly, Mathematicians are agreed in considering Laplace's Théorie nearly, if not quite, the ablest specimen of mathematical writing of his age, notwithstanding a degree of obscurity and repetition in addition to the inherent abstruseness of the subject, which render it, in the opinion of one of the most learned and extensively read of our pure mathematicians, ${ }^{2}$ "by very much the most difficult mathematical work he ever met with."

(84.) A single paragraph has been devoted to the subject Improve- of probabilities in Sir John Leslie's Dissertation, ments in its
investiga- relating to its earlier history; and the subject was so tion by popular during the last century, that there was Laplace. scarcely an eminent mathematician who did not add something to its practical development; so that Laplace may be considered rather to have enlarged widely its applications by means of his almost unexampled power in handling the calculus, than to have improved or established its first principles, or even applied it to classes of problems altogether new. We find that most of the principles of the Calculus were established by James Bernouilli, in the earliest part of the eighteenth century, who gave the first History of application of the Binomial Theorem to determine theDoctrin the probability of a particular combination of a given of Chances number of things occurring, in preference to all the other equally possible combinations. Stirling discovered a curious theorem for approximating to the continued product of the arithmetical series of numbers carried to any extent, which perpetually occurs in such calculations. Demoivre carried out Halley's application of it to the laws of mortality. Condorcet applied it to moral questions; Mitchell to natural phenomena, considered as the results of accident or design; Lagrange to errors of observation. The chief applications of the Theory of Probability are Itschief ap the following:- 1 . To chances known a priori, as plications. that of throwing two given numbers with dice, the whole range of possibilities being known with precision. 2. The calculation of the expectation of future events on a great or average scale, deduced from the past course of events observed also on a great or average scale. Of this description are the calculations of life assurance, first tabulated by Halley: 3. To find the most probable result of a number of independent observations and problems of a like kind. 4. To the proof of causation as opposed to accident or " random," derived from existing combinations of facts. 5. To the probability of testimony, and the confidence due to legal decisions. None of these inquiries are peculiar to Laplace, or originated with him. We select, however, for a brief notice (which must be confined to a few sentences) the third and fourth of these applications.

The chances are enormous against the most experienced marksman's hitting the bull's eye of a target. But if he make many shots in succession, the balls most prowill be lodor round about the spot at which he bable reaimed, and they will be ferr in sumber of of equal area drawn round the mark. The degree indepenof their scattering will depend upon the skill of the dent obsermarksman; but in all cases the most probable result will be, that the point aimed at is the centre of gravity of the shots. This may be shown to be equivalent to saying that the most probable result of any number of equally reliable observations is that which will make the sum of the squares of the outstanding errors a minimum. This rule was conjecturally proposed by Legendre's Legendre in 1806. A demonstration of its truth was method of first published by Laplace. It is of great practical least use in deducing the results of complex observations, such as those of Astronomy, and generally in combining " equations of condition" more numerous than the quantities whose value is sought to be extracted from them. In very many cases, however, a graphical 
method of interpolation will serve the same purpose, as well as save a very tedious calculation. Perhaps too much importance has been assigned to the "probable mean error" of a single observation deduced from many individual errors by the same theory. If a man has but one shot at a target, it is perfectly uncertain (by hypothesis) whether that slot be one of his best, or one of his worst, or one of his middling ones. But as there are more middling hits than very bad or very good ones, there is a certain distance which it will be safe to bet $(i, e$. for which the probability is $\frac{1}{2}$ ), that he will not exceed; though it would be a strange occurrence indeed, if he exactly struck the ring in question. This is all that is meant by the phrase " probable error ;" it is an entirely artificial number, which serves to give a sort of numerical value of the skill of the performer, but is otherwise of no importance. Its application has sometimes been strangely mistaken. Even the "rule of least squares" is often misapplied, and empirical laws altogether false have been deduced from it; for it is rare in practice that the chances of error of observation of a varying quantity are the same throughout the limits of observation.

(86.) But those applications of the doctrine of probaProbability bility which pretend to give us a measure of our belief mony and in the constancy of natural laws, in the confidence due of Design. to testimony and to the teaclings of history, in the proofs of design in cosmical arrangements, -are inquiries which, from their connection with metaphysics, religion, and morals, have had a higher interest for mankind at large than ordinary problems about cards and dice. To these Laplace paid peculiar attention; and the reputation of his name tended to create in others a belief that the analysis he so powerfully wielded could communicate a portion of its certainty to the data subjected to it, and gave a currency to many of his conclusions to which we believe them by no means entitled. Professor Playfair, one of the most ardent of Laplace's admirers, has recorded (in a criticism in the Edinburgh Review, of the works we are considering) his total dissent from Laplace's doctrine that the transmission from age to age of the historic record of a fact diminishes its credi-

Laplace's doctrines questioned. bility in a geometrical ratio. But a Cambridge mathematician and speculative philosopher of singular penetration, Mr Leslie Ellis, has most formally assailed $^{\mathrm{I}}$ the principle of nearly all Laplace's estimates of our expectation of events arising from causes unknown or assumed to be so, such, for instance, as that a common cause determined the revolution of all the planets in one direction. The subject of the metaphysics of probability evidently requires a complete reconsideration; and, owing to the singular subtlety of the matter, it is one which few persons are competent to handle. The state of health of Mr Ellis leaves us little hope of his resuming the inquiry; but two eminent mathematicians, Mr De Morgan and Mr Boole, have published considerable works chiefly bearing upon it.

As an implement bearing upon discovery in science, the Calculus of Probabilities has as yet been of little service. Whilst Laplace tries to indicate how it guided his researches connected with planetary irre- covery. gularities, every one sees at a glance that, with the data before him, common sense must have outstripped analysis. Laplace has called the doctrines of probability "good sense reduced to calculation." What is to be feared is, that the calculation should outstrip the good sense.

V. Fifthly, We are to consider Laplace's character as a general physicist and as a writer.

In the former raplace's tion than that usually attained by eminent analysts. sicist, an In fact, if we compare him with any of his own as an augeneration, we find him not only better acquainted with physical principles, and more scrupulous in taking account of them in his mathematical discussions, but even possessing skill and interest in experiments. The Calorimeter for measuring the capacities of bodies for heat was the joint invention of Lavoisier and himself; at least their memoir does not assign to either a predominant share in it, ${ }^{2}$ and their determinations of the expansions of the metals by heat seem also to have been made in cominon. His happy discovery of the chief or sole cause of the Memoirs on discrepancy between the theoretical and observed Heat, in velocity of sound (due to the heat developed by com- common pression) would alone have given him a just reputa- Lith tion, so anxiously had the matter been debated, and so much was it involved in a purely mathematical intricacy. Even in his great work on physical astronomy he takes a peculiar pleasure in embracing topics of terrestrial physics. We there find discussed the theory of barometrical measurements, the ques- On Barotion of atmospheric tides, the laws of capillary attrac- metrical tion, and the constitution of the gases. As to the first Measureof these topics he made a practical improvement on ments, the formulæ of his predecessors, so that his rules are Attraction, in fact still in use. As regards capillary attraction, \& Astronohe was materially anticipated by Young, who evi- mical Redently considered his principles to have been pirated; yet his theory, though obscured by a display of redundant mathematics, was a real improvement. His theory of tides, and that of atmospheric refractions, though closely connected with physical astronomy, were in fact not less so with the doctrines of hydro-

\footnotetext{
1 Cambridge Transactions, Vol. VIII. The writer of these pages has also given his reasons for dissenting from the arguments of Michell (which have been sanctioned by the authority of Laplace) on an astronomical question as discussed by the Theory of Probabilities. See Philosophical Magazine for December 1850.

${ }^{2}$ Dr Black, the discoverer of latent heat (who was probably well-informed), states in a letter to Watt that he belieres Laplace to have been the inventor. See Correspondence of Watt on the Decomposition of Water, p. 66.
} 
dynamics and optics; and we shall find very few important branches of general physics in which he has not left some permanent record of his interest, in the course of his career of fifty-five years of anxious devotion to science.

(89.) His largest and most systematic work, the Traite His Traité de Mécanique Céleste, in five quarto volumes, was de Mlecanique Celeste.

Far less original than the not only most ably executed, but exceedingly well timed. The applications of analysis to physical astronomy had been accumulating for nearly a century. Hundreds of meinoirs relating to them, dispersed through many volumes in different languages, written with varying ability, in various stages of scientific progress, and with differing notations, presented a mass of reading almost beyond the reach of the most resolute student. Laplace undertook to digest the whole into one body of doctrine, composed throughout on a uniform plan, with the best mathematical aids which were known at the commencement of this century. And though improvements and discoveries have been since made, the methods and most of the results of the Mécanique Céleste remain, with little variation, the preferable ones of our own time. As a work of labour, it may compete with the Principia of Newton; as an original work, it is of course immeasurably inferior. Its principles are, in fact, the same with those laid down in that immortal code, and its deductions are collected (as we have said) from the writings of Clairaut, D'Alembert, Euler, and Lagrange, as well as from the previous memoirs of the author himself. Laplace has been too sparing of his citations and acknowledgments, and a consequence of this literary avarice has been that he is sometimes considered as more of a compiler and less of a discoverer than is justly his due. For however ill he could have dispensed with the skilful preparations of his illustrious rivals and contemporaries, his preeminent sagacity furnished on several occasions the key-stone of the arch which imparted at once strength and completeness to the fabric. We have seen in the last section that though the credit of the theorems respecting the stability of the solar system is very generally attributed to Lagrange, who, indeed, principally furnished the methods, and gave great geneyet gives rality to the results, yet the capital discovery of the him justly invariability of the major axes of the planetary orbits a high re- is due to Laplace. It was he, again, who removed putation. from the theory of gravity the two greatest and most impracticable difficulties with which it had ever been assailed - the anomaly of the lunar accelerations, and the great inequalities of Jupiter and Saturn, and by so doing rendered it almost infinitely improbable that any future discrepancy should more than temporarily embarrass a theory which had tri- umphed in succession over such formidable causes of doubt. True that Lagrange, in his memoir of 1783 , had come within a single step of the first of these discoveries, and, by a process of exclusion, had almost forced attention in the right direction respecting the latter; still Laplace seized the prize in both cases, after a fair, prolonged, and arduous struggle. Now these three discoveries were the greatest in physical astronomy between those of D'Alembert and Clairaut on the precession of the equinoxes, the motion of the lunar apse, and the periodicity of comets, - and that of Leverrier and Adams on the perturbations of Uranus about a century later.

The universal testimony of mathematicians is to (90.) the effect that Lagrange was unrivalled as a pure coplace analyst, in his power of generalization, and in the with Lainherent elegance of his methods; that Laplace, grange. with nearly equal power in using the calculus, had more sagacity in its mechanical and astronomical applications, or rather, perhaps, we should say, in directing it to the discrimination of causes, and the revelation of consequences.

In other respects he differed far more widely from his illustrious compeer. He rather courted popu- His public larity, and was pleased at being considered worthy ${ }^{\text {character. }}$ of political distinction. For a short time he was one of Napoleon's ministers; but the Emperor, it is said, was more satisfied with his mathematical than with his diplomatic talents. He had none of the shyness of Lagrange, nor his repugnance to general society. He received with affability and kindness those who were introduced to him, and his attentions were afterwards recollected with gratitude by rising men of science abroad. He had a villa at Arceuil, adjoining that of Berthollet, and was one of the original members of the "Societé d'Arceuil," to whose memoirs he contributed. He exercised a powerful influence and as a in the Academy of Sciences, of which for. a time he Member of acted as dictator, and he was not very tolerant of demy of views in science opposed to his own. The undula- Sciences. tory theory of light he always opposed, and was mainly determined in doing so by the facility with which the attraction of luminous corpuscles could be subjected to calculation.

The weak point of his scientific character was one so natural, and perhaps so inseparable from his prevail ing studies, that it is not fair to criticise it too severely. analytical This was a love of analytical display in treating questions which it rather embarrassed than illustrated; and generally, a disposition to overrate the sphere of mathematical discovery. This he had in common with Euler, to whom he was very superior in physical attainments and sagacity. His language, and that of his eulogists, ${ }^{1}$ often amounts to the assumption that

1 For instance Arago says (speaking of the invariability of the major axes): "Enfin par la toute-puissance d'une formule mathèmatique, le monde materiel se trouva raffermi sur ses fondements" (Annuaire, 1844, p. 304). This is indeed the idolatry of mathematics. Many examples may be found in Laplace's writings on Probability ; which occasioned Mr Ellis to say of bim, that " to Laplace all the lessons of History were merely confirmations of the "resultats de calcul." "To the same effect was the mot of Napolcon, " that Laplace carried into the art of government the principles of the infinitesimal calculus." 
the marvellous power of analysis, in unravelling intricate consequences of admitted or assumed laws, could supply deficiencies of primary conceptions of the laws of nature, or could teach men fundamental truths in natural science.

(93.) To add to the remarkable list of Laplace's endowHis Systême ments one more; he was a perspicuous and elegant du Monde. writer. His Systême du Monde contains a popular exposition of astronomy in theory and practice entirely original in its plan and execution, and though frequently imitated, it is still perhaps the first of its class.

Laplace was born in Normandy 23d March 1749, and died the 5th May 1827, leaving in the Academy Date of he had so long honoured no one within many degrees birth an of his ability in the same peculiar walk of science.

\section{§ 3. LEGENDRE.-IvORY.-Theory of Integration; Elliptic Transcendants (Abel, Jacobi).} The Attraction of Spheroids, and Theory of the Earth's Figure. Atmospherical Refractions.

(95.) AdRien Marie Legendre was born in France in Legendre. 1752, and died in 1833 (10th January); he was consequently three years younger than Laplace, and survived him by nearly six years. He formed, therefore, an integral part of that constellation of mathematical talent of which Paris was for more than two generations the main centre. Like his illustrious compeers Lagrange and Laplace, he laboured with enthusiasm all the days of his life, and like them was engaged in editing and improving his works down almost to the day of his death, at the ripe age of fourscore.

(96.) The mathematical career of Legendre was less Distin- splendid than that of the other two whom we have an able as just mentioned. He did not possess the wonderful mathematician. powers of generalization of Lagrange, and he wanted the flexibility of mind, and the general physical knowledge, of Laplace. Legendre was very strictly a mathematician; and he has been exceeded by none in the unquenchable zeal with which he pursued subjects of a dry and even repulsive character, often till he had hunted them down by sheer force of application, or, to adopt the metaphor applied to him by Lagrange, until he carried, sword in hand, the stronghold which he besieged.

No more striking proof can be given of these statements than the unflinching pertinacity with which, during nearly fifty years (1786-1833), he studied and improved the theory of Integration, applicable to those cases frequently occurring, which involve the higher powers of the independent variable, and which do not usually admit of finite expression. Two large works, the Exercises du Calcul Integral (1811), and the Traite des Fonctions Elliptiques (1827-32), - the latter in good measure a republication of the materials of the former, - bear testimony to his diligence; and these works were almost entirely original, and contained tables of most laboured construction, calculated by himself. Hardly any mathematician entered into competition or co-operation with him until his labours were drawing to a close, when, with a liberality worthy of all commendation, he welcomed the

analytical discoveries of Abel and Jacobi, which were to give an unlooked-for extension to his own. These methods of integration, and their reference to certain properties of the Lemniscate and the Ellipse, originated in the early part of the last century with Fagnano and Euler. Legendre took up the subject exactly where Euler left it, and finally reduced the large class of expressions to which his methods are applicable to three standard forms or integrals in which the independent variable is always expressed by a circular function, and to which a numerical approximation may always be made by means of the tables calculated by himself.

ABEL, who succeeded in generalizing Legendre's methods to a far greater extent, was a native of Norway, born in 1802 (25th August), ${ }^{1}$ and died Norway, born in 1802 (25th August), and died the same
at the premature age of twenty-six (1829,26th subject; hi April). His principal memoir was presented to the personal: Institute when he was only twenty-four years old; history. and, to use the language of Mr Leslie Ellis, "when the resources of the integral calculus were apparently exhausted, Abel was enabled to pass into new fields of research by bringing it into intimate connection with a new branch of analysis, namely the Theory of Equations. The manner in which this was done shows that he was not unworthy to follow in the path of Euler and of Lagrange." 2 Legendre's eulogy of Abel was concise:- " Quelle tête celle du jeune Norvegien!" It is less agreeable to add that the life of Abel was perhaps shortened by povierty and care. Though ultimately befriended by Legendre, Poisson, and others, his first visit to Paris(in 1826) occasioned nothing but disappointment, and his great memoir (no unusual lot, for the same happened to Fresnel) lay hopelessly lost amidst the papers of the Institute for fifteen years. Much, however, to their credit, the geometers above mentioned at length addressed the King of Sweden on behalf of the rare genius his dominions contained; but in vain. Abel died neglected, unable even to print his researches, which were tardily given to the world in a collected form, at the expense of the government which refused to support

1 There is some discrepancy, as to the year of his birth, but I believe this to be correct.

2 Report on the recent progress of Analysis. British Association Report for 1846. 
him when alive. The French Academy, which had buried his memoir in their mostinaccessible "archives," decreed too late the unprecedented honour of a posthumous medal to his mother. Jacobi, the friendly rival of Abel in his discoveries, died recently, at a mature though not advanced age, at Königsberg, where he was professor.

(99.) But to return to the labours of Legendre. The theory of the figure and attraction of the earth on the at- and of other planets naturally divides itself into two traction of parts-(1.) the law of attraction of an ellipsoid on Ellipsoids, a material point without or within it ; (2.) the figure of equilibrium of a fluid subjected to no forces but the mutual attractions of its particles, and the centrifugal force due to its rotation. The latter of these problems is still imperfectly solved, the former completely so, and that mainly in consequence of the labours of Legendre and Ivory."

(100.) Though the services of Legendre are well known sometimes and admitted, the superior address of Laplace in the in part at- applications of mathematics has occasioned his reLaplace. ceiving the credit of what in some instances belonged to the former.

(101.) Maclaurin, by an exercise of synthetic skill not History of exceeded since the death of Newton, had demonthe subject. strated the attraction of an ellipsoid of revolution upon a material point anywhere within it or on its surface, as well as for an exterior point in the prolongation of its axis or in the plane of its equator. The same problem was afterwards analytically solved by D'Alembert and Lagrange. In 1782, Legendre, by a profound and complicated analysis, obtained an expression, by means of series, for the attraction of an exterior particle generally, and he was the first to imagine and employ those artifices of calculation known usually by the name of "Laplace's functions." Laplace made a step towards the simplification of the expression of exterior attractions, but the complete solution was reserved for Mr Ivory, as I shall mention below.

The other labours of Legendre need not be specified here. He co-operated in the trigonometrical survey of France, and gave the formula, known by his name, for approximately reducing a spherical to a plane triangle. He also wrote on the orbits of comets, and on the method of least squares.

JAMEs IVoRx, the most considerable British mathematician of his time, or that had appeared since Maclaurin, was born at Dundee in 1765 , and studied at St Andrews along with Sir John Leslie. The most active period of his life was passed as mathematical professor at the Military College of Marlow (afterwards removed to Sandhurst). He was essentially a selftaught mathematician, and spent much of his time in retirement. $\mathrm{He}$ fathomed in private the profoundest writings of the most learned continental mathemati- cians, and, at a period when but few Englishmen were able to understand those difficult works, he showed his capacity of adding to their value by original contributions, not unworthy of the first analysts. We pass over his earlier contributions connected with mathematics and astronomy, several of which are contained in the Transactions of the Royal Society of Edinburgh, and proceed to his most celebrated paper, published in the Philosophical Transactions for 1809, in which he completely and definitely resolves the problem of attractions for every class of ellipsoidal bodies. After what has been stated above as to the position of the problem as treated by Legendre, a few words will explain the precise import of Ivory's Theorem, one of the most celebrated mathematical results of that time.

We have seen that the attraction of an ellipsoid on a point within or at its surface had been assigned by Maclaurin. The theorem in question enables ant Theous at once to reduce the case of an exterior attracted attraction point to that of a point on the surface of the ellipsoid. of EllipSuppose an ellipsoid having the same excentricity, ${ }^{80 i d s .}$ and with the principal sections parallel to the first, but whose surface passes through the given exterior point. Let points on the surface whose co-ordinates parallel to the three axes of the respective solids are proportional to those axes be called corresponding points; then the attraction parallel to each axis which one of these bodies exerts on a point situated on the surface of the other is to the attraction of the latter on the "corresponding point" of the surface of the former, as the product of the two other axes of the first ellipsoid is to the product of the two other axes of the second. By this means the attraction on an exterior point is accurately expressed in terms of the attraction on an interior point, which is known. It is fair to add that both Legendre and Laplace had some glimpses of the principle, though they failed to apply it to the direct solution of the problem, and between the publication of their $\mathrm{Me}-$ moirs and that of Mr Ivory there elapsed nearly a quarter of a century.

Besides this paper, Ivory contributed many others (105.) on the subject of the attraction of spheroids and the His other theory of the figure of the earth, during a period of ${ }^{\text {papers. }}$ nearly thirty years; several of these were controversial, and did not add materially to the progress of the subject; others are considered as masterpieces of analytical skill. One of the last subjects which occupied his attention was the possible equilibrium of a spheroid with three unequal axes, which Jacobi had discovered.

Between the labours of Ivory and those of Legendre a great analogy subsists; for the doctrine of elliptic integrals also occupied the attention of the former.

But next to the theory of attractions, that of $A t$ mospheric Refraction most seriously engaged Irory's Atmoattention. Its great importance in astronomy, and spheric Re 
the curious mathematical difficulties which it presents, renders it very interesting to analysts. Laplace had applied to it his method of Generating Functions; Kramp had introduced into his (now scarce) treatise the almost new Calculus of Factorials; and others, like Bessel and Atkinson, had skilfully combined theory and observation for the construction of useful tables. One of the most curious results of recent enquiries into this subject is, that $\mathrm{Sir}$ Isaac Newton's table of refractions (Phil. Trans., 1721) must have been founded on a profound consideration of the problem, such as no one else thought of till a much later period, and is so numerically exact as to agree closely with the later tables, Kramp's for example. ${ }^{1}$

(108.) Mr Ivory attained the age of seventy-seven, dying on the 21st September 1842. Probably his unceasing devotion to a confined and abstruse topic of enquiry, reacting on a sensitive frame, rendered him in some degree irritable and unsocial. He was not altogether responsible for this; but students of science should recollect that diversity of occupations and interests is subservient not only to bodily health, but also to mental equanimity and vigour.

The historian of science dwells with a special in- (109.) terest on the results of Ivory's labours, when we re-His emi-

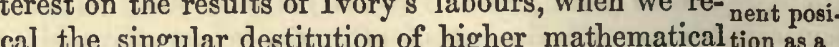
talent which had reigned in this country for so long a British M period, and which left us not only no position in the thematigreat struggle going on abroad for the advancement of physical astronomy, but scarcely even the rank of intelligent spectators.

\section{§ 4. Progress of Physical Astronomy since the publication of the Mécanique Céleste.-PoIsson.- Theory of Rotation (Poinsot).-Mr AIRY-The Solar Theory.-MM. PlanA and HANSEN- The Lunar Theory.-Physical Astronomy in America.}

(110.) Increasing like that of Gravitation, advances to perfection, physical as-the less reason have we to expect great and striking tronomy. results in the prosecution of it, and the more intense and continuous is the labour in matters of detail necessary to make any advance at all.

(111.)

As regards the general and popular view of the Its progress subject, we might pass at once from the epoch of Lapublication grange and Laplace to that of Leverrier and Adams. of the Notwithstanding, however, the necessity of extreme Mécanique compression, I must devote one short section to men-
Céleste. tioning the chief labours in connection with physical astronomy of four eminent men mentioned in our title, who may fitly be considered as the immediate successors of Laplace. Two of them will be again referred to in this discourse.

(112.). Srmeon Denis Poisson, born in 1781 , may be Poisson said truly to have been brought up at the feet of Lagrange and Laplace. He was their pupil in the first and brightest years of the Polytechnic School, where he was especially noticed by the former. He had the distinguished privilege of being literally their fellow-worker, his early memoirs having reference to their labours, and stimulating the still vigorous mind of Lagrange to the production, in his latest years, of several memoirs, which have been considered worthy of his best days. I refer more particularly to Poison the son's proof that the stability of the planetary system stability of holds when perturbations of the second order are taken into account, as has been stated in the first section of this chapter, Art. (55.) This was in 1808. Soon after, following out another of Lagrange's admirable generalizations of his theory of Arbitrary
Constants, he embraced in a common series of for- on Rotamulx the result of those mechanical laws which ${ }^{\text {tion; }}$ regulate the rotation of bodies, together with those concerned in their translation in space. This important subject (rotation) continued at intervals to engage the attention of Poisson, not only as regards the motions of the heavenly bodies on their axes, but also as a branch of common mechanics. The basis of this intricate doctrine was laid by Huygens; Euler, in a celebrated and original work, gave it a general and analytical form; D'Alembert solved by it the problems of precession and nutation; Laplace demonstrated the constancy of the time of the earth's rotation round its axis. This last problem was more fully discussed by Poisson, who showed by theory that neither can the earth ever rotate round an axis different from its present one, nor can the time of its rotation vary in consequence of any external attractions to which it is subject. These two matters are of the utmost moment; the first prevents the latitude of places from varying, and also renders impossible the extensive flooding of dry land by the waters of the ocean, which would be the evident consequence of such a change; the second assures us that the grand unit of reckoning in all ages, the invariabi basis of astronomical chronology and of physical lity of th astronomy generally, the length of the mean solar day, day. has not varied, and never will perceptibly vary under the action of known forces. Laplace had long before proved, by a comparison of ancient eclipses with modern observations, that, practically, the length of the day had not varied in 2000 years. It appears, indeed, that since the earliest recorded Chaldean 
eclipse (that of в.c. 720), the rotatory velocity has not altered by one ten-millionth part.

(113.) An eminent contemporary and rival of Poisson, M. M. Poinsot Poinsot, has added many elegant propositions to the
on Rotaon Rota

Theory of Rotation. M. Poinsot is also the author of the Theory of Statical Couples, which now forms part of all elementary treatises on mechanics.

(114.) Poisson wrote many other papers on subjects of Other physical astronomy, such, for example, as the Lunar writings of Theory, but they did not lead, on the whole, to strik-
Poisson. Theor ing conclusions. In fact, he allowed himself to be diverted from this his most natural calling, by the ambition of constructing a system of Physics mainly founded on the applications of analysis. Some bulky volumes of this series appeared, especially those on Capillary Attraction, and on the Theory of Heat. The author here shows himself as a profound analyst, but adds little to our knowledge either of principles or of important results. A similar criticism may be applied to his theory of Elastic Substances, and to his doctrine of Waves. His papers on Magnetism and Electricity will be mentioned elsewhere, but their character is somewhat similar. In Optics he was attached to the Newtonian theory. In Mechanics not requiring assumptions as to the properties of matter, he was very successful. He was eminently a solver of hard problems : his investigation of the whole circumstances of motion of a projectile in air deserves notice. Indeed every branch of mechanics received his attention, and the number of his printed papers is said to exceed three hundred. On the whole, he will, perhaps, be most generally and favourably known by the

excellent Treatise on Mechanics which he wrote for the use of advanced students. He was an eminent and diligent Professor, and his whole life was one of almost unremitting study. "La vie c'est le travail" was his reply, when urged to consult his health by reposing from his labours: and he actually died in the discharge of his duty as examinator of the Polytechnic School. This occurred on the 25th April 1840, in the fifty-ninth year of his age.

$\mathrm{Mr}$ AIRY on the perturbation by Venus of the Earth's motion.-We here detach from what we shall elsewhere have to record of the eminent services rendered to the cause of astronomy by the present Astronomer Royal of England, a notice of his chief discovery in physical astronomy, the more remarkable from being almost the only improvement in the theory of the planetary motions, as applicable to the tables, which had proceeded from an English mathematician for a very long period. Sir James South called attention, in 1826 , to a small but well marked deviation of the Sun's place from that given by Delambre's solar theory (the Sun's place or the Earth's are of course convertible terms). Mr Airy, then Lucasian professor at Cambridge, instituted, in 1827, a more extensive comparison with the Greenwich Observa- tions, and attributed the error chiefly to the assumption of erroneous masses for Venus and Mars. But prolonged study satisfied him that it arose from a "long inequality," arising from the mutual action of the Earth and Venus, similar in its nature to that mentioned in Art. (66), as detected by Laplace in the case of Jupiter and Saturn. It arises from the circumstance that eight times the orbital period of the Earth is almost equivalent to thirteen times that of Venus. Terms of the series expressive of the mutual action of the planets, which are divided by thirteen times the mean motion of the former minus eight times the mean motion of the latter (which is a very small quantity), may consequently become considerable; still more, those involving the square of this quantity. Such terms belong to the fifth and higher orders of the excentricity, and would consequently be very minute indeed, but for the casual magnitude of their coefficient. The labour of tracing out and calculating the effects of these important terms from the vast mass of algebraic developments is enormously great,-much greater than in the corresponding case of Jupiter and Saturn. As the calculations are not very likely to be repeated, Mr Airy took extraordinary precautions in their verification. The period of the inequality depends, first, as has been explained in Art. (67), upon the period required to carry the point of conjunction of the two planets completely round the circumference. This pcriod is no less than 270 years. The perturbation is of course mutual, and affects the place of Venus as well as of the Earth.

Mr Airy is also the author of investigations connected with the Figure of the Earth, and the Theory of the Tides [see Art. (82)]; and he has published valuable Tracts on Physical Astronomy for the use of students.

Sir John Lubbock's name deserves mention here as having devoted his energies, about the same time Sir John with $\mathrm{Mr}$ Airy, to intricate and laborious researches Lubbock. connected with the least inviting parts of physical astronomy, and striven to redeem England from the reproach of indifference or incapacity in respect of such inquiries. The chief object of his numerous memoirs (in the Philosophical Transactions for 1830, and following years) is to express, in a more convenient and exact manner than had been in use, the complicated serieses indicative of the perturbations as well in the Lunar as in the Planetary Theory.

The Lunar Theory: MM. Plana and HANsen.- (118.) M. Plana, an astronomer and analyst of the greatest and Hanmerit, who fortunately still does honour to the native sen on the city of Lagrange (Turin), though the author of a great Lunar number of Memoirs in the Transactions of the Turin Academy, on different points of Applied Mathematics, is, and will be, best known by his elaborate and learned treatise on the Theory of the Moon. This extends to three very bulky quarto volumes, which are, so to speak, one mass of symbols. Nothing can 
give a more impressive idea of the condition at which Physical Astronomy has now arrived, than a glance at this mountain of intellectual labour,-especially as to the intricacy, inexpressible by words, of the motions of our own satellite. Here we have a work, not much smaller in appearance than the whole Mécanique Céleste, devoted to this one object. It is not superfluous to add, that this is no chimerical undertaking - no curious puzzle_-no learned trifling; the Lunar Theory is the grand basis of the Art of Navigation. The real and main use to mankind of our companion planet is a discovery of these latter ages : her cheerful and beneficial light, which all appreciate, and all enjoy, may almost be termed a secondary boon.

(119.) Great work The merit of M. Plana is not so much that of of the for- an original geometer, outstripping the theories of Lamer.

place and Lagrange, as of a most intrepid and skilful calculator, who has contrived to place in complete order the whole mathematical and many of the arithmetical steps of the solution of one vast problem, extending to some 2000 quarto pages. The calculations he has made unaided! The details are arranged in so lucid a manner, as to court enquiry from those interested in verifying them; and though the readers of so abstruse and indeed repulsive a work must be few indeed, it has already proved of essential service in the way which was intended - the improvement of the lunar tables. The approximations by series are in all cases carried to the fifth powers of small quantities, and, in some instances, to the seventh powers. Sir John Herschel has expressed in forcible and picturesque language the nature of what is wanting to the completeness of Laplace's investigations and which M. Plana has supplied, as regards the theory of the moon's motions: - "In the Mécanique Céleste, we admire the elegance displayed in the alternate interlinking and development of formulæ, and exult in the power of the analytical methods used; but when we come to the statement of numerical results, we quail before the vast task of filling in those distant steps; and while cloud rolls after cloud in majesty and darkness, we feel our dependence on the conclusions attained to partake of superstitious trust, or of amicable confidence, rather than of clear and demonstrative conviction."1 The courage of $\mathrm{M}$. Plana did not "quail" before the serried ranks of symbolic legions. He attacked them first, and finally became their commander. But more than this; on the same high authority, "his analysis is always graceful, his combinations well considered, and his conceptions of the ultimate results to be expected from them perfectly just, and justified by the results when obtained."

I may here mention that M. Plana, in conjunction with M. Carlini of Milan, undertook the calcula- tion of the moon's motions from theory alone (that is, Lunar with only the fundamental constants required to be Tables given by observation), in compliance with a from gramme issued by the French Academy of Sciences alone. on the proposition of Laplace; and that a French Carlinigeometer, M. Damoiseau, on the same occasion, produced an independent investigation with very elaborate and valuable tables founded thereon.

M. Plana is the author of many other more circumscribed researches on important points in physical astronomy, in geodesy, and in mathematical physics.

M. Hansen, a German astronomer and analyst (122.) of great merit, has made the most recent considerable M. Hanse improvement in the theory of the moon. We will first, however, allude to an invention which is applicable to the whole theory of perturbation.

We have seen, in the first section of this chapter, that the effects of perturbation may be considered either as applicable directly to the three co-ordinates either as applicable directly to the three co-ordinates perturba-
of the perturbed body, or to the variation of the ele- tions. ments of the orbit considered as instantaneously varying; that the former method has, the advantage of being in most cases, and especially in first approximations, most direct; the latter is most applicable to secular inequalities, and has a great recommendation in the exact physical conceptions on which it is founded. M. Hansen has proposed a third method, a refinement, in fact, on that of Lagrange, in which, by an assumption purely mathematical and arbitrary, he throws the effect of perturbation entirely on the element of time, so that with the time so altered, and invariable elliptic elements, the conditions of perturbation may be satisfied, and the true place of the body may result from the calculation. Such is the general nature of the conception, which, to be carried out, requires the introduction of subsidiary terms, which serve to correct the latitude and radius vector. The advantages which are understood to follow from this highly artificial mode of proceeding are stated to be (1.) that the serieses expressing the perturbation of the co-ordinates are more convergent than in the other methods, and likewise the coefficients of the terms to be retained are more easily calculated; (2.) M. Hansen considers that his method enables him to ascertain with certainty those terms which, when fully calculated, will affect the result in a sensible manner. The inventor has applied his methods both to the Lunar and to the Planetary theory.

We have said that physical astronomy is indebted to $M$. Hansen for a notable improvement in the theory of the Moon,-the discovery, in fact, of two inequalities of long period, the existence of which had been more than suspected from observation, but which were tions.

(1)

.


not accounted for. In the infancy of the lunar theory, Euler had predicted that it would always be impossible, on account of the perturbing forces of the planets, to predict the Moon's place within $30^{\prime \prime}$; and it was a quantity of about this measure which remained outstanding after all the resources of analysis seemed exhausted. M. Poisson and Sir J. Lubbock showed that the anomaly could not be due to the solar action, nor yet to the irregularity of the Earth's figure. The planetary attractions, then, alone remained. M. Hansen discovered two independent inequalities due to the action of Venus. One of these is an indirect (or, as it is sometimes called, reflected) effect depending on the change of form of the Earth's orbit by the attraction of Venus, which, of course, modifies slightly thesolar perturbation of the Moon's longitude. It is, in fact, a secondary consequence of long inequality of Venus and the Earth investigated by Mr Airy [Art.(115)], and has the same period, namely, about 240 years. Its greatest amount is $23^{\prime \prime} \cdot 2$. The other inequality discovered by Hansen is of a still more curious and complicated kind, which goes on increasing for 2000 lunations, when it attains a maximum value of $27^{\prime \prime} \cdot 4$ in longitude (although the perturbation of the radius vector does not exceed 10 feet), after which it diminishes for an equal space of time.

By these discoveries, the movements of our refractory satellite may be considered to be, after an unprecedented amount of labour, accounted for by theory almost or quite within the limits of the present accuracy of observation. ${ }^{1}$ M. Hansen has recently been engaged in perfecting the practical details of the lunar theory, in accordance with the extensive reductions of the Greenwich observations which will be mentioned in the next chapter. He has also given the first complete theory of "Foucault's pendulum," also to be mentioned hereafter. Altogether, he stands amongst the most eminent analytical astronomers of the present day.
We have in this section selected (though not by design) representatives of the four great intellectual communities of Europe, engaged in the mighty task progress of of perfecting the theories of physical astronomy. Astronomy Poisson for France, Airy for England, Plana for Italy, Hansen for Germany. It would be easy, of course, to add the names of many others engaged in similar works, and scarcely less deserving of notice. Some of these will find a place in other chapters, and we have yet one section of this chapter to devote to the history of a discovery of rare interest, in which France and Eugland have a joint share. We may in Europe, be allowed to mention the names of $\mathrm{M}$. Damoiseau and M. Pontecoulant in France; the former known by his excellent lunar tables deduced from theory, the latter for his calculations of cometary perturbation, and his compendious treatise on physical astronomy, based on the Mécanique Céleste; in Italy, MM. Carlini and Santini. But in Germany these studies have been perhaps most systematically pursued. MM. Gauss and Encke have only not been included in this section because we find it more suitable to our plan to associate the name of the former with his theory of Terrestrial Magnetism, and the latter with the recent history of Comets. Bessel likewise was a physical as well as first-rate practical astronomer. I will here only add that these and in severe and arduous studies have at length been America. effectually cultivated beyond the limits of Europe. Mr Bowditch (born 1773, died 1838), a private Bowditch. gentleman of the United States, undertook the gigantic labour of translating and illustrating, with a complete commentary in which every difficulty is considered, and every step of analysis supplied, the Mécanique Céleste of Laplace. Since his deatl, a younger race of American mathematicians has taken up the great problems of physical astronomy, amongst whom may be mentioned Mr Walker and Mr Peirce. The latter gentleman has recently (1853) published lunar tables, embracing the latest researches of theory.

\section{§ 5. M. Leverrier-Mr Adams.-The inverse method of Perturbations. Prediction of the place and orbit of Neptune from the motions of Uranus.}

We have now to chronicle a discovery which, by general consent, stands first in the achievements of science, not only in the period now under review, but even in the long and eventful series of years which have elapsed since Newton established the doctrine of universal gravitation.

The discovery of which we speak was no less than the proof of the existence of a planet beyond the recognised boundary of our system, merely as-an inference from the perturbed motion of the outmost planet Uranus; a proof, not general or abstract, but particular and specific: "Look, on such a night, and in such a direction, and there you will see (by the telescope) a star, small indeed, but with a distinguishable disk,-that is the planet which has made Uranus move so unsteadily in its orbit;"-so spoke the mathematician; and the zealous astronomer, to whom the call was especially addressed, pointing his glass to the sky, discovered at once, that is, the same evening, a body answering almost precisely in position, as well as in brilliancy, to the oracular announcement.

1 M. Hansen has also discovered the course of a small inequality of the Moon's latitude, detected from observation by Mr Airy. His theory of the Moon's figure has been referred to in a note to Art. (57.) 
(129.)

Its great difficulty.
Since the publication of the Principia, or rather we should say since the great theory contained in that work had fully attracted the sympathies of thoughtful and able men, nearly the whole science of physical astronomy consisted in the solution of one vast and intricate problem, which has been called the "Problem of the Three Bodies." To this were bent the powers of Clairaut, Euler, Lagrange, Laplace, Plana, Hansen, and so many more. "Let three bodies be placed and move in a given manner in space and attract one another by the Newtonian law, to determine the motions as affected by their mutual influence." The problem solved independently by the two analysts whose names stand at the head of this section is this, "Given two bodies (the Sun and Uranus) and their relative motion, to find at any moment the position of a third body whose attraction shall be required to account for those motions." To have solved this new and far more difficult problem (under certain limitations) is a triumph altogether unlike in kind to any of the other brilliant successes of which we have had to speak in the preceding pages.

The great intricacy of the problem is not perhaps at the first moment fully apparent. The perturbation of the known planet (Uranus) is not the effect, either in direction or in amount, of the attraction of the body sought, either at the instant, or at any previous instant. It is an accumulated effect arising from the totality of the mutual influences of the two planets during a long space of time, and under a variety of circumstances, which circumstances it is the aim of the solution to discover. But, more than this, we do not even know the quantity or direction of the perturbative action at any moment for which the cause is sought, for we do not know the purely elliptic elements of Uranus. His motion has, by hypothesis, been always troubled by this exterior planet, and Uranus has been so short a time known and observed (only accurately since 1781) that the motion has not yet been cleared of ordinary inequalities (due to the action of the unseen body), which, therefore, are inextricably mixed up with the elliptic elements. It is absolutely necessary, therefore, to suppose not only the elements of the new planet to be unknown, but also the elements of Uranus to be severally affected by unknown errors. This nearly doubles the unknown quantities to be found.

We shall now glance at the history of these unexplained perturbations, and at the rise and growth of the idea of their being explicable by the influence of an unseen body.

After Sir William Herschel had discovered, in
1781, the planet which he called Georgium Sidus, Irregulari (afterwards named Herschel, and finally, by general ties of the consent, Uranus,) it was easy, by a few observations, motions of to ascertain its approximate orbit and distance from the sun. But the extreme slowness of its motion (it will not have completed a single revolution before 1861) made it impossible to determine its elements with precision, until it had been discovered that the records of astronomy contained about twenty observations of this body before its planetary nature was discovered; being, in fact, registered places of fixed stars where no stars exist, concurring in brightness and position with the circumstances of Uranus at those remoter periods; the earliest of these observations was one of Flamsteed, in 1690 . The first person who constructed tables of the planet was Delambre; but even at that early period, it was remarked, that the modern were not satisfactorily reconcilable with the ancient observations; and, finally, Delambre included of the latter only one, by Mayer, which he did, he tells us, "out of pure respect," although it certainly rendered the tables less exact and less durable. The longer the planet was observed, and the greater the care that was expended in analysing and combining the observations, the more clearly it appeared that the tables had only an empirical character, satisfying observations for a few years before and after the time for which they had been constructed; and that, in particular, as the nineteenth century advanced, the deviations from elliptic regularity became more and more intolerable, till the "ancient" observations were at length totally given up. This was the state of matters in 1821, after M. Bouvard of the Observatory of Paris, a most able calculator, had exhausted every resource in improving the Tables of Uranus. A few years more gave that patient astronomer the mortification of seeing his tables as obsolete as those of his predecessors, and numerous surmises were circulated as to possible explanations of the anomaly: a failure in the law of gra- Hypothese vity, cometary perturbation, a resisting medium, to accoun and, finally, the presence of an unseen planet, were for them. amongst these guesses. The last and most plausible of these hypotheses occurred to many; amongst others, to Mr Hussey in England, M. Bouvard in France, and later to M. Bessel in Germany. ${ }^{1}$ The first of these astronomers actually consulted Mr Airy, in 1834, on the possibility of predicting the place of the perturbing planet from theory, and then discovering it by observation. Mrs Somerville, in 1836, gave a precise expression to the same idea. ${ }^{2}$ From this time the subject could not be lost sight of. The errors of the tables which in 1821 were insensible, increased in 1830 to $15^{\prime \prime}$ or $20^{\prime \prime}$, and in 1845

\footnotetext{
1 Clairaut, nearly a century before, in calculating the return of Halley's comet, hinted at the possible perturbation due to a planet superior to Saturn.

2 In her Connection of the Physical Sciences.
} 
amounted to two minutes of longitude. M. Bouvard to his latest years, perhaps his latest hours, ${ }^{1}$ cherished the hope of extricating this theory from its difficulties. He also engaged his nephew M. Eugène Bouvard in the same career, who appears to have followed it with much zeal and intelligence, and in 1845 constructed new tables of Uranus. But by this time two geometers had separately and independently undertaken the problem, with the determination of finding, if possible, a physical solution of all this perplexity. The earliest in point of date was Mr Adams, a young graduate of Cambridge; the other was M. Leverrier of Paris, whose attention was directed to the subject by.M. Arago. As the researches of $M$. Leverrier, though second in point of time, occasioned the actual recognition of the planet, and thus stamped the correctness of the solution with success, we shall consider them in the first instance.

(133.) M. Leverrier is, we believe, a native of St Lo M. Levere in Normandy, a province which has been singularly rier's inves- productive of eminent men (Laplace and Fresnel tigations; were of the number). With no advantages, but the reverse, he won a high position at entering the polytechnic school, which he constantly maintained. He at first, we believe, attached himself to chemistry, but his taste for physical astronomy was soon developed, and was advanced entirely by his private efforts. It is a peculiarity of the mode of cultivating the sciences in Paris, that such abstruse and difficult studies are not merely engaged in temporarily for purposes of academial distinction, but that they actually become a "carrière" or calling, and are pursued in that methodical manner for which the French are distinguished. In 1845, when he commenced the careful examination of the theory of Uranus, M. Leverrier was already favourably known by his researches on comets, and on the orbit of Mercury, but especially by immense calculations, connected with the secular inequalities of the planets, by which his ability and hardihood in computation had been thoroughly exercised. He began his new enquiry with the method and intrepidity of calculation which distinguish him. He revised with the most minute care the observations of Uranus, and computed afresh every sensible perturbation which theory recognised as arising from known planets. This done, and having compared the most probable orbit with observations which he collected from authentic sources, and especially from the Greenwich observations which were communicated to him for this purpose, the result was, that even confining himself to observations since 1781 , arranged in eleven convenient groups (each resulting from many observed places), and attributing to each group the largest error which could be in reason allowed, and even admitting that all these errors were in the direction most favourable to the assumption, it was still impossible to account for more than one-fourth part of the observed discordances.

M. Leverrier then assumed that a perturbing planet existed beyond the orbit of Uranus, and at nearly double its distance from the sun, in conformity with the empirical law, (usually attributed to Bode the German astronomer,) which expresses with general accuracy, thus far, the arrangement of the planetary system. The law is, that the distances of the planetary orbits from Mercury are successively doubled. This assumption-(it was absolutely necessary to assume some distance to begin with) - was ingeniously confirmed by other considerations.

Leaving the perturbations in latitude out of account, he now considered each error of Uranus in longitude as the expression of a perturbation due to the action of the unknown planet, and capable therefore of algebraic expression in terms of the elements of that planet, namely its excentricity, longitude of perihelion, epoch in its orbit, and mass; but, as we have already remarked, the first three of these elements must be considered as incorrectly assumed for Uranus itself, as well as the mean distance of that planet, and, therefore, there are four unknown corrections for its elliptic elements, making in all eight quantities to be eliminated from the discordances of theory and observation. So complex an elimination cannot be directly effected; and even if it could, the result could not be depended on, as the possible error of each observation involves a fresh and important source of doubt in the conclusion. M. Leverrier proceeded, by a series of gradually restricted assumptions, to find within what limits the more important elements might be made to vary without producing effects incompatible with observation, and his attention was at first confined to the approximate mean longitude of the planet. He obtained a result after Their rea prodigious amount of tentative calculation. The sult. excentricity and position of the perihelion were then inferred. On the Ist June 1846 he announced to the Academy of Sciences that the true longitude of the expected planet for 1 st January 1847 was $325^{\circ}$, with a probable error of $10^{\circ}$. This result was immediately published in the Comptes Rendus.

Between the 1st June and 31st August 1846, when his third memoir on the perturbations of Uranus appeared, M. Leverrier busied himself in obtaining a farther approximation to the elements and place of the suspected planet. He now assumed the correction of the mean distance amongst the other quantities to be sought. By a fresh calculation he
(13.5.) how conducted : 
deduced a complete list of elements as regards longitude; and diminished the mean distance considerably. The true longitude by this calculation differed only $1 \frac{1}{2}$ from his previous estimation. He finds for the mean distance from the Sun 36 times that of our Earth (that of Uranus being 19), the period 217 years, and the mass $\frac{1}{3} \frac{1}{30}$ of the Sun's. Assuming the density of the planet to be the same as that of Uranus, he conjectures that the apparent diameter will be $3^{\prime \prime} \cdot 3$; that it will therefore have a visible disk sufficient to distinguish it from a fixed star, and that its brilliancy should equal that of a star of the 8th or 9 th magnitude. As the result of these suppositions, he found that the whole errors in the places of Uranus from 1781 to 1844 were reconciled within quantities amounting but in one instance to $5^{\prime \prime}$; that the ancient observations of the 18 th century were reconciled within $7^{\prime \prime}$ or $8^{\prime \prime}$; and the oldest observation of all, that of Flamsteed in 1690 , had an outstanding error of $20^{\prime \prime}$, a quantity very far from excessive, considering the state of astronomy at that period.

No one who read at the time the abstract of this resulting in remarkable paper in the Comptes Rendus failed to the disco- be struck with it, not only as regarded the weighty Neptune by matter, thus publicly announced, but also on account M. Galle. of the calm and well-grounded conviction which the author manifested in the truth of his bold conclusions, and the definite manner in which he gives the challenge to practical astronomers to verify or disprove them. "Since Copernicus declared" (according to the prevalent tradition) " that when means should be discovered for improving the vision, it would be found that Venus had phases like the moon, nothing," writes $\mathrm{Mr}$ Airy, "so bold, and so justifiably bold, has been uttered in astronomical prediction." M.Leverrier had hastened his calculations in anticipation of the approaching opposition of the new planet in the autumn of 1846 , but it is very doubtful whether astronomers would have made the discovery at that time, but for his personal application to M. Galle, then assistant-astronomer at Berlin, where a powerful refractor suitable for the search existed. So ardent a conviction in a manner compelled the proof which the geometer claimed, and M. Galle, whose intelligence and zeal are well known, pointed his telescope to the sky the very evening that $\mathbf{M}$. Leverrier's letter reached him. Fortunately provided with a newly published star map by Brenicker of that region of the heavens, which was not at that time diffused generally amongst European observatories, he detected that same night (the $23 \mathrm{~d}$ September 1846) a star-like body of the 8 th magnitude, not noted in the star chart, therefore a wandering body, having a manifest disk from $2 \frac{1}{2}$ " to $3^{\prime \prime}$ in diameter, and distant only fifty-four minutes of a degree from the predicted place.

(138.) It will be remarked that the discovery in question was anticipated and completed in France and Germany alone; England had no direct participation. We mustnow, however, state briefly what occurred there of a similar character at the same time, and even earlier.

Mr Joris Couch Adams, when a student at St John's College, Cambridge, in 1841, formed the design of detecting the position of a perturbing planet which should account for the anomalous motions of Uranus. He made a preliminary essay on the problem in 1843 , assuming the distance of the suspected body from the Sun to be double that of Uranus. I learn from good authority that he obtained a place for the unseen planet not very different from that which he finally adopted. Early in 1844 he obtained from Greenwich the valuable series of places of Uranus which were afterwards in like manner applied for by M. Leverrier. In September 1845, he communicated to Professor Challis the elements of the new planet's orbit (neglecting the inclination) and an ephemeris of its geocentric place; and in October he transmitted the elements also to the Astronomer Royal. Mr Adams This, it will be observed, was soon after M. Leverrier's preceded attention was first directed to the subject and nine M. Levermonths previous to his announcement of the locality similar in where the new body should be sought. Mr Adams vestigaafterwards repeated his calculation with a mean dis- tion; tance $\frac{3}{3} 0$ th less than before, and considered himself warranted, by the improvement thus produced on the residual errors, in inferring that a farther considerable-diminution of the mean distance would satisfy the observations still better. This was communicated to $\mathrm{Mr}$ Airy on the 2d September 1846 ; subsequently therefore to the publication of M. Leverrier's Elements. Mr Adams, in communicating his results (at a later time) to the Astronomical Society, with characteristic modesty says, "I mention these dates merely to show that my results were arrived at independently, and previously to the publication of $\mathbf{M}$. Leverrier, and not with the intention of interfering with his just claims to the honors of the discovery, for there is no doubt that his researches were first published to the world and led to the actual discovery of the planet by Dr Galle."

And such is no doubt the fact. The priority of $\mathrm{Mr}$ Adams in the mathematical investigation is as certain as that the researches of M. Leverrier alone produced the discovery of Neptune. Even the search for the in conseplanet which took place in August and September quence. 1846 at Cambridge by Professor Challis, was not occasioned by Mr Adams' researches only; it was the near coincidence of the longitude assigned by $\mathrm{Mr}$ Adams in the previous October with that published by M. Leverrier in June 1846 which induced $\mathrm{Mr}$ Airy to suggest this investigation of the heavens, and to offer (if need were) himself to bear the expense. Had the planet been discovered ${ }^{1}$ at Cambridge

1 The planet was indeed seen at Cambridge by $\mathrm{Mr}$ Challis, for it was recorded more than once amongst the numerous fixed stars whose places were taken down in the progress of the search; but as the comparisons of the "sweeps" were not made at the time, the discovery was anticipated by M. Galle. 
before it was at Berlin, M. Leverrier must still have had a share in the credit of success.

It is perhaps to be regretted that Mr Adams had not given his whole investigation to the world, or at least published his results, so as to arouch the confidence which he felt in his own prediction, and to throw upon practical astronomers generally the responsibility of its verification. Had he done so in 1845 , it is possible that the planet might have been discovered at the opposition of that year; but it is at least certain that $M$. Leverrier's claims to priority as regards the discovery of Neptune would have been effectually anticipated. But it is only just to our countryman to recollect the difference of his age and position. M. Leverrier was at the time about 35 years of age, and was a candidate for the substantial benefits as well as for the honour of a membership of the Academy of Sciences. Mr Adams must have been nine or ten years younger at the period of this discovery, a circumstance which enhances our admiraion at the achievement, whilst it gives an additional grace to the modest conduct of the author.

I have endeavoured to state correctly (with due regard to the limits of this essay) the main facts of the most curious case of double discovery which, perhaps, the history of science presents; and happily as to the facts, down to the minutest detail, no discrepancy of opinion ever existed. Different minds will, with perfect truth, attach more or less distinction to the two illustrious rivals,-neither of whom has for an in-

$\begin{array}{lcc}\text { Epoch of Elements, } & \text {. } & \text { Theory-Leverrier. } \\ \text { Ist Jan. } 1847,\end{array}$

(144.) The differenfes of theory and observation are so striking as to have occasioned surprise to many persons that, with data so erroneous, the perturbations of Uranus or the longitude of Neptune at the epoch of discovery should have been obtained even approximatcly.

(145.) The fact, however, is this :-that the mutual perHow an as- turbations of Uranus and Neptune are sensible for sumption of
erroneous elements planets are nearly in conjunction. The conjunction led to the (when the mutual distance is least and the attraction discovery; strongest) took place in 1822 . Now the places of Uranus from 1690 (the first observation) until 1800 can be sufficiently well represented by elliptic elements. The perturbation of Neptune became sen- stant lowered the dignity of his position by one ungenerous expression,- - but that the absolute merit of both is of the very highest character is on all hands admitted. "The names of M. Leverrier and Mr Adams," said Sir John Herschel, addressing the Astronomical Society, "which Genius and Destiny have joined, I shall by no means put asunder; nor will they ever be pronounced apart so long as language shall celebrate the triumphs of Science in her sublimest walks."

But before closing, I must briefly state how far the orbit of the planet Neptune, when discovered, realized the previsions of theory. A fortunate cir- Neptune as cumstance rendered it easy to obtain at an early a fixed star. period a correct knowledge of the elements. It seems that the planet Neptune was observed by Lalande at Paris on the 8th and 10th May 1795, and entered as a fixed star, notwithstanding a distinct change of place between the observations, actually corresponding to what the planet should have had. ${ }^{1}$ But such an oversight had been made by Lemonnier in the case of Uranus. By means of this observation of fifty years back, the orbit was easily computed. It is a singular and startling fact, that, except as regards the longitude on the orbit, the other elements computed from observation were somewhat widely different from those assigned by M. Leverrier and Mr Adams. We shall present them in a tabular view. The value of the mass in the last column is calculated from the elongation and period of a satellite of Neptune discovered by Mr Lassell.

Theory - Adams.
6 th Oct. 1846
$323^{\circ} 2^{\prime}$
$37 \cdot 24^{2}$
$299^{\circ} 11^{\prime}$
0.1206
ธते
Observation-Walker. 1st Jan. 1847. $328^{\circ} 33^{\prime}$ $30 \cdot 04$ $164 \cdot 6$ $47^{\circ} 12^{\prime}$ 0.00872 $x^{\frac{1}{8}}{ }^{3}{ }^{3}$

sible therefore only twenty years before conjunction. In this time Neptune describes (really) only $\frac{1}{8}$ of a circumference, or $45^{\circ}$, and relatively to the motion of Uranus about the same. It is evident then that even a considerable error in the period of Neptune would scarcely sensibly affect the law of perturbation during twenty years, and that the approximate determination of the place of the perturbing planet about the time of conjunction will not be much affected by the error of that assumption. Again, as to the error of mean distance, we may observe, that since the mutual action of the planets is confined within such (comparatively) narrow limits of space and time, though we might anticipate a tolerable approximation to the interval between the bodies

1 One of the observations was suppressed in the publication, and only discovered on searching Lalande's MS.

2 This was the hypothesis upon which Mr Adams made his second or corrected calculation of elements. Nevertheless, he inferred from that calculation that the mean distance might with much probability be reduced to 33.4 .

3 Pierce. Struve's mass is y 
at that time, it would be unreasonable to expect a correct determination of the form and ellipticity of the orbit of Neptune, such as might belooked for if theperturbations weresensible through the entire orbits; and in fact, by varying the position of the perihelion and the amount of excentricity, we may, for an assunied mean distance, obtain any value whatever for the interval of the two planets at a particular time. We have seen the origin of the false assumption of mean distance on the part both of $\mathrm{M}$. Leverrier and $\mathrm{Mr}$ Adams, and we find that the mathematical solution corrects to a great extent the error of that assumption by giving a correspondingly incorrect position of the perihelion, and also an exaggerated measure of the excentricity, by which two circumstances the planets of both mathematicians would have had, near the time of conjunction, a distance from the sun of only 32 or 33 radii of the earth's orbit, the true distance being about $\frac{1}{12}$ part less. This still remaining error was palliated, and evidently might for a time have been completely masked, by assuming a mass of Neptune proportionally too great, as indeed the table we have given shows was the case.

That the discovery of Neptune took place at the time when it did was no accident. The conjunction of Uranus and Neptune, when alone the perturbation and why the disco very took place when of the elliptic elements is perceptible, is a rare pheit did. nomenon, occurring but once in about 172 years. The last conjunction previous to 1822 was in 1649 : we have seen that the attention of astronomers was importunately called to the subject by the irregularities in the motions of Uranus at the first conjunction succeeding its discovery.

We are indebted to Professor Peirce and $\mathbf{M r}$
Walker of the United States for many useful inves- Principal tigations connected with the orbits of Uranus and inequality Neptune; but it is to be wished that the theory were of Uranus completely re-examined, and also the problem of the and Nepinverse method of perturbations which has now become a systematic portion of physical astronomy. The near commensurability of the two periods presents a peculiar case of perturbation similar to the long inequality of Jupiter and Saturn; but as its period is no less than 4047 years, ${ }^{1}$ although the coefficient of the inequality is considerable, it will not perceptibly alter the motions of either planet except in a long course of years.

It will not be supposed that either M. Leverrier or Mr Adams could, after such a memorable triumph, abandon the pursuits of physical astronomy. The first has continued his researches on the orbits of comets and on the perturbations of the solar system; rier. and having been recently appointed to succeed $\mathrm{M}$. Arago in the direction of the Paris Observatory, we cannot doubt that he will infuse new life into its management. Mr Adams has made the discovery of some important oversights in the details of the lunar theory. One of these, with reference to Laplace's Theory of the Secular Acceleration of the Moon's mean Motion, has been referred to in a note to Art. (62). Though Mr Adams occupies no public post, and though he has declined the honours of a title, his continued residence at Cambridge must influence very beneficially the studies of the place, where some of his many friends have founded in memory of his achievements a perpetual prize for the advancement of physical astronomy, which is denominated the Adams Prize. ${ }^{2}$

Prize 
were both distinguished by modesty, simplicity, and love of truth. Of all their contemporaries of eminence, few escaped so happily from the unprofitable strife of rivalry and personal disputes, and none exhibited a more impartial desire for the advancement of the science to which they were devotedly attached. Perhaps neither was a man of lofty talent, yet they did not fail to secure deserved respect in their own day, and far more gratitude from the posterity whom they essentially benefited than falls commonly to the share of men of higher pretensions in this respect. Both their names are important links in the history of astronomy.

(150.) Nevil Maskelyne, born in 1732 , and educated at Muskelyne; Cambridge, was early attached to astronomical pursuits. After various minor services connected chiefly with navigation and the discovery of the longitude, he attained the honourable post of Astronomer Royal, which he filled from 1765 till 1811 with distinguished success. His immediate predecessor was Dr Bliss, who held the office for but three years and without distinction. Practically, Maskelyne may be said to have succeeded Bradley, probably the greatest astronomer whom England has yet seen, whose discoveries have been recorded in the last Dissertation, and whose observations, through a variety of circumstances, were destined rather for the benefit of the nineteenth century than for his own. Maskelyne wisely recollected that the observatory was mainly founded for the improvement of navigation, ${ }^{1}$ and one of his earliest labours was the establishment in 1767 of the "Nautical Almanac," a work based on the best astronomical observations and of the highest service to seamen. “During Maskelyne's long tenure of office he was entirely devoted to its duties, making himself all the most delicate observations, particularly those of the moon, and rarely quitting the observatory except to attend the meetings of the Royal Society. The perfect method and continuity of his observations give to them a great value, especially for the correction of the Lunar Tables, in which respect they are indeed without a parallel. But the regularity of their publication was not their least merit. Four large folio volumes include the patient labours of a life (for he had but one assistant). Delambre in his character of Maskelyne says, that if through some catastrophe the whole materials of science should be lost, except these volumes, they would suffice to reconstruct entirely the edifice of modern astronomy."' cal publication which had issued from Greenwich Observatory was the "Historia Celestis" of Flamsteed; Halley's, Bradley's, and Bliss's observations remained in manuscript. A like fate attended most

of the foreign astronomical observations; labour the most irksome and conscientious lie buried in piles of MS. useless to science, and which therefore might almost as well have never been made. This is particularly the case with the Parisian observations (as the impartial Delambre records with pain), commenced even before the time of Flamsteed; but which, owing to this cause principally, have remained even to the present day (and it is forty years since Delambre wrote his patriotic protest) without contributing materially to advance astronomy. Let it then be recorded to the honour of Maskelyne, that this important step of regular and full publication at the public expense was entirely due to him. His places of the sun, moon, and planets, were the foundation of the improved theories of physical astronomy, then more ably cultivated on the Continent than in Britain, and of the tables also chiefly furnished by German and French computers.

Maskelyne's more important contributions to science may be briefly stated under these two heads; -The determination of the Lunar orbit from observation, and its application to navigation ; and the determination of the local attraction of Schehallien and of the density of the earth.

I. The determination of the Lunar Orbit from $\mathrm{Ob}$ servation and its application to Navigation.-Though astronomy owes (as we have already said) much to Maskelyne in the exact determination of the places of the sun, planets, and the most conspicuous fixed stars, the comparison of the Lunar place with the tables was by far the most arduous and the most important of his undertakings. It was the most arduous, because, from the extreme complexity of the moon's motions, every part of its orbit must be narrowly watched, requiring the astronomer's presence at his instruments at all possible hours during the course of a lunation, these motions being subject to important changes which recur every nineteen years, besides others of yet far longer duration. It was Maskelyne's good fortune, and at the same time the reward of his perseverance, to watch three revolutions of the Iunar nodes. He did not content himself, however, with making observations; he contributed in every possible way to the improvement of the tables of the moon's motion. He was in constant communication with Mayer, one of the ablest astronomers of his day, and he directed the calculation of Bradley's observations by Mason, for the farther improvement of Mayer's Tables. Maskelyne's own observations, to the number of at least 5000, were used by Bürg in his excellent tables of the moon which have even yet been hardly surpassed; but their full value has only been tested recently by the

\footnotetext{
1 In the warrant appointing Flamsteed to be the Royal "Astronomical Observator," bis duty is declared to be " to rectify the tables of the motions of the heavens and tbe fixed stars, so as to find out the so much desired longitude at sea, for perfecting the art of navigation."

2. From an article on Greenwich Observatory in the Edinburgh Review, written by the author of this Dissertation.
} 
systematic reduction of all the lunar observations of Maskelyne and Pond, and a comparison with Damoiscau's tables, under the direction of the present Astronomer Royal, Mr Airy.

(153.) But, for the application of the method of lunar The Nau- distances to navigation, farther aid than the contical Alma- struction of good tables was required. This Maskenac. lyne provided by obtaining the regular publication of the Nautical Almanac, superintended by himself, and containing the distances of the moon from the principal fixed stars at predetermined hours for the meridian of Greenwich, a comparison of which with the distance observed by means of the sextant in any part of the world enabled the seaman (after proper reductions) to infer the exact Greenwich time of the observation, and thence, by comparison with the local time obtained by the usual methods, to obtain his longitude. To this, long admitted to be the best practical solution of the celebrated problem of " the longitude at sea," Maskelyne contributed probably more than any other person. His "Lunar Distances" were reprinted in the French Almanac (Connaissance des $T e m s$ ) for a considerable number of years.

(154.) II. The determination of the Attraction of ScheAttraction hallien, and of the Earth's Density.-The deviation of moun- of the plumb-line from the vertical by the neighbourtains. hood of a mountain had been pointed out by Newton ${ }^{1}$ as a direct consequence, and also as a test, of the principle that gravity resides in every part of the earth as well as in the earth as a whole. Bouguer had the merit of pointing out the form in which the experiment might be made, and of making the trial, though in a rude and insufficient manner, in the Peruvian Andes in 1738. He observed the effect of the mountain on the south side only, but at two stations unequally distant from its centre of attraction. The numerical result being (as the author himself admitted) without value, Maskelyne proposed to the Royal Society in 1772 to repeat the observation on some British mountain. A "Committee of Attraction" was named, which, besides Maskelyne, included Cavendish, Franklin, and Horsley. Cavendish, as might have been expected, took an earnest part in it. The search for a suitable hill was confided to $\mathrm{Mr}$ Charles Mason in 1773. Skiddaw and the Yorkshire Hills were first thought of, but finally Schehallien in Perthshire was preferred. ${ }^{2}$ Thither Maskelyne himself proceeded in 1774 , with his assistant Burrows, and by these two, with the aid of a local land-surveyor, the labour of the astronomical and chief geodetical operations, including the measurement of two base lines, was effected between the 30 th June and the 24th October, notwithstanding the hindrances of a most unfavourable season.

The distance between the two stations obtained with Ramsden's 9-inch theodolite, was 4364.4 feet, which in the latitude of Schehallien corresponds to $42^{\prime \prime} \cdot 94$ of latitule of latitude. The observed difference of latitude by at Schehal337 observations with Sisson's 10 -feet zenith sector lien. was $54^{\prime \prime} \cdot 6 .^{3}$ The excess, or $11^{\prime \prime} \cdot 6$, is the double attraction of the hill drawing the plumb-line towards itself at the two stations. The sine of this angle, or $I_{7} \frac{1}{8} 04$, represents the actual ratio between the double attraction of the hill and the attraction of the earth. But by the computation of the attraction which the hill ought to exert, from its figure, as determined by Maskelyne's gauges, were its density the same as that of the globe generally, this ratio should amount to ₹ $\frac{1}{9} 3$, which can only be accounted for by assuming the earth to be denser on the average than the hill of Schehallien in the proportion of 17804 to 9933. This deduction was made by Dr Hutton by means of a troublesome calculation of the summation of the attractive effects of a number of vertical prisms into which the hill was imagined to be divided. The artifices of calculation were, however, due to Cavendish (who it will be recollected was on the "Committee of Attractions,") as Mr Airy ascertained from his manuscripts. A careful lithological survey of the hill Earth's enabled Professor Playfair to deduce the probable density de mean specific gravity of the globe to be between 4.56 and 4.87 , which was somewhat greater than $\mathrm{Dr}$ Hutton assumed it.

This is the proper place to mention an experiment on the density of the Earth perhaps still more remarkable, devised by the Rev. Mr Michell, who constructed the apparatus, but first put in practice by $\mathrm{Mr}$ Cavendish in 17.97-8. It consisted in measuring the same the force of gravitation between two spheres of such end. small size that they could be moved by the hand nearer to or farther from one another. The essential part of the invention was to contrive a balance so delicate as to measure the almost inappreciable tendency of such small bodies to unite. Newton had shown that the attraction at the surface of any sphere is directly as its radius, which he observed must always be incomparably smaller than their tendency towards the earth, that is, their weight. In the largest and heaviest masses with which it has hitherto been found practicable to operate, this tendency (i56.) Michell and Caven ish's expe (1)

\footnotetext{
1 De Mundi Systemate, § 22. Newton, in a very remarkable passage of the Third Book of the Principia (Prop. X.), conjectures that "the quantity of matter in the earth may be five or six times greater than if the whole were composed of water."

2 A laughable mistake of Zach in his account of the Schehallien experiment (in his Attraction des Montagnes) is commented on by Playfair in the Edinburgh Review. In a note to the word Schehallien, Zach says, "Montagne appelée dans le pays en langue Erse Maiden pap, qui veut dire orage perpetuel." It is needless to add that these two alleged synonyms are different interpretations given by Gaelic scholars of the word. "From this inaccuracy," adds his reviewer, "his residence in London ought to have delivered him, for though he could not learn there what was Erse, he might have learned what was English."

3 Zach obtained the same result exactly by including all the observations, as Maskelyne had provisionally obtained by using only those stars on which he most depended.
} 
amounts to only a very minute fraction of a grain. How could such quantities be accurately estimated, so as not only to leave no doubt of the phenomenon of gravity thus acting on the small scale, but to deduce its amount, and honce to weigh the globe ?

(157.) Michell imagined for this purpose the balance of torThe Torsion sion, which was re-invented by Coulomb (who probably Balance.

first executed it)for the purpose of measuring electrical forces. Michell's apparatus came first into the hands of Wollaston, then of Cavendish, who made the experiment. He used a very light rod of deal, six feet long, suspended by a fine silver or copper wire, forty inches long, within a wooden case to defend it from currents of air. At each end of the lever was hung a ball of lead, two inches in diameter, and by a simple contrivance a pair of leaden spheres, weighing, together, 348 pounds, could be brought simultaneously into the neighbourhood of the balls (but outside the case), on opposite sides, so that their attractions might concur to swing the suspended lever out of the position of repose which it had previously taken up, under the action of the slight twisting force of the silver wire. A new position of rest was thus established, the small balls being pulled as much one way by the attraction of the spheres as they were urged in the opposite direction by the torsion of the wire. The position of repose being observed from a distance by a telescope (to avoid disturbance from the heat of the observer's body), the great spheres were then changed in position so as to act upon the opposite sides of the small balls, from what they formerly did. The deflection and new stable position would be as much on the other side of the zero, and the arc described would be an accurate measure of the double deflection. The force of torsion for $1^{\circ}$ of deflection is known by the time of oscillation of the lever and balls when free, and as the forces are exactly as the angles, the force corresponding to any displacement becomes known. Cavendish conducted the experiment with his usual patience, judgment, and success; he found the joint attraction of the small balls and large spheres to be about $\frac{1}{3} \delta \sigma$ of a grain, their centres being 8.85 inches apart, and he thence computed the density of the Earth to be 5.48 times that of water. Cavendish's paper is as usual a model of precision, lucidity, and conciseness. The attraction of the fixed parts of the apparatus is calculated and allowed for.

(158.) It would be difficult to mention in the whole range Baily's re- of physics a more beautiful and more important exsult. Freiberg and Baily of London. The former obtained 5.44, the latter 5.66 for the Earth's specific gravity;

this last result being worthy of much confidence from the extraordinary care taken to avoid errors and to obtain independent values of the quantities sought. ${ }^{1}$

Maskelyne continued his zeal for the promotion of astronomy to the last. He superintended the publication of 45 annual volumes of the Nautical Almanac. He left the whole of the observatory work in perfect order, and the greater part printed. He had wich. the well-earned satisfaction of finding his observations in request in every civilized country, the bases of the most useful tables, and the tests of the most advanced theories. He cultivated the friendly correspondence of astronomers in every country. Not given to change, he preserved the instruments and chief methods of the immortal Bradley; but suffi. ciently alive to the necessity of progress in the sciences, he introduced many simple but practical improvements in the art of observation. Even in his last years, satisfied that the celebrated quadrant of Bird was no longer the best instrument for its purpose, and was besides sensibly deteriorated by use, he adopted the circle instead (then recently come into notice, though first used more than a century before by Römer), and directed the construction of that by Troughton, though it was not placed at Greenwich until after his death, which occurred in 1811, in the 79th year of his age. His biographer Delambre mentions, that a considerable number of his posthumous memoirs were put into the hands of Professor Vince for publication. They have not, however, appeared.

Practical astronomy was on the Continent far behind its state in England at the period of which we speak. The various national observatories contributed comparatively little to the progress of science; but there were of course exceptions, a few of which we will here briefly notice.

The discovery of the four small planets ${ }^{2}$ Ceres, Pallas, Juno, and Vesta, the first by Piazzi, the second and fourth by Olbers, the third by Harding, gave celebrity to those astronomers, of whom Piazzi and Olbers were farther distinguished by many important labours. To the latter we owe the discovery of several comets, and one of the best methods of calculating their orbits from observation. He was a person of much simplicity of manner, made his observations with the most unpretending means in the attic of his house, and died at a great age generally respected. His firmest friend was Baron von Zach, who had no slight share in the discovery of the new planets, owing to his having instigated the association of twenty-four astronomers, chiefly in Germany, for the express pur-

1 The experiments of Cavendish are related in the "Philosophical Transactions" for 1798; those of Reich in a separate small work, entitled "Versuche uiber die mittlere Dichtigkeit der Erde," Freiberg, 1838; those of Baily in the "Memoirs of the Astronomical Society," vol. xiv. A still more recent series of experiments by Reich gives 5.58, a close approximation to Baily's result.-(Phil. Mag. March 1853.) From observations with a pendulum in Harton coal-pit in 1854, Mr Airy has obtained a density higher than any of the above.

2 In the Fifth Dissertation, page 789, Harding instead of Olbers is named as the discoverer of Vesta. 
pose of ascertaining whether no planetary body filled the void between Mars and Jupiter. To Piazzi, of Palermo, we owe a most excellent catalogue of fixed stars from observations with a moveable circle of four feet radius by Ramsden. Oriani of Milan was likewise one of the best informed practical astronomers of his time.

(162.) In France, after the death of the celebrated LaLalande. caille, perhaps Lalande (who was exactly Maskelyne's contemporary) was the most active astronomer. To him and his nephew we owe a very valuable catalogue of 50,000 stars, lately edited by the British Association. But practical astronomy was seriously neglected in France generally. The national observatory was feebly superintended by the later members of the Cassini family; of the French expedition under Maupertuis to measure the length of a degree in Lapland, the Abbé Outhier alone, it is said, knew how to use a quadrant, and the celebrated Lagrange was as ill informed until instructed by Lalande. ${ }^{2}$

(163.) But the most important labours of the French French arc astronomers at the close of the last, and at the comof the me- mencement of the present century, were in carrying ridian.

(164.) out the measurement of the arc of the meridian from Dunkirk to the Balearic Isles, with the more immediate object of fixing the length of the mètre, but contributing to the solution of far more considerable problems connected with the FIGURE OF THE EARTH. We connect this labour with the respectable name of Delambre, who was more intimately associated with it than perhaps any other person, though united with such eminent men as Méchain, Biot, and Arago.

DeLambre was the pupil of Lalande, who used to Delambre; say that his disciple was his best work. He first obhis charac- tained distinction as a computer of tables. Those of ter and the motions of the Sun, Jupiter, Saturn, and Uranus, and of the satellites of Jupiter were deservedly prized, and some of them are still the best of their class. He was a man in whom the love of truth and accuracy was conspicuous. Learned and patient, he spared no pains in acquiring knowledge, and in using it to the best purpose. As a calculator he was eminent. Physical astronomy he did not cultivate, except with a view to compare its deductions with facts. He was intimately conversant with all properly astronomical methods and formulæ. He knew the history of every problem, and the details and modifications of every astronomical instrument. $\mathrm{He}$ has embodied the results of this vast industry in a series of works (forming six quarto volumes) on the history of his favourite science, which are without a parallel for fulness and impartiality. He laboured as conscientiously to ascribe the due credit to Hipparchus and Ptolemy as to hold an equal scale between the merits of French and British astronomers.
His critical knowledge of the ancient languages (for he could speak Greek with fluency) was not more remarkable than his complete freedom from national prejudices. Both attributes qualified him pre-eminently for the office of an historian. He published also a large treatise on astronomy, and numerous memoirs on practical subjects in the Connaissance des Tems between 1788 and 1817.

Of his original labours the measurement of the French Arc of the meridian, of which he has given a full account in his Base du Systême Mètrique Decimal, is Arc. the most important. As some account of this undertaking has been given in Sir John Leslire's Dissertation, I shall state concisely a few particulars not there mentioned. Not the least singular feature of this gigantic work was the political crisis under which it was conducted. So early as August 1790, the French Constituent Assembly, on the motion of Talleyrand, desired the king to write to the English government, to represent the advantage of the two nations uniting to adopt a common unit or standard of weight and measure, which it was proposed should be done by a joint committee of the Royal Society and the French Academy. ${ }^{2}$ This application was probably never made, at least nothing came of it; but the Academy named their own committee, who, after discussing three sorts of natural standards, the length of the pendulum in lat. $45^{\circ}$ (first proposed by Huygens in his Horologium Oscillatorium), the length of a quadrant of the equator, and that of a quadrant of the meridian from the equator to the pole assumed to be elliptic,-adopted the latter, and this labour was committed to Méchain for the southern part, from Rodez to Barcelona $(170,000$ toises), and to Delambre for the northern, from Rodez to Dunkirk (380,000 toises). The southern arc was afterwards extended to Formentera in the Balearic Isles, and the whole length of the arc was found astronomically to be $12^{\circ} 22^{\prime} 12^{\prime \prime} \cdot 6$. Two bases were measured (both by Delambre), one at Perpignan of 6006 toises, the other at Melun of 6076 toises (each about $7 \cdot 3$ miles). When the length of the former was computed by triangulation from the latter, the difference of the observed and inferred amount is said to have been only ten or eleven inches.

But difficulties greater than physical obstacles presented themselves to the completion of this vast work. The excited state of the public mind during the most frenzied period of the French Revolution rendered the simplest operations matters of suspicion. The nearer to Paris that the survey was carried on, the greater were the precautions necessary. Instruments were seized, assistants arrested, and night signals had to be totally dispensed with. "The coolness and intrepidity of Delambre, added to unexampled patience, he French 
were the principal means of extricating him from his difficulties : but his danger was often imminent, and he appears to have sometimes heard the dreadful words which, as an eloquent author has expressed it, were the last sounds that vibrated in the ear of many an unhappy victim." The operations were actually suspended for a time by a decree of Robespierre and his colleagues, who deposed Delambre, along with Laplace, Lavoisier, Borda, and others, from the Commission of Weights and Measures, as being deficient in "republican virtues and their hatred of kings." They were, however, resumed, and Delambre had finished his share of the work long before his colleague Méchain, whose shorter task was conducted amidst a people rude and uneducated, indeed, yet far more to be trusted than were then those of the north. Méchain was apparently wayward and impracticable, somewhat too aged for so great a work, yet a really good astronomer. The want of agreement to within $3^{\prime \prime}$ of two sets of observations for latitude at Barcelona, the southern end of the arc at that time, led him to the suppression of one of them, and he was tormented ever after by the consciousness of the evasion, which deprived him of the tranquillity necessary to resume and complete his work, which was done chiefly by Delambre after vexatious delays. ${ }^{1}$ The error, which may be said to have cost Méchain his life, was probably owing to the instrument employed on this survey, the repeating circle of Borda, only fourteen inches diameter, with a rather weak telescope. The opinion generally entertained in Britain is, that the repeating circle was quite inadequate to the prodigious accuracy required of it, especially in the determination of latitudes. The errors of mere division are often trivial compared to those inherent in other parts of an instrument. Of these a deficiency of optical power, and the want of absolute security of the clamps, upon which the entire success of the principle of repetition depends, are amongst the most obvious. The arc was finally prolonged from Barcelona to Formentera by Biot and Arago in 1806. The conclusion of the survey was not destitute of the adventurous character of its commencement. The French astronomers ran many risks, underwent much suffering, and Arago narrowly escaped finishing his days in the dungeons of Spain.

The English survey carried on by Roy and Mudge has been also noticed in the previous Dissertation, The arc from Dunnose to Burleigh Moor amounts to $3^{\circ} 57^{\prime} \cdot 13^{\prime \prime} \cdot 1$, the measured length to 1442953 feet. An arc of parallel was also measured from Dover to Falmouth. We shall say something of its later progress in the concluding part of this essay, but we have still to regret the postponed publication of the British Arc of the Meridian, which we have no reason to doubt will bear a favourable comparison with the work of Delambre. The practical appliances, the three-feet theodolite of Ramsden for horizontal angles, and the eight-feet zenith sector of the same artist for latitudes, were unequalled instruments, and contrasted in almost every respect with the light and portable apparatus of the French. By means of the former the spherical excess of terrestrial triangles was first observed as a fact. The results of the French and British arcs taken separately concurred in showing a local curvature in this part of the world altogether anomalous, the degrees rather shortening in the northern part of each arc. This fact, which must be imputed either to large local attractions, giving errors of several seconds in the determination of latitudes, or (less probably) to a local departure in our quarter of the world from the general or mean figure of the earth, sufficiently shows the futility of the proposed method of determining a natural, recoverable standard of length. When combined with the measures of Bouguer in South America and Lambton in India, and the revised arc (measured in the beginning of this century) of Svanberg and Melanderhjelm in Lapland, the French and English measures give a general ellipticity somewhat under $\frac{1}{3} 0$, which is probably as near the truth as local inequalities admit of the determination being made.

To Delambre was confided the drawing up of the trigonometric formulæ used in the calculations of the French survey, which were published in a separate work; metric forDe Prony conducting the laborious calculation of an mulæ and altogether new set of logarithmic tables, with the tables. aid of an immense staff of computers, the results of whose labour (still in MS.) are preserved at Paris in 17 folio volumes. Delambre carried his personal exertions so far as to compute his own triangleswhich were also independently calculated by Legendre, Van Swinden, and Tralles.

As an acknowledgment of his merit, the highest indeed in their power to bestow, the Institute of France decreed to him in 1810 one of the Decennial awarded to Prizes instituted by Napoleon. But the Emperor, though professing to be the warm encourager of science, suffered some meaner motive to interfere, and refused to ratify the decision. "Ce fut," writes Dupin, "un pas dans la route qui le menait à sa chûte." After the siege of Paris in 1814 Delambre wrote a characteristic letter to his friend Moll. The tranquil spirit which had braved the horrors of the Revolution was not to be moved by the sounds of the artillery of the allied armies. In spite of the cannonade which he heard from his library, he laboured from eight in the morning until midnight; and, conscious of rectitude, he feared little the revolution of circumstances, which changing dynasties might call forth. "Labour," he says, "occupies all my time and all my faculties."

As Secretary to the Academy of Sciences for the 
His impar-Mathematical Sciences, he had to make frequent retiality- ports on the progress of those progressive branches his death. eminent academicians, both native and foreign. Both these duties he executed with his customary labour and fidelity. His private character was as amiable as his public career was distinguished. The tendency of his writings was constantly to enforce the histori- cal credibility of the Scriptures, especially with regard to the most ancient astronomical records. The value of his testimony is increased by his unusual skill in philology. He died 19 th August 1822, aged 73.

We reserve some farther remarks on the geodetic results of this period, and especially on the pendulum observations with which they were accompanied, for the fourth section of this chapter.

\section{§2. Sir William Herschel.-History of Sidereal and Telescopic Astronomy to 1820. Her- schel as an Optician.-Planet Uranus-Solar Spots-Orbits of Double Stars-Nebuloe-The Milky Way-Sun's Motion in Space.}

(172.) Sir Wm. Herschel

The eighteenth century was not, generally speaking, distinguished by original observations. The exceptions stand out with all the brighter Iustre. Amongst these, the discoveries of Sir William Herschel occupy perhaps the foremost place. Arago has affirmed that Slough is the spot on the earth's surface signalized by the most numerous discoveries, and in a certain sense this is strictly true. No one, before or since (with possibly the exception of $\mathrm{Mr}$ Hind) has added so many new bodies to the known planetary system, and this at a time when such discoveries were rarer, more unexpected, and more difficult than now. His researches on the fixed stars are not of a nature to have their importance numerically estimated.

(173.) Sir William Herschel's career was one of the his exten- longest and of the most sustained labour in the history sive works; of science. To have contributed papers - often several in one year-to the Philosophical Transactions for thirty-nine consecutive years, from 1780 to 1818 , with but two exceptions, is a feat of astonishing perseverance; but if we recollect that many of these papers contain announcements of capital discoveries, that every one of them is stored with original matter, and that the author had already passed his fortieth year when he commenced the production of this astronomical library, we cannot withhold a tribute of the warmest admiration.

(174.) early history.

England cannot claim Herschel as her own, except by adoption. He was born at Hanover in 1738, and was one of a numerous family who supported themselves chiefly by their musical talents. William Herschel, the third son, came to England in 1759 with his elder brother, and after struggling with many difficulties, found himself in comparatively comfortable circumstances as an organist at Bath. In 1774 he had executed a reflecting telescope with his own hands, and soon acquired so much dexterity as to construct instruments of ten and twenty feet in focal length. In 1780 he contributed his first paper on the variable star in Cetus to the Royal Society, and the following year (13th March 1781) discovered the erratic body, which he at first took for a comet, but which proved to be a planet exterior to Saturn; the first addition therefore to the number of the primary planets since a period of an immemorial antiquity. So fortunate a success made the name of Herschel speedily famous, and he was effectively befriended by George III., who brought him to live near Windsor, and gave him a pension. ${ }^{1}$

From this brief sketch it will appear how great were the obstacles which Herschel had to vanquish before he became a man of science, and that, besides the claims to distinction already enumerated, his knowledge and his skill were acquired in spite of every disadvantage.

Practical Astronomy naturally divides itself into two great branches, that which depends upon the use of the telescope merely; on the telescope and micrometer; and that which determines the absolute places of the heavenly bodies, and requires the aids of divided instruments and a well furnished observatory. In the century to which he belonged Herschel is the type of the telescopic observer, Bradley of the instrumental. The discoveries of Aberration and of Nutation by the latter may stand a comparison with any in the history of science, but the resolution of Nebulæ and the proof of the mutual connection of stars in binary systems are not less distinguished and original. Herschel would have gained a great reputation as an optician, merely by the wonderful improvement which he effected in the dimensions and magnifying power of telescopes, and by the skill with which he applied them to celestial observations. He would have stood still higher as an astronomer had he been merely the first observer of a new planet and of eight secondary ones, as well as of several comets, and the author of many nice

\footnotetext{
1 It may be doubted whether any other similar annuity was given at that period on scientific grounds alone, and it is difficult to estimate the amount of benefit thus conferred on astronomy, for nothing short of the entire devotion of a lifetime could have produced such results as we owe to Herschel. His proposal to name his planet after the Sovereign was a very natural expression of his gratitude at a period when no rule whatever existed on the subject. See an interesting letter from Herschel to Sir Joseph Banks (who was also a warm friend) in Weld's History of the Royal Society, ii. 146, note. Herschel was elected into the Royal Society 6th Dec. 1781.
} 
observations on the physical appearance and constitution of the sun and planets; but his great glory was in sidercal astronomy, of which he laid almost the foundations, by means of discoveries which he fortunately lived long enough to see confirmed and enlarged beyond his reasonable hopes.

In this brief sketch of Herschel's achievements (which in his own department may be said to embrace those of his age, for he had hardly an imitator, and certainly no rival) we shall, in the first place, condense into small compass what is to be said on the two former heads-his merit as an optician and as a planctary observer.

To construct good telescopes is itself no easy art, (178.) as especially if they are to be carried to a size and peranoptician. fection previously unattained. To ascertain the best composition, whether of glass or metal, to melt and to cast it in the right way, is one branch of the art which may be called generally the chemical part. To fashion the lens or mirror correctly by grinding, and to fit it for optical use by giving it an exquisite polish, is a second step requiring a peculiar mechanical skill and perseverance. To mount the telescope effectively is another and entirely different problem in mechanics; and all these the amateur astronomer must be prepared to accomplish with his own hands, unless he command the services of practical opticians to an extent rarely to be bought. Amateurs have indeed seldom succeeded except with reflecting instruments, and Herschel acted very wisely in devoting himself to their improvement at a time when the success of Dollond with achromatic telescopes had rondered the Newtonian form unfashionable. The real secret of Herschel's success was his astonishing perseverance; his determination was to obtain telescopes of twenty feet focal length or more, and of a perfection equal or superior to that of the small ones then in use. He himself relates, that whilst at Bath he had constructed 200 specula of seven feet focus, 150 of ten feet, and about 80 of twenty feet; a proof of extraordinary resolution in a man of limited means and engaged in a laborious profession. By making so great a number he could select those having the most perfect figure, especially before he had contrived a method of obtaining mechanically a parabolic form, almost with certainty. This method was not divulged. He was justified in keeping his secret, whilst he made a handsome income from the manufacture of telescopes for sale. Lord Rosse and Mr Lassell have had the merit of publishing their methods, which by their results would appear to be at least equal to Herschel's.

The usefulness of telescopes depends on two distinct qualities_-magnifying power and illuminating power. The former may be gained indefinitely by diminishing the focal distance of the eye-glass ; the latter can be had only by increasing the diameter of the object-glass or speculum, so as to collect the rays which fall upon a circle of large diameter from every point of the object examined. But in order that the whole light may be effective, the magnifying power of the eye-glass must be sufficient to condense the emergent pencil within the diameter of the pupil of the eye. The proportion in which these two qualifications are requisite to obtain the best results in astronomy is a matter involving nice questions both of theory and practice. Sir William Herschel did more than any one who preceded or followed him to solve them. On the whole, the illuminating power is perhaps the most important, whilst it is the most difficult of attainment; but in truth each class of telescopic objects have their own rule in this respect. Brilliant objects, such as Venus, and moderately bright stars, do not require large apertures; very feeble objects, as the remoter satellites and the ncbulæ, require indispensably great illumination. Five satellites of Saturn were seen by Sir W. Herschel in his great telescope by the naked eye alone, that is, without the advantage of the magnifying power of a lens. On the other hand, Sir John Herschel tells us that the satellites of Uranus cannot be clearly made out without a magnifying power of at least 300 , whatever may be the aperture of the telescope. 1

The mention of magnifying powers is calculated to mislead rather than otherwise in comparing the efficient means of different astronomers. The magnifying power used by Galileo did not exceed thirty-two Astronomy. times the diameter. Huygens, who used aerial telescopes of immense length, pushed it to 163 times. Auzout is said to have given a power of 500 to one of these gigantic instruments of 300 feet in length ; but it is easily understood that such achievements were little more than nominal, the mechanical difficulty of managing these instruments being excessive. Short professed to carry the magnifying powers of his Gregorian reflectors to 1200 , but still they yielded only trifling fruits to astronomy. It required that such instruments should be made and used familiarly, not as objects of luxury of which but one or two were ever brought into actual use. Herschel applied even to his seven-feet Newtonian (his favourite and smallest working size, having 6.3 inches aperture) powers exceeding 2000. To his largest instrument (thirty-nine feet focus) was occasionally applied a power of 6500 . The highest powers usefully employed with the gigantic achromatics of Pulkowa and of Cambridge (in America), do not exceed 1822 and 2004 times respectively.

The motion of the earth and the troubled state of

(180.) Magnify.
1 Struve found that with a small telescope, magnifying three times, and having the same aperture with the pupil of the eye $(0.2 \mathrm{inch})$, he could count nearly twice as many stars (accurately 1.83 times) as with the naked eye. This result, interesting on Beveral accounts, deserves farther enquiry.-Etudes d'Astron. Stellaire, p. 85. 
the atmosphere, especially in these climates, impose a speedy limit to the increase of magnifying power, unless in exceptional circumstances. The same objections do not apply to the increased aperture and illuminating power of telescopes. This consideration induced Herschel to construct his fortyfeet reflector, the funds being advanced by the king, on the recommendation of the Royal Society. The speculum had a useful surface precisely four feet in diameter, the thickness three and a half inches, the weight when cast above 2000 pounds. The second or plane mirror having been dispensed with, the image was thrown to one side of the object-end of the tube by means of a very slight inclination of the speculum, and was there observed directly by a common eye-piece or single lens, the observer being thus stationed with his back to the object he is viewing. This vast instrument collected a cone of rays from a distant point between six and seven times larger than the twentyfeet instrument commonly employed by Sir W. Herschel, of which the aperture was 18.8 inches. This telescope was not frequently used, partly from the rarity of sufficiently steady weather, and from the difficulty of preserving the figure of the mirror under changes of temperature. ${ }^{1}$ Its figure was also affected by flexure under its own enormous weight, and it has been found one of the greatest difficulties by those who have followed Herschel's steps to avoid this source of error. The forty-feet speculum has been religiously preserved by the filial care of Sir John Herschel, in whose possession the writer of these pages had the pleasure of seeing it, now many years since, in a state of high polish. It remains in a deposit hermetically closed, at Slough. ${ }^{2}$

Herschel made an ingenious determination of what he called the space-penetrating-power of telescopes, by which he discriminated the relative distances at which a given fixed star would become invisible in his several instruments. It was grounded on the assumption that the visibility of the object depends, first, on the density of its light reaching the eye, and secondly, on the number of rays of that density concentrated in the image. The former quantity varies inversely as the square of the given distance, the latter directly as the square of the aperture of the telescope; the limiting visible distance therefore of the radiant body will be simply as the aperture. To this he applied corrections depending on the loss of light by reflection at the specula, and by transmission through one or more eye-glasses. On this scale the space-penetrating-power of the seven-feet Newtonian being twenty, that of the twenty-feet telescope (front view) was seventy-five, and the forty-feet telescope 192. These estimations, as we shall see, form an important step in Herschel's generalizations.

Of new bodies belonging to our system he dis- covered Uranus and his six satellites, the two satellites of Saturn next to the ring, and several comets. He did not, so far as we know, even suspect the planetary character of Uranus, which he believed to be a comet. The honour of this appears to be due partly to Saron of Paris, partly to Lexell of St Petersburg. Arago supposes that Laplace had also a share, but the evidence on the whole matter is somewhat obscure. Unquestionably it was an exceedingly difficult matter to fix upon a correct orbit for a body moving with such extreme slowness within the limits of the first few weeks after its discovery, and its distance was far beyond what any one calculating a comet's path would readily assume. The original discovery was also a fortunate one, for but eleven days previously the planet was in a position apparently stationary. It has led to results of still higher interest by the prediction of the existence and place of Neptune from the irregularities of Uranus. The satellites move almost perpendicularly to the ecliptic. Strange to say, they remained for, I believe, more than half a century, unseen by any eye but that of their discoverer and his son: and two of them still stand recorded on the single authority of Sir W. Herschel. They are the least specks of light which optical power has ever made visible.

Herschel made many observations on the physical (184.) appearance of the sun, moon, and nearer planets, and the times of their rotation. Into these details we cannot now minutely enter. The papers which record them are characterized (like all those of the same author) by the faithful minuteness with which the observations are detailed, and by the mention of every influential circumstance by which the results might be affected. In the case of observations so exceedingly delicate as those of the planets, the astronomer has to keep up an incessant struggle between the anxiety to record all that he sees, and that of recording nothing more than he really and perfectly sees. One or two glimpses are not sufficient. Herschel at one time imagined that the new planet had one or more rings which unquestionably do not exist. His elaborate investigation of the figure, belts, and rings of Saturn, the incredible tenuity of the latter, and the fact of their rotation in $10^{\mathrm{h}} 32^{\mathrm{m}}$, were amongst the most interesting of his observations, and were jllustrated by drawings made with remarkable care. The edge of the ring was seen in 1789 , when it had totally disappeared in all but his forty-feet telescope, and he estimated that its thickness could not exceed 100 miles. How astonishing the magnitude and thinness of this stupendous circular plane, or rather series of flat rings! Some idea of it may be deduced from the fact that if the thickness of the paper on which this is printed be taken to represent that of the rings, their greatest diameter would correspond to nine inches. Observadiscovery of Uranus

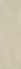

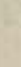

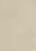

Herschel's (or Georgi. um Sidus) 
(185.)

tions on the other planets and on the moon we here pass over for want of space.

I have reserved the observations on the nature of on the Sun: the sun to this place, because everything leads us to assimilate the nature of the sun and of the fixed stars. The belief that the luminous disk of the sun is a photosphere or luminous atmosphere of great tenuity surrounding a globe of comparative density and darkness, was long anterior to Herschel, and in fact due to Dr Patrick Wilson of Glasgow, whose admirable paper on this subject was published in the Philosophical Transactions for 1774 , in which he explains the phenomena of the solar spots by apertures in the luminous atmosphere, discovering the dark nucleus below, and some shell or shells of intermediate brightness which form the penumbra. 1 These conclusions were most clearly and ably deduced from a careful observation of the changing aspect of the spots, as they move by the solar rotation from the centre to the edge of his disk. It is to be regretted that Herschel does not more pointedly refer to the discoveries of Wilson, which were more than twenty years antecedent to his first paper on the subject, and with which he could hardly fail to have been acquainted. A similar remark applies, in a less degree, to his papers generally, which rarely contain references to the observations and speculations of his predecessors. Herschel adopted Wilson's hypothesis almost literally, and his long series of patient observations on the sun, made with high powers and at an eminent risk to his eyesight, enabled him to classify the singularly varied appearances of that wonderful orb, and to draw some probable conclusions from the excessive rapidity and seeming tumult of the exterior portions of it. That the photosphere is strictly gaseous he rendered very probable, an inference confirmed by the direct observations of Arago as to the unpolarized character of its light. The singular disclosure of faint red prominences extending far beyond the disk, and observed in the total eclipses of 1842 and 1851, shows that there is still much which regards the mysterious nature of the sun within reach of direct observation; and the same may be observed of the direct experiments lately made on the heat and light of different parts of the disk, which diminish to one-half between the centre and the edge, and appear to attain a maximum at the solar equator.

A convenient, though not a strictly chronological arrangement of Sir W. Herschel's more important sidereal discoveries and speculations may be made under the following heads :-

I. Of double Stars and their mutual connection.

II. Of the Nature of Nebulæ, and the so-called Nebular Hypothesis.
III. Of the Grouping of the Stars generally in space, and the significance of the Milky Way.

IV. Of the Motion of our System in space.

I. On Double Stars. Discovery of Binary Systems.-Doulle stars were noticed as objects of curiosity even before the discovery of the telescope. The stars-orgroup of the Pleiades attracted attention from the tion; earliest times. Amongst the earliest double stars carefully observed were $\xi$ Ursæ Majoris (by Kirch, $1700) ; \alpha$ Centauri in $1709 ; \gamma$ Virginis and Castor by Bradley (1718 and 1719); Mayer made a considerable catalogue of double stars in 1756. But Lambert first announced in 1761 (in his Lettres Cosmologiques) the probability of the mutual revolution of suns, in these remarkable words (speaking of clusters of stars), "It will perhaps be decided whether there are not fixed stars which make their revolutions in no long periods round their common centres of gravity." Mitchell, in 1767 and 1784, maintained the same views, but supported them by an application of the then young science of probability, hazardous in its principle, and unquestionably wrong in its numerical solution. ${ }^{2}$

Sir W. Herschel commenced his observations on double stars with the hope of ascertaining the Annual Parallax in the manner previously indicated by parallax Galileo and James Gregory; but, as in many parallel instances, whilst he failed of his main result, he discovered unsought a phenomenon more unexpected and probably more interesting. With the micrometrical means at his disposal, he entirely failed in detecting any semi-annual fluctuation of the interval between the members of the pair of stars, but he found (in some instances) progressive and continually increasing changes both in the relative position and distance of the two. He commenced his observations in 1779 , but it was not until 1802 that he thought himself entitled to announce with confidence his discovery of the circulation of one sun round another, or rather of both round their common centre of gravity. Herschel's first list of orbital stars (Phil. Trans. 1803, where this splendid discovery was first published ${ }^{3}$ ) includes the chief examples now known; and they have all been confirmed. That of which the revolution is most rapid is $\zeta$ Herculis, which has a period of $31 \frac{1}{2}$ years, and consequently has revolved twice round since it was first observed, whilst the slow planet Uranis has not yet returned to the position of its first discovery. Herschel does not appear to have received a medal or other public recognition of this signal success.

Of the subsequent progress of this interesting inquiry we shall speak in the latter part of this chapter. . 
Greater precision has been in some respects attained, but little, if anything, added to the theory of double stars. (190.) II. On Nebuloe and the (so-called) Nebular $\mathrm{Hy}_{y}$
Nebulæ. are visible in clear but moonless nights in various parts of the sky are called nebulæ from their resemblance to light vapours moderately illuminated. Some regions of the sky abound more with them than others. The Milky Way presents to the naked eye a nebulous appearance extending over large tracts, and nebulæ more or less dense are frequent within and near its borders. These facts were well known from an early period. Some conspicuous nebulæ were described in the sixteenth century; that in Andromeda by Marius and the conspicuous nebula in Orion by Huygens. Halley added several, but the first descriptive catalogue of these objects was published by Messier in 1783 (in the Connaissance des Tems), and it forms the basis of the modern observations.

(191.) Sir W. Herschel took up the subject with his cusClassifica- tomary zeal. In 1786 he published a catalogue of tion of ne- 1000 nebulæ, which he had increased by 1802 to
bulæ;

(192.) resolvable and unresolvable.
2500. The first step to some appreciation of the nature of these extraordinary appearances was of course to classify them, and this Herschel did with such success that it may be affirmed that his classes remain the best at the present time, as generally representing the phenomena. Without entering into all his details, the following division embraces the main facts :-

A. Clusters of stars.

B. Nebulæ proper.

a. Having a certain regularity, as 1. Circular or planetary nebulæ; 2. elliptic; 3 . annular.

$\beta$. Wholly irregular, as the nebula of Orion, the Magellanic clouds, \&c.

C. Nebulous stars.

Remarks. A number of faint stars produces a nebulous haze. This is a physiological fact. Parts of the Milky Way which present this appearance to the naked eye are at once shown by a telescope of small power to be composed of stars. 1 Patches which appear nebulous in such a telescope are shown by a better one to be composed of small stars. This is called the Resolution of a nebula. Such resolutions become more and more numerous as the apertures of telescopes are enlarged. Lord Rosse's telescope has resolved several which withstood the highest power of Herschel's. One inference might therefore be that all nebulæ are clustered stars, at a greater or less distance. Such an opinion is very general, and very plausible. It may on the other hand be observed that Herschel abandoned this his earlier inference, whilst, at the same time, he proceeded diligently with the resolution of successive nebulæ, by means of his powerful telescopes. He maintained on the contrary to the last that nebulæ belong to two classes, resolvable and irresolvable. The latter he believed to be composed of diffuse self-luminous matter, more or less condeused, whilst the milky light of the former is occasioned by the blended gleam of numerous close stars. With this difference, we find his views regarding the changes in the sidereal system and the progress of the condensation and breaking up of nebulæ, nearly the same in his earlier and later papers, particularly those of 1785 and 1811.

The opinions of Herschel on these changes were the following:-Having observed that the more regular rebulæe (whether with a central star or not) presented almost invariably an appearance of gradually increasing brilliancy towards its centre, he was led to conclude that this is due to the aggregation of the self-luminous parts in virtue of their mutual gravitation; and the conclusion would so far be the same, whether the parts thus coalescing were in fact numerous distinct masses, or were like a vapour diffused through space. This was the process of condensation, which he inferred on the one hand from the law of gravity, on the other from the generally symmetrical arrangement of the luminous matter in nebulæ of the first class. In order to detect, by actual observation, the progress of condensation, he invented a simple and bighly expressive terminology, indicating the general brightness, the amount of its gradation, and the law of its gradation from the circumference towards the centre. In whatever degree Lord Rosse's late observations may be considered to deprive nebulæ of the character of symmetry which Herschel had conferred on them, the general fact of an apparent condensation in a great majority of instances cannot be denied.

The breaking up of nebulæ (also described in his paper of 1785 , as well as in his later ones) is likewise an inferred change anticipated in large irregular nebulæ, which usually exhibit bright spots or centres, with irregular vacant spaces, which sometimes seem like portions of a black and distant profundity seen through the rents of a nebulous veil. Herschel supposes that the luminous centres are also places of greatest attraction, and must gradually draw away the star-like substance from the rarer spaces.

It is curious that these speculations of Herschel (which are substantially conmmon to his papers of 1785 and 1811) harl been at least imagined at a very early period. Arago has pointed out that Tycho and Kepler conceived the New Stars of 1572 and 1604 to have resulted from a sudden condensation
(193.) IIerschel inferred their progressive condensation,

(1)

1 The group of the Pleiades presents a peculiar instance. When the eye is turned full upon it, no more than six stars (as is well known) can be counted, but when the eye is turned aside through $10^{\circ}$ or $15^{\circ}$, by a peculiarity of indirect vision by means of the lateral parts of the retina, the group appears a mass of nebulous light. This arises from an immense crowd of stars just below visibility, which, probably from the greater sensitiveness of this part of the eye, make sensible though somewhat indistinct impression. This observation as to the Pleiades, and the inference which explains by it the seeming countlessness of the stars when viewed cursorily, were published by the present writer in Brewster's Journäl for 1826. 
of diffuse celestial matter, such as the Milky Way exhibits, and Tycho alleged that the new star was surrounded by an obscure space, half as large as the moon's disk. The history of Science scarcely presents a more curious anticipation.

(196.) One practical difference between the earlier and Herschel's later views of Herschel on the nature of nebulæ, was opinions as to their distance from our system. For if the milky on the dis- aspect be due to a confusion of small stars, it is nebulæ; assumed that they must be almost incredibly distant. In his memoir of 1785 , he estimates this distance at not less than 6000 or 8000 times that of Sirius. If, on the other hand, the nebulous appearance be due to a diffusion of starry matter, the distance may be that of any order of fixed stars; and this opinion derives weight from the evident connection of some nebulæ with stars round which they seem to cluster or hang almost like a drapery. Thus in 1811 he supposed that the nebula in Orion need not be more distant than a star of the 7th or 8th magnitude.

(197.) It appears from his paper of 1802 (page 500), and their that his ideas about nebulæ were then wavering. Bechanges of tween that time and 1811 he elaborated the nebular form. hypothesis, so far as it is due to him. He seems to suppose that starry matter was once in a state of indefinite diffusion. That during " an eternity of past duration" (Ph. Tr., 1811, p. 287) it has been "breaking up" by condensation toward centres more or less remote. That the Milky Way-or at least the nebulous parts which it contained-and the dispersed nebulæ, are the relies of this former state of things. That where condensation has gone on more energetically, we have nebulæ with a gradually or rapidly increasing brightness towards the centre;-if still more energetic, a nucleus, or it may be a planetary nebula;-next a nebulous star, which he supposes our sun to be, and the zodiacal light a relic of its nebula (p. 311);-finally, the completely formed stars may be assumed to be merely consolidated nebulæ. (See pp. 284, 285, 299, 310.) This condensation, he believes, must be accompanied by rotation due to the originally irregular distribution of the gravitating particles (p. 312-319).

(198.) Theory of tion.

The proofs on which the whole of this cosmogony rests are, 1st, the gradation of appearances above described, to be collected from distinct objects in the heavens; and, $2 d l y$, supposed changes observed by him in the nebula of Orion and others, during thirty years, and even during intervals of a few years. As to the last argument, it is admitted, we believe, by the best authorities, to be without weight. The changes of aspect of such curiously faint and graduated objects, even to the same eye using the same telescope, are numberless, and may be due to the slightest-atmospheric and physiological influences. This test (as applied hitherto) is therefore generally rejected. Against the first proof much might also be urged, for which we cannot afford space. Its idea is essentially derived from the Natural History sciences, and I cannot help thinking that Herschel must have derived it from some one more conversant with these than with mechanical physics. This opinion is confirmed by a curious illustration which he uses in the same paper of 1811. "There is perhaps not so much difference between them" (viz., the whole group of nebular and quasi-nebular phenomena), "if I may use the comparison, as there would be in an annual description of the human figure, were it given from the birth of a child until he became a man in his prime" (p. 271).

A theory or hypothesis in some respects similar (199.) had been previously expounded by Laplace in the Laplace's concluding chapter of his Systême du Monde. At nebularhyleast it so far resembles it, that he puts forth Tycho's pothesisnotion of the star of 1572 being a condensation of a with Herwidely-spread stellar atmosphere (without, however, schel's. naming Tycho), and supposes that our solar atmosphere might also once have extended to the limit of the system, and that the planets were thrown off successively in the form of nebulous rings (subsequently condensed into spheres) from the equatoreal parts of this vast revolving mass during its contraction. $\mathrm{He}$ ascribes to Saturn's ring a like origin. It required all the authority of a name like that of Laplace to circulate a theory so bold, if not extravagant. He adhered, however (at that time), to the old idea of the constitution of nebulæ, which he considered to be composed entirely of stars, and to be at once the most distant and the most massive aggregations of matter in the universe. Herschel could hardly fail of being acquainted in 1811 with Laplace's views, and they probably in some degree influenced his own, particularly as to the cause of the rotation of his condensing suns. But he prudently reserved his hypothesis for sidereal objects, and did not deduce from it a planetary cosmogony. In the later editions of the Systême $d u$ Monde, Laplace quotes Herschel's observations in confirmation of his views, adopts the notion of a diffuse nebulous matter, and considers that an hypothesis arrived at by a "remarkable coincidence" in opposite directions, is thereby invested with a great probability.

Even Herschel's more limited conclusions have been very dubiously received, and notwithstanding Doubts rethe weighty adhesion of Humboldt and Arago to garding it. them, it may be affirmed that natural philosophers generally are content to leave the solution of this cosmical problem to that distant posterity which alone can hope to witness unequivocal evidence of progressive change in those wonderful objects. ${ }^{1}$

III. Of the Grouping of the Stars generally in (201.) Space, and the Significance of the Milky Way.-The The constiearlier astronomers, and particularly Halley, had tution of the the idea that the nebulæ-which abound so remark- vens, and of the Milky

1 The most intelligible account of the nebular bypothesis, in its least objectionable form, will be found in Sir John Herschel's Way in parOutlines of Astronomy, Articles 870 to 872. 
ably in the Milky Way, and which may in some sense be almost said to compose it-are the parent-matter of stars, whilst stars abound in their neighbourhood, or seem partly to compose them, in a state of incredible aggregation. That the mass of sidereal matter composing the Milky Way (which forms an irregular girdle round the whole heavens ${ }^{1}$ ) has some special reference in its locality to our solar system, was also an early idea, which, towards the middle of the 18th century, had attained to something like Kant, a definite speculation in the minds of Kant, Lambert, lambert, and other philosophers, chiefly those of Germany. Wright. Its origin is perhaps traceable to Thomas Wright of Durham $^{2}$ (1742), who affirmed that the Milky Way is a projection on the sphere of a stratum of stars, in the midst of which our Sun and system are placedsomewhat excentrically, however, towards Sirius.

(202.) I do not know - whether Sir W. Herschel derived "Herschel's his first ideas on the subject either from English or "gauges." German authors. His customary silence (which is to be regretted) on the history of his discoveries, leaves us in doubt of his originality. Be this as it may, he proceeded to test the reasonableness of the speculation, in that definite and thorough manner which was very characteristic of his genius. He proceeded to number the stars after a fashion which, if the early attempt of Hipparchus was pronounced in its day impious, might well have been accounted, even in Herschel's age, impossible. To use his own bold expression, he gauged the heavens, by counting the whole number of stars visible in the field of his 20 -feet reflector as many times as possible, and in every region of the sky which was visible from Slough, distinguishing the results of each region. As it was impossible in one lifetime to accomplish the entire survey (for the complete sphere contains 883,000 fields of $15^{\prime}$ each in diameter), he took an average for each region, and thus determined the general population of the sky in all directions. The result more than confirmed the suspicions of Wright and Lambert, and demonstrated a remarkable, and, on the whole, a steady law of decrease, from the central zone of the Milky Way in opposite directions, until we reach two poles, one in the Southern, the other in the Northern hemisphere, which are the localities poorest in stars.

This DIscovery (for it well deserves the name), which assigns a Law to the distribution of the entire visible bodies of the Universe in space, is surely one of the greatest which man has ever attained. The simplicity of the means by which it was reached, and the amount of mere mechanical and arithmetical labour which forms its sole basis, and constitutes its essence, is a powerful lesson of how little mankind has a right to scorn the humblest means of knowledge, or to attach the prerogative of genius only to the lofty flights of imagination and speculative philosophy. ${ }^{3}$ This triumph belongs to Herschel alone; his successors have commented upon and scrutinized his results; his son has "gauged" the Southern Hemisphere, with like results; but the original result is substantially confirmed. We may therefore anticipate so far as to quote the numbers denoting the "plenitude" of stars as we recede from the equator of the Milky Way in either direction, first, as calcu- as observed lated by Struve ${ }^{4}$ and Sir John Herschel, from Sir Wil- by Sir William Herschel's observations in the Northern hemi- liam and sphere; secondly, as calculated for the Southern he- Hir John misphere by Sir J. Herschel, from his own observations at the Cape of Good Hope.

\begin{tabular}{|c|c|c|}
\hline $\begin{array}{l}\text { Limits of Zone, } \\
\text { reckoning from } \\
\text { Equator of }\end{array}$ & \multicolumn{2}{|c|}{$\begin{array}{l}\text { Average Number of Stars visible in } 20 \text {-fee } \\
\text { Reflector; Aperture } 18.8 \text { inches; Field } \\
15^{\prime} \text { diameter. }\end{array}$} \\
\hline Milky Way. & $\begin{array}{c}\text { N. Hemisphere } \\
\text { (Magn. Power 157.) }\end{array}$ & $\begin{array}{l}\text { S. Hemisphere } \\
\text { (Alagn. Power 180.) }\end{array}$ \\
\hline $\begin{array}{r}0^{\circ}-15^{\circ} \\
15-30\end{array}$ & $53 \cdot 4$ & $59 \cdot 1$ \\
\hline $30-45$ & 13.6 & $13 \cdot 5$ \\
\hline $45-60$ & $8 \cdot 2$ & $9 \cdot 1$ \\
\hline $60-75$ & $5 \cdot \overline{4}$ & $6 \cdot \hat{6}$ \\
\hline $75-90$ & $4 \cdot 3$ & $6 \cdot 0$ \\
\hline
\end{tabular}

This table shows the extreme condensation of the stars round the "Galactic" equator; indeed Struve carries the average "plenitude" of the field as high as 122 stars in that plane itself.

According to Herschel's views, the whole visible firmament of stars belongs to the nebula of the Milky Way, of which our own system forms an infinitesimal part. In his paper of 1785 , on " the Constitution of the Heavens," he inferred that the general form and comparative limits of this cluster of stars might be found from his "gauges," by supposing that his telescope brought into view every star between the spectator and the surface of the cluster. Supposing the stars to be uniformly distributed within the cluster, it is easy to see that the number counted in a conical space, having an angle of $15^{\prime}$ at its vertex, would give the height of the cone, or the depth to which the spectator is immersed in the cluster, in that particular direction; and thus he endeavours to assign the solid form of the cluster and its dimensions, in terms of the distances

\footnotetext{
1 Its plane is inclined about $63^{\circ}$ to the Celestial Equator: its nodes are in $0^{\mathrm{h}} 47^{\mathrm{m}}$ and $12^{\mathrm{h}} 47^{\mathrm{m}}$ of Right Ascension.

2 In his Synopiis of the Universe, a rare work. See Professor De Morgan's account of Wright, in the Philos. Magazine for 1848, xxxii., p. 241. Wright believed that the Milky Way was a congeries of stars of a particular form, which it owes to a rotation of the whole round some central point.

3 Another remark, perhaps, deserves to be recorded. Had Hipparchus and his followers been deterred by the threatened charge of impiety from making a catalogue of stars, how great the loss would bave been, not to science alone, but to our estimate of the order and magnificence of creation. The telescope only increased our knowledge of the number of worlds, without really enabling us to approach to their limits:-the gauges of Herschel, which he at first supposed to reach to the boundary of the visible firmament, proved to him at last that the wealth of creation was unfathomable by any measure he could apply to it. "Numbers numberless" appeared in those regions where the stratum of the Milky Way is most extended.

1 Etudes d'Astronomie Stellaire.
} 
(hypothetically uniform) between any two stars. But in his later memoirs, Herschel seems to have felt the insecurity of his conclusions, and that there is no reason, but the contrary, to believe in a uniform distribution of the stars; that, at least in some directions, the Milky Way is unfathomable, or its limits unattained even by the 40 -feet telescope. (See his Memoir of 1817.) He therefore judiciously recurs to photometrical measures as the best criterion of the distance of the stars. The estimate of "the spacepenetrating power" of his telescopes, already referred to (182), enabled him to infer the relative distances at which a star would becume lost to vision aided by each telescope.

(205.)

Calling the distance of a star of the first magnitude 1 , and supposing that light takes three years to traverse it, 1 -

The distance of a star of the sixth magnitude (the limit of unassisted vision)

Dist. Light travels in will be.

Limit of 20 -feet telescope (used in gauging).

\section{2}

\section{0}

Limit of 40 -feet telescope .....................2,304

Limit of Lord Rosse's telescope ( 6 feet diameter). 3,436
Milky Way takes effect. He is therefore disposed to conclude that " our system is plunged in the sidereal stratum constituting the Galaxy, reckoning from the southern surface to a distance which corresponds to the light of a star of the 9th or 10th magnitude."2

These great and arduous enquiries occupied Sir William Herschel during nearly the whole of his scientific career, extending to almost half a century. His first observations (Struve remarks) dated from 1774, his last observations on this subject which have been published were of 1804, and his latest paper, still on the same topic, was in 1821 , when he was almost on the verge of the grave.

Excepting the continuation of his labours by his illustrious son, little has been added to our knowledge of "the constitution of the Heavens" since his y recur to the topic researches. in the latter part of this chapter. Some German astronomers of eminence have indeed tried to deduce from an assumed distribution of the stars in the Milky Way, the necessity of allowing that space is not per$2,700 \quad \ldots$ $6,912 \quad \ldots$

10,308

Yet none of these telescopes appear to touch the boundary of our nebula in the direction of its greatest extension, for fresh optical power still yields fresh harvests of stars.

In a direction perpendicular to the plane of the (206.)

Milky Way, not only is the pancity of stars remarkable, but we seem to look out, as Sir John Herschel describes it, into a starless region external to the stratum. Sir W. Herschel concluded, in 1817, that the depth of the stratum in its least dimension is such as to include all stars down to those three times more distant than the naked eye perceives. His son, 20 years later, infers, from the investigation of the southern hemisphere, that the distribution of stars is comparatively independent of direction for all those which are brighter than the 8 th magnitude, and that it is by the influence of the hosts of lesser brightness visible in the 20-feet reflector, that the very remarkable law of condensation towards the plane of the fectly transparent, and have even attempted to assign the law of extinction of light through the sidereal intervals; but having examined their reasoning with the best care I have been able to give it, I am bound to say that it appears to me very far short of conclusive. ${ }^{3}$

IV. Of the Motion of our System in Space.-That the stars are not fixed, but have " proper motions," Proper monot explicable by the gyrations of our earth round its tions of centre of gravity (which produce precession and nuta- Halley. tion), nor by its revolution round the sun (on which aberration and parallax depend), was a fact first detected by Halley from a comparison of ancient and modern observations. Though small in amount, yet as it continually increases in the same direction, this Proper Motion becomes in the course of ages an important quantity. In 2000 years, Arcturus, $\mu$ Cassiopeiæ and 61 Cygni have moved $2 \frac{1}{2}, 3 \frac{1}{2}$, and 6 times the moon's diameter.

In 1748, Bradley (in his paper on Nutation) stated the probable explanation of these apparent motions

1 These startling results appear to be necessarily true, if the following assumptions be admitted:-(1.) That the stars have on an average the same brilliancy in all regions of space. (2.) That the light received from a star is inversely as the square of the distance; or that the laws of radiation and of vis viva are rigorously true, and that there is no absorption of light in space. (3.) That the visibility of a star is exactly as the quantity of light received from it in the telescope. Probably this last assumption is the least sure. We know that magnifying power (independently of illumination) affects the result.

2 Cape Observations, p. 383.

3 I ought perhaps to state that in this passage I have referred to the reasonings of M. Struve, contrined in the latter part of his Etudes d'Astronomie Stellaire. Having taken some pains to follow the argument of that work, which professes to be "based entirely upon observation, without any arbitrary hypothesis," I was reluctantly led to the conclusion that M. Struve's assumptions, if tacit, were not the less arbitrary and questionable. Indeed it appears self-evident that no geometrical certainty can be attained as to the relative distances of the stars composing the Milky Way, but from some fundamental hypothesis respecting their magnitudes and distribution. I am persuaded that the popular writers and reviewers who have given additional publicity to the most striking and positive of M. Struve's conclusions, havē (very naturally) done so on the strength of the author's well-deserved reputation as an observer, and without attempting to analyse his reasoning, which it must be owned is sometimes obscure. My objections to M. Struve's argument were put in writing several years ago (1850), but not published except in my lectures. It was only whilst correcting the proofs of these sheets (1855) that I saw for the first time a memoir by Professor Encke in the Astronomische Nachrichten, vol. xxvi., No. 622 (published in 1848), maintaining the same view of the invalidity of M. Struve's reasoning, and questioning the hypotheses (of which M. Encke reckons five) tacitly assumed by him. The Dutch Academy of Sciences (Haarlem) has proposed the points at issue between MM. Struve and Encke as the subject of a prize question. 
Motion of to be the real displacement of our sun and system in the solar space. In 1760 Mayer gave a list of 80 stars whose system in proper motions he determined by a comparison of spaceBradley - Römer's observations. He conceived the possibility Mayer. of determining the direction of the solar motion by the changing aspect of the constellations, keeping in view, however, that the effect is complicated by real independent movements of each individual star. Lambert and Mitchell entertained similar views, but carried them to no practical result.

(211.) Here, then, Herschel appears on the field. $\mathrm{He}$ SirW. Her-proceeded in 1783 to do what his predecessors had

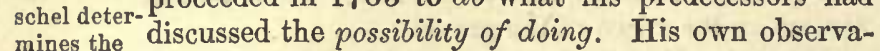
direction tions were not available in a problem like this. Takof motion. ing the best values he could obtain of the Proper Motions of only about 20 stars, he, with great sagacity and success, divined that the solar system is moving somewhere in the direction of $\lambda$ Herculis, a point in the heavens whose right ascension is $257^{\circ}$, and north declination $25^{\circ}$. This result he arrived at, almost without calculation, by a mere consideration of the changing perspective of a multitude of objects amongst which the spectator moves. Mayer had compared the change to that seen when we walk slowly through a grove of rather distant trees. Those in front will seem to widen or expand as we approach, those behind will condense towards the point we are quitting. The greatest proper motions will be in the objects on our right and left as we advance. It is not to be supposed that we find in the stars the exact counterpart of this change of place. For each star may by its own absolute movement in space either conspire with or oppose the motion due to perspective. But a combination of the whole results will make apparent the direction of that part of the motion due to a common cause, that is, to the translation of our system in space. A cleverer approximation than Herschel's was never made. He returned to the subject in 1805 , but there is reason to think that his first result was the more correct.

(212.)

As nothing essential has been added to Herschel's More recentdiscovery of the direction of the solar motion, we researches shall here refer once for all to the important confirsubject. mations which it has since received. Professor Argelander, availing himself in 1835 of the improved state of astronomy since Bradley's observations were made, and since their reduction by Bessel, considered the whole problem in a general and geometrical manner, including every well-determined proper motion $^{\mathbf{l}}$ by means of appropriate equations of condition, which, being resolved by the method of least squares, give the direction of solar motion $261^{\circ} 11^{\prime}$ of right ascension, and $30^{\circ} 58^{\prime}$ of north declination, differing respectively about $4^{\circ}$ and $6^{\circ}$ from Herschel's first numbers. Perhaps a still more convincing confirmation was obtained by Mr Galloway (Phil. Trans. 1847), from the proper motions of stars of the southern hemisphere alone, which lead to a result nearly coinciding with the above.

Sir William Herschel's leading discoveries in Sidereal Astronomy may therefore be reduced to thesehe Astromomy may therefore be reduced to these- inmary he discovery of binary systems of stars and the orbits schel's d of several revolving stars ; the discovery and classifi-coveries. cation of a prodigious multitude of nebulæ; the law of grouping of the entire visible firmament, and its connection with the great nebula of the Milky Way; and lastly, the determination of the fact of the motion of our sun and system in space, and the direction of that motion. Setting aside all that is valuable, ingenious, and noble in his farther speculations, and all that he contributed to the enlargement of our knowledge of the system of sun and planets with which we are more immediately connected, these positive discoveries will ever remain a magnificent trophy of his perseverance and success as a natural philosopher. ${ }^{2}$

When his increasing years rendered the relaxation of his arduous course of telescopic observation advisable, whilst all his other faculties were in the cal obser highest vigour, he began to devote more attention to physical enquiries less directly connected with astronomy. The nature of the emanations of light and heat proceeding from the sun was a matter on which he was forced to speculate in connection with his ideas of the sun's constitution; and even the practical enquiry as to the kind of dark glasses best fitted for defending the eye in observations of that nature, led him, in conformity with his usual habit, to a large series of experiments on solar heat, and its transmissibility through glasses of different colours, and other bodies. In the progress of these he was led Heating to examine the heating power of the different rays of rays of $t$ the spectrum, and consequently the refrangibility of heat. He then arrived at the real discovery that the place of greatest heat in a spectrum formed from the sun's rays by a flint-glass prism is considerably beyond the extreme visible red ray. But we shall return to this result in connection with the history of radiant heat. He also made some experiments on

\footnotetext{
1 A small star in Ursa Major (1830 of Groombridge's Catalogue) has an annual proper motion of 6 ".97; the star 2151 Puppis, is the greatest known, being $7 " 87$.

2 No historian of science, or biographer of Sir W. Herschel, can fail to acknowledge with gratitude the important assistance afforded by MM. Arago and Struve to a compendious acquaintance with his writings and discoveries; the former by his article on Herschel in the Annuaire du Bureau des Longitudes for 1842, the latter in his Etudes d'Astronomie Stellaire. While acknowledg. ing my obligations to these works, I shall not be suspected by any careful reader of having dispensed with a reference to the original memoirs; still less of having adopted without examination the conclusions of these eminent authors, from whom, indeed, on some important points, I differ entirely.
} 
the colours of thin plates, but to these he did not attach much importance.

(215.) The philosophical character of Herschel is almost Herschel's included in what precedes; but we will endeavour sharacter; to surn it up in a few words. He united in a remarkable degree the resolute industry which distinguishes the Germans, with the ardour and constancy of purpose which has been thought characteristic of the Anglo-Saxon. From his native country he brought with him a boldness of speculation which has long distinguished it, and it is probable that he had also a vigorous and even poetic imagination. Yet he was ever impatient until he had brought his conjectures to the test of experiment and observation of the most uncompromising kind. He delighted to give his data a strictly numerical character. Where this was (by their nature) impossible, he confirmed his descriptions by reiterated observation, in different states of weather, with different telescopes, apertures, and magnifying powers; and with praiseworthy fidelity he enabled his readers to form their own judgment of the character of his results by copious and literal transcripts from his journals. On his claims to originality we are unable in all cases to decide, owing to almost his only literary fault, that of rarely al- luding to the writings of his predecessors or contemporaries, even so far as to acknowledge their existence. The discovery of binary systems is probably that which was most absolutely his own; but even supposing the speculations of Wright, Lambert, and Mitchell were not unknown to him, this would diminish but little the substantive merit of having devised and executed the means of removing them from the regions of almost metaphysical abstraction to that of concrete reality.

His long and tranquil but ever active life corresponded happily to the nature of his pursuits, which his long required an absolute devotion of his time, and the life, means of instituting comparative observations after an interval of many years. There are not many philosophers who could have expected within their lifetime to see at least one pair of suns complete their mutual orbit.

Herschel's career at length drew to a close. He (217.) died peacefully at Slough, near Windsor, where he and dehad resided throughout the greater part of his cease. life, at the age of eighty-three, on the 23d August 1822 , one year only after the publication of lis latest memoir in the Transactions of the then recently formed Astronomical Society, of which he was the first President.

\section{§ 3. BESSEL-Mr AIRY-Modern Observatories. Fixed Star Catalogues-Planetary and Lunar Observations.}

(218.) As we approach the most recent period of the hisfodern as- tory of astronomy, I feel the increasing difficulty of a ronomical due condensation and selection of the interesting matince 1810. ter which claims our notice. Astronomy has been so generally and so zealously cultivated, that it seems almost invidious to select a few names and a few leading discoveries as the topics of discussion. It is, however, necessary to do so, and I shall be guided by the single aim of trying to specify impartially those individuals who have by their labours given most of the impress of the age to the science which they represent. In a subject like this, of almost infinite detail, the reader is not to expect a list of the names of all discoverers and improvers; it is not our business here to dwell upon mere labours of precision, to which so large a part of the most useful industry of astronomers is devoted; but to show the spirit in which these labours must be undertaken; and the general results by which they enrich the knowledge of the passing generation.

(219.) In the first section of this chapter we have sketched 'ractical (in connection with the name of Maskelyne) the stronomy state of accurate astronomy in fixed observatories, angland. down to about the year 1810. Since that period the multiplication of observatories has been very great. There has been a great improvement in instruments and in the methods of using then. But there has been an incomparably greater advance in the methods of ex- tracting trustworthy results from observations made with due care. The art of dividing instruments was carried to great perfection in Great Britain by Graham and Bird, but their preference of the quadrant to instruments of a circular form (introduced long before by Römer) retarded the progress of astronomy. Foreign observatories in the early part of this century (as in the last) sought their divided instruments from London; Piazzi and Bessel worked with English circles. Troughton was as an artist the worthy successor of Ramsden; and even to the present time British astronomers, at least, do not admit that the nice contrivances and beautiful workmanship of Repsold and of Merz in Germany, or of Gambey in France, have produced instruments worthy of more confidence than those which have been constructed at home. In the improvement of object-glasses for telescopes, however, Germany and France bear away the palm, although for many years Dollond supplied Europewith his achromatic telescopes. With regard to constancy and fidelity of observation, whether in fixed observatories or in maritime and geographical expeditions, English astronomers have never been backward; and the reputation of Greenwich Observatory in this respect (and also in the punctuality of publication) has, as we have stated elsewhere, earned the approbation and gratitude of all Europe.

But here our praise of British astronomy must 
Reduction pause. Until very recent times, with few exceptions, of observa- our observers, however industrious, have merely tions long used mechanically the apparatus put into their hands neglected. by intelligent and conscientious artists, who yet, never having occasion to apply their own handiwork to the purposes for which it was made, could not be expected to detect deficiencies of construction, nor to possess the mathematical knowledge required to remedy them. To determine in a complete and inde. pendent manner the errors, not only of adjustment, but also of construction of the instruments, and to correct them by calculations (deduced when necessary from frequent observations of their amount) is now felt to be the duty of the astronomer himself. This improvement (though, as we shall see, already partially practised even in England) was mainly due to Bessel, whose name we have placed first at the head of this section. But astronomy owes to him much more than this. He first showed that it is part of an intelligent astronomer's duty, not only to observe stars and planets, but to undergo the vastly greater labour of comparing his results with the best theories of his time, and of improving as far as possible those theories, so as to hand over each branch of science to his successor in a more perfect state than that in which he found it. In all these respects practical astronomy commenced a new epoch in Germany, and on this account principally we place the name of Bessel in a conspicuous rank.

(221.) Friedrich Wilhelm Bessel was born at Minden Bessel-his on the $22 \mathrm{~d} \mathrm{July} \mathrm{1784.} \mathrm{He} \mathrm{was} \mathrm{destined} \mathrm{for} \mathrm{a} \mathrm{mer-}$ early labours. cantile career; and it is an interesting fact that it was the expectation of an appointment as supercargo on a commercial voyage which led him to study navigation and astronomy, and finally induced him to devote his entire energies to the latter science, in which he received every encouragement from the amiable Olbers, who fortunately inhabited Bremen, where young Bessel was engaged in business. He commenced his astronomical career by reducing Harriott's observations of the comet of 1607, and was thereafter appointed assistant to Schröter at the observatory of Lilienthal. In 1810 he was remored by the Prussian government to the charge of the observatory about to be erected at Königsberg, where he spent the remainder of his life, and which he very speedily raised by his labours to almost the first rank of European observatories. In fact, he possessed, in a singular degree, the qualifications for directing a great observatory; including a thorough acquaintance with the use of instruments, with the theory of astronomy in all its branches, with the higher mathematics, and also the art and practice of calculation, as well as with many allied branches of natural philosophy.

(222.) The work by which he is perhaps best known, the His Funda- Fundamenta Astronomioe, is not grounded on his own menta $A s$ - observations, nor even those made in the same century, nor in his own country, but, what may appear singular, upon the observations of Bradley at Green- wich in 1750 and some following years. In reality,-reducno better observations had been then made. The in-tion of struments had undergone no material change during Bradley's the long and industrious, though not splendid, career tions. of Maskelyne; and Bradley was beyond question the most accomplished astronomer of the 18th century. Let us record it as a fact for the encouragement of conscientious labour in the service of science, that these precious records of the state of the heavens, of which the value seemed unknown to a whole succeeding generation (so that they were not even printed in a crude form until nearly fifty years after they were made), were disinterred, so to speak, by an illustrious foreigner, and in course of time made the basis of what, in respect of precision and method, might be called a new astronomy. Bessel was assisted in tnis work by a grant from the British Board of Longitude.

The observations especially considered by Bessel are those of the fixed stars. To apprehend their irn- Special portance, we must recollect that the foundation of an jects of accurate knowledge of the orbits, and of the perturbations of the Solar System, not to mention less apparent but not less remarkable changes of place in the stars themselves, consists in rendering perfectly comparable observations of position at one time with those made at another more or less remote. Now, not only are our transit instruments and quadrants, and circles, and clocks, affected with errors more or less unknown, so that the registered figures require correction; but, in consequence of Refraction and Aberration, we see no body whatever exterior to the earth in its real visual position; and this visual position, when found, is affected by Parallax. Besides all this, the grand points of reference in the sky, the Pole and the line of Equinoxes, are undergoing perpetual, though small, changes of position:- the first star of Aries had already, in the time of Hipparchus, wandered from the point of intersection of the Equator and Ecliptic;-consequently, longitudes and right ascensions have to be reckoned from a directional line in space altogether imaginary. The pole of the heavens wanders amongst the stars, in consequence of Nutation - the obliquity of the Ecliptic is itself changing; and, to crown all these causes of perturbation and seeming unsteadiness, the stars called fixed have (as we have formerly seen) peculiar and individual displacements not accounted for by any of the preceding causes of change. 'In short, nothing is fixed and comparable, except our measure of time. The length of the sidereal day is subject to no secular variation. The bases of Astronomy, - the Fundamenta Astronomioe, - then, evidently consist in the perfect evaluation of all these varying elements, so that the stars, if not really fixed, may become, for us at least, virtually so, - that by their aid the position of the fundamental directional lines of the sphere may at any time be found, and the relative positions of the stars themselves. After this it is easy to 
refer the places of wandering bodies to standard points.

Bessel's work includes a methodical reduction of the places of above 3000 stars observed by Bradley, and an investigation of the sun's apparent path, together with a full discussion of the principles and application of every correction, instrumental and uranographical, which such observations require, and which are applicable to all others of the same kind. It includes, therefore, dissertations on some of the most delicate points of Astronomy, and "Constants" for the different corrections, which, with slight variation, have been since employed in every observatory. Bessel devised also a remarkably simple mode of finding for each star the varying corrections of its place. The proper motions of the stars were also determined for the first time with approximate exactness, by comparing Bradley's places with more modern observations.

In subsequent years (the Fundamenta were published in 1818) Bessel contributed to the science of astronomy numerous and regular observations of the heavenly bodies; the Annals of the Königsberg Observatory were regularly published from 1814, being ( $I$ believe) the first foreign establishment which followed the example set by Maskelyne at Greenwich, with the important addition of systematic reduction.

Of the subsequent labours of Bessel we must speak and important of these, we shall refer to another section, where we shall compare it with the results obtained by other astronomers. He made interesting physical observations on Halley's comet at its return in 1835; he prepared the materials for a very extended catalogue of fixed stars, arranged in zones, more recently published by Professor Weisse. In connection with the theory of the pendulum as a measure of gravity, he repeated and extended Newton's important experiments on the uniformity of the gravitating force on all kinds of matter; he applied a new correction to the vibrations of the pendulum, and improved the method of observing them correctly; and he discussed with his habitual mathematical skill and elaborate perseverance the figure of the Earth, from the whole of the then existing observations; whilst he also directed an operation for connecting the Russian triangulations with those of western Europe,-a delicate task, which he performed with consummate skill. It will thus be seen that (without mentioning a host of minor works and memoirs) there was hardly a great department of Astronomy in which Bessel did not take a distinguished part. Even the discovery of Neptune, by calculation from the irregularities of Uranus, was contemplated as a practicable problem by the veteran astronomer in his later years. He died on the 17th March 1846; therefore only a few months previous to the publication of MM. Le- verrier and Adams' discoveries, and their triumphant consummation by M. Galle.

Even before Bessel's career as an astronomer had (227.) properly commenced, Pond, in England, had antici- Pond, the pated, in a good measure, the improvements in prac- mer-Royal tical astronomy to which we have referred in the an excelbeginning of this section. Already, in the earliest lent obyears of this century, he had made observations at his server. private residence with a comparatively small circular instrument by Troughton, which led him to detect the gradually increasing errors of Bradley's quadrant still in use at Greenwich, and to recommend the adoption of complete circles (disused since the time of Römer) and the specific examination of errors of division. These principles he carried out at Greenwich, to which establishment he was afterwards appointed. His very indifferent health prevented that incessant activity which the management of a first-rate observatory requires; nevertheless, he is justly regarded as one of the chief reformers of the practical astronomy of those days.

His successor, Mr Airy, has distinguished himself by a more active career. Endowed with very un- Succeeded common abilities, and with great physical powers of by Mr endurance, he has, from his youth, been ever foremost not only in promoting, in every one of its departments, his favourite science of Astronomy, but also many other allied subjects, particularly Optics, to which we shall have occasion to refer in another section.

Born in the county of Northumberland in 1801, Mr George Biddell AIRY acquired great distinction at Cambridge, where he graduated in 1823 , and was appointed to the charge of the Observatory there in and practi1828, after the death of Professor Woodhouse, a per- cal astroson sincerely attached to astronomy and well skilled in nomy. it, yet one who did not succeed in imparting much interest either to its theoretical or practicaldepartments. $\mathrm{Mr}$ Airy engaged in the important investigation of a new irregularity in the motion of the Earth and Venus, to which I have referred in the chapter on Physical Astronomy (114), and at the same time he undertook to publish his own Observations in regular annual volumes, including a complete comparison of the results with the best tables, thus presenting at a glance the existing errors of theory. This practice he introduced at Greenwich on succeeding Pond there in 1835; and he has pursued it ever since. But not contented with rendering the annals of the National Observatory a correct reflection of the state of the heavens in his own day, and also of the condition of Astronomical Theories, he did not rest until he had performed for the observations of his predecessors the same service, and thus produced a series of comparisons of the observed and calculated places of the moon and planets, unexampled for extent and accuracy. Beginning with Bradley's Observations in 1750 , he investigated and applied all the instrumental and uranographical corrections to each, as Bessel had done for the observations of the sun and 
fixed stars, and then calculated the tabular places for the same instants of time. This last, especially in the case of the moon, was a truly formidable task. The whole labour was methodically performed at the expense of government by a staff of no less than sixteen computers, under Mr Airy's vigilant superintendence, and it extended, first and last, over the space of a dozen years. The results, printed in the most compendious form, fill 2200 quarto pages. One consequence of this systematic reduction was, that MM. Leverrier and Adams were separately provided with the places and errors of the Tables of Uranus necessary for their great investigations; and the Lunar Theory has been enriched with Mr Hansen's latest corrections through the same facilities. The final corrections of the co-efficients of the Lunar Tables are concisely stated by Mr Airy in a paper addressed to the Royal Astronomical Society.

(230.)

Like Bessel, Mr Airy is thoroughly acquainted Greenwich with the theory and practice of mechanics; and he Greenwich has applied his knowledge both to the improvement Circle. of clocks and of proper astronomical instruments. A great and apparently successful experiment of the latter description is now taking place at Greenwich. A meridian circle (combining in effect a mural circle and transit instrument) has been erected, of great size and optical power ( 6 feet circle and $8 \frac{1}{4}$ inch objectglass). But the novelty of the apparatus is its great weight and strength. Hitherto artists and astronomers have sought to avoid the evils of unsteadiness and flexure principally by the elaborate framing and trussing of light materials (chiefly brass tubes), and (in Germany especially) by an elaborate system of counterpoises. The new Greenwich circular instrument is constructed of as few pieces as possible, of great strength and weight, and in great part of cast iron. The whole was elaborated and prepared for dividing in an engineering workshop, and with the powerful and accurate tools which modern engineers use. A man might stand upon the axis without producing the smallest bad effect. It is understood that the result is perfectly satisfactory as to steadiness and stiffness. Mr Airy has likewise invented and erected a new and most ingenious zenith tube (acting by reflection) for ascertaining the place of $\gamma$ Draconis, a star which, at its superior culmination, crosses the meridian of Greenwich almost in the zenith.

There are few projects for the improvement of astronomy in the last twenty years in which Mr Airy has not been a counsellor or participator. He has Introduc. at all times given efficient aid to those engaged in the tion of gal pursuit of science; and has been himself the first to im- vanic meport from the method of recording observations by means of a Greenwict galvanic pressure produced on a sheet of paper surrounding a cylinder; which cylinder is moved in a perfectly uniform manner by means of a connection with a clock producing uniform motion on the principle of the conical pendulum. By means of a simple contrivance the tracer follows a slightly spiral line, so that one barrel may include many hours of continuous observations. He has also established galvanically time signals, and employed them for the determination of longitudes. Besides all this, he has taken part in furthering geodetical measures, in regulating the national standards of length, and in promoting in various ways the collateral sciences. He contributed to the Encyclopoedia Metropolitana a most able analysis of the methods for determining the Figure of the Earth, and his conclusions have been very generally adopted. In the same publication he has given a theory of the Tides, already referred to $(78$, 82). The public and his own university are indebted to him for several very valuable text-books on mathematical physics.

During his incumbericy at Greenwich, Magnetical and Meteorological Observations have been added Magnetica and Meteorological Observations have been added and meto those of Astronomy, and engage the time of four teorologiregular assistants in that department. The astro-cal obsernomical assistants amount to six, besides occasional vations. computers. It may safely be affirmed that there is not a public establishment in a more complete state of efficiency than is Greenwich Observatory at this moment; and that its traditional reputation, expressed in the emphatic eulogium of Delambre, Art. (150), runs no risk of deterioration under the direction of Mr Airy.

Our limits will not allow us to refer to the nu- (233.) merous private observatories in England and public Other obones abroad which are contributing useful elements to the promotion of astronomy. It is not too much to say that the activity of Greenwich has set a generally good example. The observations at Oxford and Edinburgh have long been reduced on a systematic plan; those at Cambridge had been already so treated. The establishments where sidereal astronomy is particularly cultivated will be noticed in another section.

\section{§ 4. BoRDa-Kater-Baily. The Figure of the Earth from Pendulum Observations-" Reduc-} tion to a vacuum;" Mr Stokes. Colonel Everest-M. Struve. Latest Measures of the Earth. M. Foucault's Pendulum Experiment.

In the first section of this chapter I have mentioned the progress made in determining the figure of the earth by measurements of its surface in the early part of this century. I shall 'now advert briefly to the employment of the pendulum for the same purpose.

Clairaut had published in 1743 a very remarkable theorem which connects the force of gravity in any 
Connection latitude with the compression of the terrestrial ellipof gravity soid. ${ }^{1}$ The pendulum is the readiest and most accuand the figure of the earthTheorem. rate means of determining variations in the force of gravity; the repetition of its oscillations enabling the observer to ascertain their average duration with extreme nicety. The more rapid the oscillation the greater is the force of gravity, because the heavy body is more quickly drawn into its lowest position. Two sets of observations, made in different latitudes, would, rigorously speaking, suffice to determine the polar compression, supposing the earth to be a geometrical ellipsoid, and composed of homogeneous and concentric layers; a larger number would serve to test the accuracy of these assumptions, and would, at all events, be imperatively required to eliminate the errors of observation. (236.) It might be supposed that to swing a pendulum,
Early pen-count its vibrations, and measure its length, is a dulum ob- very easy thing. Experience shows that a more servations. difficult practical problem can hardly be proposed. Those persons, therefore, who have in a good measure overcome the difficulties, are to be regarded as promoting materially an enquiry which has always ranked amongst the most interesting connected with astronomy. Some of the observations of the eighteenth century, especially those of Lacaille at the Cape of Good Hope and of Phipps in the Arctic Regions, appear to have been made with much care; but the French astronomer Borda seems to have given the initiative to observations of a higher degree of accuracy, and his methods were ably carried out in connection with the great French meridian arc, by M. Biot, who even extended his stations to the Island of Unst in Shetland.

Jean Charles Borda, who was born in 1733 and died in 1799 , was devoted to the promotion of the exact sciences, and contributed in an eminent degree to the precision attained in physics and astronomy towards the close of the last century. The name of Borda deserves a record in the history of science, since it has been said of him, by no less an authority than Dr Young, that " he seems to have possessed a considerable share of that natural tact and sagacity which was so remarkable in Newton." His earlier researches were connected with hydrodynamics, and the resisted motion of projectiles; in later years he was chiefiy devoted to practical astronomy and geodesy. The portable repeating circle invented by him has had a very great reputation in France. He was one of the principal designers of the measurement of the French are, and was employed in its superintendence, especially as regards the measurement of the base. His experiments on the pendulum were originally undertaken ( $I$ believe) in 1790 , with a view to making it the basis of the national measures, but were afterwards subordinated to the greater schene of terrestrial measurement. Baily records a fact connected with Borda, not unworthy of mention, as a caution to observers:- the year in which many of his experiments were made is not discoverable from the account of them, though the day, hour, minute, and second, are recorded with praiseworthy fidelity. From this time pendulum experiments assumed an astronomical and geodetical importance. The apparatus of Borda consisted of a slender metallic wire, attached at one end to a knife-edge suspension, and at the other to a small brass cap, nicely fitted by grinding to a sphere of platinum which formed the bob or weight of the pendulum. The oscillations were counted by the method of " coincidences," that is, by placing the experimental pendulum in front of a good clock with a known rate, and observing after how long a time the two pendulums (having started in the same direction) again coincided in their motions by one of them having gained or lost exactly two vibrations; a method which has been used at least since the time of Bouguer. The time of vibration being thus known, the distance from the knife edges to the bottom of the ball was measured by means of a scale; and due corrections for the position of the centre of oscillation were then applied.

By English observers, an invariable pendulum of the form of a flat bar, provided with a bob or weight, has usually been preferred, and on the whole it appears to be more satisfactory. Such a pendulum, after being compared with an astronomical clock at Greenwich, or any other place of reference, is taken to different stations and its rate of vibration determined, and it is then brought back and compared once more at the point of starting. Important series of observations of this kind have been made by Colonel Sabine and Captain Foster, and also by Admiral Duperrey.

But the two individuals who have most studied (239.) the practical determination of the length of the se- Relative conds pendulum as a mechanical problem, are Kater lute measures.

1 Clairaut (besides giving a theorem which, in the case of an ellipsoid, connects the polar compression with the increase of gravity there) showed that the increase of the force of gravity from the equator will vary as the square of the sine of the latitude. This he proved to be true when the spheroid is homogeneous, or when it is composed of similar concentric layers of varying density. Laplace confirmed the result by a different analysis, and farther showed, that if the earth be composed of concentric strata which are severally homogeneous, and nearly spherical, but otherwise arbitrary in form, and if the surface be that of a fluid in equilibrium (as it practically is when we refer the earth's figure to the sea-level), there exists a necessary connection between gravity and the superficial figure, which, in the case of an ellipsoid of revolution, leads to the same relation of the square of the sine of the latitude. In this case, also, the strata are presumed to be concentric. But in a recent and remarkable paper by Professor Stokes, it is shown that the law connecting the force of gravity and the figure of equilibrinm still holds when no hypothesis whatever is made as to the distribution of the matter within the earth, provided always that it be consistent with the observed fact of the ellipsoidal figure of the earth's fluid covering. Thus the confirmation, by means of pendulum experiments, of Clairaut's Theorems cannot be regarded as a proof of the concentric arrangement of the strata, nor (so far) of the primitive fluidity of the earth. See Cambridge Transactions, vol. viii.; and for a simpler proof, Cambridge and Dublin Math. Journal, vol. iv. p. 194. 
and Baily. As regards the determination of the Earth's figure, the relative length of a pendulum vibrating seconds in different latitudes is all that is required; but the absolute length in a given place is necessary for finding the force of gravity there expressed by the acceleration of falling bodies in vacuo in one second; and as the British standard yard was at that time made to depend on the length of the pendulum vibrating seconds at London, this enquiry was mixed up with the intricate one of National Standard Measures.

(240.) Captain Henry Kater was a person of remarkable Captain mechanical skill and ingenuity, and his numerous paKater; pers in the Philosophical Transactions, between 1813 and 1828, refer chiefly to the accurate construction and use of the Pendulnu, the Balance, and certain Astronomical Instruments. He first applied to practical use the beautiful theorem of Huygens respecting the convertibility of the Centres of Oscillation and Suspension of the pendulum. A metallic bar pendulum was provided with two parallel knife-edges facing opposite ways, and upon either of which it might be swung. They were so arranged that when either was used as the point of suspension, the other nearly represented the centre of oscillation, and by means of a small adjustable weight, this condition might be accurately fulfilled; in which case (by Huygens' principle) the distance between the knife-edges corresponded accurately to the length of an equivalent simple pendulum. This elegant application was farther tested by $\mathrm{Mr}$ Baily, but it was found liable to serious practical objections. Baily dispensed with the sliding weight, and rendered the pendulum invariable: the distance of the knife-edges was adjusted by grinding.

(241.) To Kater we owe the invention of the floating ColHis Colli- limator, for ascertaining the accurate zero or level mator. points of divided astronomical instruments. The optical principle on which it depends is a very beantiful one, and the invention of Kater, with several improvements in point of form, has become the auxiliary of nearly every observatory in the world. It is one of those small but happy improvements which affect materially the progress of Science.

Francis Baily, who was born 28th April 1774, and who died 30th August 1844, was an instance of a person not endowed with extraordinary talents, yet of singular use in his generation. Those who knew Mr Baily personally, and what he accomplished for Astronomy, are best aware how rare and yet how estimable are such qualifications as his. Born of parents of a middle rank of life, educated at a country school, and at an early age thrown into the vortex of commercial life in the city of London, no probability could have appeared more remote than that he should acquire the respect of even the most accomplished Astronomers by his scientific acquirements and performances. He enjoyed the advantage, in the first place, of a perfectly sound and uninterrupted state of health until his 70th year. His intellect partook of the equable tone of his bodily powers. Method, and an and characardour which, instead of acting by impulses, seemed ter. inexhaustible and unvaried, were the secrets of his success. Mr Baily never was hurried, and he never was unemployed. He had always leisure to encourage men of like tastes, and he was never unwilling to learn from any one who had knowledge to communicate. To do one thing at a time was his first principle. When thoroughly accomplished, it was completely put aside, and a fresh subject undertaken. Whether an experiment or a calculation was found to require a month or three years, it was pursued to a successful completion, without stint or grudging. No amount of contrariety and failure in such matters was ever known to ruffle his temper, or to make his perseverance falter.

Francis Baily was known, in the first place, as the founder (in 1820) of the Astronomical Society of Founded London, an institution of signal utility, which has nomical ever since been conducted with great judgment and society. success. His accurate knowledge of past and current astronomical history, and his persanal acquaintance with most European astronomers, enabled him to conduct its affairs with great advantage.

Amongst his other acquirements, he studied history and chronology with his customary precision. A very able paper on the date of the celebrated Eclipse on Eclipse of Thales was his earliest scientific publication, ${ }^{1}$ and Catalogues the literature and phenomena of solar eclipses occupied his particular attention ever afterwards. His accounts of the Eclipse of 1820 , of the Annular Eclipse of 1836 , which he observed at Jedburgh, and the Total Eclipse of 1842, with its marvellous revelation of the rose-coloured protuberances of the solar atmosphere, are among the most interesting and classical of his writings. He promoted greatly the formation and publication of accurate Star Catalogues, and he invented, independently of Bessel, constant numbers for facilitating the calculation of Precession, Nutation, and Aberration, as affecting the places of particular stars. He also published a most useful collection of Astronomical Tables and Formulæ, and an elaborate and very curious life of Flamsteed.

I shall now proceed to notice his experiments on the Pendulum, which are more particularly connected with the present section.

Mr Baily's most important observations were on a correction of the motion of a pendulum long mis- Baily's understood. The corrections usually admitted were observathe following:-1. For temperature altering the tions. length of the pendulum. 2. For the height of the station above the level of the sea. Dr Young showed that this correction was commonly overrated, owing to the sensible amount of the attraction of the elevated land on which the experiment is made. 3. For 
the air's buoyancy. The mere resistance of the air to the motion of the pendulum may be shown to produce opposite and compensating effects in the descending and ascending semi-vibrations: but the presence of air, as of any other fluid, diminishes the total effect of gravity on the bob, and that in a degree depending on the density of the air or atmospheric pressure. But besides this obvious " reduction to a vacuum," it appears that the air acts also in a different way. Owing to its inertia and cohesion a portion of air is shoved along with the pendulum, or otherwise put in motion by it, and the force thus spent in moving air is not compensated during the two semi-vibrations. This effect was clearly ascertained and measured by Dubuat about 1786 , and described in the second edition of his "Principes d' $\mathrm{Hy}$ draulique;" but it appears to have been totally unobserved by his own countrymen and others, who (like Borda) were soon after actively engaged with the same subject. The effect was experimentally re-discovered by Bessel, and made the subject of an admirable series of experiments by Baily, which were published in the Philosophical Transactions for 1832. He vibrated pendulums of different forms in vacuo and in air (as Colonel Sabine had done to a more limited extent shortly before), and he ascertained the corrected "reduction to a vacuum" to be in many instances double of the old correction, and to depend materially on the form and density of the pendulum. It even appeared that when a convertible pendulum is swung in two positions in the manner of Kater, the correction is different in the two cases, owing to the want of symmetry, so that a pendulum convertible in air of a given density is not convertible in $v a$ cuo, nor vice versa.

Bessel and Baily agree in imputing the effect to the clinging of a mass of air to the metallic pendulum, thus rendering it in effect a pendulum of much less density, for which the ordinary correction for buoyancy will have to be increased. This view is probably rather a popular than an exact expression of the fact, and the correction need not be always proportional to that of buoyancy. Poisson has treated the question mathematically, and Professor Stokes has recently resumed the whole subject, both mathematically and practically, and considers that he has arrived at precise mathematical results in several cases. The result may probably be a revival of interest in pendulum observations, which has manifestly very much declined since the existence of this irregularity has been fully established, and the incompetency of existing rules for "reduction to a vacuum" clearly shown.

(218.) In 1833, Baily deduced from the very elaborate Result as experiments of Captain Foster in both hemispheres to Earth's $\frac{1}{209 \cdot 48}$ for the earth's compression. Colonel Sabine had found it $\frac{1}{25^{5}-40}$ from his own observations. The French and Russian observers concur in obtaining a result sensibly greater. Of these experiments, Captain Fos- ter's is by much the most extensive. Mr Baily's re- The Cavenpetition of Cavendish's experiment for determining dish expethe earth's density, has been mentioned in a previous riment. section.

In conclusion of the subject of the figure of the earth deduced from observation. I shall now briefly refer to the results of geodetical measures more recent than those noticed in the first section of this - Colonel chapter, which comprehended the great French arc. EverestIn a matter so much of detail, which will be bet- M. Struve. ter appreciated from special articles in the Encyclopoedia, I sliall best fulfil the ends of this discourse, by merely directing attention to the two most conspicuous and important of these measurements of the earth, one in India, the other in central and northern Europe; the first directed by Colonel EveREST, the second by M. STruve, the eminent astronomer of Pulkowa.

The measurement of an arc of the meridian in India by Colonel Lambton in the early part of this cen- Lambton tury, tury, has been mentioned in a former part of this rest's InDissertation, as well as in Sir John Leslie's. But its dian arc. value has been prodigiously increased by the extension of the same by Colonel Everest, who was at one time the principal assistant and coadjutor of Colonel Lambton. The arc of Lambton, extending from Punnæ (Lat. $8^{\circ} 9^{\prime} 35^{\prime \prime}$ ) to Damargida (Lat. $18^{\circ} 3^{\prime} 15^{\prime \prime}$ ), was measured after the model of the English trigonometrical survey, by means of a 100-feet steel chain for the base, a 3 -feet theodolite for the azimuths, and a 5 -feet zenith sector for latitudes. Colonel Everest pursued the triangulation in a northerly direction for some years after the death of Lambton in 1823; but losing his health, and being forced to return to Europe, he was provided, by the princely munificence of the East India Company, with an entirely new set of instruments, and with two able assistants, Captains Waugh and Renny.

He resumed operations in 1832, which were continued until 1840, and included an entirely new Difficulties measurement of an arc extending from Damargida, which it where Lambton's arc terminated, to Kaliana (Lat. was exe$29^{\circ} 30^{\prime} 48^{\prime \prime}$ ), a space of 797 miles, covering an are ${ }^{\text {cuted. }}$ of $11^{\circ} 27^{\prime} 33^{\prime \prime}$. The latest geodetical improvements were introduced. The bases were measured by General Colby's compensating bars, in which the effect of temperature is self-corrected. The signals from station to station were made during the day by heliotropes reflecting the solar ray parallel to the sides of the triangles to be measured. The azimuths were determined by a 3-feet theodolite of Troughton, in addition to that of Carey used by Lambton; the astronomical observations were made with two circles of large dimensions. 'The difficulties and annoyances experienced in conducting so vast a work, requiring such excessive accuracy, in so remote a situation, can only be judged of by referring to Colonel Everest's graphic details contained in his elaborate ac- 
count of it, published at the East India Company's expense in 1847. The heats and the rains, the dull opake atmosphere of the Doab, with its boundless and densely-wooded flats, the jungle fever and the wild animals, were natural impediments enough. But Colonel Everest, whose conscientious anxiety reached almost a nervous pitch, seems not to have been satisfied with any one instrument which the mechanicians of London could produce; but to have metamorphosed most of them, partly with his own hands, partly by native aid. It says much for his ability in this respect, that the results appear entitled to compete with all the most exquisite of the kind in Europe. His bases, for instance, about $7 \frac{1}{2}$ miles in length, when checked by intermediate triangulation above 400 miles in extent, differed in one instance by 4 , in another by 7 inches from the primary measurement. The whole extent of Lambton's and Colonel Everest's operations includes a continuous arc of $21^{\circ} 21^{\prime}$ (1477 miles), by far the greatest at that time executed.

(252.) It is indeed only rivalled in this respect by a vast Russian arcoperation very lately executed in Russia and other by M. northern countries of Europe, by which an arc of $25^{\circ}$ Struve. $20^{\prime}$, extending from the banks of the Danube to the shores of the Arctic Sea, near the North Cape, has been measured under the general superintendence and direction of that able astronomer M. Struve, whose meritorious labours in other departments of astronomy will be specified in a succeeding section. The results are still incomplete, though the operations in the field were happily concluded just in time to prevent their total frustration by the unhappy war in which Russia has since engaged.

(253.) The arcs of India and of Russia include a space Its extent. from Lat. $8^{\circ}$ to Lat. $71^{\circ}$, with the exception of only about sixteen degrees, and are unquestionably the most important which exist for the determination of the earth's figure. When to them we add the French arc of $12^{\circ} 22^{\prime}$ in a medium latitude, it will scarcely be necessary to take into account any other, at least for the Northern Hemisphere.

(254.) The following brief details of the Russian arc are taken from the provisional report of M. Struve(1852).

The southern extremity of the Russo-Scandinavian arc is Ismail on the Danube (Lat. $45^{\circ} 20^{\prime}$ ), the northern extremity is Fuglenaes on the island of Qualoe in Finnmarken (Lat. $70^{\circ} 40^{\prime}$ ). The interval from Tornea to Fuglenaes $\left(4^{\circ} 49^{\prime}\right)$ was measured by Swedish and Norwegian engineers; all the remainder by those of Russia, and, in particular, by M. Von Tenner, who, with M. Struve, has since 1816 directed the whole operation. The whole line is remarkably free from considerable inequalities of ground, and from mountain ranges, so that local attractions are probably inconsiderable. ${ }^{1}$ On the other hand, extensive forests ex-

tending over dead flats have, in the southern part of the arc (as in India), occasioned great difficulties, and compelled the erection of numerous temporary structures to overlook the country. In the north the extraordinary refractions have, as usual, created some difficulties. Ten different base lines, all at a small height above the sea, form part of the operations. It is a very satisfactory circumstance, that by the care of M. Struve and Mr Airy, the standards of length used in the Indian and Russian ares have been directly compared.

The calculation of the figure of the earth from the completed Russian arc, in combination with others has not yet been made, but it is believed that it will indicate an ellipticity somewhat greater than that generally received. The results obtained by Co-from geolonel Everest, on the other hand, by comparing his desy; arc with those of Europe, give generally small ellipticities, that is under $\frac{1}{30} 0$. The French and Indian arcs, for instance, give $\frac{1}{1} \frac{1}{6}$. Now Mr Airy had deduced 20 years ago from the best existing observations $\frac{1}{2}$; Bessel, a few years later, obtained the almost identical result of $\frac{-1}{2} \frac{1}{9}$; and Schmidt of $\frac{1}{3} \mathrm{r}$. The determinations by means of the pendulum are and from somewhat larger. The extensive observations of $\mathrm{Co}$ - pendulum lonel Sabine and Captain Foster concur in giving experian ellipticity of $\frac{1}{2} \frac{1}{8}$; but the French experiments by Duperrey and Freycinet lead to a result considerably greater. The discrepancy between the geodetical and pendulum results may of course be a real one depending on local variations of density. The astronomical determination from the lunar inequalities, which might be expected to concur with the results of the pendulum, gives (as we have seen in the chapter on Physical Astronomy, Art. 63) $\frac{1}{3} \frac{1}{6} 6$ at a mean.

I regret that my limits do not permit me to speak of the measure of degrees of longitude or arcs of parallel, as another test of the earth's figure. The results, however, cannot compete in point of certainty with those from arcs of the meridian. It may be satisfactory to add, that the British arc of parallel from Greenwich to Valentia (west coast of Ireland) sensibly accords with the earth's figure obtained independently.

In connection with the subject of the pendulum treated in the earlier part of this section, I shall here mention a very remarkable experimental observation communicated to the Paris Academy of Sciences, on dulum exthe $3 \mathrm{~d}$ February 1851 , by M. Léon Foucault, to whom we also owe some ingenious experiments on the velocity of light. It is not indeed connected with the determination of the earth's figure, but it has a connection with astronomy, inasmuch as it affords the most remarkable and direct proof of the earth's rotation round its axis.

1 In India, on the contrary, the local attractions of the Himalaya must be very sensible; indeed Mr Pratt has calculated them to be so considerable, as to have given rise to a curious speculation by Mr Airy as to the circumstances which may tend to diminish the attraction of mountain rang es.-(Phil.Trans. 1855.) 
(259.) Suppose a considerable weight suspended by a wire demon- of regular elasticity from a fixed point or stand constrating the nected firmly with the ground; and let us first imaEarth's ro- gine the place of the experiment to be exactly over
tation. the North Pole. Let the wire pendulum be swung so as to coincide with the plane of the meridian of London. As the earth rotates, the wire and the ball must evidently rotate too. But the motion of the mass originally impressed parallel to a given plane will continue in that plane, and consequently the plane of motion will coincide in the course of 12 hours with every meridian in succession, and the apparent rotation will be entirely completed in a uniform manner in 24 hours. In any other latitude than $90^{\circ}$, but greater than $0^{\circ}$, a continuous and regular apparent change of motion must also occur, since a meridian of the globe does not preserve its parallelism during diurnal motion, excepting only at the equator; consequently, at all points of the earth's surface, except at the equator, the plane of motion of the pendulum will vary uniformly in azimuth-quicker, however, in high than in low latitudes. To find this velocity, it is only necessary to decompose the rotation of the earth round its axis into two, one of which (which is alone effective) is round the vertical of the place of observation. The apparent angular motion is thus proportional to the sine of the angle of latitude. Thus, in an hour, it is $15^{\circ} \times$ sine lat.
M. Foucault's experiment was made, in the first instance, with a pendulum of steel wire from $\cdot 03$ to $\cdot 05$ Corrections inch in diameter, bearing a ball of 12 pounds weight to be atIt is desirable to make the pendulum vibrate in small arcs, in consequence of the tendency to ellipticity in the vibrations, which is necessarily accompanied by a rotation of the major axis of the ellipse, which might easily be mistaken for the influence of the earth's motion. To take account of this disturbing force, we have only to measure accurately the greater and less axes of the ellipse described. Then a revolution. of the apsides (from this cause only) will be performed in a time which will be found by multiplying the time of a double vibration of the pendulum by 8 times the square of the length of the pendulum, divided by 3 times the product of the two axes of the ellipse. This formula is due to Mr Airy. The theory of M. Foucault's beautiful experiment has been verified by numerous experiments in different latitudes.

Very recently the theory of the motion of a pendulum suspended by a thread or wire has been con- Its theory sidered in the the physical astron motion. ${ }^{1}$ It is an intricate problem of analytical mechanics. But the results show, as might have been anticipated, that all the sensible results are those which the geometrical treatment of the question indicate.

§ 5. M. Encke. Cometary Astronomy-Periodic Comets of Halley and Encke. Gambart's and Biela's Comet-Comets of 1811 and 1843. Mr Hind-New Planets or Asteroids. Mr LasSELL-Newly discovered Satellites. Mr Bond.

(262.)

Professor Encke.

Periodic Comets.

(263.)

Professor Johann Franz Encke, Director of the Observatory of Berlin, is one of the most eminent physical and practical astronomers of the present day. The author of many valuable observations and important memoirs, he is best known by those which are connected with the motions and theory of Comets. I shall therefore devote this section to an abstract of the progress of this interesting subject, and more particularly of M. Encke's discoveries and speculations. I must premise, however, that, easy and agreeable as it would be to introduce here a detailed essay on Cometary Astronomy, the design and extent of this discourse alike forbid it, and at the cost of some self-denial, I will endeavour to confine myself entirely to what is most new and characteristic in the Cometary history of later years.

Halley's Comet.-Before proceeding to describe the remarkable comet especially connected with the name of ENckE, it will be proper to resume the history of Halley's Comet where it has been left off by Sir John Leslie in his Dissertation. That Cometthe comet of 1682 -must ever remain memorable, perhaps the most so of its class, as being the first whose return was confidently predicted, in firm reliance on the Newtonian Theory of Gravity. Halley's announcement-grounded not on vague analogies, but on laborious computations-that it would reappear early in 1759, was realized almost to the letter; Its return and Clairaut, whose surprising analytical ability often in 1759. left but little to his successors to accomplish, calculated the perturbations with an accuracy which even the present state of physical astronomy has hardly exceeded. Indeed no general method for calculating perturbations in highly elliptic orbits is as yet in use, and though the methods of Clairant have been superseded by those of Lagrange and Bessel and Leverrier, the summation of the effects by the method of "quadratures" is always used,- the periodic time of the comet being divided into short intervals throughout which the elements are considered invariable, and during which the configurations and perturbing effects of the principal planets are computed with much labour.

The chief improvement in the calculation of perturbations is the introduction by Lagrange of the celebrated method of the Variation of the elements (44.) 
Its pertur- His successors have distinguished themselves chiefly bations by the praiseworthy minuteness and extent of their calculated calculations, which are amongst the most laborious,

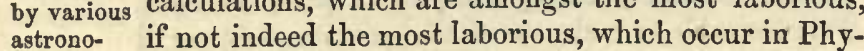
mers. sical Astronomy. The computers who calculated the return of Halley's Comet in 1835 were four in number, MM. Damoiseau, Pontecoulant, Lehmann, and Rosenberger. Their memoirs are all considered by competent judges to be excellent, but especially that of Rosenberger, who calculated more fully than the others the perturbations from 1682 to 1759 , and who has introduced a theoretical correction of some importance. Some idea of the extent of these calculations may be formed from the fact, that in some parts of the orbit the Elements were made to vary for intervals of only two days. The Comet of Halley was rediscovered at Rome on the 6th August 1835, in the Jesuits' College. The error of Rosenberger's Ephemeris was only seven minutes of arc, and the perihelion passage took place on the 16th November (civil reckoning), five days after the predicted time. Bessel states the remarkable fact, that the coincidence of the comet's path with the results of previous calculation is as close as the use of five-place logarithms in computing the perturbations would permit. All this is very creditable to the state of Astronomy; still it is infinitely less remarkable than Clairaut's approximation, only a little less close, made 77 years before. It is a matter of regret that neither in the matter of this comet, nor in any other point of theory connected with Cometary movements, have our countrymen made any considerable advance since the time of Halley.

(265.) At its return in 1835, this Comet was watched Its return until May 1836, and in the course of its long visibility was made the subject of minute and admirable telescopic researches, by Bessel in Europe, and by Sir John Herschel at the Cape of Good Hope. Their observations strictly confirmed a remark of $\mathbf{M}$. Valz, that the Tails of Comets, though evidently generated under the influence of the. neighbourhood of the Sun, yet commonly disappear at the period of closest approach. This, at least, was the case with Halley's Comet. The tail began to grow on the $2 \mathrm{~d}$ October, two months after its discovery, and diminished after the 15th (a month before perihelion). The comet reappeared in the end of January without any vestige of a tail, and then dilated in absolute bulk with incredible quickness. But before its final disappearance, on the 5 th May, the tail is supposed to have

Its peculiar been reabsorbed into the nucleus, which presented features. then a uniform circular outline. The evolution of the highly-expansible luminous matter of the tail took place from the nucleus in luminous fan-like jets, directed on the whole towards the Sun, and issuing on the side of the Comet exposed to that body. The jets harl a vibratory motion. The tail (directed from the Sun) was formed by the abrupt inflexion of these effluent jets, in a manner resembling electrical repulsion; and Sir John Herschel has not hesitated to ascribe the phenomenon to the combination of a repulsive force as respects the Sun, ("of an energy very far exceeding the gravitating force towards that luminary") with " a peculiar and highly energetic attraction to the nucleus, differing from, and exceptional to, the ordinary power of gravitation."1 The opinion that polar forces resembling magnetism or electricity are necessary to explain the phenomena of Comets, has been for some time current in Germany, and received, I bclieve, the countenance of Bessel. That there exist in the movements of these bodies anomalies so great as to render the sufficiency and completeness of the Theory of Gravity suspected by such high authorities, makes the statement of them very important, though I confess a great reluctance to share in the conclusion.

I shall terminate this notice of Halley's Comet (266.) with a table of the dates of its probable perihelion Table of passages, according to M. Laugier and $\mathbf{M r}$ Hind. ${ }^{2}$ The earlier appearances are deduced from the Chinese Annals :-

\begin{tabular}{|c|c|c|c|}
\hline $\begin{array}{l}\text { Date. } \\
\text {. c. } 11 \cdot 80\end{array}$ & $\begin{array}{c}\text { Interval. } \\
\text { Years. }\end{array}$ & Date. & $\begin{array}{c}\text { Interval. } \\
\text { Years. }\end{array}$ \\
\hline & $77 \cdot 87$ & & $77 \cdot 45$ \\
\hline D. 66.07 & $75 \cdot 17$ & A. D. 989.6 & 76.55 \\
\hline $141 \cdot 24$ & & $1066 \cdot 25$ & \\
\hline $218 \cdot 26$ & & $1145 \cdot 30$ & \\
\hline $295 \cdot 25$ & 76.99 & $1223 \cdot 52$ & 1822 \\
\hline $373 \cdot 84$ & 100 & $1301 \cdot 81$ & 1020 \\
\hline $451 \cdot 50$ & $77 \cdot 66$ & 1578.85 & $77 \cdot 04$ \\
\hline $530 \cdot 84$ & $79 \cdot 34$ & $1456 \cdot 44$ & $77 \cdot 59$ \\
\hline & $77 \cdot 96$ & $1531 \cdot 65$ & s.) ${ }^{75 \cdot 21}$ \\
\hline $684 \cdot 80$ & 70.00 & $1607 \cdot 82$ & s.) \\
\hline $760 \cdot 44$ & $\begin{array}{l}75 \cdot 64 \\
76 \cdot 82\end{array}$ & $1682 \cdot 70$ & $\begin{array}{l}74 \cdot 88 \\
76 \cdot 49\end{array}$ \\
\hline $\begin{array}{l}837 \cdot 26 \\
912 \cdot 25\end{array}$ & 74.99 & $\begin{array}{l}1759 \cdot 19 \\
1835 \cdot 87\end{array}$ & $76 \cdot 68$ \\
\hline
\end{tabular}

The differences in the above periods are attributed to the effects of perturbation. The extreme distance of the Comet's path from the Sun is $35 \frac{1}{3}$ times the radius of the Earth's orbit, which is only one-sixth part greater than the distance of Neptune, and therefore nearly within the recognized limits of the planetary system.

Comet of Encke.-Next in interest to the Comet (267.) of Halley is that discovered by Pons of Marseilles Comet of in November 1819, which was first suspected, then proved by Professor Encke, to revolve in an elliptic orbit of short period (considerably interior, in fact,

1 See Sir John Herschel's Outlines of Astronomy, and Results of Observations at the Cape of Good Hope.

2 Taken from Mr Hind's small work on Comets. 
to the orbit of Jupiter), and made the subject by him of a series of investigations altogether peculiar. On this account it bears the name of Encke, instead of that of its discoverer Pons, although M. Encke himself, with unaffected modesty, always describes it as the Comet of Pons.

Newton gave, in the Principia, his celebrated solution of the problem of determining a Comet's orbit assumed to be parabolic, from three geocentric places. This solution has been simplified and improved by Lagrange and Boscovich, and also by Olbers. Laplace gave a method for an elliptic orbit, which may represent any number of observations. Gauss, in his Theoria Motûs Corporum Celestium, treated the subject with great skill and generality. I am unable to state who first attempted to discriminate an elliptic from a parabolic cometary orbit, or to determine the period in the former from observations at one apparition only. It is evident that such outstanding differences as are irreconcilable with a parabolic orbit, will be most perceptible in the case of comets whose orbits have a tolerably short major axis, or whose period is not very great, and will be materially increased by watching a comet through a considerable part of its orbit, which the assiduous application of telescopes to every part of the hearens has of late years rendered much more frequent than formerly. Amongst others, Bessel, who has signalized himself by a capital performance in this, as in every other department of Astronomy, applied rigorous methods to determine the orbits of the comets of 1807 and 1815 ; the latter of which will very probably return to its perihelion in 1887. It is undeniable, however, that expert calculators have often been deceived in assigning orbits, even when believed to be of short period, founded upon a single apparition.

M. Encke, however, was more fortunate in the case of the first comet of 1819. Using the methods of Gauss, he showed that an Elliptic Orbit of about $3 \frac{1}{4}$ years must be admitted, and that the comet had probably been already observed in 1786 , by Méchain, in 1795 by Miss Herschel, ${ }^{1}$ and in 1805 by Pons. He investigated with great labour the effects of the planetary perturbations on this body, which, in the case of Jupiter, are occasionally very large, if that planet be in the part of its orbit near the aphelion position of the comet, when it approaches the orbit of Jupiter. The careful calculations of M. Encke for the next return in 1822 were verified by the observations of Sir Thomas Brisbane, at that time fortunately governor of New South Wales, where he had, with characteristic liberality, founded an Observatory. Since then, this body, insignificant in its physical appearance (being to all appearance a small cloud of vapour without a solid nucleus), has been detected in one or another part of the world at every revolution:namely, in $1825,1828,1832,1835,1838,1842$, Its appa1845,1848 , and 1852 ; so that the Comet has been ritions. observed at fourteen (not all consecutive) returns.

The complete establishment of the existence and pe- (270.) riodicity of a comet, quite in the interior of the planet- Its acceleary system (its greatest distance from the Sun being tion four times the Earth's distance, and its least distance but one-third of the Earth's), was a discovery in itself highly interesting. But something yet remained behind. Professor Encke, in comparing the earlier with the later apparitions of the Comet, detected a gradual acceleration of its movement, which amounted between 1786 and 1838 , to 1.8 days, on a period of about 1211 days; being about $2 \frac{1}{2}$ hours per revolution. Whatever may be the cause of this, the fact is undisputed, even by Bessel, who was indisposed to accept M.Encke's explanation. This fact, it will be observed, is unique in Astronomy. The major axis and periodic times of the planets and satellites have, as we have seen in the chapter on Physical Astronomy, no secular variation. The moon's apparent acceleration has been otherwise accounted for. M. Encke at once, attributed and at an early period, attributed the accoleration of to a resistthe Comet's mean motion to the effect of a slightly dium. resisting medium, insensible in the case of the planets, partly owing to their incomparably greater density (for this Comet appears to be one of the most loosely aggregated bodies known, being transparent to its very centre); and also, to the circumstance, that the density of the ether or resisting medium is assumed to diminish rapidly at a distance from the Sun. M. Encke supposes it to decrease in density with the square of the distance, and only to affect the Comet sensibly within 25 days preceding or following its perihelion. That the effect of resistance is to accelerate the return of the Comet is evident, by considering that the projectile force becoming gradually extinguished, the Sun's attraction must be more available to pull the body inwards at each revolution, thus shortening the major axis of the ellipse, and diminishing the time.

In order satisfactorily to arrive at any such conclusion, it was of course necessary to estimate with Perturbogreat accuracy the perturbing effects of the planets tions of on the Comet's motion; and it is not a little curious comet apand satisfactory, that the movements of this insigni- plied to ficant erratic body should have occasioned a mate- rectify the rial rectification of the masses of two of the Planets. Mercury M. Encke very early suspected that the received and Jupimass of Jupiter was too small, a fact clearly esta- ${ }^{\text {ter. }}$ blished afterwards by Mr Airy ; and in $1838 \mathrm{M}$. Encke showed that the mass of Mercury (which, not having a satellite, was little more than guessed at previously) had been assumed nearly three times too great by Lagrange. The perihelion of the Comet approaches much

1 Caroline Lucretia Herschel, sister of Sir William and aunt of Sir John Herschel, deserves a passing notice, not only as the independent discoverer of eight comets (of which five were first seen by her), but as the indefatigable and intelligent assistant of Sir William Herschel during the busiest years of his life. For this service King George III., carrying out his judicious liberality to her brother, granted her a small pension. She died at Hanover 9 th January 1848, aged 97. 
more nearly to the orbit of Mercury than the aphelion does to that of Jupiter; consequently at times the perturbations due to the former planet may be very great, and though the gravitating mass of the Comet is utterly unknown, yet since the momentary direction of its motion depends solely on the ratio of the attractive force of the Sun and Mercury, its observed course gives the means of estimating that ratio. ${ }^{1}$

The theory of a resisting medium was, on the whole, received, from this evidence of something material filling the planetary spaces. In Germany the hypothesis of resistance received the complete opposition of Bessel's high authority; who declared that " a hundred other reasons" might be found for the fact of the acceleration, which he admits to be true. Encke, in reply, reduces these 100 possible hypotheses to four, of which we shall mention only one, as seemingly important, namely, the forces exerted with so much intensity within the body of the Comet itself, as indicated by the projection of the tail. But he observes, with great sagacity, that these forces, being apparently usually excited in the line of the radius-vector joining the Comet and the Sun, can hardly be supposed to affect the periodic time. It having also been objected that Halley's Comet shows no trace of acceleration, but, if anything, of the reverse, M. Encke truly says, that its perihelion distance does not lie within the assumed limits of the denser ether.

(273.) Nevertheless, the theory of a resisting medium in and still space is not perhaps very popular, except in England. questioned. Although M. de Humboldt appears to favour it, I understand that the German astronomers in general scarcely regard it as in any degree proved.

Yet, if not true, the cardinal fact remains unexplained. The anomalous phenomena of the Tails of Comets, considered by Herschel to be altogether inexplicable by the law of gravity, demand the closest scrutiny; and one can hardly help supposing that the two difficulties may be in close connection. As the Newtonian law is now considered (since the discovery of Neptune, and the latest corrections of the Lunar Tables) to be absolutely sufficient to account for everything connected with planetary motion, the Astronomy of Comets will be looked to with increasing interest, as likely to reveal some laws of nature not otherwise to be detected. In this respect, Professor Encke's labours are likely to be more and more important in their results.

(275.) With reference to this very eminent astronomer, M. Encke we have only to add, that he has for a great many

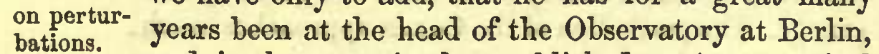
and in that capacity has published an Astronomical Ephemeris of first-rate excellence. It is as a phy- sical astronomer, however, that he will be principally remembered. Besides his admirable investigations connected with the Comet, he improved the theory of Vesta, and has very lately published a new Method of Computing Perturbations, especially for orbits considerably elliptical. Neptune was discovered at his Observatory, by the assistant astronomer, M. Galle.

Gambart's and Biela's Comet.-Jean Gambart, one of the most promising astronomers in France, died one of the most promising astronomers in France, died Gambart's
of consumption at a comparatively early age, I believe and Brela'
comet. in 1836. He was director of the Observatory at Marseilles, which, notwithstanding its very unfavourable position in the midst of the town, has acquired considerable celebrity as regards the discovery and observation of Comets. Pons, by whom Encke's Comet was found, both in 1805 and 1818, conducted the Observatory; but its mounting was as bad as its situation, and Pons used despairingly to describe his telescope as rather paralytic than parallactic. To this crippled establishment M. Gambart succeeded, and by his skill in managing his defective instruments, and by his patience in sweeping for Comets, he discovered and subsequently computed the orbits of a number of these bodies between 1822 and the period of his death. Gambart was highly esteemed, both by French and foreign Astronomers. Pons also deserves great credit for his extraordinary diligence in the discovery of Comets, and M. Valz, who still directs the Observatory of Marseilles has cultivated this and other branches of the science with success.

Gambart's most remarkable discovery was the pciodicity of the first Comet of 1826 , having detected Periodicit that body independently at Marseilles, though it had in $6 \frac{3}{4}$ year been observed some days previously in Bohemia, by Biela, an officer in the Austrian service. It is most usually called Biela's Comet, though it might with equal right be termed Gambart's, who assigned its path and predicted its return. Clausen, about the same time with Gambart, assigned it a period of about 7 years; and it was identified with former appearances in 1772 and 1805-6. Its period thus appeared to be 2460 days, or $6 \frac{3}{4}$ years; its aphelion is a little exterior to Jupiter's orbit, and its perihelion is not much within the Earth's. This Comet's orbit very nearly intersects in one place the orbit of the Earth, so that had the earth been one month forwarder in its annual course in 1832 , a collision would have taken place, or at least the Earth would have been enveloped in a cometary haze; for it is difficult to imagine a collision with a body whose tenuity is so excessive, that Sir John Herschel perceived through its entire thickness (estimated at 50,000 miles) stars of the most excessive minuteness (16th or 17th magnitude) as seen by his 20 -feet reflector. It is an interesting circumstance, that the first predicted perihelion passage, in 1832, took place within some hours of the time fixed by MM. Santini and Damoiseau, 
though the perturbations of Jupiter were, as usual, large.

Biela's Comet has been since recognised in 1846 and 1852 , but it was not seen in 1839. It does not appear to be admitted that it shows any acceleration due to a resisting medium. Its perihelion distance is, however, considerable.

At the apparition of 1846 an extraordinary circumstance occurred. When discovered in the end of November 1845 it appeared round and. single. On the 19th December it was observed by Mr Hind to be elongated, and ten days later was seen in America (and soon after at Cambridge and elsewhere) to have divided into two seemingly distinct nebulous parts. $^{1}$ These continued to subsist and move independently throughout the remainder of the apparition: the real distance of the centres being about 150,000 English miles. In 1852 the comet was rediscovered at Rome; the division into two still subsisting, but the interval of separation being increased about eight-fold.

Besides the comets of Encke and Biela, there are several others which are suspected on good grounds to have periods of from $5 \frac{1}{2}$ to $7 \frac{1}{2}$ years, their aphelia all lying in tolerable proximity to the orbit of $\mathrm{Ju}$ piter. But among these the return of only one has yet been verified by observation; namely, the comet of Faye, which, after passing its perihelion 17th October 1843, returned to it 3d April 1851, within an hour of the time predicted by M. Leverrier. The motion of comets of short period seems to be invariably direct or conformable to that of the planets. The inclination of their orbits to the Ecliptic is usually moderate.

Great Comets of 1811 and 1843.-The finest comets of the last hundred years were those of 1811 and 1843. The former was observed for a length of time altogether unusual, having been visible from March 1811 to August 1812. There is pretty good reason to think that its period is not much less than 3000 years. The comet of 1843 was even more splendid, but its flight was more rapid, and it was not favourably seen in northern latitudes. It was visible at many places in broad daylight when less than $4^{\circ}$ from the Sun, and at one time a tail $65^{\circ}$ in length could be traced. The circumstance which distinguishes this comet from all others which have been computed is the smallness of its perihelion distance, which was only $\frac{1}{2} \frac{1}{0}$ of the radius of the Earth's orbit, or the comet approached the Sun's body within one-seventh of his radius. The solar disk then subtended an angle at the comet of $121 \frac{1}{2}^{\circ}$, or the glare was equal to that of 47,000 suns as seen by us! The heat to which the comet was exposed is supposed to have exceeded 24 times that concentrated by our most powerful burning-glasses by which even rock crystal has been fused. ${ }^{2}$

Mr Hind.-Discovery of New Planets.-We have spoken in a former section (161), of the discovery of (282.) four small planets or asteroids between the orbits of of new Mars and Jupiter. They were found between the years Planets or 1801 and 1807. An interval of nearly forty years elapsed without any addition to the members of our system. In 1845 a new asteroid, Astræa, was found by $\mathrm{M}$. Hencke; the following year was distinguished by the discovery of Neptune under unparalleled circumstances; and since 1847 every year, down to the present time (1855), has added to our knowledge of the group of asteroids.

Among the discoverers of these planetary bodies $\mathrm{Mr}$ Hind has been distinguished by frequent success, under circumstances which appeared by no means peculiarly advantageous. This indefatigable observer and computer commenced (I believe) his astronomical career as one of the assistants at Greenwich, and afterwards had the sole charge of the private observatory of Mr Bishop, a wealthy citizen of London, together with the use of a fine refractor equatoreally mounted. It is within the Regent's Park, close to the smoke of the metropolis, that Mr Hind has discovered a larger number of planetary bodies than any other person living. Next to him M. de Gas- M. de Gasparis of Naples has been most successful. Unques- paris. tionably the impulse towards these new discoveries has been given by the indefatigable industry of astronomers (principally those of Germany), in constructing minutely accurate star-maps. Mr Hind is also advantageously known by the discovery of several comets, and by his ingenious observations in sidereal astronomy, especially on variable stars. I shall here give a table of the asteroids in the order of discovery as at present known (July 1855).

$\begin{array}{llll}1 \text { Ceres } & 1801 \text { Jan. 1 } & \text { Piazzi. } \\ 2 \text { Pallas } & 1802 \text { March 28 } & \text { Olbers. } \\ 3 \text { Juno } & 1804 \text { Sept. 1 } & \text { Harding. } \\ 4 \text { Vesta } & 1807 \text { March } 29 & \text { Olbers. } \\ 5 \text { Astras } & 1845 \text { Dec. } 8 & \text { Hencke. } \\ 6 \text { Hebe } & 1847 \text { July 1 } & \text { Hencke. } \\ 7 \text { Iris } & \text { Aug. 13 } & \text { Hind. } \\ 8 \text { Flora } & \text { Oct. 18 } & \text { Hind. } \\ 9 \text { Metis } & 1848 \text { April 26 } & \text { Graham. } \\ 10 \text { Hygeia } & 1849 \text { April 12 } & \text { De Gasparis. } \\ 11 \text { Parthenope } & 1850 \text { May 11 } & \text { De Gasparis. } \\ 12 \text { Victoria } & & \text { Sept. 13 } & \text { Hind. } \\ 13 \text { Egeria } & \text { Nov. 2 } & \text { De Gasparis. } \\ 14 \text { Irene } & 1851 \text { May 19 } & \text { Hind. } \\ 15 \text { Eunomia } & \text { July 29 } & \text { De Gasparis. } \\ 16 \text { Psyche } & 1852 \text { March 17 } & \text { De Gasparis. } \\ 17 \text { Thetis } & & \text { April 17 } & \text { Luther. } \\ 18 \text { Melpomene } & & \text { June 24 } & \text { Hind. } \\ 19 \text { Fortuna } & & \text { Aug. 22 } & \text { Hind. }\end{array}$

1 A similar phenomenon is related by Seneca. See Grant's History of Astronomy, p. 302.

2 See many other interesting particulars of this comet in Sir J. Herschel's Outlines of Astronomy, arts. 589, \&c. See also interesting details on the subject of comets generally in Mr Hind's and Mr Milne's works on comets, and in Mr Grant's excellent History of Physical Astronomy. 


$\begin{array}{lcl}20 \text { Massilia } & 1852 \text { Sept. 19 } & \text { De Gasparis. } \\ 21 \text { Lutetia } & \text { Nov. 15 } & \text { Goldschmidt. } \\ 22 \text { Calliope } & \text { Nov. 16 } & \text { Hind. } \\ 23 \text { Thalia } & \text { Dec. 15 } & \text { Hind. } \\ 24 \text { Themis } & 1853 \text { April 5 } & \text { De Gasparis. } \\ 25 \text { Phocæa } & \text { April 6 } & \text { Chacornac. } \\ 26 \text { Proserpine } & \text { May 5 } & \text { Luther. } \\ 27 \text { Euterpe } & \text { Nov. 8 } & \text { Hind. } \\ 28 \text { Bellona } & \text { 1854 March 1 } & \text { Luther. } \\ 29 \text { Amphitrite } & \text { March 1 } & \text { Marth. } \\ 30 \text { Urania } & \text { July 22 } & \text { Hind. } \\ 31 \text { Euphrosyne } & \text { Sept. 1 } & \text { Ferguson. } \\ \text { 32 Pomona } & \text { Oct. 26 } & \text { Goldschmidt. } \\ 33 \text { Polyhymnia } & \text { Oct. 28 } & \text { Chacornac. } \\ \text { 34 Circe } & 1855 \text { April 6 } & \text { Chacornac. } \\ \text { 35 Leucothaa } & \text { April 19 } & \text { Luther. }\end{array}$

(284.)

New Se-

condary

Planets. sell of Liverpool deserves a more lengthened tice than our limits will permit, not only as a distinguished discoverer, but as one whose success cannot be too widely made known as an encouragement to others. This gentleman, engaged in mercantile pursuits in an eminently commercial town, possessing little leisure and no enormous fortune, has contrived, in the intervals of business, to construct with his own hands telescopes which in accuracy of definition appear to rival any which art stimulated by national liberality has yet constructed elsewhere, and to use them with a degree of skill and success which has not been exceêded (nor in some respects equalled) by any astronomer whether professional or otherwise. I speak, let it be observed, of accuracy of definition, such as is necessary to display minute points of light, like the satellites of Uranus. In respect of the amount of illumination requisite for the display of many diffuse faint objects among the nebulæ, the gigantic telescopes of Herschel and Lord Rosse are of course superior.

Mr Lassel's observatory near Liverpool was erected (285.) in 1840. The principal instrument is a reflecting ments. telescope of 24 inches aperture (completed, however, only some years later), mounted equatoreally, an arrangement requiring great mechanical skill, but, as the results show, most effectually accomplished. The speculum was worked and polished by machinery con. structed by Mr Nasmyth, but principally devised by Mr Lassell, after he had examined and tried Lord Rosse's method. I should think it must be admitted to be the most perfect optical work of its kind ever made: for I believe there is no test object in existence which Mr Lassell has not seen with it; in fact he has discovered the most delicate tests himself,-the 6 th star of the group $\theta$ Orionis (though not first seen by him), a satellite of Neptune, an eighth satellite of Saturn, and several satellites of Uranus.

Not many days had elapsed after the discovery of (286.) Neptune, before Mr Lassell, directing his telescope He discoto it, perceived a satellite (as he believed) on Oct. vers a sa10, 1846. The discovery was fully made out in the Neptune, following year, and was soon after verified by the great refractors of Pulkowa and Western Cambridge (U.S.) Its period is about 5.879 days, ${ }^{1}$ and its discovery was of singular importance as leading to a knowledge of the mass of the planet.

Till 1848 only seven satellites of Saturn were admitted. The two closest to the plandt were detected and one of by Sir William Herschel in 1789, and have been Saturn. seen by very few astronomers since. During five years' residence at the Cape, Sir John Herschel never but once obtained even a doubtful glimpse of the closest with an 18-inch mirror. The third, fourth, and fifth were discovered by Cassini in 1684 ; the sixth and most conspicuous by Huygens in 1654 : the outermost by Cassini in 1671. To these an eighth satellite, intermediate in position between the two last, was added by Mr Lassell on the 19 th September 1848. By a singular coincidence, it was recognised as a satellite the very same evening by $\mathrm{Mr}$ Bond of Cambridge (in America) with the great Munich refractor. The new body was called Hyperion, in conformity with Sir John Herschel's suggestion of distin-guishing the satellites as well as the planets by mythological names. On the $22 \mathrm{~d}$ November $1850 \mathrm{Mr}$ Lassell saw at once Saturn with his whole train of eight satellites-a glorious spectacle probably enjoyed by no other astronomer. In the same month of November Faint ring Mr Bond discovered a faint or dusky ring of Saturn of Saturn interior to the two long known. It is probably ne- discovered bulous, for by Mr Lassell's observations and Mr Bond. Jacob's it appears to be trausparent.

Sir William Herschel thought that he recognised (288.) six satellites of Uranus. The second and fourth of Satellites his table have been observed by several astronomers, particularly Sir John Herschel and M. Lamont. Their periods are $8^{\mathrm{d}} 17^{\mathrm{h}}$ and $13^{\mathrm{d}} 11^{\mathrm{h}}$. To these $\mathrm{Mr}$ Lassell, aided by the fine climate of Malta (to which for two seasons he removed his telescope), has conclusively added two more : one, appearing to coincide with the closest of Herschel's, of which the period is $4^{\mathrm{d}} 3^{\mathrm{h}} 28^{\mathrm{m}}$, and one still nearer the planet revolving in $2^{\mathrm{d}} 12^{\mathrm{m}} 29 \mathrm{~s}$, the shortest orbital revolution in the solar system. Mr Lassell doubts seriously the existence of any other satellite. ${ }^{2}$ 


\section{\$.6. Sidereal Astronomy since 1820.-M. STRUVE-Double Stars. Observatories of Dorpat and Pulkowa. Sir JoHn Herschel-Orbits of Double Stars. Magnitudes of Stars. Variable Stars. EARL of ROsse-His Telescopes. Nebuloe. Henderson and BesseL-Parallax of Stars.}

(289.) As it is absolutely necessary to bring this chapter Continua- to a speedy close, and as I have already anticipated, tion of the in the account of Sir William Herschel's discoveries, Sidereal part of what relates to the recent history of the AsAstrono- tronomy of the Fixed Stars, particularly the "Conmy. $\quad$ stitution of the Heavens," and the motion of our system in space, I shall condense within brief compass a few leading facts connected, in the first place, with the Orbits of Double Stars, the Brightness of Stars, and the constitution of Nebulæ, and these subjects I shall connect with the names of the elder M. Struve, Sir John Herschel, and Lord Rosse; the second topic shall be the Parallax and distance of the fixed stars, as ascertained more particularly by the late professors Henderson and Bessel.

(290.) Friedrich Georg Wilhelm Struve has been the M. Struve. most assiduous observer of double stars since the time of Sir William Herschel. No discovery in this department can for a moment compete with the great one of the orbital revolution of one star round another. But M. Struve, by devoting his chief energies during the most active years of his life, since 1813, to the assiduous continuation of Herschel's observations, has added immensely to our knowledge of these systems, and has earned the reputation of one of the most skilful of modern practical astronomers. His most elaborate observations were made at the Russian Observatory at Dorpat, with a noble refractor by Fraunhofer, nearly 10 inches aperture, and $13 \frac{1}{2}$ feet in focal length. He has published three works on the subject of double stars, one in 1824 , one in 1837 , and one in 1852, besides minor papers. The second of these works contains the particulars of about 3000 double stars, deduced from a survey of the heavens, in which at least 120,000 stars were examined.

M. Struve's papers are distinguished by the elaboration of the reductions, and of the statistical results deduced from them. In his last publication he has made an interesting estimate of the number of true double stars in the heavens, which, it appears, is much greater in proportion to the whole number than is usually believed. But it is first necessary to distinguish those which are physically double from those which are merely apparently or optically so. The criteria on which he principally depends are(1.) the fact of observed orbital revolution; but as this is established in comparatively few instances, he very reasonably admits (2.) a common proper motion of the two components as a proof of their connection. He thus finds the evidence for physical duplicity to be much stronger for the closer double stars, and also for brighter or nearer stars, as compared with those of less magnitude. On the whole he concludes, that of
1973 double stars, 1702 , or six-sevenths of the whole, are physically connected, and that, amongst at least the brighter stars, the number of compound systems is to that of isolated stars in a ratio not less than 1 to 3 , perhaps even 1 to 2 .

M. Struve has also determined with extreme care (292.) the places and proper motions of the double stars ; M. Struve and he has formed a decided opinion, that the proper notions. motions diminish on the whole regularly with the order of magnitude, - thus confirming the criterion of increased distance from diminished brilliancy, by that of the apparent displacement of the stars by the motion of our system.

Besides these important works on Sidereal Astronomy, M. Struve is well known as the head and di- Directs the rector of perhaps the best organized observatory in observathe world, that of Pulkowa near St Petersburg, of Pulkowa. which he has published a very interesting description. Besides other noble instruments, it contains the finest refractor in Europe, that by Merz, 15 inches in diameter, and 22 feet focal length. The observations with this noble telescope are chiefly made by his son M. Otto Struve, the author of many good papers. We have seen in a former section (252), that we owe in great part to M. Struve the conduct of the most extensive trigonometrical operation ever undertaken.

Sir John Frederick William Herschel, son of (294.) Sir William Herschel, whilst conversant with almost Sir John every branch of science, has devoted himself with re- Herschelmarkable success to the cultivation of Sidereal As- mical catronomy. "Bearing a name honoured and revered reer. by all, his career at Cambridge reflected upon it fresh lustre; the variety and extent of his acquirements gave him a reputation amongst his college contemporaries, afterwards fully confirmed by the not more impartial voice of mankind at large." He was senior wrangler in 1813. "Since that time he has been indefatigable as an author:-first, in systematizing the higher mathematics, and in forwarding their study in his own university; - afterwards by treatises contributed to the Encyclopadia Metropolitana on Sound, Light, and Physical Astronomy, which still rank among the clearest, completest, and most philosophical in our language. About the same time he wrote experimental essays on different branches of Chemistry, Magnetism, and Optics, and commenced his purely astronomical investigations, chiefly on nebulæ and double stars, partly in conjunction with Sir James South, of which the details are given in different volumes of the Astronomical, and of the Royal Society's Transactions. These memoirs collectively include a complete revision of the objects of the same description catalogued and classified by Sir 
William Herschel." 1 But the most considerable monument to Sir John Herschel's love of science is the record of his four years' labours for the advancement of Sidereal Astronomy at the Cape of Good Hope, where he applied his father's methods of obSir J. Her-servation to the southern hemisphere. His Results schel's ob- of Astronomical Observations, which fill a large quarto servations volume, and which include "the completion of a of Good Telescopic Survey of the visible Heavens, commenced Hope. in 1825," form one of the most considerable and most interesting works of our time. The instruments employed were a 20 -feet reflector, of $18 \frac{1}{4}$ inches aperture, and a 7-feet achromatic, with 5 inches of aperture. With these the nebulæ and double stars of southern skies were examined and measured, and that wonderful "Gauging of the Hearens" completed, of which I have spoken in the account of Sir William Herschel (201). There is an admirable chapter on the apparent magnitude of the stars, to which I shall refer presently, and one on Halley's comet, besides other matters of interest.

Since his return to England in 1838, Sir John (295.)

Herschel has withdrawn from the labours of practical astronomy, but he continues to advance different branches of science, and to expound them by his able and lucid writings in a way which has made his authority equally respected by philosophers and by men of the world. The career of Sir John Herschel has been marked by an almost total absence of the element of ambition, so often a powerful excitement in the pursuit of discovery. Had he sought notoriety and posthumous fame, he would have confined his efforts within a more circumscribed range. But his versatile talents sought their appropriate exercise in all departments of exact science, and even (it is believed) in pursuits widely distinct from these, in natural history, belles lettres, and the fine arts. In all this he no doubt considered simply the useful and pleasurable employment of his mental activities. Truth seemed to him as desirable whether attained by the labours of others or by his own; and in his numerous writings he has expounded these with a zest which a less generous spirit might have reserved for his peculiar achievements. What he may have lost in future fame by this enlargement of his sympathies and interests, he has gained in the respect and good-will of all his contemporaries. Sir John Herschel recently filled the post of Master of the Mint, to which, like his illustrious predecessor Newton, he devoted a considerable share of his time. His general eminence as a man of science has been acknowledged by his nomination in 1855 to the distinguished honorary position of one of the eight foreign Associates of the French Academy of Sciences.

(296.) Orbits of Double Stars.-Though not absolutely On the or- the first to apply calculation to the orbits of double double stars. nected with the name of Sir John Herschel, from the ardour of his researches and the neatness of his methods. To Savary of Paris is due the merit of ascertaining the form and position of the orbit of $\xi$ Ursæ Majoris in 1827, which was followed by a more purely analytical method by M. Encke, and one chiefly graphical by Sir J. Herschel, ${ }^{2}$ in which angles of position of the component stars are used nearly to the exclusion of the more doubtful measures of distance. On the whole, these investigations not only confirm Sir William Herschel's anticipations, but render it highly probable that the relative orbits are really ellipses, and consequently that the law of force is that of the inverse square of the distance. The reader will find in Sir J. Herschel's Cape Observations a very curious discussion of the orbit of $\gamma$ Virginis, a remarkable double star, whose interval was in 1783 five seconds and two-thirds, which diminished till 1836, when the two stars appeared united in one, as seen even in the best telescopes. This was the perihelion passage of these two suns, and the angle of position must then have varied (could it have been measured) at the rate of $70^{\circ}$ per annum, or $1^{\circ}$ in 5 days. The following are some of the best ascertained periods of sidereal revolutions in years :$\zeta$ Herculis $36^{\mathrm{y} \cdot 4}$; ह Ursæ Majoris $61^{\mathrm{y}} \cdot 5$; « Centauri $77^{\mathrm{y}}$; $p$ Ophiuchi 80 or $90^{\mathrm{y}}$; $\sigma$ Coronæ Borealis 600 or $700^{\ddagger}$. M. Mädler, Admiral Smyth, and Mr Hind, have added much to our knowledge of this interesting subject.

Brightness of Stars, and Variable Stars _-Sir John Herschel has attempted by an elaborate system of On the inter-comparison to assign the correct relative bright- brightnes ness of the stars, and to give precision to the ordinary terminology of Magnitudes. His "Method of Sequences" described in his Cape Observations, appears to be one of the happiest specimens of generalization which experimental science affords. Whilst regretting the impossibility of here giving even the slightest sketch of it, I cannot but recommend it to the student of natural philosophy as a model of research. Having ascertained, in a way independent of every sort of hypothesis, the relative brightness of the stars upon the scale of Magnitudes usually adopted, but which is wholly arbitrary, Sir J. Herschel proceeds, by properly photometric methods, to give a scientific precision to this notation; and he arrives at this singular and fortunate conclusion, that by adding a small and constant correction to the received scale of Magnitudes, the numbers will represent the distarices of the respective stars from our system on the supposition of an intrinsic equality in the brightness of the stars themselves.

This subject naturally includes that of Variable (298.) Stars, which may be divided into those which under-Variable go periodic or irregular fluctuations, and the latter class may embrace new stars, and stars which have 
disappeared. The last two hundred years have not presented any such astonishing phenomena as the new stars recorded in the sixteenth and seventeenth centuries; but singular variations in the brightness of some of the most conspicuous stars, as a Orionis and $\eta$ Argûs, have been discovered by Sir John Herschel. Several stars of short and irregular periods of varying brightness were recorded towards the close of last century. But upon this very interesting subject I must content myself with referring to the details given by Professor Argelander in the third volume of Humboldt's Cosmos.

EarL of Rosse. Latest observations on Nebula.-

It is a remarkable circumstance, that as the reflecting telescope was a British invention, so the more important improvements and applications of it have been almost confined to the United Kingdom. It is also worthy of notice that the manufacture has prospered more in the hands of amateurs than of regular opticians. Sir William Herschel appeared at one time to have brought the invention to its highest perfection, but the Earl of Rosse has made an important step farther; not only by constructing a larger telescope than had been made before, but by adapting machinery driven by steam power, to the grinding and polishing of the mirror; so that the largest speculum may be finished with nearly the same accuracy and expedition as the smallest. The chef d'ouvure of Lord Rosse is a telescope of 6 feet aperture, and 53 or 54 feet of focal length. It was completed in the latter part of 1844 , and erected at Parsonstown in Ireland.

Let me here record the important fact, that neither rank nor wealth could absolve Lord Rosse from those toils and disappointments which attend all new and original efforts. There is no royal road to such triumphs. The Irish nobleman owes his success entirely to his unwearying perseverance and mechanical skill. Even his assistants were countrymen instructed by himself in his own workshops, where the very steam-engine which drives the polisher was fabricated. His labours to improve the telescope date from 1828 (when he was Lord Oxmantown), or even earlier, and they appear to have been unremitting until 1844 ; indeed I might say until the present time. Commencing with a variety of ingenious attempts to correct spherical aberration, and to overcome the extreme difficulty of procuring large castings of so excessively brittle a material as speculum-metal, they terminated in the rejection of all minor helps and expedients, and in the fortunate completion of immense mirrors at a single casting, and of correctly parabolic figure when ground and polished. The speculum of his largest telescope weighs four tons. It was polished in six hours, and its surface is con- siderably more than twice as large as that of Sir William Herschel's forty-feet instrument.

We cannot enter into the details of the methods, We cannot enter into the details of the methods, (301.) which evince no small mechanical skill and scientific Methods of ingenuity, together with a perseverance admirable in construcitself. ${ }^{1}$ I will only mention how the upper and lower surfaces of the casting were made to cool nearly equally fast. To effect this the lower surface of the mould (which naturally retains the heat more than the upper) was made of iron, a good conductor, whilst the upper surface was made of sand. This effected the purpose; but it being found that air bubbles entangled in the fluid metal could not escape beneath, and injured the casting, the iron bed was constructed of hoops set on edge and closely packed, the crevices allowing the escape of air, whilst the cooling proceeded as before; and this ingenious contrivance was perfectly successful.

Many difficulties in detail have been found in the mounting and use of so gigantic a mass, particularly Applicaon account of the distortion of the mirror by flexure. bulæ, and But these have gradually been surmounted by Lord its results. Rosse. His published observations (Philosophical Transactions, 1850) relate almost entirely to objects of the class of nebulse; and as I cannot enter into details, I may state the general results in two or three sentences. (1.) As might have been expected, maný nebulæ which resisted the power of former telescopes (for, except in rare instances, nothing greater than eighteen-inch apertures have been directed to them) have been "resolved" into stars by the six-feet speculum. (2.) The aspect of a great number of nebulæ described by the two Herschels is materially modified by the power of the telescope to embrace the fainter prolongations of these singular objects. In general, the symmetrical forms are very much cut up and confused, and in many cases vanish altogether. (3.) Instead of these, a certain species of symmetry, of a vague yet very remarkable description, has been detected by Lord Rosse, probably for the first time. It is a spiral arrangement of the nebulous coils round a centre, resembling somewhat the spiral emanations of revolving fireworks. The well-known nebula No. 51 of Messier's Catalogue shows this in a remarkable manner.

Some observations have been made upon the moon. It is much to be desired that these were continued, and that the planets could also be observed; but I believe that the climate of Parsonstown affords but few nights favourable to observation.

Henderson and Bessel. Parallax and distance (304.) of the Fixed Stars.-Thomas Henderson, at one Henderson. time government astronomer at the Cape of Good and characHope, and subsequently professor of practical as-ter. tronomy in the university of Edinburgh, and his

1 For a comparison of Lord Rosse's and Messrs Naysmith and Lassell's (subsequent) methods of mechanical grinding and polishing, see Astron. Soc. Notices, vol. ix. p. 110. In Lord Rosse's apparatus every stroke of the polisher is almost a straight line; in Mr Lassell's it never is. 
Majesty's astronomer for Scotland, was born at Dundee, in Scotland, 28th December 1798. He died at Edinburgh 23d November 1844. His career was an instance of the conquest of disadvantages of many kinds by a patient, devoted, and conscientious spirit, and of the attainment of a station of great eminence in the world of science by singleness of purpose, and an ardent love of knowledge.

(305.) He was fortunate in having $\mathrm{Mr}$ Duncan (now of Hender- St Andrews) as his instructor in mathematics at tific pro- Dundee, and it was " while employed as an attorney's gress. clerk in a provincial town that he laid the foundations of that extensive acquaintance with astronomy for which he was afterwards distinguished." It was his good fortune to attract in Edinburgh the discerning notice of Sir James Gibson-Craig and his family; through whose influence, probably, he obtained professional employments of a kind which permitted him considerable leisure, and even gave him an opportunity of forming scientific acquaintances in London. His early tastes were towards the practical calculations of astronomy, such as occultations and ephemerides; and from his merits alone he was commended and patronized by Dr Thomas Young. Had that great man lived, his promotion to a scientiAppointed fic post would have probably been earlier. As it was, to the Cape he obtained in 1831 an honourable, and, for him, Observa- lucrative appointment as astronomer at the Cape; at the sacrifice, however, of quitting his native country. In the thirteen months during which he held that situation, he performed an amount of first-rate work in practical astronomy which may bear a comparison with any similar effort. Charged with an instrument notoriously defective (Jones's circle), he had to examine its errors and their compensations; and it is no small credit to him to say that with such an indifferent tool he carried off a prize which had been the aspiration of so many astronomers before him-the determination of the parallax of a fixed star.

(306.) We must not go back to the history of this proThe paral- blem previous to the nineteenth century, when alone stars,

(307.)

had been investi-

gated by

Pond and Brinkley.

Notice of Brinkley, Bishop of

Cloyne. at Greenwich, the latter at Dublin, both with instruments of great power, being meridian circles of the largest size. It is sufficient here to note that $\mathrm{Dr}$ Brinkley attributed to some of the brighter stars, such as $\alpha$ Lyræ, a parallax of $2^{\prime \prime} \cdot 5$ (which, however, he afterwards reduced to little more than $1^{\prime \prime}$ ), whilst $\mathrm{Mr}$ Pond could arrive at no such result. There is no doubt that Mr Pond was correct. ${ }^{1}$

The first case of parallax which was determined with some certainty by the use of ordinary meridional instruments was that of $\alpha$ Centauri, a bright star of the discover southern hemisphere, which was deduced by Hender- Centauri. son from his observations at the Cape long after they had been made, and what is perhaps still more satisfactory, made without reference to this particular question. The result, which gave to this star an annual parallax of 0.91 of a second, is believed to be correct, because it has been confirmed by Mr Maclear, Henderson's successor at the Cape. It may, however, be not unreasonable to desire that the observations should be repeated in another locality, and with a different instrument; for it has not been unusual (as in the case of Brinkley) to obtain under the same circumstances perfectly consistent $t_{\text {yet }}$ erroneous, and therefore inexplicable results.

The very considerable amount of parallax in this instance, corresponding to a large proper motion $\left(3^{\prime \prime} \cdot 6\right)$, gives a strong independent probability that it is not materially erroneous; and in this respect the most competent and impartial historians of science have given full credit to $\mathrm{Mr}$ Henderson for having, by superior skill in the use of his instruments, and the happy choice of an object, made a discovery which so many eminent observers had long sought for in vain.

Subsequent to the date of Henderson's observations, but before their publication, Bessel (whose Bessel's biography we have given in Section 3 of this chap- observater) determined the parallax of the star 61 Cygni, in Cygni. a different way. It was a happy suggestion of Galileo, that if two stars be selected apparently near one another, but really disconnected, and having very unequal magnitudes (therefore probably at very different distances from our system), - and if the apparent angular distance between the two stars be measured from opposite parts of the earth's orbit, it must sensibly vary by an obvious effect of perspective. It was in pursuit of this happy idea that Sir W. Herschel discovered the fact that the connection of such pairs of stars is often real, not apparent-but it was Bessel who, guided in his choice of an object by the critical character of the

1 John Brinkley, Bishop of Cloyne, was born in England, and educated at Cambridge, where he was senior wrangler in 1788. He acted for a short time as Maskelyne's assistant at Greenwich, and was subsequently appointed Professor of Astronomy at Dublin, where he made many excellent observations, especially those on Nutation and Aberration. Brinkley ought to have been mentioned in Art. (30) of this Dissertation, as having contributed materially to the progress of the study of the Continental Mathematics in the United Kingdom. 
considerable proper motion of an otherwise unconspicuous star, first reduced Galileo's theory to successful practice. The details of this elaborate investigation have been considered by competent judges as among the happiest specimens of astronomical induction; and like Professor Henderson's, they have also had the advantage of subsequent and still more independent corroloration. Professor Johnson of Oxford, using, like Bessel, a divided object-glass micrometer (or heliometer, as it is not very appropriately called, never being applied to the sun), has obtained results for the parallax of $\alpha$ Cygni almost identical with Bessel's, which was 0.35 of a second. I am sorry that iny limits will not allow me to explain more particularly the details of Bessel's method, which, however, may be found in several modern works on astronomy.

M. Peters of the Observatory of Pulkowa has announced several additional parallaxes, all determined M. Peters. with the aid of a meridian instrument. He has even attempted to infer the mean parallax of stars of the 1st, 2d, \&c. magnitudes, which M. Struve has compared with his estimate of relative distance derived from other considerations; but the numbers of ascertained parallaxes, as well as their amount, are too small, and the anomalies still too apparent (as in the case of the star No. 1830 of Groombridge's Catalogue), and the parallactic distances too often inverted on the scale of brightness, to allow us to attach much importance to these generalizations.

\section{CHAPTER IV.}

\section{MECHANICS OF SOLID AND FLUID BODIES, CIVIL ENGINEERING, AND ACOUSTICS.}

\section{$\S 1$. WATT.-Condition of Practical Mechanics previous to the time of Watt. His genius for the application of Science to Practice. His successive Improvements on the Steam-Engine. Steam Navigation.}

J AMES WATT may be considered as the most distinguished practical man of science of the last century, or even for a much longer period. But this is not all. Few men achieve such a reputation as his without having done more than originate a great invention for the use and benefit of mankind in all ages : - He also taught men to raise the useful arts to a new dignity, - to marry thein to genuine, unpretending, and inductive science,- - to disparage ignorance and cmpiricism, and to render the labours of the workshop subservient to intellectual progress.

I have attempted, in the first chapter of this Dissertation, to place the scientific part of engineering in its due relation to pure physics, and I have compared the relation between them to that subsisting between Mathematics and Physics - the one as an instrument, the other as an end. Now it was this, in particular, which made Watt the important character he really was. He brought out the dependence of the former subjects, as Newton and others had successfully taught and demonstrated that of the latter.

To appreciate Watt's merit, we must compare the purely mechanical contrivances of the period preceding the date of his improvements on the steam-engine with those of a similar space of time succeeding it. In the former we find multitudes of contrivances on paper-ingenious, indeed, but many of which could not be executed; the greater part of the remainder could not be carried into effect through want of knowledge in the inventor. We have large promises of "semi-omnipotent engines," perpetual motions, "quintessences of motion," and the like, mingled with trivial mechanical toys; or we have elaborate diagrams of mill-gearing, lathes, fountains, and sawing machines without end, illustrated with showy and expensive plates, but destitute, for the most part, of the slightest novelty of principle, or truly mechanical skill in application. Here and there, no doubt, elegant and appropriate contrivances for communicating or sustaining motion occur; but nearly all the best forms of elementary machinery were of remote antiquity, excepting those connected with clockwork, which, including the great and truly scientific application of the principle of Isochronism, formed the only considerable step in philosophical mechanics for very many years previous to the conception of the steam-engine. The statical part of mechanics had made more progress. Masonry and Carpentry had attained a degree of perfection in many respects admirable, under the Italian and Norman architects; but the really difficult theory of machinery in motion was little understood before Watt's day, and the knowledge which then existed was unassociated with practical skill or commercial enterprize. It could be found only in profound treatises of theoretical mechanics, and in experimental courses of natural philosophy. The sources of power were almost exclusively those derived from simple gravity and the impact of fluids. It was not, indeed, the good fortune of Watt to be the first to employ the admirable 
properties of steam as a source of power; but he was almost the first to study them as a philosopher, and to apply, with exemplary patience and skill, principles sought in the laboratory, to make available the most convenient of all motive forces. This was done by means of a series of contrivances so ingeniousso strictly connected by scientific relations-as to be a model of experimental research, quite as much as a triumph of inechanical art.

(315.) Such combinations of theory and practice have now Philosophi- become far from rare. They have followed the march infused in- of physical learning, they have borrowed from it, to practical and they have contributed to it. But it was Watt mechanics who chiefly gave the happy example. Himself by by Watt. education and habit strictly a mechanic, he had the peculiar merit of apprehending the value of theory, and of acquiring a kind of knowledge then altogether uncommon amongst persons of his profession. He was no doubt a successful speculator, and a shrewd ingenious man besides; and this, his ostensible character, constituted possibly in the eyes of many his world-wide celebrity. But such considerations were little likely to influence the opinions of contemporary scientific men well qualified to judge, and least of all of eminent foreigners, who generally regard with little partiality the presumed commercial character of their insular neighbours. Dr Black, who was by no means prodigal of praise, termed the steam-engine, as improved by Watt, " an invention which is in its present state the master-piece of human skill," not "the production of a chance observation, but the result of deep thought and reflection, and really a Estimate present by philosophy to the arts." 'I Professor Roof Watt's bison, who knew Mr Watt intimately, was even more genius. enthusiastic in his appreciation of his genius; and Sir Humphry Davy, in a speech manifesting a just estimate of his peculiar merits, did not hesitate to place him on a level with Archimedes. ${ }^{2}$ But a testimony more authoritative and unbiasscd than any of these, is the fact that Watt was elected first a corresponding member of the French Institute, and finally one of the eight foreign Associates of the Academy of Sciences. This honour, to which so few can attain, which Newton once owned, and which now graces or lately graced the names of Young, Humboldt, Oersted, Brewster, and Robert Brown, is a sure passport to scientific immortality. Here, at least, no utilitarian pride, nor even the laudably patriotic emotion of gratitude to one who had proved, in more ways than one, his country's benefactor, can be supposed to have influenced in the remotest degree his election.

Having said thus much on the position to which Watt's inventions entitle him in the narrative of the history of science, we may refer with brevity to the generally well-known improvements of the steam- engine, in which they mainly consist. In Sir John Leslie's Dissertation, a space of but a few lines has been devoted to them, which seems inadequate to their importance. For details, however, we must refer to the articles in the Encyclopædia where they are explained at full length.

No doubt we cannot in strictness call Watt the inventor of the steam-engine. The grand principle of $r e n d e r i n g$ the heat contained in dering the heat contained in steam available as engive. an economical source of moving power may be traced so far back that we lose the clue altogether in the obscure, or impracticable, or simply puerile shapes in which the idea was contained. Even in the time of Worcester (1663) we must be allowed to doubt whether the history of the steam-engine had outgrown the mythical stage; Papin, indeed, proposed a piston and cylinder in which the vacuum was produced by steam instead of by the air-pump (as already suggested or practised by Guericke); but Savery (1698) was the first who constructed a steam-engine, and applied it to the drainage of mines. His invention included the two capital properties of steam, its power of producing a vacuum by condensation, and its elastic force at high temperatures. A few years later the piston-form was introduced or re-invented by Newcomen and Cawley, as well as the valuable expedient of producing condensation by a squirt of cold water injected into the cylinder; and in this condition the Atmospheric Engine remained with slight improvement for above half a century, doing the work for which it was invented,-the pumping of water out of shafts (the pump being moved by a chain attached to the end of a horizontal oscillating beam),-wherever economy of fuel was unimportant. Such was the case at coal pits, but in other mines, usually situated remote from coal, it was of comparatively little use, on account of the enormous consumption of fuel.

James WATt was born at Greenock in 1736, and, owing to feeble health, seems to have enjoyed little James. advantage from regular tuition of any kind; which Watt's was, however, to a great extent made up for by the tory, intelligent spirit with which be acquired knowledge without assistance on a great variety of subjects. At the age of nineteen he proceeded to London, where he learned mathematical-instrument making, but he soon returned to Scotland, intending to pursue that business in Glasgow. Here he met with obstacles, but finally, by the patronage of Dr Adam Smith, Dr Black, and other professors, he was established as instrument-maker to the university within the college buildings. I mention these details because they show that Watt, as early at least as 1757 , had been favourably noticed by the most celebrated professors then in Glasgow, and had received a special pledge of their good will." It is evident that the professors of Che-
(317.) Origin of

$\sqrt{2}$

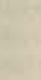

\footnotetext{
1 Lectures, i., 181.

2 Speech at Freemasons' Hall, preserved in Arago's Eloge of Watt, and in Davy's Works, vol. vii.

3 It appears from Mr Muirhead's work on The Origin and Progress of the Mechanical Inventicns of James Watt (published since the greater part of the text of this section was written), that Watt's introduction to the college took place "through the instru-
} 
and connec-mistry and Natural Philosophy (Dr Black and Dr tion with Dick), who had obtained for him his appointment, Dr Black. must have been also his chief academical employers; and we may safely conclude that Watt constructed much of Dr Black's apparatus, as he was certainly admitted to intimate and confidential intercourse with that great and amiable man. Dr Black removed to Edinburgh in 1766, but the friendship which he had extended to the young mechanic remained unaltered during their joint lives, although their personal intercourse must have been extremely slight after that time. Now Watt's first thought of improving the steam-engine dates (as we shall see) from the session 1763-4, the last but one of Black's stay in Glasgow ; their intimacy was therefore fully established by that time; and we find Watt acknowledging his "obligations to him for the information received from his conversation, and particularly for the knowledge of the doctrine of latent heat;" 1 and we also find that during Watt's experiments on steam subsequent to the last-mentioned date, Black was cognizant of them, and assisted in their contrivance. ${ }^{2}$ Moreover, Watt admits Dr Cullen's well-known experiment of reducing the temperature of ebullition under the air-pump to have been one of his starting points in the improvement of the steam-engine. ${ }^{3}$ Yet this experiment was not published until $1770,{ }^{4}$ and Watt must have heard of it from Black himself, or from some one attending his lectures, where the fact was almost certainly mentioned. Whether formally a student of $\mathrm{Dr}$ Black's chemistry class or not, it is therefore evident that Watt enjoyed advantages in the prosecution of experimental physics which nineteen-twentieths of enrolled students never attain. He had the privilege of unreserved personal intercourse, amounting at last to intimate friendship, with the first authority of the last century on the subject of Heat, and one of the most cautious and accomplished of inductive philosophers. ${ }^{5}$ Watt's eminent merits, and doubtless his merits alone, gained him this happy position; but had he remained either at Greenock, or with the optician in Cornhill, he might have failed to combine so admirably as he did the character of the practical man and the philosopher. His place in science was His posiwell typified by his position in Glasgow. His was tion in "the workshop within the College." Whilst the la-Glasgow boratories of the classes of Chemistry and Natural Philosophy must have been his familiar resort, his own rooms were frequented by the most intelligent students, including his contemporary Dr Robison, where subjects of science particularly connected with mechanics were diligently canvassed. The extent of his knowledge and the variety of his resources were fully tested, and the result, as stated by the generous pen of Robison, was a conviction of the superiority of Watt in these respects to any of his contemporaries.

It is a fact worthy of note that the immediate occasion of Watt's improvements was the commercial Watt's first consideration of economy. In 1763 or 1764 , being expericalled on to repair a model of the Atmospheric En-steam. gine in the Natural Philosophy class at Glasgow (which model is still preserved), he found the amount of steam expended in heating the cylinder at each stroke to be so great that the boiler was insufficient to supply it properly. He then commenced experiments on the amount of steam thus consumed, and on the means of diminishing it. Though the primary object was the repair of a model, it is not to be doubted that Mr Watt had in view the practical improvement of the engine on the great scale, with the use of which in the coal-fields of the west of Scotland he was probably familiar, having the intention of becoming himself a civil engineer, and being already acquainted with the writings of Desaguiliers and Belidor. ${ }^{6}$

mentality of his mother's kinsman Mr George Muirhead, who had then (1754) just exchanged the professorship of oriental languages for that of Latin." It will be seen that this refers to a period antecedent to his journey to London.

1 Letter to Dr Brewster in Robison's Mechanical Philosophy, ii., p. v.

2 Robison's Mechanical Philosophy, ii., 115, note (by Mr Watt).

3 Ibid., p. vii., and 114 , note.

4 In the Edinburgh Physical and Literary Essays, vol. ii., Dr Black (who was intimate with Cullen) knew of it at least in 1757. Lectures, i., 525.

6 It is perhaps unfortunate that Mr Watt, when on the verge of fourscore, contrary to his onn intentions, but yielding to "the representation of friends," recorded a complaint that his best friends, Black and Robison, had refused him his due share of merit in the improvement of the steam-engine, and disclaimed, as injurious, the appellation of "a pupil of Dr Black" (letter to Dr Brewster in Robison's Mechanicul Philosophy, vol. ii., p. v.) Admitting (as he does on the same page) that the doctrine of latent heat was due to Dr Black, and that he first learnt it from him, he adds, "this theory did not lead to the improvements I afterwards made in the engine," p. viii. No one ever ascribed to Black the beautiful invention of a separate condenser, but even Watt's most ardent eulogists (Lord Brougham and M. Arago) admit that the theory of latent heat " forms the key to the economical appreciation of the steam-engine." So far was Watt from being independent of Dr Black's assistance in this matter, that he himself tells us twice over, in substantially the same language, that when in the course of his experiments on the model engine in Glasgow College in $\mathbf{1 7 6 4}$ he was "at a loss to understand how much cold water could be heated by so small a quantity in the form of steam," he "applied to Dr Black, and then first understood what was called latent heat" (Robison, ii., p. v.; also p. 116, note). Mr Watt might have been the discoverer of latent heat, and the solver of his own dilemma, had not Dr Black been at hand. It is the highest compliment we can pay him to say that such an achievement was not too much to expect from him.

[These conclusions are fully substantiated by the interesting documents lately published by Mr Muirhead in the Correspondence of Watt on the Composition of Water, p. 6, and in his Mechanical Inventions of James Watt, vol. i., p. 1xxxi.; vol. ii. pp. 116, 118, 119,275 . In the last-cited passage Watt himself fixes the date and manner of his receiving from Dr Black his knowledge of the doctrine of Latent Heat. He says, "I myself never attended his (Dr Black's) lectures; but the doctor explained his doctrines to me about the year 1763."] Note added during printing.

6 See Mr Watt's own narrative in Robison's Mechanical Philosophy, ii., 113, note; and in Mr Muirhead's Mechanical Inventions of James Watt, rol. i. 
(320.) Imperfections of atmospheric engine.

(321.) Watt's principle of separate condensa tion.
When Watt discovered by means of his model that the condensation and loss of steam in the cylinder at every stroke of the piston exceeded that which is usefully employed in producing the vacuum, he proceeded very methodically to ascertain the chief numerical data or constants upon which the working of steamengines depend; as, the bulk of steam of given elasticity compared with that of the water producing it, the elasticities of steam at different temperatures, especially above the boiling point, the evaporating power of a pound of coals, and finally, the expenditure of steam and of injection-water for a single stroke. The last, the amount of cold water required effectually to condense the cylinderful of steam, appeared so very large that it at once betrayed the source of the wasteful expenditure of fuel. All the heat which was abstracted by the injection-water had to be supplied afresh at a vast expense of steam, and consequently of coal, before a fresh stroke could be made. The mere amount of steam necessary to fill the cylinder and elevate the piston was a trifle in comparison. He naturally ascribed it to the absorption of heat by the material of the cylinder, which no doubt was an important element; and he tried cylinders of different materials, and ascertained (in most instances for the first time) the specific heats of the substances used. But still the amount of injection-water remained unaccounted for, and the regulation of it was the great diffieulty of the old engine. If a tolerable vacuum was desired, the cylinder must be flooded with cold water, and all the heat abstracted must be restored before the next stroke was produced. If, on the other hand, the injection was imperfect, so that the condensed steam and water together retained a high temperature, an atmosphere of vapour so elastic spoiled the vacuum, and in many cases the working efficiency of the engine was reduced to about half of the atmospheric pressure.

The theory of latent heat, showing that steam contains as much heat as if the water yielding it had been raised through $1000^{\circ}$ of temperature, gave a clear explanation of the enormous amount of injection-water necessary. How to escape from the difficulty was another question, which was solved solely by $\operatorname{Mr}$ Watt, who, early in 1765 , first conceived the idea of retaining the cylinder always at the boiling heat, and effecting condensation in a separate vessel kept constantly as cold as possible, in which injection should also take place, and into which the steam of the cylinder would spontaneously rush, on a communication being opened at the proper instant. The success of this device was complete, and it was speedily followed by a series of admirable and mutually de- pendent improvements which Watt introduced. To keep the cylinder as warm as possible, it was first provided with a cover, through which the piston-rod passed by means of a stuffing-box. Then steam itself was employed to press down the piston, instead of atmospheric air which cooled the cylinder injuriously.

Having thus steam on each side of the piston, the step was easy to make the engine a double-acting one; so that the volume of steam which by its elasticity impelled the piston during its descent, being thrown into the condenser, produced the vacuum necessary to make the ascending stroke equally advantageous. ${ }^{1}$ But in pumping-engines, which had hitherto been the main employment of steam, or fire engines (as they were then called), it was sufficient that the power was produced in one direction, that is, to lift the pump-rods ; ${ }^{2}$ but the double action of a pushing and pulling force was evidently applicable to every sort of machinery by the use of a crank and fly-wheel. A difficulty, however, was experienced in applying this push-and-pull movement to the oscillating beam which had so long formed a part of the atmospheric engine, and which it was so convenient to use. Mr Watt obviated it with his customary saga- Paralle! city, and succeeded in inventing a connection of rigid motion. jointed links which effectually united the piston-rod with the end of the beam, the former moving in a vertical straight line, the latter in a circular arc, so that pressure was transmitted in the manner of either push or pull without the slightest practical inconvenience. No doubt the locus of the motion of the piston-rod head is not rigorously straight. It is, when fully developed, a curve of the fourth order, resembling a very elongated figure 8 ; but the portion of the curve in use is practically straight. This happy solution, the most elegant perhaps in the science of pure machinery, or kinematics, was not the result of a formal mathematical research, but presented itself to the mind of Watt, as he tells us, almost intuitively. It became from henceforth an important element of mechanical combinations.

Other methods of producing direct rotation occurred to the (323.) them were shown to be practicable by being actually working. executed in model size. But the next great practical improvement introduced by him was the princiciple of expansive working, whereby a saving of steam is produced (particularly in single-acting engines), second in importance only to the result of the separate condensation. This consists in allowing the steam to flow in upon the piston during only the first part of the stroke; the rest being completed partly

\footnotetext{
1 This happy idea seems to have been suggested by Dr Small of Birmingham in a letter to Watt, of date 5 th November 1769 "I see no reason ..... why you should not condense at both ends of your cylinder, and drive all before you, back struke and fore stroke."-Mechanical Inventions of James Watt, i. p. 81. The uncommon sagacity of Dr Small, and the important services which he rendered in many ways to Watt, come out clearly for the first time in this work.

2 So associated were these engines with the operation of raising water, that we find they were at one time used to supply common water-wheels with a constant stream, and thus to produce continued motion for mill-work, \&c.
} 
by the inertia of the moving machincry, but chiefly by the continuous though constantly diminishing elasticity of the steam as it expands and fills the entire cylinder. It is evident that, in the extreme case, the work required (in pumping) will be accomplished if the piston reach the end of the stroke just as its momentum is exhausted, and that, on the other hand, if the pressure of the steam remain constant throughout, the velocity will be a maximum just when the work being done by the machine is suddenly completed, and so much moving power will be wasted. A medium distribution produces the most favourable result in practice, and it depends entirely upon the ratio between the resistance and the effective pressure of the steam what this most favourable proportion will be. In the single-pumping engines of Cornwall, in which the economy of fuel is carried to the highest perfection, it frequently happens that not more than one-eighth of the stroke is performed under the full pressure of the steam, which acts expansively during the remainder of the stroke; but these engines, though condensing, are moved by steam of four times the elasticity of the atmosphere. The Cornish engines have been very elaborately perfected, and they have been more carefully tested in respect to performance than any others. It is perhaps not too inuch to say that they do not contain a single contrivance of any importance (beyond of course what they have in common with the atmospheric engine), which was not the unaided invention of $\mathrm{Mr}$ Watt.

In the Cornish engine we see the Energy of Heat rendered available to an extent which the inventor himself would at one time have thought scarcely credible. The combustion of a bushel of coal which in a Newcomen's engine improved by Smeaton was capable of raising $3,000,000$ pounds through one foot, in Watt's improved pumping engine raised $20,000,000$ pounds the same height. But by the indefatigable skill and perseverance of engineers the Cornish pumps now yield at least five times the last amount. This, however, is not the place to enter upon these details, nor can we stop to particularize the other and various mechanical inventions introduced by Watt in the form of valves, governors, and steam-indicators. Still less can we enlarge upon the endless and still multiplying applications of this admirable moving power, which is as capable of superseding the greatest natural forces hitherto applied by man to the useful arts, as it is adapted by its easy regulation to replace human industry in the most delicate operations; "the trunk of an elephant, which can pick up a pin or rend an oak, is as nothing to it." '2
Of the applications of the steam-engine with which (325.) $\mathrm{Mr}$ Watt was less immediately connected, its adap- Applicatation to locomotion in the case of ships in the last steam to century, and in that of railway trains in the present, navigation. have been the most striking, and fraught with consequences the most important to mankind. Of the latter we shall have occasion to speak in a future section; of the origin of steam navigation we may here say a very few words. Passing over projects which never were realized, of moving barges by steam, or other inanimate power, against wind and tide,such as those of Worcester, Papin, and Hulls,-we find that the first experiment entitled to be called successful was made by Mr Miller of Dalswinton in MillerScotland, conjointly with Mr James Taylor, tutor in Taylor. his family, who together formed the project of moving vessels by means of paddle-wheels driven by a steam-engine, and realized it with the aid of Symington, a practical engineer. As we shall also find in the case of steam-carriages, the idea of the application of the steam-engine to move ships was already a familiar one to the minds of many persons about the middle of the last century. To put it in practice with advantage was the step required. Mr Miller's first boat was launched on Dalswinton Loch in Dumfriesshire, in October 1788, and attained a speed of five miles an hour. The subject was pursued by Symington and others. In 1789 a larger vessel was propelled on the Forth and Clyde Canal. Subsequently, however, the invention languished. The want of co-operation, of capital, and ingenuity, naturally extinguishes many valuable inventions. Watt himself was only rescued from the same difficulty by the unusual intelligence of Boulton and Small, his coadjutors at Birmingham. Symington was less fortunate, as well as probably less mcritorious; and though it is well established that Fulton, who passes on the other side of the Atlantic for the inventor of steam-ships, had seen the relics of Symington's second experiment, we must do the Americans the justice to say that the application of steam to navigation first flourished in the United States. In 1807 Fulton Fulton. started a river boat with an engine of Boulton and Watt. In 1813 the example was tardily imitated on the Firth of Clyde. The subsequent improvements need not here be specified. They have been very great and striking, but with the exception of the recent substitution of the screw-propeller for paddlc-wheels, they scarcely involve any new principles. ${ }^{3}$

We may briefly close what we have to say of $\mathrm{Mr}$ Watt personally. His health was feeble from child- Personal hood, but being blessed with much calmness of tem- character per he prolonged his life to a great age, and passed through its struggles, though they were to him con-

\footnotetext{
1 The double-acting engine was planned in 1774 or 1775 . The expansion principle was first used in 1776 ; the parallel motion was patented in 1784.-Watt's Notes on Dr Robison's Article. Robison's Mech. Phil., vol. ii. 2 Lord Jefrey.

3 For farther details on the history of Steam Navigation, see that article in the Encyclopædia; and Mr Bennet Woodcroft's work on the subject, London, 1848.
} 
siderable, without serious injury. Vexatious lawsuits connected with his valuable patent rights afforded him but too much oecupation and anxiety. He wrote little, and he did not covet fame. He was fond of ehemistry as well as inechanies, and was well acquainted with the theory and practice of that science as it then existed. He put forth opinions on the chemical constitution of water which, in the judgment of some, entitle him to contest priority in the discovery with Cavendish. He must have been versed in mathematics in his early years, as we learn from his friend Dr Robison, but there is no evidence that he ever attempted a strict theory of his own engine. Nor were his successors in this respect more fortunate. The best practical writers on the steam-engine, up even to a late period, gave most rude and inaccurate rules for computing its effeets; and it is to a Frenchman, M. de Pambour, that we owe the first philosophical, and, at the same time, elementary analysis of this noble machine.

Much light has been thrown upon the character of by Mr Muirhead. ${ }^{1}$ We there see the pressure of physical infirmity, and mental despondency and indifference, under which he laboured from boyhood. We learn the accumulated difficulties, arising from the backward state of the mechanical arts in his time, which delayed for years the suecessful prosecution of his happiest, and what would appear in our day most easily realized, conceptions. We see him at times ready to abandon fame and profit for the enjoyment of the humblest competence with tranquil- lity. We find him, mechanic though he was, shrinking from possible collision with the opinions or interests of others, and in his early as in his latest days, solicitous to avoid responsibility. He had, in a word, throughout, the finely strung susceptibility of a man of genius, singularly at variance with the necessity he was under of pushing his way in the world, and of turning his inventions to the best eornmercial account. Providentially he was thrown in the way of friends to whom, by his private character, he was greatly endeared, and who supplied the elements necessary to the successful prosecution of his schemes. The sanguine zeal of Roebuck, the commercial sagaeity of the capitalist Boulton, and not least, the sympathizing friendship of Dr Small, who was well fitted by his character and attainments to mediate between Watt and the other two, were all essential to the realization of the improved steamengine.

When Mr Watt was finally relieved of the oppression and chicaneries of his opponents in the courts close of of law, he was settling down into a peaceful old age. life. He probably hoped to live over again some of the scientific passages of his youth in sympathy with his second son, Gregory, who possessed a decided taste for science, but was unfortunately early eut off. Respected and beloved by a large group of friends, many of whom survived him, and admired by a far wider circle, he died at Heathfield, near Birmingham, 25th August 1819. Statesmen, philosophers, and men of the world, united in extolling the worth of his character and the greatness of his genius.

\section{\$ 2. RoBIson.-Application of Statical Principles to Engineering-especially to Practical Masonry. Cousomb.-Friction-Force of Torsion.} practical philosopher.
The name of RoBison may perhaps not appear to be sufficiently identified with any great discovery to merit a place in this condensed sketch of the progress of science biographically illustrated. Were there no other claim, I should consider it a sufficient one to entitle him to at least a brief notice, that he was by far the most important, and, as M. Arago has justly called him, " most illustrious contributor" to the earlier editions of the Encyclopedia Britannica. But he was also a philosopher in a high sense of the word. His knowledge was multifarious in no ordinary degree. He had little of pretension to originality, yet he brought to bear upon matters of science an unfailing amount of excellent common sense, and his personal acquaintance with the Arts which may be called Philosophical far exceeded, I imagine, that of any man of his time. Though not without his prepossessions, he was generous in the highest degree in his estimation of others, who in some sense might have been considered his rivals; he was eminently patient in his study of the works of his contemporaries, and in his published writings he laboured to render the results generally accessible to ordinary readers, by means of laborious abstracts, intermingled often with highly original views; and he explained them with conscientious energy in his lectures to the students of Natural Philosophy, whom for thirty years of his life it was his pride and pleasure to instruct. Amidst these congenial labours he found little time for making prolonged original trains of experiment, though the specimens which he has almost incidentally left us give the fullest proof of his ability in this respect; and the explanation is, I have no doubt, to be found in the peculiar circumstances of his early life. Until his settlement in Edinburgh, he passed his time in a series of active pursuits, having much more of the character of stirring practical life than of literary re- 
pose or scientific contemplation. He was thirty-five years old when he began to lecture in Edinburgh; and though an excellent working age, it is rare indeed to find that the talent of discovering new truths has been formed and developed so late in life. A major part of the great steps in science have been taken at even a much earlier period.

That Robison possessed all the elements of an original thinker we shall presently endeavour to show; but had his excellences consisted alone in those we have specified, he would have been a person eminently useful in forwarding the march of science. In fact, a few more such authors in every generation would be cheaply purchased by the postponement of some second-rate discoveries. Men-and we include men of science-are in too great a hurry to push on (actuated often by a morbid love of praise) to acquire something they may call their own, whilst they are little acquainted with the important contributions of their rivals in the race of fame, and of the predecessors to whom they really owe so much of what they may choose to consider their peculiar property. To methodize knowledge from time to time -to present discoveries in a form different from that in which they were first published, and thus connect them with what is already known and what remains to be clearly proved-these are real services to science which contribute in a very essential manner to its progress. Robison was a teacher, not only to the youth of his native country, but to the men of science and of practice of all countries, and of many succeeding years.

John RoBıson was born in 1739 , entered the University of Glasgow at the early age of eleven, and graduated at seventeen. His early tastes were directed . towards Natural Philosophy. He studied Mathematics (as in after life) chiefly with reference to its applications. Whilst at Glasgow he formed the intimate acquaintance of James Watt, then a practical instrument maker. In a passage of a private paper, pub. lished first in Arago's Eloge of Watt, ${ }^{1}$ Robison, with his characteristic generosity, describes his mortification when "yet a young student" at finding him much his superior in mathematical and mechanical knowledge; but M. Arago has omitted to state that Watt was, at least, three years the senior, which at such an age, and on such subjects, might make all the difference between a beginner and a proficient. But the truth is, that with a rarely generous rivalry in excellence, each esteemed the other most ; for we find Watt bearing a similar testimony to the supe- riority of his friend and junior: "I was happy to find in him [Robison] a person who was so much better informed on mathematical and philosophical subjects than I was." 2 It was by Robison that Watt had his attention first directed to the steamengine; their correspondence appears to have been frequent during their joint lives ; and near the close of his life Watt acknowledged "his obligations to him for very much information and occasional assistance in his pursuits ;" and in the Edinburgh professor he found not only the zealous defender before a court of law of his rights as an inventor, but also the first who expounded methodically the principles and details of the steam-engine in a manner which, at least until lately, had not been superseded.

In 1758 he left Glasgow, and the following year he went to sea as tutor to a son of Admiral Knowles. His life in a man-of-war, during which he saw some active service in Canada, was favourable to the development of his practical turn of mind, and doubtless gave him an interest in seamanship, naval architecture, and other subjects, which he afterwards turned to good account; and a subsequent expedition to Jamaica, for the trial of Harrison's Timekeeper, exercised him in some of the practical parts of astronomy. He returned, however, to Glasgow in 1761, and attached himself with such success to the study of Chemistry under Dr Black that he taught the Chemical Class in the university for several sessions. ${ }^{3}$ But his active life was not at an end. In 1770 he accompanied his first patron, Admiral Knowles, to Russia, and for some years was employed, first as his secretary, superintending improvements in the marine establishment, and afterwards as professor of Mathematics in the naval school of Cronstadt. He spoke and wrote the Russian language with facility, 4 and performed his duties to the satisfaction of all. But in 1774 he and his apcould not resist the honourable invitation which he pointment received to fill the chair of Natural Philosophy in versity of the University of Edinburgh, where he spent the Edinremainder of his life, which terminated in January burgh. 1805 , amidst incessant literary occupation, even when repeated attacks of a painful disorder had prevented him from personally continuing his lectures.

This brief sketch ${ }^{5}$ of a career rather unusual for a man of science throws light upon Robison's pecu- Character liar merits. He had extensive, and then uncommon, of his aropportunities of acquiring information, of seeing vari- Encycloous countries, and of noticing their physical peculiari- poedia Brities; of being introduced to their society and literature; tannica.

1 And since, in extcnso, in Muirhead's Mechanical Inventions of James Watt, vol. i., p. xli.

2 Ibid., vol. ii., p. 293.

3 The MS. of these lectures, written with curious care, but with the use of continual abbreviations of the larger words for the sake of compression, is now in my possession, having been given to me by his son, the late Sir John Robison. I have also many others of his MSS., which for the most part seem to have been printed in some form or other. Dr Robison was an indefatigable penman, and wrote and re-wrote his lectures with great labour. Ile also made elaborate analyses of his reading, but the bad labit of contracting words remained with him through life.

- The private marks on his MSS. are often in the Kussian character.

5 A much fuller one will be found in Playfair's Works, vol. iv. 
of taking part in the actual execution of scientific designs; of studying foreign languages; and of metlodizing his knowledge for the purposes of instruction. Every one of these infiuences may be clearly traced in his writings, which will challenge comparison with those of any English writer, except perhaps Dr Thomas Young, for variety and fulness of information, and for the general soundness and strongly practical character of the mechanical knowledge with which they abound. They would also doubtless have appeared to far grcater advantage had they been the product of his most vigorous days; but he was little known as a writer until the year 1793, when he commenced a series of important contributions to the Encyclopoedia Britannica, embracing at least forty-six articles, all scientific, some of great length and elaboration, which were published during the succeeding eight years, a period when he was seriously afflicted with chronic disease. They embraced disquisitions on general philosophy, as in the articles Philosophy and Physics; of strict science, as in Astronomy, Dynamics, Projectiles, Pneumatics; of the more experimental sciences, as in Magnetism, Electricity, Sound; of the art of music (with which he was practically conversant), in Temperament, Piano, \&c.;-but his great strength lay in the articles in which just mechanical principles were applied successfully, and often with marked originality, to practice, as in Arch, Roof, Carpentry, and Strength of Materials ; Resistance, Rivers, and Waterworks; Seamanship, Steam and Steam-Engine; Machinery, Telescope, and Watchwork.

(334.) The last-named articles (most of which have leen His $\mathrm{Me}$ - collected in a compilation, edited by Sir David chanical Brewster, in four thick volumes, entitled RobiPhilosophy. son's Mechanical Philosophy) constitute a body of knowledge in civil engineering which has not yet been surpassed in clear exposition of physical principles and their application-in the extensive acquaintance it shows with the details of practice-and in proofs of elaborate and impartial study of authors, both British and foreign, on the subjects of which it treats. Several of these articles have been resorted to by the most accomplished engineers of our own time as stores of sound experience, and they have been lavishly borrowed from by some writers on similar subjects, occasionally without acknowledgment. The original matter which they contain is not always easily separated from that which is compiled. In every instance Dr Robison lays claim to less than his own share, and is so scrupulous in quoting the names of other authors, that he has unquestionably received less reputation from these Essays than he deserves. He writes like Sound a man who has himself used the saw and hammer, practical views. who might have been called upon for a scientific opinion (of far more weight than what generally goes by the name) on almost any practical subject upon which he might have been consulted; and that not merely in its general outlines, but in its strictest details; not only the design for a roof or a centre, but the scantling of the timber to be used; the minutiæ of a pump or hot-air stove; the curvatures of an achromatic object-glass; the temperament of a piano; or the angle for the pallets of an escapement. In such matters nothing important either in the theory or the practice of his times escaped lim. His opinions were very generally formed on original considerations, supported by experiments equally well devised and carried out. His method of finding mechanically the relation between the intrados and extrados of a properly balanced arch by means of suspended pieces of chain is as ingenious as it is elementary; but his observations on the manner in which stone arches, when overloaded, break up, established on direct observation and experiment, unquestionably gave a just foundation to the theory of masonry, till then so generally and erroneously treated of by mathematicians with the preposterous abstraction of the forces of friction and cohesion, the action of which in many instances vastly exceeds the direct effect of gravity.

But on this we must not dwell. Robison's articles on Electricity and Magnetism are deserving of nearly equal praise with reference to the state of knowledge of the time. Probably there was not one author tricity a in merit on these subjects, whatever his country or language, whose works he had not laboriously consulted and analysed the conclusions; whilst a multitude of ingenious experiments give evidence of the skill and patience of the writer. To Dr Robison we are, indeed, indebted for the approximate knowledge of the primary law of electric attractions and repulsions; for a careful consideration of those beautiful curves formed by iron filings round magnets, ${ }^{1}$ to which such an enlarged importance has since been given by the beautiful generalizations of Mr Faraday; and I believe likewise for the first suggestion of combining the voltaic elements in a pile or column. ${ }^{2}$ Dr Robison, though for many years secretary of the Royal Society of Edinburgh, published little in its Transactions, and almost nothing, so far as I am aware, in any periodical work. Hence his admirable adaptations of experiments were little known beyond his own class and friendly circle.

Of his more abstract mathematical writings we need say little. He was thoroughly acquainted with His exter the works and methods of Newton, and with nearly sive read all those of the same school, particularly of Bos-mathecovich. He laboured incessantly to reduce the de-matical a monstrations of the higher mechanics and astrono- quire-

\footnotetext{
${ }^{1}$ Indirectly we owe to him also the first exact determination of the inathematical properties of these curves, made at his request by Mr Playfair.-Robison's Mech. Phil., iv. 350 .

${ }^{2}$ See the Fifth Dissertation (by Sir John Leslie), p. 739.
} 
my to their simplest and most geometrical elements. But the result (as might have been foreseen) was in many cases a wearisome prolixity; and originality could not be expected in these departments without applying the continental improvements in analysis : not that Dr Robison overlooked these; but he took little pleasure in them; and as regards Physical Astronomy he adhered to the older methods. Having been so long at St Petersburg, the writings of Euler must have been familiar to him, as indeed they evidently were as far as regards all subjects connected with mechanics: he justly, however, considered Euler as a superficial natural philosopher, though an incomparable mathematician.

(337.) As a lecturer in his own department, Robison was obison as the most cminent of his time, at least in Britain. That lecturer. his courses were not considered popular will easily be understood from a slight inspection of his writings. The demonstrations were long and copious, but too rapidly delivered. "The singular felicity of his own apprehension," says Mr Playfair, "made him judge too favourably of the same power in others." The lectures must have abounded in practical details, which ordinary students rarely appreciate; and they were deficient in experiments, which unquestionably arose from no want of the ingenuity necessary either to invent or execute them. On the other hand, the effect of his discourses was greatly enhanced by his striking and encrgetic delivery, and by the stores of his memory, which often recalled the incidents of the stirring life in which he had once been engaged; and to the more thoughtful and philosophic they were rendered at once attractive and elevating in no ordinary degree by the strain of fervent thought by which they were accompanied, and the impress which they frequently bore of the pure morality and exalted piety of their author.

Apart from his local usefulness as a professor, we regard Dr Robison's place in science as eminent chiefly on account of the sagacity with which he applied knowledge to practice, and analysed complicated effects of force as manifested in engineering constructions. This he did so ably as to guide future practice, and to reflect much light on the theory of solids more or less elastic and tenacious, and subject to the intricate strains which gravity produces. The difficulty and merit of these investigations will best be gathered from the slow progress of his successors in the same field. The criticism with which his writings abound on the theories of even his more celebrated contemporaries and predecessors show remarkable acuteness, patience, and independence of thought. We may perhaps sometimes think him prejudiced, but his decisions are never uttered without an elaborate statement of reasons, nor ever sullied by the suggestions of jealousy or self-conceit. $\mathrm{Had}$ he been an accomplished analyst he must have been less distinguished in the equally important walk in which he stood pre-eminent. The limits of human life and faculties prevents universal attainment, but he was surely no. mean philosopher of whom the-sexagenarian James Watt could say, "He was a man of the clearest head and the most science of anybody $I$ have known." 2

Amongst the contemporaries of Robison was one whose acquirements were in many respects very similar to his own and second to no other philosopher of his day, to pro- and passive mote sound views in the very same branches of science. Charles-Augustin Coulomb (born 1736, died 1806) was, like Robison, addicted through life to practical enquiries, and was intimately acquainted with all the details of the civil engineering of his day. To him we owe a correct knowledge of the laws of friction in most ordinary cases, and the right application of them to the theory of machines, and to that of the stability of structures. In a very remarkable paper, published in 1776 , he analysed, from the basis both of theory and experiment, the manner in which columns of masonry give way under longitudinal pressure; not, as had previously been sup. posed, by flexure under the imposed weight, like a steel spring or a rod of deal, but by the sliding of one portion of the column over another, at an angle determined by the cohesion of the stone, and capable of being reduced to a problem of maxima and minima. This important principle is now known to apply to wrought and cast iron, and many other substances.

One of Coulomb's happiest investigations was on the force of torsion, or the resistance of wires to
twisting; which he showed to vary directly with the angle of torsion, inversely as the length of the wire, and directly as the square of its section. These mechanical principles he applied with address to determine the viscosity of fluids, and with still more consummate skill and success to the measurement of electrical and magnetical forces by the construction of a Torsion Balance, similar in principle but anterior to that employed by Michell and Cavendish for estimating the force of gravity.

Coulomb's researches on electricity (which have (341.) been only partially published) will be made the sub- His reject of discussion in another chapter of this Disser- rearches tation; they were the most sustained and elaborate Robison's. of his investigations, and display very considerable mathematical resources. In this respect he had a superiority over Robison, who, as we have seen, cul-

\footnotetext{
1 See Dr Chalmers' Life, vol. i. That great man had a peculiar veneration for Dr Robison, and is understood to have received impressions from attending his lectures which materially influenced his future life.

2 Mechanical Inventions of James Watt, ii., p. 290.
} 
tivated precisely the same branches of science which gave distinction to the career of Coulomb. When we compare the two philosophers, we find that the former was the more discursive reasoner and experimenter, and was diverted, perhaps by the copiousness of his erudition, and the attention with which he studied the works of others, from doing full justice to his own original powers. The latter excelled as a ma- thematician; he concentrated his efforts more methodically, and displayed the results to the world (so far as they were published) in a more consecutive and lucid form. In uprightness of character and high morality, the two philosophers bore a marked resemblance. They were both sufferers from bad health, and they died within about a year of each other, at nearly the same age.

\section{§ 3. Thomas Young-Strength of Materials, and Art of Construction (continued).-Telford -Introduction of Iron into permanent Structures. Suspension Bridges.-Tredgold; Mr Hodgkinson; M. Navier.-Mr Robert StePHeNSON-Tubular Bridges.}

(342.) It is a circumstance not uninstructive as to the Difficulty progress and achievements of science, that the and impor-greatest modern philosopher who preceded Newton the enquiry - Galileo-and one of the most eminent, if not into the the most eminent, of his successors-Young-should mechanical have laboured with minute and practical care, and properties with corresponding success, on a subject apparently of solids. so humble and mechanical as the Strength of Materials, and the Resistance of Beams to fracture. Newton himself condescended to swing pendulums, and to observe the collisions of elastic worsted balls. It is sufficient here to advert to the exceeding interest of enquiries which throw so much light upon the internal constitution of bodies, and in some instances intimately connect them with the laws of vibration of elastic media, to which so much of Modern Physics is intimately allied.

(343.) The eighteenth century was in this, as in so many Progress other departments of science, sluggish and mechaniduring the cal, or else abstract and ultra-geometrical. The century. learned labours of Euler and the Bernouillis on elastic curves, and the strength of pillars, were for the most part elegant mathematical amusements, and with the exception of the experiments of Musschenbroek in the earlier half of the century, and the skilful but more limited researches of Coulomb at its close, little valuable in the way of precise theory or of accurate data derived from practice had been added to this important branch of mechanical engineering.

Robison, indeed, with the peculiar tact and skill Thomas Young. which I have already ascribed to him, wrote several papers (contributed to an early edition of the Encyclopadia Britannica, and printed in his collected works) full of acute observation and reasoning, adapted to the imperfect experiments of his time, and connected by sound scientific deductions, which are still well worthy of careful perusal; but it was to the penetration of Dr Thomas Young, ${ }^{1}$ who partook strongly of Robison's mechanical tastes, whilst he surpassed him in facility of mathematical resource, that we owe a great revision of the doctrine of the strength of materials. In the "Syllabus of Lec- tures" (1802), into which he condensed, in a manner peculiar to himself, an incredible amount of positive knowledge ; in the Lectures themselves (1806), with the admirable "Catalogue of References;" and in the articles on "Bridges," and the supplementary propositions on "Carpentry," which he contributed to this Encyclopædia-we find (stated, as usual, not without some obscurity) a multitude of theorems and problems embracing the whole principles of construction, and based upon meehanical laws and the most probable interpretation of experiments.

The forces tending to alter the figure or dimensions of substances usually called solid may be thus clas- Applicasified: (1.) Extending forces, or such as produce tion of elongation in a body when applied in a direct man-solids.ner. (2.) Compressive forces. (3.) Force produ-Extension cing detrusion, or the slipping of one portion of the substance over another. (4.) Force producing flexure. (5.) Torsion or twisting force. The resistance of bodies to extension was examined by Hooke and Gravesande, and is held to be directly as the area of section of the body, and to increase directly as the amount of elongation produced, at least within certain limits. The measure of this resistance Young termed (not very happily) Modulus of Elasticity, expressing the Modulus force required to produce unit of elongation (or to Elasticity double the length) of a prism of the substance under experiment. This quantity may be measured either by the length of a depending prism of the substance which would produce the requisite strain, or more simply by the strain expressed in pounds or tons, which, supposing the elongations to increase without limit as the extending forces, would double the length of the prism under experiment. Thus, in round numbers, a bar of wrought iron an inch square will be extended $\bar{\Sigma}^{\frac{3}{\delta}} \overline{0}$ part by a pressure of one ton-hence the modulus of elasticity is about 10,000 tons. The elasticity of wrought iron remains perfect to about half the breaking weight, after which the elongations appear to double for each addition of about $\frac{1}{10}$ or $\frac{1}{12}$ of the breaking weight. Thus, in a recent experiment by $\mathrm{Mr}$ Edwin Clark, a bar of 
wrought iron, one inch square and ten feet long, extended I $\frac{8}{8 \sigma \sigma}$ of its length for every ton of weight up to 12 tons, from which point the extensions nearly doubled successively for every two tons of load, and the bar was finally torn asunder by 23 tons.

The compression of bodies proceeds (like the extension) at first uniformly with the load. Some bodies resist compression more than extension (as cast iron); some the reverse (as wrought iron). Substances give way under compression after different fashions. Hard bodies divide into prisms parallel to the compressing force; slender elastic bodies bend laterally; soft bodies bulge horizontally; bodies of a medium hardness divide into wedges, and the surfaces slide along the plane of spontaneous fissure. etrusion. Detrusion marks more particularly the mode of giving way by the sliding of surfaces in the interior of solids. Though seldom due to force directly applied, it is an important element in most cases of the rupture of semiductile solids. (347.) The force of flexure is that by which the resist-
lexure of ance of the greater number of solids is most easily sams. overcome, but which it is of most importance to re. sist; as when a beam is fastened by one end into a wall, and loaded at the other, or when it spans a horizontal space. It had not escaped the notice of James Bernouilli, Duhamel, and other writers of the earlier part of the 17th century, that the fibres on the concave side of a loaded beam are in a state of compression and not of extension, and that there is therefore a point, or rather a line, in every beam, in which the fibres are neither extended nor compressed. But the clear modification of the theory prevalent in the time of Leibnitz and Marriotte which this consideration introduced, was probably first developed by Coulomb, Robison, and Young, who in their respective publications insisted upon it with great judgment; and it is difficult to overrate its importance in mechanical engineering, although the first great canon of Galileo remains still true, that the ultimate strength of a solid rectangular beam varies as the breadth and as the square of the depth. The writings of Young and Robison did not immediately attract the attention of practical men, and Coulomb, who was by far the ablest French experimenter on subjects of mixed mechanics, seems to have done less on the theory of strains producing flexure than in the case of torsion, which he studied with so much success, and applied to such excellent purpose. Nevertheless in his memoir on the Resistance of Masonry, in the 7th vol. of the "Memoires Presentes" (1776), he had already laid down very clearly the effect of compression on a beam. ${ }^{1}$
It is, however, to Young that we owe the application of these principles in unfolding their legitimate consequences. In a series of remarkable propositions contained in the writings I have quoted (344), he assigns numerical relations between the flexure of a beam under almost every supposable circumstance, and the resistance of the material to direct strains. These results have been extensively used by all subsequent writers. They are not equally verified in all classes of substances. This, however, is not wonderful ; flexure is not due to direct compressive and extending strains alone; deformation may take place in a solid without appreciable change of density, thus giving rise to some of the nicest questions in molecular physics.

The laws of Torsion, as laid down by Coulomb, (348.) have been mentioned in the last section (340).

The mathematical investigations of Young on mechani moung's rectness and in defiance of the generalizing methods mechanical and symmetrical notation of foreign writers on such subjects. But his pre-eminent sagacity in laying hold on the salient points of the questions he discussed, and in conducting his argument to a practical conclusion, was unequalled, and deserves imitation. ${ }^{3}$

A great revival in the study of the properties of elastic matter, as regards strength, took place about (350.) the year 1820 , probably in consequence of the in- use of troduction of wrought iron into the construction of ${ }^{\text {wrought }}$ suspension bridges, which has been attended with important results.

Thomas Telford, though neither the contriver of suspension bridges, nor the introducer of them into Telford; Britain,$^{3}$ deserves notice from the superior boldness suspension and solidity of the noblest work of the kind which has yet been executed-the Menai Bridge. Telford (and the same may be said of his contemporary Rennie) was more distinguished as a man of judgment, integrity, and experience, than as eminently original or philosophical. In this respect both yield to Smeaton, who, with Watt, was the founder (each in his own department) of modern engineering. But the beautiful and truly workmanlike structure of the Menai Bridge inaugurated the era of the extensive introduction of that admirable material, wROUGHT IRON, into great permanent structures exposed to heavy strains. Cast iron had been used much earlier, as in the bridge erected at Colebrookdale in 1777 by Mr Derby, and in the very beautiful arch at Sunderland, which dates from 1796. The span of the Menai Bridge is 580 feet, the whole quantity of iron used was 2186 tons, the transverse section of the suspending chains or bars was 260 square inches, supporting a strain of $1094 \frac{1}{2}$ tons. This

1 Reprinted in the Théorie des Machines Simples, Paris, 1821.

2 A copious selection from Young's mechanical writings may be found in his Miscell. Works edited by Dr Peacock, vol. ii.

3 Captain Samuel Brown erected the first considerable chain bridge in this coustry across the Tweed in 1819. 
was a work quite unexampled at the time of its erection (1826), and showed a sagacious confidence in the employment of a material then comparatively little trusted.

(352.)

Therf never made extensive experiments on the Data of re-resistance of solids. Some special ones were indeed sistance. made under his direction on wrought iron in particular, but in general he seems to have relied upon the old ones of Musschenbroek and Buffon. The Tredgold, the old ones of Musschenbroek and Buffon. The Bodgkin- applied themselves to the practical determination son. of the data of resistance so long deficient, were Tredgold, a private engineer, and Professor Barlow of Woolwich. The data they obtained have since been generally used, not only in this, but in other countries. Tredgold's works (on Carpentry, Strength of Timber, \&c.) show a very great aptitude in applying the results of science to practice, and an acquaintance with both which is rarely attained. Mr Eaton Hodgkiuson has made many valuable additions to Tredgold's work, and has contributed an excellent paper on the strength of pillars to the Philosophical Trans actions (1840.)

To Mr Hodgkinson we are also indebted for a (353.) useful investigation (in the Manchester Transacof the tions) into the figure assumed by the chains of suscatenary. pension bridges. The elegant properties of the simple or geometrical catenary were fully investigated a century and a half since by the Bernouillis and by David Gregory, but the application of suspended structures of immense weight to purposes of utility suggested new problems. Amongst these, perhaps the most interesting was the catenary of uniform strength, in which the section of the suspending chains is made everywhere proportional to the strain which they have to resist at that particular point. Its equation was investigated by $\mathrm{Mr} \mathrm{Da}$ vies Gilbert in 1826. An elegant and valuable contribution to the geometry of catenarian curves was made by the late Professor Wallace of Edinburgh, with particular application to curves of equilibration for bridges of masonry after the ingenious manner of Robison mentioned in Art. (334).

In connection with suspension bridges, and also (354.) Navier. with researches on the yielding of elastic materials, we must record the name of M. Navier, a very eminent French engineer and writer on practical and theoretical mechanies. His work on suspension bridges (1823) is one of the earliest and best. $\mathrm{He}$ is also well known for his physico-mathematical researches on the yielding of elastic solids to pressure under given circumstances, in the course of which he came into collision with Poisson, who gave a somewhat different theory. The subject is one of extreme difficulty, owing to our ignorance of the molecular constitution of bodies; and it is believed that all these investigations were so far erroneous that they were based upon the assumption of a single constant to represent the resistance of bodies to change of form and dimension. These (form and dimension) are two very different things, and require distinct treatment. ${ }^{2}$ British mathematicians have lately paid much attention to these enquiries, with the prospect of a solid improvement in engineering theories. ${ }^{3}$

The art of bridging over great spaces has been (355.) pushed, by the requirements of the railway system, to Mr Robe an astonishing extent, and under circumstances of Stephen peculiar difficulty. I shall connect these improvements with the name of Mr RoberT STEphenson, the inventor of the Tubular Bridge, a work which, in its very simplicity, is a triumph of art, and being nothing more than a hollow beam of somewhat peculiar construction, supported at the ends, it is an admirable instance of a structure of which the stability may be easily reduced to calculation.

The wooden bridges of Switzerland were for a long (356.) time unequalled as skilful works of carpentry. During Wooden the last century the Rhine at Schaffhausen was crossed Switzerby two spans of 171 and 193 feet. At Trenton, in land and America, the river Delaware is crossed by a wooden America. bridge, of which one arch is 200 feet in span. It is on the bow principle, an elastic wooden arch, convex upwards, being skilfully braced and united to a level roadway passing through the spring of the arches. The American lattice bridge, very simply and skilfully contrived, has great firmness, owing to the depth of the framing, and exercises no horizontal thrust on the piers. The widest spanned wooden bridge in the world, 340 feet, across the Schuylkill, at Philadelphia, designed by Wernwag, combines the bow and lattice principle.

In these we might see foreshadowed in some faint degree the principle of the TuBULAR BRIDGE, the The tubu greatest discovery in construction of our day. But bridge. in reality the idea of it arose from a different consideration.

During the first ten or fifteen years of railway ex- (358.) perience, engineers had gradually acquired a correct Railway perception of the manner in which cast and wrought bridges. $^{2}$ iron may most effectually and economically be formed

1 Edinburgh Transactions, vol. xiv.

2 About twenty years ago, the present writer showed that India rubber, which possesseg to such a remarkable extent the quality which may be terned cubical flexibility, is yet scarcely at all compressible-in fact, just as much as water, and no more. Though not otherwise published, he has been in the habit of demonstrating this in his annual course of lectures.

3 Professor Stokes in Cambridge Transactions, vol. viii.; Mr Clerk Maxwell in Edinburgh Transactions, vol. $8 x$. Mr M. Rankine in Cambridge and Dublin Math. Journal for 1851 and 1852. Experimental data are still deficient; but M. Wertheim has lately published some valuable ones (which are still in progress) in the Annales de Chimie. 
into girders or beams supported at the ends, and adapted for sustaining enormous loads. Such beams were constructed, often of single castings, so as to include three portions ; an upper flarge, a lower flange, and $a w e b$, or thinner vertical plate connecting the two. The relative section of the upper and lower flange was made to vary with the material. In cast iron, which yields far more easily to tensile than to compressive strains, the lower flange should be almost incomparably greater than the upper; in wrought iron a slight predominance should be given to the upper flange for the converse reason.

(359.) Hence it will be easily understood how, when $\mathrm{Mr}$ The idea of Robert Stephenson was desired to construct a railthe tubular way bridge across the Menai Strait,- - subject to the rived from onerous condition imposed by the Admiralty, that it them. should (even at the abutments) be without lateral struts or diagonal pieces below the roadway,-he should have entertained the idea of a gigantic girder with a top and bottom flange of proportionate extent, with a deep web uniting them, or rather of two such girders placed side by side, thus forming square tubes, of which the lower flanges should constitute the bottom, the upper flanges the top, and the two webs the sides.

(360.) It is not for me in this place to explain how, step Progress of by step, the idea of a tubular bridge of wrought iron the inven- assumed the practical shape, now to be seen at Conway, and near Bangor, in North Wales. It is unfortunately notorious that there has existed an unhappy rivalry as to the share of merit due to the several persons who of necessity were jointly concerned in the completion even of the design of these astonishing works. Unfortunately for Mr Stephenson's tranquillity, the tremendous responsibility of this novel, gigantic, and costly experiment, was thrown upon lim during the very height of the commercial and engineering excitement (not unjustly called mania) which prevailed in 1845 and 1846 , on the subject of railway projects. Instead of the uninterrupted leisure which he required to superintend his preliminary experiments, to consider his plans, and perform his calculations, Mr Stephenson, as well as every other engineer of eminence was at that time engaged all day and a great part of the night in the unparalleled worry of Parliamentary contests. As a matter of course, much was trusted to able, confidential, and highly paid assistants. The experiments on models of different forms, which alone cost many thousand pounds, could not all be conducted in the presence of the chief engineer. Yet he alone was responsible for the failure or success of the plan. (361.) The comparatively great strength of tubes was
the cellu- a fact known from the time of Galileo. Their de-

fect was a liability to crumple or pucker. Round, oval, and rectangular tubes were tried, and the last (Mr Stephenson's original conception) were, as might be supposed, found to be stronger than the other two. When supported at the ends, and loaded in the middle, model tubes of this form invariably gave way at the top. How to strengthen the top against compressive strains was the question. The excessive stiffness of corrugated iron and zinc plates (long previously used for roofs) came to the engineer's assistance. A combination of two longitudinally corrugated or goffered wrought-iron plates running along the top of the model, and forming long and nearly cylindrical cells, was found to give the required stiffness with the least increase of weight. Ultimately a square arrangement of cells was adopted, principally to give facility for painting and repair. The tubes were hindered from racking by means of numerous wrought-iron frames employed to stiffen them, whose section resembled the letter $T$, and which were called $\mathrm{T}$ irons. Suitable diaphragms were also inserted at short distances along the tubes. The widest spaces to be spanned at the Menai Strait were Dimensions 460 feet, there being two intervals of this width, and and two of 230 feet. The tubes are 30 feet high and 14 strength of broad, containing a transverse section of about 1500 the bridge. square inches of wrought iron. The weight of one principal tube is about 1450 tons, and its strength, measured by the breaking load at the centre, above 2300 tons. This last number is calculated from the experiments on the breaking weight of models, one of which was on no less than a sixth of the true scale. The boiler-plates, of which the tubes are composed, are united by mechanical pressure by means of hot rivets; and it may safely be affirmed that without this ingenious and perfect method of combination (which is due to Mr Fairbairn, who had previously obtained a patent for it), the structure would have been impossible.

The success of this astonishing piece of engineering has been complete; the stiffness of the tubes, Its comwhether under constant pressure or during the rapid plete suctransit of trains, is almost incredibly great.

To Mr Robert Stephenson is clearly due the credit of undertaking, on his sole responsibility, a project $\mathrm{Mr}$ Steof equal boldness and novelty, and of contriving, not phenson the perhaps in every detail, but in its totality, the means inventor; by which so signal a triumph of art and of science was carried into effect, an honour to his own age, and a lesson to posterity. To Mr Fairbairn and Mr Hodg-assisted by kinson, his assistants, selected by himself, much praise Messrs is also due for the manner in which the experiments and Hodgwere managed, and the principles established by these kinson. educed. Mr Fairbairn, a practical engineer of Manchester, well known for his experience and sagacity, gave to Mr Stephenson, as a matter of honour, the full benefit of both; and his confidence in the result helped no doubt to sustain the manly courage of his principal amidst a storm of opposition. Mr Hodgkinson, well known for his able enquiries into the strength of pillars and girders of different forms, conducted the mathematical enquiries, and determined the relative strengths of the models. His confidence in the result was less encouraging than that of his coadjutor, which serves to show the greatness 
of the responsibility of the engineer-in-chief. As neither Mr Fairbairn nor Mr Hodgkinson could have incurred any just blame had the vast structure when on the eve of completion doubled up under its own weight, and blocked up, perhaps for ever, the navigation of half the Menai Strait, so neither can they possibly claim more than a subordinate share in the success of the undertaking. ${ }^{1}$

(364.)

Commission on railway bridges.

(365.)

I shall here only refer to the work of Mr Edwin Clark, the resident engineer of the Britannia Bridge, for farther details of its principles and construction, and to the report of a royal commission (published in 1849) on the application of iron to railway structures, for many curious researches connected with the subject of this section. In particular, we find a theoretical and practical solution of the very delicate problem of the influence of the speed of passing loads on the deflection of bridges, to which Professors Willis and Stokes, and ColonelJames,R.E., are contributors.

$\mathrm{Mr}$ Robert Stephenson is the son of Mr George Stephenson, who will be mentioned in a succeeding section. He was born in 1803; educated (in part) at Other the University of Edinburgh, under Leslie, Hope, and works of Jameson; he long occupied the chief position in the Mr Stelocomotive factory established by his father at Newcastle, having in the first place constructed under his direction the celebrated "Rocket" engine which gained the prize at the opening of the Liverpool and Manchester railway. To his own exertions, both before and after that period, the locomotive owes much of its present perfection. He surveyed and principally carried through the London and Birmingham railway, the second grcat line in the kingdom; and he has been engaged in a large proportion of the most remarkable engineering works connected with railways, both in this country and abroad. $\mathrm{He}$ has personally superintended the construction of railways amidst the blowing sands of Egypt, and in Norway with its heavy winter snows and deeply frozen soil. His high personal character, both for skill and integrity, has everywhere procured him the respect and confidence of his profession and of the public.

\section{§ 4. BRUNEL.-Self-acting Machinery.-The Thames Tunnel.-Mr BABBAGE's Calculating Engines.}

(366.)

Marc Isam nel.

(367.) His early history.
Sir Marc Isambart Bruned, born at Hacqueville in Normandy on the 25th April 1769, was one of the most inventive mechanicians and engineers of his day. As his genius gave a strong impression to contemporary art, we associate his name with the progress of civil engineering in the earlier part of the present century, particularly in connection with mechanism. Like most of his eminent coevals in the same profession, he had not the benefit of a scientific education; but he more than most of them supplied its defects by a singular capacity for correct induction and by great mechanical ingenuity. Though a native of France, it was in Great Britain that his talents were to find their full scope, and it became his thoroughly adopted country.

Disgusted by the horrors of the first revolution, he quitted France in 1793 in the capacity of a common sailor, a position far below that which either his birth or his intellect entitled him to hold, yet in which he made himself remarked by his excellent disposition and mental superiority. His destination was New York, where in 1794 he commenced his career as a civil engineer, his boyish tastes having already indicated this as his natural calling. He executted some considerable works, and planned many more; it is stated that he there devised the essential parts of his block machinery. About 1799 he decided on settling in England.

It is probable that his talents and ingenuity alone recommended him to a government employment at a Enployer time when the mere fact of his being a Frenchman lish gomust have acted as a powerful obstacle to his suc- vernmen cess. Those who recollect the vivacity and bright intelligence of even his later years, will understand that in his more active days it must have been difficult to refuse Brunel at least a hearing. And it is to the credit of Lord Spencer, then one of the Lords of the Admiralty, and of General Sir Samuel Bentham, inspector of naval works, that Brunel was engaged in 1802 to superintend the erection of his celebrated block machinery.

The invention of self-acting machinery to supersede the work of artisans was of course not new. The saw-mill and the spinning-jenny were already in

\footnotetext{
I It has been alleged that Mr Stephenson's original proposal to allow the suspension chains (which were primarily intended to be used in putting together the tubes in their final positions) to remain in aid of the rigidity of the structure, manifested a want of confidence in his own great idea. But a dispassionate consideration of his evidence before the committee of the House of Commons would alone clearly show (independent of Mr Stephenson's declarations on the subject) that he was forced into the admission that the chains might give an ulterior guarantee against miscarriage of the whole plan, simply to save the bill from being thrown out by the not unnatural incredulity of those to whom a proposal so new, so gigantic, and affecting the lives of so many persons, as well as so great pecuniary and other interests, was for the first time and suddenly proposed. Besides this, even his own coadjutors did not all entirely support him. Mr Hodgkinson, whose character for scientific knowledge carried great weight with the committee, recommended in bis report the ultimate additional security of chains.
} 
use, but the invention of Brunel was not less important as creating an epoch in art. Not only is it possible to execute in a comparatively short time, and with a prodigious economy, objects such as blocks and pulleys, which are required in vast numbers and precisely alike, but the nicety and accuracy of the manufacture is thereby increased, and owing to the facility with which inanimate force may be concentrated on machinery, works which transcend the power of unaided muscular labour are as surely and exactly executed as those of smaller dimensions. For example, by no enlargement of the common turning-lathe would it be possible to construct an accurately turned iron steam-cylinder 8 feet or more in diameter, which is yet readily executed under the direction of a very ordinary workman by means of steam power and self-acting machinery. (370.) The block-machinery at Portsmouth consists of a
he block- series of engines impelled by steam, and by means of achinery; which thematerials of wood and metal employed in the construction of ships'blocks are reduced to exact forms in graduated sizes, and are finally put together with very little manual labour. These machines, with the exception of the turning-lathe and circular saw, were wholly new, and, it is stated, were devised in part by General Bentham, who gave to Brunel at least the benefit of his advice and previous experiments. In some of them we have the first germ of implements now used by every machine-maker in the kingdom; and the ingenuity of the movements, and the variety of effects produced, earned for this great invention a just celebrity. Such a beginning could not have been made without the aid of government. . To construct the tools was an expensive and troublesome business, and to start the manufactory cost L.53,000, which was speedily saved by the economy of the pro-

cess. In the course of a year 140,000 blocks of no less than 200 different patterns were produced, and the number of workmen was diminished in the proportion of about 11 to 1 . As a reward, Mr Brunel received L.16,000, being two-thirds of the first year's saving, itself a sufficient proof that he was the bona fide inventor of this admirable apparatus, whatever hints he may have received from his immediate superior.

So successful an experinent produced ultimately, though with characteristic slowness, its effect on the mercantile world ; nearly twenty years elapsed before such a splendid example of ingenious economy and artistic precision was at all generally imitated. Yet before his death, Sir Marc Brunel saw the fruit of his ingenuity almost indefinitely multiplied in the workshops of London, Manchester, Glasgow, Newcastle, and Birmingham, and highly appreciated if less extensively imitated abroad.

The more we reflect on the comparative state of the arts now and a century ago, the more we shall find reason to estimate highly the introduction of correct and scientific ideas of machinery and of tools for con- structing other machines and structures. It was, in fact, the necessary complement of the invention of the steam-engine. Watt contrived the mighty Heart which was to give a new impulse to social life, Brunel and others of the same stamp added limbs and muscles, whereby its energies were rendered thoroughly practical. The sixteenth, seventeenth, and part of the eighteenth centuries, had given to the world designs for countless mechanical contrivances, often highly original and ingenious. But many were grounded on fallacies, and others belong to the class of elaborate trifles. At that period the slide-rest of the turning-lathe, the planing machine, and the circular saw, were practically unknown; at least the two former, which are incomparably the most important inventions of their class, and which belong to no certain author, having almost imperceptibly come into use-the slide-rest about the Slide-rest end of the last century, the planing machine as and planlately as about 1820 . The former of these readily ing maforms surfaces of revolution with geometrical accuracy, the latter plane surfaces, and in either case the application to metals is most important. The circular saw and slide-rest form part of Brunel's series of machines, and he afterwards constructed the former on a very great scale for the manufacture of wooden veneers. To them he added the mortising machine, and these, it will be seen (together with the planing engine), form the staple of the magnificent and varied apparatus with which, driven by the gigantic power of steam, our mechanical factories are now so generally provided. We again repeat that the triumphs of art in which our generation glories, our railroads, our locomotives, our crystal palaces, and our steam navies, would have been impossible feats but for the improvement of tools and the substitution of steam for muscular power.

Every one is, however, aware that Brunel owed his reputation to other achievements as well as his im- TheThames provements of mechanical tools. The Thames Tun-Tunnel. nel will ever be considered as his most arduous triumph. It is a structure of exquisite firmness laid in a quicksand. It will endure like the cloace of regal Rome, when the palace and the cathedral have crumbled to dust. Yet here also we perceive that it was Brunel's exquisite mechanical tact and ingenuity which enabled him to succeed. The problem of the tunnel is not one of balancing vaults; the statical conditions of stability are simple enough, and it was not in the solution of such that Brunel peculiarly excelled. The practical problem was to introduce a rigid tube of brick horizontally into the middle of a quaking mass of mud; and the solution was the invention of a tool which should enable men to make the excavation and to proceed with the building in safety. It was the shield which carried the shield. the tunnel under the Thames, - a moveable vertical frame of cast iron, provided with thirty-six cells, in each of which a man was placed with a pick to ex- 
cavate the area required for the construction of the tunnel. By a simple but most ingenious contrivance, every part of the face of unstable clay was firmly supported by boards which leaned upon the frame or shield, which, in its turn, pressed against the part of the brickwork of the tunnel already completed. Each workman could remove one or more of these small boards at pleasure, and excavate a short way into the yielding mass before him, then advance the boards and sustain the slippery face. When the whole face had thus undergone piecemeal excavation, the frame or shield was moved bodily forwards by powerful screws, and the bricklayers brought up the masonry behind, which was then beyond the reach of injury.

(374.) The idea of the shield was derived, it is stated, from Completion a specimen in the arsenal at Chatham, showing the of the tun- operations of a testaceous worm which bores under
nel. water, and which nature has provided with a protective covering. But the analogy is certainly indirect, since water could hardly retard the operations of such an animal. Repeated irruptions of the Thames several times drowned the work, which was as often abandoned and renewed, but every difficulty was met by fresh resources on the part of the engineer. The failure of funds was a far more serious obstacle, and government at last came to the aid of an undertaking of such consummate ingenuity that its completion was deemed due to the honour of the nation. The tunnel was commenced on the 2d March 1825,

Death of and finished 25th March 1843. Brunel survived the Brunel.

(375.)

Variety of his works. completion of his great work above six years, dying on the 12 th December 1849, aged 81 .

We have not in this brief sketch glanced at one half of his ingenious projects and successful enterprizes. Scarcely any branch of his multiform profession but received some improvement at his hand. The discovery of the condensation of several gases in 1823 , by Mr Faraday, suggested to Brunel their application as a moving power; and his want of success did not arise from any deficiency on his part of skill or forethought. He was one of the first to construct a roof of extreme lightness, somewhat resembling those now in use for railway stations. He erected a suspension bridge in the Isle of Bourbon on an original plan; and he pointed out with characteristic shrewdness how much of the stability of arches depends upon the cohesion of the parts, so that the vault may in some cases be entirely dis. pensed with.

It will be understood that we have selected Sir Marc Brunel as the representative of a class, the eminently mechanical engineers, a class now extensively multiplied, and amongst whom his son, $\mathrm{Mr}$ Brunel, occupies an eminent position.
It cannot be expected in an essay like the present that I should enter into the details of the variety of mechanical inventions which have now become so numerous, and which have been marked by every gradation of originality and resource. But as illustrating a class of contrivances altogether different from those of Brunel, though like them tending to produce a great influence on the improvement of the mechanical arts, I will briefly refer to the Calculating Machines of $\mathrm{Mr}$ Babbage, which have at different times excited the interest of the public and of scientific men.

Mr Babbage was a fellow student at Cambridge with Sir John Herschel and Dean Peacock, and along with them he contributed by his writings and personal efforts to introduce into that university the improved Continental mathematics. A few years after leaving college he originated the plan of a machine for calculating tables by means of successive orders of differences, and having received for it in 1822 and the following year the support of the Astronomical and Royal Societies, and a grant of money from government, he proceeded to its execution. I It is believed that Mr Babbage was the first who thought of employing mechanism for computing tables by means of differences; the machine was subsequently termed the difference engine. In the course of his proceedings Mr Babbage invented a mechanical notation (described in the Philosophical Transactions for 1826), intended to show the exact mutual relations of all the parts of any connected machine, however complex, at a given instant of time. He also made himself acquainted with the various machines used in the arts, with the tools used in constructing them, and with the details of the most improved workshops. Employing Mr Clements, a skilful mechanist, a portion of the calculating machine, very beautifully constructed, was brought into working order, and its success so far answered the' expectations of its projector. But, notwithstanding several additional grants from government, the outlay on this most expensive kind of work soon exceeded them. The part actually constructed is now placed in the Museum of King's College, London; it employs numbers of nineteen digits, and effects summations by means of three orders of differences. Though only constituting a smiall part of the intended engine, it involves the principles of the whole. The inventor proposed to connect with it a printing apparatus, so that the engine should not only tabulate the numbers, but also print them beyond almost the possibility of error.

At this stage (1834) Mr Babbage contrived a machine of a far more comprehensive character, which The and he calls the Analytical Engine, extending the planticalenso as to develop algebraic quantities, and to tabu- gine. late the numerical value of complicated functions. when one or more of the variables which they contain are made to alter their values. Had this engine been constructed, it would necessarily have superseded what had already been done. Government were not unnaturally startled by this new proposal, and as about the same time Mr Babbage's relations to 
Mr Clements were broken off, the difficulties of the affair became insurmountable, and the construction of either engine has for some years been in abeyance. The opinions of men of science are not unanimous as to the great practical importance of calculating tables by machinery, but the improvements of mechanical contrivance which the joint skill of $\mathrm{Mr}$ Babbage and Mr Clements introduced into enginecring workshops are unquestionably of great importance to the arts. Though the details of $\mathrm{Mr}$ Babbage's plans have not been published, there can be no doubt that, whether economical or not as substitutions of machinery for human labour, they were devised with remarkable skill and ingenuity, and even on this account inerit preservation. ${ }^{1}$

(380.) Recently (1855) attention has been directed in M.Scheutz' London to a simple and effective Difference Engine engine. constructed and patented by M. Scheutz, confessedly on the principles of $\mathrm{Mr}$ Babbage, though without an acquaintance with his mechanical contrivances. The result is stated to be satisfactory. The engine deals with fifteen digits or figures, and with four orders of differences. Only eight figures are preserved in the result, the others being reserved to prevent errors arising from the accumulation of still lower digits omitted. The engine not only computes with facility and accuracy, but, by means of steel punches impressing lead, provides for the perpetuation of the numbers in the form of stereotyped plates. The workmanship of the whole requires no particular nicety of execution, is not liable to derangement, and can by scarcely any contingency produce inaccurate results.

Before closing this section, we may advert to improvements in the theory of machines by those who Foreign have regarded it rather from the geometrical side writers on than from that of routine practice. Our French of maneighbours have been distinguished in this respect. chines. Carnot and De Prony, MM. Hachette, Poncelet, and Morin, have been or are accomplished mechanists in this respect; and in the French repertories we must look for some of the earliest good scientific descriptions of machinery, even when of English invention.

In England, besides Mr Babbage, Professor Willis of Cambridge has shown a peculiar aptitude in this English department, and has published a very valuable work ${ }^{\text {anthors. }}$ on machinery, regarded in a strictly geometrical sense. ${ }^{2}$ To $\mathrm{Mr}$ Moseley we are likewise indebted for some valuable contributions to the theory of engineering.

\section{§ 5. Trevithick.-George Stephenson.-The Locomotive Steam-Engine.-Rise and Progress of Railways.-M. de Pambour on Locomotives.}

(383.) The locogine and railway.

\section{(384.)}

Of all the inventions which have powerfully affected the interests of mankind, none have been more slowly perfected, or can be less certainly traced to a single individual as the inventor, than those of the Locomotive engine and the Railway. These two great and essentially connected portions of the greatest mechanical and commercial effort of any age or country had their origin in obscurity. Each appeared several times to be rising into the importance it deserved, but failing the concurrence of the fortunate circumstances which are necessary to give permanence to invention, was once more forgotten and was left for re-discovery at a happier epoch.

With regard to Steam-Carriages, passing over still earlier speculations, we find that Dr John Robison, at the age of twenty-one, published a design for a steam-carriage in the Universal Magazine for November 1757 , and that he also directed Mr Watt's attention to the steam-engine in the same year, with a view to this very application. The cylinder of the proposed machine was an inverted one, and Watt actually made a rude model on Robison's suggestion. ${ }^{3}$ From this time the steam-carriage seems never to have been long lost sight of by mechanical speculators. It was included in a patent by one Moore, a linen draper, in $1769 .^{4}$ In the same year it is stated that Cugnot, a native of Lorraine, actually constructed a steam-carriage, which, like the nearly contemporary but unsuccessful efforts of his countrymen to effect steam navigation, fell speedily into oblivion. About 1773 Edgeworth of Edgeworthstown urged the construction of steam-carriages, and at a later period expressed, in terms of unequivocal anticipation, the triumph arising from their connection with railways. "I have always thought," he wrote in 1813 , "that steam would become the universal lord, and that we should in time scorn post-horses. An iron railroad would be a cheaper thing than a road on the common construction." At Soho the movement of carriages as well as of boats by steam never was or could be forgotten. In Watt's patent of 1784 the steam-Watt and carriage forms the seventh article, and in the same Murdoch, carriage forms the seventh article, and in the same and Watt's establishment, made a model, acting by high-pressure steam, which drove a small waggon round the room. Hence it required no prophetic power in Darwin, the intimate friend of Watt, to

1 For historical details connected with Mr Babbage's engine, see Weld's History of the Royal Society, vol. ii. An account of the principles and action of the Difference Engine may be found in the Edinburgh Review for July 1834; and those of the Analytical Engine in Taylor's Scientific Memoirs, vol. iii.

2 Principles of Mechanism. Camb., 1841.

Mechanical Inventions of James Watt, i. 52. 
write those often quoted lines in the Botanic Garden (canto i. line 290) :-

"Soon shall thine arm, unconquered Steam, afar Drag the slow barge, or drive the rapid car."

(385.) A somewhat longer pause now occurs. But in Trevithick 1802 we find Richard Trevithick, a Cornish "cap-

and Vi-
vian; their tain " of a mine, taking out a patent along with Vipatent in vian for the high-pressure steam-engine, and apply1802. ing it specifically and practically to the movement of carriages or waggons along a railway at Merthyr Tydvil in South Wales. Mr Muirhead informs us that Trevithick saw Murdoch's model at Redruth in Cornwall. But admitting this, it is plain that the idea was much older still, and also that many years elapsed without its ever being brought practically to bear until the year 1804, when Trevithick's locomotive was actually used.

(386.) Richard Trevithick appears to have been one of Account of the most ingenious men of his time; but (from the Trevithick. scanty notices which I have been able to collect ${ }^{1}$ ) to have been also of an inconstant speculative disposition, which prevented him from bringing any of his numerous inventions to perfection. Yet he had the good fortune, which so many inventors have missed, of meeting with partners able and willing to assist him in carrying out his designs. Amongst these was Andrew Vivian, with whom in 1802 he took out the patent already mentioned for the construction of highpressure engines, and their application to the movement of carriages along rails or common roads. As

Applies high-pressure steam to locomo tives on it, as well as the ingenious invention of the four-way railways in cock, from an old scheme of Leupold's, but he over1804. came for the time the prejudice which had always existed even in the mind of Watt against its adoption. His earliest engine is stated to have been connected with a common stage coach which ran on the streets of London; but his more successful and important effort was made in dragging waggons along the Merthyr Tydvil railway in South Wales, which was successfully tried on the 21st February 1804, when the engine drew carriages containing ten tons of bar iron for a distance of nine miles at the rate of five miles an hour. This was unquestionably the first successful example of this modern species of locomotion.

(387.) If we look more closely at the means by which it Account of was accomplished, we find still more reason to com-
bis engine. mend the sagacity of the inventor. and to wonder at the interval of nearly thirty years which elapsed before the general adoption of his plan. "A square iron case containing the boiler and cylinder was placed behind the large or hinder wheels of the carriage, and was attached to a frame supported from the axles of those wheels. The cylinder was in a horizontal position, and the piston-rod was projected backwards and forwards in the line of the road towards the front of the carriage. Across the square frame, supported by the wheels of the carriage, an axle was extended reaching a little beyond the frame on each side; this axle was cranked in the middle, in a line with the centre of the cylinder, and a connecting-rod passing from the end of the piston turned this axle round, and produced a continued rotatory motion of it when the piston was moved backwards and forwards in the cylinder; upon both ends of this axle cog-wheels were fixed, which worked into similar cog-wheels upon the axles of the wheels of the carriages, so that when a rotatory motion was produced in the cranked axle by the piston-rod it was communicated to the axle of the larger or hinder wheels of the carriages, and these wheels being fixed upon and turning round with the axle, gave a progessive motion to the carriage. Upon one end of the axle was fixed a flywheel to secure a rotatory motion in the axle at the termination of each stroke." ${ }^{2}$

We here find the cranked axle and the horizontal cylinder of modern locomotives, both of which were departed from by Trevithick himself, probably in consequence of difficulties of execution. When we add to this plain description, that the fly-wheel was furnished with a break, that the boiler had a safetyvalve or a fusible plug beyond the reach of the engineer, and that the patent includes the production of " a more equable rotatory motion, .... by causing the piston-rods of two cylinders to work on the said axis by means of cranks at a quarter of a turn asunder," it is scarcely too much to say that nothing material was added to the design of the Locomotive until the invention of the tubular boiler in 1829.

The Merthyr locomotive blew up, and the preju- (389.) dice against high-pressure steam revived. The in- Treviventor, in the meantime, diverted his attention to thick's en other schemes, and continued his profession as a plodes. Cornish mining engineer.

A singular circumstance opened for Trevithick a new sphere. A Spanish-American gentleman named His subse Uvillé, who was engaged in working the silver mines reer and of Peru, came to England in 1811, with a view to disappoin discover an engine fit for draining those mines whose ment. high elevation rendered condensing steam-engines working under atmospheric pressure comparatively inert. In London he met accidentally with a model of Trevithick's engine, and having carried it to the heights of Pasco in Peru, and being satisfied with its work, he did not rest until he had returned to England and transported nine high-pressure engines in 1814 to the scene of operations. In 1816 Trevithick himself followed, with coining engines and purifying furnaces of his own contrivance. Had he

\footnotetext{
1 It is stated that the Society of Civil Engineers have in vain proposed a medal for a biography of Trevithick.

2 Wood on Railways. This description corresponds with Trevithick's specification and drawings.-Repertory of Arts, \&c., Sccond Series, vol, iv.
} 
been a prudent man his fortune was now made; but it is stated that about 1827 he returned to this country impoverished and disappointed. I am unacquainted with his further history.

In the meantime the locomotive engine, which Trevithick had long abandoned to its fate, was becoming known in the hands of a man perhaps of less genius but of greater sagacity and perseverance.

George Stephenson, civil engineer, was born in 1780 near Newcastle, of respectable persons in the humblest rank of life. His father was either a common pitman or otherwise employed about the collieries of the district, and young Stephenson, without any advantages of education, began to labour for his bread at an early age. His work appears to have been always connected with the machinery of the pits above ground, and not with their excavation. Thus he rose gradually to be an engine-man at the wages Ilis early of twelve shillings a week. This was at Killingworth difficulties, near Newcastle, where he showed considerable mechanical ingenuity, and gradually gained the confidence of his employers. Having married in 1802, he had a son born the following year, the present Mr Robert Stephenson, M.P., whom he brought up with the tenderest care, and whom he ever and justly regarded with a father's pride. In order to bestow upon him the advantage of that education of which he had himself felt the want, it is stated that he made money at extra hours by mending his neighbours' clocks and watches, and finally, in more prosperous days, sent his son to complete his education at the University of Edinburgh. George Stephenson never acquired much book-learning himself, but by natural sagacity and observation he attained to a sound knowledge of mechanical principles. We do not claim for him, however, the character of great inventiveness. His skill rather lay in perceiving how far methods and contrivances already known might be pushed to an advantageous result. He possessed that shrewd decision which ingenious persons often want, enabling him to detect what is truly valuable in the numerous mechanical schemes which at any time are afloat, and to devise the means of realizing them. He also possessed that confidence in his own judgment which is necessary to carry out principles to their legitimate extent, but from which feebler or less practical minds usually shrink.

Not to interrupt the principal topic of this section, I will here only mention that in 1815 he set about inventing a safety lamp for mines at a time when the recent heavy loss of life in his own neighbourhood had excited general attention; insomuch that Sir $\mathrm{H}$. Davy had been specially invited, by a meeting of persons interested, to propose a remedy. I shall in another place speak of the result; but in the meantime I may state, that George Stephenson made some experiments of his own, which, leading him in the same track which Davy followed, that of admitting the foul air to the lamp through long narrow tubes, might in the end have led him to a construction analogous to that of the safety lamp. As matters stood, it is not surprising that his efforts, though highly meritorious, led him slowly and uncertainly towards the goal which Davy, having once sighted, arrived at with that rapid instinct in which he has never been surpassed. Stephenson was left behind, but was rewarded by a handsome gift offered by his local admirers, who, in doing so, naturally rather considered the difficulties overcome by their humble neighbour than the strictly comparative merit of the two inventions.

But it is of the locomotive and of the railway that we have here to speak.

The former, we have seen, had been brought to considerable perfection by Trevithick. An engine on his plan had been constructed and used by Mr Blackett of Wylam in Northumberland, near the place where Stephenson resided, and was the basis of his improvements. Blackett's engine had two cylinders, an addition often ascribed to Stephenson, but which, as we have said, was included in Trevithick's patent. What he saw of the performance of this machine appears to have convinced Stephenson, once for all, of the groundlessness of an opinion which then and for long after haunted the minds of railway engineers. This opinion was, that the adhesion between the wheels of a locomotive engine and the smooth iron surfaces of the rails must be insufficient to allow the impulsion of the train, at least with any degree of velocity, or up the smallest inclination. Trevithick dismisses had a scheme for increasing the adhesion, and this the fear of ideal improvement was the subject of repeated pa-insufficient tents, some of a singular nature, between 1802 and ${ }_{\text {the rails. }}^{\text {adhesion to }}$ 1824, one of which, Blenkinsop's, provided a cogwheel in the engine working into a rack on the rail, which was actually in use down at least to 1830 . It is rather a singular thing that men spurning theories, as was the fashion of the engineers of that day, and especially those of Smeaton's school, should have thought as little of an appeal to experiment on so simple a matter as did the followers of Aristotle in the seventeenth century, when Galileo offered to convince them that light and heavy bodies fall equally fast. Forgetting that direct friction is always large, and that it varies in proportion to the pressure, these practical men could not get over their first impression, that iron must slide on iron long before a heavy train could be set in motion. It was characteristic of Stephenson's decision of character, that he dismissed all doubts on the subject so soon as his observations seemed distinct, and that he did not hesitate to carry out his belief to its consequences, and to maintain his confidence in the locomotive engine against all antagonists.

I shall not stop to particularize Stephenson's first (396.) improvements on the locomotive, which were rather G. Stephen in detail than in principle. He saw clearly all along son's im. that if it was to work at high speeds he must in every ments. 
possible way diminish the vibrations and strains to which it was subject, and which would otherwise rapidly wear out the machinery. For this purpose he proposed to connect the engine with its carriage by means of steam acting on six pistons in lieu of springs. But perhaps his most material improvement consisted in the very simple one of throwing the waste The steam. high-pressure steam as a blast into the chimney, blast. which was found to increase enormously the force of the fire, and the evaporating power of the boiler. Engines having this improvement, and with two vertical cylinders, as constructed in 1818, are, or were lately (1854), still atwork on the Killingworth railway dragging coals at the rate of five or six miles an hour.

(397.) One of Stephenson's clear practical opinions was Rejects the this, - that the locomotive and the railway are part steam-car- of one mechanism, and must be adapted to one anriage on roads.

(398.)

Origin of railways.

other. He was not a friend to steam-carriages on common roads, and the event proved his sagacity.

If the idea of a locomotive belongs to no one man, still less does that of a railway, which being one of the most elementary of mechanical contrivances, may be traced, under some modifications, almost indefinitely backwards, as a means of conveying heavy loads with facility. Hence it was at first confined chiefly to quarries and collieries, especially in underground passages or drifts. The gauge of these subterranean railways, or tram ways, was only about 18 inches. The material of the rails was first wood, then cast iron, finally wrought iron, as being less liable to wear and to accident. The wrought iron rail, though not absolutely new, was first generally introduced in 1820. About the same time, or rather sooner, the rails began to be made plain, that is, without any vertical guide or flange to prevent the wheels of the carriages from leaving the rail, and the flange was transferred to the wheels of the locomotive. Even this was not new, for it had been used by Jessop in 1789. The weight of the rails has been constantly on the increase. The original castiron rails weighed only $15 \mathrm{lb}$. a yard; the malleableiron rail in 1821 weighed about $28 \mathrm{lb}$., then 35 , afterwards 64 , and now rails of $80 \mathrm{lb}$. a yard are generally used.

(399.) One of Stephenson's first cares was to make his Stephenson railways solid and level, and to prevent jerks at the adsem to the junction of the rails. The gauge he adopted, or the locomotive. interval between the rails (now generally used, except on the Great Western Railway and its branches), was $4 \mathrm{ft} .8 \frac{1}{2}$ inches, and was derived from the accidental width of the parent railways in Northumberland. Like Watt and all other innovators, his great diffculty was to get the machinery of his locomotives properly made, and the great railway movement of 1825 was anticipated by the establishment in 1820 of an engine factory at Newcastle, which, till after the opening of the Liverpool and Manchester Railway in 1831, remained the only one, and for long afterwards the best of its class. 'The cranked axle contrived by Trevithick, and abandoned because it could not be properly welded, was now restored; the heavy locomotive was placed on strong but easy steel springs, wrought iron was skilfully introduced into the wheels of the carriages, and the whole machinery was made to work with precision, and to combine a degree of resistance never before anticipated with comparative lightness. The factory was established in 1821, and the first passenger locomotive was started on the Darlington and Stockton Railway in 1825.

I ought, perhaps, to apologize for these details, but they illustrate so well the exceedingly gradual progress of mechanical invention, that $I$ have thought them worthy of montion here. ${ }^{I}$ The subsequent history of the locomotive and the railway is more generally known. From the date of 1825 , both grew and flourished; the railway first and most steadily; the locomotive was introduced more cautiously, and met with much opposition; its triumph was almost entirely due to the steadiness of George Stephenson.

The year 1825, so fertile in speculation, produced (401.) a series of projects for railways to an extent not com-Railway monly known, since few of them came into existence ${ }^{\text {specula- }}$ or were even commenced for many years later. The 1825 . projected capital of these companies amounted to not less than L. $30,000,000$ or L. $40,000,000$. But the only considerable undertaking which was at that time seriously supported was the railway from Liverpool to Manchester, and on that battle-field were fought the great questions of the superiority of railways to common roads, - of high to low velocitios of transport, - and of locomotives to fixed engines.

On these three important points, George Stephenson (402) the pract commercial men, who had entire confidence in his roads. sagacity, he had to maintain the conflict almost singlehanded against general and professional prejudice. With respect to the Railway, he had long decided in his own mind against the use of steam-carriages on common roads. This conclusion was scientifically based on his own experiments on the friction of waggons on railways made in conjunction with $\mathrm{Mr}$ Nicholas Wood, civil engineer at Newcastle, as far back as the years 1815 and 1816. A simple dynamometer Stephenof Stephenson's invention was used, and by means of son's expe it the two fundamental propositions were established, riments on that the friction is directly as the pressure, and that it of trains. is quite independent of velocity (at leastwhen the speed was moderate). It may be said that these propositions were already known; but, besides that probably Stephenson and Wood were equally unacquainted with the writings of Coulomb, they could not have

1 I have found many curious details of the early history of railways in a series of articles on the life of George Stephenson in the Civil Engineer Journal for 1848 and 1849. I am indebted to Mr Robert Stephenson, M.P., for many interesting particulars respecting his father's inventions. 
dispensed with verifying his results under circurnstances so peculiar as those of a railway and a train of carriages. The necessity of doing so was manifested by the opposition, and even ridicule, with which the idea of friction being in this case independent of velocity was received, showing, as has been correctly observed, "how small was the amount of science at that time blended with engineering practice." The friction of even the indifferent railways of those days. amounted to only $10 \mathrm{lb}$. per ton of load ; consequently an incline of only 1 foot in 100 would increase by one-half the resistance to the motion of a carriage on a railway. Hence Stephenson determined to construct railways having only the smallest inclinations, and to use fixed engines for higher slopes. Witb respect to common roads, he showed by powdering even a level railway with sand, that the most powerful locomotives then in use speedily came to rest; this, with the previous objection, overruled in his mind the possibility of advantage in that case.

With low gradients and small resistances, together with the proved invariability of friction with speed, there necessarily came into Stephenson's mind the practicability of using high velocities. At a very early period (1816) he spoke in one of his patents of conveying goods " at nearly double the rate at which they were then usually carried along railways," in other words, at 10 or 12 miles an hour, and this he states " with no hesitation, speaking from experiments already made," referring, no doubt, to those on friction made along with Wood about this time. Yet after nine years of farther experience, his old coadjutor Wood deserted him on this grand point, and in the first edition of his book on Railways (1825, p. 290) he disclaims the "ridiculous expectation" that locomotives will be seen to travel at " 12,16 , 18 , or 20 niles an hour," and scorns " the promulgation of such nonsense." Even in 1829 he reported to Messrs Walker and Rastrick, who were referees on the Liverpool and Manchester Railway, "that no locomotive engine should travel more than 8 miles an hour." If at this comparatively late period perhaps the most practised railway engineer in England held these opinions, and that with the full knowledge of his friend Stephenson's matured convictions (which it is difficult to believe were not pointed at in this paragraph), we may imagine the opposition which the plans of the latter were likely to meet with from interested or even indifferent persons.

At last a company was formed, and funds provided to construct the Liverpool and Manchester Railway. It is unnecessary to state how successfully Stephenson conquered the engineering difficulties of the line, and refuted the predicted impossibility of crossing the Chat Moss. In every respect this railway became a model for those which succeeded, and in essentials very little has been added by 25 years' experience on lines of the same gauge. But now came the struggle as to how this beautiful road was to be worked; - with horses, - by means of fixed engines, -or by locomotives. It was not without a struggle that Stephenson gained his point. Even in 1829 the prejudices of the engineering profession were still strong against the locomotive. And it is curious to read in the contemporary documents with what distrust they were regarded. The clumsy expedient of a series of stationary engines $1 \frac{1}{2}$ miles apart, dragging the trains by ropes, would probably have been adopted to the disgrace of the age, but for the energy of Stephenson and his commercial friends. A competition The locoof locomotives was at last agreed to, which took motive place on October 6,1829 , on a level piece of rail- competiway at Rainhill near Liverpool. Though the makers 1829 at of engines had their energies hampered by various Rainhill. needless conditions (particularly as regards the weight of the engines, under the mistaken notion, that velocity could only be combined with lightness), several excellent engines appeared; but the " Rocket" made at Stephenson's factory at Newcastle, not only gained the prize, but far exceeded in its performances the limits assigned in the programme. It weighed $4 \frac{1}{4}$ tons, and dragged a gross load of 17 tons, at the rate of 15 miles an hour, but moved itself with a velocity of 35 miles an hour. The "Norelty" of Messrs Braithwaite and Ericson was also very successful. The prize was awarded to Stephenson, and this success was mainly due to the admirable invention of the multi-tubular boiler, imagined by $\mathrm{Mr}$ Booth, and carried out by Stephenson. To distribute the water of the boiler in tubes, and allow the heat of the furnace to act around them, was an idea as old as the time of Watt, but it did not succeed. To carry The multithe hot air of the furnace through tubes surrounded tubular by water, was the more successful arrangement of boiler. Booth and Stephenson, to the right working of which the draught occasioned by the steam-blast in the chimney was essential. The idea, it is said, had occurred both in France and America, but it certainly remained practically inefficient, perhaps on account of the want of draught.

This invention, by increasing almost without limit (405.) the evaporating power of the boiler, which is the Stephenkey to the efficiency of a locomotive, completed for cess, the time the skilful improvements on locomotives and railways, which, as has been seen, we owe mainly to Stephenson. The comparatively trifling ameliorations which have occurred in either, and the stereotyped character of even the minor arrangements, such as those of stations and of passenger carriages, show how much the sagacity of the engineer had anticipated the accommodation of the public. ${ }^{1}$

I here close my account of Mr Stephenson and of and lis con.

${ }^{1}$ I do not overlook of course the modifications introduced in the broad-gauge system of the Great Western Railway. Mr Brunel indeed tried to show how far he could deviate without positive injury from Stephenson's plans; in some points, perhars, he did so with advantage, yet, on the whole, the resuits do not shake Stephenson's position as the commanding engineer of his time. 
the railway system. The locomotive engine will ever remain as an invention entirely distinct from those created by the genius of Watt. It is in reality independent of his great principle of separate condensation. It has a power of adaptation and a perfection of performance more astonishing than any contrivance of our time, except perhaps the electric telegraph. In 1552 , Jerome Cardan took twenty-three days to travel from London to Edinburgh; that the same should be done 300 years later in eleven hours, and every day, is a fact as striking as any which the progress of science presents. Whilst to Trevithick and George Stephenson we are mainly indebted for these results, the theory of the steam-engine is still in ar- rear. To M. de Pambour we are indebted ${ }^{1}$ for by M. de Pam far the best account of the elements of force contained bour on th in the locomotive, and of the resistances which they theory of have to overcome; but it is not to be doubted that tives. much yet remains to be done in this direction.

George Stephenson died 12th August 1848, at the Ge of sixty (407.) age of sixty-eight, and generally respected for his Stephen private character as well as for his talents. His son dies in had the honour of completing the second great railway work in Britain, from London to Birmingham, and by the invention of the tubular bridge, described in a previous section, he has added the most important, as well as the most scientific auxiliary to the extension of railways since 1830 .

\section{§ 6. Hydrodynamics, Dubdat, Venturi, Professor Stokes.-Friction and Resistance of Fluids. MM. Weber, Mr Scott RUssell.-Propagation of Waves. Infuence on Canal Naviga- tion. MM. Fourneyron and Poncelet.-Improved Hydraulic Machines; Turbine. Reference to the subject of Capillary Attraction.}

(408.) There are few subjects less adapted for the kind Progress of of discussion which I have adopted in this DissertaHydrody- tion than Hydrodynamics, whether in its abstract or namics. practical form. Not only is it difficult to fix upon individuals who, in the more recent progress of the subject, have attached themselves to it in so especial a manner as to have impressed the science with the individual character of their own minds, but the progress made in either branch has been of a remarkably fragmentary kind, usually bearing upon the solution of individual problems, and tending to the improvement of particular machines. It must be owned, too, that however important in practice may be the efficiency of a water-wheel or the discharge of a pipe, the solution of such problems does not present the attractive interest which attaches to many less difficult ones in Natural Philosophy. As regards more general problems of which a direct solution from first principles might appear possible, it has been found that these are as yet so limited as to give a character (which is generally admitted) of great barrenness to the theoretical investigations.

While, then, I shall endeavour, in conformity with my general plan, to preserve something of the biographical character in the history, suppressing details and many minor and even some important steps, this section will necessarily have somewhat of a fragmentary character, and its deficiencies must be supplied by a reference to the numerous articles of the Encyclopædia which treat more or less fully of these subjects and their history.
1. Friction and Resistance of Fluids.-The first name I shall mention is that of the Chevalier DuBUAT, who has the signal merit of having attributed due importance to, and of having considered with much sagacity, the effects of the friction of fluids against their own particles, and against the sides of the solid bodies used to confine them. He had the full advantage of an acquaintance with the Abbe Bossut's excellent experiments and judicious writings, but he was, I believe, the first who succeeded in ascribing to the different forces which act on fluids in a state of uniform motion their effective share in determining their velocity. I refer particularly to the discharge of pipes and of rivers. Du-Theory of buat showed that when the motion of water in such rivers. circumstances becomes uniform, the accelerating force which acts is the measure of the total resistances to the fluid motion, whether arising from the inequalities of the bed or the viscosity of the fluid. These resistances are assumed to be proportional to the square of the velocity. Since they are exactly in proportion to the length of the pipe or channel, and since the moving pressure increases in the same proportion, the velocity is independent of the length of the pipe, whilst the inclination remains the same-a simple result not previously noticed. In the case of a pipe the head or superincumbent pressure may be divided into two parts; one, requisite to force the water into the tube with the requisite velocity, which is independent of the distance to be travelled; the other, which balances the resistance due to the length of the pipe, which for a given diameter varies as the length, or if the slope be constant is independent of the length.

The relation of the area of the stream to the peri- (411.) meter or rubbing surface of the channel is then taken Mean hy. into account. This ratio is called by some writers the depth. mean hydraulic depth. The manner in which Dubuat derives his formula (which I shall not here set down) from direct experiment, guided by a few general notions of theory, is a very good specimen of this kind 
of induction, without any pretence whatever of proceeding upon the mathematical theory of fluids in motion. The same views were ably expounded and enlarged by Dr Robison in his excellent article on Rivers and Resistance in this Encyclopredia, through which principally the theory of Dubuat became known in this country. Indeed, viewed apartfrom the technical value of the enquiry, the research as to the natural economy of rivers is a wonderful and striking branch of terrestrial physics, and one which had long afforded a subject of anxious and perplexing enquiries to Italian engineers from the time of Leonardo da Vinci, though comparatively little studied elsewhere. The collected treatises of Italian authors form an important body of hydraulic information. ${ }^{1}$ The rivers of the north of Italy, like those of Holland, conveying vast masses of water charged with mud under very feeble slopes to the sea, present a formidable difficulty which compels attention, - - vast territories being increasingly subject to inundation, as the beds of the rivers are raised by deposition above the general level of the soil.

Dubuat's formulas have been modified, and made to represent experiments better, by subsequent writers, particularly by De Prony, Langsdorf, Eytelwein, and Thomas Young; and the discharge from mere orifices has been discussed by numerous authors who have given empirical co-efficients to represent the phenomena. But little has been added to a philosophical view of the real laws which govern the fluid motion in this case. Nevertheless Savart and Magnus have made some ingenious observations on the constituent parts of effiuent streams.

The viscosity or imperfect fluidity of water is the property most difficult to be taken into account in these and other hydraulic problems. It is that which causes the velocity of a stream to diminish from the surface to the bottom, and from the centre to the sides ; these proportions were also sought by Dubuat. Each layer of water in motion exerts a dragging or "tangential force" upon other layers, which from any cause are comparatively quiescent; and the experiments of VENTURI about the end of the last century, showing that a stream in motion draws towards it the particles of still water with which it may be in contact, with a force sufficient to overcome considerable hydrostatic pressure, attracted much attention. About the same time Coulomb, with his usual address, made experiments on the friction of fluids by observing the rapidity with which cylinders oscillating by means of torsion in different fluids had their briginal motion destroyed.

(414.) Intimately connected with the friction, and conseThree cases quent mutual action of fluids, is the resistance which sistance to they offer to the passage of solid bodies through them,

${ }^{1}$ Raccolta' di Autori che trattano del moto dell' acque, 10 vols., 1822-26; and Nuova Raccolta, 7 vols., $1823-45$. See also the admirable methodized catalogue of writers on Hydraulics in the second volume of Young's Lectures on Natural Philosophy.

2 Vol. ix. part ii.-On the effect of the internal friction of Fluids on the motion of Pendulums.

3 On the friction of Fluids in motion. Camb. Trans., vol. viii., part iii.

and this favourite problem of mathematicians was treated by Dubuat, in his inductive way, in an able and practical manner. Three cases may be specified on account of their theoretical or practical importance :- 1 st, In the case of a body oscillating like a pendulum, with small velocities, the body being immersed in a resisting medium; $2 d l y$, The resistance to vessels floating on and propelled through water; $3 d l y$, The resistance of the air to projectiles whose velocity is very great.

Under the first head Dubuat made those ingenious (415.) motice, notice, which I have mentioned in the section on the pendulums. Pendulum in the chapter on Astronomy, Art. (246). The cause of the neglect of these striking and original observations has probably been correctly stated, by saying that in them the pendulum was made subservient to hydraulic experiments, and not the theory of fluids to the improved use of the pendulum; and they were therefore overlooked by those whose studies were connected with the pendulum and its applications. The fact observed by Dubuat was, that a large mass of air (or of water in the corresponding case) is carried along with a pendulum in motion, and affects in a sensible manner the time of vibration, quite independent of the diminution of gravity due to the buoyancy of the pendulum. The moved mass of air was proved by hanging a film of worsted from an arm a foot long in advance of the moving sphere, when it was found to be but slightly driven by the inertia of the air through which the pendulum moved. Dubuat significantly calls the mass of moved air " the prow" of the moving body, and it is easy to anticipate the sort of effect which such a graduated condition of the surrounding air from motion to absolute rest would produce.

But the most surprising thing is, that mathematicians should have attempted to compute the effect, Professor or should have been in any degree successful in doing Stokes's soso; yet after the preliminary efforts of Poisson and fines the Green, Professor STokes has introduced for the first index of time a correct definition of the "index of friction" of friction. a fluid, and after great labour has succeeded in finding exact expressions for the motions of a solid sphere and cylinder. This investigation may be found in a very elaborate paper in the Cambridge Transactions, ${ }^{2}$ in which he solves the equations found by him in a previous paper, ${ }^{3}$ in the cases of pendulums having the forms just mentioned. Another interesting result of his investigation is the immense effect of fluid friction in retarding the fall of minute rain drops, which he states to be such as to explain satisfactorily the suspension of clouds. In the second part of the paper I have first cited Mr Stokes proceeds to compare his theory with the observations on the pen- 
dulum mentioned in Art. (246), and the agreement appears satisfactory. With reference to Coulomb's experiments on oscillating disks there remains some doubt whether the theory applies satisfactorily to viscid fluids such as oil.

(417.) On the very difficult and still empirical subject of Naval ar- naval architecture as regards forms of least resistchitecture. ance, I shall not here speak; but one very interesting case of the resistance of fluids in canals will be noticed in the course of this section-Arts. (422), \&c.

(418.)

Resisted

motion of

projectiles.

The theory of military projectiles has perhaps received no improvement so considerable during the last 100 years as by the previous experiments of Robins, and the invention of the Ballistic Pendulum referred to in the preceding Dissertation; though the experiments of Borda in France, and of Hutton in this country, have of course increased the technical precision of artillery. Poisson has considered, in a mathematical way, some of the simpler cases of projectiles moving with a moderate velocity.

II. Experiments on Waves.-MM. WEBER-Mr
Russell.-Whilst the theory of the dilated and compressed waves which constitute sonorous vibrations in elastic fluids was being successfully investigated by Lagrange and Laplace, the case of waves in water, due to a disturbance of hydrostatic pressure only, was attacked by the same mathematicians with far less success. It is generally allowed that the more recent and abstruse researches of Poisson and $\mathbf{M}$. Cauchy, though very valuable as improvements in pure mathematics, have been also singularly barren of valuable results admitting of the very desirable confirmation of direct experiment. The greatest of all hydrodynamical problems, that of the Tides, is, as has been seen in the second section of the chapter on Physical Astronomy, Art. (69) \&cc., so excessively complex as to be the last, instead of the first, which analysis might have been expected to resolve. When, on the other hand, we see the light which has been thrown even upon it by experiments on a comparatively small scale, we learn to value them in proportion to their rarity.

The brothers ERNST HeINRICHWEBER and WILHELM WEBER, known for many ingenious experiments in physics, and particularly in magnetism, are the authors of a useful book on waves. ${ }^{1}$ They made experiments on the velocity of waves in glass troughs; by means of which they determined in several cases the velocity of the wave with different depths of fluid; they also ascertained mechanically the form of the wave, which they found to be that of the curve of sines. But the most important of the Webers' experiments is probably the determination of the motion of individual particles of the water, which they ascertained by watching from the exterior of the glass trough the curve described by minute floating particles as the wave passed over them. The usual form of the trajec- Trajectory tory is an ellipse having the greater axis horizontal, the of a parwhole ellipse being described when the wave includes a ridge followed by a depression of the surface; but if there be only an elevated wave propagated, then a semi-ellipse is described in the direction in which the wave moves, and the particle returns to rest in a new position; if the wave be a hollow one the converse takes place. The limits of oscillation diminish with the depth of the fluid, particularly the vertical limits; consequently at some depth the motion of the particles is nearly in a short straight line. The rapidity of the degradation of the individual motions depends on the relation between the length of a wave compared to the depth of the fluid: where the wave is very short compared to the depth of fluid, the ellipses near the surface become circles, and the motion rapidly disappears beneath; when the length of the wave is great compared to the depth of fluid, the motion of the particles is nearly the same from top to bottom. The conclusions of theory are on the whole confirmed by these experiments.

The experiments of Mr John Scott Russell, so far as we shall notice them, refer to two closely connected subjects:- the transmission of waves, and the resistance of water to ressels propelled through ments on it as affected by waves. They are principally to be waves. found in the Edinburgh Transactions, vol. xiv., and the British Association Report for 1837. The velocity of waves in troughs of different depths and sections was ascertained by allowing the wave to be reflected at either end of the trongh, which does not in any way affect the time of its propagation, and gives great facility for accurate observation, as the distance travelled over may thus become large, at the same time that all casual and interfering waves are gradually eliminated. The instant of the passage of the wave was ascertained by reflecting the light of a candle vertically upwards from its horizontal summit. The length of the trough was 20 feet, but since the wave was made to traverse it as often as 60 times, a distance of 1200 feet was really observed. The waves were single or "solitary" waves, either "positive" produced by a gush of water from behind a sluice, or "negative" by withdrawing water suddenly. Mathematicians seem not agreed as to whether or not the "solitary" waves of Mr Russell are to be treated as a species apart. ${ }^{2}$ Unquestionably it has long been known that a partial wave either positive or negative may be propagated without any farther disturbance of the fluid. Theory also shows that the velocity of a wave long in proportion to the depth of the water is nearly as the square root of the depth; but it does not clearly ap-

\footnotetext{
1 Wellenlehre auf experimente gegrïndet, oder über die Wellen tropfbarer Flüssigkeiten mit anwendung auf die Schall und Lichtwellen. Leipzig, 1825.

2 Compare Mr Airy in the article "Tides and Waves," Encycl. Metropolitana; Prof. Stokes in British Assoc. Report for 1846, and $\mathrm{Mr}$ Earnshaw in Cambridge Trans., vol, viii.
} 
pear whether the disturbed or undisturbed depth is Compared to betaken. The analysis of Mr Airy seems to show with theory that a depth somewhat greater than that due to the land and utmost effect of disturbance is to be preferred. $\mathrm{Mr}$ land and utmost effect of disturbance is to be propell also made experiments on the propagation of waves in channels of different forms of section. Professor Kelland has given a very simple expression for the velocity of the wave in this case, ${ }^{1}$ which on the whole agrees with experiment.

(422.) Sometime about the year 1830, attention was Peculiar drawn to a singular fact connected with the resisteffect of
wave trans-ance of water in the case of canal navigation. It mission on was first noticed I believe in Scotland, probably on canal navi- the Forth and Clyde Canal. It amounted to this, gation. that whereas at moderate or rather slow velocities, the resistance to a boat increases with the square of the velocity,-after a certain point, not differing very much from 7 miles an hour, the resistances not only cease to increase according to the same rapid law, but actually diminish to some extent when the. speed is greater. Different experiments were made by the canal proprietors with a view to meet in some degree the active competition by railways, then commencing. Mr Russell was employed by the directors of the Forth and Clyde Canal, and to his experiments we now refer. It appears from his tables that the resistances increased on the whole faster than the squares of the velocities up to $7 \frac{1}{2}$ miles an hour, when they suddenly diminished between $7 \frac{1}{2}$ and $8 \frac{1}{2}$ miles an hour by one-fifth part in one experiment, and by no less than one-third in another. ${ }^{2}$ It was not until about 12 miles an hour, that the resistance reached the same amount as at $7 \frac{1}{2}$ miles.

The occurrence of this singular transition was attended with a phenomenon easily noticeable. In every ordinary case a boat in a canal drives a wave before it, which is in fact a heap of water resisting the boat by increasing the pressure against its bows, which wave may be called a forced wave, having this peculiarity that it travels with the speed of the boat and never quits it; whilst a free wave, by whatever cause excited, is propagated at a rate depending only on the dimensions of the canal, particularly its depth. Now the diminished resistance takes place when the boat is by the force of traction partly drawn out of the water, and lifted up upon the wave to which its own motion gives rise. It is said to ride upon the wave, and the head of water pressing against its bows is visibly diminished. The most advantageous rate of transport was found to be about one-third greater than that required merely to mount the wave, which last depends principally on the depth of the canal. Thus on three different canals, $3 \frac{1}{2}, 5 \frac{1}{4}$, and 9 feet deep, the most advantageous velocities were 8,11 , and $15 \frac{1}{2}$ miles an hour. The actual velocities of the free wave were ascertained by Mr Russell in an in- genious and satisfactory manner. When the boat is dragged to most advantage, the draught of water is less at the stem and stern than in the centre. All these circumstances have been very ingeniously and satisfactorily explained by Mr Airy in his paper on Tides and Waves, articles (404-411).

Many persons (amongst whom are Colonel Henry Beaufoy, Mr Scott Russell, and the American (424.) builders) have bestowed much attention on the forms ships.

of vessels for ensuring speed, especially by the avoidance of waves of various kinds generated by steamvessels in motion. Every one who can compare the performance of such vessels during the last twenty year's, and the still surface which waters navigated by steam vessels now present, as if they were merely cut open and closed again before and after the passage of the ship, instead of being tossed into dangerous billows consuming uselessly the propelling force, will readily admit that, however imperfect the theory, practical art has made real progress in this direction.

III. Improved Hydraulic Machines-Turbine.Before concluding this section, I will refer to the Improved most considerable improvement made of late years bydraulic in the application of hydraulic pressure to motive machinepurposes, and I shall couple it with the names bine.

of two French engineers, MM. Fourneyron and Ponceler, the former the inventor of the Turbine (the machine referred to), at least in its improved practical form; the latter an important contributor to the useful application of hydraulics, an accomplished mathematician, and the author of several standard works connected with industrial mechanics.

The defects of common vertical water-wheels, whether overshot or undershot, are so great and Defects of so notorious, that only their simplicity, and the common fact that in very many cases water-power costs wheelsnext to nothing, and may be squandered with im-Barker's punity, could justify their use. The advantage of mill. using the simple pressure of a fluid as a moving power had been foreseen in that application of re-action called Barker's Mill; which, though well known in models, was seldom if ever applied in practice. Mathematicians were, however, aware that it offered important advantages. Of late years a patent has been taken out in Scotland for a modification of it, which is found, I believe, to work well.' But the Turbine or horizontal water-wheel imagined by Burdin and Fourneyron, and brought to a high state of perfection by the latter about the year 1833, appears to exhaust all that is valuable in this mode of applying water.

Referring to other parts of the Encyclopædia for the details, I may here explain generally that Fourneythe Turbine consists of two parts, one a fixed cy- ron's turlinder or drum of small height compared to its diameter; the other a portion of a cylinder exterior to the former, and moveable round it, so that

1 Telocity $=\sqrt{g_{b}}$; where $A$ is the area of section of the canal, $b$ the breadth of the water at the surface, and $g$ the accelerating effect of gravity. Edinburgh Trans. vol. xiv.

2 E'din. Trans., vol. xiv., p. 48. 
the inner surface of the moveable cylinder is all but in contact with the circumference of the fixed cylinder. Further, water under a greater or less hydrostatic pressure, and moving with the consequent velocity, is introduced at the centre of the fixed cylinder, and conveyed to its circumference by channels of a peculiar form constructed by means of vertical partitions or guides of continuous curvature, from which the water is discharged against float boards within the external cylinder, which are also curved, but the curvature is turned the other way so that the partitions in the first cylinder are where they terminate nearly perpendicular to the internal surface of the moveable or second cylinder where they commence. These latter partitions or float-boards terminate exteriorly in a direction nearly tangential to the outer cylindric surface where the water ennerges.

(428.)

Principle of greatest advantage in waterwheels.

(429.) Power of adaptation of the turbine.
The hydraulic principle of greatest possible advantage to which these Turbines are made as nearly as possible to approximate is this, that the water shall enter the moveable apparatus without shock, and shall quit it without velocity, being simply left behind by the wheel as it escapes from it. Since both these conditions cannot absolutely be fulfilled, the first part of the condition is left imperfect. Some secrecy is, I believe, still maintained as to the forms and dimensions of these machines; but their actual efficiency has been most thoroughly tested by means of De Prony's Friction Dynamometer ${ }^{1}$ by Colonel Morin, a most competent authority. His experiments leave no doubt of the admirable qualities of these machines. In particular, the useful effect compared to the theoretical effect represented by the fall of water expended rises higher than probably in any other hydraulic machine, being under favourable circumstances about eighty per cent. Now water-wheels moved principally by the shock of the fall seldom, in the most advantageous conditions, realize thirty-five per cent., often not seven per cent. This superiority of action of the Turbine is due entirely to the approximation which it gives to the theoretic condition above mentioned of perfect efficiency.

But what is not less striking in the performance of this machine, is the variety of circumstances under which it acts advantageously; however great may be the variation in the size and velocity of the wheel, the height of fall, and the power disposable. Turbines have been made of as small diameter as 2 feet with the enormous fall of 350 fect, making 2300 revolutions per minute. They work with nearly equal advantage (relatively to the power expended) whether the supply of water be small or great. They may be completely drowned or buried under water to a considerable depth without any sensible variation in their efficiency, thus preventing any inconvenience from floods.

It is remarkable that, with these manifest advantages, the Turbine has been so sparingly introduced at least in this country. No doubt the first establishment of it is attended with considerable expense.

M. Poncelet, an active and intelligent officer of (431.) Gérie, and member of the Institut, is favourably M. Ponceknown by his hydraulic observations and inventions, let-efflux as well as by his skilful investigation of the effects breastof machines, and his excellent works and memoirs wheels. on several subjects. He has investigated with much patience and geometrical nicety the form and discharges of spouting fluids, and was one of the first to improve materially the ordinary water-wheels, by introducing a kind of breast-wheel (which thirty-five years ago was scarcely known in France) in which the water is conveyed without shock into compartments on the descending side, from which again it was allowed to escape with all its acquired velocity spent, or nearly so. The efficiency of these wheels is equal to about two-thirds of the power expended. Before the Turbine had been finally improved by $M$. Fourneyron, M.,Poucelet had invented an engine on the same principle, in which the water enters at the circumference of the horizontal wheel, and is delivered at the centre.

I am aware how imperfect this section remains as a history of Hydrodynamics. But I must again Capillary refer to special articles on the subject, the plan of attraction the Dissertation not admitting of farther detail. As - Young nothing material has been added to the doctrine of place. Capillary Attraction since the publications alluded to in Sir John Leslie's Dissertation (although M. Poisson has written a treatise on the subject), I will for the sake of compression not enlarge upon it. I do so with the less regret, because I cannot regard the excessive mathematical illustration which it has received as altogether justified by the certainty and due appreciation of the physical principles involved, such as can alone give to applied mathematics their distinctive value. The theory of Laplace, so far as it was based on novel grounds, was anticipated by Dr Young, and gave rise to several controversial articles by that most eminent philosopher, of which an account will be found in Dr Peacock's Life of Young, pages 199-210, as well as a most excellent review of the subject of Capillary Attraction, which, indeed, by its candour and completeness, supersedes anything which I should have felt disposed to say on the subject.

\footnotetext{
1 Gaspard de Prony, born in 1755, was an eminent engineer, especially in the department of hydraulics, and the author of a voluninous work entitled Nouvelle Architecture Hydraulique; but his originality was not great enough to authorize his being placed among the leaders of his age. His simple invention of the Frein Dynamometrique, or friction dynamometer, is the one by which perhaps he will be longest remembered. It consists of an iron collar with tightening screw, which may be clasped on a horizontal wooden arbor connected with uniformly revolving machinery. A lever, to which a weight may be applied, is attached so as to form part of the collar. The clasping screw being moderately tightened, the collar and lever are of course carried round by the machinery until a weight is applied sufficient to check the velocity, and to generate an amount of friction which is in fact the useful effect of the machine for that velocity, and which is determined by the momentum of the weight overcome in one second. De Prony was a most amiable man, and died in 1839 , in the 84 th year of his age.
} 


\section{§ 7. Progress of Acoustics. Chladni-Savart. Laplace's Correction of the Theory of Sound. Vibrating Plates and Acoustic Figures. Cagniard de la Tour's "Sirène."}

(433.) The mathematical theory of the propagation of Mathema- sound, considered as a branch of analytical mechatical theory nics, made far greater progress during the eighteenth of the pro- century, in harmony with the general character of of sound. the science of that period, than the inductive doctrines of acoustics. Newton here, as in other departments, overstepping the limits of knowledge of his day, left a legacy of toil to his immediate successors. Lagrange had the most distinguished good fortune in reducing the theory of aërial tremors under their most general conditions to the laws of mechanics by the calculus of partial differentials ; and Laplace supplied the link which was wanting to reconcile the result with the known mechanical properties of air. As the former of these matters belongs more properly to the period of the previous Dissertation, and as the beautiful discovery of Laplace has been more especially touched upon by Sir John Leslie, it will be sufficient here to recall the fact that the spring of air, or the effort by which it tends to reexpand under sudden compression or to contract to its former bulk when suddenly dilated, is increased by the heat extricated in the former case, as well as by its absorption in the latter. And as sonorous pulsations are held to consist of a series of compressed and rarified waves whose velocity is affected by the recoil of air, it appears certain that the velocity must be increased by this circumstance, though it is difficult to determine experimentally the exact amount.

The revival of experimental acoustics is due to Ernst Chladni a native of Saxony, but of Hungarian extraction, born in 1756. Little had been done in this department since the time of Sauveur, who ascertained the nature of the harmonic vibrations of strings, and that of Daniel Bernouilli, who considered the analogous case of organ pipes. We are indebted to Chladni for two classes of original experimentshis measure of the velocity of sound in a variety of bodies by peculiar and ingenious methods; and his observations on the vibrations of plates by means of the ingenious expedient of strewing them with sand and other powders.

We shall say a few words under each of these heads :-

I. Chladni observed the velocity of sound in air a flute of metal" which was sounded by means of the elastic fluid required, and the resulting note enabled him to determine by an easy calculation the speed of propagation of the tremor. This method (using an organ pipe) has been more lately resorted to by Dulong for the purpose of deducing the properties

of different gases with respect to heat, by ascertaining from experiment the co-efficient in Laplace's correction for the velocity of sound. Chladni was also probably the first to notice the longitudinal oscillations of strings and rods which always yield a note immensely sharper than the lateral vibrations. By means of the former he ascertained the velocity of sound in a variety of woods and metals, in some of which it is no less than seventeen times greater than in air. These observations are not only interesting in themselves, but as throwing light on the constitution of solid bodies. To Chaldni we likewise owe a knowledge of the twisting vibrations of rods, which exhausts the modes of vibration of such bodies. To connect theoretically the periods of these different kinds of movement, has been a favourite problem with recent mathematicians, but has not even yet been quite successfully performed.

The determination of the velocity of sound in water, an experiment by no means difficult, was re- In water. served for MM. Colladon and Sturm, who observed it on the Lake of Geneva, and found it to be 4708 English feet per second, a result closely conforming to the theoretical amount deduced from Oersted's observation on the compressibility of water.

II. But Chladni's experiments on the vibrations of plates are of still greater interest and originality. Though it has been affirmed that Galileo strewed the vibraThough it has been affrmed that Galileo strewed tion of sand or light substances upon the sounding boards plates. of musical instruments, ${ }^{1}$ Chladni deserves the entire credit of rendering this an exact method of ascertaining the nodal lines or points of rest in bodies vibrating in a stable or permanent manner. He first applied it to plates round, square, or of different figures, supported horizontally and caused to vibrate by applying a violin bow to their edges. Dust or fine sand strewed or sifted uniformly over such a plate, arranges itself in a variety of beautiful figures, being Acoustic tilted from the greater part of the surface, and heaped figures. up on those parts which are at rest in consequence of the vibratory motion of adjacent parts taking place simultaneously in opposite directions; just as the nodal points of a string vibrating harmonically are without motion on the same account. The number and variety of figures thus producible in the same plate is very great, and corresponds, as Chladni clearly saw, to different simple harmonical vibrations of the elastic plate, being accompanied by their appropriate notes; or by the superposition of several such modes of vibration, and of the corresponding sounds. The tract published by him in $1787 \mathrm{en-}$ tituled, Entdeckungen über die Theorie des Klanges, contains numerous figures of these appearances, which, 
more recently, Savart found the means of preserving by transferring them to sheets of gummed paper. There are few experiments in physics of a more striking character, or which so plainly reveal minute and complex motions so small and so rapid as to be difficultly appreciated otherwise. Mr Faraday and $\mathrm{Mr}$ Wheatstone have pursued the same enquiry experimentally, and the latter has satisfactorily deduced the figures of Chladni's square plates from the mechanical superposition of simple modes of vibration which are symmetrical and isochronous. (Phil. Trans. 1833.) By so doing he has succeeded better than the mathematicians, whose results on this subject have been very little practical.

(438.)

In bodies possessing unequal elasticity.

(439.) Chaldni on meteoric bodies.

(440.)

Young-

Robison.

(441.)

Savart.

Propagation of sound in masses of घir, \&.c.
Chladni was the first to make experiments on the vibrations of bodies whose elasticity varies in different directions. Thus he cut plates out of different kinds of wood, and found the nodal curves unsymmetrical in different directions depending on the course of the fibres. The experiments were naturally afterwards employed to illustrate the theory of ellipsoidal waves on the undulatory hypothesis of Optics.

The experiments of Chladni procured for him the especial notice of Napoleon, by whose wish one of his works was translated into French. He died in 1827 , and besides his acoustical discoveries, he will be remembered by the sagacity and boldness with which he maintained the popular opinion of the fall of heavy meteors from the sky, contrary to the prevalent scepticism of philosophers. Chaldni's success in establishing this important fact in natural history is due, like his other physical inductions, to the constancy and simple-mindedness with which he attached himself to a strictly definite enquiry.

We must not here enlarge upon the ingenious and important investigations of Dr Thomas Young connected with acoustics. Being chiefly connected with his admirable Theory of Light, they will be noticed in the chapter on Optics. The peculiarly practical and sagacious views of Dr Robison connected with the Theory of Musical Instruments and Acoustics generally, must also be passed over.

In Feltx Savart we find a man like Chladni who was especially devoted to a single and circumscribed branch of science-acoustics. His published researches are almost all detached notices in the Annales de Chimie, with a few memoirs in the publications of the Institute; and whilst they are very interesting, exact, and instructive, I doubt whether it would be possible to place the results in a connected and comprehensive view before the reader. They are therefore rather to be sought in the special articles of the Encyclopædia devoted to the subject. In general they may be stated to refer to the following topics:-(1.) To the manner of propagation of sound through the air and through liquids, with an attempt to explore the manner in which sounds spread in apartments of different forms; an enquiry as difficult as it is important; (2.) To the generation and audibility of musical notes. Previous authors (for example, On the pro Chladni, Biot, and Wollaston) having differed mate- duction of rially as to the range of audibility of repeated equi- musical distant impressions which affect the human ear as musical notes, Savart used a simple method, no doubt original to him, but anticipated I believe by Robison, in which a card is held near or touching a revolving wheel, and the number of impulses (each double) given to the air by every tooth as it passes the card, is readily measured. He thus found that a note occasioned by 24,000 double vibrations in a second is perfectly audible; and, at the other limit of the musical scale, from seven to eight equidistant beats constitutes a sound having a distinct pitch. According to Savart, two consecutive double impulses of whatever duration are sufficient to convey to the ear the sensation of pitch. But a more elegant and accurate instrument for the numeration of sonorous pulsations is the Sirène Sirène of of M. CAGNIARD DE LA Tour, unquestionably one of M. Cagnithe most exact and satisfactory additions lately made ard de la to our experimental apparatus. In it a current of air is repeatedly interrupted and renewed, giving rise to a series of impulses similar to those of the toothed wheel; and this apparatus is ingeniously contrived, so as to maintain its own motion, and record its indications. It is by far the most accurate known method of ascertaining the pitch of a given note. It may also be worked with water. Robison had also the merit of the primary idea of the Sirene, by making a stopcock revolve rapidly whilst applied to a tube emitting a blast of air. (3.) Savart extended the researches of Chladni by means Savart on of sand to many new cases, and with interesting the vibra results; in particular he exhibited the effects of the solids. unequal mechanical elasticity of crystals cut in different directions. He has also examined with great care and ingenuity, the nature of the vibrations which occasion the accumulations of sand on the nodal lines of plates, and he comes to the conclusion that they are determined by simultaneous transverse and longitudinal movements (the latter of which are parallel to the surface of the plate). In proof of this he shows that in long rods or hollow cylinders, the position of the nodes is intermediate and opposed upon the contrary sides of the rod or hollow cylinder. Savart made many experiments on the communication of vibrations from one body to another; showing that the molecular movements generally preserve their parallelism, so that a longitudinal vibration of one body may give rise to transversal movements in another; and he applies this to the theory of musical instruments.

Savart was born at Mezières in the department of Ardennes, on the 30th June 1791 ; and died some- Biographiwhat prematurely on the 16 th March 1840 . He cal notice had some peculiarities of temper, amongst which was his unconquerable prejudice to everything English. He did not even acknowledge the intimation 
of his election as a foreign member of the Royal Society of London. It is to be regretted that Savart never published a connected account of his observations. He had caused to be collected at the Collège de France, where he was professor, an unequalled collection of acoustical apparatus, a great deal of which was contrived by himself, and where he delivered extensive courses of lectures on this subject alone.
Several English philosophers, in particular Professor Willis, Mr Hopkins, and Mr Wheatstone, have written several important detached memoirs on par- acoustical ticular practical points for which we must special treatises. ${ }^{1}$ The first and last of these gentlemen, together with some Germans, have approximated in some degree to the formation (on empirical principles) of a speaking machine.

\section{CHAPTER V.}

\section{O P T I C S.}

\section{§ 1. Thomas Young.-The Undulatory Theory of Light.-Its history from the time of Hooke and Huygens.-The Law of Interference.-Its application to Diffraction-to the Rainbow -and to other subjects.-The Theory of Polarization referred to another section.}

(444.) mall pro gress of optics in the 18th ventury.

The history of Optics in the eighteenth century is one of the blankest pages of scientific story; at least if we allow Bradley's discovery of aberration to be (as it really is) rather an astronomical than an optical discovery. The most notable advance was unquestionably the invention of the achromatic telescope as narrated in the Fifth Dissertation, ${ }^{2}$ founded on the proof of Newton's oversight in the matter of dispersion. The construction of refracting telescopes made rapid advancement in the workshop of Dollond, whilst reflecting telescopes, in the hands first of Short, but far more conspicuously, of Sir William Herschel, were shown to be capable of making unimagined discoveries. The geometrical theory of optical instruments was also greatly improved; but all this led to little increased knowledge concerning light itself. If we except the valuable though imperfect treatises of Bouguer and Lambert on the subject of photometry, and a paper by $\mathrm{Mr}$ (now Lord) Brougham in the last years of the century, recalling attention to the inflexion of light, the history of Physical Optics (as that part of the science touching more immediately the nature and qualities of light is now usually termed) is almost a blank from the publication of the Optics of Nervton in 1704 to that of Young's papers almost one hundred years later.

It is not therefore from overlooking Young's predecessors that we open our review of the recent progress of optics with his discoveries. We here meet with a man altogether beyond the common standard, one in whom natural endowment and sedulous cultivation rivalled each other in the production of a true philosopher; nor do we hesitate to state our belief that since Newton, Thomas Young stands unrivalled in the annals of British science.

$\mathrm{He}$ was born at Milverton in Somersetshire on the 13th June 1773, and his biographers dwell with com- His early placency on the prodigies of his youth, uncertain as education such attainments confessedly are in stamping the and attaingreatness of the future character. At the age of fourteen he had learned (principally for amusement) seven languages besides his own, and besides had made a point of mastering every subject, whether in science or miscellaneous knowledge, which he had once determined upon prosecuting. Thus, whilst studying botany he resolved to learn how to make a microscope, but finding in Martin's Opties the notation of fluxions, he becaine his own preceptor in that branch of analysis. "He acquired a great facility in writing Latin. He composed Greek verses which stood the test of the criticism of the first scholars of the day, and read a good deal of the higher mathematics. His amusements were the studies of botany and zoology, and to entomology, in particular, he at that time paid great attention." 3 Dr Young's education was almost completely private. Having been brought up according to the tenets of the Society of Friends, he had not thought of going to Cambridge

1 See also Herschel on Sound (Encyc. Metrop.); and Whewell's History of the Inductive Sciences, vol. ii.

2 It may be mentioned, however, that the credit usually ascribed to Dollond must be divided, at least, with Mr Hall, a private gentleman of Worcestershire, who not only imagined but constructed achromatic telescopes as early as 1733 (Gentleman's Magazine, 1790, and Phil. Mag., vol. ii.) The improvement by Dr Blair of Edinburgh has been alluded to in Sir John Leslie's Dissertation. It consisted in enclosing fluids in the object giass, of such composition as to disperse the several rays of the spectrum in the same proportion to one another (though not to the same absolute amount) ag the glass with which it was combined; thus rendering the achromatism more perfect.

3 Memoir of the Life of Thomas Young, M.D. 8vo, 1831. The present section of the Dissertation was written shortly before the publication of the Life and Miscellaneous Works of Dr Thomas Young, for which the public is indebted to Dr Peacock, Dean of Ely, the possession of which would have materially facilitated my task. Wherever Dr Peacock's information has 
Residence (which would have been his natural destination), and at Edin- entered the university of Edinburgh as a medical stuburgh; dent at the age of twenty-one. He had already declined the overtures of such distinguished patrons as Windham and Burke, resolving to devote himself to the pursuit of science, for which a medical education seemed to him a fit entrance; - his studies being made under the more immediate advice of his uncle, Dr Brocklesby. He attended Black's lectures in Edinburgh; whether he was known to Robison I am not aware, though I should be inclined to infer that he was from the terms in which Robison speaks of Young when criticising his strictures upon Smith's Harmonics. Robison disagreed with him on this point, and also about the nature of light, yet he speaks of Young and of his paper on Sound with very marked respect. More than a year before his enrolment at Edinburgh (which took place in autumn 1794), he communicated to the Royal Society of London a paper on vision, of which we shall presently give some further account; and was elected a fellow of the Society when just of age.

(447.) From Edinburgh he proceeded to Göttingen where at Göttin. he graduated; acquiring the German language, and gen and at leaving a vivid impression of his astonishing versabridge. tility of talent and powers of memory. Early in 1797 he returned to England, and soon after entered himself at Emmanuel College, Cambridge, in order to comply with the requisitions of the London College of Physicians, and thus to obtain a license to practice. For the next few years his time was divided much between Cambridge and London. $\mathrm{He}_{e}$ was now twenty-three years of age, and his mental habits too much formed to bend to the rules of Cambridge study. When the master of his college introduced him to the fellows, he is reported to have said, "I have brought you a pupil qualified to read lectures to his tutors," and such, no doubt, was the fact. We hear little of his occupations at Cambridge, but we can hardly doubt that his private studies then ranged over the vast fields of erudition which he afterwards proved that he had made so completely his own; and we cannot doubt that he was then preparing the groundwork of his theory of optics, although his discovery of interference was certainly not made at Cambridge, and probably in London after his settlement there in $1800 .^{1}$ His first paper on sound and light is dated from Cambridge in July 1799.

I have entered into these details because they (448.) Character throw light on the peculiarities of Young's chavestiga- racter and attainments. He was to a great degree tions. self-educated; and his studies in consequence may be called desultory, though none would dare to call them superficial. Mathematicians may consider his acquaintance with their science as not technically complete, yet one of them admits that " he could make a small amount of mathematics go farther than any one else." Had he been a consummate analyst it is unlikely that we should have had in him the author of the undulatory theory, the difficulties of which in its earlier stages made it unpalatable to Laplace, Poisson, and the most considerable French mathematicians. Having thought out for himself every one of the multifarious subjects with which he grappled, his writings have a striking force and originality, and his reports of the labours of others are almost invariably drawn fiom a study of their original works. His earliest principle was, that what one man has done another may accomplish; and one of the many respects in which he resembled his great predecessor Newton, was unbounded confidence in the powers of "patient thought." Not that heconfined the desire to excel to purely intellectual matters. What he found it worth while to do at all, he thought it worth doing well. He chose to be first-rate in dancing and in equitation;-his penmanship was (in his early days) as scrupulously elegant as his scholarship.

In 1801 Young was appointed Professor of Natural Philosophy at the Royal Institution, where he was the colleague of Davy. Young had not the qualifi- Lectures or cations of a popular lecturer, and the most important Philosophy result of his short connection of two years with the Royal Institution was the publication in 1807 of his Lectures on Natural Philosophy, in two large quarto volumes. It is a work peculiarly characteristic of the author; and is rather adapted for reference by the scholar, than to be studied as an elementary treatise. Its condensation is such as to render it in many places obscure; though when read by one conversant with the subject, its comprehensiveness and precision are surprising. It is a truly admirable monument of labour and genius combined. Embracing the arts as well as the whole of natural philosophy, it seems to include the mention of everything connected with his vast subject from the simplest tool of the artisan to the highest speculations of Newton and Lagrange; and yet it is evident, by the masterly manner in which he handles it, that the author had made all this mass of knowledge completely his own. The catalogue of references with which it closes indicates an extent of bibliographical research which would have done honour to any one who had made that an exclusive object of study. Even the plates are drawn with a studious care, betokening well his own mechanical talent.

enabled me to improve the text I have not hesitated to use it. The facility of consultation afforded by the collection of Dr Young's widely scattered writings is a most important aid to all future students of science, and one which cannot fail to raise still higher the great reputation of their author.

1 See Sect. vii. of the article Polarization in this Encyclopædia, where, in a bracketted interpolation by the translator, Dr Young, he speaks of this fundamental experiment being made "in the room and at the table on which he is now writing." This must have been in Welbeck Street, London. 
Many of the figures, though of hackneyed subjects, are represented in a novel manner; there seems not a line drawn at random. Such figures often illustrate better than pages of description, the clearness with which an author has conceived to himself the necessary results of his own principles. An example of this may be found in Plate XXX., fig. 442 of the first volume, which, as Arago relates, served to demonstrate to him, when he visited $\mathrm{Dr}$ Young in 1816, that he and Fresnel had been anticipated on one point more than they believed. ${ }^{1}$

The absence of algebraic formulæ from this work was as characteristic of Dr Young as their copious introduction into articles which he subsequently contributed to the Quarterly Review. He had decided upon writing a book without symbols, and he wrote it, though it gave additional trouble both to himself and the reader.

We shall now proceed to trace the progress of the undulatory theory of light, the greatest physicomathematical discovery of our time, in the establishment of which Young acted the leading part.

Undulations of Light-Hooke and Huygens.The idea of accounting for the effects and modifications of luminous impressions by disturbances propagated through a very elastic medium was by no means new at the commencement of the present century. We do not, indeed, attach much importance to the so-called anticipations of Grimaldi and Hooke in the seventeenth century. The former, amongst his valuable experiments on the deflection of light and fringes of shadows, had used an expression as to illumination being diminished by the addition of light, which is true in fact, and is a correct deduction from the law of interference as we now understand it. Hooke, in his Micrographia, asserts light to consist in "quick, short, vibrating motion ;" but his explanation of refraction by it is altogether erroneous; and his application of it to the doctrine of the colours of thin plates, though admitted by the candid Young to be an anticipation (unknown to him at the time) of his own, has in it no more than a germ of truth (like so many of Hooke's ingenious hints, afterwards claimed by him as discoveries), which yet only explains the fact on which it is founded by means of an additional and gratuitous assumption. The germ of truth in Hooke's writings is this, that the colours in question depend upon a mixture of the light reflected at the first surface of the thin plate with "a kind of fainter ray" propagated from the second surface backwards: the gratuitous assumption is, that " this compound or duplicated pulse does produce on the retina the sensation of a yellow ;" why it does so is not explained. This was in 1664 , before even Newton was acquainted with the analysis of white light. Hooke's idea that yellow, or any other colour, was the result of the conflict of pulses simultaneously reaching the eye, was an assertion, admissible, perhaps, at that time, as expressing a fact; but surely not a proof of interference producing reinforcement or annihilation of light, as taught by Young. I an not aware that Hooke ever even reiterated his opinions on this subject after Newton had analysed the phenomena experimentally, and shown that the colours of thin plates result from the superposition of bright and dark rings of different prismatic hues, each with its appropriate diameter. It was then apparent that colour was only an indirect effect of interference.

But whatever may be thought of the theories of Hooke, those of Huygens deserve a far more eminent Huygens place in history. Having already been succinctly ad- Traité de la verted to in Professor Playfair's dissertation, we will only observe that the Traité de la Lumière (1690) is an admirably composed and reasoned treatise on the phenomena of light on the undulatory hypothesis. The uniformity of its propagation through the celestial spaces, its rectilinear course in ordinary circumstances, the laws of its reflection and refraction, are there explained with a degree of elegance and precision which ought to have excited (we must think) general attention and assent, but for the ascendancy of Newton's authority, and the astonishing and beautiful nature of the experiments on which his theories were based; whereas Huygens referred to few experiments except those of the simplest kind, and the phenomena of colour were (for good reasons) left chiefly out of view. ${ }^{2}$ Such being the case, Huygens may fairly be considered as the author of the undulatory theory, which he supported by such convincing proofs.

The fundamental principle of the Huygenian (454.) doctrine was the same as that which Hooke ad- Fixplanamitted, which was probably far older than his time, tion of namely, that all space, including the interior of double retransparent bodies, contains an ether whose pulsa-fraction. tions communicate the sense of light to the eye, as waves in air convey to the ear impressions of sound. To this he added the assumption, that in refracting media, such as glass, the pulsations are retarded; whereas in Newton's theory, as is well known, the propagation of light is assumed to be fastest in dense media. The "law of the sines" in refraction, is deduced as a consequence; and one of the prettiestapplications made of it is to the phenomena of atmospherical refraction. But the most important features of the whole investigation are these two-(1), the

\footnotetext{
1 The reference of Arago, in his original éloge of Young, is to p. 387 of the Lectures, obviously by mistake. In the first volume of his (Arago's) collected works it is corrected into p. 787, which is as certainly correct. I have supposed fig. 442 to be more probably the one referred to than 445, to which Dr Peacock refers (Life of Young, p. 389).

2 Nevertheless the Huygenian Theory of Light was propounded as the subject of a thesis at St Andrews by David Gregory, whilst professor of matbematics there, witbin about a year of its publication (including also the Newtonian doctrine of gravity), -a pleasing proof of the activity which then reigned in that university. Principal Lee possesses the original progranım.
} 
principle that the impression of a wave of light on a screen is to be found by considering the simultaneous effect of the wavelets propagated from all the points disturbed at any previous moment (which points form what is called the front of a wave). It is only when these partial impressions concur that they are strong enough to affect the sense of vision;-(2), the representation of the phenomenon of double refraction by a wave of two sheets, or the simultaneous propagation of spherical and spheroidal waves in one and the same medium. Of the last of these doctrines I shall speak in the next section in connection with the discoveries of Malus. With respect to the former I may here observe that it gives the only satisfactory explanation of the primary difficulty of the undulatory hypothesis, - namely, that a beam of light admitted by a hole in a screen pursues a rectilincar course afterwards, instead of spreading sideways, as do waves in water, and waves of sound. Huygens shows, on elementary and convincing principles, that the lateral impressions of the wave are rapidly extinguished by the want of concurrence of the impulses which they communicate to the ether. This is necessarily true when the breadth of the aperture is such as to exceed vastly the length of a wave; and such is always the case with light, but rarely in any other instance. It is, in short, only immediately in front of the aperture that the disturbances originating in every part of the front of the original wave embraced within the aperture, concur in producing an accordant movement on the ether.

This principle, more fully stated, by which every

luminiferous disturbance of the ether is considered as the resultant of all the pre-existing disturbances to which it is due, constitutes what is sometimes called the principle of Huygens, of which I shall have more to say hereafter.

Neither at the time of its publication, nor for more than a century afterwards, was the value of these reasonings understood. It would be beside our present object to discuss Newton's opinions; but it is too certain that he did not allow Huygens' arguments on the undulatory nature of light to have any weight with him. Not that he was averse (as is often supposed) to the presence of Ether as modifying the corpuscular theory of light; on the contrary, in many of his minor writings he speaks of its existence as all but certain, and as a requisite adjunct to the corpuseular hypothesis to which he had been led by the facts of reflection and refraction. ${ }^{1}$ But he never adjusted the terms of a compro- mise, and must be held, we think, to have left behind him no substantive theory of light worthy of the name. The question never perhaps very seriously engaged his attention after the publication of Huygens' book ; and we know that about that time his intellectual energies received a shock which left him indisposed for the fatigue of constructing new theories, and still more disinclined to publish them.

Euler, though he professed to defend an undulatory theory of light, treated the subject in a point of Euler. view chiefly mathematical, and betrayed that unconcern about physical theories which characterized a mind stecped in geometric abstractions. He even retrograded ; for he did not maintain the Huygenian explanation of the cause of detinite shadows.

Young on the Undulatory Theory-Diffraction. -One hundred and ten years after the publication of the Traité de la Lumiere of Huygens, Dr Thomas Young re-opened the theory of light or "Physical Op tics," as he termed it. His experiments and reasonings tion. will be found in a series of papers in the Philosophical Transactions for $1800,1801,1802$, and $1803 .^{3}$ These memoirs are of no great length, and deserve the most careful study. They are perhaps among the clearest and plainest of Young's writings, although blamed at the time for defects precisely the reverse. They are eminently marked by penetration, profound induction, and candour of argument. Starting from his studies in acoustics, the transition to optical questions is extremely gradual. Young was, characteristically, a good musician in practice, as well as a profound one in theory, and his paper of 1799 is prineipally acoustical. In it he attaches consequence to showing that the divergence of sound from the direction of its emission is slower and less complete than it is commonly believed to be, and he applies the analogy to the existence of rays of light and definite shadows. In one short seetion he sums up the chief points of optical doctrine which lead him to prefer the theory of Huygens to that of Newton. Amongst the facts better explained by waves than corpuscles, we find reckoned Inflection and the Colours of thin plates. But all this is stated in a very general way, evidently rather as a conclusion towards which his mind had for some time been tending, than as the result of demonstrative proofs. In his paper of 1801 the undulatory doctrine is methodically expounded in a series of propositions, accompanied by proofs. The accurate definition of shadows is shown to be possible and natural on that theory, as well as the usual phenomena of reflection, refraction, and total reflection. become so general and comprehensive of other hypotheses as to leave little room for new ones to be invented." And again,"Do not the most refrangible rays excite the shortest vibrations [of the retina], the least refrangible the largest?"-Birch's Hist. of the Royal Society, quoted in Young's Lectures, ii. 615, 617. Sir D. Brewster (Life of Newton, 1855, vol. i. p. 148) considers that some passages in the later editions of Newton's Optics show that he had departed from any theory of undulations.

2 The Optics, though published in 1704, had been written principally in 1675 and 1687 (see Preface).

a They may be more conveniently consulted as reprinted in the second volume of his Lectures on Natural Philosophy, and in his Miscellaneous Works, vol. i. 
The partial reflection which always accompanics refraction is strongly and justly insisted on as an obvious consequence of the theory, while it requires a separate hypothesis on Newton's. But the chief weight is claimed for the evidence from the colours of heated surfaces, of thin plates, and of diffracted shadows, all of which the author explains by the mixture of two portions of light conveying to the same particle of ether at the same time either accordant or opposing motions, thus redoubling or destroying the light. Of these the splendid iridescent colours reflected by surfaces having fine equidistant lines drawn upon them, admit of the most elementary and striking explanation. The reflected image of a luminous point viewed in a mirror thus cut up by parallel lines, consists of one common reflection and numerous lateral images which are coloured, and in which the angles of incidence and reflection are not equal, thus contradicting one of the axioms of common optics. Young showed that the scattered waves of light recover the faculty of appearing when the surface of the plate is seen under such an angle that foreshortened intervals between the scratches amount severally to the length of one undulation or a multiple of it; for then the waves of light scattered by the reflecting surface will not come entire to the eye, but each will have a part systematically suppressed by the non-reflecting space of the groove, so that the remainders being nearly in one phase, concur in making a general impression. This experiment, therefore, literally presents us with the paradox that by suppressing half the light, the remainder is not suffered to be extinguished by it. The different colours appear reflected at different angles, because the obliquity must vary in order to be accommodated to their several wave-lengths, and each colour undergoes several repetitions corresponding to breadths representing the successive multiples of a wave-length.

Precisely similar in their origin are the coloured rays scattered by fibrous substances when held between the eye and a small point of light. If they be numerous and all of the same diameter, such fibres will suppress symmetrically portions of waves, and suffer the oblique effect to be perceptible. Dr Young most ingeniously applied this principle to construct an Eriometer or measurer of the fineness of fibres. The diffracted light of any order and colour from a distant flame will be seen at an angle with the principal or white image about four and a half times greater when viewed through the down of the beaver, than in the case of Southdown wool; being the inverse proportion of the diameters of the fibres which compose them.

(460.) Fundamental Law of Interference.-But the criFundamen-tical and characteristic experiment of interference in tal law of its simplest shape was published two years later, in
interfe- the Bakerian Lecture for 1803.' A small hole being made with a needle point in a piece of paper applied to a window-shutter, and a sumbeam being directed upon it by means of a mirror from without, a cone of light is thrown into a darkened room. A slip of card one-thirticth of an inch wide being held in the sunbeam, its shadow was observed on the opposite wall or on a moveable screen. There were seen fringes of colour exterior to the shadow on each side, such as Newton had described, and on which $\mathrm{Mr}$ Brougham and others had made experiments. But besides these, the narrower and less conspicuous fringes seen in the interior of the shadow, and first described by Grimaldi, were found by Young to have this remarkable property, that they disappeared the moment that the light passing either edge of the eard was intercepted, whilst the exterior fringe was not at all affected by that circumstance excepting on the side where the light was stopped.

Young at once perceived the significance of this admirable fact. The existence of light within the shadow at all, was evidently due to the bending of tion-bands the wave round the opaque edge; but the alternation of light and dark spaces required the union of the two lights from opposite edges, which, immediately behind the centre of the obstacle, must have described exactly equal paths, and therefore united in the same phase; but a little way either to the right or left of the centre the phases were discordant, and complete and effectual annihilation of the light resulted. In fact, when the experiment is performed under favourable circumstances, the result of the union of the light is perfect blackness in these places, but if half the light is stopped the dark spaces become luminous!

This splendid paradox may also be demonstrated without any bending round the edges of bodies, and consequently without any inflexion in Newton's sense lights proof the word; and this simplifies the conditions mate- bands. rially. In order to effect this, Fresnel (many years after) produced interference bands by allowing light emitted from a very small luminous point (an image of the sun formed by a lens of short focus) to fall upon two mirrors touching at the edge, and inclined to one another at an angle very little less than $180^{\circ}$. $\mathrm{By}$ the common principles of reflection there will be a space beyond the mirrors where the light reflected from the respective mirrors overlaps, and except in a single line within that space, the paths of the two rays meeting in any point will be different. When this difference amounts to a whole number of undulations, an exalted brightness results; when the undulations arrive in opposite phases or the centres of one set of waves concur with the ends of the other, blackness results. The experiment may even be made with a single mirror which a ray of light just grazes, and after reflection mixes with the direct light 
which had in the first instance passed clear of the mirror. ${ }^{1}$ Here evidently we have the required condition of double illumination with difference of paths. The same effect is obtained without either Inflexion or Reflection by refraction through an excessively flat prism.

(463.) It is an easy matter (comparatively) to assign the Length of lengths of a wave of light, from the intervals of the the waves interference lines, or still better from the elongations of coloured of the coloured images produced by striated surfaces, the intervals of the striæ being given. Newton's measures of the intervals between the lenses producing coloured rays, gave Dr Young the following for the number of undulations contained in an inch producing each colour :

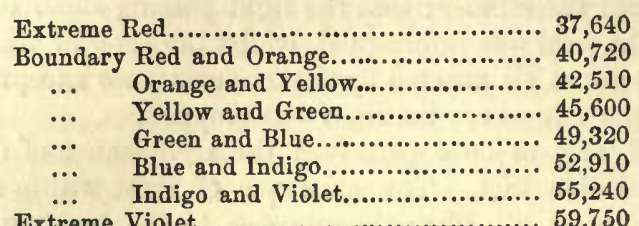

Extreme Violet..............................59,750

Now the velocity of light is known, that is, the rate of propagation of a disturbance in ether; but the duration of an impulse, or rather the interval between two successive impulses striking the eye and producing the effect of colour, is the time that an impulse Number of takes to travel over the length of a wave. It is easy vibrations to see how almost infinitely short this must be : 460 in a second. millions of millions of such impressions in a second of time go to make up the sensation of redness, 735 millions of millions that of violet light.

(464.) It might be supposed that Young's discovery and Opposition its application excited the notice and applause of all to Young's persons interested in optics. This was very far from views. being the case. Though he brought it several times in succession and in different forms before the Royal Society of London, there is no evidence, so far as I at present know, of his having then obtained a single adherent. Davy was no optician; Wollaston was too cautious to commit himself, though probably giving a tacit assent; Cavendish was aged, and besides had attended less to this subject than to most others ; Sir William Herschel had only lately taken up physical optics, and that with reference to the qualities of the spectrum least connected with Young's observations. At the Royal Institution Young vainly attempted, in the elaborate course of lectures which he there delivered for two years (1801-3) on natural philosophy and the arts, to arouse a popular interest in the unveiling of these mysteries. The $a b$ struseness of his discourses scared that mixed audience, and his colleague Davy, in a letter, incidentally observes that Young would be satisfied if any one would even offer criticisms on his opinions. Criticism of a certain kind, however, he soon got in aburidance. The Edinburgh Review, in its second number, under- took to crush at once the theory of Young and his reputation as a philosopher, and this (in singular contrast to its avowed principles), not by argument, but by an appeal to the weight of prescriptive authority in favour of the Newtonian hypothesis, concluding with an admonition to the Royal Society to cling to its old standards and old celebrities, and to save its Transactions from degenerating into volumes of miscellanies. This attack, paltry as it was, seriously prejudiced the reception, or even the dispassionate consideration of Young's views. His anxious vindication put forth in a separate pamphlet was unread, and the doctrine of interference was first understood and relished in France ten years later.

Theory of the Rainbow. - It is a matter of interest in several points of view that the phenomenon of the rainbow, which gave the first suspicion of the vary- bow inco ing refrangibility of light, and which, when explained plete wit and reduced to calculation by Newton, so convin-out the cingly proved the truth of the doctrine of the compo- doctrine site nature of white light, was destined in the hands rence. of Young and of his successors to yield one of the most refined evidences of the extensive application of the doctrine of interference. The general fact of the arrangement of colour in the primary and secondary bows Newton accounted for. But the spurious or Spurious supernumerary bows occasionally seen within the bows. primary, and far more rarely beyond the secondary, consisting of reddish and greenish bands, remained unexplained. The brilliancy of any given portion of the rainbow depends upon the deviation of the sun's rays by two refractions and one reflection, approaching to a limit which it cannot overpass. But except at this precise limit an amount of scattered light will reach the eye, which, though not reflected under the most favourable circumstances, yet is still sufficiently intense to be visible. This light must be composed, as Young showed, of two portions, entering the eye in the same direction, but which have pursued different paths within the drop, and which never coincide except at the extreme geometrical limit before mentioned. When one of these paths differs from the other by the length of half a wave of the particular kind of light considercd, darkness will result, but a feebler maximum will be again attained when the interval rises to a whole wave-length, or to two or more. Hence these consequences follow-first, that the bright part of each colour is limited by its self-destruction to a narrow band, and thus the purity of prismatic colour so striking in a well-formed rainbow is preserved; secondly, that each colour may attain (by interference) a second and a third maximum, corresponding in fact to the position of the spurious bows; thirdly, that these phenomena of perfect definition of the primary and secondary bows, and of repeated maxima in the supernumerary bows, depend essentially on this condition, that the drops of falling rain shall approach to

I This experiment is usually (and justly) ascribed to an eminent and amiable British philosopher. But it had already been performed by Fresnel with a special object. See Ann. de Chimie et de Physique, second series, xv. 382. 
a general equality of size. For the effects of interference depend on the precise diameter of the drop; if these be very various the resulting positions of maxima and minima will be altogether confused. Dr Young pointed out that to accord with the phenomena the falling drops must be about $\frac{x}{70}$ of an inch in diameter.

(466.) I have merely indicated the nature of an arguDelicacy of ment of extreme interest and beauty. It would be diffithe pheno- cult to cite (except perhaps in the science of physical astronomy) a more complete specimen of gradual inductive research. Here is a phenomenon - the rainbow-as familiar as it is beautiful. Even a partial insight into its cause confers a certain reputation upon one individual (De Dominis), its farther explication gave Newton one of his most popular triumphs. It is then found that the rainbow is not so simple a fact as was supposed, and that Newton's theory accounts for only its broader features. Then, as in the theory of gravity, a long period of uncertainty ensues; but observations are continued. A perfect rainbow is found to be one of the rarest of natural phenomena, instead of the commonest. Not above two or three individuals have ever seen, or at least described one. Then comes Dr Young, with his theory of interference and diffraction. This theory not only accounts for the spurious bows, but for the precise appearance of the principal ones, which, but for it, would have been different from what Newton supposed. Finally, after being canvassed for more than two centuries, the theory of Young is carried out into its rigorous consequences by Mr Airy ${ }^{1}$ and Professor Stokes ${ }^{2}$ (who must first invent a new mathematical method for the purpose) and illustrated by the ingenious experiments of M. Babinet and Professor Miller $;^{3}$ until at last we begin to believe that we understand this matter completely.

Exterior Fringes of Shadows. - I have mentioned only generally Young's application of his theory to the coloured fringes observed by Grimaldi and Newton to surround the outline of bodies, as thrown in shadow by a luminous point upon a distant screen. I have done so because Young's explanation was imperfect, not to say incorrect. But as it would be inconvenient to discuss the subject here, I shall briefly indicate its history and result. $\mathrm{Dr}$ Young expresses himself more obscurely in his paper of 1801 on this point than on any other, indicating three possible explanations. In 1803, however, he distinctly adopts the opinion that the periodical colours in question are due to the interference of direct light passing near the opaque edge with a portion of light very obliquely reflected from that edge; and he enters into calculations to show that such a theory represents sufficiently well Newton's measures. But it is unaccountable that Young should have been satisfied with the belief that the screens employed should in every case have reflected an appreciable quantity of light (or indeed any light at all) in the required direction. It might be conceivable in the case of a cylindrical wire or a cylindrical hair; but how could a film of gold-leaf or a slip of paper receiving the light on its broad side furnish such a degree of oblique illumination? It is wonderful that Young's intuitive sense did not perceive that the portion of a luminiferous wave passing near an opaque edge, is deficient on one side of the interfering wavelets which are necessary to make the boundary of the shadow definite, and to extinguish the laterallyspreading light. In short, he did not allow to Huygens' principle (see art. 455) the full breadth of its application-a discovery made some years later by Fresnel, who has the credit of first explaining these exterior fringes.

That great philosopher (the worthy rival of Young in this career of discovery) found the means of computing, on strict geometrical principles, the sum total nation of of the disturbance produced at any point of a them due by the whole effective portion of a luminiferous wave partially stopped by an obstacle of a given form. The principle of the calculation is simple enough. The origin of the light being distant, the front of the wave is considered as flat when it breaks against the opaque body. Its front is then divided (in thought) into small elementary portions, each of which is considered as the source. of a disturbance propagated as from a new origin. The effect of each wavelet is calculated in terms of the co-ordinates of its origin, and of the point where its effect is to be considered. The sum of all these simultaneous effects is collected by integration, a process which unfortunately is only rigorously possible in a limited number of cases. Some of these cases were solved with great ingenuity by Fresnel, and compared with observation. The re sult was extremely satisfactory. Yet it is curious to observe that Young's explanation, if it had had a sufficient physical basis, leads to nearly similar results. - In the case of an indefinite opaque body with a straight edge, the illumination precisely at the boundary of the "geometrical" shadow is, on Fresnel's theory, one-fourth of what it would have been were the body removed. Within this line the light dies away gradually, having no maxima or minima. Without it, a series of dark and light bands occur, which rapidly blend into a uniform illumination. The same theory leads to results as to the position of the interior bands which are also somewhat different from the simpler calculation of Young, and still more conformable to experience. Amongst the most singular of these results is this (which is perfectly confirmed by observation), that the shadow of a small round opaque body (as a spot of tin foil) is illuminated by a speck of diffracted light at its centre precisely as bright as if the disk were removed! How, after 
this, can we talk of light as moving in straight lines only?

We must now advert to the peculiar and disadvan-

(469.)

Young's farther re searches chiefly anonymous. tageous manner in which Dr Young laid his farther researches before the world. The optical papers in the Philosophical Transactions, ending with 1803, were the last which he published connected with his theory in that work, although he continued to be Foreign Secretary until his death twenty-five years afterwards, and although during all that time he never ceased to extend and perfect his views on the subject of his predilection. The explanation of this paradox is to be found principally in the strictness with which he interpreted the allegiance which he owed to the medical profession. He had determined to be a practical physician ; his early principles of action prevented him from doing anything by halves ; and all experience affirmed, that to gain confidence as a physician in the metropolis he must cultivate sparingly, and as it were by stealth, the studies of abstract science and of philology in which he delighted. Unquestionably he was also disgusted by the absence of one single supporter amongst the members of the great society referred to,- by the injurious petulance of the then popular critical journal, -and by the impossibility under which he laboured of communicating orally his knowledge to a general audience in an interesting and acceptable manner. The result of all this was the suppression of many of his opinions, and the publication of others in so concealed and uninviting a form that they remained for years nearly buried and unknown to men of science. He contributed a series of articles on subHis articles jects connected with light to the Quarterly Review; in the Quarterly Review, and we may well smile at the abstruse and really obscure dissertations on detached points of scienceoften unmercifully loaded with algebra-thus interspersed with articles of popular criticism for the entertainmen! of the reading public. From some of these papers we may readily gather the soreness which he felt at the cold reception of his discoveries. Farther and still more important original speculations were contained in a series of anonymous papers (sixtythree in number) on a vast variety of subjects, both in science and philology, contributed to the Encyclopadia Britannica. It is not in a work such as this that we usually look for the first publication of great and original views : the articles being anonymous could only very gradually attract notice by their intrinsic merit; and the obscurity of some of those written by Young rendered this difficult enough. But it is most fortunate that he was induced thus to write: many of his most original thoughts must have been lost but for these concealed repositories. In the articles in the Quarterly Review, for example, we watch with interest the impression which contemporary discoveries made upon his mind. The spheroidal wave of extraordinary refraction is explained by unequal elasticity of the crystal in different directions ; $;^{1}$ the discovery of polarization by reflection is received with characteristic candour, as giving a temporary blow to the undulatory theory ${ }^{2}$ whilst in a later paper the cause of chromatic polarization is convincingly deduced from the principle of interferences, and in the space of two lines the peculiar coloured laminæ occurring in Iceland spar, which had been noticed by several experimenters, are accounted for. ${ }^{3}$

From about 1815 the optical discoveries of Young were so intimately connected with those of his younger friend and rival Fresnel, that it seems best to defer our account of them until we consider (in $\$ 3$ of this chapter) the peculiar researches of Fresnel, which $\$ 3$. ultimately rendered the phenomena of polarization the most impregnable position of the partizans of the undulatory theory. The first great step was the conception of transversevibrations of ether, as constituting polarization. This, as we shall see, was first published by Young. It is to be regretted that the tardy and imperfect publication of Fresnel's memoirs on the one hand, and the resolution of Young to adhere to an anonymous and indirect mode of announcing his discoveries, on the other, render the history of the subject sometimes obscure. The correspondence between them, first fully published by Dr Peacock, throws some light upon it; but several important letters have not been recovered.

I had intended devoting a portion of this section to Dr Young's important and ingenious researches on the Physiolog physiology of vision. But the length to which it has al- of vision ready extended obliges me reluctantly to omit it. I also refer to the chapter on mechanics (Art. 344, \&c.) for some notice of his masterly reasonings on the principles of carpentry and the flexure of elastic substances. They are characterized by. directness of purpose and a consummate command of ordinary mathematics, unaccompanied by any pretension to symbolical display ; - it might be added too, by the obscure conciseness of Dr Young's habitual style. His researches (preceding and anticipating those of Laplace) on capillary attraction have also been referred to (432), as well as his masterly investigation of the tides $(80,81)$. It interpredoes not belong to this treatise to speak of his disco- tation of very of the interpretation of hieroglyphics in certain hieroglycases which gave the first real impulse to this obscure but interesting subject. The successes of Champollion, Rawlinson, and others, in similar undertakings, must logically be connected with the first great step of decyphering the polyglot stone of Rosetta. It may safely be affirmed that no philologer ever before made such a discovery in science as the law of interference, and that no natural philosopher ever made such a step in the interpretation of a lost tongue as the formation (up to a certain point) of an Egyptian alpliabet.

We cannot close this imperfect sketch of one of the greatest ornaments of our age and nation, without olarized erred to

.


adding, that in private life Dr Young was exemplary; endued with warm affections, philosophic moderation, and high moral and religious principles. His office as secretary to the Board of Longitude (the only public promotion he received), was attended not only with immense labour in editing the Nautical AImanac, but with vexatious contentions, which in his, as in so many other cases, tended to diminish his usefulness and even shorten his life. To the petty persecutions with which he was assailed, it was owing that the health which the unbroken study of fifty years had not impaired, at length gave way, and he $d$ death. died yet in the prime of intellect, the 10th May 1829 , within a few months of his honoured associates and friends, Wollaston and Davy. He had been elected two years previously one of the eight foreign associates of the Academy of Sciences of Paris.
Dr Young's philosophical character approached in many important particulars to that of Newton. With Philosoph much of the inventive fire of Davy, and of the rea- cal characsoning sagacity of Wollaston, he combined an amount. of acquired learning, and a versatility in its application, far superior to both. We do not ascribe to him an intuitive insight so rapid and almost divine as that which distinguished the author of the Principia abbve all other men, nor had Young the same strictly mathematical ability; but like Newton, whatever he did was practical and sound; nothing was done for show, nothing omitted through haste. "The power of patient thought" was the lever with which he moved the world. His self-confidence was great but unobtrusive. He attained, as he himself said, all the main objects to which he had looked forward in life, "such fame as he valued, and such acquirements as he might think to deserve it."

\section{§ 2. Malus.-Discovery of the Polarization of Light by Reflection.-Early History of Double Refraction and Polarization.}

Etienne Louis Malus was born at Paris on the 23d July 1775 , and died on the 24th February 1812 , after a too brief but brilliant career. His principal discovery, that of the polarization of light by reflection, is so intimately connected, both historically and by the nature of the case, with double refraction, that I shall briefly sum up the scanty progress of that singular subject previous to his time.

It was known to Bartholin of Copenhagen, about 1669 , that Iceland or calcareous-spar has the property of dividing a ray of light, which falls upon it in almost any direction, into two; one of which is refracted according to the usual law, but the other in an extraordinary manner, which was first analyzed by Huygens - a problem of great difficulty, in which Newton not only failed, but he also erred in continuing to pronounce Huygens' solution false. The solution was this, that there is one direction in the crystal parallel to which both the rays (called the ordinary and the extraordinary) move in a similar and uniform manner. In other directions their propagation may be expressed by considering the ordinary ray within the crystal to be due to a spherical wave (the centre of which coincides with the point of incidence), whilst the extraordinary ray corresponds to a flattened spheroidal wave concentric with the former, and having its axis coincident with a diameter of the sphere, and parallel to the mineralogical axis of the crystal. Both rays, on the Huygenian hypothesis, move slower than in air, but the extraordinary ray everywhere faster than the ordinary ray, excepting only in the axial direction. A perfectly plain though necessarily complex construction was given by Huygens for the purpose of tracing both rays in the course of their refraction, founded on this idea.
Newton's opposition to Huygens' law as a statement of fact left it for more than a century under Confirmed partial doubt. Haüy is stated to have verified it, or ton; at least to have shown that it approached nearer to the truth than Newton's ; but Dr Wollaston first reestablished it in 1802 by conclusive experiments, which, however, he found it impossible to connect by a law until the previous generalization of Huygens had been pointed out to him,-most probably by $\mathrm{Dr}$ Young.

It was several years later that Malus directed his attention to the subject, unaware of what had been and also by accomplished by Wollaston 1801 from the unfortunate French expedition to Egypt, where he was engaged as an officer of engineers, and had ruined his health through fatigue and the insalubrity of the climate. He was an accomplished mathematician, having acted as professor both at the Polytechnic School and that of Metz, and was of course a member of the Institute of Cairo. On his return to France, during the intervals of his military duties, he occupied himself in the composition of an elaborate analytical treatise on optics, which had already occupied his attention in Egypt. This led him to the subject of double refraction, and he verified by numerous experiments the accuracy of Huygens' law. La Place wrote a paper on the mathematical law of the velocity of the extraordinary ray, in which he introduced the idea of a repulsive force emanating from the axis of the crystal; but it may be truly affirmed that the notion of a spheroidal undulation so happily introduced by Huygens is the only one which really fits the case; and by the very impossibility of expressing the facts intelligibly without it, gives an undisputed advantage to that theory.

A prize having been proposed by the Academy of

(476.) 
Essay on double refraction.

(479.) Huygens' discovery of polarization by calc spar.

(480.) Malus dislarization by reflection.
Sciences for the theory of double refraction, Malus wrote a second paper, which was crowned, but which, however admirable as a specimen of mathematical address, added little to what was previously known.

Malus had already, in the end of 1808 , announced a property of light which, if not absolutely new, was entirely so with reference to the circumstances in - which it was produced. The polarization of light was in reality discovered by Huygens previous to 1680. He had observed that the two rays into which common light is divided in passing through Iceland spar have a singular diversity of character, which Newton afterwards described as an opposite polarity. Huygens showed that if two rhombs of doubly refracting spar are laid symmetrically one upon the other, the " extraordinary" ray yielded by the first is extraordinarily refracted by the second, and the "ordinary" ray from the first is ordinarily refracted by the second. But when we revolve the upper rhomb as it lies upon the lower one through a right angle, a remarkable change appears. The extraordinary ray escaping from the first is now ordinarily refracted by. the second, and vice versa, so that the qualities-of the two rays differ but only so far as this, that either may be assimilated to the other by making it (or the crystal from which it derives its properties) revolve round ninety degrees. A definite notion of such a distinction may be formed by imagining a musical string vibrating at one time in a vertical, at another in a horizontal, plane. If we could possibly imagine light to consist of vibrations of this description, the two rays of Iceland spar might be conceived the one to vibrate in a plane passing through the axis of the crystal, the other in a plane perpendicular to that. Such light might truly be said to have acquired the property of having sides. In the language of Newton, it is polarized.

The discovery of Malus consisted in showing that light may acquire properties identical with those of either ray yielded by refraction through Iceland spar, by the very simple process of simple reflection at a particular angle from any transparent body. Thus for a surface of water he found this angle to be $52^{\circ} 45^{\prime}$ with the perpendicular, and for glass $54^{\circ} 35^{\circ}$. The reflected light in either case has exactly the property of the ordinary ray transmitted by a crystal whose principal section (that is, a section passing through the axis of the crystal) is parallel to the plane of reflection. Consequently this light will be acted on by a doubly refracting crystal placed in its way precisely as if it had emerged from a similar crystal; and, on the other hand, if the two rays emerging from a crystal be incident on water or glass as above mentioned, the one will be copiously reflected from the surface, whilst the other will not be reflected at all, but pass entirely into the transparent substance. Farther, as might be expected, light thus polarized by reflection, when it falls on a second similar reflecting surface at the same angle as before, will be copiously reflected if the planes of reflection coincide, but will refuse to be reflected in an appreciable degree when the planes of reflection are perpendicular.

This phenomenon was detected by Malus by casually (481.) observing that a ray from the setting sun reflected Occasion from a distant window, and viewed through a piece ${ }^{\text {of it. }}$ of Iceland spar, afforded but a single image in two positions of the latter. The experiment attracted universal attention, and became the germ of a series of optical discoveries almost unprecedented for their beauty and variety; yet most of the experiments may be made quite as well when light is polarized by the method of Huygens as by that of Malus. Nevertheless the former had remained a sterile fact for 130 years. Upon such trifling circumstances does the progress of knowledge often depend.

Malus survived his discovery only four years, and saw but the borders of that land of promise which helo had pointed out to others. A few results he however cosines. obtained, which are worthy of notice. Thus he found that in every instance where light is polarized in any plane there is also produced a certain proportional amount of light polarized in the perpendicular plane. Arago afterwards proved the very important fact, that these two portions are universally equal. Huygens had shown in the experiment of the two rhombs, that when their positions are neither symmetrical nor perpendicular, each ray emerging from the first is duplicated by the second. When the principal sections of the rhombs are inclined $45^{\circ}$, the duplicated rays are equally bright; as they approach parallelism or perpendicularity, one pair of the rays brighten and the other pair is enfeebled. Malus ascertained the law of change of brightness, which is the same for rhombs of spar or for plates of glass whose planes of reflection vary whilst the angle of reflection remains constant. In either case the intensity of the light varies as the square of the cosine of the angle formed by the principal sections of the crystals or the planes of reflection of the plates. This important law, the best established in photometry, has been applied by Arago to the measurement of light in many instances, but the details were unfortunately not made public before his decease.

To Malus is also due the discovery of the polarization of light by common refraction. When light is in- Other dis cident on glass or water, the refracted beam contains coveries. precisely as much polarizedlight as the reflected beam, but oppositely polarized. The metals were at first believed by Malus to polarize no appreciable quantity of light. He afterwards found that at great incidences the reflected light is partly polarized. ${ }^{3} \mathrm{He}$ likewise ascertained the fact of the "depolarization"(as it was termed) of light by many crystals, and also by organized substances, such as hair, horn, and whale- 
bone. He does not appear to have noticed the phenomena of colour accompanying such depolarization, though he arrived at it so nearly that Arago, in all probability, anticipated him by presenting a memoir on the subject to the Institute just one week previous to Malus's announcement of what he had observed (19th August 1811). In less than six months later Malus was no more.

The writings and discoveries of Malus present evi- dence of great talent, but of far less fertility of com- Character bination than those of Fresnel, presently to be no- of Malus. ticed. He maintained the Newtonian theory of light. His reputation amongst his intimates was extremely high, and it was generally believed, that had he survived, his discoveries would have extended much farther. To him was applied Newton's saying on the death of Cotes, - "If Cotes had lived we should have learned something."

\section{§ 3. FRESNEL.-The Undulatory Theory of Light continued.-Diffraction.-Transverse Vibra- tions; Young.-Polarization and Double Refraction explained.-Lighthouse Illumination.}

Augustin Fresnel was born at Broglie, in France, 10th May 1788, of a feeble constitution, and he continued throughout his too short life a prey to attacks of bad health. As a boy, his slow apprehension and uncertain memory gave no indication of the maturity of his judgment. He entered first the Polytechnic School, then that of Ponts et Chaussées. His fidelity to the Bourbon cause occasioned his being harshly treated by Napoleon, and he retired to Normandy in the beginning of 1815 , to pursue the scientific studies which he had always loved.

Diffraction.-The theory of light in particular at tracted his attention, and he had a steady belief that the Newtonian doctrine was erroneous, though in ignorance, as it appears, of the undulatory doctrines of Hooke, Huygens, and Young. The phenomena of diffraction, or the coloured fringes which are seen in the interior of the shadows of opake bodies when illuminated by a minute source of light, attracted his attention as most proper for deciding the delicate question of the molecular or undulatory character of light. The results of his experiments were detailed in a memoir confided in the first place to his friend Arago, and by him communicated to the Institute of France (October 1815). This remarkable paper contained much which Dr Young had already discovered, and the explanations of the experiments which it described, both new and old, by the theory of undulations, were common to both. Dr Young having anticipated the publication by at least a dozen years, there could be no question of priority; but it is equally certain that Fresnel was unaware of what Young had done until it was pointed out to him by Arago. His memoir, which was published in great part in the Annales de Chimie for 1816 , contains much which is interesting. The mode of observing the diffraction bands directly by means of a lens, without the intervention of a screen, was equally new and important. The observation that the interior fringes of the shadow of a narrow body, such as a wire, disappear when the light is intercepted on either side of the wire, leading to the conclusion that the union of the light from both sides is neces- sary for their occurrence, was (as we have seen) one of Young's capital experiments. The explanation of Newton's rings, by the interference of the light reflected from two adjacent surfaces, though partly anticipated by Hooke, was equally important. Numerous measures of the distances of the exterior diffraction bands from the geometrical shadow, as formed by homogeneous red light, are then given and compared with theory. Here Fresnel was on original ground. These accurate numerical comparisons, afterwards pursued to a greater extent, constituted one of the most important bases of the new theory. In obtaining them he was materially aided by Arago, who, though considerably his senior, generously assisted him in every respect, and gave him the full advantage of his station as a member of the Institute, and of his experience.

Fresnel's first memoir on diffraction justly excited so much notice that the subject was proposed by the Second me. soir. ImAcademy of Sciences in 1817 for one of their prizes. proves The new essay which Fresnel then wrote was, as pro- upon bably had been anticipated, the successful one. In Young's this memoir he made an important step, by showing theory. that the exterior fringes in diffraction shadows do not depend (as Young had supposed) upon the interference of the direct light with that reflected at a great obliquity from the edge of the diffracting body, but from the interference of the different elementary undulations which proceed from the disturbed surface forming the front of the grand wave. Decomposing the front of the wave into small portions after the manner of Huygens, he computed the disturbance produced by the integral effect of the whole at a given point of the screen where the picture of the shadow fell and was submitted to examination, and he fouud that such integral effects have a periodic character, presenting points of maximum and minimum disturbance, or of greatest and least illumination as we recede from the geometrical shadow. These distances being measured in homogeneous red light were found to agree with the results of an arduous computation, requiring, as will easily be seen, an intimate acquaintance with the integral calculus and much skill in 
using it. Such Fresnel possessed, though he always refers with great modesty to his limited facility of employing the higher mathematics.

Transverse Vibrations - Young and Fresnel.(488.) Transverse vibrations -Young and Fres. nel.

Considerable obscurity hangs over the first publication of this important discovery. A clear and impartial abstract of the facts will be found in the second volume of Dr Whewell's History of the Inductive Sciences, and some further documentary evidence, including interesting letters which passed between Young and Fresnel, have more recently been published in the Life and Works of the former, edited by Dr Peacock. The difficulty of apportioning the credit between Young and Fresnel partly arises from the unfortunate system of imperfect publication, or non-publication, adopted on professional grounds by the former, and partly from the grievous delays imposed upon the latter by the opposition with which his opinions and experiments were received at the French Academy of Sciences. This continued to the very close of Fresnel's career. His greatest work was not published in the Memoirs of the Institute until six years after date; another was mislaid for above twenty years, and even the hardy friendship of Arago sometimes almost recoiled before the storm of opposition which the novelties of his associate were sure to excite in the minds of the dominant mathematical section. ' It is quite impossible to say precisely at what period Young first imagined that the differences of oppositely polarized rays of light might be explained by perpendicularity in the directions of vibration of the ethereal molecules, which he compared to the vibrations of a cord in which the elementary movements are at right angles to the direction of wavepropagation. It seems evident that Young was not possessed of this idea in 1814, when he partly explained depolarization in a few pages of an article in the Quarterly Review. It is equally certain that he announced it to Arago (with whom he became personally acquainted in 1816) in January, 1817; and that he then speaks of it as an idea which apparently had recently occurred to him, most probably since their interview. ${ }^{1}$ Arago and Fresnel had already, in 1816, made experiments demonstrating that rays oppositely polarized do not produce dark bands by their interference, a memorable discovery, requiring very great nicety for its satisfactory proof, which, however, was completely attained. It was this observation which (naturally) gave Fresnel the first idea of transverse vibrations, and it is much more than probable that Young worked out a similar solution of the great problem, in consequence of the account of these experiments which he received from Arago in the summer of $1816 .^{2}$ Be this as it may, Young and Fresnel unquestionably imagined the theory separately, but Young first announced it, Fresnel being discouraged by the doubts of Arago, and by his awe of the Institute. As clearly, the experiment of non-interference was. the first which gave a colour to so bold an assumption, and in the details of its application to double refraction Fresnel had the undivided merit. What is not least worthy of notice in the affair, is that neither of the amiable rivals (Young and Fresnel) ever published a word in disparagement of the other, nor a single unfriendly reclamation of priority.

The doctrine of transverse vibrations being allowed, (489. its applications and severest tests were twofold, 1st, Applied To the phenomena of ordinary reflection and refrac- of light. tion including the polarization produced by these operations; and $2 \mathrm{dly}$, to double refraction and the universally concomitant polarization. In these bold speculations and laborious inductions, Fresnel was nearly alone. Young did not appear as a competitor; even his friend Arago, though sympathizing with and proud of his success, was not associated with him.

Laws of Reflection and Refraction.-With re- (490. gard to the reflection and refraction of light, its in- Laws of ito the reflection and tensity has to be defined, and also its condition as to and ref polarization. The fundamental laws of the direction tionof the rays are not affected by this theory. Rigorous Fresnel mathematicians who then doubted the possibility of transverse vibrations having more than a transitory existence, if they existed at all, could not be expected to supply the theory of their reflection and refraction at the bounding surfaces of different media. Fresnel, however, guided by probable mechanical analogies, with an intuitive insight worthy of Newton himself, gave a formula for the intensity of reflected transverse vibrations, both when the plane of vibration of the molecules is in the plane of reflection, and when it is perpendicular to it; and he conceived common light to act as if equally composed of both sets of vibrations. His formulæ embrace the non-reflection of polarized light at the critical angle, under the circumstances explained in the last section. It is a most remarkable fact that these inferences by Fresnel as to the numerical relations of the intensity of the reflected to the incident light through all angles of incidence, anticipated almost every trustworthy photometrical measure; and from their singular though indirect accordance with many phenomena, they have been generally accepted as an expression of a natural law of great complexity, even by those who were not favourable to the theoretical views on which they are based.

The modifications of the state of polarization of light which takes place by reflection, was equally

1 "I have also been reflecting on the possibility of giving an imperfect explanation of the affection of light which constitutes polarization, without departing from the genuine doctrine of undulation." He then refers to "a transverse vibration propagated in the direction of the radius, the motion of the particles being in a certain constant direction with respect to that radius; and this," he adds, "is a polarization."-Young's Miscell. Works, vol. i., p. 383.

2 Peacock's Life of Young, p. 390. 
embraced in Fresnel's theory, and equally (though unknowingly) confirmed by Sir. D. Brewster's laborious observations. But on one point Fresnel himself obtained a signal triumph. Having deduced expressions for the intensity of refracted light, on pushing them to the limit where refraction out of a denser into a rarer medium becomes impracticable because the light undergoes total internal reflection, the formulæ became affected by the multiplier $\sqrt{-1}$, and were unsusceptible of arithmetical evaluation. In endeavouring to attach a meaning to these expressions, it occurred to him that as the intensity of the totally reflected ray undergoes no change with the angle of incidence, the expression in question might in some way determine the alteration of the phase of the wave (the position and direction of motion of the molecules under consideration) which took place at the instant of reflection. Now, admitting this as likely, it appeared that the phase would vary not only with the angle of internal reflection, but with the plane of polarization of the ray. It had previously been shown by Arago and by himself, that when two oppositely polarized rays meet or interfere, though there is then no destruction of the light, there is usually a remarkable change in its character. There is one position of the interfering wave relative to the primary one in which the combination produces light polarized in a plane exactly intermediate between the planes of previous polarization. If either ray be now accelerated by half a wave-length on the other, the new plane of polarization becomes perpendicular to the former; but if the shift of either of the primary rays amounts to only one quarter of a wave-length, the motion of the molecules takes place in a circle, and the undulation has a helical form. Now, Fresnel tested his hypothesis concerning totally reflected light by calculating the circumstances of incidence which should produce an effect equivalent to this ; and the result completely verified his bold conjecture. The apparatus employed is called Fresnel's Rhomb, which transforms plane-polarized light into light equally reflexible in all azimuths, yet not common light, because it possesses properties which common light does not (such as displaying the rings in crystals); this is termed circularly polarized light.

Theory of Double Refraction.-The difficulty of 作 action ex- in showing how a spheroidal wave might be propagated. Young had already shown, in 1809, that it would result from supposing a lamellar arrangement of the crystalline molecules so that the ether was differently elastic in a direction parallel to the axis than in a plane or planes perpendicular to that line. ${ }^{1}$ Huygens had shown something similar in accounting for terrestrial atmospheric refraction. The difficulty was, to account for two waves travel- ling at the same time through the same portion of matter with unequal velocities. The moment that the idea of molecular movement transverse to the line of propagation was admitted, it was easy to see that no contradiction was involved in the idea. Tro waves might simultaneously travel in the same direction, and through the same medium, provided the molecular displacements were in different planes. So happy a solution could hardly fail to strike such minds as those of Young and Fresnel with the impress of conviction. A closer analysis confirmed the probability. Iceland spar (or rather the ether imprisoned within it) is conceived of as a medium of uniform elasticity in all planes perpendicular to the axis, but of a different and greater elasticity in any direction parallel to the axis. It is shown to result from this, that in the direction of the axis alone is the motion of light independent of the plane of the vibrations of which it is composed, and consequently no separation of rays occurs. When a ray moves parallel to what may by an analogy be called the equatoreal plane of the crystal, its undulation will, generally speaking, be resolved into two whose vibrations are parallel and perpendicular to that plane, and which travel with different velocities though in the same direction. If the ray take any other direction through the crystal, both the direction and velocity of the divided rays differ. The form of the extraordinary wave is exactly the spheroid of Huygens.

But what are we to conclude concerning those crystals (of the discovery of which we shall speak in $\$ 5$ ) Theory of tals (of the discovery of which we shall speak in $\$ 5$ ) crystals once assumed that the elasticities must in that case with two vary in three rectangular directions, and he proceeded to calculate the manner of propagation of a wave through a medium thus constituted. I had proposed to attempt some explanation of the steps of his most ingenious and profound argument, but I find it incompatible with the space at my disposal, and at any rate hardly to be apprehended without the use of symbols or figures. For these reasons I shall merely state the results. When the medium presents unequal elasticities in three rectangular directions, the surface of the wave consists of two sheets each travelling with its peculiar velocity. But neither of these being spherical, the result cannot be expressed by the ordinary law of refraction. In two directions within the crystal, the wave surfaces coincide, or the two rays coalesce. These directions are evidently the optic axes, and the wave surface in their neighbourhood has very interesting geometrical and physical properties which have been elucidated by British philosophers, as will be noticed in another section. The true optical axes cannot exceed two, and when two of the three elasticities become equal, they merge into one. This is the

1 Young's reasoning (Quarterly Review 1809, and Works, vol. i. p. 228) is based on an experiment by Chladni on the differing velocity with which sound is propagated in wood, depending on the direction of the fibres. 
case in Iceland spar and similar crystals. At the same time the wave surface degenerates into the united sphere and spheroid. The equation to the wave surface was deduced by Fresnel in an indirect and somewhat tentative manner. It was demonstrated by Ampère directly, but inelegantly. M. Cauchy, Mr Archibald Smith, and Professors Sir W. R. Hamilton and Maccullagh gave more complete and elegant solutions.

(494.)

confirmed

by experi ment.

(495.)

Fresnel submitted his theory (as usual) to experiment. He found that in topaz, which is a biaxial crystal, neither ray follows the law of common refraction. The plane of polarization (which is always perpendicular in the two rays) follows very nearly indeed, by theory, the law which M. Biot had assigned by experiment. Fresnel thus stated the ground of his conviction of the truth of his theory, and it would be difficult to express more appropriately the characteristics of a just hypothesis:"The theory which we have adopted, and the simple construction which we have deduced from it, present this remarkable character, that all the unknown quantities are at once determined by the solution of the problem; - the velocity of the ordinary and extraordinary rays, and their respective planes of polarization. Physicists who have studied with attention the laws of nature, will admit that this simplicity and these intimate relations between different parts of the same phenomenon present a great probability in favour of the theory by which they are established."

The memoir on double refraction was received Reception, with much incredulity and partial applause. It was of Fresnel's not to be supposed that a theory in opposition to
theory; that innagined by Newton, and received with almost general assent for more than a century and a half, would not meet with many opponents; but in the case of double refraction and polarization it was also essentially coupled with the idea of transverse vibrations, whose exact mechanism was admitted on all hands to be extremely obscure. Laplace, now more than seventy years of age, opposed the new opinion to the last. His reason for doing so was eminently characteristic of the great geometer- "it was one to which analysis could not be applied without much difficulty ;" to which Fresnel replied, "that it was still harder to believe that the laws of nature were arrested by such obstacles." Poisson, as might have been expected, was equally opposed to the undulatory doctrine, for he was still less of a physicist than Laplace. His standing argument against it was its inability to explain dispersion. M. Biot, also a keen supporter of Laplace, was still more strongly compromised to the theory of emission. The inertia of such authorities at the Institute retarded of course the growth of Fresnel's reputation at home, notwithin Eng. standing the great weight of his friend Arago's opiland.
Fresnel were first most generally and liberally acknowledged; as, singularly enough, Young had received almost the first expression of sympathy in his optical discoveries from France. In 1825 Fresnel received the distinguished honour of being elected a foreign member of the Royal Society of London, only two years subsequent to his election into the Institute, and whilst his greatest paper was as yet known only by an abstract. In 1827 he received the Rumford medal from the same body. This recognition of his merits was due, as we learn on the authority of Dr Young (who was then Foreign Secretary of the Royal Society) to the influence of Sir John Herschel, at that time and afterwards a zealous supporter of the undulatory theory of light, and by whom it became first generally known in England through the medium of his admirable Essay on Light. Dr Young, though present, was silent; "from being," as he himself tells us, " too much interested in the subject" on account of his personal share in the matter. In announcing this distinction officially to Fresnel (then in the last stage of consumption), Young characteristically observed, "I too should claim some right to participate in the compliment which is tacitly paid to myself in "common with you by this adjudication; but considering that more than a quarter of a century is past since my principal experiments were made, I can only feel it a sort of anticipation of posthumous fame which I have never particularly coveted."2

I have stated in the opening of the section that Fresnel, who was attached to the Bourbon cause, had Fresnel's retired to Normandy near the close of Napoleon's system of career. On the re-establishment of the monarchy in illumina1815 he was recalled from his retreat and appointed tion. to an office in the departments connected with his profession as an engineer; but in 1817 he was brought to Paris with the express view of giving him more facility in his researches. In 1819 he was placed on the Commission for the Management of the Lighthouses of France (of which he afterwards became Secretary), and he entered with ardour on the application of his favourite science cf optics to the duties of his profession and the benefit of mankind.

The use of lenses in place of reflectors for preventing the indefinite dispersion of the light employed, and the effectual concentration of it in the direction where it will be most useful, was not altogether new. The construction of immense lenses of glass of no great thickness, formed by grinding out a series of concentric refracting surfaces having a common focus, had also been proposed by Buffon, and the idea of constructing these rings or échélons separately and then uniting them had been suggested by Condorcet in his éloge of Buffon, as well as at a later period 
by Sir D. Brewster. These proposals were all alike unknown to Fresnel, who had the grand merit, in a case of this kind, first, of carrying his happy idea into effectual execution, and secondly, of giving it a wonderful extension by the invention of a multitude of other forms of refracting and totally reflecting apparatus, till then unimagined as well as unexecuted. ${ }^{1}$ In 1823 the lighthouse of Corduan, at the mouth of the Garonne, was furnished with the new lenticular system, which was very skilfully executed by Soleil of Paris. The illumination was provided by means of a beautiful and powerful lamp with several concentric wicks, the joint invention of Fresnel and Arago, which gave twenty-five times the light of the best Argand then in use. The system was found to work so well that it was speedily extended in France, then to Holland, and in the third place to Scotland, principally through the energy of the late Robert Stevenson and the present Mr Alan Sterenson, his son, to the latter of whom we owe the best and most compendious treatise on the subject of lighthouses, ${ }^{2}$ as well as the noblest exemplification of it in the Skerryvore Lighthouse, erected by him in 1843. The same small work contains the details of Fresnel's admirably ingenious applications of the principle of refraction to the distribution of light under almost every circumstance, which were not, however, published by their inventor. (498.) In 1824, consequent upon his exertions as exa-
s prema- miner at the Polytechnic School, Fresnel had the feath. first seizure of the malady which brought him to the grave at the premature age of 39 , on the 14th July 1827. Eight days previously to his death he had received at the hands of Arago the Rumford medal before referred to, which his distinguished friend
Malus had obtained under like melancholy circuinstances 16 years before.

From what has been stated it will appear that Fresnel eminently possessed the qualities requisite for original investigation. So finely balanced a combination of mathematical skill and attainment with profound inductive sagacity has rarely been witnessed. Had Young not happened to precede him. there can be no question but that he would have made the undulatory theory entirely his own. Fresnel was superior to Young in the talent for devising and executing critical experiments, in which indeed he showed a degree of skill equally rare and admirable. It is hoped that his surviving brother, M. Leonor Fresnel, who is well qualified for the task, will collect his scattered papers and edit them, with a suitable biography. The éloge of Fresnel, written by the man most competent to render him justice-Arago-remained more than twenty years among the unedited papers of that philosopher, and has only appeared since his death in 1853. The cause of this suppression was one of those partly political and partly personal disputes which seem almost inseparable from the proceedings of the Institute. The éloge was announced to be read just two days before the Revolution of 1830 burst forth ; Arago could not persuade himself at such a moment to discuss the merits of the Theory of Double Refraction without committing himself also on the politics of the day. Disputes arose, friendships cooled, and the unlucky work was returned to the author's desk. Hence no biography of Fresnel appears in the publications of the Institute, but his reputation will be treasured in France and elsewhere, when the more conspicuous laurels of many of his compeers are withered and half-forgotten.

\section{§ 4. ARAG0. ${ }^{3}$-Short Account of his Scientific Career-He discovers the Colours of Polarized Light-Laws and Theory of Depolarization; M. Biot; Young; Fresnel.-Non-interference of oppositely Polarized Rays-Rotatory Action of Quartz.-M. Foucault's Experiment on the Velocity of Light.}

(500.) Dominique François J Jan Arago, one of the most generally known of the philosophers of the half century just elapsed, though the author of a large number of miscellaneous writings which since his death have been edited in a collected form, has not left an amount of positive contribution to any one of the sciences at all in proportion to the reputation for ability which he very justly enjoyed.

He was born at Estagel near Perpignan, on the 26 th February 178f, and the ardent temperament of a native of the south was one of his chief character- istics. From a fragment of his early history which he left behind him, it appears that he educated hinself almost without assistance, and that when he was admitted to the Polytechnic School in 1803 (consequently at the age of 17 ), he was intimately acquainted with the chief writings of Lagrange, had studied the Mécanique Céleste, and had consequently in his possession far more mathematical knowledge than would have been required of him on leaving that celebrated institution. From the Polytechnic School he passed into the position of Secre-

1 One of Fresnel's lenses was used in 1821 for the geodetic operations connecting France and England, and the light was observed at a distance of fifteen marine leagues one hour after sunset.

2 Rudimentary Treatise on Lighthouses, by Alan Stevenson. Weale, 1850. See also the Account of the Skerryvore Lighthouse, with numerous plates, in 4 to, 1848.

3 I may perhaps be thought to give, Arago too prominent a place in the history of Optics. If so, it has arisen in part from

(499.) Character of Eresnel. 
tary to the Paris Observatory. He pursued with M. Biot experiments on the refraction of the gases, and in 1806 the two young philosophers were despatched to the south of France and to Spain to continue the triangulation interrupted by the death of Méchain. ${ }^{1}$ Geodetical The next three years were spent by Arago in a series observa- of voluntary and involuntary journeys, perils by land tions; and sea, from robbers, and from the Spanish government and populace, such as have been rarely equalled, - perhaps never in the pursuit of science. The declaration of war against France rendered his stay either in Valentia or in the Balearic Isles impossible, and he was conveyed in disguise from Majorca to Algicrs, whence he twice essayed to reach Marseilles, but was once driven back to Africa by a storm, once made prisoner by a Spanish corsair. After great suffering, he at length reached France in Jụly 1809, carrying with him the precious record of his geodetical operations. From this time his promotion was assured, and his life became tranquil and inactive, although the deep attachment which he formed with Baron Humboldt immediately on his return to France would probably have induced him to accompany that enterprising traveller to Central Asia, had that journey ever been accomplished. At the early age of 23 Arago attained the position of Member of the Institute, and was again attached to the Paris Observatory, of which at a later period he became director. $\mathrm{He}$ took a very active share in the proceedings of the Academy of Sciences, and became one of its secretaries in 1830 .

From the period of his election to the Institute, (502.) Arago's career was destitute of stirring incidents, but was, from first to last, devoted chiefly to science. It is certain, however, that he was deficient in that power of continuous application, to which alone great discoveries are commonly due. Full of ingenious, original, and even profound conceptions, he shunned the labour of realizing them. His appointment to the charge of the Observatory of Paris was perhaps unfortunate. Well versed in the theory of astronomy, the minute drudgery of observation and the control of numerous assistants, was altogether uncongenial to him. It was a duty imperfectly fulfilled, to say the least, for 40 years: and it is needless to add how much the consciousness of habitual neglect of a duty deadens the faculty of useful application to anything else.

If the science of astronomy then owes little to Arago, beyond the part which he took in geodetical

observation, what are we to consider his chief claims IIis optica to a place in this history? I have no hesitation in labours of saying that they are to be found in connection with most imthe discoveries and labours of his attached friends, Malus and Fresnel, and therefore we group them together in this chapter. Arago not only himself made some important optical discoveries in 1811 and the following years, but he was instrumental, as we have seen, in a very important degree, in calling forth the genius of Fresnel, and in obtaining a public recognition of the labours of Young; a service not the less worthy of note because of its eminently disinterested character. The undulatory theory of light, one of the greatest triumphs, if not the greatest, of our age, stands where it does in no slight degree through the instrumentality of Arago.

One of his most considerable discoveries was that of the colours which crystallized bodies develop in Colours of white light polarized before incidence on the crystal, crystallize and afterwards transmitted through a rhomb of calc- polarized spar. These colours, by their order, the singular man- light. ner of their occurrence and disappearance, and in certain cases by their extraordinary and beautiful forms, offered a problem at once the most attractive, the most definitely marked, and the most seemingly inexplicable which had been met with in optics for much more than a century. They exemplified a new mode of analyzing light, evidently connected with the molecular forces concerned in crystallization; and for their display it was necessary that light should be in that peculiar and yet mysterious state called polarized.

The substances he employed were principally selenite, rock-crystal, and mica. When plates of the first and last of these minerals, formed by their natu- of ral cleavage, are placed in a beam of polarized light, and the light transmitted by them is then analyzed, by being passed through a doubly-refracting prism or thrown on a screen after reflection at the critical angle from glass, splendid colours are the result. These colours vary with the thickness of the plate, with itsinclination to theincidentlight, and, what is most remarkable, they vary in intensity by merely turning the plate of selenite round in its own plane. When only this last motion is made, there are two positions of the plate where no colour results, the light passing through unchanged; and these positions are at right angles to one another. At all intermediate angles colours appear, the light is said to be depolarized, and this depolarization is most complete when the plate is moved $45^{\circ}$ from

the difficulties inseparable from the biographical system which I have adopted. My intention was to have thrown together the labours of Malus, Fresnel, and Arago into one section. But having written the different portions separately, there seemed so much precision and facility of explanation to be derived from treating of them consecutively, that I sacrificed, in some degree, the biographical principle to that of systematic classification; placing under the name of Malus what referred to the empirical laws of double refraction; under that of Fresnel the doctrine of transverse vibration (though mainly due to Young); and under Arago the discoveries of Young, Fresnel, Biot, and others, relative to the great subject of chromatic polarization, to which he gave the first impulse. The establishment of the undulatory theory, principally due to Young, Fresnel, and Arago, I have considered as deserving of a more detailed and systematic treatment than almost any other of the numerous discoveries of which I have to speak in this Dissertation. I may add, that the biography of Arago not appearing in its alphabetical place in the Encyclopadia, M. Arago being still alive at the date of the publication of that part of the work, it has been incumbent upon me to enter in to more details than I should otherwise have done. 
the positions in which the light passed through unchanged.

The theory of this simple yet admirable experiment is one of the happiest examples of Fresnel's mechanical explanation of double refraction. But it was not attained by a single step, nor effected by a single hand. Malus had observed the fact of depolarization, and the existence of perpendicular neutral axes in the crystalline plate. Arago added to this the knowledge of the phenomena of colour, which Sir David Brewster also observed independently somewhat later. He also invented a particular theory to explain them by what he termed moveable polarization. But it was not a successful effort. M. Biot assiduously studied the empirical laws of these periodic colours, and traced their dependence on the thickness of the interposed plate, according to a law similar to that of Newton's rings. Young made an important step farther. $\mathrm{He}$ attributed the colour to the interference of the ordinary and extraordinary rays into which the incident light was divided on entering the crystalline plate; and he showed by accurate calculation that the retardation of the slower-moving of the two rays during their passage through the plate, did in fact produce the difference of phase necessary for developing the tints observed by their reunion on leaving the plate, according to the usual laws of interference; and he showed that this theory coincided with M. Biot's rules. ${ }^{I}$ But even this was not enough to explain the facts. It was not clear why the colours due to doublyrefracting plates should not be seen without reaching the eye through an analyzer, or calc-spar prism. To Arago and Fresnel jointly we owe the important reply to this difficulty, which in fact forced upon the latter the idea of transversal vibrations. Their joint experiments (mentioned in the last section, art. 488), had shown that oppositely polarized rays cannot interfere unless they have, first, a common origin and a coinmon plane of polarization; and, secondly, unless they be reduced to a common plane of polarization (that is, analyzed) before falling on the eye or the screen: so that the theory of the colours of crystallized plates is, briefly, as follows :-

Polarized light is represented by transverse vibrations of ether, the particles moving all in one plane. The crystallized plate has, we will suppose (in order to take the simplest case) one axis of double refiaction, and the direction of that axis is in the plane of the lamina. When the vibrations of the light falling on the crystallized plate are either wholly parallel or wholly perpendicular to this axis, the light is transmitted without alteration, either as an extraordinary or as an ordinary ray, and it is then reflected or not by the analyzer, as would have been the case had it not been transmitted at all through the crystal of selenite. But if the axis of the crystal be inclined, suppose $45^{\circ}$, to the plane of vibration of the incident polarized ray, the vibration is mechanically resolved into two, which are oppositely polarized. After traversing the thickness of the crystallized plate with the different velocities due to the motion of the ordinary and extraordinary rays, they are reunited at emergence, but in altered relative phases, so that by their union they form a beam no longer polarized (unless by an exception) in the same plane as at first, nor indeed plane-polarized at all, but most likely performing elliptical or circular vibrations, which, again, falling on the analyzer, are reflected or transmitted, or partly both, in a manner quite different from what would have happened to the light unchanged by crystalline transmission.

White light becomes coloured because the state of (508.) polarization of the emergent ray depends on the differ- Cause of ence of length of path for the two rays which underwent crystalline separation within the plate, and also on the length of a wave of light (for this determines the phase of polarization at emergence). But the wave of light varies in length for each colour, consequently every colour has its maximum under different circumstances, and if the incident light be white, the light falling after reflection on a screen will present mixed tints similar to those of Newton's rings.

This extraordinary property of a crystallized plate (which, in common light, appears equally transparent Similar and homogeneous in every direction), of modifying phenolight, or dyeing it with the most gorgeous colours, radiant when the plate is merely turned in its own plane, is heat. one of the nicest tests of the polarization of the light, and has been used to detect the analogous polarization of radiant heat, and the concomitant phenomenon of double refraction, which, except in the case of the heat accompanying the solar rays, has not yet been independently recognized. ${ }^{2}$

Arago applied his discovery to the construction of a polariscope, for estimating the feeblest Arago's poamount of polarization; and he used this instrument for some very interesting experiments on the polarization of the light of the sky (which is sunlight polarized by reflection from the atmosphere), and on that of different incandescent and reflecting surfaces. He also found that the moon and the tails of comets send light to the eye which is slightly polarized, thus betraying its borrowed origin. But that of the sun, being absolutely neutral, is only comparable (according to Arago) to the light arising from incandescent vapours, thus distinguishing the sun from a solid or liquid globe.

We cannot do more than allude to Arago's other (511.) optical papers and experiments. He was, probably, Experithe only Frenchman of his time who was well ac- Newton's quainted with Young's discoveries. The explana- rings and 
on the ro- tion by the doctrine of interference of the colours tatory ac- of Newton's rings received an important confirtion of mation from an experiment of Arago's, which proved quartz. them to arise from the inixture of the peneils of light reflected at the two neighbouring surfaces. He pressed a lens of glass against a plate of metal, in which case the central spot is white or black when light polarized perpendicularly to the plane of incidence is reflected at an angle greater or less than the polarizing angle for glass; and the rings vanish altogether at the polarizing angle;-results which have been found conformable to the undulatory theory. ${ }^{1}$ He also discovered the peculiarity of the rays transmitted along the axis of a crystal of quartz. These depolarize light, or produce colours similar to those of crystallized plates, varying aecording to a well-marked law with the thickness of the plate. The most singular fact is, that by turning round the analyzing plate, no position of neutrality is found, but a series of colours similar to those of Newton's scale succeed one another. Arago showed that this effect is due to a rotatory motion of the plane of polarization within the crystal. The rotation is greater for the violet than the red ray; this was shown by M. Biot, who also discovered that in some specimens the rotation takes place from right to left, in others from left to right-a peculiarity connected with certain crystallographic modifications, as was first shown by Sir John Herschel.

(512.) MM. Seebeck and Biot discovered an analogous Rotation of property in oil of turpentine, and in various saccharine the plane fluids, an observation which, in manyeases, allows the of polariza- substitution of an instantaneous optical, for an operose tion in chemical test. Fresnel has shown that the phenoinena of quartz may be represented on the supposition that the rays traversing the axis consist of two rays circularly polarized in opposite directions, and travelling with different velocities; and Mr Airy suceeeded in calculating, by the aid of this fundamental hypothesis, ${ }^{2}$ a number of most beautiful and complicated phenomena, such, for example, as those which oceur when plates of right and left handed quartz are superposed. Maccullagh has shown how the generation of elliptical or circular vibrations may be deduced from the general equations of motion, but he has not invented a mechanical theory to explain them. This is a point of the very highest interest inasmuch as Dr Faraday has succeeded, for the first time, in inducing artificially in a substance the power of rotating the plane of polarization by the presentation of it to the poles of a most powerful magnet. ${ }^{3}$ Arago's experiments on the non-interference of
Retarda- lays of light oppositely polarized, being undertaken tion of light in conjunction with Fresnel, have been already re- ferred to, arts. 488 and 506 ; but to Arago alone is in dens9 due the ingenious idea of interposing a thin slip of media mica or blown glass in the path of one of the inter-proved by fering pencils, and observing the displacement of the interference-bands, which is always towards the side of the interposed slip, showing that the movement of the wave has been slower within the denser medium.

Arago continued to attacl great importance to the obtaining of a still more direct proof of this fact, which Experihe considered as a crucial one between the rival hypo- ment to the theses of Newton and Huygens. In his last ycars he bame effect had the satisfaction of witnessing the accomplishment cault. of it, with the result he anticipated, and by a method which he had himself indicated. In 1838, he had already indicated the application of $\mathrm{Mr}$ Wheatstone's beautiful invention of the revolving mirror, ${ }^{4}$ as a means of measuring intervals of time incredibly short, in order to compare the velocity of light in air, and in a correspondinglength of water. He even caused an apparatus to be partly prepared, but we have seen that Arago's forte was rather in suggesting than in completing researches. After his increasing failure of sight rendered it physically impossible that he should ever realize his own idea, it was skilfully adopted by $\mathbf{M}$. Foucault (the author of the admirable experiment with the pendulum, demonstrating the earth's motion $^{5}$ ), who, by an ingenious combination of fixed and revolving mirrors, succeeded in 1850 in demonstrating the retardation of light in a tube of water only $6 \frac{1}{2}$ feet long, and with a velocity of rotation of the moveable mirror not exceeding 200 turns in a second (a rapidity four times less than had already been obtained by $\mathrm{Mr}$ Wheatstone). The rotation was produced by means of the Sirène of M. Cagniard de la Tour, acting by steam. The velocity was thus raised to 1000 revolutions. It was afterwards, however, carried by MM. Fizeau and Breguet to 2000 revolutions. It will be understood, froin the account of the method, as applied to the measurement of the velocity of electricity, in another chapter, that the retardation is shown by the displacement of the image of a minute object seen through the water, relatively to the image of the same object seen in air. If light moves faster in water (as Newton imagined), the displacement of the water-image will be (let us say) to the right; but if slower (as Huygens and Young believed), it will be to the left. The calculated displacement, with 800 revolutions in a second, was .004 inch on the first supposition, and $\cdot 003$ in the opposite direction in the second, quantities easily visible with a high magnifying power. The result, as has been stated, confirmed Arago's original experiment of 1815 on the displacement of the interference fringes.

1 Sir W. Herschel first formed Newton's rings between glass and metal. Arago's experiment was reproduced (unknowingly) by $\mathrm{Mr}$ Airy in 1831, who first explained it fully in the undulatory sense. Camb. Trans., vol. iii.

2 With this addition, that rays inclined to the axis are elliptically polarized, and that with a greater ellipticity as the inclination increases.

3 See the chapter on Electricity, § 5 .

4 See Electricity, § 6.

5 See the chapter on Astronomy, Art. (258). 
(515.)

(519.)
To complete our biographical notice, we will here just allude to M. Arago's principal researches unconnected with optics. One was on the magnetization of iron filings, and the formation of a temporary magnet, by means of a helical conductor of electricity ; ${ }^{1}$ the other was the important observation of the seeming magnetism of copper, and other non-magnetic metals, when put in rapid rotation near a permanent magnet. Neither of these happy experiments was carried out by their author. The former was left in the hands of Ampère, Sturgeon, and Henry; the latter was only rightly understood and valued when it was engrafted by Mr Faraday on his splendid series of researches on Magneto-Electric Induction."

Arago is fairly entitled to be regarded as having proved the long-suspected connection between the aurora borealis and the freely suspended magnet; and this in the face of urgent contestation. ${ }^{3}$ His contributions to Meteorology (founded rather upon the observations of others than upon his own) were of considerable importance, and several of his popular papers, appended to the smaller Almanac (Annuaire) of the Board of Longitude, contain a great deal of well-digested and curious information.

As Secretary of the Academy of Sciences in the
Mathematical Department (in which office he suc-Arago as ceeded Fourier, in 1830), the duty devolred on him secretary of of writing the biographies of eminent deceased mem- the Acabers of the Academy. He bestowed extraordinary Sciences. pains on these compositions, and strove to render them popular without sacrificing their scientific character. In this difficult attempt he was not always successful. Abrupt transitions, piquant anecdotes, paradoxical arguments, and political allusions, appear, at least to the English reader, to be unacademical adjuncts to the history of contemporary discovery. The special pleader is too often visible, and even the occasional sacrifices of the strong spirit of nationality by which he was commonly actuated, to some chivalrous adjustment of the rights of discovery, do not always carry conviction to the mind of the reader. The Eloge of Watt, probably the most popular, appears to us far from being the best of these biographies. Those of Sir W. Herschel and of Dr Young are ably executed, and display much research and candour.

After having been for three years almost withdrawn from science by lingering disease, and nearly His death. complete blindness, Arago expired at the Observatory of Paris, on the 2d October 1853, aged 67.4

\section{§5. Sir David Brewster-Progress of Experimental Optics-Laws of Polarization-Double Refraction produced by Heat and Compression-Discovery of Biaxal Crystals-Laws of Me- tallic Reflection-Absorption of Light; and Lines of the Solar Spectrum; FRAUNHOFER.- Seebeck; M. Biot.}

We have pleasure in ranking amongst the foremost promoters of the science of optics in its surprising revival in the earlier part of this century, a philosopher who still lives amongst us and pursues with ardour the investigations of his youth.

Sir David Brewster was born at Jedburgh, in Scotland, on the 11th December 1781. He was educated for the Scottish Church, and having entered the University of Edinburgh at a very early age, pursued his studies under Robison, Playfair, and Stewart, and formed the friendship of those eminent men. Amongst fellow-students of no common distinction who at that time frequented the college lectures, and of whom not a few were destined to signalize themselves in literature, science, and the career of politics, he formed the particular acquaintance of Mr, now Lord Brougham, and through him was led to study the inflection of light, and to repeat Newton's experiments. This was in 1799 ; nor did he afterwards lose sight of a science which he was so signally to improve. The distraction of other occupations, the calls of his profession, and his indifferent health prevented, however, any vêry constant application to optics: and the part of the subject he then principally studied was rather connected with the use and theory of instruments than with physical optics in the sense in which we have explained it. This is evident from his first separate publication in 1813, " on new Philosophical Instruments," which, though containing many ingenious and valuable suggestions, fell short of the importance of his subsequent publications.

Sir David Brewster's genius was first called forth by the announcement of Malus's great discovery in 1808 of the polarization of light by reflection. attention to But for the unfortunate political relations of ran. physical and England at the time, which prevented. to a degree which now appears almost incredible, the transmission of even the most interesting scientific facts from one country to the other, our countrymen would have borne a larger share in the discoveries which immediately followed; and it would have been an easier task to apportion with historical accuracy what was due to each. As the French philosophers remained long in ignorance of the discoveries of Davy, and were anticipated in every important step in voltaic science, so Malus and Arago pursued and published researches and bril-

\footnotetext{
1 See Electricity, $\$ 4$.

2 Electricity, § 5 .

3 No doubt the fact had been already distinctly noticed by Hjorter and Celsius at Upsala in 1741 . See Hansteen, Mag. netismus der Erde.

- The article Polarization of Lignt in this Encyclopadia, the production of Arago, contains an excellent review of many of the topics of this Section.
} 
liant discoveries which, literally for years, remained unknown in England to those most interested and solicitous to learn them. Thus Sir D. Brewster learned first in February 1814 that Malus had in March 1811 published the discovery of the polarization of light by refraction, which he also had made ; whilst Arago's experiments on coloured polarization were likewise unknown to him through the same want of international communication.

(522.) The work on Philosophical Instruments, menTreatise on tioned above, contains, besides what its name more new philo- particularly imports, numerous observations on resophical fractive and dispersive powers, including the discoments. very of substances more refractive than diamond, and less so than water. It also describes the property of some agates to transmit light polarized in only one plane. The imperfect, polarization of light by metals and by a serene sky liad been anticipated by Malus and Arago.

From this time (1813) Sir David Brewster became (523.) His researches on a regular contributor to the London Philosophical optical sub. Transactions, which, as well as those of Edinburgh, jects.

\section{(524.)} of the most fraction, and other quantitative laws of phenomena. important.

\section{duced by heat and pressure.}

III. The discovery of crystals with two axes of double refraction, and many of the laws of their phenomena, including the connection of optical structure and crystalline forms.

IV. The laws of metallic reflection.

V. Experiments on the absorption of light.

(525.) I. Malus had failed to discover a connection beLaw of po* tween the angle at which light is completely po-
larization by reflec- larized by reflection, and the other known optical tiun. properties of bodies. In 1814, Sir D. Brewster discovered the beantiful and simple law, "that the index of refraction is equal to the tangent of the angle of polarization." He had suspected it much sooner, but he had been baffled by the irregular results obtained by reflection from glass, whose surface he fourd to undergo an almost imperceptible chemical change. He further observed that it is only in bodies of low refractive power that the polarization is sensibly complete, a result of great importance, which has been too much overlooked until the recent and valuable paper of Jamin on the same subject. He deduced as a corollary, that at the maximum polarizing angle the incident and refracted rays are at right angles to one another, and also Malus's experimental result that the rays reflected from the first and second surfaces of plates are simultaneously polarized. He further discovered the fact that light inay be completely polarized (as to sense) by a sufficient number of reflections at any angle, and drew the conclusion that the whole light undergoes some change at each reflection, in opposition to the view of Malus, who maintained that, except at the polarizing angle, a portion of the light is polarized, and the rest is unchanged.

Sir David Brewster independently observed the polarization of light transmitted obliquely through glass, Inperfect and he calculated the number of plates necessary to polarizpolarize it with sensible completeness. All these researches he resumed some years after (Phil. Trans. 1830), endeavouring to give a photometric estimate of the effects of reflection and refraction under all circumstances. The results as regards partially polarized light may still be considered as subject to doubt. His skill in obtaining a mathematical representation of the phenomena was again displayed in a number of laws connecting the experimental results. ${ }^{1}$

II. Malus had observed that a vast number of substances depolarized light more or less completely; and Arago found feeble traces of chromatic polarization in some specimens of glass. But the more in heated definite characters of the beautiful phenomena of glass not perfectly annealed (which proved to be of unexpected importance) were noticed, independently, by Sir D. Brewster and Dr Seebeck of Nurnberg. The latter had priority in publication, ${ }^{2}$ but the former correctly referred them to their immediate causethe constraint produced by rapid cooling. Sir D. Brewster noticed that the unannealed glass which forms what are called Prince Rupert's Drops, had a remarkable power of depolarization; and he also observed subsequently that the plates of glass between which he was in the habit of squeezing heated wax and resins, for the purpose of optical examination, transiently communicated tints to polarized light. These observations, duly developed, proved on the one hand that glass (and generally refracting

1 Thus he found that the effect of refraction on the plane of polarization of the incident light may be expressed by this simple formula- $\operatorname{cotan} a^{\prime}=\operatorname{cotan} a \cos \left(i-i^{\prime}\right)$, where $a$ and $a^{\prime}$ are the azimuths of the planes of polarization of the incident and refracted rays measured from the plane of reflection, and $i$ and $i^{\prime}$ the angles of incidence and refraction. This result, admirably verified by experiment, is also confornable to Fresnel's theory.

2 In Schweigger's Journal for 1813 , vol, vii. I have not been able to find in this paper (which contains the first account of the beautiful symmetric coloured figures displayed in cubes and cylinders of glass) the smallest trace of the true cause of the phenonsenon, viz., the sudden or partial cooling of the glass. 
solids not usually possessed of any polarizing action) acquired such propertics by being suddenly and unequally cooled; and on the other, that such substances undergoing partial changes of temperature, possess the same property. These phenomena are exceedingly beautiful, and easily displayed. It is sufficient to place a rectangular piece of glass, somewhat thick, with one of its longer edges in contact with a hot iron, and placing it between a polarizing and analyzing plate, to incline the heated edge $45^{\circ}$ to the plane of polarization. Bands of light and shade are seen to traverse the glass parallel to the same edge, and simultaneously appear also on the side farthest from the heated metal. They pass gradually into rich coloured tints diffused with geometric regularity. In the case of cubes or cylinders of glass, suddenly, and therefore unequally cooled, the phenomena are permanent, and the colours splendid, being arranged in patterns which may be made to resemble those of natural crystals.

Sir David Brewster at first ascribed these effects to the direct effect of the heat in the glass upon light, and compared its sinultancous influence over a whole plate to a polar influence. Another curious discovery, also due to him, leads to the simpler conclusion, that in every case the development of a polarizing structure is due to the varying tension (transient or permanent) into which the particles of the glass are thrown by local expansion or by irre. gular cooling. This discovery was, that similar effects may be produced in jellies and soft transparent substances by the effect of pressure, and that even glass itself, when strained in any way by mechanical force, shows depolarizing bands; and crystals may have their peculiar optical phenomena altered by pressure. Fresnel, not satisfied with inferring that the chromatic display is due to a doubly-refracting structure communicated to the glass, contrived, by an ingenious arrangement of prisms, actually to exhibit the separation of the images under the action of powerful pressure.

III. Possibly the most remarkable of Sir David Brewster's discoveries, - at all events, that which probably cost him most labour to develope, - was that there are crystals possessing two axes of double refraction, and showing many remarkable phenomena, which indicate a connection between optical structure and crystalline form. No one before him suspected the existence of a doubly-refracting structure differing from that so ably investigated by Huygens and Malus in Iceland spar. In 1813 Sir David Brewster had discovered coloured rings in topaz when viewed by polarized light. Though intimately connected with M. Arago's observation of colours in crystallized plates, these interesting phenomena had a still more extraordinary and geometrical character. When a plate of topaz, split by natural clcavage, was presented between the polarizing and analyzing plate (or rhomb of calc-spar), and at the same time inclined in a certain manner, the colours were no longer in broad shcets, but, if viewed closely, they arranged themselves in oval rings of great beauty, presenting orders of mixed colour analogous to those described by Newton when formed between convex glasses, and they were traversed by dark or white brushes as the analyzing plate was held in the dark or bright position. A second such system was observed inclined at an (apparent) angle of $65^{\circ}$ in the same plate.

Dr Wollaston afterwards (1814) discovered a phenomenon equally beautiful in calcareous spar, of Phenomena which Sir D. Brewster had already perceived traces of polarizain some other crystals. Concentric with the posi- by uniaxal tion of the axis of double refraction (or optic axis), crystals. in a crystal of that mineral cut with two parallel faces perpendicular thereto, a series of perfectly symmetric and exquisitely coloured rings are seen in polarized light, having a white or black cross traversing them, according to the position of the analyzing plate. This magnificent phenomenon, which (excepting, of course, the rings of biaxal crystals mentioned auove) has perhaps no parallel in optical science, is seen in the most perfect manner possible in an apparatus constructed solely of Iceland spar, cemented by Canada balsam. A Nicol's single-image prism ${ }^{1}$ is uscd to polarize, another to analyze the light, and between them is a plate of calcareous spar properly cut. It is a truly astonishing paradox to see the union of three perfectly transparent and colourless crystals display by their union such an exquisite combination of form and colour. The pole or centre of the rings in calc-spar coinciding with the axis of double refraction, of necessity suggested the idea that topaz, which shows two systems of rings arranged round two poles, must possess two axes of double refraction; in other words, that there must exist within the crystal two directions (not mere lines), parallel to which a transmitted ray emerges without subdivision into two pencils.

This probable conjecture was verified by careful (531.) observation, not only in topaz, but also in a vast Double sysvariety of other crystals which were found by Sir tems of toDavid Brewster much more commonly to possess two \&c. than one system of rings. Amongst the earliest examples which he observed were nitre, inica, acetate of lead, and Rochelle salt. Of these the first is exceedingly remarkable for the small inclination of the axes (about $5^{\circ}$ ) which permits both systems of rings to be readily observed at once. It was not, however, for some years (1817) that he reduced these most

1 The invention of a most ingenious person, the late Mr William Nicol of Edinburgh. One of the doubly-refracted rays is thrown completely out of the field by undergoing total reflection at the surface of a film of Canada balsam in contact with the spar, whilst the other less refrangible ray proceeds quite isolated, and with scarcely any loss of brilliancy. It is of almost universal application in this branch of Optics. 
curious phenomena to anything like a law. It was evident almost from the first, that the axes in question (which he termed axes of no polarization) are only the resuitarts of remoter fundamental actions of the crystalline constitution. For example, these axes vary in position according to the colour of the light used to display them; their position within the crystal varies (as was shown by Mitscherlich) with the temperature of the body, nor is it obviously related to any of the geometrical lines of crystalliza-

Law of the tion. Sir David Brewster succeeded in finding the tints. law of the tints expressed upon the surface of a sphere of which the directions of the two axes form diameters. M. Biot expressed the law more elegantly by saying, that the tint developed by a biaxal crystal in any ray, is proportional to the product of the sines of the angles which the ray in question makes with the two optic axes. The tints are consequently arranged round the two poles of the axes, in a series of curves resembling the figure 8 , having each this property, that the product of the sines of the angular distances of each point of one curve from the two poles is equal to a constant quantity. Such curves are called lemniscates, and are beautifully seen in nitre, especially when viewed by homogeneous light.

(532.) A series of researches of the most elaborate Relation of description led Sir David Brewster to this addioptical cha- tional and admirable discovery, viz., that the optical racters to characters of single refraction, double refraction forms. with one axis, and double refraction with two axes, have reference invariably to the primitive crystalline form of the mineral, and that the complexity of the optical character is as invariably related in degree to the complexity of the crystalline figure. Cubical and regularly octahedral crystals (as rock salt and fuor spar)being possessed of perfect symmetry in three principal directions, possess also simple refraction. Crystals with one predominant line or axis of symmetry -as rhombohedrons, octahedrons with square bases, right prisms with square or hexagonal bases-have a single axis of double refraction. Such, for instance, are Iceland spar, zircon, ice, beryl. Finally, all crystals unsymmetrical in the three principal directions, including prisms and octahedrons whose bases are not square, and those which are oblique, have two axes of double refraction. The rarity and minuteness of many crystals, the difficulty of cutting them, and when cut, of detecting their optic axes, evidently made the research one of extreme labour, yet highly remunerative, not only through the discovery of the general principle, but by the vast amount of beautiful and varied optic displays witnessed in the course of it. Sir David Brewster was nearly, if not quite, alone in this research, and after a short resistance on the part of some mineralogists, his principle of discrimination of primitive forms of crystallization by optical characters has been perfectly established. reflect is very peculiar. Malus, who at first believed Laws of that they were incapable of polarizing it in any de-metallic gree, afterwards changed his opinion, and inge- reflection. niously suggested that whilst transparent bodies reflect, at the polarizing angle, light polarized only in one plane, metals reflect rays oppositely polarized and then mixed. Sir David Brewster has the merit of having, after several unsuccessful attempts, deduced the leading empirical laws of metallic polarization, having been partly guided (as he states in his paper in the Philosophical Transactions for 1830) by Fresnel's remarkable experiments on circular polarization produced by total reflection in glass (see Art. 491). Having found qualities somewhat analogous in light reflected one or several times from metallic plates at various angles depending on the nature of the substance, he gave to the light so reflected the name of elliptically polarized light. It was afterwards satisfactorily proved by $\mathbf{M r}$ Airy that the light so named by Sir D. Brewster is in fact identical in its qualities with the elliptically polarized light of Fresnel.

The subject of metallic polarization is rather too abstruse to be explained in a popular way; and the Metallic phenomen with depolarizing plates of dif-polarizaferent metals are not so well known as, according to their discoverer, they deserve to be. My limits only permit me at present to state that the care and accuracy of Sir D. Brewster's results are unquestionable; that they have formed almost the sole data upon which M. Cauchy and other mathematicians have based their theories of metallic reflection; and that, by generalizing the more limited views entertained by Fresnel as to the constitution of media and the nature of reflected light, they have been mainly instrumental in fixing the later views of optical writers as to the precise phenomena of polarization as produced, not only by metals, but by other substances. To these views I shall briefly advert in the next section. briefly. White light is coloured or analyzed by of light.
The laws of the reflection of light at crystallized surfaces have also been studied by Sir D. Brewster. In this case observation is still in advance of theory.

V. Of Sir David Brewster's experiments on the absorption of light we must speak much more (536.) refraction (as in a prism); by simple interference, as in Newton's rings; by double refraction combined with polarization. But it is also decomposed in a way which, primarily at least, seems different from all these,-by passing through coloured, or ratner, colouring media, whether solids, liquids, or gases, as red glass, ink, chlorine. This, the most familiar mode of coloration, is the most difficult to account for, and has been (on account of its obscurity) less studied than the others. In some instances, the complementary colour (that which, added to the transmitted tint, makes up white light) is entirely absorbed or lost; in other cases it is reflected at or near the first surface of the medium. Sometimes

IV. The action of metals on the light which they 
(537.)

(538.)

the transmitted light is made up of different portions of the spectrum curiously blended, whilst rays intermediate in the order of refrangibility are wholly stifled. Many crystals have the curious property of dichroism, that is, of transmitting light of different colour's in different directions. All these facts have been very carefully studied by Sir David Brewster.

But the most remarkable phenomenon to be noticed under this head is the wonderful action of nitrous acid gas upon light. ${ }^{1}$ When a beam, either of sunlight, or the light of a lamp, is passed through a bottle containing a small quantity of fuming nitrous acid, the light enierges of a tawny orange colour, which may be deepened indefinitely by heating the acid. If this light be then analyzed by a common prism, a wonderful spectacle is seen. The spectrum appears traversed by countless bands or dark spaces, whilst the blue and violet colours are nearly absorbed. The effect of the gas, then, is this, - to stifle or absorb countless minute portions of light seemingly selected at random from every part of every colour in the whole spectrum. Some of these deficient rays are broad and palpable, but most of them are so fine as to be visible only with the telescope. To understand the full import of this discovery, it is necessary to describe first the lines of Fraunhofer.

Joseph Fraunhofer, born in Bavaria, of humble parents, in 1787, raised himself by his unassisted efforts to be the first practical optician of the day. He had also the merit of devoting his leisure and the fine apparatus at his command to the observation and discovery of many optical phenomena, particularly those diffractive colours produced by fine gratings, which are known under the name of Fraunhofer's spectra. ${ }^{2}$ His principal discovery, however, was (in 1814) that of countless deficient or dark lines in the solar spectrum, resembling those which, as we have mentioned, were afterwards observed by Sir D. Brewster, to be produced in any kind of light by the action of nitrous gas. The deficient rays of solar light had, indeed, been observed still earlier (in 1802) by Dr Wollaston, but he counted only a very few of the more conspicuous ones; he described them mercly incidentally, and (unusually with him) seems not to have perceived the great value of the discoveryboth in a theoretical and practical point of view.

Fraunhofer's beautiful map of the spectrum, traversed by lines of every grade of darkness, and clustered with every conceivable variety of distribution, was published in the Munich Transactions. He counted 590 lines, but Sir D. Brewster states that he has carried the number to 2000. Like the stars, they are probably countless. These lines characterize solar light. The light of the fixed stars and that of the electric spark hare their peculiar deficiencies different from those of our sun. These were discovered by Fraunhofer, as well as their occurrence in certain coloured flames. The order and number of the lines is, in each case, independent of the kind of prism used; but the angular distribution of the deficient rays varies with the material. Thus an oil of cassia prism expands most in proportion the less refrangible end of the spectrum; while water and sulphuric acid act with disproportionate dispersive energy on blue and violet light. This property of substances had been already studied by Sir D. Brewster with his usual diligence; but the importance of Fraunhofer's discovery was this, that the lines (the larger of which he distinguished by letters of the alphabet) furnish landmarks which define special rays of light invariably recognisable under all circumstances, which the vague description of their tints is quite incompetent to do. This enabled, on the one hand, the practical optician to discover the kinds of glass most fit for achromatic combination; and, on the other, it afforded precise numerical measures of the quality of dispersiveness in bodies which have been partly already, and will yet much more become, tests of some of the more obscure and difficult portions of the theory of light, - those, namely, which are connected with dispersion and absorption. Fraunhofer was, after Dollond, the most eminent and scientific manufacturer of achromatic telescopes, of which he vastly increased the aperture. He died at Munich in 1826.

Returning to Sir David Brewster's discovery of the artificial production of analogous lines or deficient Action of rays in light from any source, its importance is easily nitrous gas perceived:-for, in the first place, it so far accounts earth's atfor the strange phenomenon of the deficient rays mosphere of the sun's light, by showing that it may be caused on the by a gas resembling nitrous acid gas in its properties existing in the solar atmosphere; and, farther, if so astonishing a result of absorption is ever to be explained by theory, the first step is to be able to produce the phenomenon at pleasure, and to examine the qualities of the bodies producing it. The phenomena of coloured flames which possess standard deficient rays, present perhaps a closer analogy to the sidereal spectra. Sir David Brewster has farther found that the absorptive action of the earth's atmosphere (detected by the varying character of the spectrum for different angular altitudes of the sun) increases the number and also the breadth of these lines.

Intimately connected with, and nearly of the same date as these experiments, was an observation of Sir D. Brewster's, which has received less general assent Brewster's than any other of the numerous and important ones the spec-

1 Edinburgh Transactions, vol. xii. (1833).

2 The peculiarity of these spectra is this, that they consist of pure colours, whilst almost all interference-colours are, like those of Newton's rings, mixed and impure. One result is very remarkable. Fraunbofer obtained his spectra of such brilliancy as to be able to measure the position of the dark lines (an evidence of their exceeding purity), thus obtaining a standard spectrum in which the material of the prism bas no influence whatever in varying the ratio of the dispersion of the various colours. 
which we owe to his genius. It is an analysis of the coloured light of the (so-called) homogeneous rays of the pure spectrum by the specific action of absorbing substances. Sir D. Brewster believes that he has separated the homogeneous orange of Newton into red and yellow, the green into yellow and blue; and that, in fact, each of the three prinuary colours exists at every point of the spectrum. But as grave doubts have been thrown on the results, especially by the recent careful experiments of $\mathrm{Helmholz}$, I shall not further insist upon them here. ${ }^{I}$ Still less can I take notice of a multitude of microscopic researches on a variety of objects in the animal, vegetable, and mineral kingdom, and on the physiology of vision, with which Sir D. Brewster has filled a multitude of memoirs, each bearing testimony to the zeal and acuteness by which his researches are directed.

Sir David Brewster received, in 1816, jointly with Seebeck, one of the great prizes of the Institute; he also received, in succession, all the medals in the gift of the Royal Societies of London and Edinburgh, and he is an honorary member of the principal academies of Europe. In particular, he is one of the eight associate-members of the French Academy of Sciences. To metenrology he has been a valuable contributor, having discussed in an able paper the law of the distribution of temperature over the globe, and pointed out the near coincidence of two regions or centres of greatest cold in the northern hemisphere, with the magnetic poles. His papers are so numerous, and their variety is so great, as to render an enumeration, even of those containing what may reasonably be termed discoveries, impossible within our limits. Few persons have made with their own eyes so vast a number of independent observations ; few have ever observed better, or recorded their observations more faithfully. He has discovered (as we have partly seen) a multitude of laws of phenomena of the greatest importance in the construction of a theory, but he has not been forward in proposing such a theory. Neither the moveable polarization of Biot, nor the transverse undulations of Young and Fresnel, received his cordial assent. Generally speaking, he has been favourable to a corpuscular theory of light, without, however, attempting to render the Newtonian view mechanically consistent with the astonishing variety of complex phenomena which he aided in discovering, and which would evidently require it (to say the least) to be completely remodelled. His scientific glory is different in kind from that of Young and Fresnel; but the discoverer of the law of polarization, of biaxal crystals, of optical mineralogy, and of double refraction by compression, will always occupy a foremost rank in the intellectual history of the age.

Before closing this section I shall add a few words respecting the discoveries of MM. Seebeck and Biot, which have a very close relation to those of Sir David Brewster.

Thomas SeEbeck was born in 1770 . We have seen that he was one of the discoverers cf the depola- Seebeck. rizing structure of heated and compressed glass (527). In 1816 he observed, independently of $\mathrm{M}$. Biot, the property of oil of turpentine and other fluids to rotate the plane of polarization of light transmitted through them, thus acting similarly to a crystal of quartz on a ray passing along its axis (512). Previously to these discoveries he had repeated Sir William Herschel's experiment on the position of maximum heat in the spectrum, and found it to vary with the material of the prism. When the science of electro-magnetism was created by Oersted in 1819, his attention became chiefly directed to that class of phenomena, and in 1823 he was fortunate enough to discover thermoelectricity. He also wrote many papers on allied subjects. He was a skilful observer, but deficient in the power of physical analysis. He died in 1831.

M. Вгот, at the time I write, the oldest member (I) (I believe) of the Academy of Sciences, and one of M. Biotthe veterans of European science, was born at Paris rous rein 1774 , and has lived to the age of 80 , a life of searches. almost unintermitted intellectual lâbour. It is impossible not to be touched by the evidence of such unconquerable love of knowledge. He was, if I mistake not, one of the original pupils of the Polytechnic School; and his talents being first developed in an almost purely mathematical direction, he attracted the notice of Laplace, who introduced him to the Institute, and by whom he was always befriended. In 1802 he published a work on curves and surfaces of the second degree, and was the first after Lambert who thought of applying mathematics to the theory of conducted heat. From this time his attention was almost exclusively directed to the applied sciences, and the number and variety of his experiments and writings almost baffles enumeration. Descriptive and practical astronomy, the theories of sound, of light, of the voltaic pile, of terrestrial magnetism, of electromagnetism, of heat, radiant and combined, have been the subjects of his studies and writings. We find him in the earlier part of his career associated with Gay Lussac in his first aeronautic expedition, and with Arago in the geodetical and astronomical operations of the great arc of the meridian. He afterwards carried the pendulum to the Island of Unst, the northmost land in Shetland; and he made original experiments on the propagation of heat and of sound. He wrote a voluminous treatise on descriptive and practical astronomy, one still more elaborate on general physics, and a vast number of miscellaneous papers in the Journal des Savans and the Biographie Universelle. His original memoirs in the Transactions of the Academy are usually very long and elaborate, his calculations and empirical formula 
laboriously accurate. One of his papers fills an entire volume of the Academy's Memoirs. Eren the astronomical hieroglyphics of the Egyptians, and the chronology of Chinese eclipses, have drawn from his pen learned treatises; and he has expounded the labours and discoveries of his countrymen and others with almost as much care and effort as if they had been proper to himself. But his subject by predilection was optics, and here he made his most considerable discovery, and that which he has followed out with most minute industry, namely, the rotatory action of fluids, in which he had Seebeck for a co-discoverer. (See Art. 512.) He studied the colours of crystallized plates with excmplary patience, and as we have seen in the preceding section, by his accurate observations on the law of the tints, prepared the way for the theory of transverse vibrations; but his own doctrine of moveable polarization, which he imagined to explain them, made no impression on the progress of science. He was the first who divided doubly-refracting crystals into positive (as quartz), and negative (as calcareous spar). In the former the extraordinary wave is a prolate spheroid, and inclosed within the ordinary spherical wave; in the latter the spheroid is oblate, and exterior to the sphere. He also discovered (very approximately) the law regulating the plane of polarization of the rays in biaxal crystals.

M. Biot has for about half a century been an active professor and member of the Institute. His researches, always marked by precision, are perhaps deficient in bold conjecture and happy genoralization. They are conducted with a mathematical stiffness which allows little play to the fancy, and in hypothetical reasoning he rarely indulges. His style is formal yet diffuse, and consequently somewhat repulsive to the student. His works are consequently not easily read, and have contributed less to the progress of knowledge than the scrupulous care often evinced in their compilation might seem to warrant. Yet the name of Biot will be ever associated with devotion to science, and especially with the progress of optics in our own day.

\section{§ 6. Mr Airy, Sir William R. Hamilton, and Professors Lloyd and Maccultaagh.-Confirma- tion of Fresnel's Theory-Investigation of the Wave Surface completed; Conical Refraction. -M. Cauchy. Mechanical Theory of Elastic Media, and of Ordinary and Metallic Reflection; M. Jamin.-Theory of Dispersion; Professor P_well.}

(547.) It would not be possible, in one short section, to rogress of do justice to the various improvements and additions re undula- which the undulatory theory of light_- the joint crea-y since tion of Huygens, Young, and Fresnel-has received resncl. since the nearly simultaneous decease of the two lastnamed plilosophers. But while a vast amount of labour and of mathematical and experimental skill has been thus expended, of which it would be in vain to attempt within our limits to give an account, we may pause upon two or three of the more conspicuous results of these researches, which, in conformity with the plan of this dissertation, may give a tolerable idea of their general tendency.

Looking at the history generally, we find one curious peculiarity in the progress of this remarkable theory. Its origin in the seventeenth century was unattended with sympathy or success. It received little support, and was well nigh forgotten for more than an hundred ycars: it was then resumed(we might almost say re-invented) in England, but it romained unpopular and almost unknown until re-echoed from a foreign land; while in France itself the views of Fresnel were (with one or two exceptions) as little appreciated as those of Young had been in England. From this period England became the place of its chief development; and with the exception of one eminent philosopher, M. Cauchy, its supporters and extenders, whether by analysis or experiment, have belonged to Great Britain, ${ }^{1}$ a few of the most conspicuous of whom are named at the head of this section.

The attention of the British public was forcibly (549.) called to the theory of Young and Fresnel, by an Sir J. IIer able treatise on Light, contributed by Sir John schel and Herschel in 1827 to the Encyclopoedia Metropolitana. The excellent method, Iucid explanations, and intelligent zcal which marked this essay compelled the notice of men of science, too long deterred from the study of the fragmentary and abstruse writings of Young. It was followed four years later by a most able and precise mathematical exposition of the theory, and its application to optical problems, by Mr AIRY (now Astronomer Royal), who was then Plumian Professor at Cambridge, and who introduced this part of optics as a branch of study in that university. Whilst the excellent tract on the undulatory theory (published in 1831 in his Mathematical Tracts) opened up the subject in a most accessible form to British mathematicians, his original papers in the Cambridge Transactions confirmed the doctrines of Fresnel by a number of new and admirably contrived experiments, some in connection with interference, some with polarization, and all were confronted with the rigorous results of the mathematical theory. The paper on Quartz, and that on the Rainbow, have been already referred to (art. 466, 512). The writings of Mr Airy and of Sir John Herschel have continued to be the 
main sources of information on this subject, and on physical nptics generally, not only in this country but on the Continent. It is remarkable that in France, which possesses so many admirable scientific books, there should not exist a single good treatise un optics. Had not Mr Airy's attention been necessarily withdrawn from optics to astronomy, it is very evident that the theory of light would have received from him many farther important additions.

(550.) Whilst an impulse was thus given to the matheSir Wm. matical theory of light in the University of Camton and Dr bridge, a similar progress was being made in the Iloyd. sister University of Dublin, where three of her most eminent professors, Sir William RowaN Hamilion and Professors Lloyd and Maccullagh devoted themselves energetically to its improvement and verification.

(551.) Tothe two former of these we owe the prediction and Conical re- ocular demonstration of the most singular and critical fraction in of all the results of Fresnel's theory. Sir William crystals. Hamilton, a geometer of the first order, having undertaken the more complete discussion of the wave surface of Fresnel (see Section Third of this Chapter), to the equation of which he gave a more elegant form than heretofore, ${ }^{1}$ ascertained the exact nature of that surface, and consequently the exact direction of refracted rays in the neighbourhood of the "optic axes." It had been shown by Fresnel that, in the case of crystals with two axes, a plane section in a certain direction cuts the two sheets of the wave surface in a circle and in an ellipse, which necessarily intersect each other in four places. (See the annexed figure.) In the lines joining these four points with the centre of the figure the velocity of the two rays is equal. Now the cusps or sharp inflections of the wave surface in these particular directions, occur not only in the particular plane of section which we have considered, but in any section of the wave surface passing through these lines of equal ray-velocity. In the figure, therefore, of the compound sheet there is not a furrow, as Fresnel had supposed, but a pit or dimple, with arched sides something like the flower of a convolvulus, and the surfaces meet at the bottom of the pit at a definite angle. Let the circle and ellipse, in the annexed figure, represent the section of the wave surface we have described; then $\mathrm{OP}$ is the line of uniform propagation, and $\mathrm{P}$ is the bottom of the conoidal pit M $\mathrm{P}$ N. Now suppose a slender ray of light to move through the crystal in the line $\mathrm{OP}$, and to emerge into air at a surface of the crystal cut perpendicular or nearly so to the direction of single ray-velocity $O P$. If we confine our attention at first to the plane of the figure only, that ray having intersecting

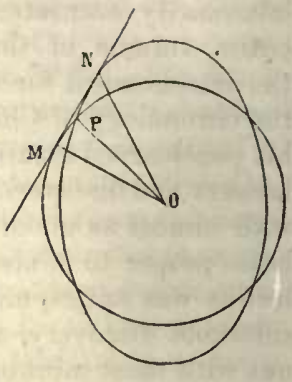
tangents both proper to the wave surface, would give rise, on Huygens' construction (art. 475), to two emergent rays inclined at an angle. But since this is the case, not only in the plane of the figure, but (as has been stated) in any plane passing through the ray in question, the emergent light must form a conical luminous sheet, the angle of the cone being determined by the refractive properties of the crystal. This beautiful and unexpected result was verified with great skill and address by Dr Lloyd in the case of Arragonite, which is a biaxal crystal, and he found the position, dimensions, and conditions of polarization of the emerging cone of light to be exactly such as theory assigns. When all the necessary corrections are attended to, the angle of the cone of light is about $3^{\circ}$. There is another case of conical (or it might be called cylindrical) refraction, which occurs nearly in the same portion of a crystal, which was predicted and discovered in like manner, but which we will not stop to particularize. ${ }^{2}$ The observations of Dr Lloyd have been extended by M. Haidinger to the case of Diopside, a crystal also having two optic axes.

Every one capable of appreciating such evidence, will feel the irresistible impression which so curious Other an anticipation, so accurately fulfilled, gives us of the works of positive truth of a theory admitting of such verifications. The names of Sir W. Hamilton and Dr Dr Lloyd. Lloyd will be lianded down to posterity in connection with this admirable discovery. But they have also other claims to our respect, to which we can here only refer in the most general terms. The former has generalized the most complicated cases of common geometrical optics by a peculiar analysis developed in his essays on "Systems of Rays" (Irish Academy Transactions, vols. xv.-xvii.) ${ }^{3}$ To Dr Lloyd

\footnotetext{
1 The equation is$a^{2} x^{2}$ $\frac{a^{2} x^{2}}{x^{2}+y^{2}+z^{2}-a^{2}}+\frac{b^{2} y^{2}}{x^{2}+y^{2}+z^{2}-b^{2}}+\frac{c^{2} z^{2}}{x^{2}+y^{2}+z^{2}-c}=0$.

2 It was shown by Sir W. Hamilton that the tangent plane $M$ N touches the wave surface, not in two points merely, but in a circle of contact; consequently, the perpendicular to this tangent plane, OM, is the direction of one of the optic axes (or the velocity is the same for both portions of the compound wave). Hence a ray incident externally so as to be refracted along this perpendicular, will at entrance spread into a hollow cone interior to the crystal, and on emergence at a parallel face each portion of the ray recovers a direction parallel to its primitive direction, and a luminous hollow cylinder is the result. See Dr Lloyd, in the Irish Academy Transactions, vol. xvii., and Sir W. R. Hamilton's third supplement to his "Systems of Rays" in the same vol.

$3 \mathrm{Sir}$ W. R. Hamilton is also a discoverer in pure analysis and its connection with geometry. Following up the ideas of Mr Warren on the geometrical significance of the symbol $\sqrt{-1}$, as indicative of direction, Sir W. Hamilton has developed the theory of a new class of imaginary quantities, which he terms quaternions, by means of which he contrives to express simultaneously the direction in space and magnitude of a line or form; and this calculus he has applied to the solutions of problems of geometry and physical astronomy. The quaternion appears to express something even beyond this; and this redundancy has been considered as a difficulty by some mathematicians. The superfluous number is considered by Sir W. Hamilton as representing time in mechanical problems.
} 
we are indebted for several interesting experimental papers on optics, for an able and impartial review of the progress of the science, ${ }^{1}$ and for an excellent elementary treatise on the Wave Theory, which forms by far the best popular introduction to the subject.

Closely associated in his pursuits, as in personal friendship, with Sir W. R. Hamilton and Dr Lloyd, was James Maccullagh, a native of Tyrone, born in 1809 , and who died prematurely and unliappily October $24,1847 .{ }^{2}$ His first paper was communicated to the Royal Irish Academy at the age of 21 . It was one of the earliest original contributions of this country to the development of the theory of Fresnel. The construction of the wave surface in biaxal crystals was simplified and improved; and in 1835 a second paper appeared, in which geometrical constructions of great elegance were employed for the farther investigation of the subject. Mr Maccullagh next attacked the theory of the undulations of ether in quartz crystals, to which he gave a mathematical expression (see art. 512). In 1838 he published a paper on the laws of crystalline reflection, in which he adopted certain hypotheses, such as that the vibrations of the ethereal particles, in the case of polarized light, are parallel to the plane of polarization (contrary to Fresnel's opinion), and that the density of ether is the same in all media. In a subsequent memoir on the dynamical theory of reflection and refraction, he arrived at similar results with fewer physical assumptions, and by a more purely mathematical treatment of the subject. Subsequent researches, presently to be mentioned, have diminished the value of these theoretical investigations.

(554.) Numerous mathematicians of eminence at home echanical and abroad entered upon the same arduous enquiry. bratory To attempt to deduce from the hypothetical constitu-

tion of a very rare highly-elastic medium, together with the known dynamic laws, the various complicated facts of optics, was a problem whose difficulty was only equalled by its indefinite character. For how little do we know of the molecular constitution of such fluids as air and water? How much less then of a fluid (if we may so term it) almost infinitely rarer, and incapable of being inclosed, measured, or weighed? The bure possibility of transversal undulations was long contested by very able mathematicians ; and conceding it, the mutual influence of such an ether and the particles of gross matter (as shown by reflection and refraction) must, it would seem, for ever remain problematical. Yet, however gratuitous or even erroneous our reasonings about such ultimate questions may be, there is no doubt a real benefit in obtaining from them at least a mathematical congruity with observed facts. The progress of science shows that there is a practical usefulness in this step. On the other hand, we must not be discouraged to find that there are so many handles to the matter, that even the most profound thinkers may conceive that they have reached the proposed end by different and incongruous routes. Besides the mathematicians whom I have mentioned, many others were in the field; for the result of tremors propagated through elastic unedia of different kinds is an enquiry of excessive generality, and forms a part of many branches of science besides optics. MM. Cauchy, Navier, Poisson, Coriolis, Green, Kelland, and Neumann are amongst those who attacked the problem. For a series of years memoirs rose fast and thick on this favourite battle-field; and even a skilful mathematician might find it no small penance to discuss the merits of the various hypotheses and the solidity of the respective deductions which were proposed.

It must be owned that a great part of this vast mathematical toil has been without immediate result $t_{\text {ing on op- }}$ in optics. It is by comparing the conclusions ar-tics. rived at by authorities of seemingly equal weight, that we learn the difference between a stable physical induction and a clever mathematical hypothesis. Maccullagh, for instance, maintains the vibrations of ether to be parallel to the plane of polarization; Fresnel and M. Cauchy ${ }^{3}$ that they are perpendicular. The first mathematician considered that the vibrations are wholly transversal, the last believes that the normal vibrations have also their share in affecting the phenomena, whilst Poisson denies that transversal vibrations can be propagated to a sensible distance. Maccullagh finds that, to account for metallic reflection, the indices of refraction of mercury and silver are 15.0 and 35.0 , whilst Cauchy makes them but 1.77 and 0.34 . One theorist assumes that the density of ether is the same in all bodies, another that it is greatest in a vacuum, and others precisely the reverse; one that vibration is not accompanied by change of density, another that it is $;^{4}$ and so on in almost endless variety. A matured opinion can only be formed after the results of the various assumptions, and their congruity with facts, have been more thoroughly worked out. Already two of Maccullagh's essential postulates have lost much of their plausibility; that respecting the direction of the vibrations in polarized light has been probably decided by Professor Stokes in favour of Fresnel's opposite view, by an admirably devised experiment on the effect of diffraction on polarized

1 British Association Reports for 1834.

2 It is unnecessary to suppress the fact that Mr Maccullagh died by his own hand, under the pressure of a fit of despondency, brought on (it is believed) by over-work; a fate happily extremely rare amongst students of exact science. I say it is needless to suppress the fact, because it infers no blame. Mr Muccullagh was an amiable, pious, and exemplary man. Irresponsible insanity was, of course, the cause.

3 This is M. Cauchy's last view ; for some years he adopted the opposite one, which he then surrendered with great frankness.

4 If, however, the vibrations were wholly transversal, it seems to be admitted that they would not affect the density of the medium. 
ravs ${ }^{1}$; the existence of normal vibrations seems to be proved by the ingenious experiments of M. Jamin, showing that at no angle is light perfectly polarized by reflection. The more we see of these diversities arising in the progress of science, the less are we disposed to found on merely mathematical conclusions from an assumed constitution of elastic bodies ; the more, on the other hand, do we admire the admirable sagacity of Fresnel-the real Newton of modern optics-few of even the least of whose suggestive anticipations have fallen to the ground.

(556.) M. Cauchy as one of the ablest and most prolific mathematical -his vast writers of this century. Besides numerous and imcal labours. portant memoirs, on nearly every branch of pure and applied mathematics, published in the Journal de l'Ecole Polytechnique, and the Memoirs of the Institute, he has published, in a separate form, Exercises des Mathematiques in two series of volumes; and for many years scarcely a weekly meeting of the Academy of Sciences occurred without a mathematical memoir of this prolific author being laid on the table, and subsequently printed in the Comptes Rendus. The integral calculus and other parts of analysis form the subjects of a large part of these writings; but the theory of hydrodynamics in the earlier, and of optics in the later part of his career, are largely represented. So diffuse and desultory a mode of publication has been little favourable to those who wish to make themselves acquainted with what has been accomplished by M. Cauchy. The scientific world is indebted to Abbé Moigno in France, M. Radicke ${ }^{3}$ in Germany, and Professor Powell ${ }^{4}$ in England, for analyzing in part his optical labours. As the present brief notice is evidently inadequate to include even the most superficial view of the whole, I shall say a few words upon two of his theoretical researches on light, which have attracted most general attention. The first is upon the theory of Reflection and Refraction, framed so as to include the phenomena of metallic reflection; the second is upon the Dispersion of Light.

I shall first mention some seemingly exceptional facts to the ordinary laws of polarization by reflec- Theory of tion. As early as 1814 (Philosophical Transactions, reflection 1814 , p. 230), Sir David Brewster had remarked that and refracsuch highly refractive substances as Realgar, Diamond, cluding the and Chromate of Lead, do not polarize, at any angle, case of the whole of the reflected light. Mr Airy afterwards metals. showed that light reflected from diamond near the maximum polarizing angle, possesses qualities resembling those of light reflected from metals. The same view was more generally stated by Mr Dale; ${ }^{5}$ and last of all, M. Jamin showed that all transparent M. Jamin. substances polarize elliptically the light which they reflect, - the difference of "phase" of the two component vibrations increasing from $180^{\circ}$ at a perpendicular incidence, to $360^{\circ}$ at an incidence of $90^{\circ}$; and that the laws of reflection at transparent surfaces, as also in the case of metals, depend upon two constants - the index of refraction and the coefficient of ellipticity. And he has determined in numerous cases the values of these constants.

Thus Fresnel's theory of reflection requires undoubted modification. It only holds true for substances whose index of refraction is nearly $1 \cdot 46$, that is held to arise from the existence of vibrations by $\mathrm{M}$. (called normal) in the direction of transmission of the Cauchy. luminiferous wave, such as those which produce the effects of Sound in air, and which produce certain effects on Light at the bounding surfaces of two media. In the theory of Fresnel, as also in those of Maccullagh and Neumann, this influence is neglected. To Green and to $\mathbf{M}$. Cauchy belongs the merit of laying down a more comprehensive theory. Mr Green's theory, published in $1837^{6}$ (not long before his death), is so far incomplete that it involves only one constant. M. Cauchy's investigations, published two years later, 7 embrace the phenomena of metallic reflection by the introduction of the two constants mentioned above, thus completing the theory of reflection and refraction both for transparent and metallic surfaces.

The fact of the unequal refrangibility (dispersion) of light has ever been felt to be one of the most real as well as prominent difficulties in admitting

- See Moigno Rep. d'Optique, p. 1385. principle as the basis of our reasoning. . . The principle selected as the basis of the reasoning contrined in the following paper is this: In whatever way the elements of any material system act upon each other, if all the internal forces exerted be multiplied by the elements of their respective directions, the total sum for any assigned portion of the mass will always be the exact differential of some function. But this function being known, we can immediately apply the general method given in the Mecanique Analytique, and which appears to be more especially applicable to problems that relate to the motions of systems of an immense number of particles mutually acting on each other. One of the advantages of this method, of great importance, is, that we are necessarily led by the mere process of the calculation, and, with little care on our part, to all the equations and conditions which are requisite and sufficient for the complete solution of any problem to which it may be applied."

A consideration of the candid admissions of the preceding paragraphs (especially the last scntence) will lead the reader to see bow short a way a theory of so general a kind-the chief characteristic of which consists in eluding every troublesome physical enquiry - can go towards explaining the relations of Iight to Matter; yet it may be of use by indicating the kind of solutions which more restricted hypothesis may be expected to give of the laws of phenomena.

7 Comptes Rendus de l'Acad. des Sciences. 
the undulatory theory. That theory, in its simple form, enables us indeed to explain clearly enough the refraction of light owing to a change of velocity in the wave as it passes from one medium to another; but it assigns no reason why that change of velocity should be different for light of various colours, in other words, of different wave-lengths. The corpuscular theory, on the other hand, furnishes at least a plausible explanation, by assuming a variable attraction between refracting media and the molecules composing the different rays.

When, however, undulationists were pressed on the subject, it was easy to see the direction in which at least a plausible explanation might be sought. In the usual form of equation for vibrations in air, given by Lagrange, the integration is effected by assuming the intervals between the particles evanescent compared with the length of a wave. This is perfectly true in the case of sound, and all sounds appear in consequence to travel uniformly. But should it fail in the case of light, that is, should the intervals of the ethereal particles bear some sensible ratio to that very small quantity, the length of a wave, what would be the result? M. Cauchy has made out, by a very complex analysis, that in this case the longer waves will travel most rapidly, and consequently be least refracted. Several other writers, especially Professor Kelland, obtained similar results; and Mr Airy, by very simple, though only approximate, considerations, showed the dependence of refraction on the length of a wave. ${ }^{1}$ M. Cauchy's memoir appeared in 1835 at Prague, in a bulky and abstruse form: the mathematical investigations are very long and complex, the numerical verifications scarcely less so. ${ }^{2}$ The indices of refraction, observed by Fraunhofer, for different lines of the spectrum, in different kinds of glass, together with the corresponding wave-lengths for these rays, formed the principal data for comparison. But others have since been obtained by Rudberg and Professor Powell, and carefully compared with M. Cauchy's theory by the latter, and the coincidence appears satisfactory. ${ }^{3}$ But in estimating the value of this coincidence, it is to be observed that of 7 indices of refraction observed for each substance, 3 must be used to ascertain the constants in the formula, and only 4 remain to be calculated.

One difficulty, however, remains. If the rays of (561.) light travel through space with variable velocities, the Non-disimages of the stars would present tails of colour in- persion of consistent with observation. M. Cauchy eludes this free space. difficulty by the following hypotlesis respecting ethereal media:-The existence of transversal vibrations (according to him) requires that the law of force between particle and particle of ether must not be intermediate between the inverse $2 \mathrm{~d}$ and inverse 4 th power of their distance. In the former case the force is an attractive, in the second a repulsive one. In the first, the velocity of propagation depends on the length of a wave, in the second it is independent of it. [The first case, too, alone will be consistent with permanent longitudinal vibrations.] Consequently if we suppose that in free space the particles of ether are arranged in close order, and exert a repulsive force on each other, no dispersion results; but in refracting media, supposing the distance of the molecules increased, and the mutual action attractive, then dispersion occurs. ${ }^{4}$

\section{\$ 7. Ritter.-Chemical Rays of the Spectrum.-Niepce; Daguerre; Mr Talbot. Art of Helio- graphy or Photography-Daguerreotype-Calotype.-Professor STOKES. Chemical Rays ren- dered visible-Fluorescence.}

A very curious chapter of the history of Light remains to be written, respecting the chemical energies which it is capable of exerting, or which at least are found in those parts of the solar rays which are dispersed by a prism. These are in part luminous and partly invisible under ordinary circumstances, the latter possessing these chemical qualities in a still higher degree than the others. Though not perhaps very closely associated with the optical discussions of the previous sections, it seems impossible to separate this part of the subject from the rest, since the rays called Chemical may, as we have reason to think, be reflected, refracted, polarized, absorbed, and made to interfere like visible light; and farther, because to the extreme limit of their sensible action they may, by certain treatment, be made visible to the eye. The art of photography, though belonging quite as much to chemistry as optics, being a means of inquiry into the qualities of the solar radiations invaluable to the natural philosopher, cannot by any means be excluded from a sketch, however general, of the progress of physical science.

J. W. Ritrer, Professor of Chemistry at Jena, and well-known for his numerous contributions Ritter. to the earlier progress of voltaic electricity, has the merit of having first clearly pointed out in 1801 the separate existence of chemical rays in the spectrum which extend beyond the most refrangible or

1 In all these cases the expression for refrangibility depends on the ratio of the sine of an arc to the arc itself; which are again includes the ratio of $\Delta x$, the distance of the molecules to $\lambda$, the length of a wave. When $\Delta x$ becomes very small, the first ratio becomes unity.

2 A portion of these researches had, however, been printed in Paris (privately, I believe) in 1830.

3 Powell on the Undulatory Theory. 1841.

4 Moigno, Repertoire d'Optique, p. 128. 
violet rays (when the sun's light is decomposed by a prism), in the same manner as the invisible rays of heat were found by Sir Wm. Herschel to extend beyond the visible red. Scheele had indeed previously noticed that the power of the sun's light to decompose and blacken salts of silver increased rapidly from the red towards the violet ray; and there is little doubt that Herschel's discovery suggested that of Ritter, of the independent or non-luminous rays of the spectrum.

(564.) Ritter attributed to these rays a deoxidizing quaChemical lity. Dr Wollaston, who also made experiments on rays of the the subject, and discovered the specific action of the
different rays on gum-guiacum, prudently suggested the more comprehensive term of chemical rays. Various other denominations have been proposed, which need not be here dwelt upon; all that can be said is, that deoxidation does not represent the solar action completely. Whether it be really an independent principle in the sun's rays which causes these effects, or merely light and its modifications, is, as in the corresponding case of heat, yet undecided. But it is remarkable that, as shown by M. E. Beequerel, the discontinuity of the luminous spectrum producing "Fraunhofer's Lines" exists equally for the chemical rays.

(565.) Soon after Ritter's first experiments, Dr Young They in- proved the Interference of the obscure chemical terfere; prays (Phil. Trans., 1803), a conclusion successively polarized. claimed since by different physicists. Berard in 1812 showed that these rays are polarized by reflection. Seebeck observed that the different rays impressed different colours upon salts of silver: and M. Edmond Becquerel long afterwards showed that the red and yellow rays, though incapable of commencing chemical action, in some instances have the power of continuing it when once excited by the more refrangible rays.

(566.) In $1835 \mathrm{Mrs}$ Somerville made some interesting Mrs So-, experiments on the permeability of different bodies merville's to the chemical rays, similar to those of Melloni on experiments. the heating rays (see chap. VI., $\$ 8$ ), and she found great and seemingly capricious variations in this respect.' The account of them was addressed to Arago, and published in the proceedings of the French Academy. She found that green glass, coloured by copper, intercepted entirely the chemical rays, yet this was not due so much to its coloux as to its other qualities (which are also peculiar as regards radiant heat), for the emerald transmits the same rays. Red glass stops most of the chemical rays, whilst the garnet transmits them. Though white glass has generally been considered very transparent for these rays, Mr Stokes has shown that it entirely stops those of the very highest refrangibility, which are readily transmitted by quartz. Sir John Herschel and Mr Hunt have made manyinteresting experiments with coloured media on the particular parts of the chemical spectrum absorbed, but a great deal remains to be done, especially as regards the nature of the substances employed.

But the great impulse given to this subject was derived from the invention or discovery of the beautiful art of Photography.

In $1802 \mathrm{Mr}$ Thomas Wedgewood and Sir Humphrey Davy succeeded in forming pictures of objects laid on paper prepared with nitrate of silver, and in wood and taking profiles (silhouettes) by means of shadows. They proposed to obtain similar effects by means of the camera obscura, but their paper was not sufficiently sensitive. The effectual bar to their proceedings was, however, this : that they could discover no means of fixing the shadows which they had obtained, or preventing the whole surface of the paper from being gradually blackened by exposure to light.

In 1814 J. NicePhore NiePce, a retired proprietor at Chalons sur Saone, ${ }^{2}$ entered into a similar enquiry, but by methods quite different. He employed the solar effect upon resinous bodies, and some at least of his pictures were executed on plates of pewter or of rolled silver. They were mostly copies of engravings, and the light parts corresponded to the lights of the originals. He, however, at length succeeded in fixing impressions of views in the camera obscura, thoughin an imperfect manner, and after very long exposure. The pictures thus obtained had this in common with more perfect processes, that the luminous impression was first brought into view by a chemical process subsequent to exposure in the camera. In 1825 Nicephore Niepce becanie associated with Daguerre, who had previously been engaged in Daguerre. the same research; they agreed to communicate the results of their several experiments. The result, as is well known, was the invention of the DAGUERrEoTYPE, not improperly called after Daguerre, who seems rcally to have worked it out almost entirely for himself, after the death of Niepce in 1833; whilst so patient and determined was Daguerre in
(567.) History o photography.

(568.) Wedge(a) 
(570.)

keeping his secret until brought to perfection, that he did not even show his results until early in 1839 , when the numerous specimens he had to exhibit rivalled in delicacy anything that the art has since produced. ${ }^{1}$

The daguerreotype is depicted in the camera on a plate of silver, conted with an evanescent film of iodide, by exposure for a short time to the vapour of iodine. After the light has acted, and the plate has been withdrawn from the camera, no trace of a picture is visible until it has been exposed to the vapour of mercury, which, by its peculiar action on the places where light has acted, produces a correct picture of the object (or positive image, as it is called, light answering to light). It is then fixed, as it is called, by a bath of dissolved hypo-sulphite of soda, which has the property of removing the iodide of silver wherever the light has not acted. This singular and elaborate process has since been but slightly modified by various plans for rendering the iodide more exquisitely sensitive. To M. Niepce belongs the credit (1) of having fixed an impression of light, (2) of using metal plates, (3) of forming a picture by means of a camera obscura; to Daguerre, on the other hand, the novel and ingenious use of vapours instead of washes, and the whole succession of operations in the daguerreotype. When the French government acquired for the public (the French public, however, only) a right of property in the invention, they marked their sense of the share of merit of the inventors, by awarding to Daguerre 6000 , to M. I. Niepce 4000 francs per annum.

Mr W. H. Fox Talbot, a Wiltshire gentleman of great ingenuity and perseverance, and well acquainted with mathematics and physics, applied himself in 1834 to the problem of fixing shadows, in entire ignorance of what Wedgewood and Davy had attempted. He used paper washed with the nitrate of silver, and soon succeeded in obtaining impressions of lace and leaves of plants, but without fxing the shadows. This great step he, however, made a year or two later: a wash of iodide of potassium, or of common brine, was found to effect it. The announcement of his success and of his methods was called forth early in $1839^{2}$ by the first reports of Daguerre's discovery. His method then consisted in dipping writing paper alternately in nitrate of silver and common salt, drying between the operations, and afterwards fixing the image. An unnatural or negative picture was thus obtained, the lights of na- ture being darkened on the paper, and vice versa; but the truth was restored by pressing the drawing thus obtained against a second prepared sheet of paper, and exposing it to light, when the natural lights and shades were of course obtained. This derived impression is called a positive. This process has evidently several advantages over Daguerre's ;such as, that paper is used instead of metal plates; and that from a single impression (negative from nature) copies may be at leisure indefinitely multiplied. Pictures were thus obtained by $\mathrm{Mr}$ Talbot with the camera obscura.

Mr Talbot's chief improvement on his first method he called the Calotype (1841), and consisted in Progress. washing the paper successively with nitrate of silver, iodide of potassium, ${ }^{3}$ and gallo-nitrate of silver. It is then exposed in the camera, but no impression appears until again washed with the gallo-nitrate of silver. It is then fixed with bromide of potassium, or with hypo-sulphite of soda, as in Daguerre's process.

By a subsequent invention, $\mathrm{Mr}$ Talbot has obtained what he justly calls an instantaneous process. ${ }^{4}$ An Instantaimage was formed in a camera of a revolving wheel, neous proto which was affixed a printed bill; the room being darkened, and the wheel made to revolve with the speed of 200 revolutions in a second, and being then illuminated by an electric spark, a legible impression of the printing was obtained. We doubt if, in the whole history of physics, a more astonishing result is recorded. Thus Mr Fox Talbot, by his rare energy, brought his iuventions almost to perfection. Numerous competitors, of course, appeared on the field, and obtained many interesting results. The only one of much importance to the art of photography is the substitution of a film of iodized collodion on a glass plate, for the prepared paper in the first or negative process.

The daguerreotype and calotype processes, though seemingly so different, have much in common:-(1) Analogy a sensitive surface has to be prepared (iodide of silver of the two is the basis in both); (2) it is exposed in the camera ; (3) the picture (still invisible) is developed; (4) it is fixed. In the calotype the printing process for obtaining positives must be added to these.

The chemical theory is very far behind the art of phe most important steps of these Chemical photography. The most important steps of these theory imtained by a kind of divination after a multitude of ${ }^{\text {perfect. }}$ failures. The salts of silver are of a highly decomposable nature, the iodides and bromides pecu-

\footnotetext{
1 The present writer had the benefit of seeing Daguerre's marvellous productions, and making his acquaintance at Paris, through the courtesy of M. Arago, while the secret was still preserved, and the public interest was excited to the highest pitch. A bout the same time he saw M. Isidore Niepce, son of the first photographer, and the specimens in his hands, as well as those in the possession of Mr Bauer of Kew, with whom they had been left by M. Nicephore Niepce, when he visited England in 1827 and exhibited them at the Royal Society. One of the latter was engraved on a plate resembling pewter.

2 Communicated to the Royal Society of London, 3lst January, and printed in the Philosophical Magazine for March.

3 Mr Talbot says (Phil. Mag., March 1839, p. 203) that Sir H. Davy had recommended iodide of silver as a sensitive substance; but in his ea:lier experiments he had found it the contrary. But now, like Daguerre, he requires no inmediate visible action of light, but developes it by a subsequent reaction.

4 The process is described in Hunt's Researches on Light, 2d edit., p. 140.
} 
liarly so. The action of light appears to be to reduce the metallic silver, at least partially, an operation which is completed in the daguerreotype process apparently by the affinity of the mercurial vapour for iodine and for oxygen. ${ }^{1}$

(576.) If anything were wanting to show the impossibility Importance of separating the scientific arts from the history of of the pho- science itself, it would be the case before us. The tographic art of photography is far in advance of the theory, art. art it constitutes in itself the greater part of what we know in a highly interesting branch of Natural Philosophy-the chemical agency of the spectrum. The surfaces of Talbot and Daguerre are the philosophical tools by which farther discoveries can alone be made.

(577.) So intimately connected with the discovery of the Chemical Chemical Rays of the spectrum, is that of revealing rays ren- their existence (at least the existence of rays of the sible.
spectrum equally refrangible with those possessing chemical properties) to the sense of sight, that we may conveniently include in this section a very brief notice of Professor Stokes' remarkable experiments.

(578.) Mr George Gabriel Stokes, a fellow of Pembroke Professor College, Cambridge, and senior wrangler in 1841, Stukes. at present holds the distinguished position of Lucasian Professor of Mathematics. It is almost needless, therefore, to state that his inathematical talents are generally acknowledged, and that he has displayed them by a ready application to nany difficult problems in optics and mechanics which had not previously been accomplished. I may refer, however, to the integration of complex differential equations occurring in the theory of the flexure of solids (see art. 364 ), and in that of the rainbow (466), and in his elaborate investigation of certain cases of the friction of fluids (416). But it is more to our present purpose to observe that he combines this profound and technical command of analysis with singular skill in the experimental department of optics; - not merely in investigations closely connected with the wave theory, and expressible by mathematical formulæ, ${ }^{2}$ but in those which depend on the judicious questioning of nature by critical experiments not necessarily quantitative;-such, in short, as Newton discusses in his Optics; and indeed since Newton himself occupied the Lucasian chair, there have been perhaps few philosophers who have shown so remarkable an aptitude for both kinds of research.

(579.) In 1852 Professor Stokes announced that the reShows that frangibility of light, which has hitherto been consithe refran- dered its most inherent and invariable quality, may libht may in some circumstances be altered. The fundamental bechanged. experiment is this:-A beam of solar light is caused to form a pure and highly dispersed spectrum by Fluorespassing through two or three prisms in succession, cence. associated with a lens. The spectrum is made to fall on a glass vessel containing a solution of sulphate of quinine (a colourless fluid). Whilst the red, orange, and indeed the greater part of the luminous spectrum, pass through as if the fluid had been merely water, from about the middle of the violet "the path of the rays is marked within the fluid by a skyblue light, which emanates in all directions from the fluid, as if it (the medium) had been self-luminous." This appearance extends far beyond the visible violet, the presence of the invisible rays (called chemical) becoming disclosed by the reaction of the quinine; and the dark bands, both of the visible and the (usually) invisible spectrum, are marked by obscure planes traversing the mass of diffuse light. When the light thus emanating from the interior of the quinine is examined by a prism, it is found to consist of rays of various colours and refrangibilities corresponding to those of the ordinary spectrum ; from which Mr Stokes concludes that, since only violet rays and the invisible rays beyond them could have furnished or excited this emanation, the rays of light themselves have been transformed into others, with a lower degree of refrangibility, and possessing the corresponding optical properties. In conformity with this explanation, Professor Stokes has considered and accounted for a number of curious phenomena described by Sir David Brewster and by Sir John Herschel ; called by the former " internal dispersion," by the latter " epipolic dispersion," all of which, so far as they involve anything peculiar, may be reduced to the general principle that certain bodies by their action on light have the power of lowering the refrangibility of the rays incident upon them, whether belonging to the visible or invisible spectrum-that is, of emitting rays of a lower, while under the excitement of rays of a higher refrangibility. Hence the phenomenon has been termed the "degradation" of light. Mr Stokes has also called it "fuorescence," a word involving no hypothesis, being derived from the characteristic action of a certain kind of fluor-spar described by Sir David Brewster.

Many substances, solid and fluid, are found to pos- (580.) sess these qualities. ${ }^{3}$ Ainongst others, and in the Substanees highest degree, glass coloured yellow by oxide of possessing uranium, called in commerce canary glass, and solu-perty. tions of horse-chestnut bark.

By using sources of light richer than sunlight in (581.) the highly refrangible rays; by also using quartz for Remarkthe prisms instead of glass (which has the power ment with of absorbing these to a considerable extent)-Pro- electric

I I may refer to a short but clear paper on the theory nnd practice of photography by the Rev. W. T. Kingsley, in the Journal of the Photographic Society, vol. i., August 1853. Mr Kingsley is one of the most scientific and practical improvers of the art.

2 This he has also done in a beautiful paper on the effect of polarization in modifying the phenomena of diffraction Camb. Trans., vol. ix.

3 The greater part of these bad been detected by Sir D. Brewster, with his usual acuteness and perseverance, in the course of his researches on "internal dispersion." The colouring matter of green leaves is one of the most remarkable. 
fessor Stokes has obtained a result truly astonishing. In the case of the light derived from a voltaic arc, produced by the battery of the Royal Institution with metallic poles, the visible spectrum formed upon uranium-glass extended no less than six or eight times the length of the ordinary spectrum. If, by way of contrast, a porcelain tablet be used as a screen, the spectrum terminates at the usual point. ${ }^{1}$

(581.) [I have already (art. 471) expressed my regret Mr Wheat- that the limits of this Essay prevent me from devotion-the ing a section to the physiological part of Optics; itereo- but I cannot close the chapter without at least naming Mr Wheatstone's beautiful invention of the Stereoscope, as by far the most interesting contribution recently made to the theory of vision, regarded in a point of view not strictly anatomical. Although Mr Wheatstone's paper was published in the Philosophical Transactions for 1838 , and the Stereoscope became at that time known to men of science, it by no means attracted, for a good many years, the attention which it deserves. It is only since it received a convenient alteration of form (due, I believe, to Sir David Brewster), by the substitution of lenses for mirrors, that it has become the popular instrument which we now see it, but it is not more suggestive than it always was of the wonderful adaptations of the sense of sight.]

\section{CH A P T E R V I.}

\section{HEAT, INCLUDING SOME TOPICS OF CHEMICAL PHILOSOPHY.}

\section{§1. BuACK.-Latent and Specific Heat.-Irvine.-Hutton.-Doctrines of Heat applied to some Natural Phenomena.}

Down to the close of the 18th century, the science of Heat was studied and advanced mainly by chemists, and it was in all respects treated as a branch of Chemistry ; a position of which we still find traces in the introduction of the doctrines of heat (even of radiant heat) into most of our approved treatises on Chemistry. This circumstance brings us, in this chapter, into close contact with the most illustrious chemical names of the second half of the last century and of the first years of the present. Such were Black, Cavendish, Lavoisier, and Dalton. Of the last and two first it may be doubter whether they were not as prominent discoverers in Physics as in Chemistry. Davy occupies a similar position. It was not, indeed, until the 19th century had made some progress that Chemistry assumed a strongly distinctive position of its own, and began to attain that large development and complex character of detail which render it a science now hardly accessible to those who do not devote to it their almost undivided attention. In the days of Black and Cavendish it was otherwise; and in the first section of the present chapter I shall attempt to give an outline of the characters of the very remarkable men who then advanced simultaneously the doctrines of Physics and Chemistry ; referring, of course, chiefly to the former, but not entirely to the exclusion of the latter portion of their researches, particularly with respect to the atomic and gaseous theories of Dalton, which have a strongly physical aspect. I have elsewhere noticed the barrenness of the greater part of the 18th century in contributions to the experimental sciences; the temptation is therefore the greater to dwell a little even on the personal history of men so celebrated and influential as Black, Cavendish, and Dalton.

JoSEPH BLACK was born at or near Bordeaux in France, in 1728. His biography, little eventful and Black-his almost exclusively academic, has been recorded in eminence some detail by his companions and friends Adam mist. Ferguson and John Robison (the former of whom was a relation), in the preface to the posthumous publication of his Lectures on Chemistry. It is sufficient for me to state that he entered the University of Glasgow as a student in 1746. Being destined for the medical profession, he removed in $\mathbf{1 7 5 0}$ or 1751 to Edinburgh, where be benefited especially by the lectures of Cullen, a most eminent physician, and the author of a beautiful experiment on the cold produced during evaporation. Before Black graduated (in 1754) he had entcred upon a course of chemical experiments connected with the causticity of many earthy bodies, which ended in his first (and perhaps most famous) discovery of the existence of fixed air or carbonic acid gas as an essential constituent of marble and other solids, together with a train of important consequences. Few inaugural dissertations have been so interesting to science as that on Magnesia, printed at Edinburghin 1754, which contained these results. But on this purely chemical question we will not enlarge. 
(584.) His disco. veries of latent and specific heat.
The discoveries of Black with which we are chiefly concerned are those of latent and specific heat. The former, at least, is Black's sole and exclusive property. When we look back to the state of the science of heat in the first half of the 18th century, we are surprised at the exceeding slowness of its progress. The thermometer had been improved by the use of the freezing and boiling temperatures of water for its fixed points, and the adoption of mercury in its construction by Fahrenheit; the correspondence of its scale with true increments of heat had been tested, though imperfectly, by Brooke Taylor; and Dr Martine of St Andrews had published an ingenious work (the best of its period) on the expansion of different liquids, and on some kindred subjects; but in general no great step was made until Black, in 1757 , or previously, ${ }^{1}$ concluded that during the melting of snow or ice, a great quantity of heat enters into the body without affecting the thermometer in an appreciable degree. The heat therefore spends itself or is absorbed in effecting liquefaction. Black called it latent (as opposed to sensible) heat. He was led to this discovery by the very simple observation of the extreme slowness with which ice is melted by the applieation of an amount of heat which would have raised the temperature of water to an enormous extent; the thermometer plunged in the thawing ice remaining stationary until it is entirely reduced to water. When that occurs, heating immediately commences according to the usual laws. One of Black's original experiments clearly illustrates his mode of procedure. $\mathrm{He}$ suspended equal weights of ice at $32^{\circ}$, and of water as near as might be at the same temperature, in two thin glass vessels 18 inches apart, in a spacious room having a temperature of $47^{\circ}$. The vessel containing water rose $7^{\circ}$ in temperature in half an hour, but the equal weight of ice had not wholly melted, nor had its temperature even slightly increased until 21 half hours had elapsed. Whence Black concluded (approximately) that as much heat is requisite merely to thaw ice as would raise an equal weight of water through $7 \times 21$ or Its amount. $147^{\circ}$; a result almost corrrect, although the experiment in this form is manifestly not unexceptionable. The converse process of freezing shows how prolonged must be the application of cold to discharge water at $32^{\circ}$ of its latent heat, or heat of liquefaction, and to convert it into ice.

Nothing is more admirable in these results than the light they throw upon certain natural processes. Were instant liquefaction the result of the smallest application of heat to snow at $32^{\circ}$, we should many times a year be the victims of uncontrollable floods; and did water instantly become ice on its temperature reaching the freezing point, our lakes and rivers would be rapidly consolidated to the very bottom on occasion of every frost, and animal life would be impossible.

The analogy of the gradual formation of steam in boiling, to the gradual liquefaction of ice, was so (586.) evident as to lead Black to conclude, without any heat of special experiment, that a great deal of heat becomes vapour. latent during the conversion of liquids into vapour. It appears to have been in $\mathbf{1 7 6 2}$ that he attempted to determine roughly the amount, by a method similar to that just described for the heat of liquefaction. He compared the time required under the action of a uniform source of heat to raise the temperature of a certain quantity of water from $50^{\circ}$ to the boiling point, with that required to boil it away; and inferring that heat was continually combining with the water at the same rate, he estimated the latent heat, or heat of vapour, to be as great as would have raised the temperature of the water had that been possible by $810^{\circ}$. This was in October $1762 ;^{2}$ about two years later his pupil, Irvine, made experiments under his direction with a still and refrigeratory, by which Experihe estimated the heat given out by steam during its Irvine and reconversion into water. $\mathrm{Mr}$ Watt prosecuted the Watt. same inquiry soon after. ${ }^{3}$ How happily $\mathrm{Mr}$ Watt applied the doctrine of latent heat thus brought experimentally under his notice, to the improvement of the steam-engine, has been already recorded.

One thing strikes us very much on a review of these discoveries of Black; it is the great interval (587.) which separates the clear perception of a fact from singularly the explanation of it; or, in other words, from the overlooked clear expression of the more general fact which em- Black. braces it, although when once given, the explanation may seem to be almosi expressed in the very enunciation of the fact. Nearly a century before Black, the lynx-eyed Hooke had noticed that water during the process of congelation remained unaltered in temperature, and that the same takes place when it boils, and this observation had been numberless times verified in ascertaining the fixed points of thermometers; yet no one before Dr Black had even guessed that the heat which enters into ice during liquefaction, and into water during vaporization, remained as it were a constituent of the water and the steam thus formed, and could at any time be recovered by a converse process. Numberless instances occur in the history of science in which it requires the utmost attention to appreciate the importance and difficulty of drawing such seemingly obvious conclusions.

The other great discovery of Black, Specific Heat, was in like manner a correct interpretation of Specific a fact already known. Boerhave and Fahrenheit heat. had found that when quicksilver and water were mixed together, their temperatures being different, the heat of the mixture was not, as might have been expected, the average of that of its ingredients, whether 
the volumes or the weights of those ingredients were equal. "Quicksilver, whether it were applied hot to cold water or cold to hot water, never produced more effect in heating or cooling an equal measure of the water than would have been produced by water equally hot or cold with the quicksilver, and only two-thirds of its bulk." Of course, when equal weights were used, the inequality of effect was still more striking, for, from the great density of quicksilver, it required no less than 30 times the weight of the water mixed with it in order that it should contribute in an equal degree to the production of a mean temperature. Strange to say, the interpretation of this important experiment remained to be made by Dr Black after half a century. He taught that temperature is an effect of heat, which is neither the same in all bodies nor in the same body under differing circumstances; that the superior effect of the water to the mercury in determining the temperature of the mixture was caused by the fact, that it is the nature of quicksilver to require a smaller anount of heat to raise its temperature through one degree than an equal volume or weight of water would require under like conditions. Black made many experiments to satisfy himself of the constancy of this property in bodies; and with the assistance of Irvine, ${ }^{1}$ probably ascertained its numerical value (the amount of heat necessary to raise an unit of mass of water through one degree of temperature being the standard) in different cases; but he left the subject chiefly in the hands of that observer and of Watt. The former gave the name of capacity for heat to this property, which was afterwards more happily termed specific heat by Gadolin, who made many experiments on the subject, as did also Cavendish and Adair Crawford whose theory of animal heat, published in 1778 , turns entirely on this property of bodies.

Irvine had the merit, such as it was, of proposing a theory on which his term capacity for heat was principally founded, which occasioned for many years after, although now comparatively forgotten, much discussion. He assumed that the changes in the temperature of bodies, whether by alteration of their mechanical condition or by chemical combination, were due to a change in the capacity for heat of the substance or the mixture. He also assumed that the total amount of heat contained in a body is proportional to the amount of heat necessary to raise its temperature through one degree (for example, a pound of mercury contains altogether one-thirtieth part of the entire heat contained in a pound of water). From these principles he deduced the temperature of absolute zero or privation of heat as follows:-When sulphuric acid and water are mixed together, the tempcrature rises. This rise, according to him, is caused by the capacity for heat of the mixture being less than the average due to its ingredients. He has, therefore, got the ratio of the whole heat in the bodies before mixture and after mixture. He has also got the number of degrees of temperature corresponding to this difference. Having the ratio of these quantities, and also their difference, the quantities themselves, or the whole amount of heat expressed in degrees of temperature before and after mixture, become known, and the temperature of absolute privation of heat is also known. He applied the same reasoning, with great ingenuity, to explain the latent heat of liquids and vapours, which he ascribed to their increasing capacities. He thence deduced other values for the absolute zero; but whereas all these determinations ought to have agreed, at least approximately, they were found by later experimenters, especially by Lavoisier and Dalton, to differ so widely-even by several thousand degrees-that, since the time of the latter, this ingenious theory has been nearly abandoned, at least as far as the search after the zero is concerned; although undoubtedly change in the specific heat of bodies is often an important element in determining their temperature.

To return to Dr Black. From 1756 to 1766 he (590.) filled the chair of Chemistry and Medicine at Glas- Black as a gow, where he also practised as a physician. In lecturer1766 he left Glasgow for Edinburgh, to succeed Dr ter. Cullen as Professor of Chemistry - a position which he held, with great credit to himself and with benefit to the University, till his death in 1799. His health, during the greater part of that time, was feeble, owing to a pulmonary affection, which often interrupted his lectures, and, it is stated, prevented him from engaging in severe study without immediate injury. Though he published one or two papers during these thirty years, they were of comparatively trifing importance. His influence on science was chiefly exerted through the medium of his pupils and of his intercourse with general society. His lectures are described by those who had the good fortune to hear them as inimitable of their kind-grave, dignified, and so interesting as to rivet the attention. "Perfect elegance as well as repose was the phrase by which every hearer and spectator naturally, and as if by common consent, described the whole delivery."2 It is probable, also, that in his private intercourse with his pupils, he inspired them with that love of research which distinguished his own early days, and his taste for neat and accurate experiment could hardly fail of being imparted to a certain extent. Yet we may be permitted to regret that his constitutional indolence, almost apathy, had perhaps as great a share as bad health in the interruption of his career of discovery. Even when quite a young man, his most interesting conclusions were so gradually evolved, that he himself had difficulty afterwards in fixing their date; and some of them were delayed even for years, until he found time to 
make the requisite experiments. So cool a temperament was not likely to grow warmer as age advanced. Almost as indifferent to the honours of discovery as his stoical contemporary Cavendish, unlike him he enjoyed in a high degree intercourse with the congenial society which Edinburgh at that time afforded. He loved to converse infinitely better than to write; especially when he could converse intimately with such men as Adam Smith, David Hume, Adam Ferguson, Principal Robertson, Dr Jolın Robison, John Home, Clerk of Eldin, and Dr Jarnes Hutton.

(591.) Hutron was perhaps Black's dearest friend, and Dr Hutton may be mentioned here (episodically) as no ordinary intimate thinker in Natural Philosophy, as well as in Geology friendhis theory of rain and vapour. and Metaphysics. Besides the "theory of the Earth," which will ever bear his name, and which, after various transmutations in name and form, is now by far the most widely prevalent, his theory of Rain was an ingenious and important speculation. Other branches of Meteorology also claimed his attention, particularly, as might have been expected, those which are connected with the temperature of the earth. He was one of the first who drew conclusions from the temperature of springs, with regard to change of climate due either to increased latitude or to increased height above the sea. His hygrometer, in which the dampness of the air was estimated by the coolness due to evaporation, was unquestionably the first suggestion of a method now in general use. His ideas on the constitution of matter were bold and ingenious, though not on all points tenable. They resembled those of Boscovich, though independent of them. He published a voluminous treatise on several subjects in Natural Philosophy, and a still more formidable one on the Principles of Knowledge, neither of which attracted much attention at the time, and have been long forgotten; yet it is not unlikely that some of his speculations in metaphysics might be worth the labour of re- examination. His friend and commentator, Playfair, (whose style was as remarkable for perspicuity as $\mathrm{Dr}$ Hutton's was the contrary) has drawn the following lively contrast between the characters of Hutton and Black, which may properly conclude this notice:"Ardour and even enthusiasm in the pursuit of Contrast science, great rapidity of thought, and much anima- of Black tion distinguished Dr Hutton on all occasions and HutGreat caution in his reasonings, and a coolness of head which even approached to indifference, were characteristic of $\mathrm{Dr}$ Black. On attending to their conversation, and the way in which they treated any question of science and philosophy, one would say that Dr Black dreaded nothing so much as error, and that Dr Hutton dreaded nothing so much as ignorance; that the one was always afraid of going beyond the truth, and the other of not reaching it. The curiosity of the latter was by much the more easily awakened, and its impulse most powerful and imperious. With the former, it was a desire which he could suspend and lay asleep for a time; with the other, it was an appetite that might be satisfied for a moment, but was soon to be quickly renewed. . . . . Each had something to give which the other was in want of. Dr Black derived great amusement from the vivacity of his friend, the sallies of his wit, the glow and original turn of his expression; and that calmness and serenity of mind which, even in a man of genius, may border on languor and monotony, received a pleasing impulse by sympathy with more powerful emotions."1

Black died on the 6th December $1799 .^{2}$ His death, (592.) as recorded by his kinsman, Adam Ferguson, ${ }^{3}$ was Black's one of the most touching on record. It succeeded his death. customary state of health by an interval inappreciably short, and, as appeared by the accompanying circumstances, without the slightest physical emotion. The philosophic composure of his whole life was mirrored in the serenity of its close.

\section{§2. Cavendish. ${ }^{4}$ - His Singular Character and Attainments-Eminent Chemical Discoveries- Observations on Heat and on other Branches of Physics-LavoIsIER-The Calorimeter- Theory of Combustion and of Oxidation.}

Cuvier has justly remarked, in his biography of

(593.) Cavendish, that he had to struggle in his scientific Cavendish. career against obstacles much more rarely encoun-

which beset the progress of genius cramped by poverty and neglect. Cavendish was the descendant of one of England's noblest families, and he was likew ise tered, and perhaps less easily overcome, than those the possessor of enormous wealth; yet neither of

\footnotetext{
I Playfair's Biographical Account of Dr James Hutton, Works, vol. iv.

2 Muirhead's Correspondence of James Watt. Introd., p. xxii. ${ }^{3}$ Preface to Black's Lectures, by Robison, p. Ixxiv.

4 I find an apology almost necessary for introducing at some length the biography of Cavendish into a chapter professedly on Heat, his positive discoveries connected with which were less notable than in some other departments. But besides that his position in the first rank of chemists naturally indicates his place to be between Black and Dalton, I felt a wish to bring out the relief of the striking intellectual characteristics of those three remarkable men, by placing them in juxtaposition. I may add that these three sections were the earliest written of this Dissertation, at a time when I had hoped to interweave into its composition more of the purely biographical character of each period of scientific history than I found it afterwards practicable in all instances to carry out. I trust, however, that it may be found a not unwelcome variety amidst the abstruser details of science. In the case of Cavendish, too, so various are his claims on our notice, that it was inevitable to recur to them in different chapters, especially in those on Astronomy and Electricity. It was not, therefore, really material under which bead the more strictly personal details were given to which $[$ have alluded.
} 
these powerful temptations could withdraw him even for an lour from the course of study which he had marked out; and which constituted for him at once labour and relaxation, the end of living, and almost life itself.

The Honourable Henry Cavendish, son of Lord Charles and Lady Anne Cavendish, was born at Nice, October 10, 1731.1 He entered St Peter's College, Cambridge, 24 th November 1749, where he resided during the usual terms for above three years, when he ought naturally to have graduated, which, however, he never did. That he had pursued at least his mathematical studies with ability and success is, however, nearly certain from the firm hold which he ever after retained of them. He joined the Royal Society of London in 1760, and published his first paper in their Transactions in 1766. From the time of his leaving Cambridge for some years his personal history is not known, though he probably resided in London.

The subsequent history of Cavendish is the history of his studies and his discoveries. The latter were published in concise memoirs, written with scrupulous precision, and all printed in the Philosophical Trans actions. If collected (which they have not been) they would fill but an insignificant volume, yet include all the requisites to establish a first-rate reputation. His studies no doubt were enormous, for they occupied every disposable moment of a life prolonged almost to fourscore. They may be guessed at (in addition to the published results) from his manuscript remains, a few of which have been edited recently, but the larger part remains in manuscript in the possession of his heirs. Few if any branches of exact science were unfamiliar to him; and his published papers include astronomy, mechanics, electricity, heat, chemistry, and meteorology, besides which he cultivated mathematics and geology. His reputation is one of those which may be called in a peculiar sense European or universal, because it marked a great epoch in science to which the publication of his writings materially contributed. That epoch was when chemistry became a science of weight and measure. Cavendish was a weigher and measurer almost by nature, and entirely so by habit. It appears from his note-books that he took the most scrupulous pains to ascertain and record the quantities of the ingredients employed in every experiment, even though they might be immaterial to the result; and his whole life was methodical in the same proportion. The immense importance of Cavendish's labours to the progress of science in his day is found in the unanimous testimony of his contemporaries ; and notwithstanding the extreme retirement in which he lived, and the rarity of his appearances as an author, he was generally regarded as perhaps the leading man of science in England of his day; and his good opinion was considered by contemporary philosophers as their highest praise. Sir Humphry Davy's opinion of him (recovered and published by Dr Davy from a manuscript lecture) represents that of such of his countrymen as were qualified to form one, and the writer of it was usually fastidious, in his judgments of others :- "It may be said of Mr Cavendish, what can perhaps hardly be said of any other person, that whatever he has done has been perfect at the moment of its production. His processes were all of a finished nature. Executed by the hand of a master, they required no correction; and though many of them were performed in the very infancy of chemical philosophy, yet their accuracy and their beauty have remained amidst the progress of discovery, and their merits have been illustrated by discussion, and exalted by time."

After this eulogium of so competent a judge, I shall be satisfied by a simple enumeration of those Chemical chemical discoveries which were the most splendid discoveries results of his career, but which hardly come within dish. the immediate scope of this dissertation. To him we are inainly or entirely indebted for the knowledge of hydrogen as a distinct elastic fluid or gas; of the exact constitution of the atmosphere, and the won. derful constancr of its ingredients ; of the composition of nitric acid; and finally, according to the opinion of most persons (at least till lately), of the non-elementary nature of water, and of its precise constituents. This last and crowning discovery has indeed been contested, and made the subject of a prolonged and bitter scientific controversy, which hardly could be said to exist until the contemporary generation who witnessed the facts, and also the succeeding one, had passed away. For Cavendish received until his death, and for nearly thirty years after, the unquestioned tribute of at least the primary merit in so great a step in science. The topics more peculiar to the present essay (sufficiently extensive besides) enable us to dispense with the unwelcome task of once more analyzing a controversy purely personal, and which has almost filled volumes. I shall content myself with stating two considerations, which must have great weight in turning the balance of the evidence (supposing it balanced) in Cavendish's favour. The first is founded on the behaviour of Watt himself; the second on the known character of Cavendish.

As to the former consideration, I would remark that Watt withdrew the letter to Priestley which he had (597.) submitted to the Royal Society and which contained versy on his views respecting the composition of water, before discovery it had been read at the meetings of that body. ${ }^{2}$ The position of causes of this suppression are candidly stated by water. Watt in a letter to Sir Joseph Banks, published by Mr Muirhead, ${ }^{3}$ in which he states that he " thought

1 Life of Cavendish, by Dr George Wilson; a valuable biography, which has been printed in the series of publications of the "Cavendish Society," and thus unfortunately has had but a limited circulation.

2 Correspondence on the Composition of Water, p. 30. 
Argument it prudent to delay the publication until he should from have considered it [the theory of the composition Watt's be- of water] more maturely, and have made some exhaviour. periments to determine the proof or falsehood of it." This, it must be owned, was not the language of a man who had acquired that amount of conviction which is needful,-not to broach a theory,- - but to hold fast by it against all opposition. With the caution which formed part of his character, Watt was unwilling to hazard his reputation by adhering to a doctrine which appears at the time to have received no support from his scientific friends, especially Dr Black. Having read the correspondence published by $\mathrm{Mr}$ Muirhead, I cannot doubt that Watt, whatever his private opinions might continue to be, would never have urged his views on an unwilling public, but would have finally suppressed the letter to Priestley, had not the experiments and claims of Cavendish at home, and of Lavoisier in France, reanimated all his zeal for the assertion of his opinion. This indeed Watt ingenuously admits in the letter to Sir Joseph Banks just cited, where he states that the fact of similar theories having since been supported by philosophers of first-rate abilities, removed his second objection to publication. As the suppression of his paper would have relieved Watt of all the responsibility of error, it seems impossible to allow him the advantage - of which that suppression deprived him-of anticipating the date of his matured conviction; and to this conviction we have his own evidence that Cavendish's publication as well as certain additional experiments of his own influentially contributed. Watt, in after life, may be said to have tacitly relinquished to Cavendish the honour which, in the first irritation of the conflict of their claims, he showed nodisposition to do; it is, therefore, reasonable to infer that, on reflection, he saw good reasons for doing so. By this I mean that he suffered judgment to be passed in favour of Cavendish's claim in the writings of many of his eminent contemporaries, without attempting publicly to correct the all but universal impression which they made. In one instance, he almost homologated this adverse judgment:- In the article on Steam, written by Robison, and revised by Watt in his last years and after Cavendish's death, this passage appears, - "This is fully evinced by the great discovery of Mr Cavendish of the composition of water;" I from which it must be concluded, first, that Robison, the intimate friend of Watt, and the almost chivalrous defender of his fame, believed Cavendish to be the true discoverer ; ${ }^{2}$ secondly, that Watt, in commenting on this article in 1814, permitted the fact to be thus transmitted to posterity. For, in his numerous animadversions on other parts of the same papers, he gives (as I have pointed out in the note to art. 318) free expression to the sensitiveness which he felt lest Dr Black should derive any credit to which he was not entitled in connection with the steam-engine, but he suffers the passage just quoted to pass without remark. Such being the case, and waiving all purely chemical discussion, I an of opinion that Watt's friends should have left the matter as he was content to leave it.

With reference to the argument from Cavendish's character, I would remark that whilst the claim of Argumer Watt cannot be maintained without impeaching the from the honour and integrity of his rival, and showing that he of caven. stooped to subterfuge in order to appropriate to him-dish. self a discovery due to another-it would yet be difficult to find in the whole range of scientific history (without excepting the venerable name of Newton), an individual so devoted to knowledge for its own sake, so indifferent to the rewards of discovery, so averse to the publication of what he felt to be important, and knew to be original, so insensible to the voice of praise when applied to himself, so ardent in acquainting himself with the labours of others, and so liberal in assisting them. Such a man was Cavendish; and that he should stoop even to the common artifices of little minds for exalting his own reputation at the expense of others, would itself be incredible; how much more the insinuation (grounded solely on alleged circumstantial evidence) that in doing so he disregarded the plainest dictates of honour and justice.

Black and Cavendish were nearly contemporaries, the former having been born only three years earlier. But I doubt whether these two men, so congenial in their studies, so different in almost every circum- on latent stance, whether of fortune or temperament, ever met. and speci They had this, however, in common, that they published their discoveries and observations with reluctance, and were fastidious in the manner of doing so. Black's experiments, both in chemistry and on heat, preceded those of Cavendish, and with regard to the latter subject (heat) it is probable that Cavendish pursued investigations and made original experiments, in which he had been unknowingly anticipated by the Scotch professor. His researches on latent and specific heat (though he did not employ these terms) appear to have been subsequent to Black's, but to have preceded those of Crawford and Wilcke; and it is not impossible that his was the earliest, or one of the earliest, determinations of the amount of heat absorbed in the conversion of water into steam, for which he obtained various results between $923^{\circ}$ and $982^{\circ}$, a mean of which would be very near the

\footnotetext{
I Robison's Mechanical Philosophy, ii., 21.

2 The article WATER in the early editions of the Encyclopadia Britannica has been quoted as the production of Robison, of which there does not appear to exist any proof, whilst the probability, as shown in the text. lies all the other way. I learn upon the best authority that the proprietors of this Encyclopocdia have no clue to the authorship of that article, and that it is not included in the lists of Robison's known contributions. The part relating to the present question was expunged in the Fourth Edition, and a reference made to the article Chemistry where Cavendish received the credit of the discovery.
} 
truth. But it is needless to dwell upon these observations, however original, because they were voluntarily suppressed by the author, and have only recently been brought to light from his manuscripts. ${ }^{1}$ What he did publish in connection with this subject was a paper on the construction and graduation of meteorological instruments, especially thermometers, and others on the temperature at which mercury freezes, and on freezing mixtures. The former of these papers was, as might be expected, far in advance of its age in the degree of exactness which was shown to be attainable in the construction of thermometers, and scarcely even now can it be considered as obsolete. The papers on freezing mercury finally corrected the exaggerated notion at first entertained of the extreme cold at which that metal becomes solid, and also contain valuable views on the subject of congelation, and fixed the latent heat of water at $150^{\circ}$. He calls this, "generation of heat" during liquefaction, objecting to Black's term as relating " to an hypothesis, depending on the supposition that the heat of bodies is owing to their containing more or less of a substance, called the matter of heat; and as I think Sir Isaac Newton's opinion that heat consists in the internal motion of the particles of bodies much the most probable, I chose to use the expression, heat is generated." 2

Two of Cavendish's most important researches re(600.) fer to the attraction and density of the earth, and to the we earth's mathematical theory of electricity. The former (which, traction, in principle, was derived from the Rev. John Michell) felectri- has been already analyzed in the chapter on Astroity. nomy (art. 156). The latter will be more conveniently referred to in our chapter on Electricity.

Cavendish's publications extended over the greater part of his active life, but those on chemistry and electricity, on which his fame principally depends, do not extend beyond the year 1775 ; the date of his paper on the density of the earth is 1798 . He died 24th February 1810, at the age of 79 . He appears to have.exercised scarcely less influence by his general derotion to science, than by his specific discoveries, great and original as they were. In 1782, when Playfair met him incidentally in London, he described him as being generally looked up to as one possessed of talents confessedly superior, and as the only nember of the Royal Society who then united the knowledge of mathematics, chemistry, and experimental philosophy. The absolute devotion of his life to inquiries the most abstractly scientific, whilst he showed an entire indifference to the luxuries which his wealth might have commanded, and the social station to which his birth entitled him, could not fail to inspire respect for his character, as well as to obtain the homage of mankind for pursuits so dignified and so generally disregarded. Many curious aneedotes are related of the annoyance which the inevitable accumulation of his unspent income occasioned. He no doubt would have distributed more liberally what he so little valued, but for the amount of time and inquiry which such a course must have compelled him to withdraw from his beloved pursuits. Some instances of his generosity are on record, and others, no doubt, will never come to light. M. Biot's epigrammatic description of him will probably long remain applicable,- " Il était le plus riche de tous les savans, et probablement aussi le plus savant de tous les riches."

This isolation of interest was doubtless due, quite as much to a constitutionally morbid temperament, as to a real misanthropy. He avoided even the most casual intercourse with his fellow men, excepting only when it was likely to bear the immediate fruit of scientific information. He almost never visited his relatives, and his heir paid him a visit of a few minutes once a year; but he frequented regularly the social meetings of the Royal Society Club, and the evening reunions of Sir Joseph Banks. But he came, not to participate, but to increase his stores ; if he spoke, it seemed to be by inadrertence, and he was silenced by a question, or even by a look. "A sense of isolation from his brethren made him shrink from their society, and avoid their presence; but he did so as one conscious of an infirmity, not boasting of an excellence. He was like a deaf-mute sitting apart from a circle, whose looks and gestures show that they are uttering and listening to music and eloquence in producing or welcoming which he can be no sharer. . . . He was one of the unthanked benefactors of his race who was patiently teaching and serving mankind, whilst they were shrinking from his coldness, or mocking his peculiarities. . . Such was he in life, a wonderful piece of intellectual clock-work, and as he lived by rule he died by it, predicting his death as if it had been the eclipse of some great luminary, .... and counting the very moment when the shadow of the unseen world should enshroud him in its darkness."3

I shall only add, that Cavendish was elected one of the eight Associates of the French Institute in 1803. This is a distinction perhaps the highest, of a formal kind, to which a scientific man can aspire, and was given at a time when, as an Englishman, he must have felt it to be peculiarly honourable.

The philosophical character of Cavendish resembled in many respects that of Newton; and with but a slight ${ }_{\text {His philo- }}^{(604 .)}$ modification of its secondary ingredients, he might sophical have been, perhaps, another Newton in experimental character. physics. His singular incommunicativeness, and the absence of a laudable ambition to perpetuate his name by the establishment of great theories, are perhaps the main reasons why his reputation, except in chemistry, did not stand yet higher than we find it.

\footnotetext{
1 By the Rev. W. Vernon Harcourt in British Association Report for 1839. Compare Wilson's Life of Cavendish, p. 446.

2 Phil. Trans. 1783.

3 Wilson's Life of Cavendish.
} 
A man indifferent to external relations pursues even his studies at a disadvantage; and the patient labours of so long a life devoted to a single object, would perhaps have told to greater effect had he published the results more frequently, and had he communicated more freely with those qualified to interchange their views with his. His excellent mathematical education, and his unusual skill in experiment, combined with a habit independent of either, but not less valuable, of patiently drawing inferences, might have placed him in the first rank as a discoverer in Heat, in Electricity, or in Opties, ${ }^{1}$ each of which sciences was so soon to take a surprising step in advance. In several of his important researches, he was more or less anticipated; a circumstance which his cold nature would perhaps scarcely have allowed him to make an effort to prevent. Black preceded him in most of his excellent experiments on heat; Epinus in his theory of Electricity; Watt was at least close on his traces in suspecting that water consists of oxygen and hydrogen; and the admirable experiment on gravitation had been devised by his friend Michell, and was not improbably recalled to his recollection, by the happy use of the torsion balance by Coulomb. It is given but to a few to achieve great discoveries, nor is the longest life always the most productive. Cavendish had his share, and some of the most considerable of these were even made later in life than is usual amongst experimentalists. ${ }^{2}$ It is no mean eulogy of him to say, that the purity and ardour of his pursuit of truth were never exceeded, and that had he been more ambitious of praise, he might have stood as pre-eminent in mathematical physics as he did in chemistry.

(605.) Antoine Laurent lúvoisier stands in intimate Lavoisier. connection with Cavendish, as well by the nature of his pursuits generally, as by the brilliancy and importance of his chemical discoveries, which were nearly contemporaneous with those of Cavendish. He was born in 1745 , and suffered by the guillotine on the 8th May 1794, without even the shadow of a misdemeanour. He was attached to the sciences of Heat and Chemistry, which he prosecuted with admirable success. He happily availed himself of the discoveries of Black, Priestley, and Cavendish, as well as his own, to establish the important chemical theory which has immortalized him. It is to be regretted that he was not always just in citing the English writers from whom he so freely borrowed. Such looseness was, however, common at that period, and (unfortunately) has continued to be so in France even to our own time.

(606.) Lavoisier's more important papers may be classed His contri- under two heads; those referring more immediately butions to to the subject of Heat as a branch of physics; and
heat and chemistry. those of a more strictly chemical character, principally in support of the "Oxygen-Theory," and con- sequently also intimately connected with the doctrine of heat considered in reference to its most ordinary source, Combustion.

He published in 1772 a paper on the Latent Heat of Water, ont Heat of Steam, which, in general, merely reproduce ${ }_{\text {rimeter- }}^{\text {heal }}$ the views of Black. In the memoirs of the Aca- rimeter demy of Sciences for 1780 (published 1784), we heat. find an important paper on Heat written in conjunction with Laplace, in which the calorimeter is described, though not under that appellation, together with its applications and their results. The principle of the calorimeter is too well known to require to be detailed here; but the authors of the joint memoir refer, with commendable precision, to the previous labours of Wilcke of Sweden, who first employed the melting of snow to measure the quantities of heat given off by bodies in cooling. To Laplace and Lavoisier, is, however, due an important addition which could alone impart any value to the results, - that of the external chamber of ice which prevents fusion taking place by the contact of the external air and by radiation. The French philosophers were not so successful in eliminating the other source of inaccuracy specified by Wilcke, which arises from the difficulty of drawing off the whole of the melted water. Sir John Herschel has of late years proposed what seems to be an important improvement on the calorimeter, by filling the interstices of the snow or ice with water, and estimating the quantity of the former melted by the contraction of volume of the compound mass. I am not aware that it has been as yet practised. I have stated in Art. (88) one ground on which the idea of the calorimeter (so far as not anticipated by Wilcke), may be probably ascribed to Laplace. Another is to be found in the fact that, in the opening of the description of the method in the paper which we are considering, Laplace writes of himself in the first person.

Of the memorable revolution which Lavoisier introduced into chemistry, more immediately in con-Chemical nection with the subject of combustion, I cannot be theory of expected to speak here at length. It is well known tion and that the early chemists entertained more correct oxidation. views as to the calcination of metals than those prevalent during the greater part of the eighteenth century under the influence of Stahl's theory of Phlogiston; and that Lavoisier, in the first instance, only led chemists back into the right road by insisting that the increase of weight observed when metals are calcined in air, must be due to some ponderable substance associated with the metal and derived from the air, and not to the escape of an imaginary spirituous substance, endued with positive levity, and termed Phlogiston. But it required the progress which had already been made in pneumatic chemistry by Black and Priestley, and especially the discovery of oxygen

1 This last subject seerns to have been comparatively indifferent to him.

2 His discovery of the composition of water was made when he was about fifty years of age. 
by the latter (a fact sparingly alluded to by Lavoisier), to give to the true theory a stable foundation. That metals calcine, and that flames burn by the aid of the vivifying principle of the atmosphere abstracted from it, and which adds its weight to the compounds produced, was the primary step made by Lavoisier; to which, by cautious, yet rapid inductions, he added the knowledge of oxygen as the usual (he believed the sole) acidifying principle, and demonstrated the true nature of carbonic acid. To these various phenomena thus happily reconciled, he added the theory of respiration, confirming it by the effects observed in air which has undergone that process. These, and many other consequences of the "oxygen theory," were developed in a numerous series of admirable memoirs. The reception of it was anything but in- stantaneous; and the hesitation and delay which occurred, enable us, as Dr Whewell has well remarked, to estimate the force of mind which was required to promulgate the theory, - as the subsequent course of discovery, and its infinite applications in practice, best attest its importance.

Lavoisier was still occupied in extending the conclusions of his chemical doctrines, when he was His unforovertaken by the unprovoked sentence to a violent death. death. Like Archimedes, he begged a short respite for the completion of experiments in which he was immediately engaged, but he was silenced by the brutal reply that " the Republic had no need of philosophers." He left a name equal to any in the science of his time, and adorned by the memory of public and private virtues.

\section{§3. DaLTon.-Theory of Gases and Vapours.-Law of Expansion by Heat.-Atomic Theory of Chemistry.-GUY-LUSSAC.}

John Dalton, the chief author of the theory of chemical equivalents or the A tomic Theory (as he preferred to call it), and of many important researches on the constitution of elastic fluids, was born at Eaglesfield, near Cockermouth in Cumberland, on the 5th of September 1766.1 His parentage was humble, and his family belonged to the sect of Quakers, whose tenets he, to the close of his life, professed. Had we wished to invent a striking antithesis in the personal histories of the cultivators of science, or to illustrate merely the various soils on which rich crops of discovery may be reared, we could scarcely have imagined more striking contrasts than in the social positions and advantages of Black, Cavendish, and Dalton - three of the names which we have selected in illustration of the history of physics of their age. intrasted Cavendish we have seen connected with one of the ith those noblest families in England, and wealthy almost beCavenish and lack.

yond the dreams of the covetous; spending his life in or near London, and enjoying every facility of direct communication with the first scientific celebrities at home and abroad;-Black, almost the beau ideal of an Academic, not wealthy indeed, but surrounded by all the opportunities of study, of information, and of social intercourse which he desired; passionless almost to a fault; admired by his pupils and friends ; enjoying, in short, all the advantages which education and a literary position can afford for the prosecution of a favourite study;-Dalton, on the other hand, poor and hardly winning a well-earned subsistence by private tuition, from the time he was himself a child until near the close of his long career, - with few friends, a scanty education, and a scantier library, - attaining, through his unaided and long almost unheeded efforts, and by means of an apparatus constructed entirely by himself, a position in the world of science unquestionably not second to that of either of his more highly-favoured contemporaries.

At the age of thirteen he had commenced the arduous office of an instructor; and from-1781 to 1792 pursued the same occupation in a humble sphere at Kendal, where he fortunately became acquainted with Mr Gough, a blind gentleman of some fortune, who devoted his time to the prosecution of science in nearly all its branches, and particularly of mathematical subjects, of which he has left an enduring record in many ingenious papers, published chiefly in the Manchester Transactions and in Nicholson's Journal. He patronized young Dalton, giving him free access to his library and apparatus, and receiving from him in return the benefit of his assistance in prosecuting his experiments. Dalton always recognised (as he had unquestionably good reason to do) the merits of his patron, and the importance of the advantages which he had derived from his advice and example. Indeed, without some such fortunate concurrence of circumstances (and something similar may be noted in the history of nearly all selfeducated men), it could hardly have been hoped that Dalton would have been so well grounded in the mathematical principles of at least some branches of Natural Philosophy as he probably was. For, though his discoveries bear mainly on the science of chemistry in the wide sense in which it was then understood, yet geometrical precision is after all their fundamental characteristic; and whether in treating of the constitution of a gas, or of the scale of a thermometer, or of the composition of a salt, it is evident that numbers and ratios were the ideas predominating in

1 Life of Dalton, by Dr Henry, in the publications of the Cavendish Society. The present section was originally written before the appearance of this biography. 
his mind. In this he resembled Cavendish; but he did not resemble him, and in one sense may be said to have surpassed him, in the boldness, we might call it audacity, with which on a frequently too slender foundation of facts Dalton established, to his own satisfaction, by reasoning almost as much $d$ priori as that of the mathematician, comprehensive physical theorems expressing the laws of phenomena.

The more important of these refer to the constiDalton's writings. tution of gases and vapours, together with their relations to heat, and to the combinations of bodies by chemical affinity. We shall endeavour to give some account of these researches separately. They are to be found detailed in the Memoirs of the Literary and Philosophical Society of Manchester (particularly the fifth volume), and in Dalton's New System of Chemical Philosophy, of which three successive parts appeared in 1808, 1810, and 1827 , of which the first is the most original and important.

(613.) Research on gases and vapours.

I. We shall first speak of his researches connected with gases and vapours. It had been noticed by Priestley and others that when gases exercising apparently no chemical action upon one another, are mixed in a confined space, they become, after the lapse of a longer or shorter time, completely intermingled, and that without any regard to even great differences in their density, the particles of the lighter gas diffusing themselves contrary to gravity through those of the denser, and causing them in their turn to ascend. The uniform composition of our atmosphere, in all circumstances and at all heights, is a striking example of this property. In explanation of it, Dalton had in 1801 arrived at the conclusion, " that the particles of one gas are not elastic or repulsive in regard to the particles of another gas, but only to the particles of their own kind." The fundamental experiment on which this singular conclusion was based was the following:- That the quantity of vapour of water (which, when purely elastic, may be considered as a gas) which can exist uncondensed in a given space, depends solely upon the temperature, and is independent of the presence of air and of its own density. Thus water, or any other evaporable liquid, being introduced into a space containing air, or any other gas, of any density, but subject to a constant external pressure, the space in question becomes damp by the evaporation of the liquid; now the amount of the latter converted into vapour depends, according to Dalton, upon the single circumstance that the vapour yielded by it must have the precise elasticity due to its temperature.
Its elastic force being added to that of the dry air, the whole will expand until equilibrium is restored with the constant pressure without, and this will occur as soon as the elasticity of the dry air alone (proper to its increased volume), added to the elasticity of the vapour alone (depending solely on its temperature), are together equal to the pressure which they have to support. In short, to use the precise enunciation of Dalton himself, "in all cases the vapour rises to a certain force, according to temperature, and the air adjusts the equilibrium by expanding or contracting as may be required."1

The importance of this law (easily verified in the particular case) is readily perceived. Not only did Theory it affect the results of almost every experiment in hygrome pneumatic chemistry, but it rendered a new theory of ${ }^{\text {try. }}$ hygrometry indispensable. The older theory of Halley, Leroy, and Franklin was, that the direct affinity between air and water drew up a portion of the latter into the former with the aid of heat, whilst De Saussure (Essai sur l'Hygrométrie, 1783) believed that the conversion of water into vapour took place first and independently by the action of heat, and that it was then drawn up into the atmosphere by the attraction of the gases which compose it. Dalton's experiments show that the air and the vapour mix without the slightest mutual interference or reaction. He founded thereupon an excellent practical method of determining the amount of vapour in the atmosphere. He first formed a table of the elasticities of watery vapour or steam for all temperatures between $32^{\circ}$ and $212^{\circ}$; and so simple and accurate were his methods, at least for the lower degrees of the thermometer, that his numbers are still received as amongst the best we have. He operated merely with a carefully constructed barometer, into the vacuum of which he introduced a few drops of water, and raising the temperature by means of a tube embracing it, and which could be filled with water at pleasure, he observed carefully the depression of the mercurial column. The elasticities thus determined commenced with 0.2 inches of mercury at $32^{\circ}$ up to 30 inches at $212^{\circ}$. These numbers, consequently, represent the utmost elasticities of vapour which can exist either in air or without air at the corresponding temperature. If we attempt to add more vapour, or to lower the temperature, in either case moisture will be deposited. Hence to find the quan-Dew-poin tity of vapour in the atmosphere when not absolutely ment. damp, it is sufficient to ascertain the temperature at which it becomes so. This Dalton did by filling a

\footnotetext{
1 The exact expression of the effect is this, $v=\frac{p}{p-f}$, where $p$ represents the pressure expressed in inches of mercury upon a given volume (equal to unity) of dry air; $f$ the force of the vapour in vacuo at the temperature of experiment, also in inches of mercury; and $v$ the volume which the mixture of air and vapour occupies under the given pressure $p$ after saturation. It is evident that $p-f$ being the pressure due to the elasticity of the dry air apart from the vapour, when we affirm that the volume (1) becomes $\frac{p}{p-f}$, we in effect affirm that Mariotte's (or Boyle's) law connecting volume and pressure holds true for air which is mixed with vapour, just as though vapour were absent or its space void.
} 
thin glass with cold spring water, or by adding, if necessary, the solid nitrate of ammonia, until the temperature fell so far that dew began to be deposited on the surface. This excellent method constitutes in fact the dew-point hygrometer. It has received various forms from $\mathrm{Mr}$ Daniell and others, but the original one is probably the best.

These important laws and deductions being fully understood, the theory of the mixed dry gases follows as a simple corollary. For it merely asserts of them what has been admitted in the case of steam, that they may diffuse themselves, and exert their elastic forces quite independently of one another; so that, for example, in our atmosphere the total pressure is made up of the partial elasticities of the oxygen, nitrogen, and carbonic acid which compose it, each acting to the same amount as if it alone had existed in the space which it occupies. In his essay of 1801, Dalton stated his view of these facts thus:- "The particles of one elastic fluid may possess no repulsion or attractive power, or be perfectly inelastic with regard to the particles of another."

Discoreries so practical could not fail to excite immediate attention, especially at a time when the researches of chemists were earnestly directed towards the gases. "The facts and experiments," Dalton tells us in his Chemical Philosophy, "were highly valued; some of the latter were repeated and found correct, and none of the results controverted; but the theory was almost universally misunderstood." Its opponents were Berthollet, Thomson, Henry, and others, and the replies of the author are contained in the work just cited. But he appears to have felt the force of the objection, "Can it be conceived that an elastic substance exists which adds its. volume to that of another, and which, nevertheless, does not act on it by its expansive force?"-for in his chemical philosophy he abandons the comparison of gaseous particles to similar magnetic poles which repel each other, but are inert towards non-magnetic matter, and allowing that heat is the primary cause of repulsion in all gases, ascribes their diffusion contrary to gravity to the dissimilar size of their spherical molecules. "The particles of one kind being from their size unable to apply properly to the other, no equilibrium can ever take place amongst the heterogeneous molecules. The intestine motion," he adds, " must therefore continue till the particles arrive at the opposite surface of the vessel against any point of which they can rest with stability, and the equlibrium at length is acquired, when each gas is uniformly diffused through the other." It may be seriously doubted whether this theory of the facts will bear examination, at least no attempt has been made to demonstrate it on mechanical principles. The subject has been allowed to remain during more than forty years of unequalled activity in such speculations without material light ,being thrown upon the proximate causes of these wonderfully general and simple truths. Yet it can hardly be doubted that the mechanical theory of the gases and vapours is capable of a great extersion, and even of being illustrated by simple experiments. But the attention of chemists has been withdrawn from the physical bearings of their science by the prodigious increase in the number of compounds which they have had to analyze and classify. The most important sequel to Dalton's discoveries has probably been that of Professor Graham, whose ex-Mr Graperiments prove that gases when separated by a ham's law porous partition permeate it in both directions, until of diffuthey have mixed in proportions which are inversely sion. as the square root of their density. This law clearly shows the purely mechanical causes by which the diffusion is effected.

A discovery of Dalton, which has scarcely been considered second in importance to those we have Dalton on mentioned, is that the rate of expansion of all gases the expanby heat is the same. Thus thermometers of air, hydro-gases by gen, and carbonic acid would all mark the same degree heat. when plunged in the same medium; whilst the mercurial thermometer shows a more rapid expansion, at higher temperatures, if the air thermometer be taken as the standard. This important fact was soon after but independently announced by Gay-Lussac of Paris. Dalton's publication dates from 1801. In his Chemical Philosophy, he gives his view of the difficult subject of a true thermometric scale; which he does not suppose to be correctly represented by the simple expansion of any known substance; but that the gases expand with true increments of heat in a very slow geometrical progression, whilst liquids expand as the squares of the true temperatures from their freezing points. These and other laws equally arbitrary have not received support from later and more precise experiments; and in the second volume of his work (1827) he freely acknowledges the correction of his hypothesis by the more recent French experiments.

II. I now proceed to the other part of Dalton's labours on which his reputation is principally based. Dalton's a clear assertion and experimental esta-Theory. blishment of the Atomic Theory or doctrine of chemical equivalents. I introduce it here on account of its important bearing on all physical questions in which the constitution of matter and the forces acting at minute distances are involved.

Early opinions on the constitution of matter and (619.) chemical combination.-Two opinions have prevailed Early opifrom the very earliest times respecting the constitu- nions on tion of body:-that which supposes its entire homo- tution of geneousness, and that which allows that it consists matter. of material parts or atoms separated by void spaces; these parts or atoms being indivisible. This last is the doctrine of Democritus and Epicurus, and in modern times of Bacon, Newton, and Dalton. ${ }^{1}$ The former opinion has been held by Leibnitz, and many

\footnotetext{
1 The reader will find ample details on the opinions of the older philosophers in Daubeny's Introduction to the Atomic Theory,
} 
writers of the recent German school. Abstracting as far as possible from the more purely metaphysical difficulties (such as those which the consideration of Leibnitz's law of continuity introduces), we may perhaps be justified in stating that, whilst the objections urged against the existence of atoms fall upon our inability to conceive and describe the properties of these individual ultimate parts in a consistent manner,- the objections to the other notion meet us at an earlier stage, and seem to defy any clear conception of the nature or possible existence of compound bodies, or of bodies in two states of consistence. Admitting atoms-whilst acknowledging our inability to describe them individually - we can clearly enough conceive the phenomena of condensation, rarefaction, evaporation, \&c., and also of the combination of elements in compounds possessing distinct properties; but excluding them, or allowing that matter is penetrable by matter throughout, is it possible to conceive clearly of such a compound, - as for example, of the perfect diffusion of two gases in the same space, yet each gas retaining its individuality so completely as to admit of easy and complete seBoscovich. paration from the other? The theory of Boscovich, which has been sufficiently touched upon by Sir John Leslie in the previous Dissertation, was intended, no doubt, to reconcile the two opposing theories, and it cannot be doubted that it is in many respects an ingenious solution. Yet it is essentially (as Professor Robison maintains) a corpuscular or atomic doctrine, and it farther appears to be difficultly reconcilable to the doctrine of inertia ; for how can a finite number of unextended physical points, though they may be the centres of intense forces, constitute a finite aggregate mass? Nevertheless this speculation has been on the whole favourably received, and in our own time seems to have been adopted by so eminently practical a philosopher as Dr Faraday.

About Dalton's idea of atoms, however, there can without reference to the position of the atoms of any of the others. But a chemical compound consists of molecules or complex atoms, each composed of two or more ultimate particles of the constituents firmly united by a chemical force, and these complex molecules act towards one another exactly as simple ones might do. The general notion of chemical forces or affinities (as they were perhaps first called by Geoffroy, a French chemist) appears to have been apprehended in two different senses corresponding to the atomic or non-atomic theory of body. For the former proceeds on the assumption of direct attractions or repulsions (push-and-pull forces, as they have been called) uniting some and tending to separate others, thus assimilating completely chemical with mechanical forces. The other school adopts the word affinity as expressing a mode of action of matter upon matter totally distinct from that of force producing motion in its particles, to which it is difficult to give an intelligible form, much more to prove that the assumption is warranted by the facts. 1 In this state of matters the choice between an opinion perhaps erroneous, and one which assumes no definite shape, can hardly, to the practical philosopher, remain long doubtful; when new facts shall have enabled him to express intelligibly a new hypothesis, it will be time enough to adopt it.

Dalton's Atomic Theory.-I shall first briefly state the general facts or laws to which Dalton gave an universal application, and then briefly refer to the un laws of cical co doubted anticipation of part of them by earlier chemists. bination. For the sake of distinctness the facts of the atomic theory may be thus enumerated:- 1 . That when two bodies unite, not merely by mechanical mixture but through a chemical affinity of the elements, - two or more ingredientsforming a wholewith new properties,- - these ingredients are invariably found to exist in constant proportions. For instance, the carbonate of lime invariably consists of 44 parts by weight of carbonic acid and 56 of lime, the slightest addition of either element remaining uncombined, or only mechanically mixed with the chemical product. 2. In many instances, however, more than one chemical combination can be formed between two or more elements, and in the simplest cases, where the elements are two in number and one remains constant in quantity whilst the other increases in amount, a fresh chemical union of the particles does not occur until one of the ingredients reaches precisely double the amount for him a uniform diffusion of the atoms of each gas throughout the space occupied by the whole, and

and in Whewell's Philos. of Inductive Sciences, vol. i. Newton's conjecture is expressed in these words :- " All things considered, it seems probable that God, in the beginning, formed matter in solid, massy, hard, impenetrable, moveable particles, of such sizes, figures, and with such other properties, and in such proportion to space, as most conduced to the end for which he formed them. And that these primitive particles being solids, are incomparably harder than any bodies compounded af them; even so very hard as never to wear or break to pieces; no ordinary power being able to divide what God himself made one in the first creation." Horsley's Newton, vol. iv., 260, quoted by Daubeny.

1 A profound and subtle thinker of our own time (Mr Leslie Ellis, of Trinity College, Cambridge) has made a definite suggestion as to a possible form of chemical forces, viz., that they may not be such as are directly exerted between a particle $\mathrm{A}$ and a particle $B$, but only by their presence enable $A$ to act on $B$, or bear the same relation to force (common force) as force in mechanics does to the motion which it causes. Thus, the science of mechanics would include-lst, Kinematics, or pure motion depending on equations of the first order; $2 d$, Dynamics, depending on equations of the second order ; $3 d$, Chemical, Vital, \&c., forces, depending on equations of higher orders. Camb. Trans., viii., 604, \&c. 
which had chemically combined with the other; and if it happen that fresh compounds are formed possessing new qualities, then the varying ingredient reaches 3,4 , or 5 times the amount which it had in the first combination; or more generally the proportions by weight of the combining elements may be exactly represented by whole numbers. 3. "If two bodies combine in certain proportions with a third, they combine in the very same proportions with each other." This is called the law of reciprocal proportion. Thus, suppose that one ounce of a body A, saturates or forms a chemical combination with two ounces of B, 5 of $\mathrm{C}$, and 11 of $\mathrm{D}$; then if $\mathrm{B}, \mathrm{C}$, and D are capable of combining, it will be in the exact proportions of the numbers 2, 5, and 11 . Hence a single number being determined for each body, determines all its possible combinations with other known bodies. 4. It may perhaps be added as an independent fact, that when complex bodies combine with other bodies, no matter whether simple or compound, the combining number of the complex body is the sum of the numbers representing the constituents. Thus the combining number of lime is 28 ; but lime consists of calcium 20 and oxygen 8 . So also the combining number of water is 9 ; but water consists of hydrogen 1 and oxygen $8:$ and the combining number of hydrate of lime is 37 , the sum of the 28 parts of lime and 9 of water of which it is composed.

The discovery of these laws has been termed by

Sir John Herschel the most important, after the laws of mechanics, which the study of nature has yet disclosed. No slight or transient reputation is due to him who first clearly apprehended and taught them. Nor must we be surprised to find several claimants to a share of the honour. It is the invariable history of all great generalizations, that they lave been partly anticipated; and it may serve to moderate the selfesteem of even the greatest discoverers, that however high may be their individual merits, they are in some sense the mere exponents of the aggregate knowledge of their contemporaries. The laws of motion were partially anticipated before the time of Galileo, and could not have remained much longer undefined; and even the unparalleled discoveries of Newton must, in all probability, have ere long been made piecemeal by the united energy of his contemporaries and immediate successors. The steam-engine was not the sole creation of Watt, nor was Davy the first to apply the voltaic battery to chemistry. In like manner, Dalton's laws of chemical combination were published at a happy moment, which gave them speedy acceptance with the active chemists of his day; whilst those who had seen with sufficient clear- ness portions of these laws twenty or thirty years before, 'addressed a scientific public by no means prepared to appreciate their value, or to feel a conviction of their generality. Had Wenzel, ${ }^{1}$ Higgins, ${ }^{2}$ and Wenzel, Richter ${ }^{3}$ individually apprehended the great impor- Higgins, tance of the definite and multiple combining pro- and Richportions which they announced, - had they felt the theory of them to constitute the very foundations of chemistry,- they would not have rested until they had verified it in numerous details, and applied it to the various purposes of speculation and practice, as Dalton did. But whether from want of energy, or from ill fortune, their ideas sunk into entire oblivion; and the ingenuity and social position of Berthollet were giving a currency to opinions respecting chemical forces which tended to undo even the far more elementary notions of the constancy of elective affinities, at the time when Dalton's researches were unostentatiously brought before the world. His first insight into the theory of chemical combinations dates from the year 1803. It was expounded by him both in conversation and by lectures in 1804, at which date Dr Thomas Thomson recorded the results of a conversation held with him at Manchester, which, three years later (evidently with Dalton's approbation), he published in his work on Chemistry. Finally, Dalton himself, in 1808, announced the principles of his theory at no greater length than five pages "on Chemical Synthesis" in his Chemical Philosophy.

Now, it is to be observed, that Dalton's views were all along expressed in the language of a strictly Ato- Importance mic theory. Compounds are only chemically com- mic Theory plete, when one or several atoms of an element com- to the probine with one, two, or more atoms of another. Any gress of superfluity of either element remains uncombined, or chemistry. mechanically mixed. All the other parts of the laws of combination readily lead to the same idea, and, in fact, find in it their simplest expression. There is no wonder, then, that Dalton firmly believed in the physical existence of his atoms, and that the new properties of compounds are due to the peculiar modification of the most elementary parts into which bodies can be divided without a loss of those properties, that is, without decomposition. He figured these elementary molecules by uniting the symbols of their constituents, and by so doing, may be said to have laid the foundation of those algebraic systems of technical notation, which speak to the eye only in another way from Dalton's diagrams, and which have been of such eminent service to the chemist. Nor must we think too lightly of a hypothesis which served so materially to aid in realizing

1 Lehre von der Verwandschaft der Körper, 1777. He shows that in a double decomposition the new compounds are chemically perfect.

2 A Comparative View of the Phlogistic and Antiphlogistic Theories, 1789, by Mr William Higgins, points out the multiple combining proportions of sulphur and oxygen, and of nitrogen and oxygen, but only incidentally. Dr Bryan Higgins, a relative of Mr W. H., had published, in 1786, a work said to contain the idea of definite atomic combinations.

- Anfangsgrüinde der Stöchyometrie, 1792. He gave a series of numbers representing the combining proportions of different elements. 
a discovery of such magnitude. Dalton's earlier researches were far more physical than chemical; and it is evident that the effort of representing to his mind, geometrically and atomically, the condition of mixed gases and vapours, led him to form clear ideas on the definiteness of chemical combinations. The delay in publishing his views, no doubt depended on his desire to present them to the public in the form of a somewhat wide induction, although it is certain that his own opinion was fully formed from a knowledge of only one or two cases of multiple proportions, and especially the combinations of hydrogen and carbon. It cannot be fairly urged as onclusive against the theory of atoms, that cases - ceur difficult to reconcile with the author's formal statement of it. There is no great theory - not even that of gravitation itself-which has not met with similar apparent contradictions.

(624.) The reception of the views of Dalton was someIts recep- what gradual, yet might be called rapid, considering tion in the obscurity of the author, and his provincial resiEngtand, dence. The energy of Dr Thomson of Glasgow contributed more than any other circumstance to compel the attention of chemists. He personally brought it under the notice of Wollaston and Davy; and the former, who, by his habits of precise thought and accurate experiment, as well as from his extensive chemical knowledge, would of all others have been the most likely to see its importance and probability, was doubly predisposed in its favour by having been himself for some time in possession of facts illustrating the numerical laws of combination similar to those which Dalton possessed. Wollaston published these in the Philosophical Transactions for 1808, in which he expressly states, that Dalton had anticipated him in the results of his enquiry into multiple combinations of elements. Davy, as might have been expected, was less prepared to accept a doctrine having the form of a mathematical law; he did so, however, after a short resistance. In his Chemical Philosophy he ascribes most if not all the merit of it to Higgins, and is supposed to have looked coldly upon Dalton's growing fame; but it is gratifying to add, that in almost his last appearance in public as president of the Royal Society, when presenting Dalton with the first royal medal, he should have expressed himself in terms of cordial praise.

(625.) In France the new doctrine soon spread, notwithand France. standing its violent contradiction to the theories of sac's law of Berthollet. Gay-Lussac was amongst its earliest volumes. and most enlightened advocates; and he had the good fortune to add, in 1809 , a new law to the principles of chemical combination, which is, that the gases, in uniting chemically, combine in equal or multiple volumes, and when any condensation occurs after they have united, it amounts to an exact fraction $\left(\frac{1}{2}\right.$ or $\left.\frac{1}{3}\right)$ of their joint bulk. This was the only addition made for a very long period to Dalton's laws, even if we consider the theory of Isomorphism and of Substitutions to take the same rank; and as it evidently includes the idea that the atomic weights of the gases have a simple numerical relation to their densities, it confirms Dalton's views of the great simplicity and uniformity of constitution of those bodies. In Sweden the doctrine of definite proportions found one of its earliest advocates in Berzelius, and his analyses contributed perhaps more than those of any other chemist to its perfect establishment.

It is not to be concluded, however, that the atomic or theoretical part of Dalton's laws obtained the same currency with the conditions of chemical combination which they serve to define. Wollaston and Prout were perhaps the most favourably disposed to the doctrine of atoms, though the former invented the term " chemical equivalents" to escape from the theoretic inference, and the latter believed that Dalton's law was only a portion of a more complicated one regulating chemical combinations. Wollaston even sought evidence in favour of ultimate atoms, from considerations of a purely mechanical kind, such as the existence of a limit to the atmosphere (Phil. Trans. 1822). We may however admit, with those who have taken an opposite view, that the finite extent of the atmosphere is "consistent with a continuous mathematical law suitably assumed, and without reference to atoms at all ; if, indeed, we can imagine a medium varying enormously in density, yet possessing perfect continuity of body. But we will not enlarge farther on these almost metaphysical considerations.

During the period from his settlement in Man- (627.) chester in 1793 , to the publication of his Chemical Dalton's Philosophy in 1808, Dalton was occupied in tuition, history first in the Mosley Street Institution, where he lec- continue tured on mathematics and natural philosophy for six years, and afterwards, privately, in a very humble and unpretending manner. His speculations and experiments gradually became more and more strictly chemical; and, aware that his atomic theory was to be the great foundation of his fame, he spared no pains in illustrating it by numerous analyses. Contemporary chemists have testified to the ingenuity and fidelity of these. Yet, isolated as he was, and unacquainted perhaps with those niceties of manipulation which are suggested by the experience of professional chemists, and rapidly communicated in great eities, his numerical conclusions were often inexact. Probably he felt some discouragement from this, at well as from the indifferent reception of the later parts of his Chemical Philosophy, in which he had to admit the inaccuracy of his theoretical scales of heat and expansion. At all events, his publications became more scanty and less original, though he was still near the meridian of life. The reality of his discoveries had been somewhat coldly acknowledged, and he felt little temptation to adventure himself in a more bustling arena, for which his habits and circumstances seemed to unfit him. Nevertheless, he had been, as

(626.) 'Chemic equivaents." 
early as 1816, elected a corresponding member of the Institute of France-Wollaston being then probably the only other English name on the list. ${ }^{1}$ Dalton found his way to Paris in 1822, and was agreeably surprised by the distinction with which he was received by the most eminent members of the Academy of Sciences. Perhaps this first personal recognition of his exalted station, as a man of science, had something to do with the tardy adjudication to him four years later of one of the medals of the Royal Society of London. In 1830 he was elected one of the eight associates of the Academy of Sciences in the room of Sir Humphry Davy.

(628.) In 1833, at the age of sixty-seven, he received a pension from government, up to which time he had maintained himself in the way already mentioned, with the utmost simplicity and contentment. Even in his lifetime it was impossible for his eulogists to forbear from some reference to this essential part of his really philosophic character. " Mr Dalton has been labouring," says Sir Humphry Davy, "for more than a quarter of a century with the most disinterested views. With the greatest modesty and simplicity of eharacter, he has remained in the obscurity of the country, neither asking for approbation, nor offering himself as an object of applause." " There is little doubt," says Dr Thomson, "that Mr Dalton, had he so chosen it, might, in point of pecuniary circumstances, have exhibited a much more brilliant figure. But he has displayed a much more noble mind by the career which he has chosen; equally regardless of riches as the most celebrated sages of antiquity, and as much respected and beloved by his friends, even in the rich commercial town of Manchester, as if he were one of the greatest and most influential men in the country." All who had the good fortune to know him personally-to see him, as the writer of these pages has done, in his modest school-room, and surrounded by his unpretending apparatus - will own that these eulogies are and death. not overdrawn. His latter days were spent in cheerfulness and comfort; he expired on 27 th July 1844 , having nearly completed his seventy-eighth year.

The philosophical character of Dalton may be bricfly summed up. He had immense vigour of conception, and an ardent love of truth. He was thoroughly devoted to the pursuit of science during his long career, and he evidently sought and expected no higher reward than the insight which he obtained into the laws of nature. His mind, like his frame, was of a strongly masculine character, and happily exempt from nervous sensibility and other like infirmities of genius. Whilst he held his own opinions with tenacity, and criticised freely those of his opponents, there is not a trace of acrimony in any of his writings; and he always spoke in terms of high respect both of those who pursued science in a similar direction with himself, and (what was more difficult) likewise of those who, having the good fortune to hold more conspicuous positions, showed him the smallest degree of kindness, which he always gratefully acknowledged. He was unlike Black and Cavendish, in the rapidity with which he seized on a few isolated facts, and made them the basis of an inference of great generality; this, indeed, was his leading characteristic; and he differed from them equally in the boldness with which he claimed from the public a general acceptance of his conclusions. Some of his inferences were unguarded enough, and have not been confirmed; and the reception of what were correct was naturally delayed by the evident facility with which his theories were shaped in his own mind. Most of his papers appeared in rapid succession; only the Atomic Theory was brought with some evident hesitation before the world. In all this we see the results of a vigorous imagination, united with great perseverance, in working out an idea. The imaginative element would have been more under control had his edueation been of a less irregular kind. We see the effect of an opposite turn in his eminent predecessors just named. They would have done more, had they trusted more. Dalton's discoveries may be said to have terminated at the age of forty. Though he laboured for thirty years after, the conceptive faculty seems to have spent itself in its earlier efforts.

Joseph Louis Gay-Lussac, an eminent French chemist and physicist, contemporary with Dalton, Gay- 630.$)$ has been mentioned in the course of the present sec- sac-che tion, as having discovered independently the equal mist and dilatation of the gases, and also a law of their com- physicist. binations in connection with their volumes, which was peculiar to himself. Besides these researches, science owes many useful observations in physics to his energy and talent, which, in the origin of his career, promised more of originality than his maturer life perfectly fulfilled. He was born in the old province of Limousin in 1778 , and became the pupil of Berthollet in chemical researches, and was one of the earliest and most active members of the Société d'Arceuil. In physics, he was the collaborator of MM. Biot, Humboldt, and Laplace. With the first Remarkof these philosophers he made his earliest experi-able balment in aërostation, which he repeated alone on the loon ascent. 16 th September 1804, when he attained the amazing height of 7016 mètres (23,019 English feet), an elevation previously unattained, and which in the course of the succeeding half century has only twice been touched, or exceeded by a small quantity. ${ }^{2}$ Con-

1 So stated by Dalton himself (Life by Dr Henry, p. 163); but I suspect some misapprehension. Considering the importance attached to these nominations, it is to be regretted that it is at all times difficult to ascertain who are, or have been, associates and correspondents of the Academy of Sciences.

Once by MM. Bixio and Barral in 1850, and once by Mr John Welsh in 1852 . Since this passage was written I find it stated, that, on the 10th September 1838, Mr Green and Mr Rush reached a height of 27,148 feet. See The Times of 15th Sept. 1838. 
sidering the novelty of the experiment in 1804 (only twenty-one years after Montgolfier's first successful experiment), this fact speaks strongly for his courage and zeal. These ascents were also the first undertaken with strictly scientific aims, and the observations made were highly interesting in connection with the decrement of temperature in the atmosphere, with the uniformity of composition of air at all heights, and with the question of whether the magnetic force of the earth diminishes at such elevations. This last enquiry was not conclusively answered.

With M. de Humboldt he made observations on terrestrial magnietism in Italy, and on other subjects. MiscellaBy desire of Laplace he studied the facts of capillary neous exattraction. In more immediate connection with the periments subject of the present chapter, he made some valu- Lussac. able experiments on hygrometry, on the mechanical properties of vapour of different kinds, and on the specific heat of the gases. His fame, however, mainly rests on the two investigations to which we previously referred, and on the results of his balloon journey. His later years were devoted to practical enquiries connected with chemistry, and to his official duties at the Mint. He died at Paris on the 9th May 1850. His death.

\section{\$ 4. RUMFORD.-Economical applications of Heat.-Point of Maximum Density of Water; Hope. -Friction as a source of Heat. Theory that Heat is convertible into Mechanical Energy; Mr Joule.}

(632.)

Thounson, Count of Rumford.

(633.)

His early history and studies.

THe name of Thomson, Count of Rumford, deserves a passing notice in the history of the physical sciences, if not for the absolute importance of his discoveries, at least as an instance of a class of benefactors to mankind at once in a physical and intellectual point of view. He was altogether in advance of his age in the application of correct theory to the improvement of the social condition of the lowest classes; and many of his experiments, and, indeed, discoveries, seem now at once so simple and so familiar, that we are apt to forget how entirely original they were sixty years since.

Sir Benjamin Thomson, an American by birth, a British knight, and a Bavarian or rather Austrian count, was born in the United States in 1753, and passed his earlier years almost entirely at military stations during the American war, being engaged on the British side. After the establishment of independence, he quitted his country for ever; came first to England, where he was well received, and proposing to enter the Austrian service, he proceeded as far as Munich, where, having become known to the elector of Bavaria, he was induced to settle; and having received different civil and military appointments, he devoted himself for a series of years to the improvement of the social condition of that capital. He introduced great improvements into the management of the army; the mechanical and chemical departments of the artillery had a peculiar charm for him; they were conducted on strictly scientific principles, and, in return, were made to contribute important results to science. His experiments on the heat of friction, deduced from the boring of cannon, are amongst the best we possess ; and they led him to results of considerable theoretical importance to 'which I shall presently refer.

(634.) most serviceable efforts on behalf of kind were in the treatment of the mendicant classes would not rest until he had cooked his dinner with his neighbour's smoke. with which Munich then swarmed. Salutary views of the importance of industry, order, morality, and public economy, were most happily united to a happy versatility of talent in physical research, to unwearied patience and great liberality, in effecting one of the greatest social reforms on record. The strict statistics of a great house of industry were ascertained with reference to the most seemingly insignificant details, and, in particular, all the applications of Heat to the physical wants of mankind were studied with equal assiduity and success. The wdrmih of clothing was traced to the amount of still air entangled amongst its fibres,- the dissipation of heat, whether from a thermometer or a kitchen boiler, was classified under radiation, conduction, and convection, the last and often most important of which (signifying the influence of currents in liquids and gases in conveying heat by the changing density of their parts) had hardly before been recognised, or at least made the subject of formal experiment,- the effective heat due to the combustion of different kinds of fuel, tested by a calorimeter of his own invention, - the economy of light based on an investigation of the properties of flame; - these were but a few of the trains of enquiry, of which his Mendicity House was the primary object. Charity and science went Practical hand in hand; and when we award to Watt the benefits $r$ highest honours for an invention which enabled him ${ }^{\text {sulting. }}$ to create mechanical force at an economy of twothirds of the coal previously consumed, shall we deny Rumford a civic crown for having so improved the methods of heating apartments and of cooking food, as to produce a saving in the precious element of heat, varying from one-half to seven-eighths of the fuel previously consumed ? 1 When we consider the enormous price of wood in nearly every part of the Continent, the destruction of forests which has occurred, and the consequent injury to the climate, as

1 In the hospital of Verona he reduced the consumption of wood to one-eighth. Some one wittily said of Rumford, that he 
well as to the material wealth of many districts, we are disposed to give Rumford a higher place than has generally been accorded to him. Had his excellent principles been universally carried out, some millions sterling would have been sared to every large state in Europe. Fontenelle characteristically says of a certain savant, who made experiments on nutrition, with a view to carry fasting to the utmost practicable extent, that his researches had the double aim of a place in heaven and in the academy. Cuvier, who tells the anecdote in his Eloge of Rumford, adds, that the latter had a truer claim to the questionable compliment. That science is surely not despicable by which a pound of wool, of fuel, or of food, can be made to go one-half farther than before in warming the naked and in feeding the hungry.

All Runiford's experiments were made with admirable precision, and recorded with elaborate fidelity, and in the plainest language. Everything with him was reduced to weight and measure, and no pains were spared to attain the best results. His experiments on heat, and the properties of bodies in connection with it, are the most important. He first applied steam generally in warming fluids and to the culinary art. He maintained the paradox of the non-conducting power of liquids, which, though practically true, appears not to be rigorously so. He contrived many ingenious instruments; but his thermoscope, identical with Leslie's differential thermometer, was probably of later invention, if not in some measure borrowed from it. In like manner his proofs of the maximum density point of water were unquestionably suggested by Dr Hope's beautiful experiment, although this derives its meaning from the laws of convection, which Rumford first established.

That water expands in bulk below the temperature IIope on of $39^{\circ}$ or $40^{\circ}$ Fahr. until it freezes, is a fact which had been asserted since the middle of the seventeenth century. But for 150 years its great improbability, and the unquestionable uncertainty introduced into the result by the irregular: expansion of the containing vessel or glass of the thermometer, enabled sceptics in every generation to withhold their assent. Perhaps the last who doubted was the illustrious Dalton. He allowed himself however to be convinced by $\mathrm{Dr}$ Hope's experiment, in which the temperature of the denser and rarer water is measured by two thermometers placed at the bottom and top of a cylindrical jar, and nothing interferes with the natural tendency of a fluid to arrange its particles according to their specific gravity, the lighter resting on the heavier ones. It is to be regretted that Hope did not prosecute original enquiry, for which the conception of this experiment, and the mode in which he conducted it, ${ }^{1}$ show that he had excellent qualifications. Hope was first the colleague, then the successor of Black in the chair of chemistry in Edinburgh ; and in his time probably the most popular teacher in Europe of that science. He died on the 13th June 1844, in the seventy-eighth year of his age.

Rumford's name will be ever connected with the progress of science in England by two circumstances; Rumford first, by the foundation of a perpetual medal and Prize-the prize, in the gift of the Council of the Royal Society stitution. of London, for the reward of discoveries connected with Heat and Light; and secondly, by the establishment, in 1800, of the Royal Institution in London, destined, primarily, for the promotion of original discovery, and, secondarily, for the diffusion of a taste for science amongst the educated classes. The plan was conceived with the sagacity which characterized Rumford, and its success has been greater than could have been anticipated. Davy was there brought into notice by Rumford himself, and furnished with the means of prosecuting his admirable experiments, $\mathrm{He}$ and $\mathrm{Mr}$ Faraday have given to that institution its just celebrity with little intermission for half a century.

Rumford spent his later years in Paris, where he died in 1814. The estimation in which he was then Rumford's held may be judged of from the fact, that he was last years one of the eight foreign associates of the Academy of Sciences. He was very capable of having done more for science: the versatility of his talents, the accidents of his early life, and the strong hold which principles of philanthropy and public utility always exerted over him, account for the absence of more sustained and erudite researches. But in those very particulars he deserves to be cited as a practical philosopher, as to many things in advance of his age, and a benefactor both to science and to mankind.2

In the history of pure science Rumford will be (639.) chiefly remembered by his espousing the (not new) His opitheory that heat consists in a motion of some kind nions on amongst the particles of matter, in opposition to the of heat imopinions then so prevalent amongst chemists, which portant. almost tended to regard it as an element capable of forming combinations. Rumford's view was mainly Derived based on the facts of friction, which he showed to be from expeirreconcilable with the notion of a change in the spe- riments on cific heat of the abraded matter, and to be seemingly inexhaustible so long as the force producing friction is continued. His conclusion was, that the heat then generated cannot be a substance, but an affection of body of the nature of vibratory motion. The amount of heat evolved in boring cannon is very

1 Edinburgh Transactions, vol. v.

2 Rumford married (for the second time) Lavoisier's widow; his daughter (by bis first marriage) became Madame Cuvier. Hence Cuvier's Eloge of Rumford contains the most authentic particulars of his life. Madame Rumfurd survived until a few years since, residing at Paris, where she formed a link between the savans of the age of Lavoisier, and those of the middle of the nineteenth century. 
great, an operation with which, as we have seen, he was professionally connected. In one experiment, a steel-borer pressed with a force of 10,000 lbs. against gun metal, and revolving 32 times in a minute, generated in $2 \frac{1}{2}$ hours the heat necessary to boil $18 \frac{3}{4}$ lbs. of water. It is probable that Rumford carried his views so far as to infer a necessary and constant relation between the quantity of heat generated and mechanical action expended; and if we take an estimate of horse-power more conformable to reality than the nominal horse-power of Watt $(33,000 \mathrm{lbs}$. raised 1 foot in the minute, which is too great), we shall find a tolerable approximation between his results and those now generally admitted. Davy favoured Rumford's theory, but the mechanical question remained for 40 years almost unconsidered.

(640.)

At length, about 1845, MrJoule of Manchester envived by deavoured to establish a rigorous connection between Mr Joule. the mechanical effort expended and the heat geneMechanical rated by friction; and he appears to have satisfaceffect of torily established (Phil. Trans., 1850) that in the
heat. case of water agitated by beaters, the work expended by the fall of 772 pounds through 1 foot is capable of raising the temperature of a pound of water by $1^{\circ}$ of
Fahrenheit. ${ }^{1} \mathrm{Mr}$ Joule's experiments and inferences, however, go much farther than this, namely, that in all circumstances where heat is generated, it is at the expense of a precisely similar equivalent of mechanical effect ; and conversely, that mechanical effect is never used up, without a corresponding evolution of heat, and that this is the case whatever be the fluids or other substances employed. Thus in the steam-engine the possible efficiency of the engine is only limited by the mechanical effort due to the heat given out by the condensed steam. So the heat given out by compressed air represents the force expended in compression; and even the heat produced by voltaic or magnetic electricity is that which corresponds to the work it might do. A step farther leads to the equivalence of heating effects by chemical combination to the amount of energy which, differently directed, might have been realized in the shape of work; and though a larger induction is still required to justify all the conclusions which the zealous promulgators of this comparatively new "mechanical theory of heat" have advanced, it cannot be doubted that there is a basis of important truth in the matter which well deserves farther enquiry.

\section{§ 5. Sir JoHn LeSLIE.-Establishment of certain Laws of Radiant Heat.-Pictet-Prevost.}

(641.) THE fact that heat is radiant, or passes through Sir John space in the manner of light, apparently disengaged Leslie- from any material vehicle, became known at an early the science period. Porta in the sixteenth century, and the Floof radiant rentine academicians in the seventeenth, had reflected heat. heat by mirrors. Marriotte and Newton respectively assigned some of the laws which characterize it. Lambert, in the middle of the last century, made some real advances, but it was not until the very close of that period that heat in the radiant form was carefully and systematically studied. The group of philosophers simultaneously engaged on it consisted of Leslie, Rumford, Herschel, Pictet, and Prevost. The two last named were earliest in point of date; but as we owe to Leslie by far the ablest series of experiments, and which for many years, and even to the present time, have formed part of the body of science, we shall connect his name principally with this section.

(642.) Sir John Lesure, born in 1766, completed his studies; studies at a very early age in the University of St Andrews. From boyhosd he was remarked for a decided and independent turn of character; and as his favourite studies were mathematical, he for some time pursued them to the exclusion of the classics. Ultimately, however, he attained also to a respectable knowledge of these, and by his strong natural talents, and his love of reading, he acquired an immense stock of information on all sorts of subjects. This he displayed not only in his conversation, but also in his writings on technical and purely scientific matters, in which he frequently introduced without much apology illustrations from his miscellaneous reading, and even metaphysical disquisitions.

As is frequently the case in persons addicted to (643.) natural philosophy, his first original researches were and essa connected with mathematics. Playfair, who was ${ }_{\text {matics a }}^{\text {on math }}$ eighteen years his senior, encouraged and directed matics al him; Ivory, who was almost his contemporary, and also his fellow-student at St Andrews, was perhaps no less influential in confirming his geometrical tastes. The former communicated Leslie's first original paper to the Royal Society of Edinburgh in 1788. It was on Indeterminate Equations, and was printed in their Transactions. Down to this period we have no record of his being engaged in original experiments; but it is probable that such was the case, for in 1790, and the following years, we have evidence not only of his having speculated on subjects of natural philosophy, but also that he had made experiments intended to confirm or refute prevailing theories. A paper on Electrical Theories was read to the Royal Society of Edinburgh, which, finding them reluctant to print, he withdrew, and he only

1 To Rumford, I believe, is due the attempt (in conformity with this view) to ascertain the heat developed by the friction of fluids, for instance in churning (which, I think, was one experiment proposed by him), but I have not been able to find a reference to it amongst his scattered writings. 
published it more than thirty years after, when certainly it was not calculated to advance science in a perceptible degree. An essay on Heat and Climate, read at the meetings of the Royal Society of London in 1793, had not a more favourable reception; and though published twenty-six years later in Thomson's Annals, it was refused a place in the Philosophical Transactions. The author, no doubt, attributed these rejections to the boldness with which he criticised opinions currently received, and to the novelty of the views which were shadowed forth; but something is, no doubt, to be allowed for the real immaturity of these works, the involved and even inflated style in which they were written, and the questionable evidence for some of the conclusions. In these, and in some subsequent scattered papers in Nicholson's Journal, we observe, with all the faults, yet many of the merits of those researches which afterwards made him justly famous. We find acute observation, ingenious, if not close reasoning, considerable inventiveness in imagining experiments and in constructing apparatus, and a general tendency to express physical laws in a mathematical form. It must be confessed, that these merits were united to a good deal of dogmatism, and a somewhat supercilious judgment of persons eminent in science whose years and attainments should have commanded respect. This, however, is a fault which many ardent students not very conversant with the world have had abundant occasions to regret at leisure. Whether or not he believed Sir Williain Herschel to have had some share in the refusal of his paper by the Royal Society I do not know, but it is difficult, on other grounds, to understand the bitterness with which he expressed himself as to that eminent person, in connection with his experiments on heat.

One of the circumstances which most contributed to encourage Mr Leslie's taste for experiment, was his engagement for above two years as tutor and companion in the family of the ingenious MrWedgwood. Another was the opportunities which he found or made for himself of foreign travel. With or without companions he visited, in the early period of his career, America, and most of the northern countries of Europe, particularly Holland, Germany, Switzerland, Sweden, and Norway. $\mathrm{He}$ also meditated a journey to Egypt and the East, a project reluctantly abandoned, and to which he reverted even in the last years of his life; but it was never carried into effect. Nothing, perhaps, fosters so surely a taste for science as such extended tours; and the acquaintance made under the most agreeable circumstances with foreign philosophers, and the familiarity gained with their language and experiments, contributes to it in no small degree.

We have now come to the period of Mr Leslie's life when his character and position became established, the first by the publication of his Experimental Inquiry into the Nature and Propagation of
Heat, in 1804; the latter by his appointment to the chair of mathematics in the University of Edinburgh in 1805. I shall first say a few words on his character as a mathematician.

Mathematics were, as has been stated, his earliest pursuit, and he cultivated them with great industry Mathemati. and success. His adviser, Playfair, was attached cal writto the methods of the foreign mathematicians; and ${ }^{\text {ings. }}$ Leslie no doubt acquired from him, as well as from his continental friends, a taste for the notation of Leibnitz, then hardly employed in this country, but which he uses in his work on Heat, and elsewhere. Nevertheless, his real preference appears to have been decidedly geometric. He almost always prefers demonstrations, whether in mathematics or natural philosophy, in the manner of Huygens and Newton. He could hardly be called a discoverer in mathematics; but his work on Geometrical Analysis and the Higher Curves shows much taste and knowledge, and is justly commended by Chasles and other foreign writers. His attempt to replace Euclid's Elements by a new work on Elementary Geometry was not more successful than such attempts have usually been.

Unquestionably, the bent of Leslie's mind was to physical research, in which he showed a peculiar Growing talent; and his selection of Heat was, as we have of the subhinted, well-timed; since there appeared a con-ject of ravergence of attention to the subject, such as usually diant heat. heralds some eminent discovery. The doctrines of heat in combination, of which we have already spoken, had engaged the attention of Black, Cavendish, and Lavoisier; the subject of meteorology, in which Leslie took the greatest interest, was becoining a science in the hands of De Saussure and Deluc; whilst Pictet repeated (without being aware of the anticipation) the curious observation of Porta on the apparent concentration of cold by a concave mirror. As this experiment really opened anew the subject of radiant heat, we shall dwell for a moment on Pictet's labours and their results.

Geneva was at this time nearly in the zenith of its eputation as a nursery of the sciences. The most Pictet of reputation as a nursery of the sciences. The most Geneva. of being also amongst its instructors, and the office of professor was then, as it still is, considered one of the most honourable in the state. About $1790 \mathrm{De}$ Saussure, the most eminent physical geographer of his time, was in the vigour of his intellect, and amongst his friends and coadjutors Marc-Auguste Pictet held a conspicuous place. The latter was professor in the Academy, and being a person of popular manners and great information, was known and esteemed by the learned throughout Europe. He was the author of numberless papers in a scientific journal which he edited; but his work on fire-Essai sur le Feu-published in 1791, was his principal publication. It contains some good observations on latent and specific heat; and on the power of different kinds 
of surfaces to reflect and absorb it. He noticed the different heights at which a blackened and a bright thermometer stand even when exposed to common daylight; but so far as I have observed, he did not distinguish the effect of colour in absorbing heat when that heat is accompanied by light or the reverse; and, indeed, this portion of his work stops short on the threshold of most interesting enquiries. He showed that radiant heat travels with great velocity, and observed the heating and cooling of thermometers in exhausted receivers. His work also contains observations on hygrometry, on some points of meteorology, and on the heat of friction. Indeed, its chief fault is embracing so many topics in so short a compass, thus preventing him from thoroughly examining any one of them. To Pictet is due the establishment of meteorological observations at the convent of the Great St Bernard, which are amongst the most interesting which have ever been made, and which are still continued. He died in 1825, at the age of seventy-three.

The interesting experiment of the reflection of cold (649.) moveable equilibrium of heat. led Pierre Prevost, one of Pictet's colleagues, to
devise the theory of " the Moveable Equilibrium of Heat." His idea is, that heat is a substance associated with bodies, of a highly elastic nature, and continually given off from them in proportion to their temperature; which may represent the tension of the imaginary elastic fluid. When the temperature of a body is stationary, it is (according to this view) because it receives by radiation from surrounding bodies exactly as much heat as it parts with in the same way. The general structure of this theory was sustained by the experiments of Leslie, and by some later ones on the law of cooling by Dulong and Petit, which, indeed, realize it in a remarkable manner. Prevost first published his ideas in 1791, in the Journal de Physique, and afterwards in a special work. Prevost was a man of an active and vigorous, rather than profound intellect. He was a foreign member of the Royal Society, and died in 1839 , at the advanced age of eighty-eight.

We now come to speak of Leslie's important Essay on Heat which received from the Royal Society the distinction of the Rumford medals, and which procured for him a European reputation. It is a work difficult to analyze, from the very fact, that its construction is fragmentary, and its arrangement desultory and obscure. Our limits will only allow us to mention the methods of research and their chief results. As a thermoscopic instrument, he used a modification of the common air thermometer (which last had been employed by Pictet), which, having two balls at a certain distance, connected by a bent tube containing a coloured liquid, showed the difference of temperature of the balls; and being hermetically closed, was free from the disturbing variation of at- mospheric pressure. This he called the differential thermometer. A similar instrument had been described by Sturmius in the seventeenth century. Whether Leslie had any previous knowledge of this does not appear; but, as Dr Young very correctly observes in one of his anonymous critical articles, "The principle of the differential thermometer is too simple to be called an invention, and it is only by its ingenious application that Professor Leslie has made it an object of attention." He usually employed as a source of heat a canister of block tin, filled with boiling water, and having sides with different surfaces. The radiating or emissive effect of Emissive these surfaces was measured by the rise of the ther-effect of mometer exposed to their successive influence in the different focus of a metallic reflector. The result showed a great variety of effect, varying from 100 when the surface was blackened, to 12 when it was of polished metal. The absorptive power of surfaces to nonluminous heat is also in exact proportion to their emissive power-a property which seems essential for preserving the equilibrium of like temperatures.

Another and not less important law clearly established by Leslie was this, that the radiation of heat from a plane surface takes place with unequal force emanatic in different directions. ${ }^{1}$ When the specific heating heat. power or density of the calorific rays is estimated in a direction perpendicular to the surface from which it emanates, it is found to be a maximum. At any other angle with the surface, it varies as the sine of the angle. This law (which Fourier showed later to be necessary for the equilibrium of temperature) obtains also in the case of light. Hence the apparent specific brightness or warmth of a surface is the same under whatever angle it is viewed with reference to the plane of the surface, which, when placed obliquely, contributes rays from a larger extent of surface, owing to the foreshortening, but being weaker in the same proportion from every point, the aggregate effect is the same. Some interesting experiments were made on the number of coats of isinglass necessary to effect a complete transformation of the metallic into the gelatinous surface, which was found to be considerable; and, in like manner, the reflective character of metals was only very gradually destroyed by varnishing. This observation, rightly interpreted, showed that some solids are permeated by radiant heat, a conclusion which $\mathrm{Mr}$ Leslie utterly rejected.

Another fundamental experiment less decisively (652.) proved was, that the law of radiation varies inversely $y_{\text {the inver }}$ as the square of the distance. Perhaps the most convincing, as well as the simplest proof of this has the disbeen given more recently by Melloni. If a delicate tance. thermometer or other apparatus for measuring radiant heat be confined in a case, so as only to admit rays coming within a definite angular space-and if 
the instrument be placed in front of an indefinite plane (such as a wall) hotter than itself-the rise of temperature will be precisely the same, whatever be the distance at which it is presented to the heated surface.

The influence of colour on the heating of bodies was considered by Leslie in an original manner. It was found to be effectual only when the radiations are luminous. A thermometer painted black or white (provided the texture of the surface be the same) parts with its heat, and also absorbs the heat derived from such a source as boiling water, in an almost equal degree. The effect depends chiefly on the degree of polish or condensation of the surface. But with luminous sources of heat the case is widely different. This subject had been carefully considered previously to the date of Leslie's work by Sir W. Herschel, who had studied the absorbing power of different colours on the sun's rays. Black and white form the two extremes, and Leslie availed himself of this principle to construct his photometer, which certainly (whatever may be its defects) is an elegant modification of the differential thermometer. It is an instrument having one ball of black, the other of pellucid glass, and united by a tube of the form of the letter $\mathrm{U}$, containing sulphuric acid tinged red as an indicator. As the texture of the surfaces of both balls is the same, dark heat is equally absorbed by both, and the indicating liquid remains stationary. But in the sun's rays, or even in common daylight, the dark ball becomes most heated; and it is not unreasonable to conclude, that when the source of heat remains the same, its variations of intensity are correctly shown. Leslie, however, erred in considering that it was applicable to measuring light differing in origin and quality on a comparative scale; and this error he unfortunately persevered in, after unquestionable experiments had shown its fallacy.

The Essay on Heat contains an elaborate and $r$ various including the effects of mass, surface, contact of air, rcum- currents of air, and likewise of inclosure of the coolinces. ing body in successive envelopes or thin cases; and the author ingeniously compared the results of actual experiment with formulæ based on principles more or less theoretical. But a fundamental error unfortunately runs through all this research, and shows in a striking manner the fatal influence of theoretical preconception steadily maintained through a course of experimental enquiry. He starts with the notion, that the presence of air is essential to the propagation of Heat, generally called "radiant." In fact, for radiation he usually substitutes the word "pulsation," and ascribes the effect of surface in modifying the cooling of bodies to its faculty of transmitting pulsations or tremors, more or less readily, to the vehicle of the air. He was indeed compelled to admit that air had a double agency; one "abductive," as it draws off heat by contact and by what is generally called "convection," that is by currents which the communication of heat itself produces; the other, "pulsatory," which corresponds to what is usually termed radiation, but which Leslie persisted in believing to be due to tremors propagated in air, after the manner of sound, and with the same velocity. In the concluding chapter of the work before us he considers the cooling effect of different gases, and of air of different degrees of rarefaction; and this last experiment might, one would have thought, have satisfied him of the fallacy of his opinion ; since, taking his own numbers, when air is rarefied 1024 times, the "pulsatory energy" is only diminished one-third part. In fact, it appears as if his work broke off abruptly, when the course of observation became irreconcilable with the opinions advanced in the early part of it.

After this analysis of Leslie's greatest contribution to science, I cannot afford space to dwell upon his His minor minor inventions. I pass over them, however, with writingsthe less regret because they have been fully dwelt experiupon in his "Dissertation," of which the present is a ment. continuation, and in his articles on Cold and Meteorology in the Encyclopredia. The most original and important of these was his very beautiful process of producing ice in quantity by the cold of evaporation, in the receiver of an air-pump; rendered effectual by his ingenious use of absorbent surfaces for withdrawing the vapour. This experiment was completed in 1811, and attracted much attention: It was connected with his researches on hygrometry, to which he also adapted his differential thermometer. But in the development of this difficult theory, he was less successful, nor indeed could he well be so, whilst he adhered to the old opinions respecting the affinity of air for moisture.

Having filled the Mathematical Chair from 1805 to 1819 , Leslie was in the latter year translated to that Close of his of Natural Philosophy, vacant by the death of Play- career. fair. He had a good collection of apparatus, and devised many ingenious experiments. In 1820, he was elected corresponding member of the Institute of France, and died on the $3 d$ November 1832, at the age of 66 , having received the honour of knighthood, on the recommendation of Lord Brougham, but a few months before.

In closing this brief sketch of Sir John Leslie's career, we cannot fail to observe the combination of His philounusual powers with unusual drawbacks to their sophical complete and vigorous exertion. Whilst he had the in some chief merits, he had also the most serious defects, of points dethe self-formed student. He was ardent and ambi-fective tious in the pursuit of knowledge. He must have been for many years a hard, if not a methodical student; he united good mathematical knowledge with a real love of experiment; he was gifted with a strong memory, and confident in the exercise of all his powers. Why, with so many advantages he did not achieve more, nor put forth even what he did to 
greater advantage, was mainly because he yielded to the guidance of an imagination which often carried him into fanciful speculation, yet which was strangely united with dogmatism in maintaining what he had once maintained, and a disposition to accuse others of misinterpreting nature, whenever they arrived at conclusions inconsistent with his own.

In such sciences as those which he chiefly cultivated, - sciences eminently progressive, imperfect, and dependent on experimental proof,-a just appreciation of the labours of others is one of the most essential parts of the philosophic character, whilst an absence of it infallibly condemns the dogmatic theorist to be gradually left behind, even in the paths which he had at first trod with the greatest distinction. With few exceptions, Sir J. Leslie carried his scientific views of 1804 with him to the grave. The possibility of the passing of heat, except solar and highly luminous heat, through any solid body, such as glass, though proved by Maycock, De la Roche, and Pow ell, - the existence of dark heating rays in the sunbeam, less refrangible than the red, demonstrated by Herschel, and afterwards confirmed by many others, - the doctrine of gases and vapours as laid down by Dalton,-the maximum density point of water shown so ably by Hope and Rumford,-the existence of climates in the arctic regions of which the mean annual temperature does not exceed $0^{\circ}$ of Fahrenheit, proved by Parry and his successors,all these, and many other demonstrated truths in his own peculiar walk of science, were, we fear, practically ignored by him throughout life. But we willingly leave the ungrateful task of indi- Merit of cating defects. Let us recollect rather with pleasure tal rehow much we owe to his beautiful discoveries. It is searches. perhaps not an insignificant test of their originality, that though they were generally adopted (at least to the extent which his own experiments justified), Leslie's observations were but rarely repeated, and that only in the way of general confirmation and illustration. I mean that for a great many years the path which he had opened, and the methods which he described, were not seized upon by others, as leading to a sure course of discovery. Until the time of Dulong, his experiments on cooling were perhaps never carefully resumed, and a very great number of his subjects of enquiry were only taken up thirty years after their publication, as we shall see in a future section.

The observations of Herschel on the Refrangibility of Solar Heat, I shall include in the notice of the experiments of Berard and De la Roche, to which we shall presently turn.

\section{§6. Fodrier.-Mathematical Theory of the Conduction of Heat. Lambert; Poisson.- Temperature of the Earth and of Space.}

(661.) It is stated by Arago, that when the Academy of Theory of Sciences, above a century ago, proposed as a prize subthe conduc- ject, "La Nature et la Propagation du Feu ;" adding, tion of heat "la question ne donne presque aucun prise à la géobert. metrie," - a majority of the candidates treated of the methods of preventing the burning down of houses ! It is true, however, that on that occasion, Euler sent a memoir which, though crowned, was unworthy of his genius. Lambert in his "Pyrometrie," in 1779," had the rare merit of laying the foundations of the science of conduction. He solved correctly this question:- "If a thin conducting bar of indefinite length be kept with one extremity heated to a constant degree above the surrounding space, required the temperature of any point in the axis of the bar?" The solution is, that the temperatures, or rather excesses of temperature, diminish in a geometric ratio, at distances reckoned in arithmetical progression, from the origin of the heat. ${ }^{2}$ In this solution it is assumed, (1.) That the flow of heat along the bar, is at any point proportional to the rapidity with which the temperature at that part of the bar is lessening as we recede from the source ; in other words, that the flow of heat from the hot part to the cold part, is more rapid in proportion as the difference of temperature of two sections of the bar at a given short interval is greater. (2.) That the bar parts with its heat to the surrounding space, exactly in proportion to its excess of temperature at every part.

This beginning, which perhaps like many of Lambert's other writings was not very generally known, Biot had no sequel until 1804, when M. Biot attempted to find the differential equation of the general movement of heat on the same principles. But the form which he obtained, including a mathematical solecism, betrayed some error in stating the conditions. Three years later Fourier had more success. But, conformably to the plan of this discourse, I shall premise some facts regarding his early career, which was far from commonplace.

Joseph Fourier was born in 1768, at Auxerre in France. He was of humble parentage, and being Fourierearly left an orphan, was educated by the Benedic- his early tine monks who, singularly enough, conducted with success in that town a military school. It seemed his fate to become either a priest or a soldier; yet he was neither, though ere long familiar with camps. He became first a pupil of the old normal school of

1 Pyrometrie, oder, von Maasse des Feuers und der Wärme. Berlin, 4to, 1779. This work was posthumous, and contains many riginal observations on Thermometry, Conductiọ, Solar Radiation, and Climate. 
Paris, when Lagrange, Laplace, and Berthollet were amongst the professors. He had already presented to the Academy, at the age of 21 , a paperon the numerical solution of equations, a subject of predilection with him, and to which we shall presently return. After leaving the Normal School, he was named one of the original professors of the Polytechnic School, a station of which he was justly proud, but from which he was withdrawn by the requisition to join, along with Monge and other savans, the Expedition to Egypt under Napoleon. It was the singular fancy the Egyp- of that extraordinary man, to create an Egyptian ian lnsti- Institute, of a constitution similar to that of France. ute. Fourier was perpetual secretary. But it proved little better than a waste of talent. The arts of Egypt were not regenerated, and France was despoiled of some of her ablest philosophers. Fourier had quite as much to do with battlos and treaties as with equations and experiments. Yet he often referred afterwards with partial recollection to those stirring times, and recounted, with the ardour of a somewhat garrulous temper, the valiant feats of arms which he had witnessed. Fourier edited the account of the Expedition to Egypt, and wrote the historical preface, the composition of which ultimately procuicd for him a seat in the Académie Francraisc.

(664.)

On his return to Europe, he was appointed Prefect of the Isère in 1802, and Grenoble became his home for some years. Whilst he devoted a just share of his attention to his public duties, he found time to produce his greatest work, The Analytical Theory of Heat. His first paper on this subject dates from 1807. It was communicated to the Academy of Sciences, but not printed. The subject was however proposed for a prize, to be decided in 1812, when Fourier's essay was crowned, but, strange to say, not published. The cause, it is to be feared, lay in the jealousy of the greatest mathematicians of the age. Laplace, Lagrange, and Liegendre, the committee of the Academy, whilst applauding the work, and admitting the accuracy of the equations of the movement of heat thus for the first time discovered, insinuated doubts as to the methods of obtaining them, and likewise as to the correctness of the integrations, which were of a bold and highly original kind. These disparaging hints were not supported by any precise allegations; and we can scarcely blame Fourier for feeling indignant at the tyranny of the mathematical section, and little disposed to regard with favour the few and comparatively insignificant efforts of several of its members subsequently to ratify and extend the discoveries which he had unquestionably made. The manuscript, after lying for twclve years in the archives of the Institute, where it was consulted by different persons, was finally printed, word for word, as it stood in 1812.1 Fourier's long absence from Paris in a remote provincial town, rendered this in- dignity possible at first; and afterwards, it was his misfortune to be unable to hold a political station without offence, amidst the violent intestine conflicts with which France was afflicted. He alternately displeased his old master Napoleon and the Bourbons, and the consequence was, that after the Restoration he found himself dispossessed of every employment, master of not one thousand pounds, and refused by the government even a seat at the Institute. This indigence, so honourable to himself, and this neglect, so disgraceful to others, tended, no doubt, to increase an irritability, such as intense mental exertion often produces, and which the injustice of his scientific countrymen had already aggravated. Finally, however, he received a modest post connected with the civil administration of the department of the Seine; he was also elected a member of the physical section of the Academy of Sciences, and he finally became perpetual secretary of that body.

Fourier's papers on Heat show a remarkable combination of mathematical skill with a strict and pre- His physicise attention to phyical considerations. In this cal preciexcels almost every writer of his time, and especially periments. his colleague and younger rival, Poisson. His experimental skill is not to be so highly praised, although he illustrated several of his solutions by actual trials, which he submitted to calculation, and showed to agree with theory. Their degree of precision, however, hardly allows them to be considered as tests of theory.

Fourier assumes the correctness of Newton's law, as well for communication of heat from point to point Assump. of a solid, as for the external radiation by which it tions of the parts with its heat into the surrounding space. In Analytical the former case, the flow of heat is proportional to the rapidity of the depression of temperature, in the direction in which the motion of the heat is considered; in the latter, it varies as the excess of temperature of the surface of the hot body above the surrounding space, affected, of course, by a constant depending on the radiating power of the surface. These, as I have said, were also the Postulates of Lambert's solution. Fourier's researches, fortunately perhaps, preceded for the most part Dulong and Petit's enquiry into the true law of cooling. I say fortunately, since otherwise Fourier might have been discouraged from attempting the solution of problems which are highly important even in an approximate form.

With regard to the law of radiation, Fourier had the merit of showing, for the first time, the necessity of Necessity Leslie's experimental law of the intensity of emanated of emanaheat being proportional to the sine of the angle which tion. the direction of emanation makes with the surface. This he considered both mathematically and physically. Mathematically, he showed that were this law not true, a body might be maintained for an indefinite time within an envelope of constant temperature, and 
yet never acquire that temperature, even approximately; and physically, considering that radiation proceeds not from a mathematical surface, but from a material physical stratum of an imaginable thickness, but which rapidly absorbs the emanations proceeding from the inferior particles, he proved that the attenuation due to oblique emanation will follow Leslie's law, independent of the precise rate of absorption in traversing the physical surface.

(668.) Fourier takes extraordinary pains to define and Definition justify every step of his demonstrations. He has the ing power, great merit of having first given a clear definition of conducting power, or conductivity proper, which is this :- "The number of units of heat (measured by the weight of ice which it can melt) passing in unit of time across a square unit of surface of an infinitely extended plate bounded by two parallel surfaces at unit of distance which are respectively maintained at the freezing and boiling temperatures (unit of difference of temperature)." 1 In like manner, the " exand of terior conductivity" expresses the number of units "exterior of heat parted with by unit of surface to the air and conducti- surrounding space, when the difference of their temvity."

(669.)

Fourier's Thèorie An -The problems considered by Fourier in his Thèrie alytique de Analytique, refer principally to the propagation of lachaleur. heat in homogeneous conducting solids of definite forms, and in some cases maintained in certain parts at fixed temperatures.

(670.) The number of examples fully worked out is very Instances small, but they may be referred to the following of problems by classes :- (1.) When some part of a solid has an indehim. finite source of heat applied to it, the remaining surface being exposed to the air, or having determinate temperatures maintained at certain parts. In this case, the state of the solid in regard to heat is permanent, or independent of time; and the problem is to assign the temperature of each part, and the flow of heat through that part in a given direction. (2.) To assign the temperature of every point of a solid primitively heated, either uniformly or after any assigned law, and at any given moment. (3.) To solve the last question only in the case where the cooling at the surface has been going on for an exceedingly long time.

(671.) Of the first class of problems, the slender bar heated Stationary by a constant source of heat at one end, and exposed of heat in a to the cooling influence of radiation and of the air, slender which had been treated of by Lambert, is the simplest bar. slice perpendicular to the axis of the bar, is the result on the one hand of the heat which it acquires from the hotter slice nearest to it on the side of the source of heat; and on the other, of the heat with which it parts to the slice next beyond and also to the air in contact with the exterior surface of the slice and by radiation from the same surface. The solution of this problem is that of a simple differential equation of the second order, and the result is the diminishing geometrical progression of temperature already mentioned. This has been approximately confirmed by some careful experiments of M. Biot, which indeed are nearly the best which we yet possess on the subject. But instead of drawing from them, as he does, an argument for the accuracy of the Newtonian law of cooling, the diminution of temperature along the bar is far more rapid at first, and less afterwards than that law indicates. In fact, the apparent agreement of the formula is owing to the use, in a case to which it does not correctly apply, of that often misapplied rule of the doctrine of chances-the method of least squares.

The solution of another case of stationary temperature, - an indefinite solid bounded- by three infinite In an infi. planes (two of which, B, C, are parallel, and the third, ${ }^{\text {nite solid. }}$ A, perpendicular to both) having determinate temperatures,- -requires the introduction of a species of analysis, in which Fourier acquired great dexterity, but which is of so subtle a kind as to have created doubts in the minds of the committee of the Institute to which the Memoir was referred, and to have been a source of some controversy and much discussion since. Fourier contrives to express, by an infinite trigonometrical series, the law of temperature in such a solid, which shall not only satisfy the differential equation of the equilibrium of heat, but also the conditions of temperature at the bounding planes, $\mathrm{B}$ and $\mathrm{C}$, which being zero by the problem, the value of the temperature which, up to that point was finite, suddenly comes to nothing, and has no value beyond. This problem leads to a long digression on the possibility of expressing by trigonometrical series, quantities which vary according to any conceivable law and of determining the co-efficients of the successive powers of the sines and cosines employed. The theorem to which Fourier is led, in which any function of $x$ is expressed by a series of definite integrals, including $\sin x$ and $\cos x$, is known by his name.

The problem, however, which Fourier most elaborately treated, belongs to the $2 \mathrm{~d}$ and $3 \mathrm{~d}$ class,namely, the cooling of a sphere primitively heated Movemen sphere.

1 Let $\mathrm{F}$ be the flux of heat measured as above, $\mathrm{K}$ the constant of interior conductivity, $z$ an ordinate measured across the thickness of the plate, and $v$ he temperature of the stratum of which $z$ is the ordinate; then $\mathrm{F}=-\mathrm{K} \frac{d v}{d z}$; in the permanent state the temperature varies uniformly from stratum to stratum. When the thickness and difference of temperature both are equal to unity, $\mathrm{K}=\mathrm{F}$. The expression $\mathrm{F}=-\mathrm{K} \frac{d v}{d z}$ manifestly expresses the Newtonian Law, interpreted by Fourier as stated above. 
in a given way, but so that points equi-distant from the centre have a common temperature. ${ }^{1}$ It might be expected that the symmetry of the conditions would admit of a simple solution; and such indeed was sought by Professor Playfair long before, in a paper in the Edinburgh Transactions, with reference to Geological Spcculations. It was, however, by no means sufficiently general. It is shown by Fourier, that even in the simplest supposable case-that where the temperature of the sphere was originally uniformthe resulting expression for the temperature of any stratum at any time, though capable of algebraic expression, cannot be assigned in finite terms, and no attempt has been made to evaluate it generally. The special cases which have been considered, are when the sphere is extremely small, or has been cooling for a very long time.

(674.) The extreme complication of even such apparently urces of simple cases when solved in all their generality conm in the sists in this, that the flow of heat across each part is oblem of in fact dependent at each instant on the state of heat Sphere. in each other part, and this distribution of the whole is equally unknown with the local distribution on which the movement of heat in each part depends. To this must be added the peculiarity of conditions at the surface, where the temperature undergoes an abrupt change. The law according to which the superficial particles radiate heat, is also different from that according to which they receive it from the interior. When a body uniformly heated to a considerable temperature cools in air, the subtraction of heat from the surface commences with great rapidity, the excess of temperature of the superficial particles being very much greater than it ever can be afterwards. The drain of heat from the interior of the sphere is at first nearly imperceptible. The exterior cooling will by and by become slower; and the degree in which it takes place, will depend upon the rapidity with which the conducting mass of the sphere is able to supply fresh heat to the surface; then the rate of superficial cooling will become relatively somewhat accelerated, and a fresh drain will take place from the interior towards the surface. It is only in the case of exceedingly small bodies, or those of an infinite degree of conductivity, that the body will cool according to a simple law. In all other cases, there will be characteristic periodical inflections in the course of cooling. These, no doubt, are represented in Fourier's series, if it could be numerically calculated; and it is to be desired that some attempt were made to represent it approximately. When the cooling has endured for a very long time, these gushes of heat cease altogether; the surface has attained nearly to the temperature of the surrounding space, and the drain of heat from the interior is so slow, that the progression of temperature is very gradually and slowly disturbed.

Application to the Thermal condition of the Earth. -This last case has been discussed by Fourior (675.) -This last case has been discussed by Fourier with Applicathe Farth, considered as a mass which has been once these prinat a high temperature, of which we have evidence the therin the general increase of heat as we descend in mines, mal condi or when we penetrate its crust by Artesian wells tion of the The surface of our planet receives a large amount of heat annually by absorbing the sun's rays, but parts with it by radiation into free space, to an extent which preserves a sensible uniformity of temperature from age to age. The considerations connected with the subject are these:-(1.) The proper heat of the earth and how much heat reaches the surface from the (perhaps) still incandescent interior; (2.) How much heat do we receive from the sun, what share of it enters the surface, and how far, and according to what periods does the influence of the seasons extend below the surface? (3.) What is the amount of refrigeration of the earth's surface?-how does the atmosphere affect it? and, if the cooling be due entirely to radiation, what are we to set down for the temperature of space, so as to account for the heat lost?

First, As to the proper heat of the globe. Not to go further back than the last century, the incandescence of the earth's nucleus was assumed as very pro- globe ; bable by Buffon and other popular writers, and their opinions were, on the whole, confirmed by the progress of observation. The existence of volcanoes was, of course, an obvious argument; another was, that if the earth were once hot it must be still cooling; consequently, climates are continually becoming colder, especially in the Arctic regions, which it was supposed depended most on the supply of heat from within. In evidence of this change were quoted, not only the romains of elephants found interred with flesh and skin in the midst of Siberian ice, but also the unequivocal proofs, so well known to geologists, that at a period indefinitely more remote, tropical plants of gigantic growth, and animals of a class which now frequent only southern seas, appear to have lived and flourished in high northern latitudes. But even in the time of Buffon, attention was directed to a fact yet more important for the theory of heat, namely, the increase of temperature observed in deep mines. Notwithstanding many sources of doubt and confu-manifested sion, such as the heat from candles, and from men at by the inwork in mines, from chemical changes in some cases, heat in and from the increased density of the air, the fact of mines. the increase is now well established, and also its rate of progression with more certainty than in the time of Fourier. On an average (including the best data of all, those yielded by Artesian springs), the

1 The problem of the Armil or ring, heated at one or several points, is one which offers considerable facilities for its solution, but the results are of little utility, the form being so peculiar; and even as a test of theory, the variations of temperature from point to point are insufficient under the limited conditions in which the numerical solution is practicable. 
increase appears to be $1^{\circ} \mathrm{Fahr}$., for 50 or $60 \mathrm{English}$ feet of descent. Fourier first undertook to enquire, Whether, supposing the earth to have no primitive internal heat, the continual action of the sun might not produce the increasing temperature observed. But he found, on the contrary, that there is no such tendency, and that after a long time, the temperature at any moderate depth below the surface (but beyond the varying influence of the seasons) will be constant. Now, observations on the temperature of mines and Artesian springs extend to the depth of 1700 English feet, and the influence of the seasons is usually quite extinct (in this latitude) at 50 or 60 feet. The nearly uniform increase beyond is therefore due to a source of heat within, or, what amounts to the same thing, to the relatively warmer state of the nucleus. The analysis of Fourier shows, that the variation of temperature in the successive strata of a sphere, cooling with excessive slowness, is very closely allied to the flux of heat which passes through it, and which is spent by radiation or otherwise at the Its insigni- surface. The result is exceedingly striking, and may ficant effect be considered as remarkably well established; the on climate. flow of heat from the interior contributes to raise the temperature of the surface by only one seventeenth of a degree of Fahrenheit ; or would melt annually a stratum of ice $\frac{1}{4} \frac{1}{t}$ inch in thickness. This is all the refrigeration which the earth's surface can ever suffer on this account, and in its present state of cooling, it would take millions of years even to reduce it by one half. So little ground is there for the belief of Buffon and his friends, who imagined that the destruction of animal and regetable life must rapidly ensue by reason of the diminishing central heat.

Again, the depth to which we must descend in order to reach a temperature sufficient for the supply of molten lava, is not excessive: for this depends entirely on the conductivity of the earth's crust, which we know to be very small. A familiar instance occurs in streams of recent lava, where a crust soon forms, on which a man may walk safely, yet only be separated by a foot or less from the fiery liquid. Fourier calculated that the temperature of incandescence may prevail at a depth within the earth of only about 15 English miles, without affecting the superficial temperature by more than a small fraction of a degree.

As it seems scarcely possible to ascribe some, at (678.)
Cause of secular change of climate.
Secondly, As to the effects of the sun's heat on the earth. It has been already stated that Fourier Effect o howed that below a moderate depth, the he (he heat on earth would be uniform and invariable, so far as solar earth. radiation is concerned. At smaller depths, it will vary according to the season of the year, and these variations will at all depths be gone through in the same period with the variation at the surface, which, is of course annual, but the inflections follow a peculiar law in each latitude and climate, and even in one year compared with another. Within the depth to which the influence of the seasons extends, the amount of the range of temperature continually diminishes, nearly in a geometrical progression, and at length it becomes insensible. At increasing depths, the periods of maxima and minima are continually retarded, so that at a certain depth, the earth is hottest in winter and coldest in summer. A smaller fluctuation of the same kind, and penetrating to a smaller depth, follows the diurnal range of temperature. The manner in which these interesting phenomena are connected with the conducting power and specific heat of the earth's crust, was clearly pointed out by Fourier.

Before as well as after these theoretical investigations, observations on thermometers sunk to different depths in the ground had been made, and the compare results confirm these conclusions of theory in every with exp respect. Such observations were made by De Saussure, Leslie, Arago, Quetelet, and other experimenters, including the writer of these pages, who caused careful experiments to be made for five years, on three sets of thermometers, placed in different kinds of soil and rock, extending to a depth of above 25 feet. By means of these, the conductivity of those various soils was determined with accuracy, and data afforded for subsequent enquiry. 1

Amongst the most interesting enquiries connected (681.) witb this subject, is the total quantity of heat re- Totalqu ceived in a year from the sun by the absorptive tity of h action of the earth's surface; but this does not ap- the eartl pear as yet to have been successfully answered. from the Nevertheless, M. Pouillet, from merely experimen- sun. tal data, considers that he has proved that the amount of solar radiation which reaches the earth would melt in a year an average thickness of 31 mètres of ice, all over its surface, provided that it were entirely absorbed. Poisson has obtained a result four times less, and estimates the effect of the sun in raising the temperature of the climate of Paris at 24 centigrade degrees, a result incredibly small.

Thirdly, On the temperature of space. Fourier was probably the first who introduced this idea into The tem science. The term perhaps is a doubtful one and perature liable to misconception, but the object of the enquiry spaceadmits of being precisely stated. The researches of the heat emitted 'by the change; in other words, that our sun is a variable star.

1 See Transactions of the Royal Society of Edinburgh, vol. xvi., where a history is given of the observations previously made. 
Dulong and Petit ${ }^{1}$ upon the cooling of a body by radiation only within an envelope having a temperature lower than itself, include, as a particular case, that in which the body receives nothing from the envelope,-when the radiation is entirely that of loss without any gain; and yet the cooling proceeds with a finite velocity. It could not with any propriety be said in such a case, that the temperature of the space in which the body cools is infinitely low; it cannot be said to have any temperature at all. (683.) Now, in the case of our globe and its atmosphere,
fined by we have a heated mass, suspended as it were, in space. purier. If there were no other bodies in the universe, the earth must by degrees lose its heat. We know indeed that it is cooling. The proportion of its native heat annually emitted, would melt a crust of ice $\frac{1}{4}$ th inch thick. This heat is dissipated in space. It may therefore be enquired, whether a sphere of known conducting power and of known temperature at the surface, is parting with its heat to space at a rate which supposes it to radiate without any requital, or whether it receives from space (or the bodies which space contains, independently of the sun) any portion of the heat which it thus dissipates. To solve this question, we must evidently know with great accuracy the radiating power of the surface of the earth, than which, unfortunately, no datum is more completely uncertain: and the influence of the atmosphere (which is truly a part of the earth) renders the solution still more indeterminate. It is not known what method Fourier took to arrive at a numerical result, but it is well known that he obtained it in a way which appeared satisfactory to himself, and that he often referred to it. He supposed the "temperature of space" to be $50^{\circ}$ or $60^{\circ}$ below zero on the centigrade scale, and believed he did not err in fixing it by more than $8^{\circ}$ or $10^{\circ}$. By this we understand, that after infinite ages, the earth, or any other body placed in the same situation and previously heated, would attain this temperature and no lower.

This slight sketch gives an imperfect idea of the extent and originality of Fourier's labours. But enough has been said to show, that he must rank amongst the most considerable philosophers of his day. That he excited the jealousy of the great mathematical geniuses of the previous generation, and that his new train of research, though fully accepted by those who succeeded him, has as yet received but slight extension at their hands, are facts which concur to prove his originality and merit. That he did not solve more cases of the propagation of heat, and that some of his solutions are so complicated as hardly to be such in a practical sense, show only the extreme difficulties of a subject which touches every where the boundaries of existing mathematical knowledge. In the opinion of many persons, it is to be desired that some at least of the problems of conduction were treated in a somewhat different way, and approximations obtained by the application of a less abstruse calculus. But towards this desirable result, little has yet been done.

As a pure mathematician, Fourier occupies a (685.) distinguished rank. His conversion of functions of Writings every kind into series of periodical quantities-his on pure treatment of problems, involving discontinuous laws mathema-his solution of higher differential equations-are tics.

all important additions to analysis, to the improvement of which, as he has himself very justly observed, physical problems are ever the most important avenues. Such considerations as Fourier treated of could hardly have entered into the mind of a mathematician not guided by a specific physical enquiry. His favourite subject of the solution of Numerical Equations, which brought forth his first essay-which occupied him even on the banks of the Nile-and an elaborate work on which was almost his last addition to science,-is of course one of less general interest.

His experimental abilities, as we have said, were not equal to his mathematical ; and it is to be re-Experigretted that his schemes for several practical appli- mentscations of theory seem to have been left imperfect. Style. He invented a Thermometer of Contact, an instrument for determining the conductivity of bodies, which is but little known or used, and of which the theory was left incomplete. He also joined Oersted in experiments on Thermo-Electricity. He studied with great care the principles on which his theories were based, and seems throughout to have desired to leave no doubtful step in his reasonings, nor to make any tacit or unproved assumptions. His compositions are minutely clear. To them we might apply the remark attributed to Voltaire- "Whatever is obscure is not French." If there be obscurity, it is only due to the abstruseness of the subject, not to the manner of conveying it.

Fourier succeeded Delambre as Secretary of the Ma- (687.) thematical Class of the French Academy of Sciences, His death. and wrote several Eloges. He died no the 16th May 1830 , generally respected.

We will here, in a few words, comment on the (688.) subsequent progress of the subject of the Conduction Fourier's of Heat.

Whilst Fourier's papers were still in the archives (689.) of the Institute, they were consulted by Poisson, who Poisson. published solutions of several problems based on Fourier's principles, and coinciding in result with his. As the analysis used was somewhat different, the coincidence in so new a subject was not without importance. In 1835, the Theory of Heat of the same author appeared, based on the law of cooling, discovered by Dulong and Petit, which Poisson, with 
unaccountable yet characteristic rashness, chose to consider as applicable to the communication of heat from particle to particle, as well as to its dissipation from the surfaces of bodies. But the result was worthy of the looseness of the assumptions; the terms arising from the peculiarity of Dulong's law are mostly dispensed with as the investigation proceeds; and the whole work is to be regarded rather as a mathematical exercitation, than as a serious step in physics. Having elsewhere recorded a criticism on that part of Poisson's work which treats of the Heat of the Globe, I shall not dwell farther on its defects. ${ }^{1}$

Professor Kelland has published a work ${ }^{2}$ - the only (690.) Kelland's Theory of Heat. one intended for students - on this subject, and he has likewise made a valuable report to the British Association, on the best means of comparing the Mathematical Theory of Heat with Observation. ${ }^{3}$ He has suggested, that the results of the Newtonian law of cooling may be used in combination with such an hypothesis as to the relation between quantities of heat and the temperature shown by an air thermometer, as will reconcile it with Dulong's law. But there is reason to think, that internal conductivity varies with temperature in the reverse manner of external cooling; in other words, that it diminishes as the absolute temperature increases. ${ }^{4}$

MM. Duhamel and Stokes have considered the propagation of heat in bodies which do not conduct it uniformly in all directions; and their investigations are very interesting in connexion with the beautiful observation of M. de Senarmont, that such is the case heat uniin crystallized bodies. It is easily shown in a plate formly ir of gypsum coated with a thin layer of wax, to a small all dir. part of which heat is applied.

\section{§ 7. Dulong.-The Law of Cooling.-Progress of the Science of Radiant Heat between Leslie's and Melloni's Discoveries; transmission of Radiant Heat through Glass. Herschel; De la Roche; Professor Powell.-Theory of Dew; Wells.}

(692.)

Subject of radiant heat resumed.

(693.) Dulongan eminent chemist.
The researches of Leslie on Radiant Heat, though very generally appreciated both at home and abroad, were not, on their publication, immediately repeated or extended. On some points they were seriously controverted. But the labours of most of his immediate contemporaries, of whose names we have given a few at the head of this section, were rather preparatory to the fuller developments of a later time-to be made with improved apparatus - than demonstrated discoveries. I make an exception, however, with regard to the experiments of Dulong and Petit on the laws of simple radiation and of the cooling of bodies by the contact of gases, not only because they established propositions quite new in an incontrovertible manner, but also because they introduced into this branch of science a mode of investigation so delicate and precise, and methods of reduction and of physical analysis so beautiful and convincing, as placed the whole science of Heat on a new footing. The main credit is due, we believe, to Dulong, who was one of the most estimable and accomplished philosophers of his time.

Pierre Louis Dulong was born in 1785, and showed precocious talents. He was admitted to the Polytechnic School at the age of 16 , and rose through every grade of that celebrated institution until he became "Directeur des Etudes." It is superfluous to say that he was a good mathematician. He was also a most excellent chemist; and the honour of being discoverer of the most terrific of fulminating compounds (chloruret of azote) was purchased by the loss of one of his eyes. But his experiments on Heat are those by which he will be longest remembered. The most important series of these was devoted to a rigid examination of the amount of heat radiated under different circumstances, and of that dissipated by the contact of air.

The law of Cooling.-It had been suggested by Newton, and tacitly admitted by nearly all writers on heat, Dulong including the most eminent, that simple radiation (or and Petit pulsation, in the language of Leslie) takes place in di- Newtoni rect proportion to the excess of temperature of the hot law of body above the surrounding space. Martine, and par- cooling. ticularly De la Roche, had indeed thrown doubt on the subject, and had rendered it probable that at high temperatures the velocity of cooling (which is proportional to the radiant energy) is considerably greater than Newton's law supposes. Dulong and Petit, however, first demonstrated this. They disengaged the experiment as far as possible from the influence of the contact of air, by using the most perfect exhaustion which the best air-pumps could produce; and it appeared very plainly from the result of observations made under successive degrees of rarefaction, that instead of there being the slightest appearance of radiation ceasing to take place when the vehicle

\footnotetext{
1 See Second Report on Meteorology, Brit. Assoc. Reports, 1840.

2 Theory of Heat. Camb., 1837. ${ }^{3}$ Brit. Assoc. Reports, 1841.

- This at least is the result of an experimental investigation by myself on the conductivity of iron, executed on a principle which I believe to be new, but which I have not yet been able to publish. Mr Airy and Professor Kelland are each in possession of the outline of my method; and the result noted in the text was briefiy announced by me in the Reports of the British Association for 1852. If my health permits, I shall resume and publish these experiments. I may here add, that I poirted out in 1833, from some experiments which I made at that time (Proceedings of the Royal Society of Edinburgh, vol. i. p. 5), that the metals range in the same order as conductors of Heat and of Electricity; and this law appears to be confirmed by more recent observations.
} 
of air was absent, the influence of the latter became less and less perceptible.

(695.) The principal apparatus of Dulong consisted of a balloon of thin copper about a foot in diameter, coated internally with lamp black, and placed in connection with an air-pump, so that any portion of atmospheric air could be extracted up to about $\frac{3}{4} \frac{9}{0} 6$ of the whole, and any other gas could be introduced into it. The temperature of the balloon could be nicely regulated by introducing it entirely into a water trough. Into the centre of the balloon, thermometers of different sizes, or having different kinds of surface, could be introduced. The temperature of the balloon having been first regulated, the thermometer under experiment (being itself the radiating and cooling body) was heated nearly to the boiling point of mercury, and inserted in the balloon so as to occupy the centre of it. The exhaustion and other arrangements being made, the observations on the rate of cooling of the thermometer commenced when its temperature was as high as $250^{\circ}$ or $300^{\circ}$ centigrade, equivalent to $482^{\circ}$ and $572^{\circ}$ Fahrenheit.

(696.) With respect to simple radiation, or when the velocity of effect of air in the balloon is estimated as nothing,
cooling not simply as the inaccuracy of the Newtonian law was soon apthe excess parent. Whilst the excess of temperature of the of heat. cooling body above the envelope or balloon remained constant, and the absolute temperature of both was made to vary, the velocity of cooling, instcad of being constant, increased rapidly with the temperature. Thus the excess of temperature being in every case $200^{\circ}$ centigrade, and the temperature of the balloon being $\quad \ldots \quad 0^{\circ}, 20^{\circ} ; 40^{\circ}, 60^{\circ}, 80^{\circ}$, the rate of cooling was $7 \cdot 4,8 \cdot 6,10 \cdot 0,11 \cdot 6,13 \cdot 4$. The whole of an elaborate series of observations was beautifully and satisfactorily represented by a formula admitting of this simple physical interpretation, viz., that the cooling of the thermometer is the difference between the heat which it parts with to the envelope and the heat which it receives from the envelope; and that the heat thus parted with, either by the thermometer or the envelope, varies in a geometric ratio with its temperature. ${ }^{1}$

The effect of contact of a gas in cooling the thermometer is more complex. It is independent of the texture of the surface, as Leslie had already supposed. The cooling power of a gas is proportional to a certain power of its elasticity, which varies for each; it takes place more rapidly in hydrogen than in any other known gas, which was likewise discovered by Leslie. It also comes out rigorously, that the ratio of the radiating power of different surfaces is the same at all temperatures. This ratio for glass and silver is 5.707: 1. Assuming this last principle, and also that the cooling due to the contact of air is independent of the surface, the law of cooling in vacuo may be deduced from the observed cooling of two thermometers suspended in air, and having glass and silvered surfaces respectively. Dulong and Petit found that, when they analyzed their experiments in this way, they obtained values for the radiation in vacuo almost absolutely coinciding with what direct experiment had already given. No more perfect criterion could be desired of the soundness of every link of the chain of experiment and induction.

We shall not analyze Dulong's other memoirs. They regarded matters in the science of Heat requir- Other meing the same skill in devising apparatus and in mani- moirs of pulation, the same caution in eluding errors, and the the laws same just principles of calculation as in the investi- of heat. gations already specified. They did not, however, lead to the discovery of laws so striking and so general. They included the very delicate and difficult subject of the laws of the thermal expansion of bodies, particularly that of air and of mercury, which were applied to the theory of the thermometer, the very basis of all exact knowledge in the doctrine of heat. Another referred to the specific heat of the Specific gases, an enquiry of the very greatest difficulty, in heat of which we still find physicists disagreed. Dulong gases. bethought himself of using Laplace's celebrated correction for the velocity of sound due to the heat developed during the compression of an elastic medium (art. 433), and proposed to deduce the heat thus developed, by a comparison of the observed and theoretical (Newtonian) velocity of sound, and thence to obtain the specific heat. The theoretical velocity is easily obtained from the density of a gas under a given pressure: the observed velocity was ingeniously found by sounding one and the same organ pipe with the different gases in succession, and ascertaining the pitch by the aid of Cagniard de Latour's Sirène. (441.)

One of Dulong's latest, most elaborate, and most useful labours, was ascertaining the elasticity of high- On the elaspressure steam in terms of its temperature. These $e_{\text {steam. }}$ experiments were carried as far as 24 atmospheres of pressure. In the course of them the law of Mariotte and Boyle was verified up to the same limit. The condensation of air was found to be exactly proportional to the pressure. We shall return to the subject of these later experiments of Dulong in mentioning the still more recent ones of M. Regnault.

Dulong was unfortunately lost to the world at the comparatively early age of 54 . His was the peculiar Character merit of a well-balanced scientific mind. He felt of Dulong.

1 Thus symbolically expressed:- $\quad \nabla=m\left(a^{t+\theta}-a^{\theta}\right)$;

where $V$ is the "velocity of cooling," or depression of the thermometer in centigrude degrees in one minute, supposing it to continue constant for so long; $\theta$ is the temperature of the envelope; $t+\theta$, that of the thermometer; $a$ is a constant independent of the size and surface of the cooling body, and which is $=1.0077 ; \mathrm{m}$ is a constant depending on the dimensions and surface of the body. 
the necessity of introducing into physics a degree of precision then almost unthought of. Coulomb had done something of the kind in other branches; but in Heat it was really new. It was speedily succeeded by a rapid advance of precision in almost every kind of delicate experimental research. Nowhere can the student of physics find a better model than in the celebrated memoir on the Law of Cooling, which, we may add, received, as a matter of course, from the Academy of Sciences, the prize in competition for which it had been composed. ${ }^{1}$ It is only justice to the countrymen of Dulong to say that they retain the superiority in the deduction of numerical laws from observation, which Coulomb and he conspicuously exemplified. The industry of the Germans and of the English have indeed been great; but in this particular enquiry they have not equalled in address the members of the French Academy.

In order to complete our sketch of the more important steps connecting the discoveries of Leslie with those of Melloni, we will now, going back a little in point of date, trace the origin of correct experiments on the immediate transmission and refraction of heat by solid substances.

(702.) Sir Willian Herschel having found it requisite in Observa- the course of his arduous observations on the sun to tions of Sir devise means for preventing the intense heat of its rays schel on from reaching the eye, was naturally led to observe the the heat of effectof different coloured glasses, and even of coloured the solar liquids in this respect. He also placed thermometers spectrum. in different parts of the spectrum formed by a prism, in order to discover which of the rays it was most important to exclude. The singular result at which he arrived was this, that the intensity of heat accompanying the light of the sun not only increases from the violet to the red end of the spectrum (as was already known), but is more intense quite beyond the red, and gradually diminishes in force for a long way farther. This result was keenly contested by Leslie, butwas confirmed by Englefield and Davy. Berard admitted the existence of invisible heating rays from the sun, but yet found the maximum effect within the red. Seebeck, by numerous experiments, proved that the position of the maximum depended on the nature of the prism, being found even in the yellow ray when a prism of water is employed, whilst with flint glass it always occurs in the space beyond the red. The rationale of this curious result was first discovered by Melloni, whose labours will be detailed in another section; and it was shown to depend on the different degree of absorption exercised on the heat of the several rays of the spectrum by the differing material of the prisms.

(703.)

Herschel made a very great number of experiments on the transmission both of solar and fire-heat through different kinds of glass and other bodies. But they were rough trials, giving a sort of practical test of this quality, rather than admitting of accurate estimations of the quantities of transmitted heat. It was impossible, for instance, to infer from them the degree in which the warmth induced in the glass or other medium by the heat which it absorbed, tended to raise the indications of the thermometer beyond; although such an effect was manifest from the results of the experiments themselves. Hence it was open to an objector to deny the divect transmission of radiant heat through such bodies as glass, except in the cases of the sun and of brilliant combustion, when it eannot be doubted.

Prevost had proved to his own satisfaction the immediate transmission of heat derived from bodies Important even when below the temperature of visible redness, ${ }^{\text {experi- }}$ by using thin screens of glass, and renewing them De la frequently before they could have absorbed much Roche on heat. Maycock obtained a similar result; but to the transDE LA ROCHE is due not only the establishment of mission of this fact beyond any reasinable doubt, but also the heat discovery of certain laws of its operation which are through inexplicable on any other supposition but that of glass. inimediate transinission. One of these laws, for instance, was this, that when a series of thin glasses are interposed between a source of heat and a thermometer, each successive glass transmits a larger proportion than the previous ones of the heat which falls upon it. De la Roche rightly accounted for this significant fact by assuming that heat is not homogeneous, and that the heat which has once passed through glass has lost the rays which glass most easily intercepts. He farther found that the susceptibility of heat to pass through glass increases rapidly with the temperature of its source. The experiments of De la Roche date from 1812.

The next step was made by Professor Powell of (705.) Oxford (1825). He showed that the quality of the Professor heat transmitted by glass is not the same as that Powell. of the incident heat. This he proved by ascertaining the proportion of heat absorbed by a black relatively to a white surface. This proportion was invariably increased by the interposition of glass. Mr Powell concludes that heat consists of two kinds intimately mixed. That of which the absorption depends on the colour of the surface on which it falls, is usually luminous, and is most easily transmitted by glass. That kind of heat which is equally absorbed by black and white surfaces is totally devoid of light, and is sometimes considered as pure radiant heat.

It will be sufficient here to refer to an interesting (706.) Essay on Dow, published by Dr Wells in 1815, in Wells' which he applies Leslie's experiments on the radiating theory

1 As the original paper of Dulong and Petit, in the Memoirs of the Institute, or the Annales de Chimie, is not always accessible, I may mention that it is translated almost or quite in extenso in Thomson's Annals of Philosophy, vol. xiii., and in Mr Lunn's excellent treatise on Heat in the Encyclopadia Metropolitana. 
power of different surfaces to account for the apparently capricious formation of dew in different situations,-establishing that it is moisture deposited from the lowest stratum of air upon surfaces cooled below the " dew-point," by their unrequited radiation of heat towards a clear sky. A slight wind, by continually restoring the equilibrium of temperature to the surface, prevents the deposition.

\section{\$ 8. MeLloni-Recent History of Radiant Heat-Transmission and Refraction of Heat; Pro- perties of Heat analogous to Colour.-Experiments in Great Britain on the Polarization and Double Refraction of Heat.}

(707.) Recent ob-
servations on radiant heat.

(708.) Melloniciate of $\mathrm{NC}$ bili.

(709.)

The thermo-multi-
plier, an instrument of research.

(710.)
THE length to which this chapter has already extended, must be my apology for bringing concisely to a conclusion what remains to be stated regarding the progress of the subject of radiant heat. With the exception of the excellent researches of De la Roche on the immediate transmission of radiant heat through glass (mentioned in tle preceding Section), but which that ingenious philosopher did not live to extend and complete, little of importance was done between the researches of Leslie and those of Melloni, of which we are now to speak.

Macedonio Melloni, a native of Parma in Italy, became associated as an experimenter, probably about the year 1828 or 1829 , with Nobili, a skilful and ingenious physicist of Reggio (Modena). Nobili was well known by his experiments on galvanic Electricity and on Electro-Magnetism. He was also the great improver of Schweigger's Multiplier, rendering it an instrument of precision; and to him we owe the happy and ingenious application of Thermo-Electricity to the measurement of minute effects of heat.

The Thermo-Multiplier, a thermometer of extreme delicacy, though improved by Melloni, was (as has just been stated) the invention of Nobili. It consists of two portions, a sentient part and an indicating part. The first is composed of a number of short thin bars of antimony and bismuth, arranged like a square faggot, pairs of bars being soldered together in consecutive order at the opposite ends of the faggot, so as to form a single bent metallic conductor. If the junctions exposed at one end of the faggot are subjected to heat, and those at the other end kept cool, the effect will be a thermo-electric current of considerable intensity generated by the pile. This current is conveyed by means of two wires from the opposite ends of the system, which are connected with a delicate galvanometer which forms the indicating part of the apparatus. In practice, one end of the pile, armed with a conical reflector for concentrating the rays of heat, is exposed to a calorific source whose radiant effect is to be measured, whilst the other end is carefully screened from external influences. The deviations of the galvanometer needle indicate the heating effect as on the scale of a thermometer. The precautions required in the construction and use of the instrument, and in the interpretation of its results, are too numerous to be mentioned here.

Nobili, in conjunction with Melloni, applied the thermo-multiplier (amongst other experiments) to the proof of the instantaneous transmission of heat through glass and other solid and liquid bodies.

From 1831, this enquiry was conducted nearly exclusivcly by Melloni, who about that time settled first in Geneva and then in Paris, having been com- discovers pelled, on political orounds, to quit Itoly - the wons first derful to the Academy of Sciences early in 1833, and was parency of received with marked coldness, if not incredulity, by for heat. that body. A few months later, the writer of these pages had an opportunity of seeing Melloni's experiments in Paris, and he made known their importance at the immediately succeeding meeting of the British Association at Cambridge. The Royal Society of London in no long time awarded their Rumford medal to Melloni, after which mark of foreign approbation, he first obtained a hearing from the Institute of France. The most considerable result at which he had then arrived was this: that rock-salt possesses a power unapproached by any other substance of transmitting heat of any temperature and from whatever source, with extremely little loss; and as a natural consequence of this, that heat wholly devoid of luminosity, such as that from boiling water, or even the heat of the hand, may be refracted by prisms and lenses of rock-salt exactly in the same manner as light is refracted by glass. The reality of these effects (which had excited the persevering scepticism of the Parisian savans) was demonstrated by a great number of most ingenious experiments, in which every possible source of error and confusion was avoided or allowed for.

Melloni even believed that the loss observed in passing heat of any temperature, high or low, through polished screens of rock-salt, was precisely the same; and, moreover, that it occurred entirely at the two surfaces by partial reflection, so that the solid medium was absolutely transparent for every kind of heat. It is certain that the loss is in every case small; but this almost paradoxical conclusion has not been completely confirmed by those who have repeated his experiments. The important and unexpected discovery of the nearly complete transparency of rock-salt for heat, enables us to construct complex thermotic apparatus for refracting and concentrating it, analogous to those of glass which are used in optics.

The next point clcarly made out by Melloni, was the specific action of different bodies in sifting the 
The specific rays of heat they transmit, stopping altogether certain action of kinds or qualities of heat and transmitting others. different Substances Substances, in general, transmit most readily the heat substances Substances, in gences having a high temperature; this of heat. - had already been shown to be true in the case of glass Coloration by De la Roche. That experimenter had also demonof heat. strated (as we have seen in Art. 704) that successive plates of glass intercept a constantly decreasing percentage of the heat incident upon them. This may be explained by supposing radiant heat to be, like the light of the sun or of a flame, heterogeneous, containing rays of different qualities, some of which are easily transmitted and others are wholly stopped by glass. And if we pursue the analogy with respect to other substances, we may imagine (for the sake of illustration) heat to be coloured, and that different media, though equally transparent and colourless as regards light-such as glass, rock-crystal, and ice-exercise a specific action on the rays of heat, each transmitting certain portions of the heat and stopping others. Rock-salt alone (according to Melloni) is absolutely colourless with respect to heat, transmitting all its varieties with uniform facility. Thus equally thick and equally clear plates of salt, glass, and alum, transmit, out of 100 rays of heat from different sources the following proportions :-

$\begin{array}{cccc}\begin{array}{c}\text { Heat from } \\ \text { a bright } \\ \text { flame. }\end{array} & \begin{array}{c}\text { Heat from } \\ \text { incandes- } \\ \text { cent Pla- } \\ \text { tinum. }\end{array} & \begin{array}{c}\text { Heat from } \\ \text { a surface } \\ \text { at 700 } \\ \text { Fahr. }\end{array} & \begin{array}{c}\text { Heat at } \\ 212^{\circ}\end{array} \\ \text { Rock-salt .........92 } & 92 & 92 & 92 \\ \text { Plate-glass .......39 } & 24 & 6 & 0 \\ \text { Alum ............ } 9 & 2 & 0 & 0\end{array}$

(714.) Let, however, heat which has been sifted by a plate Refrangi- of alum fall on another similar plate, then instead of beat. 9 per cent., 90 per cent. will be transmitted. On the

(715.) Explana point of maximum heat in the spectrum.

other hand, if we unite two plates of opposite transmissive qualities, as alum and green glass, the comtints (say red and green) would be opake for light. The working out of this beautiful enquiry is entirely due Melloni ; and he has published a separate work probable that the rays most easily absurbed by glass and bodies generally are the least refiangible; and this has been made certain by direct experiments by the author of this Dissertation, who, by a peculiar thod, founded on the Total Reflection of heat withi a prism of rock-salt, has obtained the following In-

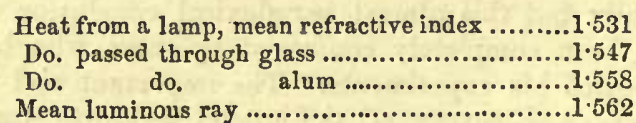

Melloni ingeniously applied the facts previously mentioned to explain the variable position of the hottest part of the spectrum, as observed by Sir
William Herschel and others. It depends on the nature of the prism employed. In a prism of rocksalt, the hottest part of the spectrum is as far beyond the extreme visible red, as the interval between that red and the yellow ray in an opposite direction.

Through the intervention of Arago and of Baron (716,) Humboldt, Melloni ultimately obtained permission to Death of return to Italy and to reside at Naples, where he spent his latter years. He ceased, however, to prosecute his researches on radiant heat with the same energy, undercircumstances of ease and comparative affluence, that he had done in the period of distress and obscurity. Nevertheless, several original papers were written by him at this period, as well as the condensed account of his earlier researches on the Coloration of Heat, of which only the first volume appeared. Melloni died of cholera at Portici, in August 1854, aged 53 .

The analogy of Radiant Heat to light, strikingly established by Melloni, with respect to the diversified refrangibility and other qualities of the various radiations emitted by one or different sources, suggests an enquiry as to the intimate nature of these two agencies. No answer is likely to be so conclusive as an appeal to the test of Polarization, which, in the case of light, has been so remarkably explained by the theory of the transverse undulations of a medium. Some years before any of Melloni's papers appeared,-indeed, before he had entered on the investigation just noticed,-the writer of the present Dissertation had attempted, by means of common thermometers, to test the polarizability of heat. The trial was not a new one; but, except in the case of the heat of the solar rays, the results seemed to be inconclusive, or were even wholly negative. Berard had, indeed, not long after the discovery by Malus of luminous polarization by reflection, repeated (in 1812) that experiment with sum-heat, and also with the heat emanating from terrestrial sources; and as he believed with success. ${ }^{2}$ I have ventured to call his experiments inconclusive, because others besides myself vainly endeavoured to repeat them. Professor Powell failed with ordinary thermometers, and at a later period Nobili announced a decidedly negative result, obtained with the thermo-multiplier. Simple radiant heat, he affirmed, is not polarizable by reflection. ${ }^{8}$

I have just referred to my own early experiments on the subject (which were likewise inconclusive), Experiin order to explain that it was natural, on hear-ments by ing of the application of the thermo-multiplier writer. to measure radiant heat, that $I$ should wish to repeat them with the new instrument. This I did in 1834. I first succeeded in proving the polarization of heat by tourmaline (which Mel-

(717.) Farly attempts to polarize radiant heat -Berard. nation is almost absolutely opake, just as a combi-
1 La Thermochrose, ou la Coloration Calorifique. Naples, 1850.

2 Memoires d'Arceuil, vol, iii., p. 5.
3 Bibliothèque Universelle, Sept. 1834. 
loni had announced did not take place) $;^{1}$ next, by transmission through a bundle of very thin mica plates, inclined to the transmitted ray; and afterwards by reflection from the multiplied surfaces of a pile of thin mica plates placed at the polarizing angle. ${ }^{2}$ I next succeeded in showing that polarized heat is subject to the same modifications which doubly refracting crystallized bodies impress upon light, by suffering a beam of heat (even when quite obscure), after being polarized by transmission, to pass through a depolarizing plate of mica, the heat traversing a second mica bundle before it was received on the pile. As the plate of mica used for depolarization was made to rotate in its own plane, the amount of heat shown by the galvanometer was found to fluc- tuate just as the amount of light received by the eye under similar circumstances would have done. This experiment which, with the others just mentioned, was soon repeated and confirmed by other observers, still remains the only one proving the double refraction of heat unaccompanicd by light; and though somewhat indirect, it will hardly be regarded by competent judges as otherwise than conclusive. Iceland spar and other doubly-refracting substances, absorb invisible heat too rapidly to be used for effecting directly the separation of the rays, which requires a very considerable thickness of the crystal. I also succeeded in repeating Fresnel's experiment of pro- Circular. ducing circular polarization by two internal reflec-polarizations. The substance used was of course rock-salt. ${ }^{2}$ tion.

\section{§ 9. M. RegnaUlt.-Numerical Laws of Expansion by Heat; Rudberg.-Vaporization; Dulong.-Latent Heat; Hygrometry.}

The limits of this Essay will not permit me to do more than allude in very general terms to the meritorious services of M. HeNRI-Victor RegnatiLt in the science of heat. In the seventh section of this chapter I have mentioned his name in connection with that of Dulong, whose researches he has prosecuted, and whose position in the Collège de France he now fills.

The attention of M. Regnault has been devoted chiefly to heat in its combinations with matter-to dilatation and vaporization. I have already said, in speaking of Dulong, that, in point of numerical precision in the results of experimental physics, the French are unrivalled. The talent which they have shown in the construction of apparatus, skill in its use, and patience in deducing results with due attention to every numerical correction, have not been equalled either in England or Germany, much less elsewhere.

We must, however, note that doubts were first thrown upon the accuracy of Gay-Lussac's coefficient Coefficient of the expansion of the gases $(0.375$ of the vo- of expanof the expansion of the gases lume at $32^{\circ}$ for the expansion between $32^{\circ}$ and $212^{\circ}$ gases and Fahrenheit) by Rudberg a Swedish philosopher, who mercury; determined a new coefficient $(0 \cdot 3645)$. M. Reg-Rudberg nault finds for air of the ordinary density a co--Dulong. efficient nearly the same as that of Rudberg, but differing slightly for the same fluid under differing pressures, and also for the various gases. ${ }^{4}$ The expansibility of all of these fluids appears to tend to the same limiting value when they are sufficiently attenuated. As a preliminary to these experiments, the expansion of mercury was ascertained by its hydrostatic equilibrium at different temperatures, as had already been done by Dulong, and with almost coincident results. The dilatation of mercury was used to ascertain that of the glass vessels employed.

The irregularity of the dilatation of glass is one of

1 Annales de Chimie, tom. Iv. (1833).

2 I was led to polarize heat by transmission through mica films from having observed the extraordinary permeability of those films to radiant heat, and from the facility of adapting them to tubes applied to the pile. The idea of using bundles of mica for reflecting heat did not occur to me until some time after. But I cannot here omit mentioning a circumstance of which I only became aware some years after the publication of my researches. In arranging my correspondence, I found some letters from Sir David Brewster, with whom I had communicated as to the best means of polarizing heat, during my earliest and unsuccessful attempts with common thermometers. In one of these letters he recommends, among other methods, the reflection of radiant heat from mica bundles. This suggestion was not put in practice; for, owing to change of residence and other circumstances, my attention was diverted to other stibjects, and only recalled, after a lapse of some years (as stated in the text), to the polarization of heat, by the invention of the Thermomultiplier. Nor was Sir D. Brewster's suggestion recollected by me until I accidentally met with it (after another long interval), in the manner which I have just stated. I am glad to have an opportunity of acknowledging the friendly assistance and encouragement in all matters of science which at an early age I received from him when I was an obscure, though ardent student, and when he was my only scientific adviser.

3 I have not thought it proper to go into farther details concerning my own experiments on radiant heat. Those who desire more information will find it in Professor Powell's Second Report on Radiant IIeat, in the Brit. Assoc. Reports for 1840 . But I may here state, that M. Melloni's first experiments on polarization were made with mica piles, furnished to him by myself in 1835 .

4 M. Regnault's experiments were published in 1841. Professor Magnus of Berlin was at the same time engaged on similar experiments, and with nearly coincident results. The following table contains the summary of all these experiments :-

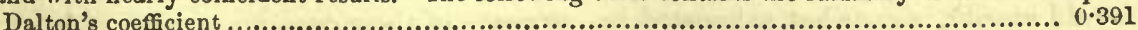

Gay-Lussac's coefficient .................................................................. $0 \cdot 375$

Rudberg's coefficient ................................................................. 0.3645

M. Regnault $\&$ coefficient (from the expansion observed under a constant pressure)........... 0.367

M. Regnault'e coefficient (from the elasticity observed under a constant volume)........... $0 \cdot 3665$

M. Magnus' coefficient (from the elasticity observed under a constant volume)................ 0.3665

Dalton's experiments wcre made between $55^{\circ}$ and $212^{\circ}$, and after allowing for the expansion of glass, he obtains for the relatire

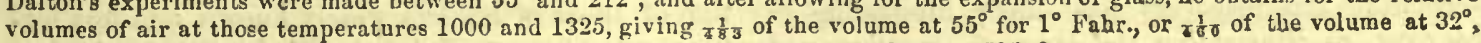
which agrees with the co-efficient given above. See Aranchestor Mfemoirs, vol. v., p. 598-9. 
Irregular the great difficulties not only of these enquiries, but dilatation generally, in constructing comparable thermometers. of glass. It varies so much with the composition of the glass as to leave a serious amount of uncertainty in the measure of temperatures above that of boiling water. M. Regnault has had the perseverance himself to graduate the thermometers which he uses.

These enquiries were in some measure introductory Dulong and $M$. Regnault on the elas ticity of steam.

(724.) M. Regnault on latent hea

(725.) to the determination of the elasticities of steam at different temperatures, which had been already ascer- tained with so much care by Dulong (nominally in conjunction with Arago and other academicians), as to leave little need for their repetition, except on the ground of the uncertainty of the indications at high temperatures of the thermometers which they used. All M. Regnault's results are most carefully expressed in terms of temperatures measured on an air thermometer corrected for the expansion of glass. They agree well with those previously ascertained by Dulong.

In connection with this subject, the important law of the latent heat of steam at different temperatures has been correctly ascertained for the first time by $M$. Regnault. Watt maintained that the sum of the sensible and latent heats of steam is constant at all temperatures : Southern and others, on the contrary, believed that the latent heat has a constant value, whatever be the temperature of vaporization. M. Regnault shows that the true law is intermediate. The latent heat diminishes as the sensible heat of steam increases, but in a slower proportion.

Our author also followed the steps of his prede- cessor Dulong in verifying the law of Mariotte and Law of Boyle on the compressibility of the gases. He found Mariotte it to be indeed very approximately true, yet not ab- and Boyle solutely so for any gas. For atmospheric air and for most other gases the compression increases rather faster than the strict proportionality to the pressures would assign. In hydrogen gas the contrary is the case. This result, together with that on the dilatation of different gases, shows (if fully confirmed hereafter) that mere simplicity or uniformity of result is not by any means a sure ground of induction as to a law of nature. That simplicity often appears to be predicable only of some abstract condition of matter which we may assign or imagine, but which we rarely if ever find realized amongst the bodies around us.

The preceding investigations, the result of an amount of minute and assiduous labour almost fearful to contemplate, are to be found collected in the 21st volume of the Memoirs of the French Academy of Sciences, which they entirely occupy.

M. Regnault has elsewhere published analogous (727.) researches on the Specific Heat of a great variety of M. Regsubstances, and on the theory and practice of Hygro nault on metry, both of which are highly inderesting and im- Ileat and portant, but which I have not here space to analyze. HygromeHe is also favourably known as the author of an try. excellent treatise on chemistry, and in fact sits in the Institute as a member of the Chemical Section. He now directs the manufactory of porcelain at Sevres; and being still in the prime of life, much may yet be hoped from his devotion to science.

\section{H A P T E R V I I.}

\section{ELECTRICITY-MAGNETISM-ELECTRO-MAGNETISM.}

\section{\$1.-Galdani.-Discovery of Galvanism; Proper Animal Electricity.-The subject revived by Nobili.-MM. Matteucci and Du Bois Reymond.}

(728.)

Galvani.

(729.) His position as a discoverer difficult to estimate.
There are few discoverers in science whose position it is more difficult to assign with accuracy than Galvani. Attaining at first, by a curious observation patiently reflected on and carefully repeated in detail, to the rank of the founder of a new science, he was so far outstripped in its applications, that his merit was soon in a measure overlooked, and his unquestionable discoveries ascribed to capricious accident.

We find that a concurrence of circumstances contributed to this result. Galvani was advanced in years, and, it would appear, somewhat exlausted in constitution, when he made his famous observation on muscular contractions. He was an anatomist, far more than either a chemist or physicist;-no blame, surely, is to be attributed to him on that account! His discovery was chiefly interesting in his eyes, as illustrating the laws of sensation and the source of nervous irritability. It was calculated to throw great light on these most abstruse enquiries. Groping to find the thread which should reveal to him that labyrinth, is it surprising that he devoted himself exclusively to those effects which gave him a real promise of success?-a promise held out still by the same facts - still the envied goal of physiologists -yet how little realized by the unremitting labours of two subsequent generations!

Again, the almost irresistible temptation of converting successful philosophers into heroes, at the Contrast 0 expense of their contemporaries, added to a less par- and Volta. donable wish to relieve the tedium of scientific discussion, by the introduction of a lively, though questionable anecdote, has induced the eulogists of Volta to exalt his unqestionable claims by the depreciation of those of his less widely known and less fortunate countryman, Galvani. Their contrasts in cha- 
racter and circumstances were sufficiently marked. Galvani was a professional anatomist and physiologist; Volta a physicist. Galvani was little known, and had probably travelled little beyond the province in which he resided; Volta was personally and advantageously known in Paris and London. Galvani, soon after his discovery, fell into undeserved political disgrace, which undermined his health; Volta lived to an advanced age, his experiments and discoveries rewarded by every honour which not only academic authority could bestow, but which the almost universal sway of Napoleon could render to his genius. Galvani died prematurely, and whilst his best observations were contested; Volta survived to nearly the latest term of human life, having witnessed the fruits of his great invention in the splendid discoveries of Davy and Oersted.

The biographical particulars of Galvani's life may be passed over in a few words. The history of his discoveries has been recently materially enlarged and corrected, by the researches of the Academy of Bologna to which he belonged, and especially by those of Professor Gherardi; it forms, together with his collected writings, a ponderous quarto volume. ${ }^{1}$ The diffuseness of the commentary, and that of Gralvani's writings also, is a defect in this compilation, which tends to weaken the unquestionable force of the evidence in his favour.

Luigr Galvani was born in 1737, and was promoted in 1762 to the chair of anatomy at Bologna, his native place, the seat of a most celebrated university. He studied and taught his science witl great success, and published several memoirs. Probably he would have become still more widely known, but that he was anticipated in some of his observations (particularly on the organ of hearing) by the celebrated Scarpa; in consequence of which, Galvani, with his customary modesty, suppressed what had already become known through that able anatomist. As early at least as 1780 (as we learn from the researches of Gherardi, and the MSS. of Galvani himself), he made experiments on muscular contractions taking place by electric influence from the electrical machine, the electrophorus, and the Leyden phial. ${ }^{2}$ The experiments were made on frogs in particular. This was ten years antecedent to the commonly alleged casual discovery of galvanism. The experiments were continued in 1781 and 1782 , when he drew up a paper (not published)
"On the Nervous Force and its relation to Electricity." 3 In 1786 he pursued the enquiry, with the aid of his nephew Camillo Galvani; and the effect of thunder-storms in occasioning muscular contractions in the frog (which he had previously noticed), was farther studied. He then designated the prepared frog as "the most delicate electrometer yet and on discovered."4 But this year was also the one of electricity his real discovery, namely, that muscular tions are sometimes occasioned by causes remote it. from any then known to be connected with electricity. Camillo Galvani, pursuing his uncle's experiments on Atmospheric Electricity, had prepared some frogs, by dividing them about the middle, and detaching a portion of the lumbar nerves from the integuments, leaving them in contact with a portion of the vertebral column which was then suspended by an iron hook. These prepared frogs were lying horizontally on the top of an iron rail of a balcony on the third floor of Galvani's house, where he was in the habit of observing the effects of atmospheric electricity. The nephew noticed that when the hook or the vertebræ were pressed on the rail by the finger or otherwise, muscular contractions ensued, which he pointed out to his uncle, who lost no time in repeating the observation, which seems to have been made early in September $1786 .^{5}$ His experiments in this and the following montl are detailed, with the exact dates still preserved, with this remarkable title in Galvani's hand-writing, - Esperimenti circa l'Eletricita dei Metalli; and the results are formally drawn up in a Latin Dissertation of 62 pages, bearing date 30 th October 1786, forming the substance of the most important section of his Commentary on the Electric Forces, \&c., published five years later, but differing from it in some important particulars. ${ }^{6}$ Thus it appears distinctly, that one metal alone -iron-was used in producing the convulsion $;^{7}$ whilst in the printed Commentary, the hooks are said to be of brass or of copper. The explanation is, that Galvani having become aware of the superior efficacy of unlike metals in contact, described the experiment, not as it was first made, but as it might be made with greater certainty. Yet, singularly enough, this Dissertation is entitled, $-D e$ animali Electricitate; showing, that in the short space of a few weeks, he had abandoncd his earlier notion of the metals being the source of the electricity, and ascribed the effects to the proper electricity of the nerves and muscles.

1 Opere edite ed inedite del professore Luigi Galvani, raccolte e pubblicate per cura dell' Accademia delle Scienze dell' Instituto di Bologna. Bologna, 1841. The copy which I use belongs to the Royal Society of Edinburgh.

2 Rapporto, \&c., p. 11 (in the work above cited).

3 Ib. p. 18

4 Ib. p. 30. The expression is remarkable, because Volta is often regarded as the first who considered the frog in the aspect of a mere electroscope.

Rapporto, pp. 33, 36.

6 Ib. p. 35,36 . It were to be desired that this MS. were published in full.

7 The passage from the MS. is conclusive,_- Ranas itaque consueto more paratas uncino ferreo earum spinali medulla perfo rata atque appensa, Septembris initio [1786] die vesperascente supra parapetto horizontaliter collocavimus. Uncinus ferream laminam (namely, the top of the iron parapet or rail) tangebat; en motus in rana spontanei, varii, haud infrequentes ! Si digito uncinulum adversus ferream superficiem premeretur, quiescentes excitabantur et toties ferme quoties bujusmodi pressio adhiberetur."-Rapporto, p. 36. 
(733.)

Of these two very opposite aspects of this remarkable experiment, it might have been reasonably enough anticipated, that one should be wholly erroneous. This, however, is not the case. Galvani was justified in both his inferences, although he unquestionably believed that only one could be true. As a physiological anatomist, he not unnaturally adhered finally to the opinion of vital or animal electricity being the cause of the phenomenon which he had observed. Volta, on the other hand, already an experienced natural philosopher, though for a short time an entire convert to Galvani's published opinions, maintained the contact of metals, unlike either in kind or in their mechanical condition, to be the source of the nervous commotion; and whilst he was enabled to support his opinion by very striking experiments, its popularity was not unjustly perhaps exalted to the highest pitch by the happy application which he made of it, to the construction of that wonderful instrument, the voltaic battery, which effectually withdrew attention for a time from the comparatively feeble and obscure effects of the electric power residing in the animal tissues.

(734.) The historian of science, well aware how far the inThe proper trinsic importance of discoveries is from depending of the frog, upon the mere magnitude of their effects, and how often the philosopher, dazzled by the splendour of a new truth, overlooks some minute concomitant phenomenon which hereafter may rival or eclipse its splendour,-readily recognises the perseverance with which Galvani maintained his theory of animal electricity as a part of the true philosophical character, and therefore as enhancing his reputation. The metallic arc which, by connecting the muscular part of the limb with the root of the lumbar nerve, occasioned the convulsion, was to be regarded, on his theory, as merely establishing equilibrium between those parts to which the vital principle had communicated an electric tension similar to that which subsists on the opposite surfaces of a charged plate or Leyden jar. Galvani gradually disembarassed his experiments from the suspicious presence of metals altogether. Taking the prepared hind-limb of a frog, with the connected nerve entering the spine, he found that when the latter was suffered simply to touch the bare surface of any muscular part, and without the Confirmed intervention of metal or any other conductor, a spasm by Baron of the limb immediately occurred.
A. von This important Humboldt. experiment was repeated and varied in 1795 , by the celebrated Baron Alexander von Humboldt, who published a paper on the subject in Gren's Journal, fully confirming Galvani's conclusions. Volta, on the other hand, admitting the facts, strove to explain them on the supposition, that they were due, like other electric currents, merely to the heterogeneous nature of two solid bodies, the muscle and the nerve brought into opposition, and moistened by a conducting liquid.
Experiments instituted after a lapse of 30 years, with the aid of new instruments, and vastly increased knowledge of electric manifestations, have conclusively demonstrated the accuracy in this respect of Galvani's reasoning in preference to Volta's.

The publication of the results already noticed did not take place till 1791 in his celebrated paper in the Bolognese Transactions. The statement popularly made by almost every writer (and which may be views. traced to Alibert, one of the earliest historians of the subject, but whose authority seems to be of little weight), is that the discovery of Galvanism was made in 1790, in Madame Galvani's kitchen, where a frog soup was being prepared for that lady's repast, she being at the time in delicate health. The absurdity of the invention is evident from the history which we have given, founded on unquestionable documents. The memoir of 1791 was the resumé of elaborate experiments continued with (apparently) little interruption for eleven years; and the most interesting results had been digested in a Latin tract five years before. All this shows at once great patience and intelligence on the part of Galvani, who, perceiving the difficulty and also the probable importance of the subject (in a physiological view) -oscillating perhaps in some measure between the two very opposite opinions regarding the source of muscular excitement which we have seen that he almost simultaneously held,--postponed for so long a time the publication of a discovery which he must have been sure would confer upon him a great reputation. By the time of his publication his views had become fixed in favour of animal electricity; and he defended it in several succeeding memoirs.

The unjust deposition of Galvani from his chair on political grounds affected seriously his health and His death energies. Perhaps his latest experiments were on the Electricity of the Torpedo. He died 4th December 1798 , aged only 61 - happy perhaps in not having witnessed the discovery of the Pile, which, by its astonishing results, was to throw into the shade Galvani's more intricate and difficult studies.

To appreciate justly Galvani's place in scientific history, we must recollect three circumstances which His disti have often been overlooked, - First, that his discoveries guished were the result of patient, ingenious, and protracted research, not of a casual observation exciting ignorant surprise. Secondly, That however deficient was Galvani's theory of animal electricity to explain all, or even the most conspicuous facts witnessed by him, it was a real discovery which has been confirmed by the latest and most scrupulous researches, and of a physiological importance which can hardly be overrated. Thirdly, Galvani's Commentary was received at the time with enthusiasm, not only from the importance of the facts which it contained, but from the

(735.) Gradual progress Galvani's 
ability shown by the author in discussing them. There was no part of Europe in which Galvani's observations were not held to bear out his theory; and the warmest eulogy of them is to be found in the writings of Volta himself, who soon adrocated a different explanation. Volta calls animal electricity " a great and luminous discovery which forms an epoch in the annals of physical and medical science," and as "proved to demonstration (ad evidenza) by many experiments well contrived and accurately executed." 1 Had there been as little novelty as has sometimes been alleged in Galvani's observations, they would not have been heard of at once, and repeated in every civilized country, nor have given birth to a new and splendid science. Nothing then in progress, either in the hands of Volta or of any one else, gave the slightest clue to the invention of the Pile, which, but for Galvani, might have been yet undiscovered.

(738.)

Revival of experiments or animal electricity - Nobili.
The grand discovery of Oersted, which gave a fresh impulse to so many branches of science, revived likewise the subject of the proper electricity of the animal tissues, which liad been well-nigh forgotten since the death of its discoverer Galvani. Twenty-nine years later, in 1827, Nobili of Florence demonstrated the existence of what has been termed "The current of the frog." We have seen that a momentary spasm is produced when a circuit is completed, including the muscle and nerve of the recently dead animal. But by the aid of that admirable instrument the Galvanometer, Nobili succeeded in showing that a continuous current of positive electricity constantly passes from the feet to the head of the frog. This he detected by placing the feet of the animal in connection with one end of the galvanometer wire, and its spine with the other (the whole being properly insulated), when the needle of the instrument was permanently deflected to the amount of $5^{\circ}$ or moreindicating the passage of a stream of electricity in the direction already mentioned, which continued for several hours after death. Strange to say, Nobili misapprehended the nature of the phenomenon, ascribing it to Thermo-Electricity, though he ought to have been undeceived by the singular intensity of the animal current, which, feeble as it is, can force its way along thousands of feet, or even some miles of fine wire. ${ }^{2}$

These experiments were renewed in 1837 by $\mathrm{M}$. Matteucci of Pisa, who has the merit of reviving the MM. Mat original and correct opinion of Galvani as to the vital ${ }_{\mathrm{Du} \text { Bois }}^{\text {teuci and }}$ source of this electricity. To his researches, and the Reymond. still later ones of Dr Du Bois Reymond of Berlin, we owe the knowledge of most of the facts as yet ascertained in this most difficult and obscure branch of enquiry, where the sources of error are so numerous as only to be eluded by consummate skill on the part of the experimenter. It appears to be established that the vital electricity exists both in the muscles and in the nerves of many, probably of all animals when living or recently dead; that therefore the frog current of Nobili is only a single case of the general muscular current, and that the latter arises from the electro-motive action of even the minutest fibres of which a muscle is composed-the general law being (according to Dr Du Bois Reymond) that positive electricity moves from the transverse section to the longitudinal section of a muscle or a nerve, or any portion of either. Finally, the last-named writer has shown, to the satisfaction of many eminent men who have witnessed his experiments, that powerful muscular contraction, whether induced by stimuli or the result of volition, tends to diminish the force of the natural muscular current. This he demonstrated first on the frog poisoned by strychnine, but afterwards on the muscles of his own arm, in which by voluntary contraction he could diminish at will the force of the natural current, which in the state of rest is directed from the shoulder to the hand.

\section{\$2. Vouta.-Progress of Discovery in Common and Atmospheric Electricity-The Electro-motive Theory-Voltaic Pile-Chemical Analogies and Decomposition-Fabbroni; Nicholson and Carlisle.}

Volta was the first among philosophers whose career lay solely in the study of electricity. Franklin and Epinus, Beccaria and Wilke, Cavendish and Coulomb, gave it only a share of their attention; but Volta was from boyhood exclusively an electrician. Such devotion deserved success, and he achieved it. He was already famous, and an honorary fellow of the Royal Society, long before his principal discovery of the Pile. A review of his career may therefore be conveniently divided into two parts-what con- cerns ordinary and atmospheric Electricity; and the new doctrine of Galvanism and the Electro-motive force.

I. Alessandro Volta was born at Como, of a noble family, in 1745. His first paper on Electricity was ad- experidressed to Beccaria at the age of eighteen. But it was mentsnot till $1775^{3}$ that he published a description of the his ElectroElectrophorus, an ingenious instrument in which a phorus and conducting body becomes electrified an indefinite number of times in succession by being brought near to an

\footnotetext{
1 Opere del Volta, ii., p. 13, 14.

2 The galvanometer of Du Bois Reymond contains 3.17 English miles of wire, in 24,160 coils.

3 See the letter to Priestley in the First volume of Volta's Works.
} 
excited cake of pitch and resin. This charge is received by induction merely; and as it expends none of the electricity connected with the pitch, the successive charges are precisely equal, a circumstance which enabled Volta very simply to give a numerical value to the amounts of electricity used in his experiments, as they were given by one, two, or more contacts with the electrophorus. The Condenser, an ingenious and useful instrument for accumulating small charges of electricity until they attain a measurable amount, or cause divergence in the electrometer, was described by Volta in 1782 in the Transactions of the Royal Society, its construction having occurred to him in following out the idea of the electrophorus. This instrument was ultimately of essential service in establishing his theory of Electro-motion. The theory of both the electrophorus and condenser had been indicated by Epinus some time before Volta constructed them; but he did not apply them in the practical way which Volta did to the improvement of his science. Volta being, besides, unquestionably ignorant of $\mathbb{E}$ pinus's labours, has been generally and justly regarded as the real inventor. Though both of these instruments depend on the principle of induced electricity, Volta never appears to have possessed correct views on that subject, but throughout his writings speaks of electrical atmospheres, and uses other phrases of the old school of electricians, showing a certain vagueness in his conception which a study of the writings of his able contemporaries, Epinus and Coulomb, would have dissipated.

(742.) But Volta's tact was unconnected with any tinge Volta's of mathematical reasoning: his experimental ability, Electrome- his caution, and his persevering devotion to one subter. ject, enabled him, however, to advance science in a different way. Even the simple Straw Electrometer which he generally used, was tested by him with such skill and care as to lead to correct results in the measurement of small quantities of electricity. M. Biot has indeed criticised his preference for so rude an instrument, which depends, mathematically considered, upon repulsions of a very complicated character; but as Volta carefully tested its comparability up to $30^{\circ}$ of divergence, and found it proportional to the force, there is no doubt that he was justified in relying on its use; and Arago, in opposition to his colleague, maintains that Volta's essay on the Straw Electrometer is one of the best examples of experimental research which can be put into the student's hands. This instrument is described in the first of a series of letters to Lichtenberg collected in the first volume of his works; the subject of these letters being the Electricity of the Atmosphere.

(743.) The important experiments of Dalibard and FrankAtmosphe- lin, repeated with fatal consequences by Richmann, ric electri- had demonstrated the perfect resemblance or rather
city. identity of lightning and electricity. Lemonnier discovered the fact of electricity being manifested when no thunderstorm threatened, and even when the sky was cloudless, and that it was subject to diurnal variations of intensity. Beccaria farther ascertained that with a clear atmosphere the electricity of the air is always positive. De Saussure, Deluc, and Volta continued the interesting enquiry. The first, availing himself of the known action of points to draw off electricity, connected his electrometer with a pointed rod two or three feet in length. Volta substituted for this the flame of a lamp producing a heated current of air, which has a wonderful power of drawing off electricity; and he suggested the employment of large fires during thunderstorms in preference to metallic conductors. Volta hesitates not to ascribe to the worshippers of Jupiter Tonans the secret intention to draw off the electricity of heaven by the action of the flames on the altar. ${ }^{1}$ Arago has ingeniously suggested that a statistical enquiry as to the frequency of thunderstorms in the neighbourhood of extensive iron-smelting furnaces might test the value of this safeguard. The straw electrometer which Volta connected with his apparatus was capable (by a gradation of instruments of greater or less delicacy) of measuring numerically the force of charges from 1000 to 2000 units.

That the chief source of atmospheric electricity is evaporation appears first to have occurred to Volta, and to have been first demonstrated by experiments made either with him or by his suggestion, at Paris in 1780 , by Lavoisier and Laplace. The history is given by Volta himself in a paper in the Philosophical Transactions for 1782 . When water is thrown upon an insulated heated body so that evaporation takes place, or when hot coals are thrown into an insulated vessel of water, the hot body is usually found to be electrified negatively. Volta has very candidly stated the inversions of effect which occasionally occur, and which still throw some doubt upon the precise significance of this very important experiment. Later experimenters have thought that absolutely pure water developes no electricity: this, however, will not affect the validity of the explanation of the origin of atmospheric electricity. To prevent misapprehension it may be observed that the astonishing developement of electricity from high-pressure steam escaping through a small aperture, as lately observed by Mr Armstrong, appears, from the experiments of Dr Faraday, to depend on an entirely different cause. Of Volta's electrical theory of Hail we cannot now stop to speak.

Volta, who understood chemistry and who always (745.) took a peculiar interest in the inflammable gases, Volta's contrived the Eudiometer which is often erroneously meter called Carendish's, having been frequently used by that philosopher. The amount of oxygen in the air poration.

(1)

\footnotetext{
2 Opere, vol. i., part 2., p. 205. Volta's contrivance dates from the beginning of 1787. Bennett imagined it independently.
} 
(746.)

is tested by mixing the latter in known proportions with hydrogen in a close vessel through which an electric spark can be passed. Detonation takes place, and the quantity of gas which has vanished (by conversion into water) measures the amountof ogygen which has combined with hydrogen in the experiment. It was for a long period employed as by far the best means of testing the purity of air.

All the preceding labours of Volta (and I do not intend to touch on any minor ones) have evidently an intensely practical character. His aim throughout was to improve the instrumental means of detecting and measuring electricity, and to detect and measure it as it occurred in practice, rather than to form theories of its nature. ${ }^{1}$ Even the discovery of galvanism, which vividly excited his interest, only partially diverted him from his scientific destiny. Volta will indeed be always remembered as the author of the plausible theory of electro-motion, and as having corrected the too exclusive doctrine of Galvani concerning the sources of electric excitement; but his real claim to immortality is the invention of the Pile. To this part of the history we therefore proceed.

II. When Galvani announced his discoveries in the Bolognese Transactions in 1791, ${ }^{2}$ Volta was Professor of Physics at Pavia, having been appointed to that post in 1774. As has been mentioned in a former section, the announcement of these researches excited the immediate attention of electricians and anatomists in every part of Europe. Of course Italy was not exempted from the general impulse. In that country physiological observations have always been prosecuted with interest and success; and indeed it has never been deficient in persons of ability, whether in physical or in purely mathematical enquiries, since the very dawn of letters, at which time Italy made so distinguished a figure in literary progress. Volta, Aldini, Valli, and Spallanzani were all, at the time of which I now speak, actively engaged, in the pursuit of science; and Galvani's opinion that the commotion of the frog by the connection of the muscle and nerve through a "conducting arc" of metal was due solely to animal electricity, was generally adopted, and by none more cordially than by - Volta in a letter and memoirs published in Brugnatelli's Journal early in 1792. These were speedily

His letters followed by two letters to Cavallo, dated October of the same year, and communicated to the Royal Society of London, in acknowledgement, as the author states, of the honour recently done him of electing him an Honorary Fellow. The title of this communication deserves notice, - "Account of some Discoveries made by Mr Galvani of Bologna, with Experiments and Observations on them ;" 3 and also the first sentence (the letters are in French),
"Le sujet des découvertes, et des recherches, dont je vais vous entretenir, Monsieur, est l'electricité animale." In the course of the paper, however, he distinctly states that whilst he agrees with Galvani in considering that the convulsions of the frog, obtained with homogeneous conductors, are due to a proper animal electricity ( $\S 12,16)$, the more powerful effects occasioned by the contact of unlike mctals are caused by "common electricity" developed at the junction, and having the nature, not of a discharge, but of a continued stream. He repeats and modifies Galvani's experiments on animals, cold and warm-blooded, and makes interesting observations on the excitement of the nerves of taste and sight by the contact of unlike metals. The conclusion of his paper is in opposition to its earlier portion. He expresses a grave doubt whether there be any vital electricity in the matter.

The induction of Volta was imperfect in this, that he did not prove that the effects which he attributed Volta rewith great probability to the contact of metals pro- ceives the duced any other recognised electric effect than the phy- Medal. siological ones. Galvani had gone very nearly as far. $\mathrm{He}$ had even hesitated between the terms animal electricity and electricity of metals; he had considered the frog as a very sensitive electrometer, exactly as Volta did; and the manner of so using and applying it is ascribed by the latter in this memoir to Galvani, who having thus invented the instrument which for years served alone to indicate the presence of the new species of electricity - and having also described accurately the influence of the heterogeneous metals in aiding the results-left to Volta only the credit of the assertion that in some instances the effect was due to the metals themselves, in others to the natural electricity of the animal frame. Under these circumstances, I think that the award of the Copley Medal by the Royal Society to Volta, rather than to Galvani, was a questionable decision: the great value of Volta's paper, at the time, was undoubtedly that it directed the attention of English experimenters to Galvani's discoveries, then quite recent and probably imperfectly known. ${ }^{4}$

Many publications followed. I shall only notice that by DrFowler of Edinburgh (afterwards of Salis- (749.), bury), which is remarkable as containing a letter by anticipaProfessor John Robison (335), who first thought of volta's increasing the effect of heterogeneous contact by using pile. " a number of pieces of zinc made of the size of a shilling, and making them up into a rouleau with as many shillings." We have here unquestionably the first idea of the pile, which moreover was actually constructed. This was in May 1793. It was only applied, however, to excite the nerves of the senses.

In various scattered memoirs, from 1793 to 1796 ,

\footnotetext{
1 His arguments as to the primary law of electric attractions and repulsions, are wholly inexact. His electrometer was unfitted for such enquiries,

2 Vol. vii. His paper was reprinted separately at Modena in 1792. 3 Philosophical Transactions, 1793.

4 See the grounds of the award stated by Sir Joseph Banks. Weld, Hist. R. Society, ii., 202.
} 
Volta's theory of electromotion.

Invention of the pile.

we find Volta gradually insisting more on the purcly mechanical nature of the electrical excitement. The last-named year produced an important letter to Gren of Halle, ${ }^{1}$ which contains the real germ of the invention of the pile, though it has been little taken notice of. We there find conducting bodies divided into two classes, primary and secondary; the first including metals, metallic ores, and charcoal; the second liquids, solutions, animal tissues, \&c. The first class he also called motors. Using the prepared frog always as an indicator, he tried the effect of combining three or more elements of the two kinds. He found that a double combination of three elements, when arranged so that their order was reversed, neutralized each other, or produced no spasm; on the contrary, when the two combinations conspired in direction, the convulsions were increased ( $\$ \S$ xii., $x \nabla ., x i x$.$) . This appears to definc the$ date of Volta's discovery of the principle of the pile -that, namely, of superadding minute effects - to be August 1796. The form of the arrangement resembled that afterwards adopted in the Couronne des Tasses. The second letter to Gren, dated the same month of August 1796, contains the important discovery (the most important abstractly of any due to Volta), that the electricity set in motion by the contact of unliko metals may, by means of the condenser (due also to him), be made evident by the usual effect of repulsion on the common electrometer:- -thus when zinc and silver are used, the former is positive, the latter Repulsion negative, and so of other metals. Tolta used for due to con- this experiment Nicholson's ingenious modification of tact of his own condenser, called a Revolving Doubler. It metals. must be owned that the experiment, in its simplest form, is difficult of repetition, and that Nicholson's instrument sometines gives delusive results. But Volta's great address in practical electricity, and his fairness in stating his results, leave no doubt of the reality of his discovery, which evidently for the first time eliminated the physiological element of Galvani's experiments, leaving the recognised mechanical effects of electricity due to the contact of unlike metals; and, therefore, deserved the highest honour which could be bestowed. Pfaff had already constructed a table of the electro-motive power of metals by their actions on the frog, in which zinc stood at one end, carbon at the other. But one of the most curious parts of the paper by Volta is the evidence of a strong suspicion which had crossed his mind, and been for a time entertained, that in his experiments with combinations of three elements-two metallic, and one humid-the electricity was developed separately at the contacts of the latter with the two former, and that the resulting current was merely the difference of the two in favour of the stronger. Truly in this whole history we may see how often first suggestions have a peculiar and intuitive worth, which reflection and controversy often only obscure! This is, of course, the case rather in the research of causes than of the means of rendering discovery practical.

Whilst Volta was thus maintaining the opinion that the electricity excited by the contact of metals Fabbron was entirely mechanical, and due to contact merely; $;$ the chemi- and whilst Galvani, his relative Aldini, and others, cal origin maintained strenuously the vital theory, in which they of galvan. were substantially confirmed by no less authorities ism. than Wells and Baron von Humboldt-a third school appeared, at first little popular, represented by $F_{A B}-$ BRONI, a Tuscan chemist and natural philosopher of no small merit. His papers published, I believe, in 1799 , though written several years previously, and

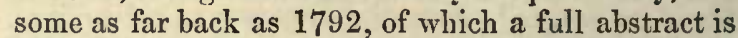
given in Nicolson's Journal, ${ }^{2}$ show great acuteness. $\mathrm{He}$ attributed the effects of the contact of metals to a chemical action developed at the place of contact. He referred to Sulzer's experiment of the taste of heterogeneous metals applied to the tongue-and to manyinstances of the rapid oxidation of heterogeneous metals in contact, when exposed to heat and moisture. Amongst others, by a remarkable anticipation of one of the most curious applications of the electro-chemical theory, he notices the oxidation of the copper sheathing of ships. Without " excluding all electrical influence from the prodigious effects of galvanism," he infers that there are chemical forces "exerted with the swiftness of lightning," to which the physiological effects, and perhaps some others ascribed to electricity, are probably due. Thus, he says, "the experiment of Sulzer is nothing more than a combustion or chemical operation, as is proved not only by its result but by its duration ; for electricity acts always instantancously, whereas the effect of chemical affinitics continues so long as the re-agents are not saturated." The weak point of Volta's theory of electro-motion is here cleverly hit. That effects indefinitely prolonged, capable of producing mechanical, chemical, and vital changes, without any mutual action between the touching bodies, save mere pressure; appears indeed a paradox startling even to a first inventor, but which, when maintained by successive generations of able men, may rank as a delusion more memorable than the phlogistic theory of the older chemists. Fabbroni did not himself pursue his ingenious speculations, but his papers, though now almost forgotten, acted powerfully on the minds of his contemporaries, as we shall see in the next section. He died at the age of 70 , in 1822 , having spent most of his life in patriotic and useful labours in his native country. ${ }^{3}$

We have seen that already in 1796 Volta had arrived at a knowledge of the principle that the electric fection of effect of the metals might be increased by combining his pile, two sets of triple elements similarly disposed, which, and its cfunknown to him, Robison had already done (749). fects. 
But three years seem to have elansed before he was led to the invention of the pile, although it is in truth nothing more than the same arrangement frequently repeated. In March 1800 he wrote from Como a letter to Sir Joseph Banks, which was printed in the Philosophical Transactions for the same year, and in which he describes the Pile and the Couronnedes Tasses. The former consisted of 20 or more copper or silver coins interlaid with as many disks of tin or zinc, and others of paper or leather, soaked in water or brine. The same order of sequence of the three elements was carefully preserved throughout; and the whole formed a vertical pile or rouleau. Several such piles could be used together. The effects were-1:Theready excitement of the common electrometer by the aid of the condenser; 2 . The production of smart shocks through the hands and arms, similar to those produced by the torpedo; 3 . The production of vivid sensations of taste, of sound in the ears, and of flashes of light. There was nothing new in these effects (it may be seen) except that their intensity was much exalted, and the verification of the metallic theory was thereby rendered more easy. Volta attributes the action to the effect of " simple contact"' of the metals, allowing to the fluid element no other share than that of conducting sufficiently, but not too rapidly, the impulse thus excited. Having an eye probably to Fabbroni's opinions, he insists on the superior effects obtained with saline and alcaline fluids, and with hot in preference to cold fluids, being explicable solely by their increased conducting power. He justly describes the effects of the pile as similar to those of an immense electric battery with a very feeble charge; only the action is continuous, instead of intermittent.

But the invention had scarcely become known in London when the importance of the pile, as an instrument of discovery, was keenly appreciated in consequence of one capital discovery.

Nicholson, a good electrician and chemist, and Carlisle (afterwards Sir Anthony), a medical man, were the first in England to construct one of Volta's piles. It consisted first of 17 , afterwards of 36 , halfcrowns, with as many disks of zinc and of pasteboard, soaked in salt water. Experimenting upon the electrical effects of the pile, they used a drop of water "to make sure the contacts" upon the upper plate. Carlisle first observed a disengagement of gas round the wire which the water moistened. Nicholson suspected it to be hydrogen, and proposed to break the circuit by enclosing water in a tube between the two wires. This was accordingly done on the $2 \mathrm{~d}$ May 1800, within a month of the arrival in England of the first four pages of Volta's letter to Sir J. Banks, which preceded the remainder by a considerable space of time. The brass wire in the water tube, which was connected with the positive end of the pile, became tarnished and black, whilst minute bubbles of gas were evolved from the other, to the amount of $\frac{1}{15}$ th of a cubic inch in $2 \frac{1}{2}$ hours. Being mixed with an equal quantity of common air, and a lighted waxed thread being applied, it exploded. It was, therefore, concluded to be hydrogen derived from the decomposition of the water whose oxygen had combined with the brass of the positive wire. ${ }^{1}$ Nicholson, it appears, was well acquainted with Fabbroni's writings on the relation of galvanism to chemical action; and in the very paper where he describes-Volta's pile and his own discovery, he expresses his astonishment that Volta should have taken no notice of Fabbroni's results, or of the rapid oxidation of zinc in contact with other metals which appears in the pile, and which had been noticed by Fabbroni in every case where two metals differing in oxidability are placed in water, and in contact with each other. The experiment was repeated at Vienna, and then by Volta himself, who called attention to an experiment by three Dutch chemists, Paets, Van Troostwyk, and Dieman, who had decomposed water by common electricity in 1789.

Volta, himself, however, did not enter with zeal upon this new career; he even left to others the Volta re. to of his battery, which was first done by the useful from $\mathrm{N}_{\mathrm{a}}$ arrangement of Cruickshank. He was now ap-poleon, and proaching his 60th year, and seems to have been from the not indisposed to pass an old age of ease, and to re- Franceceive in tranquillity the marks of distinction which were showered upon him. In 1801 Napoleon called him to Paris, attended the meeting of the Institute where Volta explained his theory of the pile, caused to be voted to him on the spot a gold medal, and sent him home with a valuable present in money. He was then made a Senator, finally a Count : he was also made an Associate of the Institute in 1802. No scientific discovery ever excited the enthusiasm of Napoleon to the same degree as that of the Pile. He even extemporized a theory of life from its phenomena, comparing the vertebral column in man to the pile, the bladder being the positive and the liver the negative pole. An eminent medical chemist, Dr Prout, has seriously maintained a somewhat similar hypothesis. The favours lavished on Volta excited, perhaps, some jealousy amongst the French philosophers; for it is remarkable how little was added in France to the progress of the revived science of electricity. The French ruler, however, had himself in some measure to blame for this ; for the rigid exclusion of foreign, and especially of English publications, for a number of years, was felt to be highly injurious, and was in vain remonstrated against by Berthollet and others.

Volta survived his great invention above a quarter of a century. He died 5th March 1827, aged 82. His death. 
(757.) His scien. tific cha. racter. History of the pile of Volta con

(759.) Cruickshanks, Wollaston Davy!
His scientific character is easily summed up. He was patient, intelligent, and devoted to science from youth to age. He had, in an eminent degree, that patience and tenacity of purpose and of interest which Newton described as the chief attributes of his own genius. He had the candour which is more especially to be desired in the experimentalist; and he wrote without pretension, and generally clearly, though not without that diffuseness which is often associated with the use of the Italian language even in matters of science. On the other hand, his intellect may be rather described as opening itself to sonviction, than as forcing its way by a native power of penetration to great results. His taste and his talent lay far more in experimental than in abstract reasoning. His explanations of the effects which he observed were often involved and obscure; yet he had a very happy talent of combination, which led him to effect what others only talked about. His instrumental inventions, including the pile, were his happiest efforts. His theories, on the other hand, were surrounded, even in his own mind, with a certain obscurity. Even the contact theory, with its manifold paradoxes, was perhaps only vigorously carried out by him under the excitement of an active controversy. The invention of the pile may, in very many respects, be placed on a par with that of the steamengine. The results of the former were indeed more interesting, immediately, to pure science; the latter to the arts of life and the needs of civilization. Yet, after half a century, this distinction can hardly be drawn with severity. The rapid pace of steam is insufficient for our demands. The electric wire conveys to its destination, ere the locomotive has time to start on its journey, tidings of joy and sorrow-life and death-of victories won, and kingdoms lost.

\section{§ 3. Sir Humphry Davy. Progress of Voltaic Electricity-Electro-Chemistry; Berzelius.- Davy's Invention of the Safety-Lamp.-WoLLASTON; his Electrical and other Observations. Contrast of his Character with that of Davy.}

The pile of Volta was, in one sense, rather a means of discovery than a discovery itself. Volta had neither a just theory of the source of power which he invented, nor was he successful in applying it to any important research. The discovery of its chemical efficiency by Nicholson and Carlisle, stimulated, as we have seen, for a short time his interest and curiosity; but he never seriously attached himself to this line of discovery. His subsequent papers are chiefly controversial, in support of the Contact theory. The generation, as well as the expenditure of chemical forces by the pile, consequently'remained, as far as he was concerned, in complete obscurity.

The invention of the pile having been communicated to the world through the Royal Society, naturally gave an impulse to English electricians and chemists. The first discoverers of its chemical energy did not however themselves prosecute their experiments to a great extent, but Cruickshanks decomposed salts and revived the metals by the voltaic current, whilst he improved the form of the apparatus in an important manner. Colonel Haldane ascertained the significant fact that the action of the pile cannot be continued in an atmosphere deprived of its oxygen. Hisinger and Berzelius, carrying out Cruickshanks' experiments, showed that, generally in the decomposition of compounds, the alcaline and metallic elements appear to be attracted towards the negative wire of the battery, and the acids to the positive one. In the mean time, Davy and Wollaston appeared on the arena, and the former especially filled so important a part in the history of science for the next twenty years, that we can hardly give his name too great a prominence in a review of the characteristics of the period. The constitution of Davy's mind was also more than usually interesting, and his career of discovery, short, brilliant, and decisive, is at once one of the most instructive and remarkable of those which we have to consider. The contrast between him and his contemporary, Wollaston, was one of those curious antitheses of really great minds which occasionally occur in such close connection, and with such prominent relief, as to compel rather than invite a comparison between them. It is an instructive lesson to observe, how natures, the most unlike, cultivated in a school the most opposite, may yet, when both directed by a common impulse to similar objects, promote the development of truth, and the cause of scientific discovery.

Sir Humphry DAvy was born at Penzance on the (760.) 17th December 1778. His was an ardent boyhood. Educated in a manner somewhat irregular, and with only the ordinary advantages of a remote country town, his talents appeared in the earnestness with which he cultivated at once the most various branches of knowledge and speculation. He was fond of metaphysics; he was fond of experiment; he was an ardent student of nature; and he possessed at an early age poetic powers, which, had they been cultivated, would, in the opinion of competent judges, have made him as eminent in literature as he became in science. All these tastes endured throughout life. Business could not stifle them, - even the approach of death was unable to extinguish them. The reveries of his boyhood on the sea-worn cliffs of Mount's Bay, may yet be traced in many of the pages dictated during the last year of his life amidst the ruins of the Coliseum. But the physical sciences-those more emphatically called at that time chemical-speedily attracted and absorbed his most earnest attention. The philosophy of the imponderables—of Light, Heat, 
and Electricity - was the subject of his earliest, and also that of his happiest essays. He was a very able chemist in the strictest sense of the word, although his ardour and his rapidity of generalizing might seem to unfit him, in some measure, for a pursuit which requires such intense watchfulness with regard to minutiæ, such patient weighings of fractions of a grain, such frequent though easy calculations. To Carendish and Dalton, his great contemporaries-to whom we may now add Wollaston-these things were a pleasure in themselves; to Davy they must ever have been irksome indispensables to the discovery of truth. But, in fact, Davy's discoveries were almost independent of such quantitative details: Numerical relations, and harmony of proportion, did not affect his mind with pleasure, which possibly was one reason of his deficient appreciation of works of art, the more remarkable from his poetic temperament. Dalton's doctrine of atomic combinations was (as we have seen) slowly and doubtfully received by him whilst Wollaston perceived its truth instantaneously. A keener relish for such relations might most naturally have led Davy to an anticipation of Mr Faraday's notable discovery of the definite character of electrical decomposition, and the coincidence of the Electro-chemical proportions for different bodies with their atomic weights.

The early papers of Davy refer chiefly to Heat, Light, and Electricity. He was, in fact, a physicist more than a chemist. Whilst yet a surgeon's apprentice at Penzance, he satisfied himself of the immateriality of heat, which he illustrated by some ingenious experiments, in which, concurring unawares with the conclusions of his future patron Rumford, he laid one foundation of his promotion. Removed to a sphere of really scientific activity at Clifton, under Dr Beddoes, ${ }^{1}$ he executed those striking researches in pneumatic chemistry and the physiological effects of breathing various gases which gave him his first reputation; researches so arduous and full of risk as to require a chemist in the vigour of life, and urged by an unextinguishable thirst for discovery, to undertake thein. Even his brilliant discovery of the effects of inhaling nitrous oxide brought no competitor into the field; and the use of anæsthetics, which might naturally have followed-the greatest discovery (if we except, perhaps, that of vaccination) for the relief of suffering humanity made in any age - was delayed for another generation. But so it was in all his triumphs. He never seemed to drain the cup of discovery. He quaffed only its fieshest part. He felt the impulse of an unlimited command of resources. He carried on rapidly, and seemingly without order, several investigations at once. As in conversation he is described as secming to know what one was going to say before uttering it, - he had the art of divining things complex and obscure. Seizing on results, he left to others the not-inconsiderable merit, as well as labour, of pursuing the details. Keenly alive as he was to the value of fame, and the applause which his talents soon obtained for him, he left enough of both for his friends ; his contemporaries, as well as his successors, were enabled to weave a chaplet from the laurels which he had not stooped to gather.

These remarks apply quite as strongly to his discoveries in the laws and facts of electro-chemical de- Removed composition-those on which his fame most securely to the rests. Promoted in 1801 to a situation in the Labo-stitutionratory of the Royal Institution in London, he attached experihimself to the study of galvanism in the interval of ments on the other and more purely chemical pursuits which electricity. the duties of his situation required. He had already, at Clifton, made experiments with the pile of Volta, and taken part in the discussion of its theory and effects, then (as we have seen) so actively carried on in Britain. In his papers of that period we find not only excellent experiments, but happy and just reasoning. The chemical theory of the pile-namely, that the electrical effects observed by Galvani and Volta are due solely or chiefly to the chemical action of the fluid element on the metals-was more strongly embraced by him then than afterwards. In November 1800 he concluded that "the pile of Volta acts only when the conducting substance between the plates is capable of oxidating the zinc; and that in proportion as a greater quantity of oxygen enters into combination with the zinc in a given time, so in proportion is the power of the pile to decompose water and to give the shock greater." $\mathrm{He}$ concludes that "the chemical changes connected with" oxidation "are somehow the cause of the electrical effect it produces." 2 His views on this subject underwent some modification afterwards. In his Elements of Chemical Philosophy, published twelve years later; we find the following statement of his opinions on the subject:- "Electrical effects are exhibited by the same bodies acting as masses, which produce chemical phenomena when acting by their particles; it is, therefore, not improbable that the primary cause of both may be the same." A little further on he adds :- "They," speaking of electrical and chemical energies, "are conceived to be distinct phenomena, but produced by the same power acting in the one case on masses, in the other on particles."3

1 Davy hit off his principal's character in a single sentence,-_ "Beddoes had talents which would have exalted him to the pin. nacle of philosophical eminence, if they had been applied with discretion."

2 Works, ii., 162.

3 Worles, iv, 119. In his Bakerian lecture (1806), he had said, "In the present state of our knowledge, it would be useless to attempt to speculate on the remote cause of the electrical energy, or the reason why different bodies, after being brought into contact, should be found differently electrified; its relation to chemical affinity is, however, sufficiently evident. Mlay it not be identical with it, and an essential property of matter?" -Works, vol. v., p. 39. 
(763.) Electro. chemical theoryBerzelius, Hisinger Davy's first Bake rian lecture.

\section{(764.)}

Davy's sagacious inductions respecting polar forces.
Electro-chemical Theory-Decomposition of the Alcalies.-In 1804 Berzelius had published, in conjunction with Hisinger, a paper on Electro-chemical Decompositions, in which he insisted on the general fact, that alcalies, earths, and combustible bodies seem to be attracted to the negative pole, and oxygen and acids to the positive. He also showed that the subdivision of bodies thus obtained was only a relative not an absolute one; for the same body may act as a base to a second, and as an acid to a third. But we must observe that results almost similar were contained in the early papers of Davy, and that Berzelius did not carry out his own principle so far as to lead to any striking discovery between 1803 (when his experiments were made) and 1806 (the date of Davy's first Bakerian lecture), during which time the science of Galvanism or Voltaism made little real progress. The numerous experimenters engaged with it were baffled by the anomalous chemical results obtained, and by the appearance of decompositions under circumstances wholly unexpected, such as appeared to threaten the existence of some of the best established chemical truths. The chemical theory of the pile, at first so plausible, presented new difficulties, and Berzelius having for a while defended it, returned to the simple contact theory of Volta. It was then that Davy seriously addressed himself to the subject, resolved to trace to their source every chemical anomaly; and this he effected in a masterly manner in his Bakerian lecture read before the Royal Society in 1806. In it he traces the unaccountable results of his predecessors to impurities in the materials used by them, or to those of the vessels in which the decompositions were made; and he brings into a far distincter light than his predecessors had done, the power of the galvanic circuit to suspend or reverse the action of even powerful chemical affinity; "different bodies naturally possessed of chemical affinities appearing incapable of combining or of remaining in combination when placed in a state of electricity different from their natural order." We here see the fundamental doctrine of the electro-chemical theory, that all bodies possess a place in the great scale of natural electrical relations to one another; that chemical reactions are intimately connected with this electric state, and are suspended or reversed by its alteration.

In the interpretation of those striking experiments, in which he caused acids to pass to the positive pole of the battery through the midst of alcaline solutions, and the converse, we find so close an approach to the theory of polar decomposition as enforced by the discoveries and reasoning of $\mathrm{Mr}$ Faraday, that it seems impossible to deny to Davy the merit of having first perceived these curious relations. "It is very natural," he says, "to suppose that the repellant and attractive energies are communicated from one particle to another particle of the same kind so as to establish a conducting chain in the fluid, and that the locomotion takes place in consequence;" and presently adds, "there may possibly be a succession of decompositions and recompositions throughout the fluid" (p. 29). ${ }^{1}$ He likewise shows (p. 21) that the decomposing power does not reside in the wire or pole, but may be extended indefinitively throngh a fluid medium capable of conducting electricity. Mr Faraday's experiments, which led him to discard the term pole, lead to the same conclusion, and are of the same character. A few pages further on in this same Bakerian lecture, Davy observes ( $p$. 42 ), that " allowing that combination depends on a balance of the natural electrical energies of bodies, it is easy to conceive that a measure may be found of the artificial energies as to intensity and quantity capable of destroying this equilibrium; and such a measure would enable us to make a scale of electrical powers corresponding to degrees of affinity." Here we see the acute presentiment of the beautiful discovery of the definiteness of electrical decompositions; as in the concluding portion of the same remarkable paper we find a clear anticipation of natural electrical currents to be discovered in mineral, and especially metalliferous deposits, since established by Mr R. W. Fox, and of the agency of feeble electric energies, long continued, in effecting geological changes, and in producing insoluble combinations of earths and metals, so ingeniously confirmed by the beautiful and direct experiments of Becquerel.

The sequel to this remarkable paper, read to the Royal Society in November 1807, contained the splen- Second B did application of the principle and methods which it kerian lecdescribed, to the decomposition of the alcalies and to composithe discovery of their singular bases,- - substances po- tion of the sessing the lustre and malleability of metals, yet so alcalies. light as to float upon water, and having the extraordinary property of becoming inflamed in contact with ice. Potassium was discovered in the Laboratory of the Royal Institution on the 6th October 1807, and sodium a few days later. The battery used contained 250 pairs of plates of 6 and 4 inches square. Such success was fitted to charm a disposition like that of Davy, and more than reward him for all his toils. To have discovered two new bodies, and opened an entirely new field of wide chemical research, would itself have been enough. But the extraordinary properties of the new bases were such as seemed to correspond to the lively imagination of the Chemist who produced them, and to transport him to an Aladdin's palace more brilliant than even his fertile imagination had ever conceived. Yet it is pleasing to remember that these popular discoveries Receives a followed, at the interval of a year, the patient and prize from able researches which led him to them, and which tute of had already been rewarded, at a period of the bitter- France. 
est international hostilities, by the scientific prize of 3000 francs, founded by the Emperor Napoleon. ${ }^{1}$

The genius displayed in these, Davy's most celebrated researches, is evident on a careful perusal of his papers; but still more from a consideration of the state of science of the time, and of the willing tribute to his merits paid by the ablest of his contemporaries. Few persons of the present day will venture to controvert the assertion of his acute contemporary, Dr Thomas Young (than whom no man was ever a less indiscriminate eulogist), that Davy's researches were "more splendidly successful than any which have ever before illustrated the physical sciences, in any of their departments "' and that the contents of the Bakerian Lectures, in particular, "are as much superior to those of Newton's Optics, as the Principia are superior to these or any other human work." 2 A not less impartial tribute to his superlative genius has been yielded by $\mathbf{M}$. Dumas, who, if I mistake not, has described Davy as being the ablest and most successful chemist who ever lived. A similar homage is paid to him by the sagacious Cuvier.

It is not within our scope to consider minutely Davy's purely chemical discoveries and experiments, though they were numerous and important, independently of those made with the aid of electricity. His proofs of the elementary nature of chlorine and iodine were amongst the most considerable in their results. But as a mere analyst, Davy had neither the leisure nor the taste for continuous plodding labour, and he therefore naturally made mistakes in chemical details. His Elements of Chemical Philosophy remained, in consequence, a fragment of an extensive work. His contemporary, Berzelius, following his steps in electro-chemical discovery, attained far greater address, and became an author of high and merited reputation, whilst his school surpassed all others in Europe in producing accomplished analysts. ${ }^{3}$

The years immediately following the publication of

(768.) Javy's ex- his Bakerian Lectures were passed by Davy in the ensive po- $u$ envied possession of the highest fame, and in the ind its ind its tranquil furtherance of his first and greatest discoveries. His lectures at the Royal Institution continued to be one of the most fashionable resorts in London, and he was freely admitted in return into the most aristocratic society; he had but to express a wish, and a voltaic battery of no less than 2000 pairs, containing 128,000 square inches of surface, was constructed for his use, by means of a liberal subscription. His health, when scriously compromised by the severity of his labours, was a matter of public concern, and its variations were announced by frequent bulletins. The copyright of his lectures on agriculture was sold for a price unexampled perhaps before or since for such a work. In 1812 he was knighted by the Prince Regent, and soon after he married a lady of fortune and accomplishments. His duties at the Royal Institution became thenceforth honorary. He had in a space of ten years attained the pinnacle of scientific reputation, and he was for the time truly happy:-unenvious of others-deeply attached to his relatives-generous of his resources-unwearied in his philosophic labours. A certain change (it must with regret be owned) came over his state of mind, tarnished his serenity, and gradually though imperceptibly weakened his scientific zeal. It was to be ascribed solely, we believe, to the severe ordeal of exuberant but heartless popularity which he underwent in London. The flatteries of fashionable life acting on a young, ardent, and most susceptible mind, mingling first with the graver applause of his philosophic compeers, and at length, by their reiteration and seductions quite overpowering it, by degrees attached Davy to the fashionable world, and loosened his ties to that laboratory which had once been to him the sole and fit scene of his triumphs. Had he been blest with a family, his course would probably have been evener and happier. Let us not severely criticise, where we still find so much to admire and to imitate. But we record the fact, for the consolation of those who, beginning the pursuit of science, as Davy did, in a humble sphere, and with pure ardour, may fancy that they are worthy of pity, if they do not attain with him the honours of wealth and title, and the homage, grateful to talent, of rank, wit, and beauty.

A research, second perhaps only to his electrochemical discoveries, remains to be noticed, as the Third pechief fruit of the third period of his life, on which we riod of his now enter; the first being his early career before settling in London; the second, that passed in the Royal Institution.

Researches on Flame-The Safety-Lamp.-The subject was, the laws of combustion, and the happy Researches invention of the safety-lamp. Though intimately the safetyconnected with the doctrine of simple heat, it may, lamp. most properly, from its chemical character, and from its connection with Davy's history, be considered briefly here. The lamentable loss of life occurring in coal mines from explosions of fire-damp or inflammable air disengaged from the workings, had for many years attracted the attention and sympathy of the public, and had likewise been carefully considered by scientific men. The explosive gas was known to be the light carburetted hydrogen. Two plans alone seemed to present themselves for diminishing the danger:-the one to remove, or chemically to decompose the fire-damp altogether ; the other, to provide a miner's lamp which, by its construction, should

1 Such was the national feeling at this time in England, that worthy people were found who considered Davy as almost a traitor, when he accepted the French prize. See Southey's Life.

2 Quarterly Review, No. 15.

3 Jöns Jacob Berzelius, the greatest analytical chemist of his day, was born in East Gothland, in the same year with Davy, and died in 1848, when he had almost completed his 69th year. He contributed, in a signal manner, to the establishment of Dalton's principle of definite chemical equivalents; but he made no single discovery of commanding importance. 
be incapable of causing explosion. The former of these modes of protection, it was soon seen, could only be palliative; the only efficient form which it took, was that of a more effectual ventilation; but the terrific rapidity with which a mine may be suddenly invaded by fire-damp, from channels opened by a single blow of the pickaxe, must prevent it from ever acting as a cure. The latter plan had as yet yielded nothing more effectual than the steel mill long used by miners, which produced an uncertain and intermitting light, by the rotation of a steel wheel against a flint, the scintillations of which were incapable of inflaming the fire-damp. The insufficiency of the light prevented it from being used, except in circumstances of known danger. The celebrated Baron Humboldt, Dr Clanny, and several others, had invented safety-lamps on different principles; but they were all clumsy and more or less ineffectual. ${ }^{1}$

(771.) At last, in the summer of 1815, the Rev. Dr History of Gray (afterwards Bishop of Bristol), then chairthe inven-
tion of the man of a committee appointed by a benevolent assafety sociation at Bishop Wearmouth for the prevention of lamp. colliery accidents, applied to Davy, who was then on a sporting tour in Scotland, requesting his advice and assistance. Sir Humphry answered the call with promptitude. On his southward journey, in the latter part of Angust, he visited the collieries, ascertained the circumstances of the danger which he had to meet, and was provided by Mr Buddle with specimens of the inflammable air for examination. Within a fortnight after his return to London, he had ascertained new and important qualities of the substance, and had already four schemes on hand for the prevention of accident. Before the end of October, he had arrived at the following principles of operation in connection with a safety-lamp. "First, A certain mixture of azote and carbonic acid prevents the explosion of the fire-damp, and this mixture is necessarily formed in the safe-lantern; secondly, The firedamp will not explode in tubes or feeders of a certain small diameter. The ingress to, and egress of air from my lantern," he adds, "is through such tubes or feeders; and, therefore, when an explosion is artificially made in the safe-lantern, it does not communicate to the external air." The effect of narrow tubes in intercepting the passage of flame, is due to the cooling effect of their metallic sides upon the combustible gases of which flame is composed $;^{2}$ and one of his first and most important observations was the fortunate peculiarity that fire-damp, even when mixed with the amount of air most favourable to combustion (1 part of gas to 7 or 8 of air), requires an unusually high temperature to induce combination. Olefiant gas, carbonic oxide, and sulphuretted hydrogen, are all inflamed by iron at a red heat, or ignited charcoal, but carburetted hydrogen does not take fire under a perfect white heat. The earliest safety-lamp consisted of a lantern with horn or glass sides, in which a current of air to supply the flame was admitted below by numerous tubes of small diameter, or by narrow interstices betwreen concentric tubes of some length; or, finally, by rows of parallel partitions of metal, forming rectangular canals extremely narrow in proportion to their length. A similar system of escape apertures was applied at the top of the lantern.

With characteristic ingenuity, Davy did not stop here. He continued to reduce at once the apertures The safety and length of his metallic guards, until it occurred fected. to him that wire gauze might, with equal effect, and far more convenience, act upon the temperature of flame, so as to reduce it below the point of ignition, and thus effectually stop its communication. The experiment was successful, and by the 9 th November 1815 , or within about ten weeks after his first experiments, an account of the safety-lamp defended by wire gauze was presented to the Royal Society. About two months later he produced a lamp entirely enveloped in metallic tissue.

There are none of Dary's ressearches which will stand a closer scrutiny than those which terminated a closer scrutiny than those which ter- as a model vation led him to conceive a happy idea obser- for similar vation led him to conceive a happy idea and to investiga-
apply it to practice. A great boon to humanity tions. and the arts was required at his hands; and without a moment's delay, he proceeded to seek for it under the guidance of a strictly experimental and inductive philosophy. Without, perhaps, a single false turn, and scarcely a superfluous experiment, he proceeded straight to his goal, guided by the promptings of a happy genius aided by no common industry. The chemical, the mechanical, and the purely physical parts of the problem were all in turn dealt with, and with equal sagacity. It may safely be affirmed that he who was destitute of any one of these qualifications must have failed in attaining the object so ardently desired, unless by the aid of some rare good fortune. We have it on Dary's own authority, that none of his discoveries gave him so much pleasure as this one. His whole character possessed in it much of a sympathizing and generous humanity; his ideas of the dignity of science were from the first (as his researches in Dr Beddoes' laboratory showed) intimately connected with the aim of advancing the welfare, and of diminishing the misfortunes of mankind : the rapidity and singular success of his investigation in the case of the safety-lamp, kept his ardent soul all alive, and afforded him the triumph of a $E u-$ reka at its completion. To these sources of inward gratification was added the unstinted meed of praise bestowed on him by his contemporaries. Playfair,

\footnotetext{
1 I have spoken in Art. 393 of the independent and ingenious efforts of George Stephenson towrards the invention of a safetylamp contemporaneously with those of Davy.

2 This fact had been ascertained some years previously, by Mr Tennant and Dr Wollaston, bat it remained anpublished, and was not applied by them to the prevention of colliery explosions.
} 
" the true and amiable philosopher," as Davy long before described him, thus proclaimed his victory in the Edinburgh Review:-After describing the course of a discovery "which is in no degree the effect of accident," he adds, "this is exactly such a case as we should choose to place before Bacon were he to revisit the earth, in order to give him, in a small compass, an idea of the advancement which philosophy has made since the time when he had pointed out to her the ronte which she ought to pursue. The result is as wonderful as it is important. An invisible and impalpable barrier made effectual against a force the most violent and irresistible in its operations; and a power that in its tremendous effects seemed to emulate the lightning and the earthquake, confined within a narrow space, and shut up in a net of the most slender texture-are facts which must excite a degree of wonder and astonishment, from which neither ignorance nor wisdom can defend the beholder."

For this truly patriotic labour, the only national testimony which Davy received was the inadequate one of a baronetcy, which was conferred on him by the Prince Regent in 1818; but his real triumph and great reward were in the enthusiastic appreciation of his entire success by those on whom he had disinterestedly conferred so great a benefit. A testimonial, in the form of a service of plate, of great value, was presented to him by the coal-owners of the north of England.

Davy's researches on flame were intimately connected with his electrical and chemical discoveries. He remodelled Lavoisier's theory of combustion, and put an end to the distinction between combustibles and supporters of combustion. Chemical combination, effected with great energy, and accompanied by a high temperature, is essential to combustion, and either element of the combination is equally entitled to the denomination of combustible. Guided by the electro-chemical theory, Davy appears to have thought that the heat of flame has an electrical origin.

But I must hasten to close this section. Among the labours of his latter years, there was none which interested Davy more, or which reasonably promised more useful results, than his plan for protecting the copper sheathing of ships from the corrosive action of sea water, by affixing plates of zinc or iron, which shouldrender the copper slightly electro-negative, and thus indispose it for combining with acid principles. It is a somewhat singular fact that Fabbroni, about 30 years before, had instanced the corrosion of copper sheathing near the contact of heterogeneous metals, as an instance of the chemical origin of galvanism. ${ }^{1}$ Davy's experiments were conducted with his usual skill and success, and the remedy only failed of general adoption on account, it may be said, of being too effectual, other and opposite injurious effects having been found to arise.

Davy was elected President of the Royal Society in 1820 , in the room of Sir Joseph Banks, who had Davy as held the office for 42 years. It was a distinguished President compliment, for the election was all but unanimous. Royal so$\mathrm{He}$ continued to communicate papers for several ciety-his years subsequently; but his energy, his temper, and, death. finally, his health began to give way-showing that the ardent labours of his youth and prime had injured his constitution. Attacked with paralysis in 1827, he spent his last years chiefly abroad, and died at Geneva (where he was buried), on the 29 th May 1829.

The character of Davy was a rare and admirable combination. The ardour of his researches, and the deep devotion of his whole being to scientific investigation, have been already proved. They had the effect of completely annihilating every baser passion. He valued property only in so far as he could apply it usefully; and his disinterestedness with respect to the fortunes which several of his practical discoveries might have honourably earned, was one of the most striking parts of his character. His fancy was discursive to a degree rarely met with in men of science. $\mathrm{He}$ continued to write poetry nearly all his life, and the tone of it was that of grave speculation, always reverting to the destiny of man and the beneficence of the Creator. His lectures were composed with care; and their effect, even as pieces of oratory, was striking. Coleridge frequented them "to increase his stock of metaphors;"-yet they were always to the point, and never degenerated into rhetorical display. For a man of such extraordinary liveliness of fancy and impetuosity of action, his mistakes were astonishingly few. After his very first experience, his publications were made with great care and judgment. His estimates of his contemporaries appear generally to have been fair and liberal, though it would be incorrect to affirm that he was universally popular among them. The combination of isolated and intense occupation in his laboratory, with excitement in the mixed society of an admiring London public, was a trial which few, if any, could have escaped better than he did; and so far as we can judge of a man from his expressed opinion of his own successes, whether recorded in his works or in his intimate correspondence, Davy must be accounted to have acquitted himself gracefully and well. $\mathrm{He}$ always spoke of the Pile of Volta as the first source of his own success. "Nothing tends so much to the advancement of knowledge as the application of a new instrument," he says; and then adds, "The native intellectual powers of men in different times are not so much the causes of the different success of their labours, as the peculiar nature of the means and artificial resources in their possession ;" a proposition 
which he applies to his own discoveries. But we may truly say with one of his biographers, that to him "the Voltaic apparatus was the golden branch by which he subdued the spirits that had opposed the advance of previous philosophers; but what would its possession have availed him had not his genius, like the ancient sybil, pointed out its use and application?"

(779.) The last, and not least, extraordinary characterisNumerous tic of Davy to which I shall now advert, was the practical highly practical turn of a mind which seemed formed in a speculative mould. Four at least of his chicf researches were of this kind-his experiments on breathing the gases; his lectures on agricultural chemistry; his invention of the safety-lamp; and his protectors for ships. No man, whose path so clearly lay in original discovery, ever left so many valuable legacies to art and to his country.

(780.) The name of Davy gave to England a distinguished Davy's as- pre-eminence in science during the first 25 years of Yociates- the century. But two others, less noticed at the time, Wollaston. were also among her worthiest sons. These were Young and Wollaston. They were all three nearly contemporaries; all lived on good terms with one another, and united in promoting natural knowledge in their several spheres. Young was Davy's early, though less successful colleague at the Royal Institution; and Wollaston was joint-secretary with him to the Royal Society. All three were originally cducated for the medical profession, and they all abandoned it for the pursuit of science. Not the least singular coincidence was in the periods of their deaths, which all occurred within the space of six months.

(781.) Our notice of Young, the first optical philosopher William of his age, belongs to another chapter. WoldAston, Hyde Wol- though an original observer in nearly every branch
laston-his contribu- of exact science, considered himself as a chemist; tions to and his observations on Electricity were amongst his Electricity, first and best contributions to science. After the impulse given to discovery by the invention of the Pile, and the proof of the decomposition of water, Wollaston undertook to compare critically the effects of galvanic and frictional electricity-a task of some nicety, and of very great importance at a time when it could hardly be considered as certain that these agents were not specifically different. By methods peculiarly his own, he produced decomposition, accompanied with separation of the elements at the respective poles by means of common electricity. $\mathrm{He}$ at the same time gave his powerful support to the purely chemical theory of the Pile.

His most important inventions were rather in$(782$.
and the other sciences. struments which, in the hands of others, were to produce important discoveries, than discoveries in art of rendering platinum malleable, which las conferred inexpressible benefits on chemistry, and on the arts connected with it. The principle of the reflection of a ray of light for measuring angular spaces, though it existed already in the single instance of the sextant, has been, since it was applied to the goniometer, adapted to a multitude of most ingenious and valuable contrivances.

Wollaston was an excellent optician, and of some of his observations I have alrcady spoken (476), (538).

The strong points of his character were precision and rare acuteness in observation, patience and caution in deduction, and habitual devotion of his time racter and energies to scientific pursuits. His foibles were with tha an excess of caution, and a certain microscopic turn of Davy. of mind which, though it sometimes rewarded him with valuable discovories, consumed his time in occupations of mechanical ingenuity, and prevented him from grappling with almost any of the great theories of his day. An exception, yet one which illustrates his character, may be found in the fact that he had all but anticipated Dalton in his discovery of the multiple combinations of salts, whilst, with his characteristic sense of justice, he disclaimed any participation in the merit (624). While Davy was delighting crowded audiences with his eloquence, his discoveries, and their wonderful results, Wollaston was pursuing his solitary experiments on a scale so small that scarcely three persons could witness them at once. While Davy was firing his potassium with ice, and making mimic volcanos heave by the oxidation of his new metals, Wollaston was extracting, by minute analyses, from the refractory and unoxidable ores of platinum, substances previously undetected, which, neither by their quantity nor their characters, could ever interest any but a man of science. While Davy was charging his prodigious battery of 2000 pairs,- the largest which has ever been constructed (a homage to his genius, provided by his numerous admirers),-Wollaston was proving, after his fashion, how similar effects could be produced by the very same agency on.a small scale; and with no greater apparatus than a shred of zinc, a fewdrops of acid, and an old thimble, he would gratify his friends by exhibiting the mimic glow of an almost microscopic wire of platinum. Davy seemed born to believe; Wollaston to doubt. Davy was a poet; Wollaston, a mathematician, or, at least, capable of becoming a great one. Davy announced his discoveries in fiery haste, and presented all their consequences and corollaries as a free gift to mankind; Wollaston (estimating more truly the rarity of the inventive faculty) hoarded every observation, turned it over and over, polished it, rendered it exact beyond the reach of criticism, and then deliberately laid it before the world. He had the coldness and the accuracy of Cavendish, but he wanted the spur of his genius, and the wide grasp

.
theinselves. One was the invention of the Reflecting Goniometer for measuring the angles of crystals, now so essential to mineralogy; another, the 
of his apprehension. Among other legitimate results of discovery, Wollaston was not unwilling to claim for his own the material profits which such researches sometimes, though rarely, yield; whilst Davy, as we have seen, spurned every possible attribution of an interested motive. Davy never made a shilling in his life, save as an author or a lecturer (except as paid assistant to Dr Beddoes); Wollaston realized a fortune by his art of working platinum. Davy was admired by thousands both at home and abroad; Wollaston was little known except to a small circle who could appreciate the resources of a mind rarely opened in confidence to any one, and of which the world was only partially informed.

Wollaston was born in 1766 , and died in December 1828. The composure of his end rivalled that of Black and Cavendish. His disorder was one of the brain. When he had lost the power of speech, his attendants remarked aloud that he appeared unconscious. Making a sign for a pencil and paper, he wrote down a column of figures, added them up correctly, and expired.

\section{§ 4. Oersted. - AMPERE. - Discovery of Electro-Magnetism-Electro-Dynamic Theory- Discovery of Thermo-Electricity; SEEBECK. The Galvanometer of Schweigger and Nobili.}

Hans Christian Oersted was born in Langeland; one of the Danish isles, on the 14th August 1777. Of him it might almost be said that "on awaking one morning he found himself famous." The single discovery of the mutual action of magnets and electric conductors gave him a celebrity which a life-long devotion to science has oftener than the contrary failed to secure.

Yet in this, and perhaps every similar case, it will be found that brilliant, and, as the world, or jealous rivals esteem it, fortunate success, was not the result of an isolated effort, but was connected with a long career of patient though comparatively obscure labour.

At the age of 20 , Oersted, whilst yet a student at the University of Copenhagen, became an author. His first publication was a prize essay on an æsthetical subject. Being intended for the medical profession, he soon after wrote some chemical papers, and, in 1801, his first " On Galvanic Electricity." But his turn of mind at this time, as well as later, was of a strongly metaphysical cast, and of course tinctured with the peculiarities of the German school as regards the study of physics, of which the title of his thesis on graduation may be given as an instance:-It was On the Architectonicks of Natural Metaphysics. His studies in voltaic electricity were made chiefly under Ritter, an obscure and mystical writer, though the author of some curious experiments on what were called Secondary Piles; and he at length obtained, in 1806, a professorship in his own university; but his associates appear to have been rather literary than scientific persons, such as Steffens, Oehlenschlager, Niebuhr, and Fichte; he also engaged in controversies of a theological tendency, which, to the end of his life, appear to have had a great attraction for him.

In 1812 Oersted visited Berlin, and published is first there a work on Chemical and Electrical forces, tend-
ritings on ing to prove their identity, which was translated into Fetricity. French by Marcel de Serres. The author afterwards
ench looked back to the period of the publication of this treatise as the dawn of his electro-magnetic discovery.
So far as I know of its contents (for I have never seen a copy), it does not contain anything beyond indefinite anticipations of the real identity of electricity and magnetism. In this, indeed, there was nothing new. Compass-needles had been seen to be reversed by lightning; electric shocks had been passed through steel without any certain effect; and Van Swinden had published a work in three volumes expressly on the subject, containing the results of a mass of ingenious failures. Nor, perhaps, can we give Oersted credit, at that early period, for a more distinct apprehension of the relation so anxiously sought for, than was possessed by several of his contemporaries. His belief is said to have been grounded on the notion, that " if galvanism be only a hidden form of electricity, then magnetism can only be electricity in a still more hidden form"-a syllogism which, if it satisfied Oersted's metaphysical friends, would hardly be accepted as demonstrative in the laboratory; and, after all, it suggests no one form of relation rather than another.

Professor Forchhammer, the friend and pupil (790.) of Oersted, states that, in 1818 and 1819, it was His discowell known in Copenhagen that he was engaged electroin a special study of the connection of magnetism magnetism. and electricity. Yet we must ascribe it to a happy impulse-the result, no doubt, of much anxious thought-that, at a private lecture to a few advanced students in the winter of 1819-20, he made the observation, that a wire uniting the ends of a voltaic battery in a state of activity, affected a magnet in its vicinity. It was in the fact of the circuit being closed, that the main difference consisted between this and previous attempts, in which galvanic pairs or batteries not connected by conductors were expected to show magnetical relations, though, in such a case, the electricity was evidently stagnant.

Some mystery hangs over Oersted's apprehension (791.) of his own experiment. It seems difficult to believe Details rethat he clearly saw its significance. Unlike Davy, ${ }^{\text {specting it. }}$ when he first saw the fiery drops of potassium flow under the action of his battery, and recorded his triumph in a few glowing words in his laboratory jour- 
nal, Oersted took no immediate measures either to complete or to publish his discovery. Some months appear to have elapsed whilst waiting for the convenience of a larger battery before he repeated the experiment with the aid of Professor Esmark and other friends. The battery then employed contained 20 twelve-inch elements, charged with water and $\frac{1}{3} \frac{1}{t}$ th of mixed nitric and sulphuric acids. The conducting wire was heated red hot, which must have rather diminished the effect than otherwise. The nature of the wire was found to be unimportant. If positive electricity passed from north to south through a conducting wire placed horizontally in the magnetic meridian, then a compass needle suspended over it had its north end deviated to the west; if under it, to the east ; if the needle was placed on the east side of the conductor, its north end was raised; if on the west side, it was depressed. Oersted further found that needles of non-magnetic substances, such as brass and gumlac, were not affected, and that the electrical efficiency depended on the quantity, not the intensity, of the current. These experiments seem to have been made in July 1820; and Oersted and his friends being now fully alive to the novelty and importance of the discovery, he circulated extensively copies of a Latin tract, dated the 21 st July, in which the effects of the "electric conflict," as he terms the presumed combination of the opposite electricities in the "conjunctive wire" upon a magnet, were described. ${ }^{1}$ " In this tract we find the following expressions:- "The electric conflict acts in a revolving manner." "It resembles a helix." "The electric conflict is not confined to the conducting wire, but it has around it a sphere of activity of considerable extent."

The effect of this pamphlet, consisting of a few Speedily pages only, was instantaneous and wonderful. The taken up author probably counted on the opportunity of develby Ampère, Arago, and with such avidity and pursued rith was seized on Davy. cess, particularly in France, that he probably gave up the race of invention in despair. Ampère had already communicated experiments to the Institute on the 18th and 26th September. Arago and Davy separately, and but little later, discovered the magnetizing power which the voltaic conductor exerted on iron filings, and the latter tried in vain the magnetizing power of common or machine electricity, which, however, was soon after shown by Arago, who enclosed steel wires in helices of copper wire, through which the discharges were passed. When soft iron was placed in such a helix, it was found to become a temporary magnet of 'great power whilst the voltaic current continued. Thus magnets of enormously greater power than any previously known were constructed; one of the first large ones was made by Professor Henry of the United States.

(793.) was far more indebted to Ampère, a professor at Paris, than to any other philosopher. I shall, therefore, introduce here some account of his discoveries before closing what I have to say of Oersted.

Andre Marie Ampere was born in 1775 at Lyon. He was an able mathematician, and wrote several Electromemoirs on Chances, and on the Integration of Par- dynamic tial Differential Equations. But with this he com- Ampere. bined a taste for, and a practical acquaintance with, the experimental sciences. He was a very good chemist, and showed himself particularly attentive to Davy on his first visit to Paris. He was also much attached to metaphysical speculation. His skill in devising apparatus and in performing experiments was eminently shown in his electro-magnetic researches ; whilst he judiciously rendered his mathematical knowledge subservient to them. In this respect he had greatly the advantage of Oersted, who appears to have been little acquainted with mathematics, and, perhaps, in common with his metaphysical friends of the German school, misapprehended their utility in physical discoveries. Three different hypotheses Various were speedily broached to represent mechanically opinions the singular kind of force mutually exerted between on the a conductor and a magnet. The first and most ob- of the ele vious was, that this action was not a push-and-pull tro-magforce, but a force producing rotation without direct ${ }^{\text {netic force }}$ attraction and repulsion, or of the nature of a couple exerted between any part of an electric current, and a small magnet or magnetic element. The second opinion was, that an electric current may be esteemed equivalent to a magnetizing force at right angles to it. The third, that a magnet is composed of elements which act as if a closed electric circuit existed independently within each of them; that is, each magnetic molecule may be replaced by a small conducting wire bent upon itself, in which some unfailing source of electricity, like a galvanic pair, keeps up, in the same direction, a constant current.

This last hypothesis, arbitrary and improbable as it may sound, was that defended by Ampère. Whilst few will be disposed to regard it as a true and complete physical picture of the condition of magnetized adopted by bodies, it seems impossible not to award to it the same sort of credit which we do to Newton's "fits of easy reflection and transmission" of light, when we find that it not only serves to represent the more obvious phenomena, but has suggested experiments absolutely new, and which turned out in accordance with the anticipation; and that, finally, by the sagacity and industry of its author it was made to include, by merely mathematical deductions, and without any complication of the hypothesis, certain experiments of a very singular kind, which at first seemed inexplicable by it. I proceed to develope a little farther this consideration. 
(796.) The theory of Ampère rejects all but push-andutual pull forces, such as are commonly recognized in meectric chanical physics. These forces are mutual, and beinductors. long to electric currents. A permanent magnet is a congeries of minute parallel and circular currents, all acting in the same direction, which is at right angles to the magnetic axis or line of force. Granting this for a moment, Oersted's experiment shows that the current in the conductor acts on the currents in the magnet; and as a magnet places itself transversely to a conductor, the currents in the magnet tend to place themselves parallel to that in the conductor. Do we then find such properties in moveable electric conductors alone? Have they any mutual action? Does that mutual action tend to produce parallelism? And if so, may it be farther analysed into direct attractions or repulsions of the several parts of the electric currents upon one another? All these questions were answered by Ampère affirmatively after due appeal to experiment. Two copper wires connected with voltaic circuits, and suspended with the requisite degree of freedom, approach when the currents have a similar direction, but are repelled when the direction is opposite in the two. When two moveable conductors are placed at right angles, or indeed at any angle, they tend to parallelism. All the usual phenomena of a magnet may be imitated by a long helix of copper wire through which electricity is made by some artifice continually to circulate. The position of the poles is the same as in a real magnet, and the name of pole is determined by the direction (right or left handed) in which the helix is wound. Such an instrument, not containing one particle of iron, is attracted and repelled by a steel magnet,-obeys the directive influence of the earth,-gives transverse motion to an electric conductor near it,-in short, does whatever magnetized iron does.

Thus, in the mutual action of electric currents (for the phenomena of static electricity are wholly of unlike) we recognize the great discovery of Ampère. A new science was formed, which he called electrodynamics, which he proceeded to develope with great skill and success. MM. Biot and Savary found that the electro-magnetic force exerted by an indefinite straight conductor and needle, varies inversely as the simple distance from the conductor; but looking to the elementary actions of each portion of the current, it will be found that this corresponds to the usual physical law of the inverse square of the distance between the magnetic and the electric element.

(798.) Whilst Ampère was pursuing his inquiries into
Ir Fara- the properties of electric currents, others were varyay and De the properties in a great variety of ways, Oersted's fundamental lectro- experiment. A great number of beautiful mechanilagnetic cal arrangements were invented, particularly by the elder De la Rive and by Mr Faraday. The latter, however, had the sole merit of effecting a most singu- lar kind of motion, that in which a magnet floating in mercury is made to revolve continuously around a central conducting wire, and in like manner a conductor may be made to revolve round a fixed magnet; nay, stranger still, a magnet acting at once as conductor and magnet, revolves with great velocity on its own axis when an electric stream is made to traverse one half of its length. These astonishing experiments, which, in an earlier age, might have founded a new sect of astronomers and replaced the theory of Vortices, offered also considerable difficulties in the application of Ampère's theory. They were, however, ulti-Accounted mately removed by Ampère himself, who analysed for by with great skill the mechanical conditions of each case, and interpreting them into the language of his theory, showed how continuous rotations might be produced, according to the laws which he had established, by electric currents alone suitably arranged; and he effected by most ingenious experimental combinations purely electro-dynamic rotations. Some other experiments, in which magnets seemed to produce a different effect from electro-dynamic cylinders, presented a more serious obstacle, which, however, was removed by a rigorous demonstration of the effects which must ensue, if we regard the elementary molecules of a magnet as very small, and consequently the entire magnet as a collection of indefinitely small and correspondingly numerous electro-dynamic cylinders. By means of four critical experiments, Ampère determined completely the elementary laws of the mutual action of currents, including that previously established by Biot and Savary in the case of a magnet and a conductor. This investigation was one of great intricacy, and was carried out with remarkable skill. Ampère had the field almost to himself, Savary making some contributions; and, what is remarkable, little or nothing has been added either to the theory, or to the deductions from it, since his death. The progress of the science of electro-magnetism has been so astonishingly rapid since the year 1820, that one set of phenomena after another has for the time attracted almost exclusive notice. The discovery of diamagnetism will probably lead to a reconsideration of Ampère's theory as applicable to all matter in a more general form.

This rapid succession of interesting topics has prevented attention from being perhaps sufficiently di-Great rected to the importance of Ampère's labours. He $\mathrm{Herit}^{\text {mef }}$ is at least as well entitled as any other philosopher Ampère. who has yet appeared, to be called "the Newton of Electricity."

Ampere was of an amiable, though rather eccentric (800.) character. His absence of mind was proverbial, and ${ }^{\text {His death. }}$ his style is somewhat cumbrous and obscure. But he was devoted to science, the promotion of which was ever his first consideration, and he evidently himself possessed great clearness in his conceptions. He died on the 17th May 1836. 
(801.) Seebeck. Rives, and $\mathrm{Mr}$ Faraday, were throwing light on of thermo- the causes, and developing the consequences of electricity. Oersted's experiment, SEEBECK of Berlin discovered in 1822 a new source of electric excitement, which has since become indirectly of very great importance. This was Thermo-Electricity. He found that when heterogeneous metals are united, either by soldering or pressure, and the junction heated, a current of electricity is established. The order of metals which produces the most energetic combinations, is wholly unlike the arrangement of the voltaic series, and has no apparent reference to any other known property of those substances. Bismuth and antimony stand at the opposite extremities of the scale, and a pair formed of them is consequently the most powerful which can be made. When heated at the junction, positive electricity passes from bismuth to antimony. In 1823, Oersted, then on a visit to Paris, united with Fourier in making experiments on this subject, and was probably the first who constructed thermo-electrical piles. Unquestionably, the most important application of these was to the construction of an instrument for measuring the effects of radiant heat, by Nobili and Melloni, of which an account has already, been given, Art. (709).

(802.) of the galvanometerSchweigger and

Nobili.

(803.) Oersted's history continued.
An application of electro-magnetism of extreme importance, was the Multiplier or Galvanometer, contrived by Schweigger, of Halle. In it the idea was first realized of measuring the power of an electric current by its effect in deviating a magnetic needle. Schweigger perceived that he could multiply the action of one and the same current, by causing it to traverse successive parallel coils of the conducting wire carried round the needle. Its sensibility was still farther, and almost indefinitely increased by Nobili's invention of rendering the needle astatic, or diminishing its natural directive power in any required degree. This he did by connecting it firmly with a second needle parallel to the first, of nearly equal strength, with its poles placed in an inverted position relatively to the other, and moving freely in a plane altogether exterior to the coil, so that whilst the directive effect of the earth's polarity is almost neutralized, the electro-magnetic effect of the coil tends to produce a similar deviation in both needles. This is one of the most precious philosophical instruthirty years in almost every electrical research or application. One of its best forms for many purposes (though hitherto little used) is the Torsion Galvanometer of Ritchie.

Oersted, of course, interested himself in this new application of his own great discovery. Indeed, having the good fortune to survive that discovery for more than thirty years, with a full enjoyment of his intellectual vigour, he had the gratification of contemplating a body of science entirely ments ever invented. It has been employed for new as its results, and a variety of useful applications scarcely less astonishing, which might, in one sense, be called his own creation. The discoveries of Ampère, Seebeck, and Mr Faraday, were all based upon his; and during those thirty years, this elegant and interesting branch of experimental physics underwent an almost uninterrupted extension, such as hardly any other affords an example of. The Electric Telegraph is one of its most direct and practical results; nor should we omit that Oersted himself proposed, as far back as 1818 , the application of electricity to blasting rocks by the very same process in which it has of late years been so usefully applied, namely, that of heating a fine wire to incandescence.

Though Oersted was the author of numerous papers connected with science down nearly to His exper the close of his life, they do not contain any impor-ments on tant discovery, and with reference to electro-mag- the comnetism, he appears to have contented himself princi- water. pally with repeating and expounding the observations of his contemporaries. But some of his experiments on other subjects deserve mention, especially those on the compressibility of water. This fact, which the Florentine Academicians had vainly sought to establish in the 17th century, had been clearly demonstrated by Canton in the middle of the $18 \mathrm{th}$, but Oersted first devised a compendious and effective apparatus for producing and measuring it more effectually. His result, that the compression amounts to 46 -millionths of the bulk, for a pressure equal to one atmosphere, agrees almost precisely with Canton's. In 1845, he considered that he had established that the heat developed by the same amount of compression is 0203 of a centigrade degree. He also made some experiments on the Law of the Compressibility of Air and upon other subjects.

The desideratum of a clear expression of the manifest alliance between Electricity and Magnetism had been so long and so universally felt, that the discover placed its author in the first rank of scientific men. the InstiThere was not even, so far as I am aware, a sus- tute of picion that he had been, however remotely or dimly, anticipated. The prize of the French Institute which had been awarded to Davy for his galvanic discoveries, was bestowed upon Oersted, and so far as I am informed, has not been since adjudicated. Hewas elected first Correspondent, and finally Associate of the Academy of Sciences. He was personally known to many of the philosophers of Europe, having made repeated. journeys in France, Germany, and England. His His scienagreeable manners and general information rendered tificcharac him popular. Sir H. Davy, who visited him at Co- ter. penhagen, describes him as " a man of simple manners, of no pretensions, and not of extensive resources." Niebuhr, however, who viewed his character in a different light, says, "I scarcely know another natural philosopher with so much intellect, and freedom from prejudice and esprit de corps." His writings were indeed too discursive. Professor Forch- 
hammer has enumerated above 200 of his publications or articles, on a vast variety of subjects ; but of all these, only a single tract of a few pages will perhaps be ultimately remembered. As I before remarked, his mind, though capable of continued application, appears to have wanted the sort of concentration which prolonged physical researches require, and the school of philosophy in which he was considered by his own countrymen as a proficient, has never been fruitful in researches based on Induction.

In November 1850, the fiftieth anniversary of his connection with the University of Copenhagen was celebrated by a jubilee. Though in his 74 th year, his activity was unimpaired, and he continued his lectures and other employments until within a few days of his death, which occurred on the 9 th of March 1851 , closing a life full of years and honour.

\section{§5. Dr Faraday.-Progress of the Theory of Electro-Chemical Decomposition-Volta-Electric Induction-Magneto-Electricity-Diamagnetism-Optical Changes induced by Magnetism.- Professor Plücker-Magneoptic Action.}

(807.) Iminent liscoveries f Dr Fara. lay.

Immeasurably the larger part of what we know with regard to the nature and laws of electricity and of its connection with Magnetism, so far as it has been developed since the discovery of Oersted, is due to the genius and perseverance of one man-MichaEL FARADAY.

This eminent philosopher was born, I believe, in 1791. He was originally " a bookseller's apprentice, -very fond of experiment and very averse to trade." In 1812 he sent to Sir H. Davy, then at the height of his reputation, a copy of a set of notes taken at his lectures, desiring his assistance " to escape from trade, and enter into the service of science." To the credit of the popular and distinguished chemist, he gave $\mathrm{Mr}$ Faraday a courteous answer, and appointed him as chemical assistant in the Laboratory of the Royal Institution in March 1813. Leaving England to travel in the autumn of the same year, Davy engaged $\mathrm{Mr}$ Faraday to accompany him as secretary and scientific assistant; they returned in April 1815, and from that time to the present $\mathrm{Mr}$ Faraday has been constantly engaged in the scientific business of the Royal Institution, which is as completely associated with his numcrous and splendid discoveries as Cambridge is with those of Newton, and Slough with those of the elder Herschel. By a rare, perhaps unexampled good fortune, that establishment, founded principally for the promotion of original research and the promulgation of discoveries, has been indebted during the first fifty years of its existence to the talents of two men only, for a succession of new scientific truths which might have done credit to a whole academy; indeed, if to the names of Davy and $\mathrm{Mr}$ Faraday we add that of Young, who here first promulgated the doctrines of the Interference of Light, there is scarcely an academy in Europe which las within the same period added so extensively to our choicest stock of original science.

(809.) Partly in consequence of his official duty of bringVariety of ing forward and explaining the most important cotemis publi- porary discoveries, partly also in consequence of his is $R e$. earches o Electricity. to expound, the variety of subjects on which Dr Faraday has made essential additions to our knowledge is so great that it is difficult to comprehend them under one section. In conformity, however, with our plan of suppressing minor facts, and insisting on the most important, I shall confine myself to a summary statement of his main discoveries connected with Electricity and Electro-Magnetism as contained in a continuous series of "Researches," published in the Philosophical Transactions between 1831 and the present time; which, when collected (as they have been in a distinct form), now fill three closely printed octavo volumes. It would be difficult to name in the history of any progressive experimental subject so large an amount of research prosecuted for so long a time in so methodical a manner and with such remarkable uniformity in plan, and with such unvarying success.

I shall only farther premise that Dr Faraday's earliest essays were naturally of a chemical charac- Electroter. In 1820 he assisted Dary, in prosecuting Oer-magnetic sted's researches on the relations of Electricity and Magnetism, and the following year he himself succeeded in producing, for the first time, the continuous rotation of a magnet round an electric conductor, and the converse rotation of the conductor round the magnet (798). These experiments were the germ of others which continued to interest philosophers as well as the curious public for a long time after. But it was in 1831 (when the author had attained his 40th rear) that the genius of Dr Faraday was displayed in a commanding manner by the appearance of his First and Second series of the Researches on Electricity, which have not perhaps been surpassed by even the most brilliant of their successors. The subject was the Induction of Electric Currents from other Currents and from Magnets. But we shall find it most convenient to take an order different from that of the discovery, and to present the main results of $\mathrm{Dr}$ Faraday's electrical labours under the following Heads of heads:- $\quad$ his chief

I. The law of definite Electro-chemical Decompo- electrical sition, and the theory of the pile connected therewith. and magII. The Induction of Electric Currents from other coveries. 
Currents and from Magnets, or the discovery of Magneto-Electricity.

III. The influence of the Magnet on all bodies, and the consequent division of substances into two classes, Magnetics and Diamagnetics.

IV. Optical changes induced by Magnetism.

I. With regard to electro-chemical decomposition and the theory of the pile, the great extent and intricacy of the subject require us to restrict our analysis to a few of the leading conclusions. The most important of these may be summed up in the following propositions :- 1 st, The amount of a decomposable substance (or electrolyte) analysed into its elements by a current of electricity depends solely on the amount of electricity passing through it, and is independent of the form of apparatus employed, the dimensions of the poles (or electrodes), the strength of the solution, or any other circumstance. It is thence inferred, with respect, for instance, to water, that the amount of it decomposed in a given time is an exact measure of the quantity of electricity set in motion in that time. $2 d$, When a substance is thus decomposed, it is a necessary, or at least a highly probable, consequence of Dalton's laws, that the elements separated are in atomic proportions to one another. But Mr Faraday also found that when several decompositions are effected at the same time by interposing different electrolytes in intervals of the same circuit, the whole of the series of elements separated bear the atomic relations to one another. Thus, to take a single case; an electric current decomposes in the same time 0.497 grain of water and 3.2 grains of protochloride of tin. Now, these are exactly the proportions of the atomic weights of those bodies. From this and numerous other cases $\mathrm{Mr}$ Faraday infers, that universally the amount of electrical action required to dissolve a combination is in a constant proportion to the force of chemical affinity by which its elements are united. The corollary seems therefore highly probable that it is one and the same force which is exerted in either case. But the conclusion as to their identity becomes almost irresistible when we add to these propositions the following: $3 d l y$, That the oxidation of one atom of zine by the acid of the battery generates precisely so much electricity as would resolve one atom of water into its elements. Thus, 8.45 grains of zine dissolved occasioned the analysis of 2.35 grains of water; but these numbers are in the ratio of 32.5 to 9 ; the equivalents or atomic weights of zinc and water.

From these strictly experimental laws, Dr Fara- day considers that he is entitled to draw these important inferences: First, that the source of voltaic electricity in the pile is chemical action solely; Secondly, that "the forces termed chemical affinity and electricity are one and the same." Itis needless to add that these conclusions, involving the very essence of the science of voltaic electricity, are supported by Mr Faraday by a great variety of collateral proofs ; and, on the whole, I cannot see that they admit of any reasonable doubt. The contact theory of Volta still, however, holds its ground in Germany, where the number of influential writers on electricity is considerable; and so perseveringly is it maintained, that it is difficult to perceive how it is ever to be dislodged. But on this wide and not very profitable controversy we cannot here enter.

There are a great many other considerations (813.) connected with the action of the voltaic battery Dr Fara. which are independent of these primary ones, and day on Inwhich are scarcely less important. Dr Faraday and Con has entered into a most elaborate experimental duction. argument to show that induction always precedes both conduction and decomposition, and that decomposable bodies or electrolytes must be all more or less perfect conductors. His views may be thus concisely summed up in his own words :- "The first effect" of the electrifying influence, whether of frictional electricity or of voltaic electricity, upon bodies, is " the production of a polarized state of their particles which constitutes induction; and this arises from its action upon the particles in immediate contact with" the excited body, "which again act upon those contiguous to them, and thus forces are transferred to a distance. If the induction remain undiminished, perfect insulation is the consequence; if the contiguous particles" thus polarized " have the power to communicate their forces, then conduction occurs, conduction being a distinct act of discharge between neighbouring particles." "In the inductive condition assumed by water" when about to be decomposed, "the discharge between particle and particle is not, as before, a mere interchange of their powers and forces, but an actual separation of them, the oxygen travelling in one direction and carrying with it its amount of force acquired during polarization, and the hydrogen doing the same thing in the other direction, until they each meet the next approaching particle, which is in the same electrical state with that they have left, and by association of their forces with it produce discharge. This action may be regarded as a carrying one performed by the constituent particles of the dielectric." ${ }^{2}$ Again, "the current is an indivisible thing; an axis of power, in every part of which both electric forces are present in equal amount."'

These views respecting the molecular progress of conduction and decomposition, though perhaps never so categorically stated as by Dr Faraday, have been, I imagine, substantially held by a majority of those who have considered the subject since the time of Davy, who first gave them a partial expression. And when Davy and others speak of the electric forces in decomposition as if they emanated from the 
poles of the battery and became enfeebled with distance from them, they used a language not quite rigorous indeed, yet expressing the actual phenomena with that general accuracy which we can alone expect in the first stages of so new and difficult an inquiry. "The sum of chemical decomposition is constant for any section of a decomposing conductor" is Dr Faraday's expression. ${ }^{1}$ So is the sum of illuminations arising from light radiating from a point, when taken across any section of its path, yet the influence is said to vary inversely as the square of its distance from the origin. The part of Dr Faraday's conclusions, however, most open to exception, is what refers to electric action at a distance, which he conceives to depend solely upon induction acting on intervening particles, which induction may take place along curved lines. It is indeed true that he has shown, by a beautiful experiment, that the interposition of different substances between an excited electric and a body capable of being electrified by induction, occasions different degrees of excitement in the latter, even when the interposed bodies are glass, sulphur, and other "non-conductors;" and this he justly refers to a peculiar power or property of bodies called "specific inductive capacity." But this is rather different from the general proposition above referred to.

(815.) Uniform nature of electricity from whataversource.

(816.)
In conclusion of this part of the subject, I must add that Mr Faraday has, with great pains and success, demonstrated the fundamentally identical nature of electricity from whatever source derived, and however differing in its usual manifestations;-such as electricity of the pile, of the common machine, or that induced by magnetic, thermal, and animal electricity. These have all common properties, producing the shock, the spark, and magnetic, chemical, and heating effects; and, except two, also producing sensible attraction and repulsion. But the disproportion of the effects of electricity, varying so much in intensity when referred to unit of quantity, is astonishing and paradoxical. The electricity which so silently and speedily decomposes a single grain of water would, when its intensity is sufficiently exalted, produce, according to Mr Faraday, "a very powerful flash of lightning," or 800,000 times the contents of a well charged Leyden battery. Again, zinc and platinum wires half an inch long and one-eighteenth inch diameter, dipped into slightly acidulated water, produce in three seconds as much electricity as a man can easily bear in the form of a shock.

II. Induction of electric currents from other currents and from magnets. - This splendid research, which dates from 1831, constitutes the discovery of magneto-electricity.

The discovery by Ampère of the attraction and repulsion of conductors conveying electric currents rapidly followed (as we have seen in Art. 796) Oer- sted's discovery of the power of electricity to affect the magnet, and the corollary from it of the magnetizing agency of electricity. This being achieved, very striking analogies led to the expectations-1. That a wire conveying a current ought to excite by induction a current in another wire near it; and, 2. That a magnet ought, under some circumstances at least, to be capable of exciting electric action. But attempts in these directions had repeatedly and signally failed, and for a reason which Mr Faraday first rendered apparent.

Having made a compound helix of two copper (817.) wires wound parallel to one another, but not touch- Volta-elecing, and rolled one within the other upon a cylin-tion. der, he found that when he transmitted a continuous voltaic current through one wire, a momentary current (tested by a galvanometer) took place in the independent helix opposed in direction to that of the primary current; but it ceased to exist instantaneously, although the primary current continued to act; and it was only on the cessation of that current that a new momentary induced current appeared, but in the contrary direction to the previous one. The same effect occurred when a wire conducting a current was mechanically brought into the presence of another wire; the approximation of the two induced an oppositely directed current, their separation a similar one. Whilst the wires were immovable no induced current took place. This he termed Volta-Electric Induction.

Mr Faraday next took a ring of soft iron, disposing two copper-wire coils round opposite portions of the ring. In passing a current through one coil, and thus magnetizing the ring, a current was induced in the other copper coil, but, as in the former case, only for an instant. When the primary current stopped, and the magnet was unmade, an opposite current shot through the secondary coil.

The transition to the next experiment was natural, but highly important. The primary coil was Magnetosuppressed; and the piece of soft iron embraced by electricity. the secondary coil was now magnetized by the inductive action of a powerful bar magnet, with which contact was alternately made and broken. At the instant of making contact a momentary current of electricity was produced in the remaining coil, and on breaking it a reversed current, also of instantaneous duration. No current existed whilst the magnet continued to be applied. The direction of the current at making contact was opposite to that which would have produced the magnetism present in the iron core; on breaking contact the current was similar to that which would have magnetized the iron. The electricity momentarily induced in the coil was tested by its action on the galvanometer, by its power to magnetize steel, to convulse a frog, and finally by the 
production of a spark. This, then, was the discovery of magneto-electricity.

The mere motion of a permanent magnet was now substituted for the induction of magnetism in soft iron. By pushing one end of a bar-magnet into the coil, electricity was developed so long as the motion continued; on withdrawing it an opposite current took place. Even the feeble magnetism of the earth induced a sensible electric current in a wire moved transversely to the direction of the dippingneedle.

By making a copper plate revolve in the neighbourhood of a powerful magnet, a continuous current of electricity may be detected passing from the centre to the circumference of the plate, and may be collected by proper means. Here, then, is a magneto-electric machine. This current perpetually present in a conducting plate revolving beneath a magnet, cannot fail (by the common laws of electromagnetism) to react on that magnet. Dr Faraday showed in the most satisfactory manner that its action is exactly what is required to explain M. Arago's experiment of " transient magnetism by rotation" - namely, to cause the magnet (if free) to follow the direction of motion of the plate (515).

On the whole, this research of Dr Faraday may be cited as one of the most original and admirably conducted which the annals of science present, and as such may be usefully recommended to the student.

III. The influence of the magnet on all bodies, and their consequent division into two classes; Magnetics and Diamagnetics.-By many, perhaps most persons, this will be regarded as the greatest of Dr Faraday's discoveries. It dates from 1846. By using electromagnets of very great power, and suspending bodies of a somewhat elongated form between the poles, he has proved that every substance, solid, liquid, or gaseous which he has put to the test, is either drawn into a line joining the poles of the magnet, as soft iron would be, returning to that line if displaced, or else it settles in a position at right angles to this, or across the line of poles. The former he calls paramagnetic or simply magnetic bodies, and their position axial; the latter diamagnetic bodies, and their position equatoreal. Bodies may be arranged in a list commencing with those most paramagnetic, diminishing to neutrality, then feebly diamagnetic, and finally the strongest diamagnetics. The following is such a list of a few solid and liquid bodies thus classified :-

Magnetics or Paramagnetics....... $\begin{aligned} & \text { Iron. } \\ & \text { Nickel. } \\ & \text { Cobalt. } \\ & \text { Manganese. } \\ & \text { Palladium. } \\ & \text { Crown glass. } \\ & \text { Platinum. } \\ & \text { Osmium. }\end{aligned}$

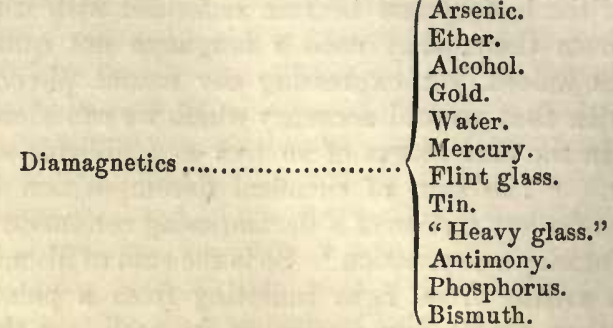

The equatoreal pointing of diamagnetic bodies evi- (824.) dently presupposes that they are longer in one di- Fundamenmension than in the others. A small bar of sili- ment and cated borate of lead, or "heavy glass," about two definition inches long, and from a quarter to half an inch of diamagbroad and deep, suspended in a stirrup of paper by six or eight lengths of cocoon silk, was the apparatus first employed by Dr Faraday. When a sphere or a cube is used, of course it cannot point. The diamagnetic action is shown in that case by the little body being repelled indifferently from either pole of the magnet, in the same manner as soft iron is indifferently attracted by either. This repulsive tendency includes the phenomenor of equatoreal pointing, and its law is thus comprehensively expressed: "The diamagnetic tendency is to move the body from stronger to weaker places of magnetic force."

The behaviour of diamagnetics in the presence of (825.) a magnet may be thus further illustrated. It is Farther what would occur if a body absolutely inert were tions. suspended in a fluid pressing upon it, that fluid being at the same time more or less magnetic, that is, more or less attracted by either pole of the magnet. The result would evidently be, that the body would seem to be repelled, and would set equatoreally for the same reason that a piece of wood plunged in water rises to the surface as if repelled by gravity. Thus Dr Fararday suspended feebly paramagnetic bodies in ferruginous solutions more magnetic than themselves, when they acted as diamagnetic bodies would do.

It is impossible for the most part to guess beforehand to which class a substance will belong. Chinaink, porcelain, silkworm gut, shell-lac, and charcoal, rank amongst paramagnetic substances; whilst sulphur, resin, wood, leather, and most animal substances, are diamagnetic. Thus, if a living man could be delicately enough suspended between the poles of a huge magnet, he would settle equatoreally.

Philosophers are not yet entirely agreed as to the (827.) precise nature of the Diamagnetic relatively to the Discussio Magnetic actions of bodies. Besides Dr Faraday, nature of MM. Weber and Edmond Becquerel abroad, and Pro- diamagnetfessors Tyndall and William Thomson in this coun- ism. try, have examined the subject both practically and theoretically in great detail. The more probable opinion seems to be, that bismuth and its analogues 

Iceland spar is repelled by the magnet. A sphere of that substance suspended by a thread, and having its axis horizontal, being placed between the poles, notwithstanding the perfect symmetry of external figure, the axis ranges itself in the equatoreal position. In some other crystals the axis is directed in the line of poles. The law of the phenomena is not yet completely made out; but so strong is the latter quality in crystals of kyanite, that a piece of that substance properly suspended will actually show a directive power under the influence of the earth's magnetism.

Probably closely allied to this fact is a similar directive tendency observed in well crystallized spe- Magnecimens of bismuth, antimony, and arsenic. This crystallic M. Plücker calls the magne-crystallic, as the former may be termed the magne-optic force; and it is often so intense as to oppose and even reverse the directive tendency which the body would have had between the poles in its massive or uncrystallized state.

It will be easily conceived that the interest created by these admirable discoveries, revealing not only new and general properties of matter, but also relations between very different branches of science, soon became general, and raised the reputation of $\mathrm{Dr}$ Faraday to the very highest rank as an experimental philosopher. If we compare his two greatest works, that on magneto-electricity, and this on diamagnetism, we find in the former perhaps a more perfect specimen of inductive sequences; in the latter, facts more independently novel and unlooked-for, and an unrivalled skill in the application and invention of experimental methods.

Passing over many less important matters, there (834.) yet remains one interesting discovery to be men-Dr Farationed, which in point of time preceded the last, day on namelychanges

IV. Optical Changes induced by Magnetism. - induced by In his 19th Series of Researches, published in 1845, magnetism. Dr Faraday announced "The Magnetization of Light and the Illumination of the Magnetic Lines of Force,"- - title which, though intended to express exactly the author's idea of his discovery, perhaps excited undue anticipations in the public mind. For here we have no direct, or even apparently direct, action of the magnetic force on a luminous ray, but only that a peculiar state is induced by magnetism in some transparent bodies which produces an action on light which they did not possess before, and which, indeed, differed in some respects from any similar action previously recognised.

Dr Faraday's leading experiment is the following : -A piece of "heavy glass," or siliceous borate of Rotation of lead, was placed lengthwise between the poles of a polarizapowerful electro-magnet. A ray of plane-polarized of a ray of light was transmitted through the glass parallel to light. the line of the magnetic poles; when the magnetic energy was fully applied, the plane of polarization of the light was found to have twisted round, similarly 
(apparently) to what occurs when polarized light passes through quartz or oil of turpentine. It is found that a great number of solids and liquids are subject to the magnetic influence in the same manner as in the case of "heavy glass," though to a smaller amount.

Now, in reasoning on this experiment, it is to be

(836.)

Resembles the action of quartz but with a difference observed that no rotation of the ray takes place unless there be a medium on which the magnetism impresses its energy. ${ }^{1}$ Some molecular change, no doubt, results, such as that which pressure gives to glass, rendering it doubly refracting and depolarizing, or, to take a still closer analogy, when heat applied to it conveys similar properties. Yet no one imagines that these experiments show a direct reaction of heat, still less of mechanical compression, upon light. Yet, with all abatements, Dr Faraday's discovery is novel and singular; the more so, that this constrained state differs from that naturally possessed by quartz and certain liquids. The state magnetically induced in a body causes the rotation of the ray to be reversed when it moves in the contrary direction; that is, its rotation is right-handed when the ray moves from the north to the south pole, but left-handed when it moves from S. to N. But in bodies in the natural state the rotation takes place towards the same hand whatever be the path of the body. Mr Airy has shown that this peculiarity admits of being mathematically expressed in a manner somewhat analogous to that imagined by Professor MacCullagh in the case of quartz (512); but it is not pretended that these formulæ convey informa- tion as to the physical conditions on which these singular phenomena depend.

Amongst Dr Faraday's contributions to science (837.) not connected with electricity, the most remarkable Dr Faraperhaps is the condensation of many gases into the day liqueliquid form by cold and pressure, of which he is the tain gases. undoubted discoverer. This fact is highly interesting both in a scientific and practical point of view. In the latter it was early applied by the late ingenious Sir M. I. Brunel as a new moving power (375), and it may not improbably yet be resorted to for that purpose. The subsequent discovery of a mode of solidifying carbonic acid by M. Thilorier is not only interesting in itself, but affords a method of producing more intense cold for experimental purposes than any other previously known.

Dr Faraday still continues his laborious and fruitful inquiries. Whilst he has attained almost every titular honour which the world of science has to bestow (including that of associate of the French Academy of Sciences), he has preserved a modesty of character and a simplicity of life which enhance the respect in which he is held by all who are admitted to his nearer acquaintance. No one has more successfully escaped the contentions which literary rivalry so often produces; and by his extraordinary skill in expounding the most difficult researches, whether made by himself or by others, he has maintained (as I have already said) the early reputation of the Royal Institution, and has immensely enlarged the circle of those who are able to admire and appreciate his successes.

\section{§6. OHM-DANIELI-Mr WheATSTONE-M. JACOBI.-Laws of Electrical Conduction;-Constant Battery;-Applications of Electricity to Telegraphs-Clocks-Motive Engines-the Electrotype.}

(839.) Vast detai of the science of electricity. Its numerous culti. vators.
We have traced in the last section the progress of electrical and of electro-magnetic discovery since the days of Davy and Oersted, as well exemplified in the pre-eminent researches of Dr Faraday. In conformity with the plan of this Dissertation (13), (14), I have, on account of their immense interest and importance, analyzed them more fully than could possibly have been done were $I$ to render similar justice to all who have distinguished themselves in the same career. Thus, France has produced in $M$. Becquerel one of her most ingenious and indefatigable experimentalists, full of devotion to science, and giving up conscientiously the whole of a long life to the cultivation of this particular department. His discovery of the efficacy of long-continued and very feeble voltaic actions to produce crystallized earthy and metallic compounds not obtainable by chemical means is highly important. Switzerland is proud of her two De la Rives, ${ }^{2}$ and Italy of not a few disciples of Volta. In Germany the number of electricians is greater than in any other country; and as they have taken the lead in obtaining correct measures of the electric forces, and in determining (in many cases) the numerical laws which regulate the efficiency of batteries and conductors, and have applied these to many important practical purposes, I shall devote a section to some account of these, as well as to the beautiful experiments of our countryman Mr Wheatstone.

Онм's Law of Electrical Conduction. - Georg (840.) Simon OHм was born in Bavaria in 1787, and was Ohm's successively professor at Cologne, Nürnberg, and theory of

\footnotetext{
1 I may here record that before the year 1835 , I suspected that there might be some immediate action between circularly polarized light and a magnetized body, and made experiments in consequence in May 1836, which, however, led to no result. I rather think, however, that these experiments deserve careful repetition under more varied circumstances.

2 MM. Becquerel and A. De la Rive have both published elaborate works on Electricity, to which the reader is referred for details on this inexhaustible subject.
} 
(841.)

Munich. He died on the 7 th July 1854. His theory of electrical conduction was not highly appreciated in Germany until it had received, in 1841, an eminent mark of approval from the Royal Society of London, by the award of their Copley medal. His principal work on the Galvanic Circuit (Die galvanische Kette mathematisch bearbeitet; Berlin, 1827) has had a somewhat peculiar fate. Accepted by only a few persons as a great discovery, it met with comparatively little attention, at least until recently; yet notwithstanding the long anticipation by $\mathrm{Ohm}$ of his results, it has been his misfortune to have their originality contested.

It scems not difficult to account for the diversity of estimation in which this work has been held. The primary fault is the author's own. He deduces the strength of a voltaic current in any given circuit, and the electroscopic excitement of each part of the circuit, by means of reasoning seemingly a priori, from certain assumed axioms submitted to mathematical reasoning. The axioms are very simple; the theory founded on them is intended to correspond to Fourier's theory of heat, of which, indeed, in point of form, it is a mere and literal copy; but as every circumstance which introduces real complication is soon left on one side, the leading propositions are almost selfevident results of the axioms. In short, the parade of mathematics is uncalled for, and the whole structure of the theory seems so slight and questionable that one is surprised that it should ever have bcen regarded as more than a clever expression of some approximately true experimental laws. It appears, indeed, that this is the simple fact; that the axioms were obtained from the results which they seem to predict, and that $\mathrm{Ohm}$ was an experimentalist before he became an author. In this guise we understand how to treat the so-called "Laws of Ohm." They are truly important empirical laws, calculated to guide the practical man in applying and measuring galvanic forces, to enable the theorist to form clearer notions of the different (often confusing) effects of these forces, and to reduce their varying energy to calculation; but we must be allowed to doubt whether $\mathrm{Ohm}$ has thrown any new light on the real first axioms of electrical excitement or transmission.

The most important of these laws refer to the numerical measure of the voltaic stream circulating in the conductor of a closed circuit. Such a closed circuit may be imagined to consist of - (1.) an excitor or battery; (2.) a conductor homogeneous or otherwise, but necessarily continuous, uniting the ends of the battery. The exciting force is derived (we will assume) from the chemical or thermo-electric action present in the battery. The electric equilibrium being disturbed, is restored more or less speedily through the medium of the conductor which connects the poles. If the conductor be good, the electricity passes rapidly through it, and does not accumulate in the battery ; if the reverse, it accumulates until it acquires power to overcome the resistance, and then it passes through in a stream less abundant, but of a higher intensity. If the construction of the battery does not permit that degree of intensity to be reached, the electricity stagnates in the battery, the conductor cannot perform its office, no effect results. The Illustrawhole maybe compared to a spout of water discharged tion. into a trough, from the bottom of which extends a long narrow horizontal pipe. The water is the electricity, the trough is the battery, the pipe is the conductor. If the pipe be very long and narrow, no water at all will pass through it until the water in the trough has attained a certain height, or has a head of pressure sufficient to overcome the resistance in the pipe. If the trough be filled to the brim without the resistance being overcome, the trough is as good as plugged, no motion takes place, the stream regurgitates. The longer the pipe the feebler the stream that passes; shorten the pipe indefinitely, and the efflux depends only on the construction of the trough. Indeed, the illustration might be pushed considerably farther. The depth of the cistern represents the electro-motive force of the voltaic combination; its area the size of the plates. By increasing the latter, we do not give the means of overcoming more resistance; but when the resistance is small, we afford a larger supply without lowering the level-i.e., the intensity.

$\mathrm{Ohm}$ regards the current as proportional to the electro-motive fore diretly, and to the resione tro-motive force directly, and to the resistances in- Resist versely ; and the latter are divided into $(\alpha)$ the re- ${ }_{\text {estimated. }}^{\text {ances }}$ sistance of the battery itself to the passage of the current; $(b)$ the resistance of the conductor. Now the latter varies as the length of the wire completing the circuit. We may therefore double its amount by doubling the length of wire joining the poles; and if we observe the strength of the current passing before and after this has been done, we have a measure of $a+b$ in the first experiment, and of $a+2 b$ in the second ; and $b$ being assumed to be known, $a$, or the comparative resistance within the battery, becomes known also.

The resistance of a standard copper wire a foot long may be taken as the unit of resistance. Mr Standard Wheatstone finds it convenient to assume a copper of resistwire a foot of which weighs 100 grains. M. Jacobi prefers a mètre of copper wire one millimètre in diameter. The resistance is as the length, and inversely as the sectional area.

To measure the current two methods have chiefly been used, and the results agree closely. One is Force of the tangent compass. A voltaic current is allowed the current to pass through a thick wire arranged in a vertical compass. circle. At the centre of the circle is placed a very short magnetic needle. When a current passes the needle is deflected; and it is easy to show that the deflecting forces are as the tangent of the angle of deflection. A double or treble force in the circuit 
produces a deflection of the needle whose tangent is double or treble the first.

(846.) The other method is by Dr Faraday's Voltameter. Voltame- The amount of water decomposed is directly as the ter. quantity of the current. The unit in this case is one cubic centimètre of gas produced from water in a minute.

These two measures agree. After being once compared, we may in all cases deduce the decomposing force of a current from its effect upon a tangent compass.

$\mathrm{Mr}$ Wheatstone has facilitated the measurement (848.) Rheostat of MM.

Wheatstone and Jacobi.

(849.) Farther deductions from $\mathrm{Ohm}$ theory.

(850.)

(851.) Fechner's experiments. ception in France and England is mainly due to the ingenious and (in many cases) independent experiments of M. Pouillet and Mr Wheatstone.

Danielis's Constant Battery. - This seems the proper place to mention an invention which has exercised a remarkable influence on the progress of prac- niell-the tical electricity-I mean the Constant Battery. I constant believe that the merit of this application is entirely battery. due to the late Professor Daniell, ${ }^{1}$ although the German writers (who manifest throughout a singular sensibility with regard to their national claims to electrical improvements) seem to claim it for their countrymen. Every battery previously constructed diminished rapidly in energy from the instant of being charged. This was chiefly due to two causes; first, to the acid becoming gradually charged with oxide of zinc ; and, secondly, to the appearance of "nascent" hydrogen arising from the decomposition of water at the copper surface where it prevented effectual conduction of the electricity. These sources of diminished effect were prevented in the following way :- Instead of a single cell containing one fluid moistening both the copper and the zinc, a double cell was formed by means of a partition of bladder or porous earthenware. The partition next the zinc was filled with dilute sulphuric acid; the partition next the copper with a solution of sulphate of copper, also acidulated. When galvanic action proceeds, both fluids are decomposed; but whilst that in the zinc cell becomes charged with oxide of zinc, it is at the same time continually acidulated by the electro-chemical transfer of acid from the decomposition of sulphate of copper in the copper cell; and the copper set free from the same combination in the form of oxide is metallically reduced by combining with the "nascent" hydrogen (the oxygen derived from the water decomposed having combined with the zinc), and the metallic copper is deposited in an ever fresh film on the surface of the copper plate. This beautiful invention was described in 1836 . Many other batteries on the same principle have been since contrived and described; sereral are more powerful, but none perhaps are so constant in their action.

Application of Electricity to the Arts-MM. Wheatstone and JACOBI.-I have selected Messrs ApplicaCharles Wheatstone and M. H. Jacobi as the repre- tion of Charles Wheatstone and M. H. Jacobi as the repre- ${ }_{\text {elion of }}$ sentatives of a numerous class of ingenious men who to the arts. have shown great felicity of invention in applying ingenious mechanism to render electric agency available in the arts. We here again find the reciprocal influence of art upon science, to which I have elsewhere

1 John Frederic Daniell, Professor of Chemistry in King's College, London, was born in 1790 . He was the author of a work on Chemical Philosophy, of Meteorological Essays, and of numerous papers in the Philosophical Transactions, many of which were connected with Voltaic Action. His work on Meteorology contributed materially to the progress of that science, as did the invention of his Hygrometer (notwithstanding certain defects in that instrument) to the theory and practice of Hygrometry. For his Constant Battery Mr Daniell recelved the Copley medal of the Royal Society. His death took place from apoplexy while attending a council meeting of the Royal Society on the 13th March 1845. 
adverted $(32$, \&c.) The requirements of practice are magnificent experiments, such as no individual and no scientific Society would think of executing for the illustration of theory. It is not in the least min purpose to transfer to these two gentlemen an exclusive merit which they need not be unwilling to share with other energetic and able competitors in the hard-run race of scientific applications. They occupy, however, perhaps the most marked and distinguished place, and the field is so wide and includes so many minute details, that it requires all our resolution to fix our eyes steadily on the most considerable acquisitions-the nobler sheaves of so prolific a harvest.

I shall connect, then, with the name of $\mathrm{Mr}$ Wheatstone, (1), the apparatus for determining the velocity of electrical conduction, (2), the electric telegraph and clock, - with that of M. Jacobi, (3), electrodynamic machines, and (4), the electrotype.

(855.)

I. The apparatus used by $\mathbf{M r}$ Wheatstone in 1834 Mr Wheat- for measuring the velocity of the passage of the elecstone on trical impulse through a good insulated conductor the velo- - such as a copper wire, deserves particular notice tric con- from its great ingenuity, and from its general appliduction. cation to the measurement of short intervals of time. Let a copper conducting wire of half a mile long be so convoluted that the middle and the two ends of the wire may be brought near together, the whole being perfectly insulated. Let the wire be slightly interrupted at these three places, and the whole put into connection at pleasure with an electric machine or battery. When contact is made, three sparks will take place. Let the two end sparks be called $\mathrm{A}$ and $\mathrm{C}$ and the middle one B. As the three sparks take place close to each other, they can easily be seen at once reflected in a small plane mirror. Let now this small mirror be put in very rapid rotation round a horizontal axis so placed that the sparks (if they occur in the suitable part of the revolution) may be reflected together to the eye. Imagine the rotation to become immensely rapid:-in Mr Wheatstone's apparatus the velocity reached 800 times in a second; consequently the mirror described $1^{\circ}$ in $\frac{1}{800 \times 360}$ th part of a second; i. e., in $28 \overline{8} \frac{1}{0 \sigma \bar{\sigma}}$ of a second. But for $1^{\circ}$ of rotation of a mirror the reflected image will describe an $\operatorname{arc}$ of $2^{\circ}$. Supposing then that all the sparks occur at the same absolute instant of time, they will be seen in one line (supposing the points of the interrupted circuit in a line), but if either spark occur later than the others by only $2 \bar{\varepsilon}_{\overline{8}, 0}^{1}, 0 \sigma$ of a second, the mirror will have revolved so much in the interval as to displace the image of that spark relatively to the others by the very palpable angular amount of $2^{\circ}$. In the copper wire half a mile long, the end sparks occurred simultaneously, whilst the middle spark occurred later by about one millionth of a second; giving a velocity of transmission (according to $\mathrm{Mr}$ W.) of 288,000 miles a second, or somewhat greater than that of light. ${ }^{1}$ The velocity in an iron telegraph-wire, ascertained lately in America with much greater accuracy, and by a different method, is only 16,000 English miles a second; but doubts have been thrown upon the correct interpretation of these experiments. Those of M. Fizeau on the telegraphic lines of France give results more conformable to Mr Wheatstone's, namely, about 70,000 English miles per second for iron, and 120,000 for copper wire. The duration of a spark drawn immediately from the battery is insensible, but in Mr Wheatstone's experiment it lasted $\frac{2 \pi, \frac{1}{\delta} \delta 0}{0}$ of a second when transmitted by a copper wire half a mile long.

II. Electric Telegraph and Clocks.-The idea of (856.) using the transmission of electricity to communicate Electric signals is so obvious as hardly to deserve the name of an invention, the prodigious velocity of common history. electricity in wires having been established by Watson before the middle of the last century. The earliest proposal of the kind appears in the Scots Magazine for February 1753, where a correspondent from Renfrew, who signs himself C. M., proposes several kinds of telegraphs acting by the attractive power of electricity, conveyed by a series of parallel wires corresponding in number to the letters of the alphabet, and insulated by supports of glass or jeweller's cement at every twenty yards. Words are to be spelt by the electricity attracting letters, or by striking bells corresponding to letters. One Lesage, in 1782, and even long before, proposed to convey twenty-four insulated wires in a subterranean tube, and to indicate the letters of the alphabet by means of the attraction of light bodies. In 1811 Sömmering suggested a similar application of voltaic electricity, chemical decomposition being the effect observed. Oersted first, and then Ampère (1820) suggested the use of magnetic deflections for the same purpose, which is nothing else than the needle telegraph in general use in England; but they contented themselves with the suggestion merely. MM. Gauss and Weber communicated signals at Göttingen in 1833 or 1834 to a considerable distance, and gave them the signification of letters. This was the first accomplishment of telegraphic communication by means of electricity, and it realized the fancy of Strada, quoted by Addison, of sympathetic magnets. It was, however, a mere appendage to a magnetic observatory, and its application and diffusion on a great scale seems to have required a distinct effort; for several years elapsed before we hear more of the telegraph.

The year 1837 is the date of the realized electric (857.) telegraph. We find three distinct claimants, of whose Telegraphs independent merits there is no reason whatever to of MM. doubt, though how much of the merit of all must be Steinheil, considered due to MM. Gauss and Weber, who first and Wheatmade the experiment, though they did not offer it ${ }^{\text {stone. }}$ 
for general adoption in a convenient form, is a matter which we need not here decide. The three independent inventors (I name them alphabetically) are $\mathbf{M r}$ Morse of the United States, M. Steinheil of Munich, and $\mathrm{Mr}$ Wheatstone of London. The telegraph of the two last resembles in principle Oersted's and Gauss's; that of the first is entirely original, and consists in making a ribbon of paper move by clockwork, whilst interrupted marks are impressed upon it by a pen or stamp of some kind brought in contact with the ribbon by the attraction of a temporary magnet, which is excited by the circulation of the telegraphic current of electricity. In the telegraphs of MM. Wheatstone and Steinheil the needle moves only to the right or left; and by the combination of a certain number of right and left motions, either with one or with two independent needles acted on at once by distinct currents, the alphabet is easily, though somewhat tediously constructed. Such, however, is the dexterity which practice gives, that forty or even more of such complex signals are transmitted and registered per minute. ${ }^{1}$

It has already been said that we claim the exclusive invention of the electric telegraph for no one individual. But of the several inventors none probably has shown such perseverance and skill in overcoming difficulties as Mr Wheatstone. ${ }^{2}$ His telegraph accordingly was in general use in England before $M$. Steinheil was able to obtain a similar success in Germany. The telegraphs of $\mathrm{Mr}$ Morse are naturally preferred in America, and they have this inestimable advantage, that they preserve a permanent record of the despatches which they convey.

There is one circumstance connected with the electric telegraph deserving of particular notice-I mean the apparently infinite conducting power of the earth when made to act as the vehicle of the return current. Setting all theory aside, it is an unquestionable fact, that if a telegraphic communication be made, suppose from London to Brighton, by means of a wire going thither, passing through a galvanometer, and then returning, the force of the current shown by the galvanometer at Brighton will be almost exactly doubled, if, instead of the return wire, we establish a good communication between the end of the conducting wire and the mass of the earth at Brighton. The whole resistance of the return wire is at once dispensed with! This fact was more than suspected by the ingenious M. Steinheil in 1838; but, from some cause or other, it obtained little publicity; nor does the author appear to have exerted himself to remove the reasonable prejudices with which so singular a paradox was naturally received. A most ingenious artist, $\mathrm{Mr}$ Bain, established for himself the principle, and proclaimed its application somewhat later; and in 1843, perhaps the first entirely convincing experiments were made by M. Matteucci at Pisa. From this time the double wire required to move the needle telegraph was reduced to a single one. The explanation of this curious fact appears to be,-not that the electricity is conducted back by the earth to its origin at the battery, - but that the molecular disturbance polarly communicated along the conducting wire to its farther end being effectually relieved by perfect communication with a vast reservoir of neutral electricity like the earth, conduction proceeds in an uninterrupted manner, and to an unlimited extent.

Of submarine telegraphs, it is sufficient to state that the isolation is obtained by inserting the conducting wires in a mass of gutta percha, and that the first on a considerable scale was sunk between Dover and Cape Gris Nez, on the French coast, in August 1851.

The applications of electricity to the measurement of time are so numerous, that I can only refer generally to the principal contrivances.

1. The simple electric clock of Mr Bain derives its maintaining power from two large plates of copper and zinc (or more simply zinc and charcoal) sunk in the earth, which affords for a very long time a continuous supply of voltaic electricity. The current is conveyed into the bob of the pendulum, where it traverses a long coil of wire; and as the pendulum oscillates, the current (by a simple shifting contrivance) is reversed at each vibration. A stationary bar-magnet is placed so that when the pendulum moves, the voltaic coil of the bob embraces the magnet, and the direction of the current is such as by the electro-magnetic reaction to strengthen and maintain the vibratory movement, which is by this means perpetuated.

2. Sympathetic clocks.-By means similar to those (863.) just explained, one standard clock anyhow regulated Sympathemay, by means of magneto-electric currents, convey tic clocks. absolutely isochronous movements to any number of affiliated clocks at any distance. Probably the first application of the kind was made by M. Steinheil.

3. American electric-registration clocks. - Mr (864.) Locke proposed to register the instant of an event American occurring in the following way: A ribbon of registra. paper being put in uniform motion, as in Morse's tion clocks, telegraph, a dot is imprinted on it every second by

1 Occasionally 18 or 20 words per minute have been telegraphed.

2 I ought to mention that the practical introduction of the electric telegraph in England is in no small degree due to the energy of Mr Fothergill Cooke, joint patentee with Mr Wheatstone for the invention. The question of the respective shares of these gentlemen in the merit of telegraphic communication was submitted, in 1841, to the arbitration of Sir Marc Brunel and the late Mr Daniell, the result of which appears to leave the preponderance of merit in some respects ambiguous; nevertheless, in a history of Science, Mr Wheatstone is clearly entitled to the pre-eminent place. Several pamphlets have also been subsequently published by the parties. It is significant that Mr Cooke admits having borrowed his idea from becoming acquainted, at Heidelberg, in March 1836, with Gauss's experiments. 
(865:)

Chronoscopes.

(866.)

Electromagnetism mover.

(867.)

\section{§ 7. Cavendish-Coulomb-Experimental Laws of the Distribution of Statical Electricity;- Mathematical Theory of the same.-PoIsson-Mathematical Theory of Statical Electricity and of Magnetism generalized. Green; Professor William Thomson.}

(869.) Having thus brought down the history of galvanic Statical theory of Electricity

or voltaic electricity, and that of the wonderful discoveries connected with it, to our own time, I shall in this section briefly notice the more intermitting progress during the same period of our knowledge of the quantitative laws which regulate the distribution of statical electricity on bodies charged with it.

(870.)

" Epinus and Coulomb," says Dr Whewell, "1 "were two of the most eminent physical philosophers of Cavendish, the last century." They laid the foundations of an and Cou- exact science of statical electricity; and a third, and lomb. extreme care in its conception and execution. He
Perhaps the most elaborate of the memoirs not strictly chemical which Cavendish published were Caventhose on electricity. The Franklinian hypothesis of dish's a single fluid in excess or in defect of its average theory and state producing the phenomena then known as elec- ments. trical, offered a tempting field to an experimental philosopher well trained in the mathematical knowledge of the day; and his paper on this subject shows assumes, as a matter of necessity, the repulsion of matter for matter at sensible distances, considered apart from the electricity always combined with it in greater or less quantity. The indifference of matter under ordinary circumstances is held to arise from the union with it of a sufficient amount of electricity to neutralize the repulsion of the matter. In short, the electric fluid is considered as a second kind of matter repelling its own particles, and attracting those still more eminent name, deserves to be connected with theirs, - that of CAvENDISH, of whose general labours I have already given some account in-the Second section of the chapter on Heat. The labours of Exinus belong rather to the period embraced in the previous Dissertation, where they have been referred to by Sir John Leslie. 
caren-

dish's

electrical theory ar experi-
ments. of other matter with a force varying inversely as some less power of the distance than the cube. Commor matter repels its own particles and attracts electric particles according to the same law. The limitation as to a power below the inverse cube of the distance is necessary, since were the decrease of force more rapid, a particle would not be sensibly affected by the repulsion of any portion of the fluid except what was placed close to it. The hypothesis of Cavendish and his mode of reasoning from it were in general the same as those of Epinus; but Cavendish was not aware of the researches of the Swedish philosopher until his own memoir was completed. The number of facts accurately ascertained concerning electricity was at that time too small to admit of very precise numerical comparisons, but the ordinary cases of attraction, repulsion, and induction, were perspicuously explained by the theory; and had the inverse square of the distance been assumed (as it very safely might have been) to represent the law of diminishing repulsion, several of the theorems would have assumed a much more definite character, as was shown by Robison. Cavendish first demonstrated in his paper of 1771 that electricity must be confined close to the surface of a spherical body. This memoir also includes a correct theory of the Leyden phial, a just approximation to the law of attraction as the inverse square of the distance, a theory of conduction, and of the distribution of electricity on insulated conductors placed at a distance but connected by a fine wire or electric canal; and it was only the prelude to other researches never published, but of which some remarkable fragments exist amongst his manuscripts. Professor William Thomson, who has partly examined these, informs me that they contain the experimental solution of problems such as the following: "To compare the quantities of electricity on a spherical conductor, and a plane disk of equal diameter connected by a long conducting wire." Cavendish found that the sphere holds 1.57 times the electricity of the plate, a result exactly coincident with the deductions of theory.

I may here (for the sake of biographical connection) mention Cavendish's paper on the Torpedo, as a remarkable instance of the explanation of an obscure natural phenomenon, by the analogous effects of an artificial imitation. The experiments of Walsh on the Electrical Fishes have been cited in the preceding Dissertation. Cavendish undertook the bold task of proving, that all the external effects of the shock under varied circumstances, might be reproduced by a combination of Leyden jars duly protected. He made an artificial torpedo, consisting of 49 jars in a frame covered with leather, and he succeeded in obtaining shocks in air as in water, exactly comparable to those obtained from the live fish by Walsh. But perhaps the most striking part of the paper is the incidental mention of several laws of electricity then certainly new, and which he had deduced from experiment. Thus, he affirms the conducting power of iron for electricity to exceed 400,000 times that of distilled water, which he states to be equivalent to the fact, that a conductor of equal diameter will transmit as much electricity if the iron be 400,000 times longer than the water-a law conformable to that of $\mathrm{Ohm}$. He farther estimates the conducting power of sea-water at 100 times, but of saturated brine at 720 times that of pure water. Again, the quantity of electricity required to raise the charge on different jars or plates to the same intensity he finds to be directly as the area of the coating, and inversely as the thickness of the plate, and he applies this just conclusion with great ingenuity to explain the surprising power of the torpedo's shock by the extreme fineness of the membranes separating the columns of the electrical organs.

Coulomb (born 1736, died 1806 ${ }^{1}$ ) was a person of (873.) less genius and less mathematical attainment than Coulonsb's Cavendish, yet he had very considerable geometrical electrical ability and much facility in applying it to the results mperiof experiments, which he conducted with the greatest ingenuity and accuracy. In the latter respect he has seldom been surpassed. His methods, and even his numbers, are still, after a lapse of more than half a century, in many cases the best we can quote. Like Cavendish, he was devoted to quantitative estimations of phenomena.

His two greatest inventions were the balance of (874.) torsion and the proof plane. In the course of his The bastrictly mechanical researches (which, as we have torsion seen in the chapter on Mechanics, Art. 339, \&c., were and the numerous and important) he ascertained the laws of proof torsion. Within the limits of perfect elasticity, he plane. found that the force is as the angle of torsion of the wire or fibre, and inversely as its length. An almost indefinite minuteness may thus be attained in the measure of forces which may be balanced by the elastic torsion of a wire. We have seen (Astronomy, $\S 1$, Art. 156) how it was applied by Michell and Cavendish to measure the gravitation of bodies. Coulomb's invention dates at least from 1784. By means of it he established (in a different and perhaps more satisfactory way than had been done by Robison in 1769) that the electric and magnetic forces vary according to the Newtonian law; ${ }^{2}$ and with the aid of the "proof plane" he obtained exact measures of the electric tension on any part of an excited body. The "proof plane" consists of a small gilt disk with an insulat-

\footnotetext{
1 For a farther account of Coulomb, see the chapter on Mechanics, § 2.

2 Besides this law, Coulomb experimentally proved two others of great importance:- 1 . That the electricity of an e'ectrified conductor resides wholly on its surface. 2. That the interior of such a conductor is in a condition absolutely undisturbed by the presence of other external excited bodies.
} 
ing handle, which being applied fat-wise to the surface of an excited body takes off a portion of electricity, which is found in all cases to be proportioned to the electric excitement of the part which it had touched; being then presented to the torsion balance properly electrified, it shows by the repulsive effect produced, the relative tension of the part of the body whence the sample was obtained.

In this manner Coulomb determined the distribution of electricity upon electrified spheres at and after contact with one another;-on spheres inductively electrified, - on rods and plates and other figures; and his results, so far as they have been compared with theory, give evidence of the care and skill with which they were obtained, allowance being in all cases made for the loss of electricity by imperfect insulation. $\mathrm{He}$ also laboured with praiseworthy diligence to compare his results with the theory which he adopted of two fluids, each attracting the particles of the other and repelling their own according to the Newtonian law. The Epinian theory admits of only one fluid, but as it assumes a repulsion between the elementary particles matter it cannot be said to gain much in simplicity, whilst the mathematical results of either hypothesis are in general the same. M. Mosotti has endeavoured recently to revive the view of Franklin and of Epinus, so as to include, after the manner of Boscovich, the entire mechanical properties of matter.

The doctrine of attractions is a complex and difficult one even when the distribution of the attracting matter, as well as the fundamental law of attraction, is known. But it becomes much more so when the distribution of the attracting matter is itself the result of the very effect which it is the object of the problem to discover. If two homogeneous spheres attract one another, molecule to molecule, by the law of gravity, the problem is easy, provided the matter be rigid, and the distribution of it therefore unchanged; but if two such spheres be charged with the mobile electric fuid (using the term as a mere abbreviation), the case is very different, for now the electricity tends to shun the nearest points of each sphere, and to accumulate itself towards the remoter parts of their surfaces. The distribution of the electricity, and also the repulsive effect at any point, are both to be found simultaneously. The calculations of Coulomb were inadequate (as has been said) to such a solution; he contented himself with computing the effect of certain simple distributions which evidently lay on opposite sides of the truth, and comparing them with the result of experiment. Though any one such comparison might avail little, the cumulative evidence of many imperfect comparisons argued favourably for the truth of the hypothesis.

At the very time that Coulomb was pursuing this inquiry, Legendre first, and then Laplace, were inventing and improving those subtle and powerful
mathematical methods at which we have glanced in the chapter on Physical Astronomy, Art. 99, \&c., Laplace, for estimating attractions by a general method. Le- and Poisgendre's principles of calculation applied to cases of ${ }^{800}$. the symmetrical distribution of the attractive substances, but Laplace escaped this restriction. The problem is reduced to finding a quantity usually denoted by the letter $V$, called by some writers the potential, for the given body or surface, the expression Potential. for which may in each case be expanded into a series, the co-efficients of the terms of which (known as Laplace's co-efficients) are connected by certain relations which are evolved from the conditions of the problem. It was M. Biot first of all, but principally Poisson, who applied this method to electrical, and subsequently to magnetical phenomena. Poisson, with great labour, succeeded in representing correctly by analysis the conditions of some of Coulomb's experiments on spheres and ellipsoids. But it must be owned that the complication of the analysis, the difficulty of applying it to any but the very simplest cases, and the considerable latitude of the errors of experiment, rendered the results rather analytical exercises than solid bases for physical induction; which may in some degree account for the manner in which Sir John Leslie mentions them in his Dissertation. Poisson (as I have elsewhere remarked) had not the talent of conducting his mathematics in a fertile direction, and usually left the fields of experimental physics on which he touched nearly as barren as he found them. But this is no reason why other mathematical reasoners may not obtain more pregnant results. We shall see in the next section that Gauss, a distinguished contemporary of Poisson, by treating the great problem of the distribution of the magnetism of the globbe (in many respects similar to those of the theory of electricity) with the utmost mathematical generality, has obtained results of great novelty and importance; that he has not only shown experimenters how to proceed, but has invented instruments for them to use. A similar step has not yet been taken in electricity. Notwithstanding the unquestionable beauty of Sir William Harris's methods of measuring electrical attractions (Phil. Trans. 1834), they are little adapted for comparison with theory, and Coulomb's experiments still remain the standard ones on the subject.

The theory of Coulomb has, however, been ably generalized by Green, a nearly self-taught mathemati- Writings cian of great originality, who died at a premature age. of $G$ reen In a memoir on electricity privately printed about Professor 1830 he generalized Poisson's methods and ap-W.Thomplied them to a number of new cases. His paper ${ }^{80 n}$. was reprinted a few years since in Crelle's Journal. To him I believe is due the term potential. Several continental mathematicians of eminence have added some steps to the theory of electricity, but probably the most important from its fertility and simplicity is a theorem discovered by Professor William Thom- 
Electrical son of Glasgow, for reducing the electrical effects of images.

(879.) Application of the theory of magnetism to correct ships' compasses. MM. Barlow and Airy. electrified spheres upon points or other spheres without them to those of electrified points in certain positions replacing the spheres, which points, from certain analogies to well known optical formulæ, Professor Thomson has designated electrical images. ${ }^{1}$

The mathematical theory of magnetism has received a highly practical application in the correction of the deviation of compass due to the local attraction of ships. Professor Barlow of Woolwich led the way in attempting practically to correct the errors thence arising. But when ships began to be constructed almost entirely of iron, the use of his "correcting plate" was found to be totally insufficient. To the Rev. Dr Scoresby, practical navigation is indebted for many ingenious observations on the magnetism of ships, and suggestions as to the means
(881.) Professor Hansteen -Magne. tismus de Erde.

Variation or decliation.

(880.)

Ristory of errestria nagnet ism.

I could hardly have intentionally selected a more characteristic example of the scientific progress of the nineteenth century than the recent history of terrestrial magnetism, even had it not accidentally formed the closing section of this Dissertation. The combination of extended methodical research in ob. taining physical data, with mathematical skill in comparing them and in deducing from them the most important results, has been attended with merited success. I regret that the unforeseen extent of this historical sketch compels me to touch with great brevity on the leading points of this research.

Professor Christopher Haisteen, of Christiania, in Norway, is the person who has given probably the greatest impulse in recent times to the efforts to methodize the facts and laws of the earth's magnetism. M. Hansteen was born 26th September 1784, and is Professor of Astronomy in the University of Christiania, and Director of the Observatory. His dissertation, entitled Magnetismus der Erde, published in 1819, which received a prize from the Royal Danish Academy, recapitulated all the authentic facts obtained by voyagers and others from the earliest times. It will be recollected ${ }^{2}$ that Halley had represented the magnetic variation at different parts of the globe by lines traced on Mercator's chart, and passing through all places where the variation (or declination) of the needle from the true north was equal ; and being well aware of the progressive (or secular) changes in the course of these lines, he of allowing for it; while Mr Airy and Mr Archibald Smith have the merit of applying the Theory of Magnetism to the case in question. Mr Airy's investigations may be found in the Philosophical Transactions for 1839 and 1855; from which it appears that the effects of local magnetism in a vessel may be speedily and effectually corrected for a given place by the use of two permanent magnets placed in rectangular positions relatively to the compass; and by the use of a mass of soft iron in a third direction. But grave doubts still remain as to the possibility of rendering such corrections permanent, and applicable in all magnetic latitudes. A great step has, however, been gained by putting the power of readily verifying the compass corrections in any part of the world in the hands of every intelligent captain.

\section{§ 8. Professor Hansteen-Baron A. von Humboldt-Gauss-Major-General Sabine-Captain Sir J. C. Ross.-Progress of our Knowledge of Terrestrial Magnetism in the present Century.}

proposed the hypothesis of two pairs of magnetic poles interior to the globe, of which one pair revolves slowly.

This hypothesis, little thought of at the time, (882.) and perhaps of little value except as a help towards IIalley's the formal representation of the facts, appears to hypothesis. have been revived by Wilcke, a Swede, whose la- charts. bours attracted the attention of Professor Hansteen first in 1807. M. Hansteen found the results of his own collections to coincide well with Halley's chart for 1700 , and also with the hypothesis of four poles, two in each hemisphere, one stronger than the other. It results from these charts that the Line of $N_{0} V a$ - Line of No riation, which, in 1600 , formed a remarkable arch-Variation. like curve, stretching from the Gulf of Mexico to near the North Cape of Norway, then descending through Central Europe to the Gulf of Guinea, had, during the seventeenth and eighteenth centuries, become gradually flattened (having passed through Paris in 1669 , and through London twelve years earlier), and at present this part of the line of No Variation is confined to the American continent and neighbouring seas. Another and more complicated branch of the same line traverses the Pacific Ocean, making a complex serpentine track through Eastern Asia and Siberia. The line of No Variation may be expected to pass through those points of the earth's surface towards which the needle converges, which are sometimes called the magnetic poles (of which more presently), and of which M. Hansteen concludes the position to be as follows: ${ }^{3}-$

\footnotetext{
1 Cambridge Mathematical Journal, 1850.

2 See Fifth Dissertation, p. 741.

3 From M. Hansteen's recent paper on the Secular Change of the Dip (Copenhagen, 1855). In this ingenious memoir it is inferred, with considerable plausibility, that the annual diminution of the dip is decreasing, and consequently that a minimum ot dip will occur in Europe before the close of this century.
} 
Pcsition of magnetic zentres or poles.

(883.)
Dist. from
Pole of Earth.
Greeng. E. from

$\begin{array}{ll}25^{\circ} 22^{\prime} & 280^{\circ} 19^{\prime}\end{array}$

, or strongest northern pole (N. America), for 1838

$n$, or weakest northern pole (Siberia), for 1829

$25^{\circ} 22^{\prime}$

S, or strongest southern pole (S. from Australia), for $1845 \ldots \ldots \ldots$ 21

s, or weakest southern pole (S. from Tierra del Fuego), for 1842. 1353

$114 \quad 33$

$131 \quad 28$

21626

* $t$ denotes the current year. This result appears very uncertain. It gives about $+6^{\prime} \cdot 5$ of annual change at the present time. The latitudes of these " points of convergence" are also varying.

Variation maps of a different kind have been constructed by the French Admiral Duperrey, in which the actual direction of the needle is represented by arrows. The above positions of the magnetic poles were deduced by Mr Hansteen from the apparent convergency of the magnetic needle towards those four points. The earlier observations appear to be more precise than might have been expected.

Professor Hanstcen also constructed charts of the lines of equal dip. In certain positions between the tropics the dip is nothing, or the freely suspended magnetic needle remains horizontal. The line connecting these places is called the magnetic equator. It is an undulating line inclined somewhere near $12^{\circ}$ or $13^{\circ}$ to the terrestrial equator, and cutting it in two points not exactly opposite, but in about $3^{\circ} 20^{\prime}$ and $174^{\circ} 30^{\prime}$ of east longitude from Paris, according to Admiral Duperrey's observations in 1825. The position of these nodes is, however, variable. The north end of the needle (as is well known) dips more and more in the northern hemisphere, until in a certain place it becomes vertical, where therefore the horizontal component of the magnetic force is nothing, and the common compass loses altogether its directive power. Similar phenomena occur in the southern hemisphere. Lines of dip of $10^{\circ}, 20^{\circ}$, \&c., may be drawn, and where the dip is $90^{\circ}$ there is a true magnetic pole. The best observations seem to show that there is but one such true pole in each hemisphere.

If we define as " pole" a spot where the needle points vertically (which is the signification adopted by Gauss and others), these points do not coincide with those of greatest intensity. On the 1st June 1831, Commander Ross (now Sir James Clark Ross) attained the true magnetic pole in the North American continent in lat. $70^{\circ} 5^{\prime} 17^{\prime \prime} \mathrm{N}$., and long. $96^{\circ} 45^{\prime} 48^{\prime \prime} \mathrm{W}$. The dip of the needle was sensibly $90^{\circ}$.

A third element not less important than Variation and Dip, though more lately brought into notice, is the Intensity of the earth's magnetism, and to this Professor Hansteen directed special attention. That the intensity of the earth's directive force may be measured by counting the oscillations of a suspended magnet in the same way that a pendulum measures gravity was known to Graham, Lambert, and others, towards the middle of the last century ; but the exploration of its variation on the earth's surface was first attempted by the officers of Laperouse in 1785, and later by De Rossel. But it was Baron Humboldt who, at the instigation of Borda, I undertook the earliest observations which have had any permanent influence on this branch of science, and who in the first years of this century determined the rela- Baron tive intensity of the earth's magnetism at Paris and Humat the magnetic equator in South America, to be in boldt's the ratio of 1.3482 to $1.0000 ;^{2}$ a result which has ${ }^{\text {unit. }}$ become, sn to speak, classical, and which the author considered as the most important result of his journey. ${ }^{3} \quad M$. Hansteen promoted the same enquiry extensively; he devised a neat and convenient apparatus for counting the oscillations of the needles, and he investigated the effects of time and temperature in altering their magnetism. ${ }^{4}$ He made numerous observations in the north of Europe, and finally undertook, between 1828 and 1830 (by the liberality of the Norwegian parliament or Storthing), an adventurous journey into Siberia, for the purpose of examining the "region of convergence" of the needle in that quarter. His account of his journey has most M. Erman. unfortunately not been published; but his companion, Professor Adolphe Erman, has given the main results, together with extensive observations entirely his own. ${ }^{5}$ This was the first magnetical expedition of any magnitude, and of a national character. It yields in importance to none which have succeeded it.

The magnetic intensity then increases from the neighbourhood of the equator torvards the Arctic Distrib Regions, and there it has two foci of greatest inten-tion of sity. A similar arrangement occurs in the southern ${ }^{\text {intensity. }}$ hemisphere, and these four points of maximum attraction or convergence of the needle constitute the "poles" of Halley and of M. Hansteen. To M. Hansteen we are indebted for the first good approximation to a general chart of the lines of equal intensity.

The supposed diminution of the magnetic intensity as we recede from the earth's surface has been Diminurepeatedly made the subject of experiment. The tion of insulated experiments of Gay-Lussac in a balloon intensity (630), and of M. Kupffer at.Mont Elbroutz, led to with no conclusive result. From a numerous series of height. 
careful observations made in the Alps and Pyrenees with Hansteen's apparatus, and studiously corrected for temperature, the present writer has found a diminution of one-thousandth part in the horizontal intensity for a vertical ascent of about 3000 feet. $^{1}$

(888.) Such are the general features of the distribution of Variations the magnetic force upon the surface of the globe. But of mag- from an early period the magnetic elcments for the netic ele- same place have been known to be variable. Such
ments. deviations from constancy are either-1. Secular ; 2 . Periodic; 3. Irregular. All the three magnetic elements (Variation, Dip, and Intensity) probably par-1 . Secu- take of these changes. The westerly Variation or Delar. clination had till 1818 been increasing in Europe since the earliest observations. This results from a complicated movement of the line of No Variation and its companion curves. While their common centre or pole in North America has been slowly advancing towards the East, a considerable portion of the system of curves in this quarter of the globe has been proceeding in a south-westerly direction nearly in a line joining Spain with South America, thus producing a complicated rotatory motion of the lines of $\mathrm{Va}$ riation, of which the first effect was to extinguish the singular loop in the curve of No Declination (including a space of easterly declination) which is shown in M. Hansteen's chart for 1600 to have occupied a large part of Western Europe. ${ }^{2}$ About 1818 the needle began to retrograde towards the east in this part of Europe. The dip has been diminishing in Europe since the earliest observations.

(889.) Graham discovered the Diurnal Variation of the nal. southern hemisphere. Near the equator (as at St Helena) the needle partakes at one season of the northern character, at another of the southern, depending on the position of the sun. (890.) To Baron. Humboldt and to Arago we are prin-
3. Irre- cipally indebted for a knowledge of great and caprigular. cious fluctuations of the magnetic elements occurring simultaneously over vast regions of the earth. These have been called magnetic storms. ${ }^{3}$ They are often connected with auroral appearances.

(891.) Next to Professor Hansteen, science is mainly indebted for the recent great extension of our knowledge of the facts and laws of terrestrial magnetism to two illustrious German philosophers, Baron Alexander von Humboldt and the late Professor Gauss.

The name of Alexander von Humboldt is as widely known as science is cultivated. The ardour Baron of his love of nature, the comprehensive interest which Alexander he takes in every department of knowledge, and the von Hu generosity of his disposition, in combination with the fortunate accidents of an unusually vigorous constitution, and an eminent social position, have combined to place him in the foremost rank of natural philosophers.

He was born on the 14th September 1769, and (893.) consequently is now (1856) in his 87 th year. He His early was a pupil of Werner in 1791, and devoted consi- history an derable attention to metallurgy. An early longing for foreign travel seems to have foreshadowed his future career, but political circumstances were, towards the close of the last century, eminently unfarourable to its accomplishment. His celebrated journey to Southern and Central America (undertaken after the failure of several other schemes) lasted from June 1799 until August 1804. We may, perhaps, be allowed to regret that an impression of the duty of presenting to the public the results of that interesting journey in their most complete form should have absorbed the leisure of so many of his most vigorous years, and should havo withheld him from other and still more important enterprizes. From 1808 to 1826 Baron Humboldt resided mainly in Paris, in the most intimate companionship with Arago, who, though much his junior, has predeceased him. He then took up his residence at Berlin, in compliance with the wish of the King of Prussia, and he soon after delivered there a course of public lectures on Physical Geography which formed the basis of his remarkable work entitled Kosmos, the composition of which has formed the chief occupation of his vigorous old age. In 1829 he made a rapid but interesting journey into Asiatic Russia, important in its results, yet imperfectly carrying out the ardent aspiration of his early years to unfold the marvellous physical peculiarities of the Eastern continent.

Baron Humboldt has contributed more to Physical (894.) Geography than any other man now living; and that, ${ }_{\text {in }}$ Physical not only by his individual efforts, but by the direction Geography and encouragement which he has given to innumerable travellers and naturalists. His career seems to have been more closely modelled upon that of De Saussure than of any other of his contemporaries or predecessors. Those branches of Physical Geography which admit of numerical treatment seem most congenial to him; and he has left more of the impress of his personal influence upon the sciences of Meteor-

\footnotetext{
1 Edinburgh Transactions, vol. xiii.

2 M. Arago gives, no doubt, an erroneous impression in stating that the westerly movement of the line of No Declination has carried it in 200 years from Paris to Philadelphia. Yet I cannot subscribe to the opinion of his able commentator that the Loop of No Declination which passed over Europe in the seventeenth century moved eastwards, and may still be traced in Asia -(British Association, Fifth Report, p. 63; Arago's Meteorological Essays, translated, p. 330). I conceive that, as stated in the text, the loop moved south-westwards, became successively an oval and a singular point, and was finally worked out previously to 1700 .

3 In September 1841 a remarkable simultaneous disturbance took place in Canada, Scotland, the Cape of Good Hope, and New South Wales. Such occurrences are now known to be very common.
} 
ology and Magnetism than upon any others which he cultivated. His conception of Isothermal Lines and his treatment of the subject of Climatology in his remarkable paper of $1817^{1}$ gave a new impulse to the former subject. His magnetical observations in his voyage to the Equator have already been adverted to. Besides this we are indebted to Baron Humboldt for directing attention to the simultaneous and seemingly accidental disturbances of the magnetic needle which take place over vast portions of the area of the globe. He commenced observations on this subject in conjunction with M. Oltmanns as early as 1806; and duxing his residence in Paris he no doubt encouraged his friend Arago in those elaborate observations on the hourly variations of the needle which, to the irreparable loss of science, remained unpublished whilst they might have been most useful. ${ }^{2}$ In 1829 simultaneous observations were made in Germany and Russia, and compared by MM. de Humboldt, Dove, and others, with interesting results. ${ }^{3}$ It is probable that the results then obtained instigated Gauss to the enlarged enquiry to which I shall immediately refer. M. de Humboldt had already addressed the Russian Government on the same subject, suggesting the registration of hourly observations in their vast territories; and these, the first systematic observations of the kind, have been continued ever since. The suggestion of "Term Days" of continuous registration of the Declination Needle was also due to Baron Humboldt. In 1836 he addressed the President of the Royal Society of London on the same subject, and his letter formed the basis of the extensive undertakings which have formed the contribution of the British Government to this great enquiry.

(895.) Baron Humboldt still lives at Berlin, enjoying the respect of all who know him, and the distinguished favour of his Sovereign.

(896.) Carl Friedrich Gauss, late Professor of Astronomy at Göttingen, belonged to that group of celebrated geometers who illustrated the commencement of this century, and of which he was for some years the last survivor; but he too has now passed away.

(897.) Gauss was born at Brunswick on the 30th April His early 1777, of humble parents, and was indebted for a hathema- liberal education to the notice which his talents protical works. cured him from the reigning duke. His earliest original researches were in the theory of numbers. His Disquisitiones Arithmetica were published in 1801 , and were as profound and original as they have always been considered obscure even by those devoted to such studies. Gauss demonstrated the possibility of geometrically inscribing a regular poly- gon of 17 sides within a circle; the only extension of geometry in this direction since the time of Euclid. His Theoria Motûs Corporum Calestium, published in 1809 , is a very remarkable treatise on the geometrical theory of the planetary orbits; in which it was shown for the first time how the elliptic elements of a heavenly body may be deduced from three observations only of longitude and latitude,an important extension of Newton's celebrated demonstration in the case of parabolic cometary orbits. In the same work the method of least squares (85) was fully unfolded in its applications to astronomy.

These and many other important labours all connected with the higher mathematics, had obtained for Gauss an exalted place among the men of science of his time, long before he commenced those re- netism. searches on Terrestrial Magnetism, in virtue of which his name is introduced into the present scction. It can hardly be doubted, however, that by these-scarcely begun before he had entered on his 55 th year-he will be hereafter chiefly remembered. His first work on the theory of magnetism ${ }^{4}$ was published in 1833, and excited very general notice, as it contained a remarkable application of the Theory of Attractions to the distribution of magnetism in a steel bar, a singularly ingenious and rigorous proof of the primary law of the magnetic force, and likewise a new and practical application of a suggestion Magnetic thrown out by Poisson, of a method by which the force in thrown out by Poisson, of a method by which the absolute magnetic directive force of the earth itself may be measure. expressed in absolute measure, irrespectively of the constancy of the magnetism in the bar which is in the first instance used to estimate it. For this purpose two kinds of observation are required,-1st, By vibrating a needle or bar of known weight and dimensions, and observing the time of its oscillation, the force pulling it into the meridian is ascertained in terms of the ordinary dynamical units. The time depends jointly on the magnetic force of the earth, and on that of the bar, or it varies inversely as the product of the two. 2dly, Another bar, B, is suspended like the first, $A$, in the magnetic meridian. $A$ is then brought to act upon $B$, so as to draw it permanently out of the meridian. The position in which $B$ rests determines the ratio of the magnetic force of the earth and of the bar A. But quantities whose product and whose ratio are given in known measures, are. also known; whence the separate intensities of the earth's and the bar's magnetism may be eliminated. ${ }^{5}$ Having thus fairly entered on a career of magnetic experiments, Gauss proceeded, in conjunction experiments, Gauss proceeded, in conjunction New magwith an experienced physicist of Göttingen, Professor ${ }_{\text {struments. }}$ Wilheln Weber (420), to invent new apparatus for

1 Memoires d'Arceuil, tom. iii., p. 462.

2 Posthumously published in bis CEures.

3 See abstract in Bibliotheque Universelle, Août 1832.

- Intensitas vis magnetica terrestris ad mensuram absolutam revocata. Gott. 1833.

6 The unit of force to which these intensities are referred is that force (in grains) which acting on unit of mass through unit of time (a second) generates in it unit of velocity (a foot). 
observing the earth's magnetism and its changes. The instruments devised by them were a Declination Instrument and a Bifilar Magnetometer. The first is a heary magnetized bar some feet in length, suspended by a bundle of parallel silk fibres. It is suffered to place itself in the magnetic meridian, and the small displacements due to hourly, annual, or irregular fluctuation, are ascertained by viewing with a telescope the reflection of a fixed scale of equal parts placed at some distance in a small mirror which is attached to the magnetic bar. The bifilar instrument is for ascertaining changes of intensity in the earth's magnetism. It is a bar like the last, suspended by two parallel threads or wires. By twisting round the points of double suspension, the bar is forced by torsion into a position at right angles to the magnetic meridian, where it is held in equilibrium by the force of the earth's magnetism, and that of the torsion of the wires. Supposing the latter force to be constant, if the former vary, the bar will change its position, which is observed by the mirror and telescope as before.

To these instruments, Professor Lloyd, of Dublin, added another for measuring the vertical component of the magnetic intensity. It is a magnet suspended horizontally on knife-edges like those of a balance. Its deflection from the horizontal line indicates variations in the vertical portion of the earth's magnetism.

The dip is determined in the usual way; the variations of dip, however, are ascertained by comparing the variations of horizontal and vertical intensity.

Gauss did not content himself with suggesting new forms of apparatus, and recommending them to others. With the active assistance of $M$. Weber, he crected, in 1833, at Göttingen, a magnetic observatory free from iron (as M. de Humboldt and Arago had already done on a smaller scale), where he watched with patience the incessant movements of the newly-constructed needles or bars. It was from the same observatory that he sent telegraphic signals to the neighbouring town, thus showing the practicability of an electro-magnetic telegraph (856). He farther instituted an association (magnetischer Verein), composed at first almost entirely of Germans, whose continuous observations on fixed Termdays extended from Holland to Sicily. 1 The marvellous coincidence of the occurrence of even the minute irregularities of the earth's magnetism was thus more fully established.

The Mathematical Theory of Terrestrial Magnetism of Gauss ${ }^{2}$ is intended to replace all arbitrary assump-

tions whatever as to the distribution of magnetism Gauss's in or over the earth. It proposes to express by an mathemaempirical law, involving as little of hypothesis as of terrespossible, the direction of the frecly suspended mag- trial magnetic needle and its directive force at any point of the netisn. earth's surface, the data being of course derived from a limited number of observations.

The theoretical assumption with which Gauss starts is merely this, - that the elementary force of magnetism varies inversely as the square of the distance, and that it is distributed over the matter of the terrestrial globe in a way or according to a law presumed to be entirely unknown.

Following up the methods employed by Laplace and others, for representing the attractions of a sphere or spheroid, he proposes to discover the form of that remarkable function (sometimes called the Potential) ${ }^{3}$ whose differential coefficients express the resolved components of the total magnetic force. This quantity $\mathrm{V}$, and also its differential coefficients (representing the attractions in given directions), may always be expressed by a series with indeterminate coefficients, which is known to converge more or less rapidly; and since the component forces or attractions are given by observation, the coefficients of the terms of the series representing them may be deduced from a comparison with the data. The convergency is greatest if the magnetic matter be disposed towards the centre of the sphere, least if it reside near its surface.

Retaining quantities of the fourth order, there are twenty-four constants, which, rigorously speaking, may be deduced from complete observations of the three magnetic elements (888) at only eight stations anywhere situated on the surface of the globe. With great patience and skill, Gauss collected as many available data as possible for determining these constants with accuracy, and he thence deduced by calculation the values of $V$, those of the declination, horizontal and vertical force, and that of the dip for all points of the globe. The beautiful charts which he caused to be constructed show a remarkable general accordance with the complex facts of magnetism as then known. But in many instances more accurate data have since been obtained applicable to the improvement of the empirical theory. Perhaps the most surprising fact which Gauss considers to be demonstrated is this, that the average amount of magnetic force associated with every cubic yard of the earth's volume (supposing the distribution uniform) is equal to that contained in six saturated steel bars each a pound in weight.

1 The volumes of their publications, from 1836 to 1839 , contain much interesting information on the history of this subject.

2 Allgemeine Theorie des Erdmagnetismus. Leipzig, 1839.

3 See Art. 877. This function $V$ represents the integral $\int \frac{d m}{\rho}$, were $d m$ is the attracting or repelling element, and $\rho$ its distance from the point acted on. Gauss represents in his charts the values of the function V at different points of the earth's surface. The lines of equal values of $\mathrm{V}$ are everywhere perpendicular to the direction of the needle, and the horizontal intensity is inversely as the distance between two adjacent lines. 
(907.) On the whole, it is no small praise to say that the Great merit of Gauss.

adopted all over the world, and his name honoured tingen (where he had resided since 1807), in great tranquillity, on the $23 \mathrm{~d}$ February 1855 , in the 78 th year of his age.

(908.) But to obtain the data of the einpirical theory with Import- exactness, it is evident that expeditions fitted out exance of national expeditions.

(909.) To Major-General Edward Sabine, of the Royal General Artillery, is mainly due the judicious management of Sabine.

(910.) But his efforts in promoting and directing the British system of national magnetical research set on foot at magnetical Baron Humboldt's instigation are of still greater expedi- importance, to which, in its earlier stages, the exerpressly with accurate apparatus, and directed at once to unany points of the habitable globe, are indispensable. Powerful governments can alone effect this and to Baron Humboldt is due (as has been shown) the praise of having vigorously pressed the importance of these upon the governments of England and of Russia. The vast marine resources of the former, and the peculiar magnetic interest of the stupendous Asiatic territory of the latter, rendered their cooperation highly important. In the result, Great Britain has accomplished by far the larger share of this vast enterprise.

these magnetic explorations, and the speedy and skilfu publication of their interesting results. During the course of an active life he has enjoyed opportunities of making extensive observations with magnetic apparatus and with the pendulum (238) throughout a great range of latitude. His experiments (carried on principally between the years 1819 and 1826) were made in Brazil, the coast of Guinea, Spitzbergen, and Arctic America. His observations on magnetic intensity are particularly valuable, and first indicated the position of a region of maximum intensity in North America considerably to the south of the magnetic pole as indicated by the dipping needle. To him we also owe a valuable Intensity Chart of the Globe, and a Magnetic Survey of the British Islands, published in the reports of the British Association for 1836 and the two following years. tions of Dr Lloyd (550) were also of essential service. Although seconded by young, able, and enthusiastic officers of the service to which General Sabine be- longs, the difficulty of starting simultaneously systematic observations (with instruments new and little understood, and dispersed to the widest possible extent over the surface of the globe), was evidently very great. Canada, St Helena, the Cape of Good Hope, and Van Diemen's Land, were selected for primary stations; and the vastly voluminous results of hourly or two-hourly observations of numerous instruments continued year by year came pouring in to the central establishment at Woolwich under General Sabine's presidency. Besides these, several nautical expeditions were fitted out for similar purposes, the most important of which was sent in 1840 under the direction of Sir James Clark Ross to approach as nearly Captain as might be to the Antarctic Pole. This voyage, whe- Sir J. C. ther in respect of the spirit of adventure displayed, the wonderful character of the natural objects revealed by it for the first time to human view, or the importance of the scientific results obtained, may rank with any of our memorable polar expeditions. By the aid of an ingenious instrument contrived by Mr Fox, trustworthy magnetical observations were made on dip and intensity (as well as on declination) even in the open sea, and thus the area contributing to our knowledge of the earth's magnetism was vastly increased. The results of these researches properly reduced have been gradually laid before the world by General Sabine in a series of memoirs of the utmost interest, contained in the Philosophical Transactions from 1840 to the present time.

With regard to the geographical phenomena of magnetism, it may now be inferred almost with cer-Geogratainty that the position of the Antarctic magnetic pole phical is in a region hitherto inaccessible, and to the south curves of of New Holland, placed somewhere near longitude intensity. $150^{\circ} \mathrm{E}$., and probably not within $17^{\circ}$ of the pole of the earth, a latitude which was reached by Sir James Ross in a longitude somewhat farther east. ${ }^{1}$ It appears also that in the southern as in the northern hemisphere there are two centres of greatest intensity, round which the curves of equal intensity form ovals, and afterwards loops like lemniscates. The stronger magnetic centres of each hemisphere are near, though apparently not coincident with, the two proper magnetic poles. The following numbers approximately represent on a scale of absolute intensity (898), in which the grain, the foot, and the second are units, the magnetic force at the four centres just mentioned; the second column contains the corresponding values on Baron Humboldt's scale, mentioned in Art. 885.

\begin{tabular}{|c|c|c|}
\hline & Absolute. & On Hnmboldt's Scale. \\
\hline North America, & - $14 \cdot 2$ & 1.88 \\
\hline - . & . $13 \cdot 3$ & $1 \cdot 76$ \\
\hline w Holland, & $15 \cdot 14$ & $2 \cdot 00$ \\
\hline ther Antarctic centre, & $14 \cdot 9$ & $1 \cdot 97$ \\
\hline
\end{tabular}

The absolute intensity at St Helena, which is not far 
from the minimum, is $6 \cdot 4$. These observations also fully confirm the progressive motion of the whole system of magnetic lines on the surface of the globe.

(912.) In Europe national, and even private, observaEuropean tories have contributed largely to our knowledge of magnetical the laws of magnetism. Among these observatories
observatories. currents may, in part at least, be of a thermo-electric character, or the effects may possibly depend upon the direct demagnetising influence of temperature, as Dr Faraday supposes (830). The secular magnetic changes will probably ever remain the most unaccountable. The greater portion of those which are obviously periodic seem to depend upon the position and efficiency of the sun in its apparent diurnal and annual course, having their crises at certain hours of the day, and certain seasons of the year. It is understood that the most recent researches leave little doubt as to a like influence arising from the moon's position. ${ }^{1}$ It appears also to be indicated (although the induction is perhaps yet incomplete) that the diurnal changes, and also those of a capricious and irregular character (magnetic storms), have a period of greatest and least intensity of fluctuation extending to about ten years, a minimum having occurred in 1843 , and a maximum in 1848 . This nearly coincides with a period of greatest and least abundance of the solar spots detected by M. Schwabe, and it is possible that there is a real connection between the phenomena. The first recognition of a ten-year magnetic period appears to be due to M. Irimont, that of a probable concurrence with the frequency of solar spots to General Sabine.

1 While correcting the press of this page, I have received an interesting paper by General Sabine (Phil. Trans., 1856), in which the dependence of the various magnetic elements on the moon's diurnal place is deduced from a very large number of observations. The change of declination in the course of the lunar day amounts to above $38^{\prime \prime}$.

Addition to Art. 283, page 860.

The following is a continuation of the list previously given of the small Planets :-

$\begin{array}{ll}\text { No. } & \text { Name. } \\ 36 & \text { Atalanta } \\ 37 & \text { Fides } \\ 38 & \text { Leda } \\ 39 & \text { Lætitia } \\ 40 & \text { Harmonia } \\ 41 & \text { Daphne } \\ 42 & \text { Isis }\end{array}$

42 Isis

Date of Discovery.
1855 Oct. 5
Oct. 5
1856 Jan. 12
Feb. 8
March 31
May 22
May 28

overers.

Goldschmidt.

Luther.

Chacornac.

Goldschmidt.

Goldschmidt.

Pogson. 


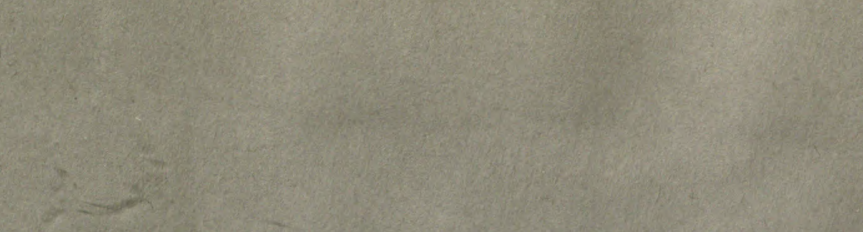

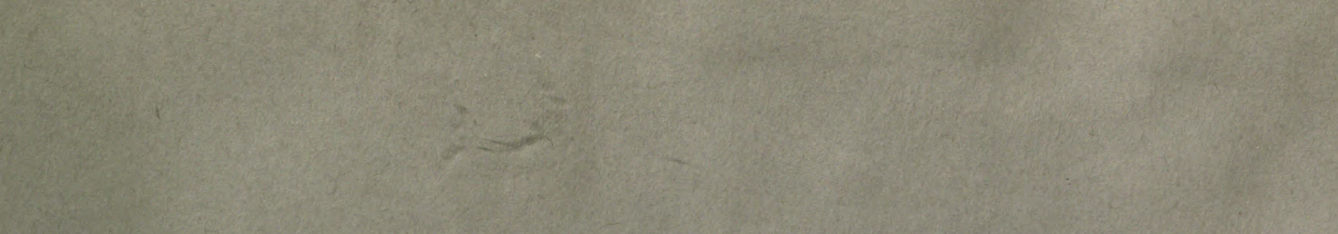

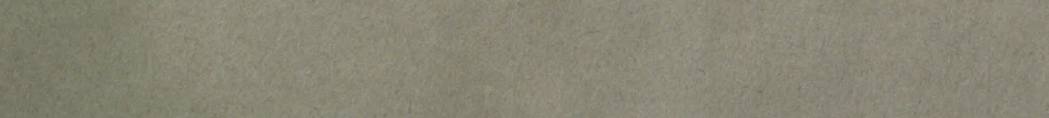

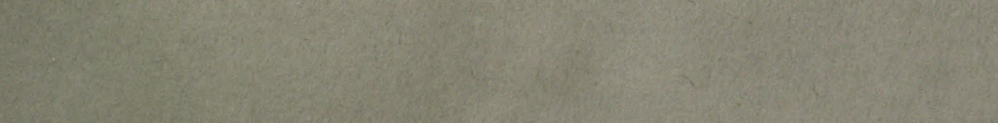

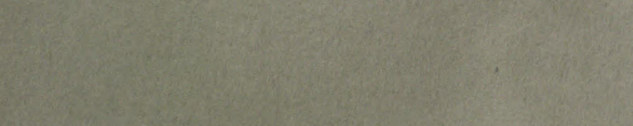

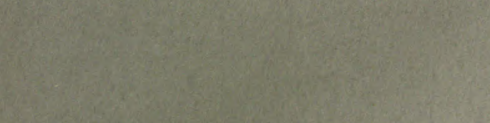

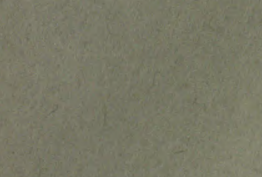

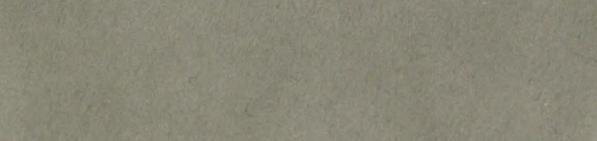

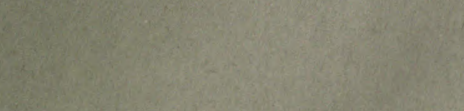

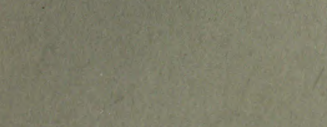

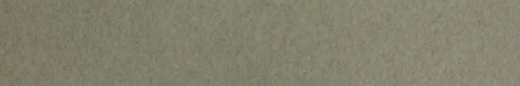




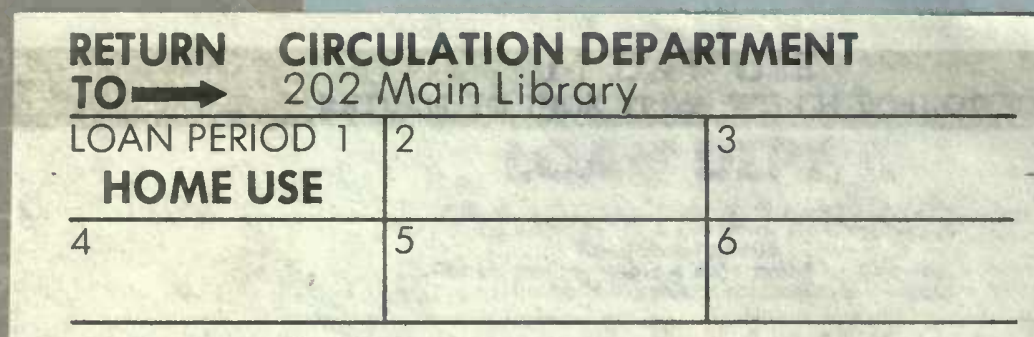

ALL BOOKS MAY BE RECALLED AFTER 7 DAYS

1 -month loans may be renewed by calling 642.3405

6-month loans may be recharged by bringing books to Circulation Desk

Renewals and recharges may be made 4 days prior to due date

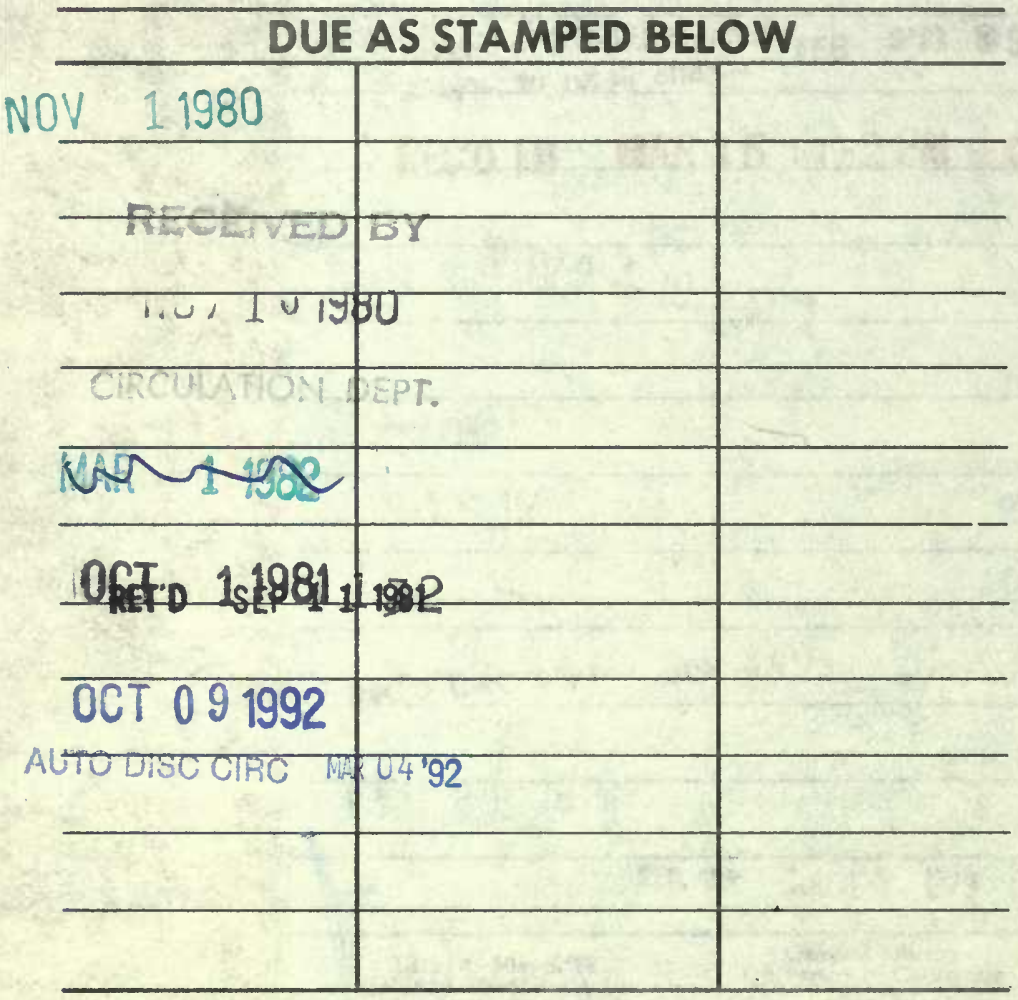

UNIVERSITY OF CALIFORNIA, BERKELEY FORM NO. DD6, $60 \mathrm{~m}, 3 / 80$ BERKELEY, CA 94720 


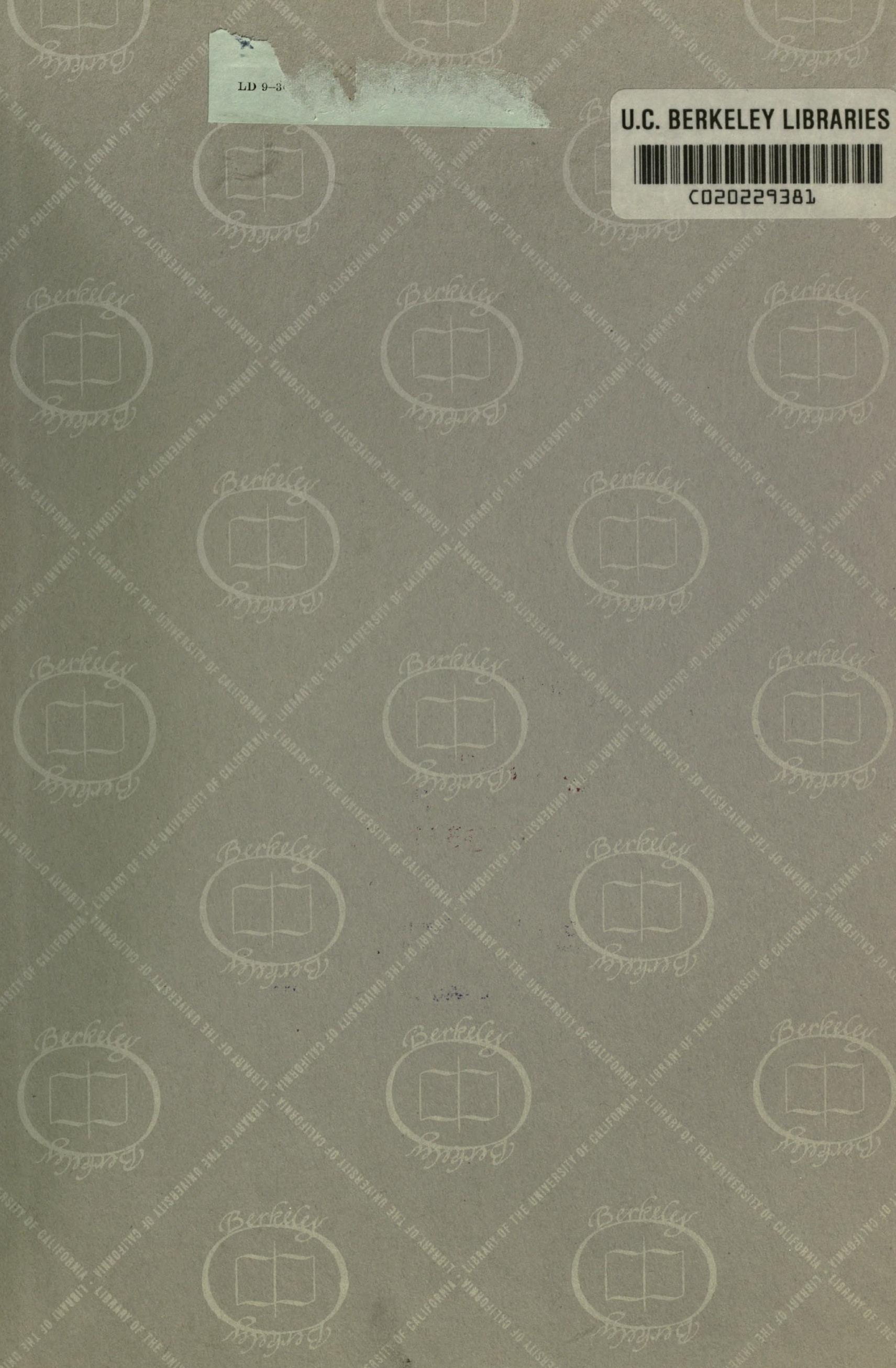


